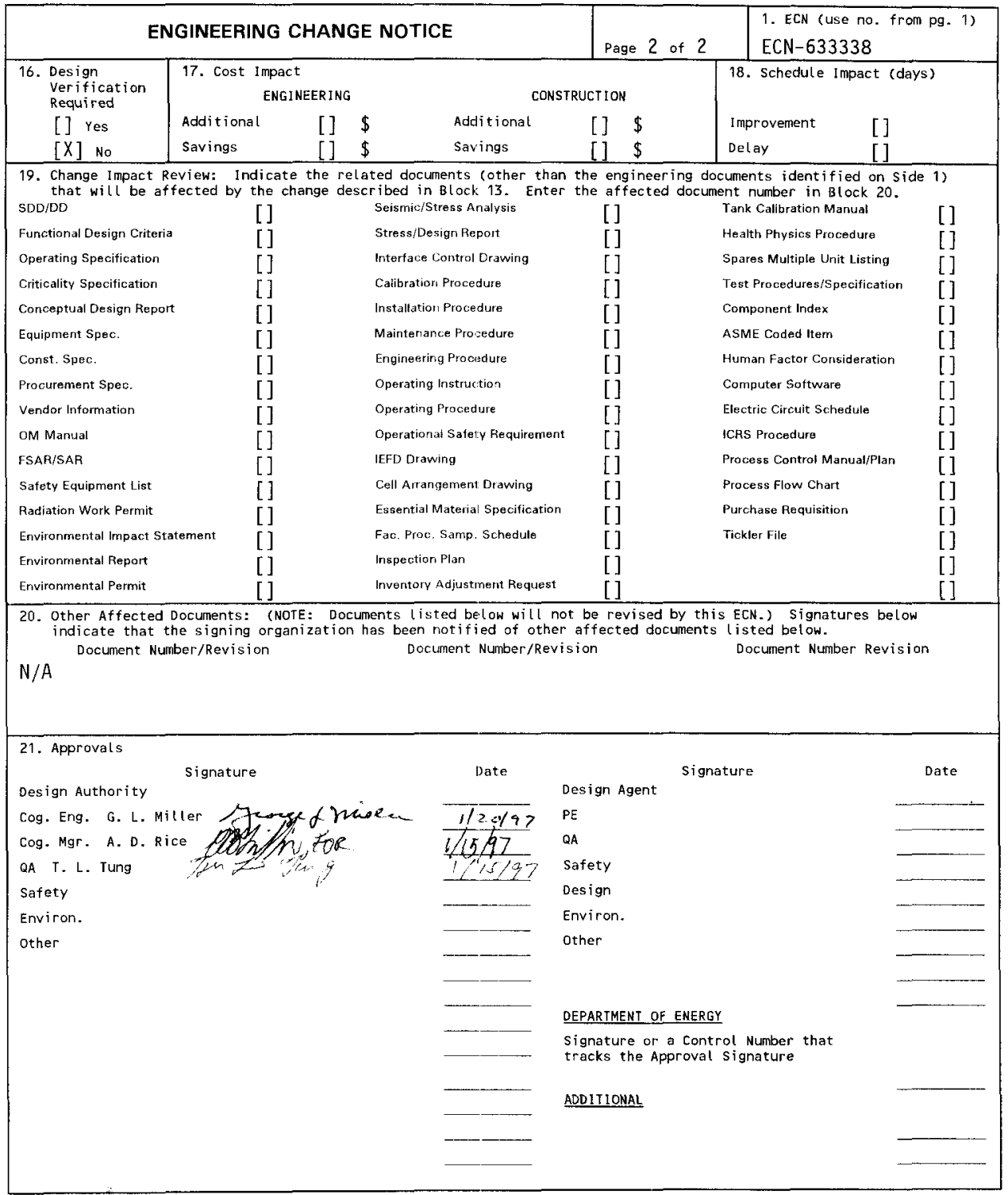




\section{Final Characterization and Safety Screen Report of Double Shell Tank 241-AP-105 for Evaporator Campaign 97-1}

\section{George L. Miller}

Rust Federal Services of Hanford, Inc., Richland, WA 99352

U.S. Department of Energy Contract DE-AC06-87RL 10930

$\begin{array}{lll}\text { EDT/ECN: } & \text { ECN-633338 } & \text { UC: 2070 } \\ \text { Org Code: } & 7576 \mathrm{~B} & \text { Charge Code: MD378 } \\ \text { B\&R Code: } & \text { EW 3120074 } & \text { Total Pages: } 1068\end{array}$

Key Words: Final, Characterization, Safety Screen, Report, Double She11, Tank 241-AP-105, 241-AP-105, AP-105, Evaporator, Campaign 97-1, 97-1

Abstract: $N / A$

TRADEMARK DISCLAIMER. Reference herein to any specific commercial product, process, or service by trade name, trademark, manufacturer, or otherwise, does not necessarily constitute or imply its endorsement, recommendation, or favoring by the United States Goverment or any agency thereof or its contractors or subcontractors.

Printed in the United States of America. Io obtain copies of this document, contact: WHC/BCS Document Control Services, P.O. Box 1970, Mailstop H6-08, Richland WA 99352, Phone (509) 372-2420; Fax (509) 376-4989.
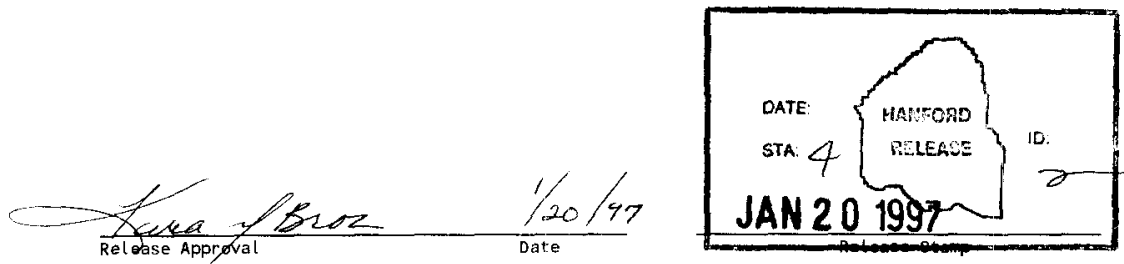

\section{Approved for Public Release}


(2) Title

Final Characterization and Safety Screen Report of Double She 11 Tank 241-AP-105 for Evaporator Campaign 97-1

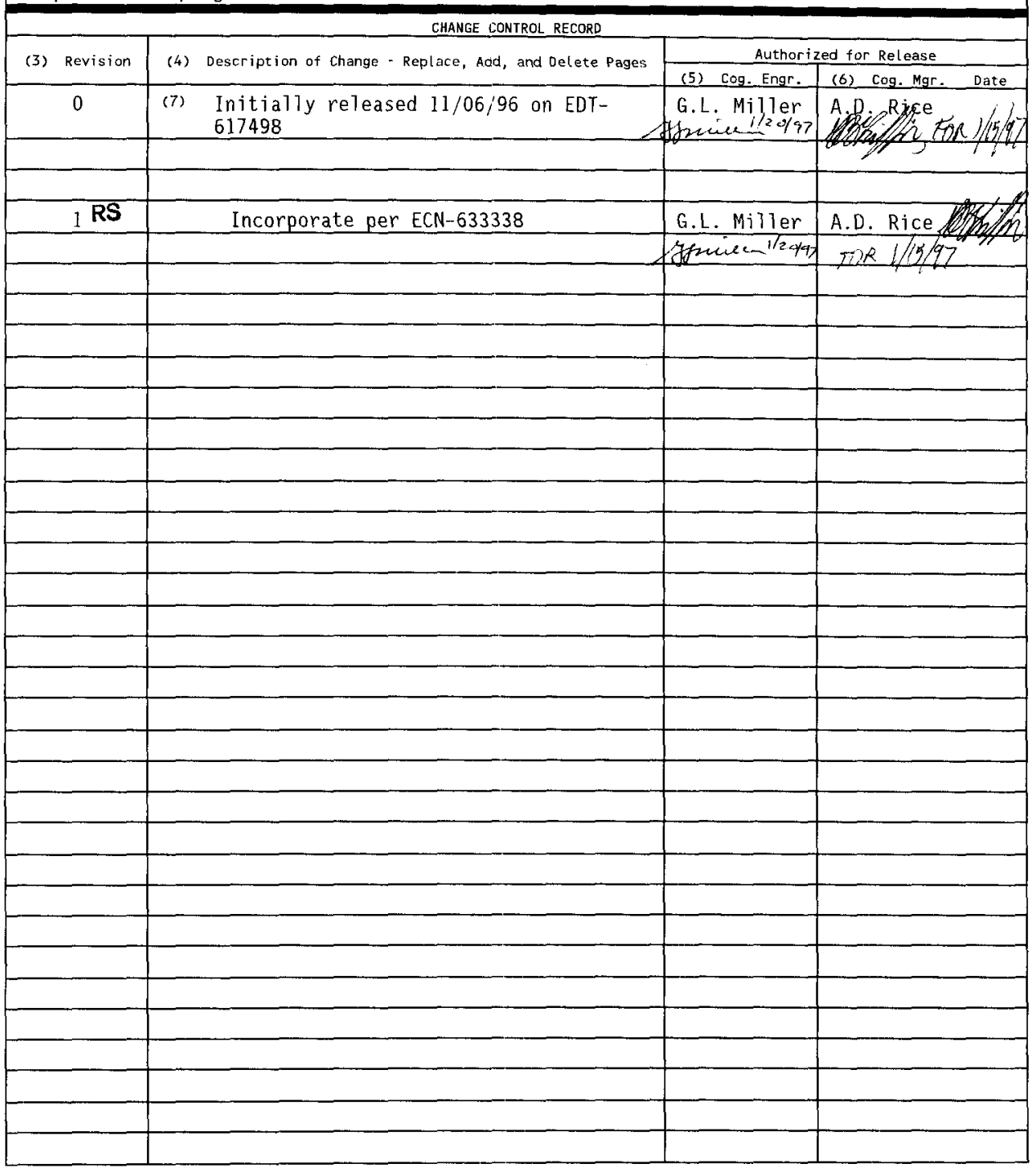




$$
\begin{aligned}
& \text { HWF-SD-WM-DP-202, REV } \perp \\
& \text { WHC-SD-WM-DP-202, REV. } 1
\end{aligned}
$$

ANALYTICAL SERVICES

\title{
FINAL CHARACTERIZATION AND SAFETY SCREEN REPORT OF DOUBLE SHELL TANK 241-AP-105 FOR EVAPORATOR CAMPAIGN 97-1
}

\author{
Project Coordinator: GEORGE L. MILLER \\ Prepared for the U.S. Department of Energy \\ office of Environmental Restoration \\ and Waste Management \\ by \\ 222-S Laboratory \\ Rust Federal Services of Hanford Inc. \\ P. O. Box 700 \\ Richland, Washington
}


HNF-SD-WM-DP- $20 \alpha$, REV. 1

WHC-SD-WM-DP-202, REV. 1

THIS PAGE WAS INTENTIONALLY LEFT BLANK 


\section{HNF-SD-WM-DP-2CQ, REV.1}

WHC-SD-WM-DP-202, REV. 1

TABLE OF CONTENTS

Narrative . . . . . . . . . . . . . . . . . . . . . . . 1

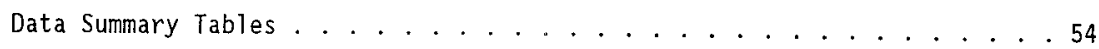

Chain of Custody Forms ..................... . . . 77

Sample Breakdowns . . . . . . . . . . . . . . . . . . . . . . 98

Sample Preparations . . . . . . . . . . . . . . . . . . . 106

Inorganic Analyses . . . . . . . . . . . . . . . . . . . . . . 110

Specific Gravity (SpG)

SpG Worklist \# $12917(48,49,47,53)$. . . . . . . . . . . 112

$\mathrm{Ph}$

ph Workl ist \# $12905(48,49,47)$. . . . . . . . . . . . 120

Hydroxide

OH Workl ist \# $12904(48,49,47,58)$. . . . . . . . . . . . 121

Ammonia

NH3 Worklist \# $(48,49,47,58)$. . . . . . . . . . . . . . . 138

Ion Chromatography

IC Workl ist \# $12912(48,49,47,58)$. . . . . . . . . . . . . . 151

Inductive Coupled Plasma Spectroscopy

ICP Work list \# $14566(50,51,52,54,60) \ldots . . . . . . . . . .163$

Total Organic Carbon

TOC Worklist \# $12910(48,49,47,53,58) \ldots . . . . . . . . .171$

Total Inorganic Carbon

TIC Workl ist \# $12909(48,49,47,58)$. . . . . . . . . . . . . . . 191

Total Carbon

TOTC Workl ist \# $12911(48,49,47,58)$. . . . . . . . . . . 215

Uranium

U Workl ist \# 12889 (47)................... 233

U Workl ist \# $12891(48,49)$. . . . . . . . . . . . . . . . . 244

U Work1 ist \# 12892 (58). . . . . . . . . . . . . . . . . . . . . . 256

Radiochemical Analyses . . . . . . . . . . . . . . . . . . . . . 267

Tritium

H3 Worklist \# $15286(47,48,49,58) \ldots . . . . . . . . . .269$ 


\title{
HNF-SD-WM-DP. 202, REV 1
}

\author{
WHC-SD-WM-DP-202, REV. I \\ TABLE OF CONTENTS (Continued)
}

A]pha, Beta (AB)

$A B$ Worklist \# $14423(50,51,52,54,60) \ldots 279$

Gamma Energy Analyses

GEA Work1 ist \# $14453(50,51,52,60) \ldots . . . . . . . .311$

Iodine 129

I129 Workl ist \#I2887 $(48,49) \ldots \ldots . \ldots . \ldots . \ldots 343$

I129 Worklist \# $15633(58)$. . . . . . . . . . . . . . . . . 373

1129 Worklist \# 15764 (47) . . . . . . . . . . . . . . 399

Carbon 14

C14 Work1 ist \#12880 (58) . . . . . . . . . . . . . . . . . 426

C14 Worklist \# $15602(47,48,49) \ldots . . . . . . . . . .4435$

Strontium $89 / 90$

Sr90 Work1 ist \# $15408(50,51,52,60) \ldots \ldots 446$

Technetium 99

TC99 Work7ist \# $15376(50,51,52,60) \ldots . . . \ldots 456$

Neptunium 237

Np237 Worklist \# $14430(50,51,52,60) \ldots . . . . . . .468$

Americium 241

Am241 Worklist \# $14424(50,51,52) \ldots \ldots . . . \ldots 49$

Am241 Worklist \# $14425(60) \ldots . . . . . . . . . . . . . .523$

Plutonium 239/40

Pu239 Workl ist \# $14433(50,52,51,60) \ldots . . . . . . . .551$

Selenium 79

Se79 Worklist \# $14439(50,51,52,60) \ldots . . . . . . . .5596$

Organic Analyses .................... 606

Volatile Data. . . . . . . . . . . . . . 608

Semi Volatile Data . . . . . . . . . . . . . . . . 757

Part II Tank 241-AP-105, Grab Samples 5AP-96-1C, 5AP-96-2C, 5AP-96-3C, 5AP-96-4 and 5AP-96-IB2, Analytical Results for the 45 Day

Report .................. 2-1

Narrative . . . . . . . . . . . . . . 2-1

Chain of Custody Forms .................. 2-11

Inorganic Anlayses . . . . . . . . . . . . . . . 2-18 


\author{
HNF-SD-WM-DP QCO2, REV. I \\ WHC-SD-WM-DP-202, REV. 1 \\ TABLE OF CONTENTS (Continued)
}

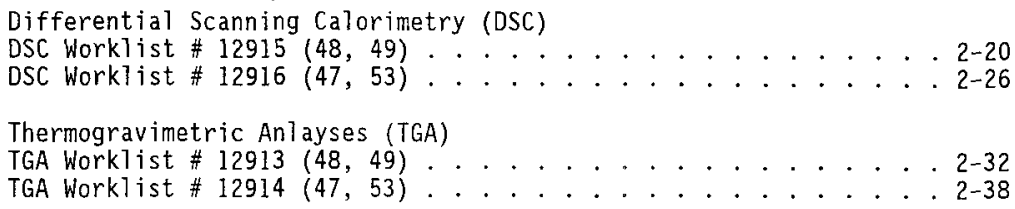

This Document consists of two sections.

Part I consists of pages 1 through 1002. Pages ii, 55, 78, 99, 107, 111, 268 and 607 were intentionally left blank.

Part II consists of pages 2-1 through 2-43. Pages 2-2, 2-12 and 2-19 were intentionally left blank.

TRADEMARKS:

Perkin-Elmer is a Registered Trademark of Research and Manufacturing Company, Inc. Mettler is a Registered Trademark of Mettler Electronics 


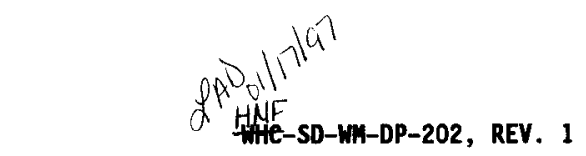

\title{
FINAL CHARACTERIZATION AND SAFETY SCREEN REPORT OF DOUBLE SHELL TANK 241-AP-105 FOR 242-A EVAPORATOR CAHPAIGN 97-1
}

\author{
CASE MARRATIVE
}

\section{INTRODUCTION}

\section{SOURCE DOCUNENTATION}

Evaporator candidate feed from tank 241-AP-105 (hereafter referred to as AP105) was characterized for physical, inorganic, organic and radiochemical parameters by the 222-S Laboratory as directed by the Tank Sample and Analysis P1 an (TSAP), References 1 through 4, and Engineering Change Notice, number 635332, Reference 5.

This data package satisfies the requirement for a format IV, final report as described in Reference 1 . This data package is also a follow-up to the 45-Day safety screen results for tank AP-105, Reference 8 , which was issued on November 5, 1996, and is attached as Section II to this report. Preliminary data in the form of summary analytical tables were provided to the project in advance of this final report to enable early estimation of evaporator operational parameters, using the Predict modeling program.

Analyses were performed at the 222-S Laboratory as defined and specified in the TSAP and the Laboratory's Quality Assurance PIan, References 6 and 7. Any deviations from the instructions documented in the TSAP are discussed in this narrative and are supported with additional documentation.

\section{SAMPLING}

The TSAP, section 2, provided sampling information for waste samples collected from tank AP-105. The "bottle-on-a-string" method was used to collect liquid grab samples from the tank. Each glass sample bottle was amber, precleaned, and contained approximately 100 milliliters. Each bottle was closed with a tefion seal cap (or teflon septum for volatile organic analysis samples).

Field blank samples were prepared by placing deionized water into sampling bottles, lowering the unclosed bottles into the riser for a period of time, retrieving them from the riser, and then closing the bottles with the same types of caps used for the tank samples.

None of the samples were preserved by acidification. Upon receipt, the sample bottles destined for organic analyses were placed in a refrigerator. No attempt was made during sampling to assure the complete filling of the bottles so as to exclude all headspace. These actions were consistent with safety procedures, which attempt to 1 imit personnel exposure to hazardous ionizing radiation. 


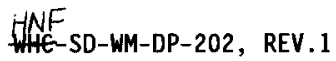

Chain-of-Custody forms were generated by the sample collector and delivered to 222-S Laboratory with the samples. Copies of these Chain-of-Custody forms are included in this data package beginning on page 78. Samples were transported to the 222-5 laboratory receiving door in shielded pigs.

Sample collection and identification data are summarized in Table 1.

\section{LABORATORY OPERATIONS}

\section{SAMPLE TRACKING}

\section{RECEIVING PROCEDURES/CHAIN-OF-CUSTODY}

Tank AP-105 samples were received into the 222-S Laboratory by the laboratory's sample custodian, who signed the Chain-of-Custody form, becoming the new sample custodian. The Chain-of-Custody form, a legal document, tracks the transfer of samples between individuals or organizations to establish sample ownership.

The pigs containing the samples were transported to a hood where the sample bottles were removed, and visually inspected. A radioactive dose rate at one inch from the side of the samples was measured by a Health Physics Technician. The measured dose rate was multiplied by five as a correction factor to compensate for sample geometry. Samples were placed in shielded containers, relabeled to include the laboratory identification number, and transferred to metal storage cabinets (or refrigerators) for storage. Because the sample dose rate was not sufficiently high, it was determined that processing the samples through the hot cell was not required. Hot cell processing of samples is required when the sample dose rate measured at the laboratory exceeds 7 rem per hour or 25 rad per hour.

\section{SAMPLE LOGGING/DATA HANDLING}

At the 222-A Laboratory, a computerized Laboratory Information Management System (LIMS) called LABCORE was used by the project coordinator to log samples directly into a computer, assign analyses to be performed on each sample, and to assign quality control parameters. Chemists and Lab Leaders generated worklists, assigning samples to batches for analysis. As analytical data were generated, the results were entered into LABCORE either manualiy or through a direct data upload from the instrumentation. The LIMS was used to periodically track the complete/incomplete status of each sample.

The procedures for boildown and mixing/compatibility will be performed on a composite of samples from AP-105. As instructed in the TSAP, Reference 1, these data are to be reported independently, with data attached to an Internal Memo. 
Table 1: Tank AP-104 Grab Sample Information

\begin{tabular}{|c|c|c|c|c|c|c|c|c|}
\hline CUSTOMER SAMPLE NUMBER & $\begin{array}{l}\text { DATE } \\
\text { SAMPLED }\end{array}$ & $\begin{array}{l}\text { SAMPLE SEAL } \\
\text { MUMBER }\end{array}$ & LABORATORY ID NUMBER & $\begin{array}{l}\text { AMALYSES TO BE } \\
\text { PERFORMED OW WASTE }\end{array}$ & " HASTE TYPE & SAMPLE LOCATION" & $\begin{array}{l}\text { SAMPLE DEPTH } \\
\text { Centi imeters }\end{array}$ & $\begin{array}{c}\text { Date Sample } \\
\text { Received }\end{array}$ \\
\hline $5-A P-1 A$ & $8 / 29 / 96$ & 10524 & $596 v 000039$ & Organic/von & Supernate & Riser $1,330^{\circ} \mathrm{N}$ & 587 & $8 / 30 / 96$ \\
\hline $5-A P-1 B$ & $8 / 29 / 96$ & 10525 & $596 \vee 000043$ & Organic/svou & Supernate & Riser $1,330^{\circ} \mathrm{N}$ & 587 & $8 / 30 / 96$ \\
\hline 5-AP-1C & $8 / 29 / 96$ & 10526 & $\begin{array}{l}\text { S96v000047, direct } \\
\text { s96v000050, acid digestion }\end{array}$ & Inorg/Red & Supernate & Riser $1,330^{\circ} \mathrm{N}$ & 587 & $8 / 30 / 96$ \\
\hline $5-A P-1 D$ & $8 / 29 / 96$ & 10527 & S96v000055 & Mixing/Boildown & Supernate & Riser $1,330^{\circ} \mathrm{N}$ & 587 & $8 / 30 / 96$ \\
\hline $5-A P-2 A$ & $9 / 3 / 96$ & 10528 & s96v000040 & Organic/von & Supernate & Riser $1,90^{\circ} \mathrm{N}$ & 221 & $9 / 4 / 96$ \\
\hline $5-A P-2 B$ & $9 / 3 / 96$ & 10529 & S96v000044 & Organic/svon & supernate & Riser $1,90^{\circ} \mathrm{N}$ & 221 & $9 / 4 / 96$ \\
\hline $5-A P-2 C$ & $9 / 3 / 96$ & 10530 & $\begin{array}{l}\text { \$96v000048 (direct) } \\
\text { \$96v000051 (acid digest) }\end{array}$ & Inorg/Rad & supernate & Riser $1,90^{\circ} \mathrm{N}$ & 221 & $9 / 4 / 96$ \\
\hline 5-AP-2D & $9 / 3 / 96$ & 10531 & 596V000056 & Mixing/Boildown & Supernate & Riser $1,90^{\circ} \mathrm{N}$ & 221 & $9 / 4 / 96$ \\
\hline 5-AP-3A & $9 / 3 / 9$ & 10532 & 596v000041 & Organic/von & Supernate & Riser $1,90^{\circ} \mathrm{N}$ & 688 & $9 / 4 / 96$ \\
\hline $5-A P-3 B$ & $9 / 3 / 96$ & 10534 & s96v000045 & Organic/svon & Supernate & Riser $1,90^{\circ} \mathrm{N}$ & 688 & $9 / 4 / 96$ \\
\hline 5-AP-3C & $9 / 3 / 96$ & 10533 & $\begin{array}{l}\text { 596v000049 (direct) } \\
\text { 596v000052 (acid digest) }\end{array}$ & Inorg/Rad & Supernate & Riser $1,90^{\circ} \mathrm{N}$ & 688 & $9 / 4 / 96$ \\
\hline $5-A P-30$ & $9 / 3 / 96$ & 10535 & 596v000057 & Mixing/Boildown & Supernate & Riser $1,90^{\circ} \mathrm{N}$ & 688 & 9/4/96 \\
\hline 5-AP-4 & $9 / 3 / 96$ & 10536 & $\begin{array}{l}\text { s96v000053 (direct) } \\
\text { s96v000054 (acid digest) }\end{array}$ & IOC \& Safety Screen & Surface & Riser $1,90^{\circ} \mathrm{N}$ & approx. 1041 & $9 / 4 / 96$ \\
\hline 5-AP-IB1 & $9 / 3 / 96$ & 10537 & S96v000058 (direct) & Inorg/Rad & Field Blank & Riser $1,90^{\circ} \mathrm{N}$ & & $9 / 4 / 96$ \\
\hline $5-A P-1 B 2$ & $9 / 3 / 96$ & 10538 & $\begin{array}{l}\text { S96v000059 (direct) } \\
\text { S96v000060 (acid digest) }\end{array}$ & Inorg/Rad & Field Blank & Riser $1,90^{\circ} \mathrm{N}$ & & $9 / 5 / 96$ \\
\hline 5-AP-OB1 & $9 / 3 / 96$ & 10539 & S96v000061 & Organic/von & Field Blank & Riser $1,90^{\circ} \mathrm{N}$ & & 9/5/96 \\
\hline 5-AP-CB2 & $9 / 3 / 96$ & 10540 & 596000062 & Organic/svon & Field Blank & Riser $1,90^{\circ} \mathrm{N}$ & & $9 / 4 / 96$ \\
\hline 5-AP-TB1 & $9 / 3 / 96$ & 10541 & s96v000042 & Organic/von & Trip 8 lank & Irip Blank & $\mathrm{n} / \mathrm{a}$ & 9/4/96 \\
\hline 5-AP-TB2 & $9 / 3 / 96$ & 10542 & 596000046 & Organic/svon & Trip Blank & Irip Blank & $n / a$ & $9 / 5 / 9$ \\
\hline
\end{tabular}

Sample Elevation is defined as the distance from the tank bottom to the mouth of the sample bottle. 


\section{SAMPLE IDENTIFICATION}

Customer generated sample identification numbers for each sample were provided in the TSAP, Reference 1. New sample identification numbers were assigned by LABCORE to each sample when logged into the 222-S Laboratory. Table 1 relates the customer identification number and laboratory identification number to each sampling location. As can be seen in Table l, multiple samples from each location were collected and given unique identification numbers to enable better sample handling and control within the laboratory, particularly with regard to organic analyses. Four bottles were provided from each of three subsurface locations to insure ample sample volume for analyses. An additional sample was collected from the waste surface for total organic carbon and safety screening analyses.

Two trip blanks were collected for organic analyses: one for VOA and one for Semi-VOA. Trip blank analyses were required by the TSAP only when those analytes required in the TSAP were observed in the field blanks.

\section{QUALITY ASSURANCE OF THE ANALYTICAL SYSTEM AND DATA}

\section{CONTROLLED PROCEDURES}

Each procedure used at 222-S for this project was a controlled procedure. All procedures were evaluated and approved for a maximum period of two years. Procedures may be revised, modified, or deleted as appropriate. Each time a procedure was revised or modified, however, a new revision/modification number was added to the procedure number. Upon review (at the end of the two year period), a procedure approval may be extended for another two year period without a change in the revision/modification number.

\section{STATISTICAL EVALUATION}

Performance data have been gathered historically on each analytical procedure for which a laboratory control standard was available. Summary statistics were calculated for the percent recovery from which estimates of the procedure precision and accuracy were obtained. These procedure control limits were/are statistically re-evaluated upon acquiring 100 additional data points, whichever occurs first. If necessary, new acceptance control limits were/are generated.

\section{REVIEW OF DATA}

Descriptive information was provided on the Worklist Data Entry sheets when unusual conditions occurred during analysis of a batch. These narrative comments were generally also noted in this case narrative unless the data were rejected and not reported.

Each analytical batch was reviewed for correctness of data at several levels. Chemists reviewed not only analytical calculations, but assured that the analytical system was performing appropriately and that the laboratory technicians were following written procedures. All analysis results, which had been entered into LABCORE, were reviewed by the cognizant chemist, verifying data accuracy and completeness. Once this process was completed, which was a step termed "validation" by LABCORE, then access to the data was 
WNF-SD-WM-DP-202, REV.1

locked. Locked data may be unvalidated or corrected during subsequent

reviews, where such modifications require an explanation plus the input of the initials of the person making the modifications as an audit trail.

All data were reviewed by the project coordinator to also verify that correct values had been entered to LABCORE from the hand written Work1 ist Data Entry sheets. Correctness of case narrative text and tables was reviewed by the project coordinator. Errors and unclear statements were subsequently corrected in the released data package.

The internal quality assurance group performed a general review of this data package, including at least a $10 \%$ review of the analytical data, where final analytical values were recalculated from the raw data to verify analytical accuracy and completeness. Case narrative text and tables were also examined for accuracy, clarity and completeness.

\section{ANALYTICAL DATA REPORTING}

\section{LABCORE REPORTS}

LABCORE has the capability of generating final reports from analytical data which were input to the system. The 45 Day Safety Screen Results report (Reference 8) was prepared using the system's "45-Day Report" format, in which all analytical results were presented per each individual sample. This format was acceptable for the Safety Screen Report, and the data were accurately presented.

The LABCORE system is currently unable to generate a complete report for all of the analytical parameters required by the TSAP. Consequently, all data were downloaded from LABCORE to spreadsheet format to enable the generation of summary data tables, where all data could be included. All data on the summary tables were checked against LABCORE hard copy data sheets to assure accuracy.

\section{SAFETY SCREEN AND PRELIMINARY REPORTS}

The TSAP, Reference 1, required delivery of a 45-day Safety Screen Report by October 20, 1996. This report (Reference 8) was released as a supporting document on November 6, 1996, which was 17 days after the required delivery date.

Preliminary summary analytical results were delivered by FAX to Treatment Systems Plant Engineering to enable early process control planning. The report format was the same as for this report. The data were considered to be preliminary because they had not been subjected to a final review. It was understood by the program that these data were neither final nor validated. 


\section{CASE MARRATIVE}

This case narrative was prepared in accordance with TSAP, sections 3 and 6 .

The intent of the case narrative within this data package is to:

- Present required analytical data,

- Evaluate the quality of these data,

- Document problems encountered while performing the analytical procedures,

- Characterize the nature of the constituents within tank AP-105, and

- Interpret, whenever possible, the relevance or impact of these findings on the evaporator program.

\section{ANALYTICAL REQUIREMENTS AND PROCEDURES}

\section{SAMPLE PRESERVATION}

The TSAP was silent regarding sample preservation requirements. No preservation of samples occurred prior to being received in the laboratory. Once the samples were "broken down" at the time of arrival at the 222-S Laboratory, all sample bottles, which were designated for organic analyses, were maintained at $4^{\circ} \mathrm{C}$.

\section{SAMPLE HOLDING TIME}

The TSAP, section 3.2, states the following:

"... the laboratory and sampling organization should strive to meet SW-846 (EPA 1986) holding times. However, adherence to SW846 holding times is not strictly required if documented cases show that additional time was required to ship, process, and analyze the samples ..."

For inorganic and radiochemical analytes, the SW-846 maximum sample holding time limits that were applicable are found in volume IA, Table 2-21 (revision 1 , July 1992). SW-846 sample holding times for organic analytes are specified in Volume IB, Chapter 4, Table 4-1. Table 2 presents these sample holding time limits. 


\begin{tabular}{|c|c|c|}
\hline Parameter & Maximum Holding Time & Preservation \\
\hline Nitrate & 48 hours & $4^{\circ} \mathrm{C}$ \\
\hline Sulfate & 28 days & $4^{\circ} \mathrm{C}$ \\
\hline Aluminum & 6 months & $\mathrm{HNO}_{3}$ to $\mathrm{pH}<2$ \\
\hline Sodium & 6 months & $\mathrm{HNO}_{3}$ to $\mathrm{pH}<2$ \\
\hline $\mathrm{pH}$ & Analyze immediately & None \\
\hline Total Organic Carbon & 28 days & $4^{\circ} \mathrm{C}$ \\
\hline $\begin{array}{l}\text { Volatile Organic } \\
\text { Analysis }\end{array}$ & 14 days & $4^{\circ} \mathrm{C}, \quad 0.008 \% \quad \mathrm{Na}_{2} \mathrm{~S}_{2} \mathrm{O}_{3}$ \\
\hline $\begin{array}{l}\text { Semi-Volatile Organic } \\
\text { Analysis }\end{array}$ & $\begin{array}{l}14 \text { days for extraction, } \\
40 \text { days for analysis of } \\
\text { extract }\end{array}$ & $4^{\circ} \mathrm{C}, \quad 0.008 \% \quad \mathrm{Na}_{2} \mathrm{~S}_{2} \mathrm{O}_{3}$ \\
\hline Total Alpha & 6 months & $\mathrm{HNO}_{3}$ to $\mathrm{pH}<2$ \\
\hline Total Beta & 6 months & $\mathrm{HNO}_{3}$ to $\mathrm{pH}<2$ \\
\hline Radium (radiological) & 6 months & $\mathrm{HNO}_{3}$ to $\mathrm{pH}<2$ \\
\hline
\end{tabular}

Agreement within the scientific community is divided with regard to reasonable sample holding times. SW-846 holding times are based on worst-case scenarios and in many cases are excessively short. Note that SW-846 protocol expects that samples are to be preserved at the time of collection for SW-846 holding times to be valid. However, tank AP-105 samples were intentionally not preserved so as to limit the exposure dosage of ionizing radiation to personnel.

Sample degradation can occur due to many factors. One of these factors, biological degradation, is typically controlled by the addition of a strong acid, creating a hostile biological environment due to extreme $\mathrm{pH}$. Relative to biological degradation of tank AP-105 samples, it can be argued that sample preservation with the use of acid was unnecessary because of high level ionizing radiation lethal to micro-organisms, as well a pH in the high extreme (ranging from 13.3 to 13.5 ).

The actual sample holding time for each sample is discussed relative to each analyte in the Results of Analyses section in this narrative.

\section{ANALYTICAL ALIQUOTS}

Because multiple samples were collected from each sample point, the probability was low that the sample would be exhausted before all of the analyses could be analyzed and results accepted. Direction was given by the project coordinator in a pre-job briefing that analytical aliquots were to be optimized to achieve the lowest detection limits whenever the analyte concentration in the sample was expected to approach the detection limit. 


\section{PREPARATIVE METHODS}

Table 2 of the TSAP specified which preparative method was to be used for each constituent. All sample preparations conformed to the TSAP specifications. Table 3 indicates the preparative procedures stated in the TSAP.

\begin{tabular}{|c|c|}
\hline Analytical Procedure & $\begin{array}{l}\text { Preparative } \\
\text { Procedure }\end{array}$ \\
\hline Differential Scanning Calorimetry (DSC) & direct * \\
\hline Thermal Gravimetric Analysis (TGA) & direct * \\
\hline pH & direct* \\
\hline Specific Gravity & direct * \\
\hline Hydroxide & direct * \\
\hline Ion Chromatography: $\mathrm{F}_{1} \mathrm{NO}_{2}, \mathrm{NO}_{3} \mathrm{SO}_{4}, \mathrm{PO}_{4}$ & direct * \\
\hline Inductively Coupled Plasma/Optical Emission Spectrometry: Al, Ma & acid digestion \\
\hline Iotal Carbon (TC) & direct* \\
\hline $\mathrm{CO}_{3}$ (TIC) & direct * \\
\hline Total Organic Carbon (TOC) & direct * \\
\hline Anmonia & direct * \\
\hline $\begin{array}{l}\text { Volatile Organic Analysis (VOA): } \\
\text { Acetone, 1-Butanol, 2-Butanone, 2-Hexanone, Methyl I sobutyl Ketone (4-Methyl- } \\
\text { 2-pentanone), 2-Pentanone, Tetrahydrofuran }\end{array}$ & direct * \\
\hline $\begin{array}{l}\text { Semi-Volatile Organic Analys is (Semi-VOA); 2-Butoxyethanol, } \\
\text { n-Tributylphosphate }\end{array}$ & direct * \\
\hline Uranium, gross (U-gross) & direct * \\
\hline Total Alphe (AT) & acid digestion \\
\hline Total Beta (TB) & acid digestion \\
\hline $238_{\mathrm{Pu},}, 239 / 240_{\mathrm{Pu}}$ & ecid digestion \\
\hline $237_{\mathrm{Hp}}$ & acid digestion \\
\hline${ }^{99} \mathrm{Tc}$ & acid digestion \\
\hline${ }^{90} \mathrm{Sr}$ & acid digestion \\
\hline${ }^{14} \mathrm{C}$ & direct * \\
\hline $3_{H}$ & direct * \\
\hline${ }^{79} \mathrm{Se}$ & acid digestion \\
\hline $129_{1}$ & direct * \\
\hline Ganma Energy Analysis (GEA): ${ }_{94}^{60} \mathrm{Co},{ }^{137} \mathrm{Cs},{ }^{106} \mathrm{Ru}, 134 \mathrm{Cs},{ }^{144} \mathrm{Ce},{ }^{154} \mathrm{Eu},{ }^{155} \mathrm{Eu}$, & acid digestion \\
\hline $243 / 244 \mathrm{Cm}$ (and $\left.{ }^{241} \mathrm{Am}\right)$ & acid digestion \\
\hline
\end{tabular}

* The TSAP, Table 2 states, "Direct liquid samples may be diluted in acid or water to adjust to proper sample size and/or pH". 


\section{WEF-WM-DP-202, REV.1}

\section{ANALYTICAL PROCEDURES}

Table 4 summarizes the analytical procedures which were used for analyses of AP-105 samples. The procedures used were the same as those cited in TSAP, Table 2, except for plutonium analyses. At the time that the TSAP was being prepared, procedure LA-943-127 was a valid, active procedure, however that procedure was inactivated when it was replaced with procedure LA-943-128 on $11 / 28 / 95$. 


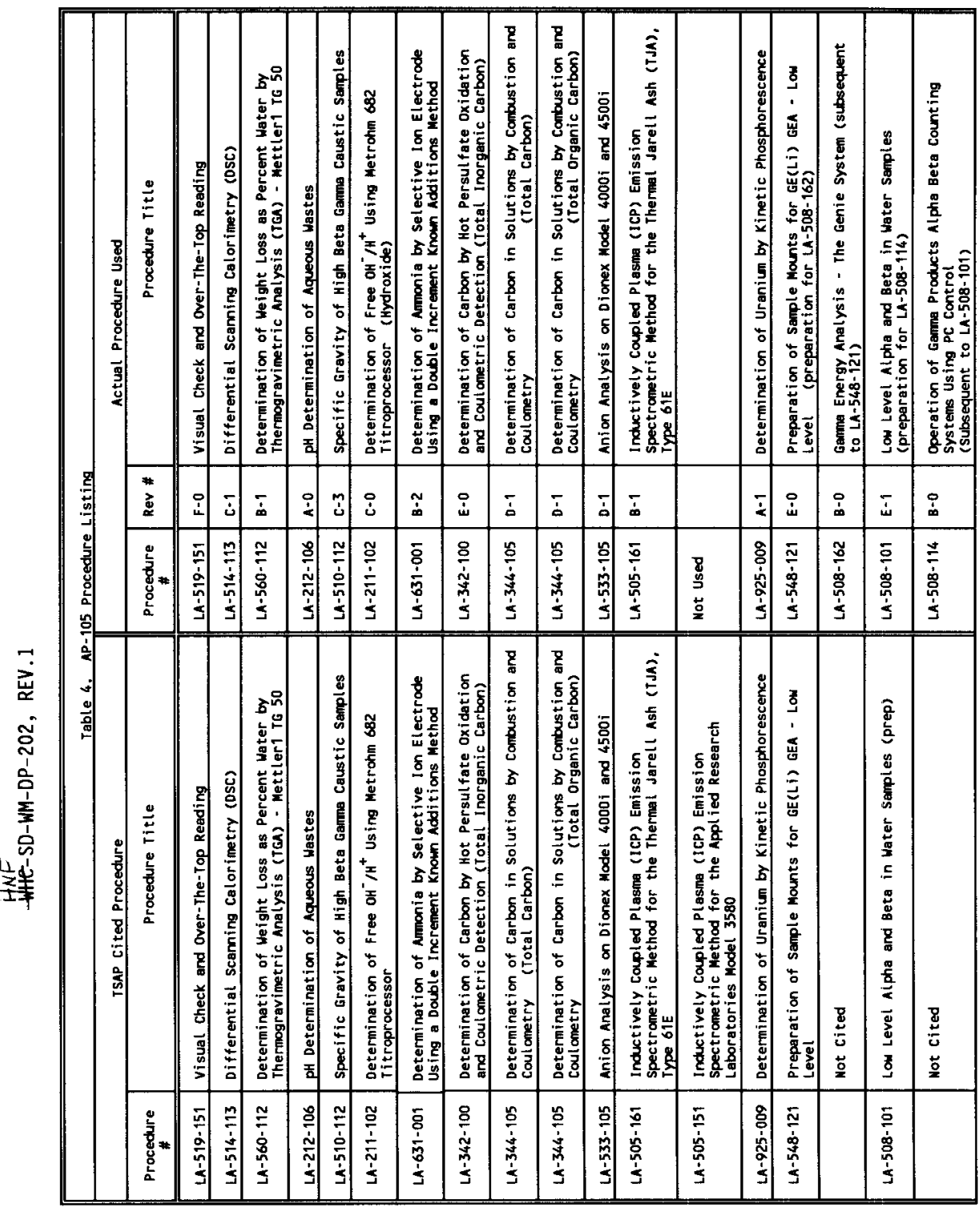


WF

WHE-SD-WM-DP-202, REV.1

\begin{tabular}{|c|c|c|c|c|}
\hline \multicolumn{2}{|r|}{ TSAP Cited Procedure } & \multicolumn{3}{|r|}{ Actual Procedure Used } \\
\hline $\begin{array}{l}\text { Procedure } \\
\#\end{array}$ & Procedure Title & $\begin{array}{c}\text { Procedure } \\
\#\end{array}$ & $\operatorname{Rev} *$ & Procedure Title \\
\hline $1 A-953-103$ & $\begin{array}{l}\text { Determination of Americium by Extraction with TRU.Spec } \\
\text { Resin }\end{array}$ & $L A-953-103$ & $B-0$ & $\begin{array}{l}\begin{array}{l}\text { Determination of Americium by Extraction with TRU.Spec } \\
\text { Resin } \\
\text { (also } \mathrm{Cm}-243 / 244)\end{array} \\
\end{array}$ \\
\hline$L A-943-128$ & Determination of Pu by Ion Exchange & $L A-943-128$ & $B-0$ & $\begin{array}{l}\text { Determination of Plutonium by Extraction with } \\
\text { iRU-Spec Resin } \quad\left(P_{U}-239 / 240 \& \mathrm{Pu}-238\right)\end{array}$ \\
\hline$L A-218-114$ & $\begin{array}{l}\text { Tritium by Lachat Micro-Dist . and Liquid Scintitlation } \\
\text { Counting (LS) }\end{array}$ & LA-218-114 & B-0 & $\begin{array}{l}\text { Iritium by Lachat Micro-Dist. and Liquid Scintillation } \\
\text { Counting (LS) }\end{array}$ \\
\hline \multirow[t]{2}{*}{ LA-348-104 } & $\begin{array}{l}\text { C- } 14 \text { in Small Volume Samples by Persulfate oxidation } \\
\text { and Liquid Scintillation }\end{array}$ & $L A-348-104$ & $c-0$ & $\begin{array}{l}\text { C- } 14 \text { in Snall Volume Samples by Persulfate Oxidation } \\
\text { and Liquid Scintillation }\end{array}$ \\
\hline & Mot cited & $L A-508-121$ & B-2 & $\begin{array}{l}\text { Operation of the Beckman Liquid Scintillation Counter } \\
\text { (subsequent to LA-218-114\&\&A-348-104) }\end{array}$ \\
\hline LA-438-101 & $\begin{array}{l}\text { Determination of Ic- } 99 \text { by Solvent Extraction and } \\
\text { Liquid Scintillation Counting }\end{array}$ & $L A-438-101$ & $0-2$ & $\begin{array}{l}\text { Determination of Tc- } 99 \text { by Solvent Extraction and } \\
\text { Liquid Scintillation Counting }\end{array}$ \\
\hline$L A-220-101$ & High Level Strontium-89,90 in Aqueous Samples & $L A-220-101$ & $\begin{array}{l}0-1 \& \\
E-3\end{array}$ & High Level stront ium-89,90 in Aqueous Sanples \\
\hline$L A-365-132$ & Determination of $\mathrm{Se}-79$ & LA-365-132 & $c-1$ & Determination of $\mathrm{se}-79$ \\
\hline LA-933-141 & $\begin{array}{l}\text { Determination of Np-237 by TiOM/TTA Extraction and } \\
\text { Alpha Counting }\end{array}$ & $L A-933-141$ & $H-1$ & $\begin{array}{l}\text { Determination of Np-237 by TiOA/TTA Extraction and } \\
\text { Alpha Counting }\end{array}$ \\
\hline LA-378-103 & Determination of Jodine-129 in Waste Tank Samples & $L A-378-103$ & $c-0$ & Determination of lodine-129 in Waste Tank Samples \\
\hline \multirow[t]{2}{*}{$L A=523-405$} & $\begin{array}{l}\text { Volatile Organics by Gas Chromatography/ Mass } \\
\text { Spectrometry Using SW-846. (VOA) }\end{array}$ & $L A-523-405$ & $A-4$ & $\begin{array}{l}\text { Volatile Organics by Gas Chromatography/ Mass } \\
\text { Spectrometry Using SW-846. (Voa) }\end{array}$ \\
\hline & Not cited & $L A-523-132$ & B-O & $\begin{array}{l}\text { Semi-micro Continuous } L \text { iquid-Liquid Extraction of } \\
\text { Semivolatiles based on SW-846 Methods }\end{array}$ \\
\hline \multirow[t]{2}{*}{ LA-523-406 } & $\begin{array}{l}\text { Semivolatile Organics by Gas Chromatography/Mass } \\
\text { Spectroscopy Based on SW-846, Method } 8270 \mathrm{~A} \text { (Semi-voA) }\end{array}$ & $L A-523-406$ & $A=0$ & $\begin{array}{l}\text { Senivolatile Organics by Gas Chronatography/Mass } \\
\text { Spectroscopy Based on SH-846, Method } 82 \mathrm{roA} \text { (Semi-VOA) }\end{array}$ \\
\hline & Not cited & LA-505-158 & $c-0$ & $\begin{array}{l}\text { Acid Digestion of Aqueous Samples and Extracts for } \\
\text { Total Metals for Analysis by FLM and ICP Spectroseopy }\end{array}$ \\
\hline
\end{tabular}


WHE-SD-WM-DP-202, REV.1

\section{DETECTION LIMITS}

Detection limits were defined for each procedure without reference to a uniform laboratory protocol to determine such limits. Some of the procedures used the reagent blank value as the detection limit. Some procedures used the concentration of the lowest standard in the calibration curve as the detection limit, and others used the EPA replicate procedure. Generally, the sample matrix was not considered in generating the detection limit and therefore would be more indicative of an "instrument detection limit", rather than a "method detection limit". Wherever possible, the detection limit was modified by the typical dilution factor of the samples to provide a more representative value relative to the samples. All of the practices described above for estimation of the detection limit are allowed as estimated quantitation limits by $\mathrm{SW}-846$ protocol.

\section{SIGNIFICANT FIGURES}

A review was made of each controlled procedure to assure compliance with any stated significant figure requirements. Generally three significant figures were reported because data were formatted into scientific notation. Specific gravity was the only procedure for which significant figures were specified for reporting. For this analysis, the reported value must have three digits to the right of the decimal in standard numerical notation (not scientific notation) format. Specific gravity results were reported with the specified number of significant digits.

\section{CALIBRATION DATA}

Raw calibration data can be found on instrumentation printouts, which were incorporated in the raw data portion of this data package for reference.

None of the 222-S analytical procedures that were required for this project specified a required correlation coefficient $\left(r^{2}\right)$.

\section{EVAPORATOR NOTIFICATION LIMITS}

To evaluate compliance with safety requirements, limits were established, whereby if any analytical results exceeded these limits, the safety screen program was to be immediately notified. The analytical results are summarized in the sample Data Summary Tables section and evaluated in the Results of Analyses section. As stated in Reference 8 , none of the notification limits were exceeded, consequently no notifications were made.

From an evaluation of the AP-105 data against the evaporator operational limits, it was determined that the only limits that were exceeded were for calculated total organic carbon and ${ }^{226} \mathrm{Ra}$. Although all ${ }^{226} \mathrm{Ra}$ results were 
WHE $=$ SD-WM-DP-202, REV.1

less than the detection limit, the operational limit was exceeded only because that limit was less than the analytical detection limit.

Notification limits, as cited in the TSAP, Table 2, are shown in Table 5. 


\begin{tabular}{|c|c|}
\hline \multicolumn{2}{|c|}{ Table 5. Notification Limits for Tank AP-105 } \\
\hline Analytical Parameter & Limit Which if Exceeded Requires Notification \\
\hline Differential Scanning Calorimetry (DSC) & Exotherms $<335^{\circ} \mathrm{F}$, and $\mathbf{\Sigma}$ exotherms $+\Sigma$ endotherms $>1$ \\
\hline Visual Determination of Organic Layer & Presence of Organic Layer \\
\hline Specific Gravity & $>1.41$ \\
\hline Hydroxide & $<170 \mu \mathrm{g} / \mathrm{ml}$, or $>170,000 \mathrm{~kg} / \mathrm{ml}$ \\
\hline Nitrite & $<506$ or $>253,000 \mu \mathrm{g} / \mathrm{ml}$ \\
\hline Nitrate & $>341,000 \mu \mathrm{g} / \mathrm{ml}$ \\
\hline Phosphate & $>9,450 \mu \mathrm{g} / \mathrm{ml}$ \\
\hline Sodium & $>184,000 \mu \mathrm{g} / \mathrm{ml}$ \\
\hline Calculated Total Orgenic Carbon & Total Carbon - Totgl Inorganic Carbon $>87 \mu \mathrm{g} / \mathrm{ml}$ \\
\hline $\begin{array}{l}\text { Analytically Derived Total Organic } \\
\text { Carbon (surface sample only) }\end{array}$ & $>2,600 \mu \mathrm{g} / \mathrm{ml}$ \\
\hline Ammonia $\left(\mathrm{HH}_{3}\right)$ & $>5,000 \mu \mathrm{g} \mathrm{NH}{ }^{2} / \mathrm{ml}$ \\
\hline Acetone & $>87,000 \mu \mathrm{g} / \mathrm{L}$ \\
\hline 1-Butanol & $>226,000 \mathrm{\mu g} / \mathrm{L}$ \\
\hline 2-Butoxyethanol & $>95,200 \mu \mathrm{g} / \mathrm{L}$ \\
\hline 2-Butanone & $>58,000 \mu g / L$ \\
\hline n-Tributylphosphate & $>10,150,000 \mu g / \mathrm{L}$ \\
\hline U-gross & $239 / 240 \mathrm{Pu}+(1.077 \mathrm{E}-10 \times \mathrm{u}-\mathrm{gross})>0.005 \mathrm{~g} / \mathrm{L}$ \\
\hline Total Alpha & $\begin{array}{l}>(0.10 \times \text { Specific Gravity) as } \mu \mathrm{Ci} / \mathrm{ml} ;>41 \mu \mathrm{Ci} / \mathrm{ml} \text { (safety } \\
\text { screen) }\end{array}$ \\
\hline $239 / 240 \mathrm{Pu}$ & $239 / 240 \mathrm{Pu}+(1.077 \mathrm{E}-10 \times \mathrm{u}$-gross $)>0.005 \mathrm{~g} / \mathrm{L}$ \\
\hline $14 \mathrm{C}$ & $>0.26 \mu \mathrm{Ci} / \mathrm{ml}$ \\
\hline${ }^{79} \mathrm{se}$ & $>0.078 \mu \mathrm{Ci} / \mathrm{ml}$ \\
\hline${ }^{90} \mathrm{Sr}$ & $>220 \mu \mathrm{Ci} / \mathrm{ml}$ \\
\hline${ }^{60} \mathrm{Co}$ & $>1.2 \mu \mathrm{Ci} / \mathrm{ml}$ \\
\hline${ }^{137} \mathrm{Cs}$ & $>800 \mu \mathrm{Ci} / \mathrm{ml}$ \\
\hline${ }^{106} \mathrm{Ru}$ & $>53 \mu \mathrm{Ci} / \mathrm{ml}$ \\
\hline $134 \mathrm{cs}$ & $>15 \mu \mathrm{Ci} / \mathrm{ml}$ \\
\hline $94 \mathrm{Nb}$ & $>0.098 \mu \mathrm{Ci} / \mathrm{ml}$ \\
\hline $154 \mathrm{Eu}$ & $>5.0 \mu \mathrm{Ci} / \mathrm{mL}$ \\
\hline $155_{\mathrm{EU}}$ & $>7.0 \mu \mathrm{Ci} / \mathrm{ml}$ \\
\hline $226_{\mathrm{Ra}}$ & $>0.033 \mu \mathrm{Ci} / \mathrm{ml}$ \\
\hline${ }^{99} \mathrm{TC}$ & $>2 \mu \mathrm{Ci} / \mathrm{mL}$ \\
\hline${ }^{129} 1$ & $>0.0026 \mu \mathrm{Ci} / \mathrm{ml}$ \\
\hline $238_{P U}$ & $>0.0013 \mu \mathrm{Ci} / \mathrm{ml}$ \\
\hline $241_{A M}$ & $>1 \mu \mathrm{Ci} / \mathrm{ml}$ \\
\hline${ }^{244} \mathrm{~cm}$ & $>0.013 \mu \mathrm{Ci} / \mathrm{ml}$ \\
\hline
\end{tabular}


HF-SD-WM-DP-202, REV.1

\section{QUALITY CONTROL REQUIREMENTS}

\section{STANDARDS}

Laboratory control standards (LCS) are required to be analyzed with each batch of analyses for SW-846 procedures. 222-S Laboratory analyzes such standards, whenever available, with each analytical batch. These standards were used as an independent confirmation of proper calibration of the analytical system. LCS standards were prepared from materials traceable to National Institute of Standards and Technology (NIST) standards and were not used for instrument calibration. The LCS standards which were analyzed were normally generated in house by a special group within 222-S Laboratory using controlled procedures. Analyte concentrations of these standards were known in advance to the analysts.

LCS control limits were typically defined as the statistical mean plus or minus three standard deviations of the existing values in the data base. For those procedures which didn't have a data base large enough to provide statistically significant data, an administratively set control limit was used.

If a standard failed to meet the control criteria, all data associated with that batch were invalidated. A new batch, including the appropriate standard and quality control analyses, was rerun. The standard associated with the new batch must pass the standard acceptance criteria before data from that batch are able to be reported.

No LCS standard was available for the following analytes: appearance, ${ }^{106} \mathrm{Ru}$, ${ }^{134} \mathrm{Cs},{ }^{144} \mathrm{Ce},{ }^{154} \mathrm{Eu},{ }^{155} \mathrm{Eu},{ }^{94} \mathrm{Nb},{ }^{79} \mathrm{Se},{ }^{226} \mathrm{Ra},{ }^{238} \mathrm{Pu}$ and ${ }^{263 / 244} \mathrm{Cm}$.

\section{BLANKS}

A reagent blank was analyzed with each batch except for visual appearance, pH, specific gravity, TGA and DSC. A preparation blank was analyzed with each batch of acid digested samples.

Table 2 of the TSAP, required that field blanks be analyzed for all analytes except for DSC, TGA, pH, specific gravity, visual appearance, and total organic carbon (on the surface sample only).

The TSAP further required that the corresponding trip blank would be analyzed if any of the organic analytes, TC or TIC were observed in the field blanks.

The 242-A Evaporator Quality Assurance Project Plan (Reference 9), section 2.5, paragraph 13, defined blank contamination as follows. 
"Contamination of blanks is indicated when any analyte exceeds $20 \%$ of the lowest sample concentration in that batch. This criterion is not valid when the sample concentration is less than 10 times the detection limit for an analyte."

Because the samples were not processed in a hot cell, a hot cell blank was neither required nor analyzed.

\section{DUPLICATE ANALYSIS}

Precision quality control criteria were specified in the TSAP for each analyte. Generally, precision was determined as the relative percent difference (RPD) between the percent recoveries of a spike and its corresponding spike duplicate. However, precision for analytes which were not able to be spiked (e.g. DSC, TGA, $\mathrm{pH}_{15}$ Hydroxide, specific gravity, ${ }^{79} \mathrm{Se},{ }^{60} \mathrm{Co}$, ${ }^{137} \mathrm{Cs},{ }^{154} \mathrm{Eu},{ }^{106} \mathrm{Ru},{ }^{134} \mathrm{Cs},{ }^{144} \mathrm{Ce},{ }^{94} \mathrm{Nb},{ }^{155} \mathrm{Eu},{ }^{226} \mathrm{Ra},{ }^{238} \mathrm{Pu}$ and $\left.{ }^{243 / 244} \mathrm{Cm}\right)$ was based on the RPD between the sample and its corresponding duplicate. Rerun requirements were not specified by the TSAP when its precision criteria were not met. No duplicate analytical data were provided for those analyses which were visually based, such as Appearance.

\section{SPIKE/SPIKE DUPLICATE ANALYSIS}

To evaluate accuracy of data, the TSAP, Table 2, specified that samples be spiked for the following analytes: $\mathrm{NO}_{2}, \mathrm{NO}_{3}, \mathrm{~F}, \mathrm{PO}_{4}, \mathrm{SO}_{4}, \mathrm{Al}, \mathrm{Na}$, Total Inorganic Carbon (TIC), Total Organic Carbon (TOC), $\mathrm{NH}_{3}$, Acetone, 1-Butanol, 2-Butoxyethano1, 2-Butanone, 2-Hexanone, Methyl Isobutyl Ketone, 2-Pentanone, Tetrahydrofuran. Tributylphosphate $U$-gross total alpha, total beta, ${ }^{239} / 240 \mathrm{Pu},{ }^{241} \mathrm{Am},{ }^{3} \mathrm{H},{ }^{14} \mathrm{C},{ }^{69} \mathrm{Tc},{ }^{90} \mathrm{Sr},{ }^{129} \mathrm{I}$, and ${ }^{237} \mathrm{~Np}$.

A minimum of one spike and one spike duplicate was performed for each of the above procedures for the one sampling event. "Sampling event" was defined as all samples collected from one tank.

Accuracy criteria as determined by percent recovery were valid only when the analyte concentration in the spiked sample was increased by at least 25 percent more than the original sample concentration.

When precision criteria were based on the difference between the spike and spike duplicate, they were valid only when the analyte concentrations of the spike and spike duplicate were greater than ten times the detection limit. This allowed the precision evaluation to be made on analyte concentrations that were within the quantifiable range.

Because spiking standards were not available, neither spikes nor spike duplicates were performed for the following analytes: yisual appearance, DSC, ${ }_{15 A}$, pH. speçific gravity ${ }_{155}$ hydroxide, ${ }^{79} \mathrm{Se},{ }^{60} \mathrm{Co},{ }^{137} \mathrm{Cs},{ }^{106} \mathrm{Ru},{ }^{134} \mathrm{Cs},{ }^{144} \mathrm{Ce},{ }^{94} \mathrm{Nb}$, ${ }^{154} \mathrm{Eu},{ }^{155} \mathrm{Eu}$, ${ }^{226} \mathrm{Ra}$, and ${ }^{243 / 244} \mathrm{Cm}$. 
HNF-SD-WM-DP-202, REV.1

Accuracy control limits were specified in the TSAP for DSC and TGA. Percent recovery of laboratory control standards was used to evaluate accuracy for these analytes.

\section{RESULTS OF ANALYSES (DATA SUMMARY AND EVALUATION)}

\section{PHYSICAL ANALYSES}

\section{APPEARANCE/HONOGENEITY}

Observations were performed on the direct (unmodified) sample. No instruments were used, consequently there was no instrument calibration. No quality control criteria were specified in the TSAP.

Analyses were performed by procedure number LA-519-151/F-0 at approximately $24^{\circ} \mathrm{C}$ on $9 / 3 / 96$ and $9 / 10 / 96$. SW-846 does not define a holding time criteria for this parameter.

From the visual appearance, these liquid samples were essentially homogeneous. Each of the samples was clear with no observable solids. No organic phase was observed. Consequently the notification limit of "No Observable Organic Layer" was not exceeded. These samples did not require heating or dilution to maintain solubility. The samples were collected in amber glass bottles, making the observation of color not possible.

\section{DIFFERENTIAL. SCANNING CALORIMETRY (DSC)}

Analyses were performed in duplicate on the direct sample using procedure/revision number LA-514-113/C-1.

No unusual instances or problems occurred during the analyses of DSC. SW-846 protocol do not specify a hold time for DSC. Sample holding times ranged from 13 to 25 days.

The TSAP requires an LCS percent recovery of 80 to 120 percent. DSC analyses of LCS standards yielded recoveries of 93.5 and 99.5 percent, meeting the TSAP accuracy quality control limits.

Endotherms were observed as expected in the standards, as well as in all samples. The sample endotherms were due mainly or wholly to the presence of water. No blank was run for DSC, because it was unnecessary. The DSC instrument is sufficiently stable that any occurrence of baseline aberration is observable on the sample thermogram. No baseline drift was seen.

No exotherms were observed in any of the samples or their duplicates, thus none of the samples exceeded the TSAP specified precision criterion of \pm 20 RPD for exotherms. Sample average endotherms ranged from 1203 to $1803 \mathrm{~J} / \mathrm{g}$, and 
endotherm precision values were 27.5 RPD, 0.9 RPD , 14.2 RPD and 3.9 RPD for samples $1 C, 2 C 3 C$ and 4 , respectively.

Because no standard exists that can be spiked into the samples, the evaluation of spike accuracy was not possible.

No notification limit was exceeded. The TSAP notification limit for evaporator operations specified that the absolute value of the ratio of exotherm to endotherm could not exceed 1, nor could any exotherm exist which occurred at a temperature less than $335{ }^{\circ} \mathrm{F}$ (safety screen).

\section{THERMOGRAVIMETRIC ANALYSIS (TGA)}

All analyses were performed in a nitrogen atmosphere in duplicate on the direct sample, using procedure/revision number $[A-560-112 / B-1$. No unusual instances or problems occurred during the analyses. SW-846 protocol do not specify a hold time for TGA. Sample holding times ranged from 13 to 25 days.

The TSAP requires an LCS percent recovery of 80 to 120 percent. TGA analyses of the LCS standard yielded recoveries for both batches of 100.1 percent, meeting TSAP accuracy quality control criteria.

Average weight percent of water in the samples ranged from 69.1 to 69.8. The grand mean TGA value of all samples was 69.4 weight percent.

The TSAP specified precision criterion of \pm 20 RPD was met with precision values ranging from 0.1 to $0.7 \mathrm{RPD}$. No blank was run for TGA.

Because no standard exists that can be spiked into the samples, the evaluation of spike accuracy was not possible.

\section{SPECIFIC GRAVITY}

Analyses were performed on the direct samples using procedure/revision number LA-510-112/C-3. No unusual instances or problems occurred during the analyses. SW-846 protocol does not specify a sample holding time for specific gravity. The sample holding times ranged from 61 to 66 days.

The TSAP did not specify an accuracy limit, however, the LCS standard for specific gravity yielded a reasonable recovery of 98.6 percent.

No blank was run for specific gravity. Precision of the sample duplicates was acceptable with values ranging from 0.5 RPD to 0.9 RPD. The TSAP precision criterion was $\pm 20 \mathrm{RPD}$.

Because no standard exists that can be spiked into the samples, the evaluation of spike accuracy was not possible. 
HNE-SD-WM-DP-202, REV.1

Average specific gravity of samples $1 \mathrm{C}, 2 \mathrm{C}, 3 \mathrm{C}$ and 4 was $1.226,1.232,1.231$ and 1.242 , respectively, with a mean of 1.233 . The TSAP specified notification limit of $>1.41$ was not exceeded.

\section{INORGANIC ANALYSES}

\section{HYDROGEN ION ACTIVITY, pH (by pH Meter/Electrode)}

Analyses were performed on the direct samples using procedure/revision number LA-212-106/A-0. No unusual instances or problems occurred during the analyses. SW-846 protocol specify the hold time for $\mathrm{pH}$ as "analyze immediately", which could be met only as a field measurement. Sample holding times ranged from 73 to 78 days.

The TSAP did not specify an accuracy limit, but the LCS standard for pH yielded a recovery of 100.1 percent.

No blank was run for $\mathrm{pH}$. The duplicate analyses resulted in acceptable precision values ranging from 0.01 to $0.04 \mathrm{pH}$ units. The TSAP precision criterion was $\pm 0.3 \mathrm{pH}$ units.

Because no standard exists that can be spiked into the samples, the evaluation of spike accuracy was not possible.

The $\mathrm{pH}$ of samples $1 \mathrm{C}, 2 \mathrm{C}$ and $3 \mathrm{C}$ was $13.31,13.49$ and 13.45 , respectively. The TSAP notification 1 imit of $<8.0$ was not exceeded.

\section{HYDROXIDE (by Titration)}

Hydroxide was performed by procedure/revision number LA-211-102/C-0 on direct samples. There did not appear to be any analytical anomalies or difficulties during the analyses of this analyte.

Sample holding times ranged from 59 to 64 days. SW-846 did not specify a sample holding time for hydroxide, consequently sample holding time requirements were met.

The autotitrator $\mathrm{pH}$ was calibrated using standards of $\mathrm{pH} 7.00$ and 10.00 . During sample analyses, all titration endpoints were within the calibration range of $\mathrm{pH} 7$ to 10 (except for the blanks as expected, where the $\mathrm{pH}$ shift was extreme with a minute addition of $\mathrm{HNO}_{3}$ titrant).

Typical titration curves were seen in the analyses of tank AP-105 samples despite the presence of sample ammonia in the samples. In earlier work (tank $A P-101$ ), it was found that a cause of major interference with the analys is of hydroxide was the presence of high ammonia concentration. 


\section{HNF}

No spikes were required by the TSAP, and no spikes were analyzed. The TSAP did not specify accuracy control limits.

Precision between the samples and sample duplicates ranging from 0.3 to 1.6 RPD was acceptable (less than the TSAP specified maximum control limit of \pm 20 RPD).

Although reagent blanks were analyzed, the procedure uses them to correct sample values, consequently it is not possible to determine an independent analytical value for a reagent blank because it is subtracted from itself to yield a result of zero. The field blank hydroxide concentration was less than the detection limit, and was determined to not be contaminated.

Sample hydroxide concentrations ranged from 31,800 to $36,100 \mu \mathrm{g} / \mathrm{ml}$. The mean hydroxide concentration of the three samples was $33,500 \mu \mathrm{g} / \mathrm{ml}$.

\section{AMMONIA (by Ion Selective Electrode)}

Ammonia analyses were performed using procedure/revision number LA-631-001/B-2 on direct samples. There did not appear to be any analytical anomalies or difficulties during the analyses.

SW-846 does not specify a sample holding time for ammonia, but should be as short as possible to prevent losses due to a high pH sample matrix. Sample holding times ranged from 71 to 76 days. For ammonia, the accepted method of preservation is to acidify samples at the time of collection to $\mathrm{pH}<2$ with nitric acid. Time was intentionally not spent to preserve these samples, so as to limit the exposure of sample collectors to excessive radiation dosage. Although biodegradation was not expected to be a significant factor for ammonia decomposition in these samples due to the high level of radioactivity, which is lethal to microorganisms, it was expected and quite likely that, due to the high $\mathrm{pH}$ of these samples, sample degradation could occur due to chemical reactions. At higher $\mathrm{pH}$, ionic ammonia reacts with hydroxide to generate $\mathrm{NH}_{3}$, which is volatile and is readily dissipated at ambient temperature. The hydroxide concentration of these samples was sufficient to cause such losses. Thus due to sample matrix conditions, it is possible that these ammonia data could be biased low.

LCS recovery was acceptable at 91.6 percent.

Spike accuracies were acceptable with percent recoveries ranging from 105 to 123 , as evaluated against the TSAP specified accuracy criteria of 75 to 125 percent recovery.

Precision was determined to be acceptable with 5 RPD between the spike and its duplicate. The TSAP specified limit for precision was \pm 20 RPD.

Ammonia concentration was less than the detection limit for the field blank and for the reagent blank, indicating the absence of blank contamination. 
WNE-SD-WM-DP-202, REV.1

Sample concentrations were low, ranging from 20 to $64.8 \mu \mathrm{g} \mathrm{NH} / 3 / \mathrm{ml}$, with a mean concentration of $49.3 \mu \mathrm{g} \mathrm{NH} / \mathrm{ml}$.

\section{ION CHROMATOGRAPHY}

All ion chromatography (IC) analyses were performed on the direct samples, using procedure/revision number LA-533-105/D-1. As was discussed above, tank AP-105 samples were not preserved. Due, however, to the characteristic high $\mathrm{pH}$ and radioactivity of the waste, the samples were not likely to be subject to biodegradation, which is generally the greatest source of nitrate deterioration.

The procedure detection limit, as shown in the summary tables for all of the IC analytes, was set at the concentration equivalent to the lowest standard within the calibration curve multiplied by the dilution factor. The sample detection limit was generated by multiplying the above detection limit by an additional factor based on the sample aliquot that was injected into the IC (that is to say, 11 or 101).

\section{FLUORIDE}

There did not appear to be any analytical anomalies or difficulties during the analyses of this analyte. Sample holding times ranged from 59 to 64 days. SW-846 does not specify a holding time for fluoride.

LCS recovery was acceptable with a value of 92.9 percent.

The accuracy control limits were 75 to 125 percent recovery. Fluoride accuracy was acceptable with a spike recovery of 112.2 percent.

Precision between the spike and spike duplicate was acceptable with a value of 12.4 RPD, meeting the TSAP acceptance 1 imits of $\pm 20 \%$.

The reagent blanks and field blank fluoride concentrations were less than the detection limits of 0.012 and $0.072 \mu \mathrm{g} / \mathrm{ml}$, respectively, indicating the absence of contamination.

Sample fluoride concentrations ranged from 254 to $348 \mu \mathrm{g} / \mathrm{ml}$ with a mean concentration of $308 \mu \mathrm{g} / \mathrm{ml}$.

\section{NITRATE}

There did not appear to be any analytical anomalies or difficulties during the analyses of this analyte. Sample holding times ranged from 59 to 64 days. SW-846 specifies a holding time for nitrate of 48 hours, consequently none of the sample holding times were met. It should be noted that for handling high 


$$
\text { WHE-SD-WM-DP-202, REV.1 }
$$

radiation dosage samples, the required operational procedures make attaining analytical results on protocol analyses within 48 hours quite unlikely.

LCS recovery was acceptable with a value of 101.0 percent.

Spike accuracy was acceptable with a recovery of 100.5 percent recovery. The TSAP specified accuracy acceptance limits were 75 to 125 percent recovery.

Precision between the spike and spike duplicate met the program's precision criterion of $\pm 20 \%$, by yielding a value of 2.0 relative percent difference.

The reagent blank nitrate concentrations were less than the detection limit of $0.139 \mu \mathrm{g} / \mathrm{ml}$, and were determined to not be contaminated. The field blank was very slightly greater than the detection limit with a concentration of 0.863 $\mu \mathrm{g} / \mathrm{ml}$. It was determined to not be contaminated.

Sample nitrate concentrations ranged from 95,000 to $102,000 \mu \mathrm{g} / \mathrm{ml}$, with a mean of $99,300 \mu \mathrm{g} / \mathrm{ml}$.

\section{NITRITE}

There did not appear to be any analytical anomalies or difficulties during the analyses of this analyte.

Sample holding times ranged from 59 to 64 days. SW-846 does not specify a sample holding time for nitrite. It is known, nonetheless, that in the environment, nitrite is chemically less stable than nitrate, suggesting that at least for environmental samples, a sample holding time of short duration is reasonable. Tank AP-105 matrix is significantly different from that encountered environmentally, and may, however, have such conditions that nitrite could be chemically stable. Nitrite concentrations are expected to change through oxidation-reduction reactions as a corrosion inhibitor.

LCS recovery was acceptable with a value of 93.0 percent.

Accuracy, as indicated by recovery of the spike, was acceptable with a recovery of 99.8 percent. The accuracy control 1 imits were 75 to 125 percent recovery.

Precision between the spike and spike duplicate met the acceptance criterion of $\pm 20 \%$, where the value was 9.7 RPD.

The reagent blanks and field blank nitrite concentrations were less than the detection limits of 0.108 and $0.648 \mu \mathrm{g} / \mathrm{ml}$, respectively. These blanks were determined to not be contaminated.

Sample nitrite concentrations ranged from 45,700 to $48,100 \mu \mathrm{g} / \mathrm{ml}$, with a mean of $46,900 \mu \mathrm{g} / \mathrm{ml}$. 


\section{HFE-SD-WM-DP-202, REV.1}

PHOSPHATE (ORTHO-PHOSPHATE)

There did not appear to be any analytical anomalies or difficulties during the analyses of this analyte.

Sample holding times ranged from 59 to 64 days. SW-846 does not specify a holding time for phosphate.

LCS recovery was acceptable with a value of 99.4 percent.

Accuracy was acceptable as indicated by recovery of the spike with a value of 93.6 percent. The accuracy control 1 imits were 75 to 125 percent recovery.

Precision between the spike and spike duplicate was acceptable with a value of 6.0 RPD. The TSAP specified precision acceptance limits were $\pm 20 \mathrm{RPD}$.

The reagent blanks and field blank phosphate concentrations were less than the detection limits of 0.12 and $0.72 \mu \mathrm{g} / \mathrm{ml}$, respectively, and were determined to not be contaminated.

Sample phosphate concentrations ranged from 1,150 to $1,540 \mu \mathrm{g} / \mathrm{ml}$ with a mean of $1,360 \mu \mathrm{g} / \mathrm{ml}$.

\section{SULFATE}

There did not appear to be any analytical anomalies or difficulties during the analyses of this analyte.

Sample holding times ranged from 59 to 64 days. The SW-846 holding time limit of 28 days was exceeded for the all samples.

LCS recovery was acceptable with a value of 103.0 percent.

Accuracy was acceptable as indicated by a percent recovery of 99.8 for the spike. The TSAP accuracy control 1 imits were 75 to 125 percent recovery.

Precision between the spike and spike duplicate was acceptable with a value of $1.3 \mathrm{RPD}$. The TSAP specified precision acceptance limits were \pm 20 RPD.

The reagent blank sulfate concentration was less than the detection limit of $0.138 \mu \mathrm{g} / \mathrm{ml}$. Although the field blank concentration was significantly greater than the detection limit, it was much less than $20 \%$ of the lowest sample concentration. Therefore, all blanks were determined to not be contaminated.

Sample sulfate concentrations ranged from 1,930 to $2,060 \mu \mathrm{g} / \mathrm{ml}$ with a mean of $2,180 \mu \mathrm{g} / \mathrm{ml}$. 
HNE-SD-WM-DP-202, REV.1

\section{TOTAL CARBON, TC (by Combustion and Coulometry)}

Total carbon (TC) analyses were performed on direct samples using procedure/revision number LA-344-105/D-1. Total carbon analys is is a subset of the procedure for total organic carbon. A maximum sample holding time for total carbon was not specified in SW-846 protocol, but is set at 28 days for total organic carbon. The actual sample holding times for TC ranged from 43 to 48 days.

The LCS recovery was acceptable with a value of 94.7 percent.

Accuracy for the spike was acceptable with a percent recovery of 107.7 . The TSAP specified range of acceptance was 75 to 125 percent recovery.

Precision between the spike and spike duplicate was acceptable with a value of $0.7 \mathrm{RPD}$. The TSAP specified range of acceptance was $\pm 20 \mathrm{RPD}$.

The field blank total carbon concentration was almost ten times the detection 1 imit of $5.5 \mu \mathrm{g} \mathrm{C} / \mathrm{ml}$, however, it was much less than $20 \%$ of the lowest sample concentration. Therefore it was determined to not be contaminated. The reagent blank concentration was detectable at the detection limit. The detection limit shown on the data summary sheet was based on a standard volume of 0.20 milliliters (an optimal value). Generally the least amount of carbon detectable was $1 \mu \mathrm{g}$. Thus, one microgram of carbon divided by $0.20 \mathrm{ml}$ equaled $5.00 \mu \mathrm{g} \mathrm{C} / \mathrm{ml}$.

Total carbon concentrations of the samples ranged from $4,650 \mu \mathrm{g} \mathrm{C} / \mathrm{ml}$ to 4,810 $\mu \mathrm{g} \mathrm{C} / \mathrm{ml}$. The mean concentration of the three samples was $4,710 \mu \mathrm{g} / \mathrm{ml}$.

\section{TOTAL INORGANIC CARBON, (CARBONATE) (by Coulometry)}

Total inorganic carbon (TIC) analyses were performed on direct samples with procedure/revision number $L A-342-100 / E-0$. There did not appear to be any analytical anomalies or difficulties during the analyses of this analyte.

A maximum sample holding time for this analyte was not specified in $\mathrm{SW}-846$ protocol, but the actual holding times ranged from 64 to 69 days.

The LCS recovery was acceptable with a value of 98.7 percent.

Accuracy for the spikes were acceptable with recoveries ranging from 100.6 to 105.3 percent. The TSAP specified range of acceptance was 75 to 125 percent recovery.

Precision between the spike and spike duplicate was acceptable with an RPD of 2.5. The TSAP specified range of acceptance was $\pm 20 \mathrm{RPD}$.

The field blank carbon concentration was slightly greater than the detection limit of $5 \mu \mathrm{g} \mathrm{C} / \mathrm{ml}$. The reagent blanks had a TIC concentration of $1.90 \mu \mathrm{g}$ 


\section{SD-WM-DP-202, REV.1}

$\mathrm{C} / \mathrm{ml}$, which was much less than $20 \%$ of the lowest sample concentration. Consequently all blanks were determined to not be contaminated.

In calculating the sample concentrations, an instrument blank value was subtracted from the sample value. Values shown on the data summary sheet for each reagent blank were uncorrected because, if the result blank value was subtracted from the instrument blank, it could yield a corrected blank value less than zero.

Carbonate (TIC) concentrations of the samples ranged from $2,510 \mu \mathrm{g} \mathrm{C} / \mathrm{m} 1$ to $2,740 \mu \mathrm{g} \mathrm{C} / \mathrm{ml}$. The mean concentration of the three samples was $2,680 \mu \mathrm{g} \mathrm{C} / \mathrm{ml}$.

\section{TOTAL ORGANIC CARBON, TOC (by Combustion and Coulometry)}

Total organic carbon (TOC) analyses were performed on direct samples using procedure/revision number LA-344-105/D-1.

A maximum sample holding time of 28 days was specified in SW-846 protocol for total organic carbon. The sample holding time was not met with holding times ranging from 42 to 47 days.

There did not appear to be any analytical anomalies or difficulties during the analyses of this analyte

LCS recovery was acceptable with a value of 94.0 percent.

Accuracy for the spike was acceptable with a percent recovery of 90.2. The TSAP specified range of acceptance was 75 to 125 percent recovery.

Precision between the spike and spike duplicate was acceptable with an RPD of 0.8 . The TSAP specified range of acceptance was \pm 20 RPD.

The reagent blank's total organic carbon concentration was $0.70 \mu \mathrm{c} / \mathrm{ml}$. Using the definition of contamination provided in Reference 9 , as discussed in the "Blanks" section above, it was determined that the blank was not contaminated because the TOC concentration of the blank was less than $20 \%$ of the lowest sample concentration $(1,440 \mu \mathrm{g} C / \mathrm{ml})$. The field blank was only slightly greater than the detection 1 imit. Therefore, both blanks were determined to not be contaminated.

TOC concentrations of the samples, including the tank surface sample, ranged from $1,440 \mu \mathrm{g} \mathrm{C} / \mathrm{ml}$ to $1,530 \mu \mathrm{g} \mathrm{C} / \mathrm{ml}$. The mean concentration of the four samples was $1,490 \mu \mathrm{g} \mathrm{C} / \mathrm{ml}$.

\section{CALCULATED TOTAL ORGANIC CARBON}

The analysis of total organic carbon by direct oxidation is subject to potential underestimation of the total concentration of organic carbon due to 
losses of purgable (volatile) organics (if present at a significant concentration) during the acidified sparging phase of sample preparation. The total organic carbon concentration of samples can also be obtained as the difference in concentrations between two individual analyses: total carbon and total inorganic carbon (carbonate). This calculation method is useful as a comparative check against TOC by direct oxidation, as shown in Table 6 .

Table 6. Comparison of Calculated TOC with Analytically Derived TOC

\begin{tabular}{|c|c|c|c|c|c|}
\hline $\begin{array}{l}\text { Sample ID } \\
\text { Number }\end{array}$ & $\begin{array}{c}\text { Sample } \\
\text { Description }\end{array}$ & $\begin{array}{c}\text { Total } \\
\text { Carbon } \\
\mu \mathrm{C} / \mathrm{ml}\end{array}$ & $\begin{array}{c}\text { Total } \\
\text { Inorganic } \\
\text { Carbon } \\
\mu \mathrm{g} \mathrm{C} / \mathrm{ml}\end{array}$ & $\begin{array}{c}\text { Calculated } \\
\text { TOC } \\
\text { (TC-TIC) } \\
\mu \mathrm{g} \text { C/ml }\end{array}$ & $\begin{array}{c}\text { TOC by } \\
\text { Direct } \\
\text { oxidation } \\
\mu \mathrm{g} \mathrm{C} / \mathrm{ml}\end{array}$ \\
\hline S96V000058 & $\begin{array}{c}\text { 5AP-96-IB } \\
\text { field blank }\end{array}$ & 50.6 & 7.0 & 46.6 & 7.7 \\
\hline $596 V 000047$ & $5 A P-96-1 C$ & 4670 & 2740 & 1930 & 1500 \\
\hline S96V000048 & $5 A P-96-2 C$ & 4650 & 2780 & 1870 & 1480 \\
\hline S96V000049 & $5 A P-96-3 C$ & 4810 & 2510 & 2300 & 1530 \\
\hline
\end{tabular}

The notification limit for calculated TOC was $>87 \mu \mathrm{g} \mathrm{C} / \mathrm{ml}$. The limit was exceeded by all tank samples. From Table 6 , it appears that although the differences between calculated TOC and TOC analys is results were rather large, it is possible that they are within expected experimental error. It was inferred from these data that the amount of purgable (volatile) organic material lost during TOC analyses ranged from about 400 to $800 \mu \mathrm{g} \mathrm{C} / \mathrm{ml}$ per sample.

\section{INDUCTIVELY COUPLED PLASMA/ENISSION SPECTROSCOPY (by ICP)}

An acid predigestion was performed on ICP samples prior to analysis. Analyses were performed by procedure/revision numbers LA-505-161/B-1. There did not appear to be any analytical difficulties during analysis of these analytes. The only anomaly that occurred for the ICP analysis was that a spike duplicate was not prepared nor analyzed. Although this is contrary to the direction of the TSAP, it did not cause the data for aluminum and sodium to be rejected. This occurred because both of these metals exceeded the concentration limit for which spiking was feasible. As an alternative, a serial dilution was provided for these analytes to give some measure of the test's accuracy.

All metals other than aluminum and sodium were reported voluntarily, and would have been required only if secondary safety screening analyses were required. Because these results were not required by the TSAP, there was no requirement to hold these data to TSAP's spike duplicate criterion. Duplicate sample 


\section{WE-SD-WM-DP-202, REV.1}

analyses were provided as a measure of precision for each sample, however there was no requirement to do so in the TSAP.

Sample holding times for ICP analyses ranged from 62 to 67 days. SW-846 protocol require that the holding time for these metals may not exceed six months. All ICP analyses were completed within the required holding time.

An undigested blank and standard was used to initialiy calibrate the ICP instrument and to check calibration on a continuing basis. A digested reagent blank included with each batch, however, was used to determine the extent of blank contamination introduced during sample preparation (and by inference, the estimated amount of sample contamination due to digestion).

ICP accuracy evaluation criteria for LCS standards were based on the undigested initial calibration verification (ICV) standards.

Interelement data corrections were automatically performed for spectral interferences from calcium on iron, manganese, and silicon; from chromium on iron, silicon and uranium; from iron on chromium, and manganese; from potassium on silicon; from sodium on silicon and nickel; from antimony on nickel; and from uranium on aluminum, chromium, iron, manganese, nickel, and silicon.

The linear concentration range was determined for ICP analytes. The maximum concentration within the linear range (defined as the highest concentration in which the percent recovery of a standard deviates less than five percent from 100 percent with a 5 second signal integration time) was $1,000 \mu \mathrm{g} / \mathrm{ml}$ for aluminum and $1000 \mu \mathrm{g} / \mathrm{ml}$ for sodium.

Although the elements, chromium, iron, Manganese, nicke1, silicon and uranium, were not required to be analyzed (because they were not primary safety screening analytes), they were analyzed with aluminum and sodium to maximize analytical efficiency in the case that data for the secondary analytes would be required.

\section{ALUMINUM}

Percent recovery of the undigested LCS standard was acceptable with a value of 89.8 percent recovery.

Spike accuracy was not acceptable with value of 66.6 percent recovery, however this measure was invalid because the sample concentration exceeded $1000 \mu \mathrm{g} / \mathrm{ml}$. The TSAP specified acceptance limits for a spike were 75 to 125 percent

recovery. A serial dilution was provided as an alternative to the spike. The serial dilution was acceptable ( $<5 \%$ difference) with a 2.3 percent difference.

Precision between the samples and their duplicates was good with values

ranging from 0.6 to 6.1 relative percent difference. The TSAP specified 1 imit 


\section{HWF-SD-WM-DP-202, REV.1}

for spike duplicate precision was \pm 20 RPD, but was not specified between the samples and their duplicates.

The aluminum concentration of the preparation blank was less than the detection limit, and was only slightly greater than the detection limit for the field blank, indicating the absence of contamination.

Average aluminum concentrations of the samples ranged from 17,200 to 17,500 $\mu \mathrm{g} / \mathrm{ml}$, with a mean of $17,400 \mu \mathrm{g} / \mathrm{ml}$.

\section{CHROMIUM}

Percent recovery of the undigested LCS standard was acceptable with a value of 88.6 percent recovery.

Spike accuracy was acceptable with a value of 89.6 percent recovery. The TSAP was not explicit in specifying acceptance limits, however it was assumed that they were consistent with those for aluminum and sodium, which were 75 to 125 percent recovery.

Precision between the samples and their duplicates was good with values ranging from 0.0 to 5.2 relative percent difference. The TSAP did not specify precision acceptance limits between samples and their duplicates. It was also not explicit in specifying acceptance limits for spike duplicate precision, however it was assumed that they were consistent with those for aluminum and sodium, which were \pm 20 RPD.

The chromium concentrations of the field and preparation blanks were less than the detection limit, indicating the absence of contamination.

Average chromium concentrations of the samples ranged from 210 to $214 \mu \mathrm{g} / \mathrm{ml}$, with a mean of $213 \mu \mathrm{g} / \mathrm{ml}$.

\section{IRON}

Percent recovery of the undigested LCS standard was acceptable with a value of 91.0 percent recovery.

Spike accuracy was acceptable with a value of 91.8 percent recovery. The TSAP was not explicit in specifying acceptance limits, however it was assumed that they were consistent with those for aluminum and sodium, which were 75 to 125 percent recovery.

Precision between the samples and their duplicates was not able to be determined because all values were less than the detection limit. The TSAP was not explicit in specifying acceptance limits for spike duplicate precision, however it was assumed that they were consistent with those for aluminum and sodium, which were $\pm 20 \mathrm{RPD}$. 


\section{垈E-SD-WM-DP-202, REV.1}

The iron concentrations of the field and preparation blanks were also less than the detection limit, indicating the absence of contamination.

Because all sample concentrations were less than the detection limit, the average iron concentrations of the samples were not able to be calculated. A11 sample iron concentrations were less than $12.5 \mu \mathrm{g} / \mathrm{ml}$.

\section{MANGANESE}

Percent recovery of the undigested LCS standard was acceptable with a value of 86.4 percent recovery.

Spike accuracy was acceptable with a value of 87.0 percent recovery. The TSAP was not explicit in specifying acceptance 1 imits, however it was assumed that they were consistent with those for aluminum and sodium, which were 75 to 125 percent recovery.

Precision between the samples and their duplicates was not able to be determined because all values were less than the detection limit. The TSAP was not explicit in specifying acceptance 1 imits for spike duplicate precision, however it was assumed that they were consistent with those for aluminum and sodium, which were \pm 20 RPD.

The manganese concentrations of the field and preparation blanks were also less than the detection limit, indicating the absence of contamination.

Because all sample concentrations were less than the detection limit, the average manganese concentrations of the samples were not able to be calculated. All sample manganese concentrations were less than $2.5 \mu \mathrm{g} / \mathrm{ml}$.

\section{SODIUN}

Recovery of the undigested LCS standard was acceptable with a value of 104.6 percent recovery.

When sample sodium concentrations exceed $1000 \mu \mathrm{g} / \mathrm{ml}$, the normal control 1 imits for accuracy determination are not applicable. This occurs because the instrument's detector is overwhelmed with the intensity of the signal at extremely high analyte concentrations, causing detector insensitivity to even large concentration differences. The sodium concentration of each sample was determined to exceed $1000 \mu \mathrm{g} / \mathrm{ml}$, consequently an alternative evaluation of accuracy was applied using serial dilution. Following serial dilution a dilution RPD value was determined. The formula for this calculation follows:

$$
\text { Dilution RPD }=\frac{[\text { initial conc. }]-([\text { serial di]'n conc. }] \times \text { dilution factor })}{\text { [initial concentration] }} \times 100
$$


Using a dilution rather than a spike has advantages and disadvantages as a data quality evaluation tool. Although an evaluation of the deviation between the actually derived concentration and the expected concentration following dilution does not definitively indicate the degree of matrix interference within a sample, it does establish whether or not the analysis was performed within the linear portion of the calibration curve. It should be understood, however, that matrix interference is generally insignificant when the analyte is present in such high concentrations in the sample. Conversely, a spike is not particularly useful when the initial sample concentration is very high. To be distinguishable above the initial sample concentration, spiking must generate a final concentration at least 25 percent greater than the initial concentration, yet this frequently places the analyte concentration within the region of calibration non-linearity. The result is that percent recovery is significantly underestimated. For example, accuracy for sample $1 \mathrm{C}$ was not acceptable as indicated by the spike recovery of -3.4 percent. As an al ternative, the serial dilution method was applied to sodium (and to a) uminum, the only ICP analytes in which the sample concentrations exceeded $1000 \mu \mathrm{g} / \mathrm{ml})$. Dilution RPD values less than five percent indicate that measurements are within the linear portion of the calibration curve. Sample 1C had a serial dilution value of 2.7 percent difference, which was within the 5\% acceptance limit.

Precision between the spike and spike duplicate was meaningless and was consequently not determined. The TSAP specified criterion for precision was $\pm 20 \mathrm{RPD}$. Precision was determined, however, between the tank samples and their duplicates, where the values ranged from 0.0 to 11.5 RPD.

The preparation blank concentration was about five orders of magnitude less than the mean of sample values, indicating the absence of contamination. The field blank's sodium concentration was three orders of magnitude less than the lowest mean sample concentration, consequently it too was determined to not be contaminated.

Average sample sodium concentrations ranged from 112,000 to $113,000 \mu \mathrm{g} / \mathrm{ml}$, with a grand mean of $113,000 \mu \mathrm{g} / \mathrm{m} 1$.

\section{NICKEL}

Percent recovery of the undigested LCS standard was acceptable with a value of 91.2 percent recovery.

Spike accuracy was acceptable with a value of 92.4 percent recovery. The TSAP was not explicit in specifying acceptance limits, however it was assumed that they were consistent with those for aluminum and sodium, which were 75 to 125 percent recovery.

Precision between the samples and their duplicates was not able to be determined because all values were less than the detection limit. The TSAP 
was not explicit in specifying acceptance limits for spike duplicate precision, however it was assumed that they were consistent with those for aluminum and sodium, which were $\pm 20 \mathrm{RPD}$.

The nickel concentrations of the field and preparation blanks were less than the detection limit, indicating the absence of contamination.

Because the concentrations for the samples were less than the detection limit, the grand average nickel concentration was not able to be calculated. All sample concentrations were less than $5.0 \mu \mathrm{g} / \mathrm{ml}$.

\section{SILICON}

Percent recovery of the undigested LCS standard was acceptable with a value of 97.6 percent recovery.

Spike accuracy was unacceptable with a value of 65.4 percent recovery. The TSAP was not explicit in specifying acceptance 1 imits, however it was assumed that they were consistent with those for aluminum and sodium, which were 75 to 125 percent recovery. Significant error can occur in this procedure due to digestion of the samples in glassware which contains silicone.

Precision between the samples 2C, 3C, 4 and IB and their duplicates was good, ranging from 1.0 to 8.6 relative percent difference. The RPD of 24.6 for sample 1C was not good. The TSAP was not explicit in specifying acceptance limits for precision, however it was assumed that they were consistent with those for aluminum and sodium, which were \pm 20 RPD for the spike duplicate.

The silicon concentration of the preparation blank was less than the detection limit, indicating the absence of contamination. The field blank was only 85 percent of the lowest sample concentration. This is interpreted to mean that the samples were impacted by the field conditions (presumably the glass container itself) and the true concentration is likely to be significantiy less than the analytically determined value.

Average silicon concentrations of the samples ranged from 84.0 to $88.0 \mu \mathrm{g} / \mathrm{ml}$, with a grand mean of $85.3 \mu \mathrm{g} / \mathrm{ml}$.

\section{URANIUM}

Percent recovery of the undigested LCS standard was acceptable with a value of 85.6 percent recovery.

Spike accuracy was acceptable with a value of 88.2 percent recovery. The TSAP was not explicit in specifying acceptance limits, however it was assumed that they were consistent with those for aluminum and sodium, which were 75 to 125 percent recovery. 
Precision between the samples and their duplicates was not able to be determined because all values were less than the detection limit. The TSAP was not explicit in specifying acceptance limits for spike duplicate precision, however it was assumed that they were consistent with those for a 7 uminum and sodium, which were \pm 20 RPD.

The uranium concentrations of the field and preparation blanks were less than the detection limit, indicating the absence of contamination.

Because the concentrations of the samples were less than the detection limit, the grand average uranium concentration of all samples was not able to be calculated. Al1 uranium concentrations were less than $125 \mu \mathrm{g} / \mathrm{ml}$.

\section{TOTAL URANIUM (by Kinetic Phosphorescence)}

The chemical (not radiochemical) analyses for total uranium were performed on direct samples using procedure/revision number LA-925-009/A-1. There did not appear to be any analytical anomalies or difficulties during the analyses of this analyte.

Sample holding times for uranium ranged from 14 to 59 days. A maximum sample holding time was not specified for this analyte in SW-846 protocol.

The LCS standard recoveries were acceptable with values ranging from 100.2 to 104.5 percent.

Spike accuracy was determined for three samples. All spikes were acceptable with results ranging from 87.4 to 108.8 percent recovery. The TSAP specified requirement for spike accuracy was 70 to 130 percent recovery.

Precision between the three spikes and spike duplicates was acceptable with values ranging from 1.8 to 5.5 relative percent difference. The TSAP specified criterion for precision was $\pm 20 \mathrm{RPD}$.

The preparation blank concentration was two orders of magnitude less than the mean of sample concentrations, and the field blank was less than the detection limit, indicating the absence of contamination.

Average sample concentrations for total uranium ranged from $14.1 \mu \mathrm{g} / \mathrm{ml}$ to 18.5 $\mu \mathrm{g} / \mathrm{ml}$, with a mean of $16.4 \mu \mathrm{g} / \mathrm{ml}$. These data were consistent with the analytical results for uranium by ICP.

\section{RADIOCHEMICAL ANALYSES}

\section{TOTAL ALPHA (by Proportional Counter)}

Total alpha analyses were performed on acid predigested samples (assuring that the analyte was fully dissolved to facilitate analyte detection) using 


\section{WE-SD-WM-DP-202, REV.1}

procedure/revision number LA-508-101/E-1. Sample holding times ranged from 57 to 62 days. The required maximum sample holding time for total alpha activity as specified in SW-846 protocol is six months. Tank AP-105 analyses for total alpha were analyzed within the required holding time. There were no analytical anomalies or difficulties.

LCS standard recovery was acceptable with a value of 103.0 percent recovery.

The accuracy of the spike (performed on the field blank) was acceptable with a recovery of 87.7 percent. The TSAP specified criterion for spike recovery was 70 to 130 percent.

Precision between the spike and spike duplicate was acceptable with 4.1 RPD. Precision between the tank samples and their duplicates was not able to be calculated because all sample activities were less than the detection limit. The TSAP specified criteria for precision for both the spike/spike duplicate and the samples/sample duplicates was \pm 25 RPD.

Alpha activities of the field blank and reagent blanks for the tank samples were less than the detection limit, indicating the absence of contamination for all blanks.

Al1 sample total alpha activities less than their detection limits, ranging from 0.00505 to $0.0102 \mu \mathrm{Ci} / \mathrm{ml}$. The notification limit specified in the TSAP was to be calculated as ">0.10 x specific gravity". Table 7 compares the calculated notification limit against sample alpha activities. The alpha notification limit was not exceeded for any of the samples.

\begin{tabular}{||c||c|c|c||}
\hline \multicolumn{2}{|c|}{ Table 7. Comparison of Alpha Activities and Action Limits } \\
\hline \hline Sample Number & $\begin{array}{c}\text { Average Specific } \\
\text { Gravity }\end{array}$ & $\begin{array}{c}\text { Calculated } \\
\text { Notification } \\
\text { Limit }\end{array}$ & $\begin{array}{c}\text { Sample Alpha } \\
\text { Activity, } \\
\mu C i / m i\end{array}$ \\
\hline \hline $5 A P-96-1 C$ & 1.226 & 0.1226 & $<0.0102$ \\
\hline $5 A P-96-2 C$ & 1.232 & 0.1232 & $<0.0102$ \\
\hline $5 A P-96-3 C$ & 1.231 & 0.1231 & $<0.00505$ \\
\hline $5 A P-96-4$ & 1.242 & 0.1242 & $<0.00505$ \\
\hline
\end{tabular}

\section{TOTAL BETA (by Proportional Counter)}

Total beta analyses were performed on acid predigested samples (assuring that the analyte was fully dissolved to facilitate analyte detection) using procedure/revision number LA-508-101/E-1. Sample holding times ranged from 57 to 62 days. The required maximum sample holding time for total beta activity 
WFE-SD-WM-DP-202, REV. 1

as specified in SW-846 protocol is six months. Tank AP-105 analyses for total beta were analyzed within the required holding time. There were no analytical anomalies or difficulties.

LCS standard recovery was acceptable with a value of 100.3 percent recovery.

Accuracy for the total beta spike (performed on the field blank) was acceptable with 101.3 percent recovery. The TSAP specified limits for accuracy were 70 to 130 percent recovery.

Precision between the spike and spike duplicate was acceptable with 3.9 relative percent difference. The TSAP specified limits for precision between spikes was \pm 25 RPD. Precision between the samples and their duplicates ranged from 0.0 to 5.5 RPD. Precision between the field blank and its duplicate yielded a $31.9 \mathrm{RPD}$, exceeding the $\pm 25 \mathrm{RPD} 1 \mathrm{imit}$. However, the blank's activity was only slightly greater than the detection limit and may be attributable to counting error, thus the result is invalid.

The field blank had an activity that was slightly greater than the detection limit. The preparation blank beta activity was three orders of magnitude less than the sample activities. It was determined that neither blank was contaminated.

Average beta activities for the samples ranged from 108 to $111 \mu \mathrm{Ci} / \mathrm{ml}$, with an average activity of $109 \mu \mathrm{Ci} / \mathrm{ml}$.

\section{GAMMA ENERGY ANALYSES (GEA)}

Samples were acid predigested and analyzed using procedure/revision number LA548-121/E-0. Sample holding times ranged from 77 to 82 days. Except for radium-226 with a maximum holding time of six months, there were no specified maximum holding times in SW-846 protocol. A11 GEA analytes were, therefore, within the required holding time criteria. There did not appear to be any analytical anomalies or difficulties during these analyses.

The GEA procedure does not use an LCS quality control standard for every isotope; the LCS standard contained only ${ }^{60} \mathrm{Co}$ and ${ }^{137} \mathrm{Cs}$. Qual ity control parameters for all of the GEA analytes were expressed relative to these two isotopes.

The ${ }^{137}$ CS LCS standard recovery was acceptable with 96.3 percent recovery. The ${ }^{60}$ Co LCS standard recovery was al so acceptable with 99.2 percent recovery.

The GEA procedure is sufficiently free of matrix interference with analyte quantitation that the procedure does not require spiking to assess matrix effects. However, another quality control parameter, percent counting error, was of significance. It was determined and reported when the sample analyte activity was above the detection limit. 


\section{FE-WD-WP-202, REV.1}

Precision evaluation was based on the difference between the samples and their corresponding duplicates. The TSAP specified control limit for precision for all GEA analytes was $\pm 20 \mathrm{RPD}$.

\section{CESIUM-137 (by GEA)}

${ }^{137} \mathrm{Cs}$ precision value for sample $1 \mathrm{C}$ was acceptable with $1.8 \mathrm{RPD}$.

The counting error for all samples ranged was 0.3 percent.

${ }^{137}$ Cs activities in the preparation blank and field blank were less than the detection limit, indicating the absence of contamination.

Sample ${ }^{137} \mathrm{Cs}$ activities ranged from 111 to $114 \mu \mathrm{Ci} / \mathrm{ml}$, with a mean value of $112 \mu \mathrm{Ci} / \mathrm{ml}$. These activities were less than the notification limit specified in the TSAP.

\section{CESIUM-134 (by GEA)}

${ }^{134} \mathrm{C}$ s precision RPD between sample $1 \mathrm{C}$ and its duplicate was not able to be determined because the sample activity values were less than the detection 1 imit.

Sample counting errors were not able to be determined because the samples had activities less than the detection limit.

Preparation blank activity was less than the detection limit, indicating the absence of contamination. ${ }^{134} \mathrm{Cs}$ activity in the field blank was also less than the detection limit, indicating the absence of ${ }^{134} \mathrm{Cs}$ contamination.

All sample ${ }^{134} \mathrm{Cs}$ activities were less than $0.0559 \mu \mathrm{Ci} / \mathrm{ml}$. These activities were significantly less than the notification limit specified in the TSAP.

\section{CERIUM/PRASEODYMIUM-144 (by GEA)}

${ }^{144} \mathrm{Ce}$ is counted with ${ }^{144} \mathrm{Pr}$ because the two isotopes are indistinguishable by GEA analysis. ${ }^{144} \mathrm{Pr}$ is the decay daughter product of ${ }^{144} \mathrm{Ce}$. The combined activity was determined from the ${ }^{144} \mathrm{Pr}$ gamma energy 1 ine when the parent and daughter were at secular equilibrium.

${ }^{144} \mathrm{Ce} / \mathrm{Pr}$ precision values between samples and their duplicates were not able to be determined. ${ }^{144} \mathrm{Ce} / \mathrm{Pr}$ values for each sample and sample duplicate were less than the detection limit.

Sample counting errors were indeterminable because all sample activities were less than the detection limit. 
WE-SD-WM-DP-202, REV.1

${ }^{144} \mathrm{Ce} / \mathrm{Pr}$ activities for the preparation blank and field blank were less than the detection limit, indicating the absence of contamination.

Average sample ${ }^{144} \mathrm{Ce} / \mathrm{Pr}$ activities were not able to be calculated because all were less than the detection 1 imit. The ${ }^{144} \mathrm{Ce} / \mathrm{Pr}$ activity for all samples was less than $0.731 \mu \mathrm{Ci} / \mathrm{ml}$.

\section{COBALT-60 (by GEA)}

${ }^{60} \mathrm{Co}$ precision values between samples and their duplicates were not able to be determined because activities for each sample and sample duplicate were less than the detection limit.

Sample counting errors were indeterminable because all sample activities were less than the detection limit.

${ }^{60}$ Co activities for the preparation blank and field blank were less than the detection limit, indicating the absence of contamination.

Average sample activities were not able to be calculated because all were less than the detection limit. The ${ }^{60} \mathrm{Co}$ activity for all samples was less than $0.00757 \mu \mathrm{Ci} / \mathrm{ml}$. These activities were significantly less than the notification limit specified in the TSAP.

\section{EUROPIUM-154 (by GEA)}

${ }^{154}$ Eu precision values between samples and their duplicates were not able to be determined because activities for each sample and sample duplicate were less than the detection limit.

Sample counting errors were indeterminable because all sample activities were less than the detection limit.

${ }^{154}$ Eu activities for the preparation blank and field blank were less than the detection limit, indicating the absence of contamination.

Average sample activities were not able to be calculated because all were less than the detection limit. ${ }^{154}$ Eu activity for all samples was less than 0.0263 $\mu \mathrm{C} i / \mathrm{ml}$. These activities were significantly less than the notification limit specified in the TSAP.

\section{EUROPIUN-155 (by GEA)}

${ }^{155}$ Eu precision values between samples and their duplicates were not able to be determined because activities for each sample and sample duplicate were less than the detection limit. 


\section{SD-WM-DP-202, REV.1}

Sample counting errors were indeterminable because all sample activities were less than the detection limit.

${ }^{155} \mathrm{Eu}$ activities for the preparation blank and field blank were less than the detection limit, indicating the absence of contamination.

Average sample activities were not able to be calculated because all were less than the detection limit. ${ }^{155}$ Eu activity for all samples was less than 0.204 $\mu \mathrm{C} i / \mathrm{ml}$. These activities were significantly less than the notification limit specified in the TSAP.

\section{NIOBIUN-94 (bY GEA)}

${ }^{94} \mathrm{Nb}$ precision values between samples and their duplicates were not able to be determined because activities for each sample and sample duplicate were less than the detection limit.

Sample counting errors were indeterminable because all sample activities were less than the detection limit.

${ }^{94} \mathrm{Nb}$ activities for the preparation blank and field blank were less than the detection limit, indicating the absence of contamination.

Average sample activities were not able to be calculated because all were less than the detection limit. ${ }^{94} \mathrm{Nb}$ activity for all samples was less than 0.0141 $\mu \mathrm{Ci} / \mathrm{ml}$. These activities were significantly less than the notification limit specified in the TSAP.

\section{RUTHENIUM/RHODIUM-106 (by GEA)}

${ }^{106} \mathrm{Ru}$ is detected in the presence of its daughter, ${ }^{106} \mathrm{Rh}$ because the two isotopes are indistinguishable by GEA analysis. Radioactivity values were shown in the spread sheet as the sum of $\mathrm{Rh}^{106}$ and $\mathrm{Ru}^{106}$ activities at secular equilibrium.

${ }^{106} \mathrm{Ru} / \mathrm{Rh}$ precision values between samples and their duplicates were not able to be determined because activities for each sample and sample duplicate were less than the detection limit.

Sample counting errors were indeterminable because all sample activities were less than the detection limit.

${ }^{106} \mathrm{Ru} / \mathrm{Rh}$ activities for the preparation blank and field blank were less than the detection limit, indicating the absence of contamination.

Average sample activities were not able to be calculated because all were less than the detection 1 imit. ${ }^{106} \mathrm{Ru} / \mathrm{Rh}$ activity for all samples was less than 
SD-WM-DP-202, REV.1

$1.13 \mu \mathrm{Ci} / \mathrm{ml}$. These activities were significantly less than the notification limit specified in the TSAP.

\section{RADIUH-226 (by GEA)}

${ }^{226}$ Ra precision values between samples and their duplicates were not able to be determined because activities for each sample and sample dupl jcate were less than the detection limit. Because the detection limit for ${ }^{226} \mathrm{Ra}$ is characteristically very high by the GEA procedure, it is not the procedure of choice for samples with low activity.

Sample counting errors were indeterminable because all sample activities were less than the detection limit.

${ }^{226} \mathrm{Ra}$ activities for the preparation blank and field blank were less than the detection limit, indicating the absence of contamination.

Average sample activities were not able to be calculated because all were less than the detection limit. ${ }^{226} \mathrm{Ra}$ activity for all samples was less than 1.45 $\mu \mathrm{Ci} / \mathrm{ml}$. Despite being less than the detection limit, all sample results exceeded the notification limit of $>0.033 \mu \mathrm{C} i / \mathrm{ml}$. Failure to provide sample results that were less that the notification limit occurred because of the relatively high ${ }^{137} \mathrm{Cs}$ activity in the samples. To minimize the excessive background, dilutions of the sample were required, causing the detection limits to be increased by the sample dilution factor.

\section{TRITIUN (by Lachat/Liquid Scintillation)}

This procedure was performed on the direct sample using procedure/revision number LA-218-114/B-0. Sample holding times ranged from 89 to 94 days. A required sample holding time was not specified in SW-846 protocol for this analyte.

Performance on the LCS standard was acceptable with a recovery of 114.5 percent.

Spike accuracy was acceptable, being within the TSAP specified acceptance criteria of 70 to 130 percent recovery, with a value of 101.9 percent recovery.

Precision, as measured by the relative percent difference between the spike and spike duplicate, was within the TSAP specified acceptance limit of \pm 25 RPD with a value of 2.7 RPD.

The counting error for all samples ranged from 0.5 to 5.3 percent.

The activities of the field and reagent blanks were only slightly greater than the detection limit, indicating the absence of contamination. 
HF-SD-WM-DP-202, REV.1

${ }^{3} \mathrm{H}$ activities for the tank samples ranged from 0.00104 to $0.0276 \mu \mathrm{Ci} / \mathrm{ml}$, with a mean activity of samples of $0.0111 \mu \mathrm{Ci} / \mathrm{ml}$.

\section{CARBON-14 (by Liquid Scintillation)}

This procedure was performed on the direct sample using procedure/revision number LA-348-104/C-0. Sample holding times ranged from 48 to 107 days. A required sample holding time was not specified in SW-846 protocol for ${ }^{14} \mathrm{C}$. For the analytical run that included the field blank, the chemist noted, "Although (reagent) blank activity was high, it appears to have had no effect on the sample or duplicate results. Spike and Spike duplicate indicate no interferences."

The LCS standard recoveries were within acceptance limits with values of 90.2 and 75.2 percent. The statistically derived upper and lower control 1 imits were $110.32 \%$ and $58.55 \%$, respectively.

Spike accuracy was acceptable, being within the TSAP specified acceptance criteria of 70 to 130 percent recovery with values of 85.8 and 86.7 percent recovery.

Precision performance between the spikes and spike duplicates met the TSAP specified acceptance 1 imit of $\pm 25 \mathrm{RPD}$, having a values of 0.7 and $0.9 \mathrm{RPD}$. Precision between sample IC and its duplicate was good with 6.3 RPD.

The counting error for tank samples was reasonable for liquid scintillation analyses, ranging from 1.0 to 1.3 percent.

The ${ }^{14} \mathrm{C}$ activity of the reagent blank for the tank samples was less than the detection limit. The activity of the field blank's reagent blank was slightly greater than the detection limit. Consequently is was determined that all blanks were not contaminated.

Average ${ }^{14} \mathrm{C}$ activities for the tank samples ranged from 0.000213 to 0.000398 $\mu \mathrm{Ci} / \mathrm{ml}$, with a mean activity of samples of $0.000330 \mu \mathrm{Ci} / \mathrm{ml}$. These activities were significantly less than the notification limit specified in the TSAP.

\section{SELENIUH-79 (by Ion Exchange/Dist/Liquid Scintillation)}

${ }^{79}$ Se analysis was performed on acid digestions of the samples. The digestion generated soluble selenium needed for full recovery of the analyte, and also produced an acid matrix which was required for this procedure.

${ }^{79} \mathrm{Se}$ analyses were performed using procedure/revision number LA-365-132/C-1. Sample holding times ranged from 99 to 104 days. A required maximum sample holding time was not specified in SW-846 protocol for this analyte. There did not appear to be any analytical anomalies or difficulties during the analyses of this analyte. 
HF-SD-WM-DP-202, REV.1

${ }^{79}$ Se activity was based upon calibration with a ${ }^{14} \mathrm{C}$ standard, since both nuclides have approximately the same beta energy. This was necessary because no ${ }^{79} \mathrm{Se}$ standard exists.

Isotopic recovery through the preparative procedure was estimated gravimetrically by the use of a carrier for both the sample and the blanks. Carrier recoveries were good for the field blank, samples and duplicate with values ranging from 79.5 to 93.5 percent.

The counting error of ${ }^{79} \mathrm{Se}$ for all samples ranged from 2.9 to 3.7 percent.

The ${ }^{79}$ Se activities of the field blank and reagent blanks were less four times greater than the detection limit, indicating the absence of contamination.

The precision met the TSAP specified acceptance criteria of \pm 25 , where the RPD for sample IC was 12.6. Precision for ${ }^{79} \mathrm{Se}$ is based on the difference between a sample and its duplicate.

${ }^{79} \mathrm{Se}$ activities ranged from $0.0000331 \mu \mathrm{Ci} / \mathrm{ml}$ and $0.000274 \mu \mathrm{Ci} / \mathrm{ml}$. The average ${ }^{79} \mathrm{Se}$ activity of a11 samples was $0.000211 \mu \mathrm{Ci} / \mathrm{ml}$. These activities were significantly less than the notification limit specified in the TSAP.

\section{STRONTIUM-89/90 (by Separation/Proportional Counting)}

This procedure was performed on acid digestions of the samples using procedure/revision numbers LA-220-101/D-1 and E-3. Sample holding times ranged from 96 to 101 days. A required sample holding time was not specified in SW-846 protocol for this analyte. There did not appear to be any analytical anomalies or difficulties during the analyses of this analyte.

The ${ }^{89 / 90} \mathrm{Sr}$ LCS standard percent recovery of 97.9 was within acceptance limits.

Spike accuracy (measured on the field blank) was acceptable, being within the TSAP specified acceptance criteria of 75 to 125 percent recovery, with a value of 102.9 percent recovery.

Precision as measured by the relative percent difference between the spike and spike duplicate was within the TSAP specified acceptance limit of \pm 20 RPD with a value of 10.7 RPD.

The counting error was low for samples $1 \mathrm{C}$ and $2 \mathrm{C}$ with 2.6 to 2.5 percent, respectively. The counting error was high for sample 36 was high at 99.0 percent because the sample activity was less than the minimum detectable activity. For the field blank, the counting error was high with a value of 177.0 percent, which is expected because of the low number of counts detected for a blank. Sample carrier recoveries were acceptable, ranging from 89.5 to 91.7 percent. 
WNF-SD-WM-DP-202, REV.1

The activity of the preparation blank was less than the detection limit, and the field blank activity was only slightly greater than the detection limit. Consequently, both blanks were determined to not be contaminated.

${ }^{89 / 90} \mathrm{Sr}$ activities for the samples ranged from 0.00103 to $0.316 \mu \mathrm{Ci} / \mathrm{ml}$. The mean activity of all samples was $0.210 \mu \mathrm{Ci} / \mathrm{ml}$. These activities were significantly less than the notification limit specified in the TSAP.

\section{TECHNETIUN-99 (bY EXTRACTION/LIQUID SCINTILLATION)}

${ }^{99} \mathrm{Tc}$ analyses were prepared by performing an acid predigestion of samples. Digestion was performed to fully dissolved the analyte, facilitating analyte detection. Analyses were performed using procedure/revision number LA-438$101 / \mathrm{D}-2$. Sample holding times ranged from 94 to 99 days. A required maximum sample holding time was not specified in SW-846 protocol for this analyte.

There did not appear to be any analytical anomalies or difficulties during the analyses of this analyte.

${ }^{99}$ TC LCS standard recovery was acceptable with 84.7 percent recovery.

Accuracy as evaluated by percent recovery of spike was acceptable, with a value of 98.7 percent. The TSAP specified limits for accuracy were 75 to 125 percent recovery.

Precision performance, as measured by the relative percent difference between the spike and its duplicate, was acceptable at 1.3 RPD. The TSAP specified limit for precision was \pm 20 RPD.

Sample tracer recoveries ranged from 70.7 to 72.2 percent, which were acceptable. The counting error for all samples ranged from 1.3 to 9.6 percent and was also of acceptable quality.

The ${ }^{99} \mathrm{Tc}$ activities of the field blank and preparation blank were less than the detection limit and were determined to not be contaminated.

${ }^{99} \mathrm{TC}$ activities for the tank samples ranged from 0.0558 to $0.0644 \mu \mathrm{Ci} / \mathrm{ml}$ (significantly less than the notification limit of $>2 \mu \mathrm{Ci} / \mathrm{ml}$ ). The mean activity for all samples was $0.0598 \mu \mathrm{Ci} / \mathrm{ml}$.

\section{IODINE-129 (by Distillation/Ion Exchange/GEA)}

${ }^{129} \mathrm{I}$ analysis was performed on direct samples using procedure/revision LA-378$103 / C-0$. Sample holding times ranged from 93 to 120 days. Several reruns were performed for this analyte, causing the sample holding times to be extended significantly. Required maximum sample holding times are not specified in SW-846 protocol for this analyte. 
Accuracy performance, as determined by the recovery of the LCS standard, was acceptable with values for the three runs of 75.0,72.5 and 67.9 percent recovery. The statistically derived upper and lower control limits for acceptable LCS standard recovery were 121.84 and 49.19 percent, respectively.

The accuracy control limits specified in the TSAP were 75 to 125 percent recovery. Accuracy was acceptable for the spike for only sample $2 C$ with a percent recovery of 79.3. For samples IC and IB, the spikes had slightly lower recoveries of 71.4 and 67.9 percent, respectively. Achieving percent recoveries for the spikes that are within the upper and lower LCS control limits is statistically probable because the standard recovery typically represents the best analytical results possible. Therefore, al though two of the spikes representing different runs failed to meet the customer specified acceptance criteria, the data presented are within the laboratory's technical acceptance limits.

The precision control 1 imit specified in the TSAP for spike duplicates was \pm 20 RPD. Precision between the spikes and their duplicates was acceptable with values of 8.3, 1.1 and $0.6 \mathrm{RPD}$. Two samples which were analyzed in duplicate also yielded comparable results with values of 3.5 and $2.8 \mathrm{RPD}$.

Sample carrier recoveries were marginally acceptable, ranging from 47.5 to 61.3 percent.

Counting errors were reasonably good, ranging from 0.0 to 3.3 percent.

Activities of the field blank and reagent blanks were less than the detection limit, indicating the absence of contamination.

${ }^{129} \mathrm{I}$ activity for the samples ranged from 0.000115 to $0.000140 \mu \mathrm{Ci} / \mathrm{ml}$, with an grand average of 0.000125 , which did not exceed the notification limit of $>0.0026 \mu \mathrm{Ci} / \mathrm{ml}$.

\section{NEPTUNIUM-237 (by Extraction/Internal Proportional Counter)}

${ }^{237} \mathrm{~Np}$ analyses were performed on previously acid digested samples, using procedure/revision LA-933-141/H-1. Sample holding times ranged from 81 to 86 days. A required maximum sample holding time was not specified in SW-846 protocol for this analyte. There did not appear to be any analytical anomalies or difficulties during the analyses of this analyte.

Accuracy performance, as measured by percent recovery of the LCS standard, was acceptable with 67.0 percent recovery, despite seeming to be extremely low. Internal control limits for the ${ }^{237} \mathrm{~Np}$ LCS standard were 43.78 percent to 114.25 percent. The limits are empirically derived and set at \pm 3 standard deviations from the mean of the historical data. 


\section{WNE-SD-WM-DP-202, REV.1}

Accuracy for the spike was acceptable with a percent recovery of 82.6. The TSAP specified range of acceptance was 75 to 125 percent recovery. A rerun was neither requested nor performed.

Precision performance was acceptable between the spike and spike duplicate with a 0.6 relative percent difference. The TSAP specified acceptance limit was $\pm 20 \mathrm{RPD}$.

The counting errors were quite high for all samples, ranging from 183 to 474 percent, because the sample activities were less than the detection limit.

Activities of the field blank and preparation blank were less than the detection limit, indicating the absence of contamination.

${ }^{237} \mathrm{~Np}$ activities for the samples were less than the detection limit, ranging from $<0.00120$ to $<0.00170 \mu \mathrm{Ci} / \mathrm{ml}$.

\section{PLUTONIUM-239/240 (by Ion Exchange/Alpha Energy Analysis)}

${ }^{239 / 240} \mathrm{Pu}$ analyses were performed using procedure/revision LA-943-128/B-0 on samples which had been previously digested. There did not appear to be any analytical anomalies or difficulties for these analyses.

The sample holding times ranged from 71 to 76 days. A required maximum sample holding time was not specified in SW-846 protocol for this analyte.

Recovery of the LCS standard was acceptable with a result of 94.0 percent.

Accuracy performance was acceptable for the spikes with percent recoveries of 88.6 and 91.9 percent. The TSAP specified acceptance 1 imits for $239 / 240 \mathrm{Pu}$ accuracy were 70 and 130 percent recovery.

Precision between the spikes and their duplicates was acceptable with 2.3 and 3.7 RPD. The TSAP specified acceptance 7 imit for precision was \pm 25 RPD.

${ }^{236} \mathrm{Pu}$ tracer recoveries were acceptable with values ranging from 81.3 to 91.3 percent. The sample counting error for tank samples ranged from 10.4 to 11.2 percent.

The field blank and preparation blank had activities less than the detection 1 imit, indicating the absence of contamination.

The tank sample ${ }^{239 / 240} \mathrm{Pu}$ activities were all less than $0.000202 \mu \mathrm{Ci} / \mathrm{ml}$, and did not exceed the TSAP specified notification limit. 


\section{WE-WM-DP-202, REV.1 \\ PLUTONIUM-238 (by Ion Exchange/Alpha Energy Analysis)}

${ }^{238} \mathrm{Pu}$ analyses were generated concurrently with ${ }^{239 / 240} \mathrm{Pu}$ data in the $\mathrm{Pu}$ procedure. Acid digested samples were prepared and then analyzed using procedure/revision LA-943-128/B-0. There did not appear to be any analytical anomalies or difficulties with these analyses.

The sample holding times ranged from 71 to 76 days. A required maximum sample holding time was not specified in SW-846 protocol for this analyte.

The assessment of accuracy for this method was based on the recovery of the ${ }^{239} \mathrm{Pu}$ LCS standard because no ${ }^{238} \mathrm{Pu}$ standard was available. Percent recovery of the ${ }^{239} \mathrm{Pu}$ LCS standard was acceptable with a result of 94.0 .

An evaluation of accuracy and precision based on a spike and spike duplicate was not possible because a spiking standard was not available. A sample was not analyzed in duplicate as specified in the TSAP to evaluate the precision. However, because all sample results were less than the detection limit, a RPD could not have been calculated even if a duplicate analysis had been performed.

${ }^{236} \mathrm{Pu}$ tracer recoveries were acceptable with values ranging from 88.5 to 91.3 percent. The counting error for all samples ranged from 9.7 to 100.0 percent.

Field blank and preparation blank activities (based on $\mathrm{Pu}^{239 / 240}$ ) were less than the detection limit, indicating the absence of contamination.

${ }^{238} \mathrm{Pu}$ activities of the tank samples were all less than $0.000202 \mu \mathrm{Ci} / \mathrm{m} 7$. Reported "less than" values for the ${ }^{238} \mathrm{Pu}$ detection 1 imit were generated using $20 \mathrm{dpm}$ for the sample activity. Sample analytical values did not exceed the notification limit.

\section{AMERICIUM-241 (by Extraction/Alpha Energy Analysis)}

${ }^{241} \mathrm{Am}$ analyses were performed on acid digested samples using the newly developed procedure/revision LA-953-103/B-0. There did not appear to be any analytical anomalies or difficulties for these analyses.

The sample holding times ranged from 63 to 75 days. A required maximum sample holding time was not specified in SW-846 protocol for this analyte.

Percent recovery of the LCS standard was acceptable with a value of 88.2 .

Accuracy performance was acceptable for the spikes with percent recoveries of 88.2 and 91.6 percent. The TSAP specified acceptance 1 imits for ${ }^{241} \mathrm{Am}$ accuracy were 70 and 130 percent recovery.

Precision between the spikes and their duplicates was acceptable with 1.9 and 7.2 RPD. The TSAP specified acceptance limit for precision was \pm 20 RPD. 


\section{WHF-SD-WM-DP-202, REV.1}

${ }^{243} \mathrm{Am}$ tracer recoveries were good, ranging from 84.1 to 91.9 percent.

The field blank and preparation blanks had activities less than the detection limit, indicating the absence of contamination.

${ }^{241} \mathrm{Am}$ activity was less than the detection limit for all samples, with all values less than $0.000308 \mu \mathrm{Ci} / \mathrm{ml}$. These "less than" values were determined using 5 percent of the ${ }^{243} \mathrm{Am}$ tracer peak as the ${ }^{241} \mathrm{Am}$ peak. The notification limit of $>1 \mu \mathrm{Ci} / \mathrm{ml}$ was not exceeded by any sample.

\section{CURIUH-243/244 (by Extraction/Alpha Energy Analysis)}

${ }^{243 / 244} \mathrm{Cm}$ analytical data were generated as an analytical by-product of ${ }^{241} \mathrm{Am}$ analysis, using the ${ }^{241}$ Am procedure/revision LA-953-103/B-0 on acid digested samples. There did not appear to be analytical anomalies or difficulties for $243 / 244 \mathrm{Cm}$ analyses.

The sample holding times ranged from 63 to 75 days. A required maximum sample holding time was not specified in SW-846 protocol for this analyte.

${ }^{243 / 244} \mathrm{Cm}$ and ${ }^{241} \mathrm{Am}$ were analyzed simultaneously using the same procedure, however there was no ${ }^{244} \mathrm{Cm}$ standard or spike available for the evaluation of $243 / 244 \mathrm{Cm}$ accuracy. Because of this, ${ }^{241}$ Am was used as a surrogate LCS standard, and ${ }^{243} \mathrm{Am}$ was used as a tracer to estimate accuracy. ${ }^{241} \mathrm{Am}$ standard recovery was acceptable with a value of 88.2 .

To verify that the procedure could appropriately measure ${ }^{244} \mathrm{~cm}$, the counter was calibrated for efficiency. It was determined that the counting efficiency was uniform across the entire energy counting spectrum.

The ${ }^{243 / 244} \mathrm{Cm}$ counting error for the samples and field blank was 100.0 percent. ${ }^{243} \mathrm{Am}$ tracer recoveries were good, ranging from 84.1 to 91.9 percent.

Evaluations of accuracy and precision based on a spike and spike duplicate were not possible because a spiking standard was not available. A11 samples were analyzed in duplicate to assess $243 / 244 \mathrm{Cm}$ precision. An RPD value could not be calculated for any of the samples, however, because the activities for all samples and their duplicates were less than the detection limit.

The activities of the field and preparation blanks were less than the detection limit, indicating the absence of contamination.

The ${ }^{243 / 244} \mathrm{Cm}$ activity of al1 samples was less than $0.000308 \mu \mathrm{Ci} / \mathrm{ml}$. These "Tess than" values were determined using 5 percent of the ${ }^{243}$ Am tracer peak as the ${ }^{243 / 244} \mathrm{Cm}$ and ${ }^{241} \mathrm{Am}$ peaks. No sample exceeded the notification limit of $>0.013 \mu \mathrm{Cj} / \mathrm{ml}$. 


\section{- itW F-SD-WM-DP-202, REV.1}

\section{ORGANIC ANALYSES}

Although Total Organic Carbon is an organic analysis and would generally be discussed in this section, it was placed in the inorganic section. This placement enabled consolidating the discussion of total carbon, total inorganic carbon and total organic carbon and their interrelationships in the characterization of AP-105 waste.

The matrix for tank AP-105 samples as defined by SW-846 was concentrated aqueous waste. Sample holding times for both volatile organic analysis (VOA) and semi-volatile organics (Semi-VOA) were defined in volume 1 , section B, chapter 4, table 4-1 of SW-846, as 14 days prior to extraction. For semivolatiles, extracts must be analyzed within 40 days following extraction.

Analytical accuracy was based on the percent recovery of target compound spikes. Analytical precision was based on the relative percent difference between these spikes and their duplicates. Accuracy and precision acceptance criteria for each compound were given Table 2 of the TSAP.

To enable interpretation of summary data for VOA and Semi-VOA compounds, Table 8 defines the qualifiers, called Q-flags, shown on Form I in the Organic Raw Analytical Data section and on the Data Summary Tables.

\begin{tabular}{|c|l||}
\hline \multicolumn{1}{|c|}{ Table 8. Data Qualifier Definitions } \\
\hline \hline Q-FLAG & \multicolumn{1}{|c|}{ DEFINITION } \\
\hline \hline$U$ & $\begin{array}{l}\text { Indicates the compound was analyzed for but not detected; the U- } \\
\text { flagged concentration number is the quantitation limit. }\end{array}$ \\
\hline $\mathrm{J}$ & $\begin{array}{l}\text { Indicates an estimated value for the target or tentatively } \\
\text { identified compounds; spectra meet criteria but response is below } \\
\text { the quantitation limit for the target compounds. }\end{array}$ \\
\hline $\mathrm{B}$ & Compound was found in the blank. \\
\hline $\mathrm{D}$ & Analysis was performed on a diluted sample. \\
\hline $\mathrm{E}$ & Indicates that quantitation was above the calibration range. \\
\hline $\mathrm{N}$ & $\begin{array}{l}\text { Indicates the presumptive evidence of a compound. This flag is } \\
\text { only used for tentatively identified compounds, where the } \\
\text { identification is based on a mass spectral 1ibrary search. }\end{array}$ \\
\hline $\mathrm{X}$ & Indicates nitration product of the acid surrogate. \\
\hline
\end{tabular}

Raw data can be found in the Raw Analytical Data section under Organic Analyses. 
WNTE-SD-WM-DP-202, REV. 1

VOLATILE ORGANIC ANALYSIS, VOA (by GC/MS)

The samples were analyzed using procedure LA-523-405/A-4, a purge and trap/gas chromatograph-mass spectrometer instrumental method. A narrative by the cognizant chemist was provided in the raw data section of the data package which gives additional information.

A $0.1 \mathrm{ml}$ aliquot of the sample was combined with $4.9 \mathrm{ml}$ of 1 ab blank water in the purge vessel. Hanford wastes have historically been difficult to analyze because of the carry-over of foam into the trap from the VOA sparging vessel. As a consequence, the sample aliquot was limited to the largest aliquot (0.1 $\mathrm{ml}$ ) that was not expected to produce foaming above the vessel during sparging. Sample holding times ranged from 22 to 26 days. The SW-846 holding time for VOA is 14 days.

The Internal Standard compounds (IS) were bromochloromethane, 1,4difluorobenzene and chlorobenzene-d5.

The initial calibration data met all acceptance criteria for the SW-846 method except for the Bromoform response factor, which was slightly low. The Bromoform response factor did, however, meet CLP acceptance critera. The lower response factor for Bromoform does not impact the data generated for the target compounds specified by the program for this project.

None of the target compounds exceeded the maximum percent difference of \pm 40 percent for continuing calibration.

The System Monitoring Compounds (SMC), also called surrogates, were toluene$\mathrm{d} 8$, bromofluorobenzene, and 1,2-dichloroethane-d4. The LCS control 1 imits which were applied to $V O A$ analyses were those specified in the Contract Laboratory Program (CLP) Statement of Work (SOW), August 1991, for System Monitoring Compounds (SMC). The LCS standard for VOA is defined as the method blank, which contains internal standards and surrogates and is analyzed at the start of each 12 hour analysis window. The acceptance criteria used are located on the Recovery Report page for each sample in the Raw Analytical Data section and in the Data Summary Tables section (shown as surrogates).

Recovery of all surrogates was acceptable, meeting the recovery 1 imit criteria.

The Matrix Spike compounds (MS), which were analyzed, were the same as the target analytes which were required by the TSAP in Table 2. All target analytes met the TSAP's spike accuracy acceptance limits. Table 9 indicates the control limits specified in the TSAP for the VOA compounds.

Precision performance was evaluated for each target compound as the relative percent difference between the matrix spike for sample IA and its duplicate. The RPDs for all targets were acceptable with values less than or equal to 4 . 
The concentrations of all target compounds in the field blank, trip blank and reagent blank were less than the quantitation 1 imit and consequently were determined to not be contaminated.

\begin{tabular}{||c|c|c||}
\hline \multicolumn{2}{|c|}{ Table 9. TSAP Specified Control 1imits for V0A Compounds } \\
\hline Target Compound Name & $\begin{array}{c}\text { Precision Control } \\
\text { Limits }\end{array}$ & $\begin{array}{c}\text { Accuracy Control } \\
\text { Limits }\end{array}$ \\
\hline Acetone & \pm 25 RPD & $40-110 \%$ Recovery \\
\hline 1-Butanol & \pm 25 RPD & $30-110 \%$ Recovery \\
\hline 2-Butanone & \pm 25 RPD & $40-110 \%$ Recovery \\
\hline 2-Hexanone & \pm 25 RPD & $40-125 \%$ Recovery \\
\hline $\begin{array}{c}\text { Methyl Isobutyl Ketone } \\
\text { (4-Methyl-2-pentanone) }\end{array}$ & \pm 25 RPD & $40-110 \%$ Recovery \\
\hline 2-Pentanone & \pm 25 RPD & $40-125 \%$ Recovery \\
\hline Tetrahydrofuran & \pm 25 RPD & $30-110 \%$ Recovery \\
\hline
\end{tabular}

The compound Methyl Isobutyl Ketone (MIBK) is specified as an analyte in the TSAP. The preferred name for this compound is 4-Methyl-2-pentanone.

All raw data, including calibration information, were furnished as instrument printouts.

No target analytes were observed in any of the samples above the notification limit. Acetone was observed in all three samples with the concentrations ranging from 490 (estimated) to $550 \mu \mathrm{g} / \mathrm{l}$. Information on Tentatively Identified Compounds (TIC) is available in the raw data section.

\section{SEMI-YOLATILE ORGANIC ANALYSIS, SEMI-VOA (bY GC/MS)}

The samples were extracted with a semi-micro continuous liquid/liquid extractor using procedure $L A-523-132 / B-0$. The sample extracts were analyzed by a gas chromatograph/mass spectrometer instrument using procedure LA-523406/A-0. A narrative by the cognizant chemist was provided in the raw data section of the data package which gives additional information. That narrative noted that there were difficulties with acid surrogate recoveries, recoveries of acid matrix spike compounds (non-target) in samples, and contamination by numerous non-target compounds of the blank. Several observations of color changes and precipitate formation were also recorded during the extractions.

The SW-846 specified holding time (Table 2) of 14 days for extraction was exceeded for all samples. The sample holding times for extraction ranged from 
78 to 90 days. The SW-846 specified holding time of 40 days after extraction for analysis was not exceeded for any sample. The sample holding times for analyses ranged from 7 to 14 days. It was not possible to meet the SW-846 short holding time for extraction of Semi-VOAs because of the extra procedural steps necessary to handle radiological material. The TSAP required that the trip blank be analyzed only after target compounds were detected in the field blank. Because target compounds were detected in the tank samples, however, it was decided to also analyze the trip blank.

LCS control limits for the Semi-VOA compounds were administratively set to the criteria listed in procedure LA-523-406/A-0 for surrogates. The LCS standard for Semi-VOA is defined as the method blank, which contained internal standards and surrogates. The internal standards were 1,4-Dichlorobenzene-d4; Naphthalene-d8, Acenapthene-d10; Phenanthrene-dI0; Chrysene-dl2 and Perylene-d12. The surrogates (SMCs) were 2-Fluorophenol; Phenol-d5; 2Chlorophenol-d4; 1,2-Dichlorobenzene-d4; Nitrobenzene-d5; 2-Fluorobiphenyl; 2,4,6-Tribromophenol and Terphenyl-d14. The surrogate acceptance criteria are located on Form II SV-l in the Organic Raw Analytical Data section and on the data summary tables.

2-Butoxyethanol and tri-butylphosphate had acceptable percent differences of -4.7 and -4.2 , respectively for the continuing calibration check.

All analytes had percent relative standard deviations which were acceptable (less than 15\%) for the initial 5 point calibration except for 4-Nitrophenol.

The acid surrogate recoveries were affected by the sample matrices. The nitration of phenolic surrogates and other phenolic compounds in Hanford tank waste samples is expected. Such nitration does not occur in the blanks or other non-tank waste matrices. For example, 2-Fluorophenol can be seen to be nitrated to form 2-Fluoro-4-nitrophenol and 2-Fluoro-6-nitrophenol. Surrogate nitration products, flagged on form $1 F$ with an $X$, were found in all three samples. Acid surrogate recoveries for the trip blank and field blank were acceptable.

Acceptable tributylphosphate (TBP) recoveries of $91.6 \%$ and $88.0 \%$ were obtained in the matrix spike (MS) and duplicate (MSD), respectively. The TSAP specified limits for accuracy for TBP were 40 to 125 percent recovery. Precision between the MS and MSD was 4 RPD. 2-Butoxyethanol had acceptable accuracy (with MS and MSD results of 88.0 and 100 percent recovery). The precision between the MS and MSD (with 13 RPD) was also acceptable, meeting the TSAP control limit of \pm 25 RPD.

All of the samples and blanks met the internal standard criteria, with the following exception: Perylene-d12 (the last eluting internal standard) was present below the lower acceptance limit for all three tank samples. The loss of this internal standard had no impact, however, on any of the surrogates or target analytes, because they used other internal standards for quantitation. 
WNE-SD-WM-DP-202, REV.1

Raw data, including calibration information, were furnished in the Raw Data section.

Many Tentatively Identified Compounds (TIC's) were observed in the AP-105 samples. An attempt was made to identify some of the surrogate nitration products, which were flagged with an $X$ on the $1 F$ forms. The Tibrary was unable to produce even poor quality hits on many peaks. On the others, the library match was considered very tentative, and no attempt was made to verify the TIC with an external standard. The chemist's narrative notes that many of the contaminants which appear as TICs in the samples and blanks appear to be coming from degradation of the stabilizer in the methylene chloride extraction solvent. Consequently these compounds are artifacts of the procedure but are not true components of the tank samples. Sample TICs were flagged with the letter "B", when these TICs were also found in the method blank.

\section{CONCLUSIONS}

The major constituents in the samples taken from tank AP-105 were (not surprisingly) water, aluminum, sodium, hydroxide, nitrate, nitrite, phosphate, sulfate and carbonate. The concentration of total organic carbon was also present at significant concentration relative to other tank waste constituents. ${ }^{137} \mathrm{Cs}$ activity accounted for essentially all of the total beta activity.

No total alpha activity was detected in any of the samples, which was consistent with the observation that ${ }^{239 / 240} \mathrm{Pu},{ }^{238} \mathrm{Pu},{ }^{24 \mathrm{Pm}} \mathrm{A},{ }^{243 / 244} \mathrm{Cm}$ and ${ }^{237} \mathrm{~Np}$ were also not detected. Iron was not detected in any of the samples, whereas it is frequently found in tank samples.

Calculations were performed to evaluate the waste composition with respect to the waste compatibility corrosion rules. The data generated in Table 10 indicate that the waste in all three tank samples meets the waste compatibility corrosion specifications. 

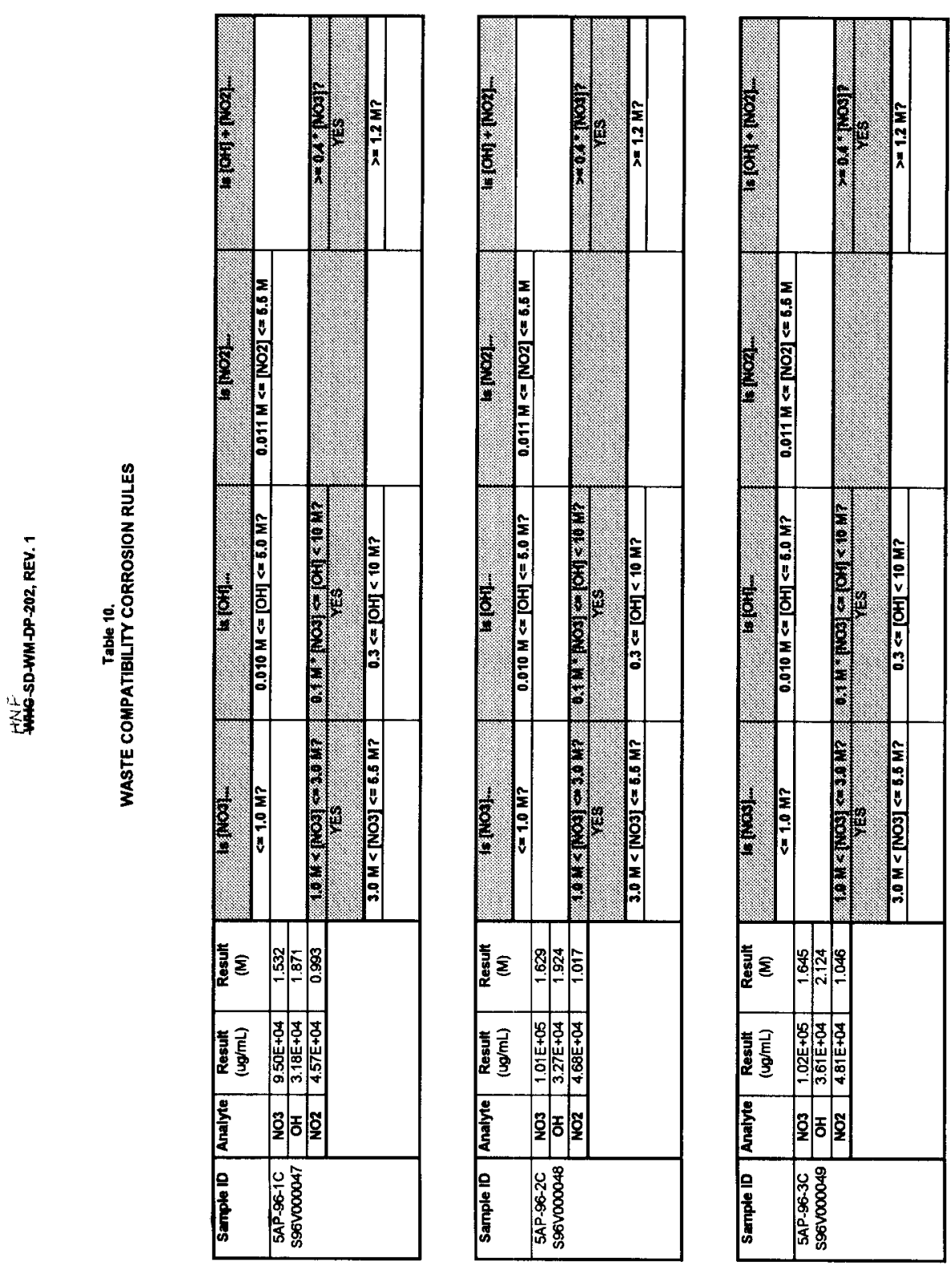


\section{References}

1. WHC-SD-WM-TSAP-091, REV. 0, "Tank 241-AP-105 Grab Sampling and Analys is Plan", dated April 18, 1996, Westinghouse Hanford Company, Richland, WA 99352 .

2. WHC-SD-WM-TSAP-091, REV. 0-A, "Tank 241-AP-105 Grab Sampling and Analysis Plan", dated June 24, 1996, Westinghouse Hanford Company, Richland, WA 99352.

3. WHC-SD-WM-TSAP-091, REV. 0-B, "Tank 241-AP-105 Grab Sampling and Analysis Plan", dated August 27, 1996, Westinghouse Hanford Company, Richland, WA 99352.

4. WHC-SD-WM-TSAP-091, REV. 0-C, "Tank 241-AP-105 Grab Sampling and Analys is Plan", dated October 15, 1996, Lockheed Martin Hanford Company, Richland, WA 99352.

5. Engineering Change Notice, No. 635332, Albert A Kruger, Data Assessment and Interpretation, dated August 26, 1996, Westinghouse Hanford Company, Richland, WA 99352.

6. WHC-SD-CP-QAPP-016, Rev. 1, "222-S Laboratory Quality Assurance PIan", dated July 31, 1995, Westinghouse Hanford Company, Richland, WA 99352.

7. WHC-SD-CP-QAPP-016, Rev. 1A, "222-S Laboratory Quality Assurance Plan", dated August 31, 1995, Westinghouse Hanford Company, Richland, WA 99352.

8. WHC-SD-DP-202, Rev. 0, "Tank 241-AP-105, Grab Samples 5AP-96-1C, 5AP96-2C, 5AP-96-3C, 5AP-96-4, and 5AP--96-IB2, Analytical Results for the 45 Day Report", dated, November 5, 1996, Rust Federal Services of Hanford, Inc., Richland, WA 99352.

9. WHC-SD-WM-QAPP-009, Rev. 2, "242-A Evaporator Quality Assurance Project Plan", dated May 4, 1995, Westinghouse Hanford Company, Richland, WA 99352 . 
HE-SD-WM-DP-202, REV. 1

Data Summary Tables 
HNSSD-WM-DP-202, REV, 1

THIS PAGE WAS INTENTIONALLY LEFT BLANK 
WNE-SD-WM-DP-202, REV. 1
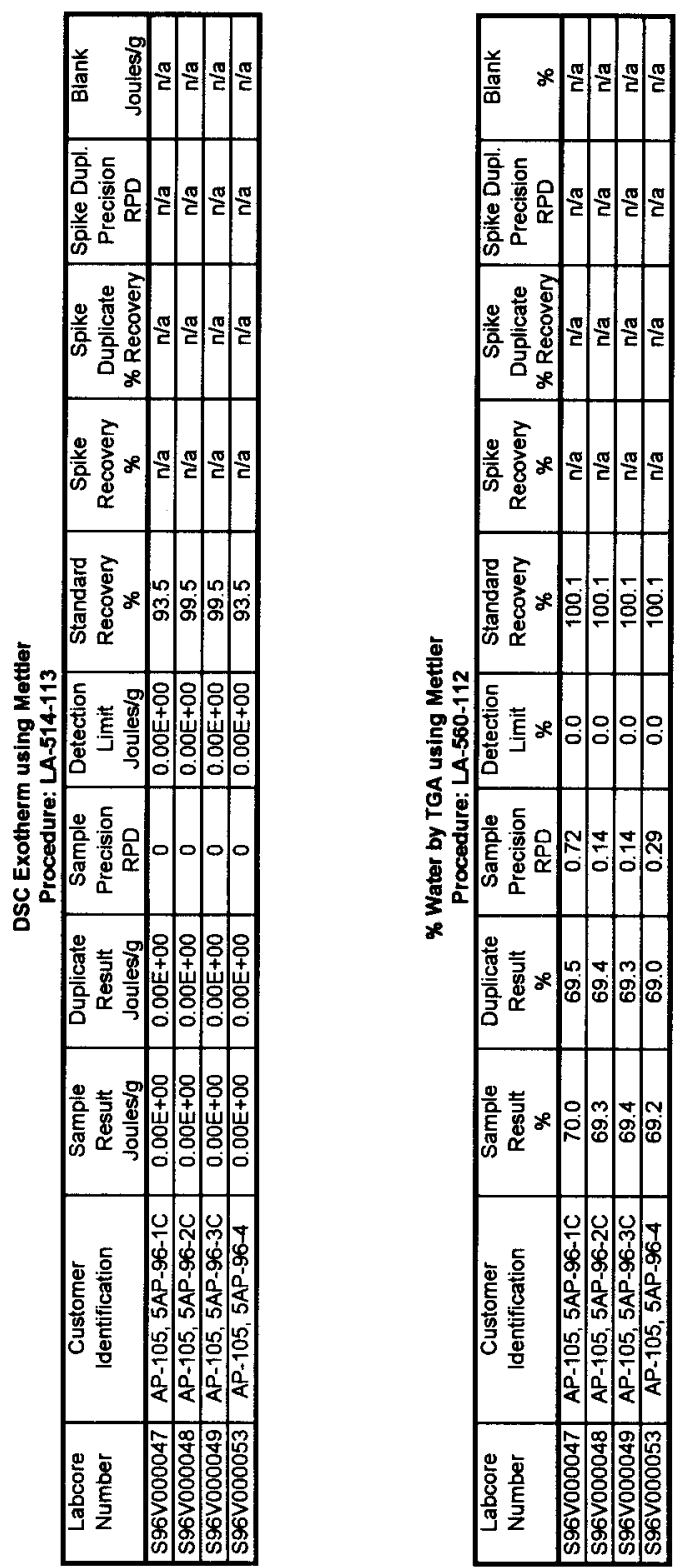
HNESD-WM-DP-202, REV

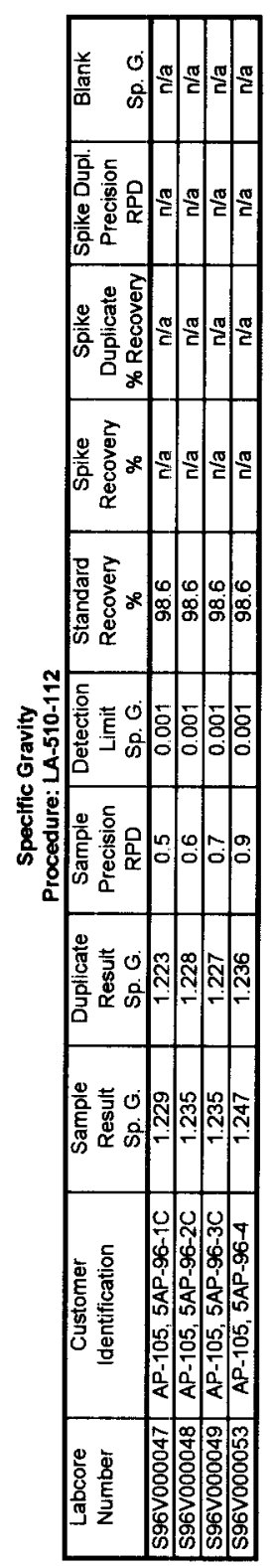


HESD-WM-DP-202, REV. 1

OH- by Pot. Titration

Procedure: LA-211-102

\begin{tabular}{|c|c|c|c|c|c|c|c|c|c|c|}
\hline $\begin{array}{l}\text { Labcore } \\
\text { Number }\end{array}$ & $\begin{array}{c}\text { Customer } \\
\text { Identification }\end{array}$ & $\begin{array}{c}\text { Sample } \\
\text { Result } \\
\mu g / m L\end{array}$ & $\begin{array}{c}\text { Duplicate } \\
\text { Result } \\
\mu \mathrm{g} / \mathrm{mL}\end{array}$ & $\begin{array}{c}\text { Sample } \\
\text { Precision } \\
\text { RPD }\end{array}$ & $\begin{array}{c}\text { Detection } \\
\text { Limit } \\
\mu g / m L\end{array}$ & $\begin{array}{c}\text { Standard } \\
\text { Recovery } \\
\%\end{array}$ & $\begin{array}{c}\text { Spike } \\
\text { Recovery } \\
\%\end{array}$ & $\begin{array}{c}\text { Spike } \\
\text { Duplicate } \\
\text { \% Recovery }\end{array}$ & $\begin{array}{c}\text { Spike Dupl. } \\
\text { Precision } \\
\text { RPD }\end{array}$ & $\begin{array}{l}\text { Blank } \\
\mu \mathrm{g} / \mathrm{mL} .\end{array}$ \\
\hline S96V000047 & AP-105, 5AP-96-1C & $3.18 \mathrm{E}+04$ & $3.13 E+04$ & 1.6 & $2.50 \mathrm{E}+03$ & 98.6 & $n / a$ & $n / a$ & $n / a$ & $<4.20 E+01$ \\
\hline S96V000048 & AP-105, 5AP-96-2C & $3.27 \bar{E}+04$ & $3.32 E+04$ & 1.5 & $2.50 \mathrm{E}+03$ & 98.6 & $n / a$ & $\mathrm{n} / \mathrm{a}$ & $n / a$ & $<4.20 E+01$ \\
\hline S96V000049 & AP-105, 5AP-96-3C & $3.61 E+04$ & $3.60 E+04$ & 0.3 & $2.50 \mathrm{E}+03$ & 98.6 & $n / a$ & n/a & n/a & $<4.20 \mathrm{E}+01$ \\
\hline$\$ 96 \vee 000058$ & AP-105, 5AP-96-IB & $<6.30 \mathrm{E}+01$ & $<6.30 E+01$ & $\mathrm{n} / \mathrm{a}$ & $6.25 E+01$ & 98.6 & $n / a$ & $n / a$ & $n / a$ & $<4.20 \mathrm{E}+01$ \\
\hline
\end{tabular}

4

pH Direct

Procedure: LA-212-106

\begin{tabular}{|c|c|c|c|c|c|c|c|c|c|c|}
\hline $\begin{array}{l}\text { Labcore } \\
\text { Number }\end{array}$ & $\begin{array}{c}\text { Customer } \\
\text { Identification }\end{array}$ & $\begin{array}{c}\text { Sample } \\
\text { Result } \\
\text { pH }\end{array}$ & $\begin{array}{c}\text { Duplicate } \\
\text { Result } \\
\mathrm{pH}\end{array}$ & $\begin{array}{c}\text { Sample } \\
\text { Precision } \\
\text { RPD }\end{array}$ & $\begin{array}{l}\text { Detection } \\
\text { Limit } \\
\text { pH }\end{array}$ & $\begin{array}{c}\text { Standard } \\
\text { Recovery } \\
\text { (Expected pH } \\
\text { minus Observed pH) }\end{array}$ & $\begin{array}{c}\text { Spike } \\
\text { Recovery } \\
\%\end{array}$ & $\begin{array}{c}\text { Spike } \\
\text { Duplicate } \\
\% \text { Recovery }\end{array}$ & \begin{tabular}{|c|} 
Spike Dupl. \\
Precision \\
RPD
\end{tabular} & Blank \\
\hline S96V000047 & $A P-105,5 A P-96-1 C$ & 13.31 & 13.35 & 0.3 & 0.01 & 0.01 & $\mathrm{n} / \mathrm{a}$ & $n / a$ & $n / a$ & $\mathrm{n} / \mathrm{a}$ \\
\hline S96V000048 & AP- $105,5 A P-96-2 C$ & 13.49 & 13.48 & 0.1 & 0.01 & 0.01 & $n / a$ & ก/a & $\mathrm{n} / \mathrm{a}$ & $n / a$ \\
\hline S96V000049 & $A P-105,5 A P-96-3 C$ & 13.45 & 13.46 & 0.1 & 0.01 & 0.01 & $n / a$ & $n / a$ & n/a & $n / a$ \\
\hline
\end{tabular}


WHE-SD-WM-DP-202, REV. 1

Ammonia by ISE-Std Additions Procedure: LA-631-001

\begin{tabular}{|c|c|c|c|c|c|c|c|c|c|c|}
\hline $\begin{array}{l}\text { Labcore } \\
\text { Number }\end{array}$ & $\begin{array}{c}\text { Customer } \\
\text { Identification }\end{array}$ & $\begin{array}{l}\text { Sample } \\
\text { Result } \\
\mu \mathrm{g} / \mathrm{mL}\end{array}$ & $\begin{array}{c}\text { Duplicate } \\
\text { Result } \\
\mu \mathrm{g} / \mathrm{mL}\end{array}$ & $\begin{array}{c}\text { Sample } \\
\text { Precision } \\
\text { RPD }\end{array}$ & $\begin{array}{c}\text { Detection } \\
\text { Limit } \\
\mu \mathrm{g} / \mathrm{mL}\end{array}$ & $\begin{array}{c}\text { Standard } \\
\text { Recovery } \\
\%\end{array}$ & $\begin{array}{c}\text { Spike } \\
\text { Recovery } \\
\%\end{array}$ & $\begin{array}{c}\text { Spike } \\
\text { Duplicate } \\
\text { \% Recovery }\end{array}$ & \begin{tabular}{|c} 
Spike Dupl. \\
Precision \\
RPD
\end{tabular} & $\begin{array}{l}\text { Blank } \\
\mu g / \mathrm{mL}\end{array}$ \\
\hline S96V000047 & AP-105, 5AP-96-1C & $2.00 E+01$ & $n / a$ & $n / a$ & $5.00 \mathrm{E}+00$ & 91.6 & 123 & $\mathrm{n} / \mathrm{a}$ & $n / a$ & $1.36 E+00$ \\
\hline S96V000048 & AP-105, 5AP-96-2C & $6.32 \mathrm{E}+01$ & $n / a$ & $n / a$ & $5.00 \mathrm{E}+00$ & 91.6 & 116 & 122 & 5 & $1.36 E+00$ \\
\hline S96V000049 & AP-105, 5AP-96-3C & $6.48 \mathrm{E}+01$ & n/a & $n / a$ & $5.00 E+00$ & 91.6 & 121 & $\mathrm{n} / \mathrm{a}$ & $n / a$ & $1.36 E+00$ \\
\hline S96V000058 & AP-105, 5AP-96-1B & $<5.00 E+00$ & $n / a$ & $\mathrm{n} / \mathrm{a}$ & $5.00 E+00$ & 91.6 & 105 & $n / a$ & $n / a$ & $1.36 E+00$ \\
\hline
\end{tabular}


$H N F$

WHG-SD-WM-DP-202, REV. 1

Ion Chromatography by Procedure: LA-533-105

Fluoride-IC-Dionex $4000 / 4500$

\begin{tabular}{|c|c|c|c|c|c|c|c|c|c|c|}
\hline $\begin{array}{l}\text { Labcore } \\
\text { Number }\end{array}$ & $\begin{array}{c}\text { Customer } \\
\text { Identification }\end{array}$ & $\begin{array}{l}\text { Sample } \\
\text { Result } \\
\mu \mathrm{g} / \mathrm{mL}\end{array}$ & $\begin{array}{c}\text { Duplicate } \\
\text { Result } \\
\mu g / m L\end{array}$ & $\begin{array}{c}\text { Sample } \\
\text { Precision } \\
\text { RPD }\end{array}$ & $\begin{array}{c}\text { Detection } \\
\text { Limit } \\
\mu g / m L\end{array}$ & $\begin{array}{c}\text { Standard } \\
\text { Recovery } \\
\%\end{array}$ & $\begin{array}{c}\text { Spike } \\
\text { Recovery } \\
\%\end{array}$ & $\begin{array}{c}\text { Spike } \\
\text { Duplicate } \\
\text { \% Recovery }\end{array}$ & $\begin{array}{c}\text { Spike Dupl. } \\
\text { Precision } \\
\text { RPD }\end{array}$ & $\begin{array}{l}\text { Blank } \\
\mu g / m L \\
\end{array}$ \\
\hline S96V000047 & AP-105, 5AP-96-1C & $3.23 \mathrm{E}+02$ & $n / a$ & $\mathrm{n} / \mathrm{a}$ & $2.54 E+01$ & 92.9 & n/a & $\mathrm{n} / \mathrm{a}$ & $n / a$ & $<1.20 \mathrm{E}-02$ \\
\hline S96V000048 & AP-105, 5AP-96-2C & $2.54 E+02$ & n/a & $\mathrm{n} / \mathrm{a}$ & $4.97 \mathrm{E}+01$ & 92.9 & 112.2 & 113.0 & 12.4 & $<1.20 \mathrm{E}-02$ \\
\hline S $96 \mathrm{~V} 000049$ & AP-105, 5AP-96-3C & $3.48 \mathrm{E}+02$ & $\mathrm{n} / \mathrm{a}$ & $\mathrm{n} / \mathrm{a}$ & $2.54 \mathrm{E}+01$ & 92.9 & $n / a$ & n/a & $n / a$ & $<1.20 \mathrm{E}-02$ \\
\hline S96V000058 & AP-105, 5AP-96-IB & $<7.20 \mathrm{E}-02$ & $\mathrm{n} / \mathrm{a}$ & $n / a$ & $7.20 \mathrm{E}-02$ & 92.9 & $\mathrm{n} / \mathrm{a}$ & $\mathrm{n} / \mathrm{a}$ & $n / a$ & $<1.20 \mathrm{E}-02$ \\
\hline
\end{tabular}

Nitrite-1C - Dionex $4000 / 4500$

\begin{tabular}{|c|c|c|c|c|c|c|c|c|c|c|}
\hline $\begin{array}{l}\text { Labcore } \\
\text { Number }\end{array}$ & $\begin{array}{c}\text { Customer } \\
\text { identification }\end{array}$ & $\begin{array}{l}\text { Sample } \\
\text { Result } \\
\mu \mathrm{g} / \mathrm{mL}\end{array}$ & $\begin{array}{c}\text { Duplicate } \\
\text { Result } \\
\mu g / m L\end{array}$ & $\begin{array}{c}\text { Sample } \\
\text { Precision } \\
\text { RPD }\end{array}$ & $\begin{array}{c}\text { Detection } \\
\text { Limit } \\
\mu g / m L\end{array}$ & $\begin{array}{c}\text { Standard } \\
\text { Recovery } \\
\%\end{array}$ & $\begin{array}{c}\text { Spike } \\
\text { Recovery } \\
\%\end{array}$ & $\begin{array}{c}\text { Spike } \\
\text { Duplicate } \\
\text { \% Recovery }\end{array}$ & $\begin{array}{l}\text { Spike Dupl. } \\
\text { Precision } \\
\text { RPD }\end{array}$ & $\begin{array}{l}\text { Blank } \\
\mu g / m L\end{array}$ \\
\hline S96V000047 & AP-105, 5AP-96-1C & $4.57 E+04$ & $n / a$ & $n / a$ & $2.29 E+02$ & 93.0 & $\mathrm{n} / \mathrm{a}$ & na & $\mathrm{n} / \mathrm{a}$ & $<1.08 \mathrm{E}-01$ \\
\hline S96V000048 & AP-105, 5AP-96-2C & $4.68 \mathrm{E}+04$ & $n / a$ & $\mathrm{n} / \mathrm{a}$ & $4.47 E+02$ & 93.0 & 99.8 & 90.7 & 9.7 & $<1.08 \mathrm{E}-01$ \\
\hline S96V000049 & AP-105, 5AP-96-3C & $4.81 \mathrm{E}+04$ & $\mathrm{n} / \mathrm{a}$ & $n / a$ & $2.29 E+02$ & 93.0 & $n / a$ & $n / a$ & $n / a$ & $<1.08 E-01$ \\
\hline S96V000058 & AP-105, 5AP-96-1B & $<6.48 E-01$ & $n / a$ & $n / a$ & $6.48 \mathrm{E}-01$ & 93.0 & $\mathrm{n} / \mathrm{a}$ & na & $\mathrm{n} / \mathrm{a}$ & $<1.08 \mathrm{E}-01$ \\
\hline
\end{tabular}

Nitrate by IC-Dionex $4000 / 4500$

\begin{tabular}{|c|c|c|c|c|c|c|c|c|c|c|}
\hline $\begin{array}{l}\text { Labcore } \\
\text { Number }\end{array}$ & $\begin{array}{c}\text { Customer } \\
\text { identification }\end{array}$ & $\begin{array}{c}\text { Sample } \\
\text { Result } \\
\mu g / m L\end{array}$ & $\begin{array}{c}\text { Duplicate } \\
\text { Result } \\
\mu g / m L\end{array}$ & $\begin{array}{c}\text { Sample } \\
\text { Precision } \\
\text { RPD }\end{array}$ & $\begin{array}{c}\text { Detection } \\
\text { Limit } \\
\mu g / \mathrm{mL}\end{array}$ & $\begin{array}{c}\text { Standard } \\
\text { Recovery } \\
\%\end{array}$ & $\begin{array}{c}\text { Spike } \\
\text { Recovery } \\
\%\end{array}$ & $\begin{array}{c}\text { Spike } \\
\text { Duplicate } \\
\text { \% Recovery }\end{array}$ & $\begin{array}{c}\text { Spike Dupl. } \\
\text { Precision } \\
\text { RPD }\end{array}$ & $\begin{array}{l}\text { Blank } \\
\mu g / m L\end{array}$ \\
\hline S96V000047 & AP-105, 5AP-96-1C & $9.50 E+04$ & $n / a$ & $n / a$ & $2.95 E+02$ & 101.0 & $n / a$ & $n / a$ & $n / a$ & $<1.39 \mathrm{E}-01$ \\
\hline S96V000048 & AP-105, 5AP-96-2C & $1.01 E+05$ & $n / a$ & $n / a$ & $5.76 E+02$ & 101.0 & 100.5 & 101.8 & 2.0 & $<1.39 \mathrm{E}-01$ \\
\hline S96V000049 & AP-105, 5AP-96-3C & $1.02 E+05$ & $n / a$ & $\mathrm{n} / \mathrm{a}$ & $2.95 E+02$ & 101.0 & $n / a$ & $\mathrm{n} / \mathrm{a}$ & $n / a$ & $<1.39 E-01$ \\
\hline S96V000058 & AP-105, 5AP-96-1B & 8.63E-01 & $\mathrm{n} / \mathrm{a}$ & $\mathrm{n} / \mathrm{a}$ & $8.34 E-01$ & 101.0 & $n / a$ & $\mathrm{n} / \mathrm{a}$ & n/a & $<1.39 \mathrm{E}-01$ \\
\hline
\end{tabular}

Phosphate-1C-Dionex 4000/4500

\begin{tabular}{|c|c|c|c|c|c|c|c|c|c|c|}
\hline $\begin{array}{l}\text { Labcore } \\
\text { Number }\end{array}$ & $\begin{array}{l}\text { Customer } \\
\text { Identification }\end{array}$ & $\begin{array}{l}\text { Sample } \\
\text { Result } \\
\mu g / m L\end{array}$ & $\begin{array}{c}\text { Duplicate } \\
\text { Result } \\
\mu g / m L\end{array}$ & $\begin{array}{c}\text { Sample } \\
\text { Precision } \\
\text { RPD }\end{array}$ & $\begin{array}{c}\text { Detection } \\
\text { Limit } \\
\mu \mathrm{g} / \mathrm{mL}\end{array}$ & $\begin{array}{c}\text { Standard } \\
\text { Recovery } \\
\%\end{array}$ & $\begin{array}{c}\text { Spike } \\
\text { Recovery } \\
\%\end{array}$ & $\begin{array}{c}\text { Spike } \\
\text { Duplicate } \\
\text { \% Recovery }\end{array}$ & $\begin{array}{l}\text { Spike Dupl. } \\
\text { Precision } \\
\text { RPD }\end{array}$ & $\begin{array}{l}\text { Blank } \\
\mu \mathrm{g} / \mathrm{mL}\end{array}$ \\
\hline S96V000047 & AP- $105,5 A P-96-1 C$ & $1.15 \mathrm{E}+03$ & $n / a$ & n/a & $2.54 \varepsilon+02$ & 99.4 & $n / a$ & $\mathrm{n} / \mathrm{a}$ & $n / a$ & $<1.20 E-01$ \\
\hline S96V000048 & AP-105, 5AP-96-2C & $1.39 \mathrm{E}+03$ & $\mathrm{n} / \mathrm{a}$ & $n / a$ & $4.97 E+02$ & 99.4 & 93.9 & 93.9 & 6.0 & $<1.20 \mathrm{E}-01$ \\
\hline S96V000049 & AP- $105,5 A P-96-3 C$ & $1.54 \mathrm{E}+03$ & $\mathrm{n} / \mathrm{a}$ & $n / a$ & $2.54 E+02$ & 99.4 & $\mathrm{n} / \mathrm{a}$ & n/a & n/a & $<1.20 \mathrm{E}-01$ \\
\hline S96V000058 & AP- 105,5 AP- $96-1 B$ & $<7.20 \mathrm{E}-01$ & $n / a$ & $n / a$ & $7.20 \mathrm{E}-01$ & 99.4 & $\mathrm{n} / \mathrm{a}$ & $\mathrm{n} / \mathbf{a}$ & $\mathrm{n} / \mathrm{a}$ & $<1.20 \mathrm{E}-01$ \\
\hline
\end{tabular}

Sulfate by IC-Dionex $\mathbf{4 0 0 0 / 4 5 0 0}$

\begin{tabular}{|c|c|c|c|c|c|c|c|c|c|c|}
\hline $\begin{array}{l}\text { Labcore } \\
\text { Number }\end{array}$ & $\begin{array}{c}\text { Customer } \\
\text { Identification }\end{array}$ & $\begin{array}{l}\text { Sampie } \\
\text { Result } \\
\mu g / m L\end{array}$ & $\begin{array}{c}\text { Duplicate } \\
\text { Result } \\
\mu g / m L\end{array}$ & $\begin{array}{c}\text { Sample } \\
\text { Precision } \\
\text { RPD }\end{array}$ & $\begin{array}{c}\text { Detection } \\
\text { Limit } \\
\mu g / \mathrm{mL}\end{array}$ & $\begin{array}{c}\text { Standard } \\
\text { Recovery } \\
\%\end{array}$ & $\begin{array}{c}\text { Spike } \\
\text { Recovery } \\
\%\end{array}$ & $\begin{array}{c}\text { Spike } \\
\text { Duplicate } \\
\text { \% Recovery }\end{array}$ & $\begin{array}{c}\text { Spike Dupl. } \\
\text { Precision } \\
\text { RPD }\end{array}$ & $\begin{array}{l}\text { Blank } \\
\mu g / m L\end{array}$ \\
\hline S96V000047 & AP-105, 5AP-96-1C & $1.93 E+03$ & $n / a$ & $\mathrm{n} / \mathrm{a}$ & $2.93 E+02$ & 103.0 & $n / a$ & n/a & $\mathrm{n} / \mathrm{a}$ & $<1.38 E-01$ \\
\hline S96V000048 & AP-105, 5AP-96-2C & $2.54 \mathrm{E}+03$ & $n / a$ & $\pi / a$ & $5.72 E+02$ & 103.0 & 99.8 & 101.1 & 1.3 & $<1.38 \mathrm{E}-01$ \\
\hline S96V000049 & AP-105, 5AP-96-3C & $2.06 \mathrm{E}+03$ & $n / a$ & $\mathrm{n} / \mathrm{a}$ & $2.93 E+02$ & 103.0 & $\mathrm{n} / \mathrm{a}$ & $n / a$ & $n / a$ & $<1.38 \mathrm{E}-01$ \\
\hline S96V000058 & AP-105, 5AP-96-IB & $1.47 \mathrm{E}+01$ & $n / a$ & $\mathrm{n} / \mathrm{a}$ & $8.28 E-01$ & 103.0 & $n / a$ & n/a & $n / a$ & $<1.38 \mathrm{E}-01$ \\
\hline
\end{tabular}


WAEE-SD-WM-DP-202, REV. 1

Aluminium HCP-Acid Digest-Liq

Procedure: LA-605-151/161

\begin{tabular}{|c|c|c|c|c|c|c|c|c|c|c|c|c|}
\hline $\begin{array}{l}\text { Labcore } \\
\text { Number }\end{array}$ & $\begin{array}{c}\text { Customer } \\
\text { Identification }\end{array}$ & $\begin{array}{l}\text { Sampie } \\
\text { Result } \\
\mu g / m L \\
\end{array}$ & $\begin{array}{c}\text { Duplicate } \\
\text { Result } \\
\text { pg/mL }\end{array}$ & $\begin{array}{c}\text { Sample } \\
\text { Precision } \\
\text { RPD }\end{array}$ & $\begin{array}{c}\text { Detection } \\
\text { Limit } \\
\mu \mathrm{g} / \mathrm{mL}\end{array}$ & $\begin{array}{c}\text { Standard } \\
\text { Recovery } \\
\%\end{array}$ & $\begin{array}{c}\text { Spike } \\
\text { Recovery } \\
\%\end{array}$ & $\begin{array}{c}\text { Spike } \\
\text { Duplicate } \\
\text { \% Recovery }\end{array}$ & $\begin{array}{c}\text { Spike Dupl. } \\
\text { Precision } \\
\text { RPD }\end{array}$ & $\begin{array}{c}\text { Preparation } \\
\text { Blank } \\
\mu \mathrm{mL}\end{array}$ & $\begin{array}{c}\text { Serial } \\
\text { Dilution } \\
\mu g / \mathrm{ml}\end{array}$ & $\begin{array}{c}\text { Serial } \\
\text { Dilution } \\
\text { \% Difference }\end{array}$ \\
\hline$\$ 96 \mathrm{~V} 000050$ & AP-105, 5AP-96-1C & $1.74 E+04$ & $1.75 E+04$ & 0.6 & $1.25 E+01$ & 89.8 & 66.6 & n/a & na & 1.31E-01 & $1.78 E+04$ & 2.3 \\
\hline S96V0000S1 & AP-105, 5AP-96-2C & 1.77E+04 & $1.67 E+04$ & 5.8 & $1.25 E+01$ & 89.8 & $\mathrm{n} / \mathrm{a}$ & n/a & $n / a$ & 1.31E-01 & Na & Na \\
\hline S96V000052 & AP-105, 5AP-96-3C & $1.72 E+04$ & $1.76 \mathrm{E}+04$ & 2.3 & $1.25 \mathrm{E}+01$ & 89.8 & $\mathrm{n} / \mathrm{a}$ & n/a & n/a & 1.31E-01 & $\mathrm{n} / \mathbf{a}$ & $\mathrm{n} / \mathrm{a}$ \\
\hline S96V000054 & AP-105, 5AP-96-4 & $1.77 \mathrm{E}+04$ & $1.73 E+04$ & 2.3 & 1.25E+01 & 89.8 & n/a & Na & na & 1.31E-01 & $n / a$ & $\mathrm{Na}$ \\
\hline \$96V000060 & AP-105, 5AP-96-1B & $7.42 \mathrm{E}+\infty 0$ & $6.98 E+00$ & 6.1 & $2.50 \mathrm{E}+00$ & 89.8 & $\mathrm{Na}$ & $\mathrm{n} / \mathrm{a}$ & na & 1.31E-01 & na & n/a \\
\hline
\end{tabular}

Chromium KCP-Acid Digest-Liq

Procedure: LA-505-151/161

\begin{tabular}{|c|c|c|c|c|c|c|c|c|c|c|c|c|}
\hline $\begin{array}{l}\text { Labcore } \\
\text { Number }\end{array}$ & $\begin{array}{c}\text { Customer } \\
\text { Identification }\end{array}$ & $\begin{array}{l}\text { Sample } \\
\text { Result } \\
\mu g / m L\end{array}$ & $\begin{array}{c}\text { Duplicate } \\
\text { Result } \\
\mu \mathrm{g} / \mathrm{mL}\end{array}$ & $\begin{array}{c}\text { Sample } \\
\text { Precision } \\
\text { RPD } \\
\end{array}$ & $\begin{array}{c}\text { Detection } \\
\text { Limit } \\
\mu g / m L\end{array}$ & $\begin{array}{c}\text { Standard } \\
\text { Recovery } \\
\%\end{array}$ & $\begin{array}{c}\text { Spike } \\
\text { Recovery } \\
\%\end{array}$ & \begin{tabular}{c|} 
Spike \\
Duplicate \\
\% Recovery \\
\end{tabular} & $\begin{array}{c}\text { Spike Dupl. } \\
\text { Precision } \\
\text { RPD } \\
\end{array}$ & $\begin{array}{c}\text { Preparation } \\
\text { Blank } \\
\mu g / \mathrm{mL} \\
\end{array}$ & $\begin{array}{c}\text { Serial } \\
\text { Dilution } \\
\mu \mathrm{mL}\end{array}$ & $\begin{array}{c}\text { Serial } \\
\text { Dilution } \\
\% \text { Difference } \\
\end{array}$ \\
\hline S96V000050 & AP+105, 5AP-96-1C & $2.13 E+02$ & $2.13 E+02$ & 0.00 & $2.50 E+00$ & 88,6 & 89.6 & n/a & na & $<1.00 \mathrm{E}-02$ & $2.18 \mathrm{E}+02$ & 2.3 \\
\hline $596 \mathrm{~V} 000051$ & AP-105, 5AP-96-2C & $2.16 \mathrm{E}+02$ & $2.05 E+02$ & 5.2 & $2.50 E+\infty 0$ & 88.6 & $\sqrt{a}$ & Na & n/a & $<1.00 E-02$ & Na & $\mathrm{N} / \mathrm{a}$ \\
\hline S96V000052 & AP-105, 5AP-S6-3C & $2.11 E+02$ & $2.15 E+02$ & 1.9 & $2.50 \mathrm{E}+00$ & 88.6 & $\mathrm{n} / \mathrm{a}$ & n/a & Na & $<1.00 \mathrm{E}-02$ & Na & $n / a$ \\
\hline S96V000054 & $A P=105,5 A P-96-4$ & $2.16 \mathrm{E}+02$ & $2.11 \mathrm{E}+02$ & 2.3 & $2.50 \mathrm{E}+00$ & 88.6 & $\mathrm{na}$ & $\mathrm{n} / \mathrm{a}$ & $\mathrm{n} / \mathrm{a}$ & $<1.00 E-02$ & $\mathrm{Na}$ & $n / a$ \\
\hline $596 \mathrm{~V} 000060$ & AP-105, 5AP-96-1B & $<5.00 \mathrm{E}-01$ & $<5.00 \mathrm{E}-01$ & $\mathrm{n} / \mathbf{a}$ & $5.00 \mathrm{E}-0 \mathrm{I}$ & 88.6 & $n / a$ & na & $\mathrm{n} / \mathrm{a}$ & $<1.00 E-02$ & $n / a$ & $\mathrm{Na}$ \\
\hline
\end{tabular}

Iron KCP-Acid Digest-Liquid

Procedure: LA-605-151/161

a)

\begin{tabular}{|c|c|c|c|c|c|c|c|c|c|c|c|c|}
\hline $\begin{array}{l}\text { Labcore } \\
\text { Number }\end{array}$ & $\begin{array}{l}\text { Customer } \\
\text { Identification }\end{array}$ & $\begin{array}{l}\text { Sample } \\
\text { Resutt } \\
\mu \mathrm{g} / \mathrm{mL}\end{array}$ & $\begin{array}{c}\text { Duplicate } \\
\text { Result } \\
\mu g / \mathrm{mL}\end{array}$ & $\begin{array}{c}\text { Sample } \\
\text { Precision } \\
\text { RPD }\end{array}$ & $\begin{array}{c}\text { Detection } \\
\text { Limit } \\
\mu g / m L\end{array}$ & $\begin{array}{c}\text { Standard } \\
\text { Recovery } \\
\%\end{array}$ & $\begin{array}{c}\text { Spike } \\
\text { Recovery } \\
\%\end{array}$ & $\begin{array}{c}\text { Spike } \\
\text { Duplicate } \\
\text { \% Recovery } \\
\end{array}$ & $\begin{array}{c}\text { Spike Dupi. } \\
\text { Precision } \\
\text { RPD } \\
\end{array}$ & $\begin{array}{c}\text { Preparation } \\
\text { Blank } \\
\mu \mathrm{g} / \mathrm{mL}\end{array}$ & $\begin{array}{c}\text { Serial } \\
\text { Dilution } \\
\mu g / \mathrm{mL}\end{array}$ & $\begin{array}{c}\text { Serial } \\
\text { Dilution } \\
\% \text { Difference }\end{array}$ \\
\hline S96V000050 & AP-105, 5AP-96-1C & $<1.25 E+01$ & $<1.25 E+01$ & $\mathrm{na}$ & $1.25 E+01$ & 91.0 & 91.8 & $\mathrm{n} / \mathrm{a}$ & $\mathrm{n} / \mathrm{a}$ & $5.00 \mathrm{E}-02$ & $<6.25 E+01$ & $\mathrm{n} / \mathrm{a}$ \\
\hline s96v000051 & AP-105, 5AP-96-2C & $<1.25 E+01$ & $<1.25 \mathrm{E}+01$ & n/a & $1.25 E+01$ & 91.0 & $\mathrm{n} / \mathrm{a}$ & Na & n/a & $<5,00 \mathrm{E}-02$ & N/a & $\mathrm{n} / \mathrm{a}$ \\
\hline S96V000052 & AP- 105,5 AP-96-3C & $<1.25 E+01$ & $<1.25 E+0 t$ & $\mathrm{n} / \mathbf{a}$ & $1.25 E+01$ & 91.0 & $\mathrm{Na}$ & na & $n / a$ & $<5.00 \mathrm{E}-02$ & Na & n/a \\
\hline s96V000054 & AP-105, 5AP-96-4 & $<1.25 E+01$ & $<1.25 E+04$ & na & $1.25 E+01$ & 91.0 & n/a & $\mathrm{na}$ & $n / a$ & $<5.00 \mathrm{E}-02$ & na & $n / a$ \\
\hline S96V000060 & AP-105, 5AP-96-1B & $<2.50 E+\infty$ & $<2.50 \mathrm{E}+\infty$ & n/a & $2.50 E+\infty 0$ & 91.0 & $n / a$ & $\sqrt{a}$ & $\mathrm{n} / \mathrm{a}$ & $<5.00 \mathrm{E}-02$ & na & $\mathrm{n} / \mathrm{a}$ \\
\hline
\end{tabular}

Manganese +CP-Acid Digest-Liq

Procedure: LA-605-161/161

\begin{tabular}{|c|c|c|c|c|c|c|c|c|c|c|c|c|}
\hline $\begin{array}{l}\text { Labcore } \\
\text { Number }\end{array}$ & $\begin{array}{l}\text { Customer } \\
\text { Identification }\end{array}$ & $\begin{array}{l}\text { Sample } \\
\text { Result } \\
\mu \mathrm{g} / \mathrm{mL}\end{array}$ & $\begin{array}{l}\text { Duplicate } \\
\text { Result } \\
\mu \mathrm{g} / \mathrm{mL}\end{array}$ & $\begin{array}{c}\text { Sample } \\
\text { Precision } \\
\text { RPD }\end{array}$ & $\begin{array}{c}\text { Detection } \\
\text { Limit } \\
\mu \mathrm{g} / \mathrm{mL}\end{array}$ & $\begin{array}{c}\text { Standard } \\
\text { Recovery } \\
\%\end{array}$ & $\begin{array}{c}\text { Spike } \\
\text { Recovery } \\
\%\end{array}$ & $\begin{array}{c}\text { Spike } \\
\text { Dupllicate } \\
\text { \% Recovery }\end{array}$ & $\begin{array}{l}\text { Spike Dupl. } \\
\text { Precision } \\
\text { RPD }\end{array}$ & $\begin{array}{c}\text { Preparation } \\
\text { Blank } \\
\text { yg/mL }\end{array}$ & $\begin{array}{l}\text { Serial } \\
\text { Dilution } \\
\mu / \mathrm{mL}\end{array}$ & $\begin{array}{c}\text { Serial } \\
\text { Dilution } \\
\text { \& Difference }\end{array}$ \\
\hline 5967000050 & AP- 105,5 AP-96-1C & $<2.50 E+00$ & $<2.50 E+\infty 0$ & $\mathbf{n a}$ & $2.50 E+00$ & 86.4 & 87.0 & n/a & $n / a$ & $<1.00 \mathrm{E}-02$ & $<1.25 \mathrm{E}+01$ & n/a \\
\hline s96V000051 & AP-105, 5AP-96-2C & $<2.50 E+\infty$ & $<2.50 E+00$ & na & $2.50 E+00$ & 86.4 & n/a & n/a & $\mathrm{n} / \mathrm{a}$ & $\angle 1.00 \mathrm{E}-02$ & na & n/a \\
\hline S96V000052 & AP-105, 5AP-96-3C & $<2.50 \mathrm{E}+\infty$ & $<2.50 \mathrm{E}+\infty$ & $n / \mathbf{a}$ & $2.50 \mathrm{E}+00$ & 86.4 & $n / a$ & na & n/a & $<1,00 E-02$ & na & $\mathrm{n} / \mathrm{a}$ \\
\hline s96v000054 & AP-105, 5AP-96-4 & $<2.50 \mathrm{E}+\infty$ & $<2.50 E+\infty 0$ & nia & $2.50 \mathrm{E}+00$ & 86.4 & n/a & Na & $\mathrm{n} / \mathrm{a}$ & $<1,00 \mathrm{E}-02$ & na & $\mathrm{na}$ \\
\hline S96V000060 & AP-105, 5AP-96-1B & $<5.00 \mathrm{E}-01$ & $<5.00 E-01$ & na & $5.00 \mathrm{E}-01$ & 86.4 & n/a & $n \sqrt{a}$ & $n / a$ & $<1.00 E-02$ & n/a & n/a \\
\hline
\end{tabular}


HEs

SD-WM-DP-202, REV. 1

Sodium HCPAAcid Digest-Liquid

Procedure: LA-505-151/161

\begin{tabular}{|c|c|c|c|c|c|c|c|c|c|c|c|c|}
\hline $\begin{array}{l}\text { Labcore } \\
\text { Number }\end{array}$ & $\begin{array}{c}\text { Customer } \\
\text { Identification }\end{array}$ & $\begin{array}{l}\text { Sample } \\
\text { Result } \\
\mu g / m L\end{array}$ & $\begin{array}{c}\text { Duplicate } \\
\text { Result } \\
\mu g^{\prime} / m L\end{array}$ & $\begin{array}{c}\text { Sample } \\
\text { Precision } \\
\text { RPD }\end{array}$ & $\begin{array}{c}\text { Detection } \\
\text { Limit } \\
\mu g / \mathrm{mL}\end{array}$ & $\begin{array}{c}\text { Standard } \\
\text { Recovery } \\
\text { \% }\end{array}$ & $\begin{array}{c}\text { Spike } \\
\text { Recovery } \\
\%\end{array}$ & $\begin{array}{c}\text { Spike } \\
\text { Duplicate } \\
\text { \% Recovery }\end{array}$ & $\begin{array}{l}\text { Spike Dupl. } \\
\text { Precision } \\
\text { RPD }\end{array}$ & $\begin{array}{c}\text { Preparation } \\
\text { Blank } \\
\mu g / m L\end{array}$ & $\begin{array}{l}\text { Serial } \\
\text { Dilution } \\
\mu 9 / \mathrm{mL}\end{array}$ & $\begin{array}{c}\text { Serial } \\
\text { Dilution } \\
\text { * Difference }\end{array}$ \\
\hline S96V000050 & AP-105, 5AP-96-1C & $1.13 E+05$ & $1.13 \mathrm{E}+05$ & 0.0 & $2.50 \mathrm{E}+01$ & 104.6 & -3.4 & $\mathrm{n} / \mathrm{a}$ & $n / a$ & 9.85E-01 & $1.16 \mathrm{E}+05$ & 2.7 \\
\hline S96V000051 & AP-105, 5AP-96-2C & $1.16 \mathrm{E}+05$ & $1.09 \mathrm{E}+05$ & 6.3 & $2.50 E+01$ & 104.6 & $n / a$ & $\mathrm{na}$ & $\mathrm{na}$ & 9.85E-01 & $\mathrm{n} / \mathrm{a}$ & na \\
\hline S96V000052 & AP-105, 5AP-96-3C & $1.10 \mathrm{E}+05$ & $1.13 E+05$ & 2.7 & $2.50 \mathrm{E}+01$ & 104.6 & $\mathrm{n} / \mathrm{a}$ & $\mathrm{Na}$ & na & $9.85 \mathrm{E}-01$ & $\mathrm{n} / \mathrm{a}$ & na \\
\hline S96V000054 & AP- 105,5 AP-96-4 & $1.14 \mathrm{E}+05$ & $1.12 \mathrm{E}+05$ & 1.8 & $2.50 E+01$ & 104.6 & $n / a$ & $\mathrm{n} / \mathrm{a}$ & $n / a$ & $9.85 E-01$ & $\mathrm{n} / \mathrm{a}$ & na \\
\hline S96V000060 & AP-105, 5AP-96-18 & $4.90 \mathrm{E}+01$ & $5.50 E+01$ & 11.5 & $5.00 E+\infty$ & 104.6 & $\mathrm{n} / \mathrm{a}$ & $\mathrm{N} / \mathrm{a}$ & $n / a$ & $9 . \overline{85 E-01}$ & $\mathrm{n} / \mathrm{a}$ & $\mathrm{n} / \mathrm{a}$ \\
\hline
\end{tabular}

Nickel HCP-Acid Digest-Liquid

Procedure: LA b05-151/181

\begin{tabular}{|c|c|c|c|c|c|c|c|c|c|c|c|c|}
\hline $\begin{array}{l}\text { Laboore } \\
\text { Number }\end{array}$ & $\begin{array}{l}\text { Customer } \\
\text { Identification }\end{array}$ & $\begin{array}{l}\text { Sample } \\
\text { Result } \\
\mu \mathrm{g} / \mathrm{mL}\end{array}$ & $\begin{array}{c}\text { Duplicate } \\
\text { Result } \\
\mu \mathrm{g} / \mathrm{mL}\end{array}$ & $\begin{array}{c}\text { Sample } \\
\text { Precision } \\
\text { RPD }\end{array}$ & $\begin{array}{c}\text { Detection } \\
\text { Limit } \\
\mu \mathrm{g} / \mathrm{mL}\end{array}$ & $\begin{array}{c}\text { Standard } \\
\text { Recovery } \\
\%\end{array}$ & $\begin{array}{c}\text { Spike } \\
\text { Recovery } \\
\%\end{array}$ & $\begin{array}{c}\text { Spike } \\
\text { Duplicate } \\
\text { \% Recovery }\end{array}$ & $\begin{array}{c}\text { Spike Duph. } \\
\text { Precision } \\
\text { RPD }\end{array}$ & $\begin{array}{c}\text { Preparation } \\
\text { Blank } \\
\mu g / m L \\
\end{array}$ & $\begin{array}{c}\text { Serial } \\
\text { Dilution } \\
\mu \mathrm{g} / \mathrm{mL}\end{array}$ & $\begin{array}{c}\text { Serial } \\
\text { Dilution } \\
\% \text { Difference }\end{array}$ \\
\hline$\$ 96 \mathrm{~V} 000050$ & AP-105, 5AP-96-1C & $<5.00 E+00$ & $<5.00 E+\infty 0$ & na & $5.00 E+00$ & 91.2 & 92.4 & $\mathrm{n} / \mathrm{a}$ & $\mathrm{n} / \mathrm{a}$ & $<2.00 E-02$ & $<2.50 \mathrm{E}+01$ & $\mathrm{n} / \mathbf{a}$ \\
\hline S96V000051 & AP- $105,5 A P-96-2 C$ & $<5.00 \mathrm{E}+00$ & $<5.00 E+00$ & na & $5.00 \mathrm{E}+00$ & 91.2 & $\mathrm{n} / \mathrm{a}$ & $n / a$ & $n / a$ & $<2.00 \mathrm{E}-02$ & $n / a$ & $n / a$ \\
\hline S96V000052 & AP- $105,5 A P-96-3 C$ & $<5.00 E+\infty$ & $<5.00 \mathrm{E}+00$ & ra & $5.00 E+00$ & 91.2 & $\mathrm{n} / \mathrm{a}$ & n/a & na & $<2.00 E-02$ & $\mathrm{~N} / \mathrm{a}$ & na \\
\hline S96V000054 & AP- $105,5 A P-96-4$ & $<5.00 E+00$ & $<5.00 E+\infty 0$ & na & $5.00 E+00$ & 91.2 & $\mathrm{na}$ & $\mathrm{n} / \mathrm{a}$ & n/a & $<2.00 \mathrm{E}-02$ & $\mathrm{~N} / \mathrm{a}$ & Na \\
\hline S96V000060 & AP-105, 5AP-96-1B & $<1.00 E+\infty$ & $<1.00 E+\infty$ & $n / \mathbf{a}$ & $1.00 E+\infty 0$ & 91.2 & $n / a$ & $n / a$ & $n / a$ & $<2.00 E-02$ & $\mathrm{n} / \mathrm{a}$ & n/a \\
\hline
\end{tabular}

Silicon HCPAcid Digest-Liq

Procedure: LA-605-151/161

\begin{tabular}{|c|c|c|c|c|c|c|c|c|c|c|c|c|}
\hline $\begin{array}{l}\text { Labcore } \\
\text { Number }\end{array}$ & $\begin{array}{l}\text { Customer } \\
\text { identification }\end{array}$ & $\begin{array}{l}\text { Sample } \\
\text { Result } \\
\mu \mathrm{g} / \mathrm{mL}\end{array}$ & $\begin{array}{c}\text { Duplicate } \\
\text { Result } \\
\mu \mathrm{g} / \mathrm{mL}\end{array}$ & $\begin{array}{c}\text { Sample } \\
\text { Precision } \\
\text { RPD }\end{array}$ & $\begin{array}{c}\text { Detection } \\
\text { Limit } \\
\mu \mathrm{g} / \mathrm{mL}\end{array}$ & $\begin{array}{c}\text { Standard } \\
\text { Recovery } \\
\%\end{array}$ & $\begin{array}{c}\text { Spike } \\
\text { Recovery } \\
\%\end{array}$ & $\begin{array}{c}\text { Spike } \\
\text { Duplicate } \\
\text { \% Recovery } \\
\end{array}$ & $\begin{array}{c}\text { Spike Dupl. } \\
\text { Precision } \\
\text { RPD } \\
\end{array}$ & $\begin{array}{c}\text { Preparation } \\
\text { Blank } \\
\mu / \mathrm{mL}\end{array}$ & $\begin{array}{c}\text { Serial } \\
\text { Dilution } \\
\mathbf{\mu x / m L}\end{array}$ & $\begin{array}{c}\text { Serial } \\
\text { Dilution } \\
\text { \% Difference } \\
\end{array}$ \\
\hline S96V000050 & AP-105, 5AP-96-1C & $9.44 E+01$ & $7.37 E+01$ & 24.6 & $1.25 E+01$ & 97.6 & 65.4 & n/a & $\mathrm{n} / \mathrm{a}$ & $1.17 E+\infty$ & $9.70 E+01$ & \begin{tabular}{|l|l|}
2.8 \\
\end{tabular} \\
\hline S95V000051 & AP-105, 5AP-96-2C & $8.44 E+01$ & $8.36 E+01$ & 1.0 & $1.25 E+01$ & 97.6 & na & $\mathrm{n} / \mathrm{a}$ & $\mathrm{Na}$ & 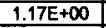 & $\mathrm{n} / \mathrm{a}$ & na \\
\hline S96V000052 & AP-105, 5AP-96-3C & $8.36 E+01$ & $8.69 E+01$ & 3.9 & $1.25 E+01$ & 97.6 & $n / a$ & $n / 2$ & $n / 2$ & $1.17 E+\infty$ & $n / a$ & $\mathrm{n} / \mathrm{a}$ \\
\hline $596 \mathrm{~V} 000054$ & AP-105, 5AP-96-4 & $9.09 E+01$ & $8.51 E+01$ & 6.6 & $1.25 E+01$ & 97.6 & $n / a$ & n/a & n/a & $1.17 \mathrm{E}+\infty 0$ & na & $\mathrm{n} / \mathrm{a}$ \\
\hline s96v000060 & AP-105, 5AP-96-19 & $6.82 \mathrm{E}+01$ & $7.43 E+01$ & 8.6 & $2.50 E+00$ & 97.6 & na & na & $\mathrm{na}$ & $1.17 E+\infty$ & $n / a$ & na \\
\hline
\end{tabular}

Uranium +CP-Acid Digest-Liq

Procedure: LA-505-151/161

\begin{tabular}{|c|c|c|c|c|c|c|c|c|c|c|c|c|}
\hline $\begin{array}{l}\text { Labcore } \\
\text { Number }\end{array}$ & $\begin{array}{c}\text { Customer } \\
\text { Identification }\end{array}$ & $\begin{array}{l}\text { Sample } \\
\text { Result } \\
\text { Ho/mL }\end{array}$ & $\begin{array}{c}\text { Duplicate } \\
\text { Resulk } \\
\mu g / \mathrm{mL}\end{array}$ & $\begin{array}{c}\text { Sample } \\
\text { Precision } \\
\text { RPD }\end{array}$ & $\begin{array}{c}\text { Detection } \\
\text { Limit } \\
\mu \mathrm{g} / \mathrm{mL}\end{array}$ & $\begin{array}{c}\text { Standard } \\
\text { Recovery } \\
\%\end{array}$ & $\begin{array}{c}\text { Spike } \\
\text { Recovery } \\
\%\end{array}$ & $\begin{array}{c}\text { Spike } \\
\text { Duplicate } \\
\text { \% Recovery }\end{array}$ & $\begin{array}{l}\text { Spike Dupl. } \\
\text { Precision } \\
\text { RPD }\end{array}$ & $\begin{array}{c}\text { Preparation } \\
\text { Blank } \\
\mu g / \mathrm{mL}\end{array}$ & $\begin{array}{c}\text { Serial } \\
\text { Dilution } \\
\mu g / m L\end{array}$ & $\begin{array}{c}\text { Serial } \\
\text { Dilution } \\
\text { \% Difference }\end{array}$ \\
\hline S96V000050 & AP-105, 5AP-96-1C & $<1.25 E+02$ & $<1.25 \mathrm{E}+02$ & na & $1.25 E+02$ & 85.6 & 88.2 & na & $\mathrm{N} / \mathrm{a}$ & $<5.00 \mathrm{E}-01$ & $<6.25 E+02$ & $n / a$ \\
\hline S96V0000S1 & AP-105, 5AP-96-2C & $<1.25 \mathrm{E}+02$ & $<1.25 E+02$ & $n / 2$ & $1.25 E+02$ & 85.6 & $n / a$ & na & $\mathrm{n} / \mathrm{a}$ & $<5.00 \mathrm{E}-01$ & $\mathrm{n} / \mathrm{a}$ & na \\
\hline S96V000052 & AP-105, 5AP-96-3C & $<1.25 E+02$ & $<1.25 E+02$ & $n / a$ & $1.25 E+02$ & 85.6 & $\mathrm{n} / \mathrm{B}$ & $\mathrm{na}$ & na & $\angle 5.00 \mathrm{E}-01$ & $\mathrm{na}$ & Wa \\
\hline S96v000054 & AP-105, 5AP-96-4 & $<1.25 E+02$ & $<1.25 E+02$ & $\mathrm{n} / \mathrm{a}$ & $1.25 \mathrm{E}+02$ & 85.6 & $\mathrm{Na}$ & $\mathrm{Na}$ & $\mathrm{n} / \mathrm{a}$ & $<5.00 \mathrm{E}-01$ & na & $\mathrm{Na}$ \\
\hline S96V000060 & AP-105, 5AP-95-18 & $<2.50 \mathrm{E}+01$ & $<250 E+01$ & $\pi / \mathbf{a}$ & $2.50 E+01$ & 85.6 & $n / a$ & $\mathrm{n} / \mathrm{a}$ & $n / a$ & $<5.00 E-01$ & $n / a$ & $\mathrm{n} / \mathrm{a}$ \\
\hline
\end{tabular}


TIC by Acid/Coulometry

Procedure: LA-342-100

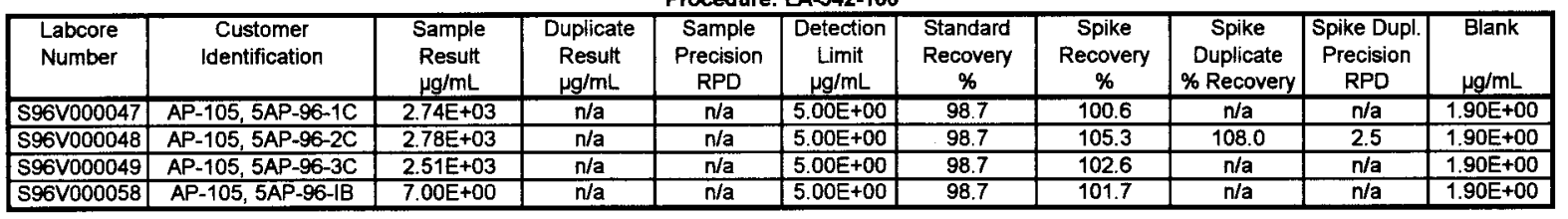

Tot. Organic Carbon by Coul.

Procedure: LA-344-105

\begin{tabular}{|c|c|c|c|c|c|c|c|c|c|c|}
\hline $\begin{array}{l}\text { Labcore } \\
\text { Number }\end{array}$ & $\begin{array}{c}\text { Customer } \\
\text { Identification }\end{array}$ & $\begin{array}{l}\text { Sample } \\
\text { Result } \\
\mu g / m L\end{array}$ & $\begin{array}{c}\text { Duplicate } \\
\text { Result } \\
\mu \mathrm{g} / \mathrm{mL}\end{array}$ & $\begin{array}{c}\text { Sample } \\
\text { Precision } \\
\text { RPD }\end{array}$ & $\begin{array}{c}\text { Detection } \\
\text { Limit } \\
\mu \mathrm{g} / \mathrm{mL}\end{array}$ & $\begin{array}{c}\text { Standard } \\
\text { Recovery } \\
\%\end{array}$ & $\begin{array}{c}\text { Spike } \\
\text { Recovery } \\
\%\end{array}$ & $\begin{array}{c}\text { Spike } \\
\text { Duplicate } \\
\text { \% Recovery }\end{array}$ & $\begin{array}{c}\text { Spike Dupl. } \\
\text { Precision } \\
\text { RPD }\end{array}$ & $\begin{array}{l}\text { Blank } \\
\mu g / m L\end{array}$ \\
\hline S96V000047 & AP-105, 5AP-96-1C & $1.50 \mathrm{E}+03$ & $n / a$ & $\mathrm{n} / \mathrm{a}$ & $5.50 \mathrm{E}+01$ & 94.0 & $\mathrm{n} / \mathrm{a}$ & $n / a$ & $n / a$ & 7.00E-01 \\
\hline S96V000048 & AP-105, 5AP-96-2C & $1.48 \mathrm{E}+03$ & $\mathrm{n} / \mathrm{a}$ & $n / a$ & $5.50 \mathrm{E}+01$ & 94.0 & 90.2 & 89.5 & 0.8 & $7.00 \mathrm{E}-01$ \\
\hline S96V000049 & AP-105, 5AP-96-3C & $1.53 E+03$ & $n / a$ & $n / a$ & $5.50 E+01$ & 94.0 & $n / a$ & $\mathrm{n} / \mathrm{a}$ & $\mathrm{n} / \mathrm{a}$ & $7.00 \mathrm{E}-01$ \\
\hline S96V000053 & $A P-105,5 A P-96-4$ & $1.44 E+03$ & $n / a$ & $n / a$ & $5.50 \mathrm{E}+01$ & 94.0 & $\mathrm{n} / \mathrm{a}$ & $n / a$ & $n / a$ & 7.00E-01 \\
\hline S96V000058 & AP-105, 5AP-96-IB & $7.70 \mathrm{E}+00$ & n/a & $n / a$ & $5.50 \mathrm{E}+00$ & 94.0 & $n / a$ & $\mathrm{n} / \mathrm{a}$ & $n / a$ & $7.00 \mathrm{E}-01$ \\
\hline
\end{tabular}

Total Carbon by Coulometry

Procedure: LA-344-105

\begin{tabular}{|c|c|c|c|c|c|c|c|c|c|c|}
\hline $\begin{array}{l}\text { Labcore } \\
\text { Number }\end{array}$ & $\begin{array}{c}\text { Customer } \\
\text { Identification }\end{array}$ & $\begin{array}{c}\text { Sample } \\
\text { Result } \\
\text { Jg/mL }\end{array}$ & $\begin{array}{c}\text { Duplicate } \\
\text { Result } \\
\mu \mathrm{g} / \mathrm{mL}\end{array}$ & $\begin{array}{c}\text { Sample } \\
\text { Precision } \\
\text { RPD }\end{array}$ & $\begin{array}{c}\text { Detection } \\
\text { Limit } \\
\mu \mathrm{g} / \mathrm{mL}\end{array}$ & $\begin{array}{c}\text { Standard } \\
\text { Recovery } \\
\%\end{array}$ & $\begin{array}{c}\text { Spike } \\
\text { Recovery } \\
\%\end{array}$ & $\begin{array}{c}\text { Spike } \\
\text { Duplicate } \\
\text { \% Recovery }\end{array}$ & $\begin{array}{c}\text { Spike Dupl. } \\
\text { Precision } \\
\text { RPD }\end{array}$ & $\begin{array}{l}\text { Blank } \\
\mu g / \mathrm{mL}\end{array}$ \\
\hline S96V000047 & AP-105, 5AP-96-1C & $4.67 E+03$ & n/a & n/a & $5.50 E+01$ & 94.7 & $\mathrm{n} / \mathrm{a}$ & $n / a$ & $n / a$ & $5.00 \mathrm{E}+00$ \\
\hline S96V000048 & AP-105, 5AP-96-2C & $4.65 E+03$ & $n / a$ & $n / a$ & $5.50 E+01$ & 94.7 & 107.7 & 107.0 & 0.7 & $5.00 \mathrm{E}+00$ \\
\hline S96V000049 & AP- 105,5 AP-96-3C & $4.81 E+03$ & $\mathrm{na}$ & n/a & $5.50 E+01$ & 94.7 & $\mathrm{n} / \mathrm{a}$ & $n / a$ & $n / a$ & $5.00 \mathrm{E}+00$ \\
\hline S96V000058 & AP-105, 5AP-96-1B & $5.06 \mathrm{E}+01$ & $\mathrm{n} / \mathrm{a}$ & $n / a$ & $5.50 \mathrm{E}+00$ & 94.7 & $\mathrm{n} / \mathrm{a}$ & $n / a$ & $n / a$ & $5.00 \mathrm{E}+00$ \\
\hline
\end{tabular}


HASGD-WM-DP-202, REV. 1

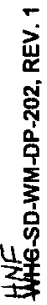

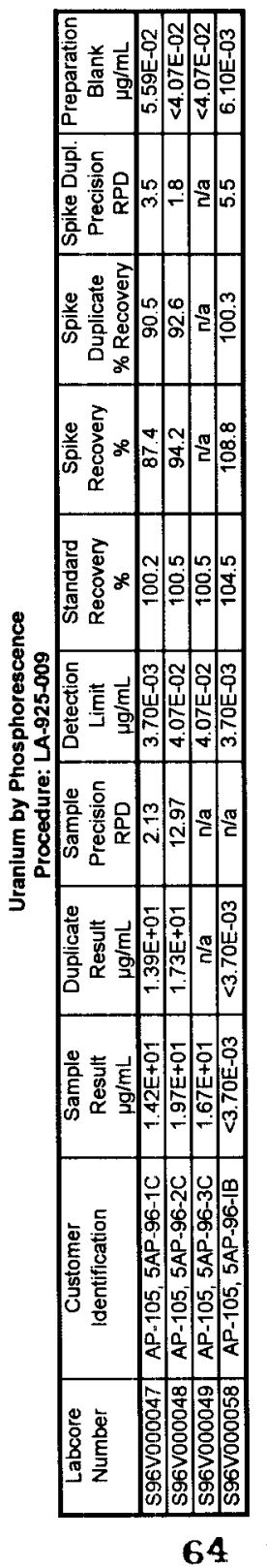


WNFE-SD-WM-DP-202, REV. 1

Apha in Llquid Samples

Procedure: LA-508-101

\begin{tabular}{|c|c|c|c|c|c|c|c|c|c|c|c|}
\hline $\begin{array}{l}\text { Labcore } \\
\text { Number }\end{array}$ & $\begin{array}{c}\text { Customer } \\
\text { Identification }\end{array}$ & $\begin{array}{l}\text { Sample } \\
\text { Result } \\
\mu \mathrm{Ci} / \mathrm{mL}\end{array}$ & $\begin{array}{c}\text { Duplicate } \\
\text { Result } \\
\mu \mathrm{Ci} / \mathrm{mL}\end{array}$ & $\begin{array}{c}\text { Sample } \\
\text { Precision } \\
\text { RPD }\end{array}$ & $\begin{array}{c}\text { Detection } \\
\text { Limit } \\
\mu \mathrm{Ci} / \mathrm{mL}\end{array}$ & $\begin{array}{c}\text { Standard } \\
\text { Recovery } \\
\%\end{array}$ & $\begin{array}{c}\text { Spike } \\
\text { Recovery } \\
\%\end{array}$ & $\begin{array}{c}\text { Spike } \\
\text { Duplicate } \\
\% \text { Recovery }\end{array}$ & $\begin{array}{l}\text { Spike Dupl. } \\
\text { Precision } \\
\text { RPD }\end{array}$ & $\begin{array}{c}\text { Preparation } \\
\text { Glank } \\
\mu \mathrm{Ci} / \mathrm{mL}\end{array}$ & $\begin{array}{c}\text { Counting } \\
\text { Error } \\
\%\end{array}$ \\
\hline S96V000050 & AP-105, 5AP-96-1C & $<1.02 \mathrm{E}-02$ & $<6.76 \mathrm{E}-03$ & n/a & $1.21 E-02$ & 103.0 & $n / a$ & n/a & तa & $<1.02 E-02$ & 500 \\
\hline S96V000051 & AP-105, 5AP-96-2C & $<1.02 \mathrm{E}-02$ & $<5.05 E-03$ & na & $1.21 E-02$ & 103.0 & n/a & na & n/a & $<1.02 E-02$ & 500 \\
\hline S96V000052 & AP-105, 5AP-96-3C & $<5.05 \mathrm{E}-03$ & $<5.05 E-03$ & na & $1.21 E-02$ & 103.0 & n/a & $\mathrm{Na}$ & Na & $<1.02 \mathrm{E}-02$ & 500 \\
\hline S96V000054 & AP-105, 5AP-96-4 & $<5.05 \mathrm{E}-03$ & $<5.05 E-03$ & n/a & $1.21 E-02$ & 103.0 & na & $\mathrm{Na}$ & na & $<1.02 \mathrm{E}-02$ & 500 \\
\hline S96V000060 & AP-105, 5AP-96-1B & $<2.50 \mathrm{E}-05$ & $<4.19 E-05$ & na & 5.97E-05 & 103.0 & 87.7 & 91.4 & 4.1 & $<1.02 \mathrm{E}-02$ & 500 \\
\hline
\end{tabular}

on

Beta In Llquild Samples

Procedure: LA-508-101

\begin{tabular}{|c|c|c|c|c|c|c|c|c|c|c|c|}
\hline $\begin{array}{l}\text { Labcore } \\
\text { Number }\end{array}$ & $\begin{array}{c}\text { Customer } \\
\text { Identification }\end{array}$ & $\begin{array}{c}\text { Sample } \\
\text { Result } \\
\text { ¡Ci/mL }\end{array}$ & $\begin{array}{c}\text { Duplicate } \\
\text { Result } \\
\mu \mathrm{Ci} / \mathrm{mL} \\
\end{array}$ & $\begin{array}{c}\text { Sample } \\
\text { Precision } \\
\text { RPD }\end{array}$ & $\begin{array}{c}\text { Detection } \\
\text { Limit } \\
\mu \mathrm{Ci} / \mathrm{mL}\end{array}$ & $\begin{array}{c}\text { Standard } \\
\text { Recovery } \\
\%\end{array}$ & $\begin{array}{c}\text { Spike } \\
\text { Recovery } \\
\%\end{array}$ & $\begin{array}{c}\text { Spike } \\
\text { Duplicate } \\
\text { \% Recovery }\end{array}$ & $\begin{array}{l}\text { Spike Dupl. } \\
\text { Precision } \\
\text { RPD }\end{array}$ & $\begin{array}{c}\text { Preparation } \\
\text { Blank } \\
\mu \mathrm{Ci} / \mathrm{mL}\end{array}$ & $\begin{array}{c}\text { Counting } \\
\text { Error } \\
\%\end{array}$ \\
\hline S96V000050 & AP-105, 5AP-96-1C & $1.08 \mathrm{E}+02$ & $1.08 E+02$ & 0.0 & 3.71E-02 & 100.3 & $\mathrm{n} / \mathrm{a}$ & $\mathrm{n} / \mathrm{a}$ & na & $1.59 \mathrm{E}-01$ & 1.2 \\
\hline S96V000051 & AP-105, 5AP-96-2C & $1.12 E+02$ & $1.06 \mathrm{E}+02$ & 5.5 & $3.71 \mathrm{E}-02$ & 100.3 & $n / a$ & $n / a$ & $\mathrm{n} / \mathrm{a}$ & $1.59 \mathrm{E}-01$ & 0.4 \\
\hline S96V000052 & AP-105, 5AP-96-3C & $1.07 E+02$ & $1.08 \mathrm{E}+02$ & 0.9 & $3.71 \mathrm{E}-02$ & 100.3 & $\mathrm{Na}$ & $n / a$ & $\mathrm{Na}$ & $1.59 E-01$ & 0.4 \\
\hline S96V000054 & AP-105, 5AP-96-4 & $1.11 \mathrm{E}+02$ & $1.10 \mathrm{E}+02$ & 0.9 & $3.71 \mathrm{E}-02$ & 100.3 & $n / a$ & $n / a$ & n/a & 1.59E-0t & 0.4 \\
\hline S96V000060 & AP-105, 5AP-96-1B & $3.02 E-04$ & $2.19 E-04$ & 31.9 & $1.84 E-04$ & 100.3 & 101.3 & 97.4 & 3.9 & $1.59 \mathrm{E}-01$ & 47.1 \\
\hline
\end{tabular}


WHE-SD-WM-DP-202, REV. 1

Gamma Energy Analysis by Procedure LA-548-121

Ce/Pr-144 by GEA

\begin{tabular}{|c|c|c|c|c|c|c|c|c|c|}
\hline $\begin{array}{l}\text { Labcore } \\
\text { Number }\end{array}$ & $\begin{array}{c}\text { Customer } \\
\text { Identification }\end{array}$ & $\begin{array}{c}\text { Sample } \\
\text { Result } \\
\mu \mathrm{Ci} / \mathrm{mL}\end{array}$ & $\begin{array}{c}\text { Duplicate } \\
\text { Result } \\
\mu \mathrm{Ci} / \mathrm{mL}\end{array}$ & $\begin{array}{l}\text { Sample } \\
\text { Precision } \\
\text { RPD }\end{array}$ & $\begin{array}{c}\text { Defection } \\
\text { Limit } \\
\mu \mathrm{Ci} / \mathrm{mL}\end{array}$ & $\begin{array}{c}\text { Co-60 } \\
\text { Standard } \\
\text { \% Recovery }\end{array}$ & $\begin{array}{c}\text { Cs-137 } \\
\text { Standard } \\
\text { \% Recovery }\end{array}$ & $\begin{array}{c}\text { Preparation } \\
\text { Blank } \\
\mu \mathrm{Ci} / \mathrm{mL}\end{array}$ & $\begin{array}{c}\text { Counting } \\
\text { Error } \\
\%\end{array}$ \\
\hline S96V000050 & AP-105, 5AP-96-1C & $<7.20 \mathrm{E}-01$ & $<7.27$ E-01 & $\mathrm{n} / \mathbf{a}$ & $7.20 \mathrm{E}-01$ & 99.2 & 96.3 & $<1.22 \mathrm{E}-03$ & $n / a$ \\
\hline S96V000051 & AP-105, 5AP-96-2C & $<7.31 \mathrm{E}-01$ & $n / a$ & $n / a$ & 7.31E-01 & 99.2 & 96.3 & $<1.22 \mathrm{E}-03$ & $\mathrm{n} / \mathrm{a}$ \\
\hline S96V000052 & AP-105, 5AP-96-3C & $<7.23 \mathrm{E}-01$ & $n / a$ & $n / a$ & $7.23 \mathrm{E}-01$ & 99.2 & 96.3 & $<1.22 \mathrm{E}-03$ & $\mathrm{n} / \mathrm{a}$ \\
\hline \$96V000060 & AP-105, 5AP-96-IB & $<5.24 \mathrm{E}-02$ & $\mathrm{n} / \mathrm{a}$ & n/a & $5.24 E-02$ & 99.2 & 96.3 & $<1.22 \mathrm{E}-03$ & $n / a$ \\
\hline
\end{tabular}

Cobalt -60 by GEA

\begin{tabular}{|c|c|c|c|c|c|c|c|c|c|}
\hline $\begin{array}{l}\text { Labcore } \\
\text { Number }\end{array}$ & $\begin{array}{c}\text { Customer } \\
\text { Identification }\end{array}$ & $\begin{array}{c}\text { Sample } \\
\text { Result } \\
\mu \mathrm{Ci} / \mathrm{mL}\end{array}$ & $\begin{array}{c}\text { Duplicate } \\
\text { Result } \\
\text { jCi/mL }\end{array}$ & $\begin{array}{c}\text { Sample } \\
\text { Precision } \\
\text { RPD }\end{array}$ & $\begin{array}{c}\text { Detection } \\
\text { Limit } \\
\text { jCímL }\end{array}$ & $\begin{array}{c}\text { Co-60 } \\
\text { Standard } \\
\% \text { Recovery }\end{array}$ & $\begin{array}{c}\text { Cs-137 } \\
\text { Standard } \\
\text { \% Recovery }\end{array}$ & $\begin{array}{c}\text { Preparation } \\
\text { Blank } \\
\text { jCi/mL }\end{array}$ & $\begin{array}{c}\text { Counting } \\
\text { Error } \\
\%\end{array}$ \\
\hline S96V000050 & AP-105, 5AP-96-1C & $<6.07 E-03$ & $<7.31 E-03$ & $\mathrm{n} / \mathrm{a}$ & 6.07E-03 & 99.2 & 96.3 & $<1.14 \mathrm{E}-04$ & $\mathrm{n} / \mathrm{a}$ \\
\hline S96V000051 & AP-105, 5AP-96-2C & $<7.57 \mathrm{E}-03$ & $\mathrm{n} / \mathrm{a}$ & $\mathrm{n} / \mathrm{a}$ & $7.57 E-03$ & 99.2 & 96.3 & $<1.14 \mathrm{E}-04$ & $n / a$ \\
\hline S96V000052 & AP-105, 5AP-96-3C & $<6.71 \mathrm{E}-03$ & $n / a$ & $\mathrm{n} / \mathrm{a}$ & 6.71E-03 & 99.2 & 96.3 & $<1.14 \mathrm{E}-04$ & $\mathrm{n} / \mathrm{a}$ \\
\hline S96V000060 & AP-105, 5AP-96-IB & $<6.71 \mathrm{E}-03$ & $n / a$ & $n / a$ & 6.71트-03 & 99.2 & 96.3 & $<1.14 \mathrm{E}-04$ & $n / a$ \\
\hline
\end{tabular}

\section{Cesium-134 by GEA}

\begin{tabular}{|c|c|c|c|c|c|c|c|c|c|}
\hline $\begin{array}{l}\text { Labcore } \\
\text { Number }\end{array}$ & $\begin{array}{c}\text { Customer } \\
\text { Identification }\end{array}$ & $\begin{array}{c}\text { Sample } \\
\text { Result } \\
\mu \mathrm{Ci} / \mathrm{mL}\end{array}$ & $\begin{array}{c}\text { Duplicate } \\
\text { Result } \\
\mu \mathrm{Ci} / \mathrm{mL}\end{array}$ & $\begin{array}{c}\text { Sample } \\
\text { Precision } \\
\text { RPD }\end{array}$ & $\begin{array}{c}\text { Detection } \\
\text { Limit } \\
\mu \mathrm{Ci} / \mathrm{mL}\end{array}$ & $\begin{array}{c}\text { Co-60 } \\
\text { Standard } \\
\text { \% Recovery }\end{array}$ & $\begin{array}{c}\text { Cs-137 } \\
\text { Standard } \\
\text { \% Recovery }\end{array}$ & $\begin{array}{c}\text { Preparation } \\
\text { Blank } \\
\mu \mathrm{Ci} / \mathrm{mL} \\
\end{array}$ & $\begin{array}{c}\text { Counting } \\
\text { Error } \\
\%\end{array}$ \\
\hline S96V000050 & $-96-1 C$ & $<5.50 \mathrm{E}-02$ & $<5.46 \mathrm{E}-02$ & $n / a$ & $5.50 \mathrm{E}-02$ & 99.2 & 96.3 & $<1.01 E-04$ & $n / a$ \\
\hline S96V000051 & AP-105, 5AP-96-2C & $<5.59 \mathrm{E}-02$ & $n / a$ & $n / a$ & $5.59 \mathrm{E}-02$ & 99.2 & 96.3 & $<1.01 \mathrm{E}-04$ & $n / a$ \\
\hline S96V000052 & AP-105, 5AP-96-3C & $<5.55 E-02$ & $n / a$ & $n / a$ & $5.55 \mathrm{E}-02$ & 99.2 & 96.3 & $<1.01 \mathrm{E}-04$ & $n / a$ \\
\hline S96V000060 & AP-105, 5AP-96-IB & $<4.18 \mathrm{E}-03$ & $\mathrm{n} / \mathrm{a}$ & $\mathrm{n} / \mathrm{a}$ & $4.18 E-03$ & 99.2 & 96.3 & $<1.01 \mathrm{E}-04$ & $n / a$ \\
\hline
\end{tabular}


HNF

WHG-SD-WM-DP-202, REV. 1

Gamma Energy Analysis by Procedure LA-548-121

Cesium- 137 by GEA

\begin{tabular}{|c|c|c|c|c|c|c|c|c|c|}
\hline $\begin{array}{c}\text { Labcore } \\
\text { Number }\end{array}$ & $\begin{array}{c}\text { Customer } \\
\text { Identification }\end{array}$ & $\begin{array}{c}\text { Sample } \\
\text { Result } \\
\mu \mathrm{Ci} / \mathrm{mL}\end{array}$ & $\begin{array}{c}\text { Duplicate } \\
\text { Result } \\
\mu \mathrm{Ci} / \mathrm{mL}\end{array}$ & $\begin{array}{c}\text { Sample } \\
\text { Precision } \\
\text { RPD }\end{array}$ & $\begin{array}{c}\text { Detection } \\
\text { Limit } \\
\mu \mathrm{Ci} / \mathrm{mL}\end{array}$ & $\begin{array}{c}\text { Co-60 } \\
\text { Standard } \\
\% \text { Recovery }\end{array}$ & $\begin{array}{c}\text { Cs-137 } \\
\text { Standard } \\
\% \text { Recovery }\end{array}$ & $\begin{array}{c}\text { Preparation } \\
\text { Blank } \\
\mu \mathrm{Ci} / \mathrm{mL}\end{array}$ & $\begin{array}{c}\text { Counting } \\
\text { Error } \\
\%\end{array}$ \\
\hline S96V000050 & AP-105, 5AP-96-1C & $1.10 \mathrm{E}+02$ & $1.12 \mathrm{E}+02$ & 1.8 & $0.00 \mathrm{E}+00$ & 99.2 & 96.3 & $<2.91 \mathrm{E}-04$ & 0.3 \\
\hline S96V000051 & AP-105, 5AP-96-2C & $1.14 \mathrm{E}+02$ & $\mathrm{n} / \mathrm{a}$ & $\mathrm{n} / \mathrm{a}$ & $0.00 \mathrm{E}+00$ & 99.2 & 96.3 & $<2.91 \mathrm{E}-04$ & 0.3 \\
\hline S96V000052 & AP-105, 5AP-96-3C & $1.12 \mathrm{E}+02$ & $\mathrm{n} / \mathrm{a}$ & $\mathrm{n} / \mathrm{a}$ & $0.00 \mathrm{E}+00$ & 99.2 & 96.3 & $<2.91 \mathrm{E}-04$ & 0.3 \\
\hline S96V000060 & AP-105, 5AP-96-IB & $<1.49 \mathrm{E}-02$ & $\mathrm{n} / \mathrm{a}$ & $\mathrm{n} / \mathrm{a}$ & $1.49 \mathrm{E}-02$ & 99.2 & 96.3 & $<2.91 \mathrm{E}-04$ & $\mathrm{n} / \mathrm{a}$ \\
\hline
\end{tabular}

Europium-154 by GEA

\begin{tabular}{|c|c|c|c|c|c|c|c|c|c|}
\hline $\begin{array}{l}\text { Labcore } \\
\text { Number }\end{array}$ & $\begin{array}{c}\text { Customer } \\
\text { Identification }\end{array}$ & $\begin{array}{c}\text { Sample } \\
\text { Result } \\
\mu \mathrm{Ci} / \mathrm{mL}\end{array}$ & $\begin{array}{c}\text { Duplicate } \\
\text { Result } \\
\mu \mathrm{Ci} / \mathrm{mL}\end{array}$ & $\begin{array}{c}\text { Sample } \\
\text { Precision } \\
\text { RPD }\end{array}$ & $\begin{array}{c}\text { Detection } \\
\text { Limit } \\
\mu \mathrm{Ci} / \mathrm{mL}\end{array}$ & $\begin{array}{c}\text { Co-60 } \\
\text { Standard } \\
\text { \% Recovery }\end{array}$ & $\begin{array}{c}\text { Cs-137 } \\
\text { Standand } \\
\% \text { Recovery }\end{array}$ & $\begin{array}{c}\text { Preparation } \\
\text { Blank } \\
\mu \mathrm{Ci} / \mathrm{mL}\end{array}$ & $\begin{array}{c}\text { Counting } \\
\text { Error } \\
\%\end{array}$ \\
\hline S96V000050 & AP-105, 5AP-96-1C & $<2.36 \mathrm{E}-02$ & $<2.82 \mathrm{E}-02$ & $n / a$ & $2.36 \mathrm{E}-02$ & 99.2 & 96.3 & $<3.45 E-04$ & $n / a$ \\
\hline S96V000051 & AP-105, 5AP-96-2C & $<2.41 \mathrm{E}-02$ & $n / a$ & $n / a$ & $2.41 \mathrm{E}-02$ & 99.2 & 96.3 & $<3.45 \mathrm{E}-04$ & $n / a$ \\
\hline S96V000052 & AP-105, 5AP-96-3C & $<2.63 E-02$ & $n / a$ & $\mathrm{n} / \mathrm{a}$ & $2.63 E-02$ & 99.2 & 96.3 & $<3.45 \mathrm{E}-04$ & $n / a$ \\
\hline S96V000060 & AP-105, 5AP-96-IB & $<1.53 E-02$ & $n / a$ & $n / a$ & $1.53 \mathrm{E}-02$ & 99.2 & 96.3 & $<3.45 \mathrm{E}-04$ & $n / a$ \\
\hline
\end{tabular}

Europium- 155 by GEA

\begin{tabular}{|c|c|c|c|c|c|c|c|c|c|}
\hline $\begin{array}{l}\text { Labcore } \\
\text { Number }\end{array}$ & $\begin{array}{c}\text { Customer } \\
\text { Identification }\end{array}$ & $\begin{array}{c}\text { Sample } \\
\text { Result } \\
\mu \mathrm{Ci} / \mathrm{mL}\end{array}$ & $\begin{array}{c}\text { Duplicate } \\
\text { Result } \\
\mu \mathrm{Ci} / \mathrm{mL}\end{array}$ & $\begin{array}{c}\text { Sample } \\
\text { Precision } \\
\text { RPD }\end{array}$ & $\begin{array}{c}\text { Detection } \\
\text { Limit } \\
\mu \mathrm{Ci} / \mathrm{mL}\end{array}$ & $\begin{array}{c}\text { Co-60 } \\
\text { Standard } \\
\text { \% Recovery }\end{array}$ & \begin{tabular}{|c|} 
Cs-137 \\
Standard \\
\% Recovery \\
\end{tabular} & $\begin{array}{c}\text { Preparation } \\
\text { Blank } \\
\mu \mathrm{Ci} / \mathrm{mL}\end{array}$ & $\begin{array}{c}\text { Counting } \\
\text { Error } \\
\%\end{array}$ \\
\hline S96V000050 & AP-105, 5AP-96-1C & $<2.02 \mathrm{E}-01$ & $<2.04 \mathrm{E}-01$ & $\bar{n} / \mathrm{a}$ & 2.02E-01 & 99.2 & 96.3 & $<3.02 E-04$ & $n / a$ \\
\hline S96V000051 & AP-105, 5AP-96-2C & $<2.05 \mathrm{E}-01$ & $\mathrm{n} / \mathrm{a}$ & $n / a$ & $2.05 E-01$ & 99.2 & 96.3 & $<3.02 E-04$ & $n / a$ \\
\hline S96V000052 & AP-105, 5AP-96-3C & $<2.04 \mathrm{E}-01$ & $n / a$ & $n / a$ & $2.04 \mathrm{E}-01$ & 99.2 & 96.3 & $<3.02 \mathrm{E}-04$ & $\mathrm{n} / \mathrm{a}$ \\
\hline S96V000060 & AP-105, 5AP-96-IB & $<1.80 \mathrm{E}-02$ & $n / a$ & $n / a$ & $1.80 \mathrm{E}-02$ & 99.2 & 96.3 & $<3.02 \mathrm{E}-04$ & $n / \mathbf{a}$ \\
\hline
\end{tabular}



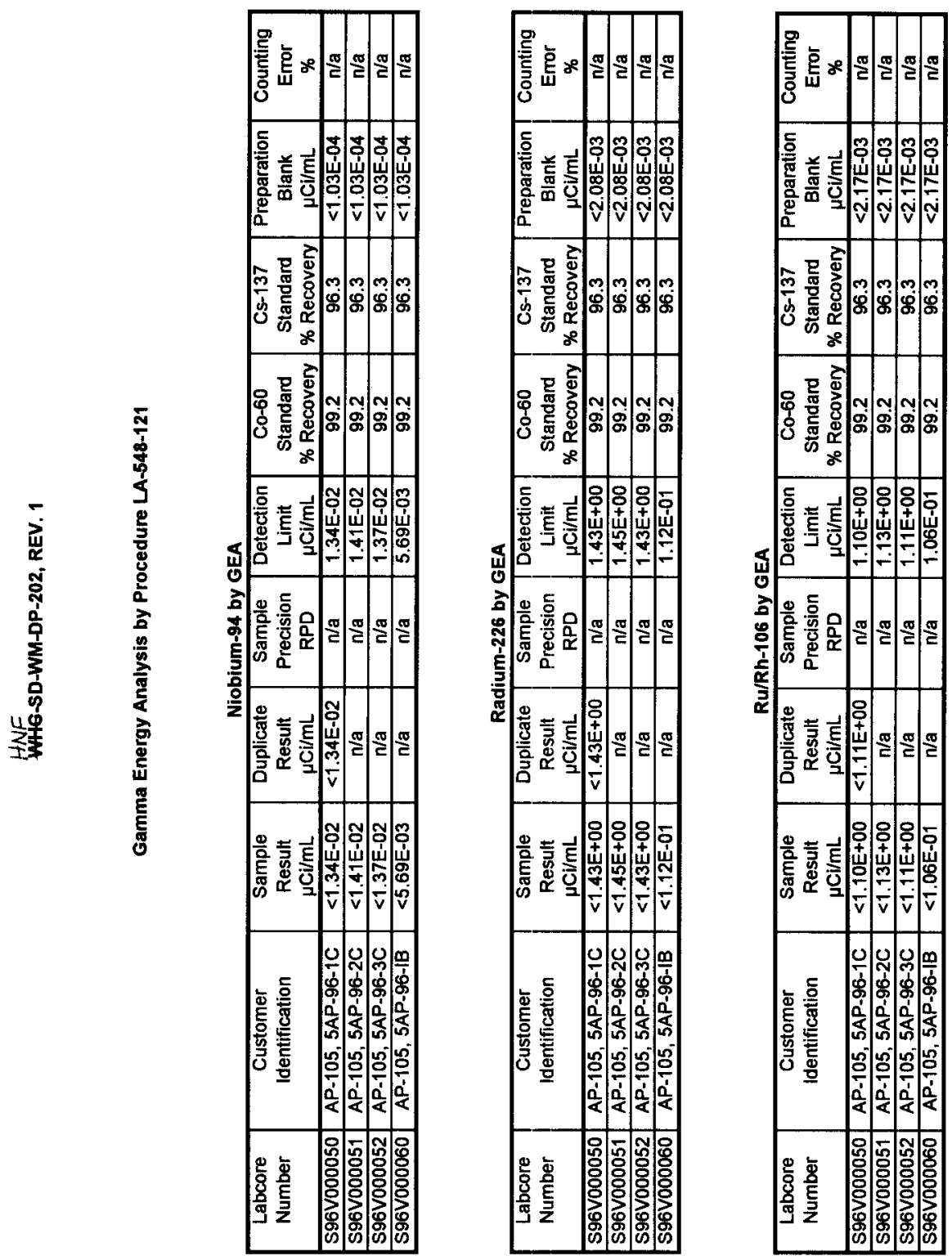

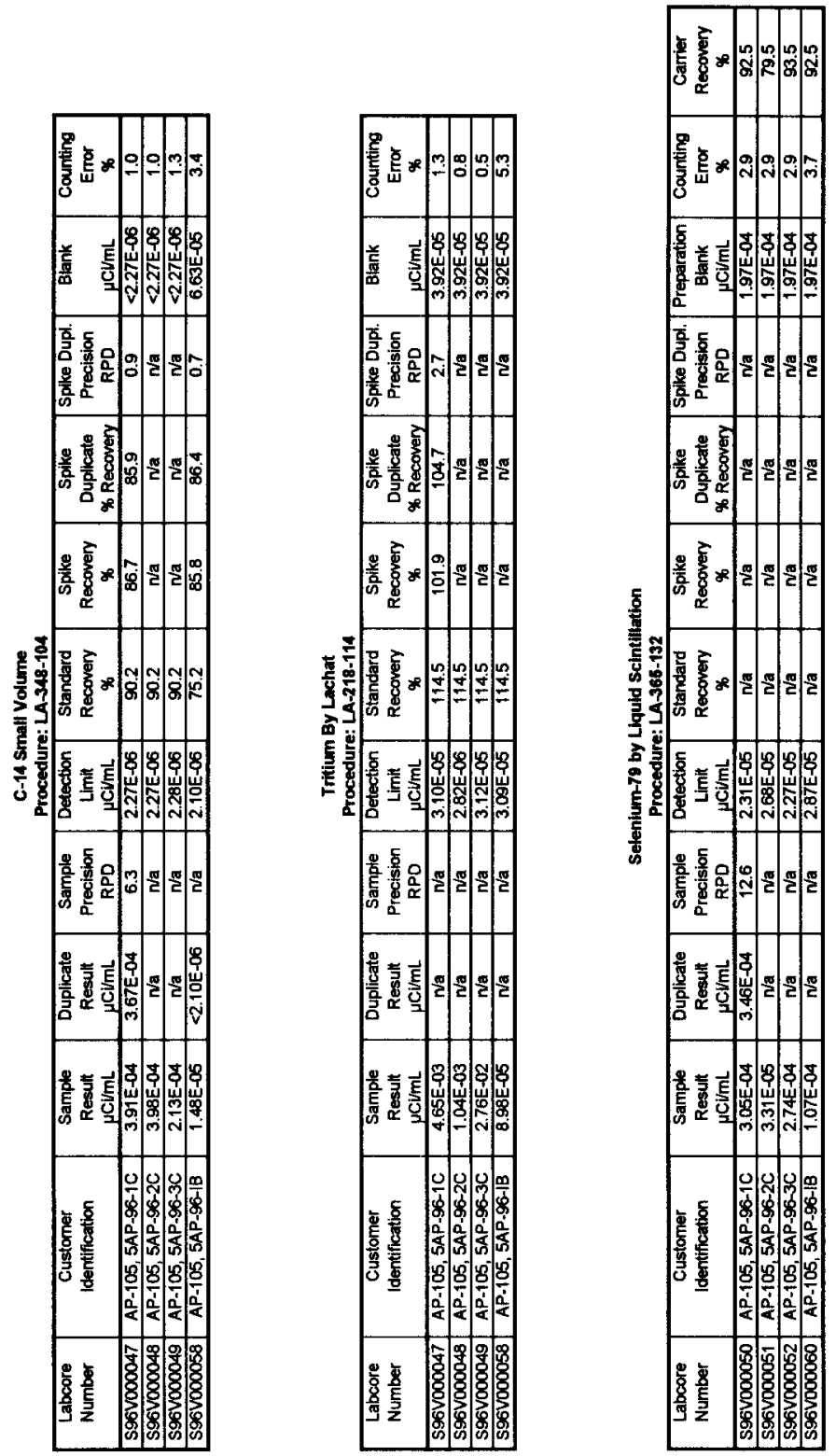
WFE-SD-WM-DP-202, REV. 1

ํํㄹ
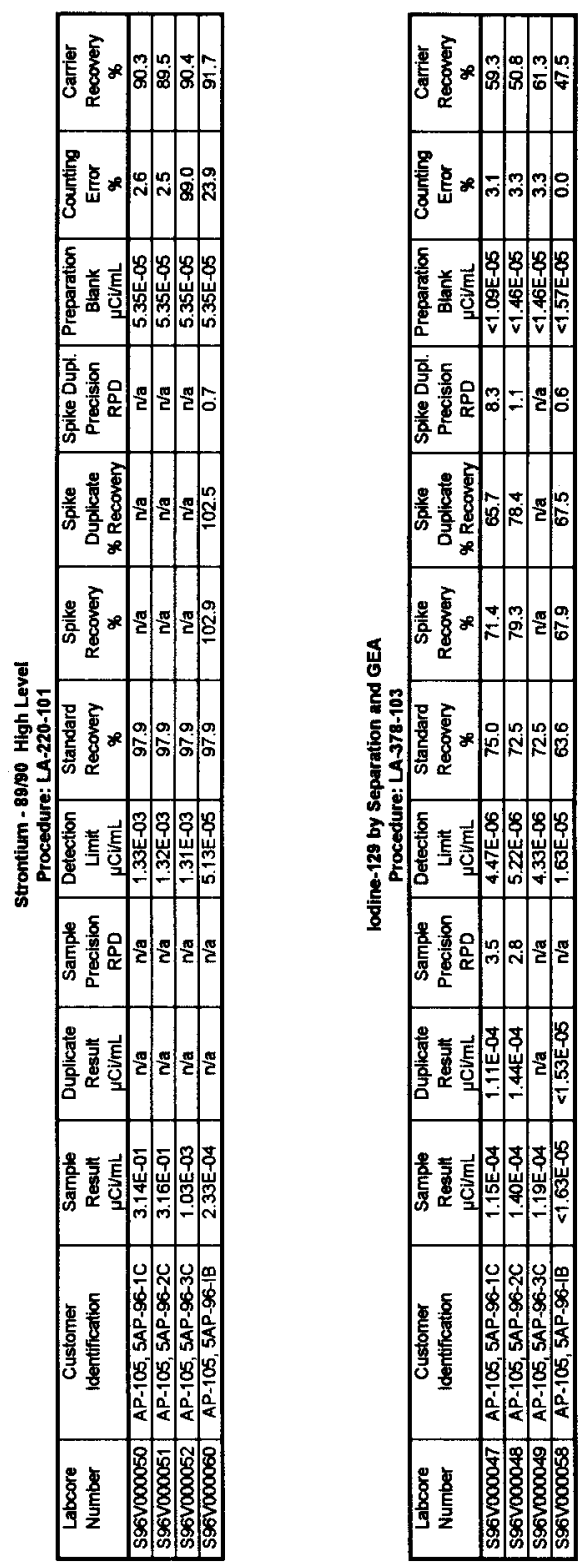
WNE-SD-WM-DP-202, REV. 1
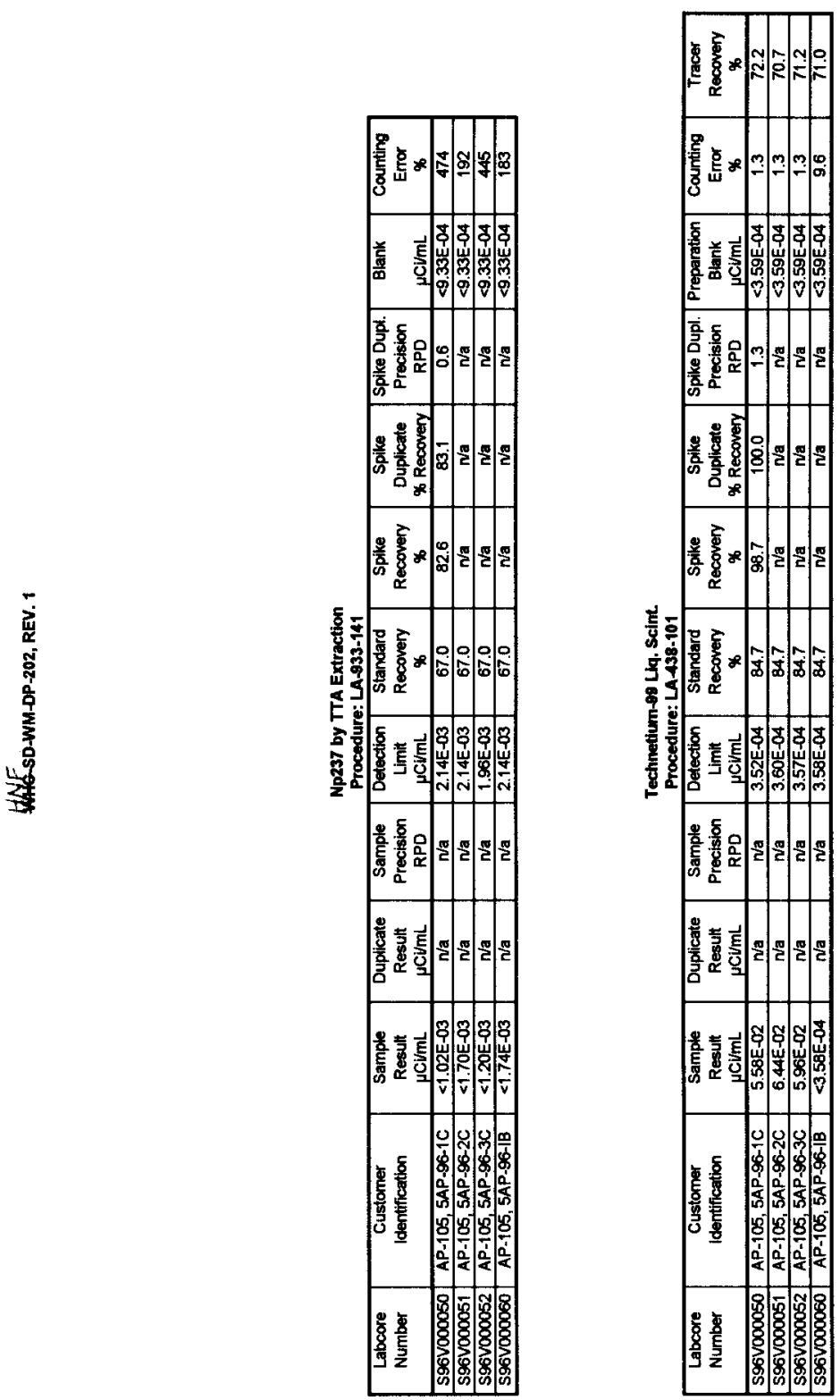

$\because 1$ 
WNF.SD-WM-DP-202, REV. 1

Pu-238 by lon Exchange

Procedure: LA-943-128

\begin{tabular}{|c|c|c|c|c|c|c|c|c|c|c|c|c|}
\hline $\begin{array}{l}\text { Labcore } \\
\text { Number }\end{array}$ & $\begin{array}{c}\text { Customer } \\
\text { Identification }\end{array}$ & $\begin{array}{l}\text { Sample } \\
\text { Result } \\
\mu \mathrm{CimL}\end{array}$ & $\begin{array}{c}\text { Duplicate } \\
\text { Result } \\
\mu \mathrm{Cl} / \mathrm{mL}\end{array}$ & $\begin{array}{c}\text { Sample } \\
\text { Precision } \\
\text { RPD }\end{array}$ & $\begin{array}{c}\text { Detection } \\
\text { Limit } \\
\mu \mathrm{C} / \mathrm{mL}\end{array}$ & $\begin{array}{c}\text { Pu-239 } \\
\text { Standard } \\
\text { \% Recovery }\end{array}$ & $\begin{array}{c}\text { Spike } \\
\text { Recovery } \\
\%\end{array}$ & $\begin{array}{c}\text { Spike } \\
\text { Duplicate } \\
\text { \% Recovery }\end{array}$ & $\begin{array}{c}\text { Spike Dupl. } \\
\text { Precision } \\
\text { RPD }\end{array}$ & $\begin{array}{c}\text { Preparation } \\
\text { Blank } \\
\mu \mathrm{C} / \mathrm{mL}\end{array}$ & $\begin{array}{c}\text { Counting } \\
\text { Error } \\
\%\end{array}$ & $\begin{array}{c}\text { Pu-236 } \\
\text { Tracer } \\
\text { \% Recovery }\end{array}$ \\
\hline 5961 & $\mathrm{P}-96-1 \mathrm{C}$ & $<1.77 \mathrm{E}-04$ & $\mathrm{na}$ & n/a & $1.77 \mathrm{E}-04$ & 94.0 & $\mathbf{n a}$ & n/a & $n / a$ & $<1.69 \mathrm{E}-04$ & 9.7 & 91.3 \\
\hline 596 & $5 A P-96-2 C$ & $<2.02 \mathrm{E}-04$ & na & $n / \mathbf{a}$ & 2.02E-04 & 94.0 & $\mathrm{n} / \mathrm{a}$ & na & $\mathrm{Na}$ & $<1.69 \mathrm{E}-04$ & 6.9 & 81.3 \\
\hline S96V & $5 A P-96-3 C$ & $<1.87 \mathrm{E}-04$ & $\mathrm{na}$ & na & $1.87 \mathrm{E}-04$ & 94.0 & $\mathrm{n} / \mathrm{a}$ & $\mathrm{n} / \mathrm{a}$ & $\mathrm{n} / \mathrm{a}$ & $<1.69 E-04$ & 100.0 & 88.5 \\
\hline S96V000060 & AP-105, 5AP-96-1B & $<1.77 \mathrm{E}-04$ & na & $\mathrm{n} / \mathrm{a}$ & $1.77 \mathrm{E}-04$ & 94.0 & $\mathrm{n} / \mathrm{a}$ & $\mathrm{n} / \mathrm{a}$ & $\mathrm{na}$ & $<1.69 \mathrm{E}-04$ & 11.5 & 90.5 \\
\hline
\end{tabular}

is

PU-2391240 by TRU-SPEC Resin

Procedure: LA-943-128

\begin{tabular}{|c|c|c|c|c|c|c|c|c|c|c|c|c|}
\hline \multicolumn{13}{|c|}{ Procedure: LA-943-128 } \\
\hline $\begin{array}{l}\text { Labcore } \\
\text { Number }\end{array}$ & $\begin{array}{c}\text { Customer } \\
\text { Identification }\end{array}$ & $\begin{array}{l}\text { Sample } \\
\text { Resulit } \\
\mu \mathrm{Ci} / \mathrm{mL}\end{array}$ & $\begin{array}{c}\text { Duplicate } \\
\text { Result } \\
\text { HCl/mL }\end{array}$ & $\begin{array}{c}\text { Sample } \\
\text { Precision } \\
\text { RPD }\end{array}$ & $\begin{array}{c}\text { Detection } \\
\text { Limit } \\
\mu \mathrm{Ci} / \mathrm{mL}\end{array}$ & $\begin{array}{c}\text { Pu-239 } \\
\text { Standard } \\
\text { \% Recovery }\end{array}$ & $\begin{array}{c}\text { Spike } \\
\text { Recovery } \\
\%\end{array}$ & $\begin{array}{c}\text { Spike } \\
\text { Duplicate } \\
\text { \% Recovery }\end{array}$ & $\begin{array}{l}\text { Spike Dupl. } \\
\text { Precision } \\
\text { RPD } \\
\end{array}$ & $\begin{array}{c}\text { Preparation } \\
\text { Blank } \\
\mu \mathrm{Ci} / \mathrm{mL}\end{array}$ & $\begin{array}{c}\text { Counting } \\
\text { Error } \\
\%\end{array}$ & $\begin{array}{c}\text { Pu-236 } \\
\text { Tracer } \\
\text { \% Recovery } \\
\end{array}$ \\
\hline S96V000050 & AP-105, 5AP-96-1C & $<1.77 E-04$ & $n / a$ & $n / a$ & $1.77 E-04$ & 94.0 & 91.9 & 88.6 & 3.7 & $<1.69 E-04$ & 11.2 & 91.3 \\
\hline $596 \mathrm{~V} 000051$ & AP-105, 5AP-96-2C & $<2.02 E-04$ & $n / a$ & na & $2.02 E-04$ & 94.0 & $\mathrm{Na}$ & $n / a$ & $\mathrm{n} / \mathrm{a}$ & $<1.69 \mathrm{E}-04$ & 10.4 & 81.3 \\
\hline S96V000052 & AP-105, 5AP-96-3C & $<1.87 \mathrm{E}-04$ & na & $\mathrm{n} / \mathrm{a}$ & 1.87E-04 & 94.0 & 88.6 & 86.6 & 2.3 & $<1.69 E-04$ & 10.5 & 88.5 \\
\hline S96V000060 & AP-105, 5AP-96-1B & $<1.77 \mathrm{E}-04$ & $n / a$ & $\mathrm{n} / \mathrm{a}$ & 1.77E-04 & 94.0 & $\mathrm{n} / \mathrm{a}$ & n/a & $\mathrm{n} / \mathrm{a}$ & $<1.69 E-04$ & 100.0 & 90.5 \\
\hline
\end{tabular}




\section{$H N F$}

AHE-SD-WM-DP-202, REV. 1

Am-241 by Extraction

Procedure: LA-953-103

\begin{tabular}{|c|c|c|c|c|c|c|c|c|c|c|c|c|}
\hline $\begin{array}{l}\text { Laboore } \\
\text { Number }\end{array}$ & $\begin{array}{c}\text { Customer } \\
\text { Identification }\end{array}$ & $\begin{array}{l}\text { Sample } \\
\text { Result } \\
\mu \mathrm{Ci} / \mathrm{mL}\end{array}$ & $\begin{array}{l}\text { Duplicate } \\
\text { Result } \\
\mu \mathrm{Ci} / \mathrm{mL}\end{array}$ & $\begin{array}{c}\text { Sample } \\
\text { Precision } \\
\text { RPD }\end{array}$ & $\begin{array}{c}\text { Detection } \\
\text { Limit } \\
\mu \mathrm{Ci} / \mathrm{mL}\end{array}$ & $\begin{array}{c}\text { Standard } \\
\text { Recovery } \\
\text { \% }\end{array}$ & $\begin{array}{c}\text { Spike } \\
\text { Recovery } \\
\text { \% }\end{array}$ & $\begin{array}{c}\text { Spike } \\
\text { Duplicate } \\
\text { \% Recovery }\end{array}$ & \begin{tabular}{|c|} 
Spike Dupl. \\
Precision \\
RPD
\end{tabular} & $\begin{array}{c}\text { Preparation } \\
\text { Blank } \\
\mu \mathrm{C} V / m L\end{array}$ & $\begin{array}{c}\text { Counting } \\
\text { Error } \\
\%\end{array}$ & $\begin{array}{c}\text { Am-243 } \\
\text { Tracer } \\
\text { \% Recovery }\end{array}$ \\
\hline $596 \mathrm{~V} 000050$ & AP-105, 5AP-96-1C & $<2.69 E-04$ & $<3,00$ E-04 & $\mathrm{n} / \mathrm{a}$ & $2.69 \mathrm{E}-04$ & 88.2 & $n a$ & $\mathrm{n} / \mathrm{a}$ & $n / a$ & $<2.48 \mathrm{E}-04$ & 100.0 & 88.3 \\
\hline S96V000051 & AP-105, 5AP-96-2C & $<3.08 \mathrm{E}-04$ & $<3.56 \mathrm{E}-04$ & $\mathrm{n} / \mathrm{a}$ & $3.08 E-04$ & 88.2 & $\mathrm{na}$ & n/a & $n / a$ & $<2.48 \mathrm{E}-04$ & 10.1 & 84.3 \\
\hline S96V000052 & AP-105, 5AP-96-3C & $<2.61 E-04$ & $<3.58 E-04$ & $\mathrm{n} / \mathrm{a}$ & $2.61 E-04$ & 88.2 & 88.2 & 82.1 & 7.2 & $<2.48 \mathrm{E}-04$ & 100.0 & 91.9 \\
\hline S96V000060 & AP-105, 5AP-96-1B & $<3.02 E-04$ & $<3.04 E-04$ & $\mathrm{n} / \mathrm{a}$ & $3.02 \mathrm{E}-04$ & 88.2 & 91.6 & 89.9 & 1.9 & $<3.19 \mathrm{E}-04$ & 100.0 & 84.1 \\
\hline
\end{tabular}

w

Cm-243/244 by Extraction

Procedure: LA-953-103

\begin{tabular}{|c|c|c|c|c|c|c|c|c|c|c|c|c|}
\hline $\begin{array}{l}\text { Labcore } \\
\text { Number }\end{array}$ & $\begin{array}{l}\text { Customer } \\
\text { Identification }\end{array}$ & $\begin{array}{l}\text { Sample } \\
\text { Result } \\
\mu C i / m L\end{array}$ & $\begin{array}{c}\text { Duplicate } \\
\text { Result } \\
\mu \mathrm{Ci} / \mathrm{mL}\end{array}$ & $\begin{array}{c}\text { Sample } \\
\text { Precision } \\
\text { RPD }\end{array}$ & $\begin{array}{c}\text { Detection } \\
\text { Limit } \\
\mu \mathrm{CimL}\end{array}$ & $\begin{array}{c}\text { Am-241 } \\
\text { Standard } \\
\text { \% Recovery }\end{array}$ & $\begin{array}{c}\text { Spike } \\
\text { Recovery } \\
\%\end{array}$ & $\begin{array}{c}\text { Spike } \\
\text { Duplicate } \\
\text { \% Recovery }\end{array}$ & $\begin{array}{c}\text { Spike Dupl. } \\
\text { Precision } \\
\text { RPD } \\
\end{array}$ & $\begin{array}{c}\text { Preparation } \\
\text { Blank } \\
\mu \mathrm{Ci} / \mathrm{mL}\end{array}$ & $\begin{array}{c}\text { Counting } \\
\text { Emor } \\
\%\end{array}$ & $\begin{array}{c}\text { Am-243 } \\
\text { Tracer } \\
\text { \% Recovery }\end{array}$ \\
\hline S96V & $1 \mathrm{C}$ & $<2.69 E-04$ & $<3.00 E-04$ & $n / a$ & $2.69 E-04$ & 88.2 & $n a$ & $\mathrm{n} / \mathrm{a}$ & na & $<2.48 E-04$ & 100.0 & 88.3 \\
\hline S96V000051 & AP-105, 5AP-96-2C & $<3.08 E-04$ & $<3.56 \mathrm{E}-04$ & n/a & $3.08 E-04$ & 88.2 & $\mathrm{n} / \mathrm{a}$ & $\mathrm{n} / \mathrm{a}$ & $\mathrm{Na}$ & $<2.48 \mathrm{E}-04$ & 100.0 & 84.3 \\
\hline S96V000052 & AP-105, 5AP-96-3C & $<2.6$ & $<3.58 \mathrm{E}-04$ & n/a & $2.61 E-04$ & 88.2 & Na & $n / a$ & n/a & $<2.48 E-04$ & 100.0 & 91.9 \\
\hline S96V000060 & AP-105, 5AP-96-1B & $<3.02 \mathrm{E}-04$ & $<3.04 E-04$ & $\mathrm{f} / \mathrm{a}$ & $3.02 E-04$ & 88.2 & n/a & $\mathrm{n} / \mathrm{a}$ & $\mathrm{n} / \mathrm{a}$ & $<3.19 \mathrm{E}-04$ & 100.0 & 84.1 \\
\hline
\end{tabular}


HNE-SD-WM-DP-202, REV. 1

VOA

1-Butanol

Procedure: LA-623-405

\begin{tabular}{|c|c|c|c|c|c|c|c|c|c|c|c|c|c|}
\hline \multirow{2}{*}{$\begin{array}{l}\text { Labcore } \\
\text { Number }\end{array}$} & \multirow{2}{*}{$\begin{array}{l}\text { Customer } \\
\text { Identification }\end{array}$} & \multirow{2}{*}{$\begin{array}{c}\text { Sample } \\
\text { Result } \\
\mu g / L\end{array}$} & \multirow{2}{*}{$\begin{array}{l}\text { Sample } \\
\text { Result } \\
\text { Qualifier }\end{array}$} & \multirow{2}{*}{$\begin{array}{l}\text { Quantikation } \\
\text { Limit } \\
\mu g / L\end{array}$} & \multirow{2}{*}{\begin{tabular}{|c|} 
Continuing \\
Calibration \\
* Difference
\end{tabular}} & \multicolumn{3}{|c|}{ Surrogate } & \multirow{2}{*}{$\begin{array}{c}\text { Spike } \\
\text { Recovery } \\
\%\end{array}$} & \multirow{2}{*}{\begin{tabular}{|c|} 
Spike \\
Duplicate \\
\% Recovery
\end{tabular}} & \multirow{2}{*}{$\begin{array}{l}\text { Spike Dupl. } \\
\text { Precision } \\
\text { RPD }\end{array}$} & \multirow{2}{*}{$\begin{array}{l}\text { Blank } \\
\mu g / \mathrm{L}\end{array}$} & \multirow{2}{*}{$\begin{array}{l}\text { Blank } \\
\text { Result } \\
\text { Qualifier }\end{array}$} \\
\hline & & & & & & $\begin{array}{c}\text { TOL } \\
\text { \% Recovery }\end{array}$ & $\begin{array}{c}\text { BFB } \\
\text { \% Recovery }\end{array}$ & $\begin{array}{c}\text { DCE } \\
\text { \% Recovery }\end{array}$ & & & & & \\
\hline S96V000039 & AP-105, 5AP-96-1A & $2.50 E+04$ & $\mathrm{U}$ & $2.50 E+04$ & 8.6 & 104 & 99 & 106 & 100 & 100 & 0 & $5,00 E+02$ & $\mathbf{U}$ \\
\hline $596 \mathrm{~V} 000040$ & AP-105, 5AP-96-2A & $2.50 E+04$ & $\mathrm{U}$ & $2.50 \mathrm{E}+04$ & 8.6 & 99 & 100 & 107 & na & $\mathrm{n} / \mathrm{a}$ & $\mathrm{Na}$ & $5.00 \mathrm{E}+02$ & U \\
\hline S96V000041 & AP-105, 5AP-96-3A & $2.50 E+04$ & U & $2.50 E+04$ & 8.6 & 100 & 100 & 106 & na & $\mathrm{Na}$ & $\mathrm{n} / \mathrm{a}$ & $5,00 E+02$ & $\mathrm{U}$ \\
\hline $596 \mathrm{~V} 000042$ & AP-105, 5AP-96-TB & $2.50 \mathrm{E}+04$ & $\mathrm{U}$ & $2.50 \mathrm{E}+04$ & 8.6 & 100 & 100 & 106 & na & $n / \mathbf{a}$ & n/a & $5.00 E+02$ & $\mathrm{U}$ \\
\hline $596 V 000061$ & AP-105, 5AP-96-OB & $2.50 \mathrm{E}+04$ & $\bar{U}$ & $2.50 E+04$ & 8.6 & 100 & 100 & 105 & n/a & n/a & $\mathrm{n} / \mathrm{a}$ & $5.00 \mathrm{E}+02$ & $\mathrm{U}$ \\
\hline
\end{tabular}

2-Hexanone

Procedure: LA-523-405

\begin{tabular}{|c|c|c|c|c|c|c|c|c|c|c|c|c|c|}
\hline \multirow{2}{*}{$\begin{array}{l}\text { Labcore } \\
\text { Number }\end{array}$} & \multirow{2}{*}{$\begin{array}{c}\text { Custorrer } \\
\text { Identification }\end{array}$} & \multirow{2}{*}{$\begin{array}{c}\text { Sample } \\
\text { Result } \\
\mu g / L\end{array}$} & \multirow{2}{*}{$\begin{array}{c}\text { Sample } \\
\text { Result } \\
\text { Qualifier }\end{array}$} & \multirow{2}{*}{$\begin{array}{c}\text { Quantitation } \\
\text { Limit } \\
\mu g / \mathrm{L}\end{array}$} & \multirow{2}{*}{$\begin{array}{c}\text { Continuing } \\
\text { Calibration } \\
\% \text { Difference }\end{array}$} & \multicolumn{3}{|c|}{ Surrogate } & \multirow{2}{*}{$\begin{array}{c}\text { Splke } \\
\text { Recovery } \\
\%\end{array}$} & \multirow{2}{*}{\begin{tabular}{|c|} 
Spike \\
Duplicate \\
\% Recovery
\end{tabular}} & \multirow{2}{*}{$\begin{array}{l}\text { Spike Dupl. } \\
\text { Precision } \\
\text { RPD }\end{array}$} & \multirow{2}{*}{$\begin{array}{l}\text { Blank } \\
\mu g / L\end{array}$} & \multirow{2}{*}{$\begin{array}{c}\text { Blank } \\
\text { Result } \\
\text { Qualifier }\end{array}$} \\
\hline & & & & & & $\begin{array}{c}\text { TOL } \\
\text { \% Recovery }\end{array}$ & $\begin{array}{c}\text { BFB } \\
\text { \% Recovery }\end{array}$ & $\begin{array}{c}\text { DCE } \\
\text { \% Recovery }\end{array}$ & & & & & \\
\hline S96V000039 & AP-105, 5AP-96-1A & $5.00 E+02$ & $\mathrm{U}$ & $5.00 \mathrm{E}+02$ & 7.4 & 104 & 99 & 106 & 92 & 96 & 4 & $1.00 E+01$ & $\mathrm{U}$ \\
\hline S96V000040 & AP-405, 5AP-96-2A & $5.00 \mathrm{E}+02$ & $\mathrm{U}$ & $5.00 E+02$ & 7.4 & 99 & 100 & 107 & $\mathrm{na}$ & na & $\mathrm{Na}$ & $1.00 \mathrm{E}+01$ & $\mathrm{U}$ \\
\hline $596 \mathrm{~V} 000041$ & AP-105, 5AP-96-3A & $5,00 \mathrm{E}+02$ & $\mathrm{U}$ & $5.00 E+02$ & 7.4 & 100 & 100 & 106 & na & $\mathbf{n} / \mathbf{a}$ & $\pi \sqrt{a}$ & $1.00 \mathrm{E}+01$ & $\bar{U}$ \\
\hline S96V000042 & AP-105, 5AP-96-TB & $5.00 E+02$ & $\mathrm{U}$ & $5.00 E+02$ & 7.4 & 100 & 100 & 106 & Na & na & na & $1.00 \mathrm{E}+01$ & $\mathrm{U}$ \\
\hline S96V000061 & $A \bar{P}-105,5 \mathrm{AP}-96-\mathrm{OB}$ & $5.00 \mathrm{E}+02$ & $\bar{U}$ & $5.00 \mathrm{E}+02$ & 7.4 & 100 & 100 & 105 & Na & na & na & $1.00 E+01$ & $\mathrm{U}$ \\
\hline
\end{tabular}

it \begin{tabular}{|l|l|l}
\hline S96V000061 & AP $-105,5 A P-96-O B$ \\
\hline
\end{tabular} $5.00 \mathrm{E}+02$ $5.00 \mathrm{E}+02$

\section{Pentanone}

Procedure: LA-623-405

\begin{tabular}{|c|c|c|c|c|c|c|c|c|c|c|c|c|c|}
\hline \multirow{2}{*}{$\begin{array}{l}\text { Labcore } \\
\text { Number }\end{array}$} & \multirow{2}{*}{$\begin{array}{c}\text { Customer } \\
\text { Identification }\end{array}$} & \multirow{2}{*}{$\begin{array}{c}\text { Sample } \\
\text { Result } \\
\mu g / L\end{array}$} & \multirow{2}{*}{$\begin{array}{l}\text { Sample } \\
\text { Result } \\
\text { Qualifier }\end{array}$} & \multirow{2}{*}{$\begin{array}{l}\text { Quantitation } \\
\text { Limit } \\
\mu g / \mathrm{L}\end{array}$} & \multirow{2}{*}{$\begin{array}{c}\text { Continuing } \\
\text { Calibration } \\
\text { \% Difference }\end{array}$} & \multicolumn{3}{|c|}{ Surrogate } & \multirow{2}{*}{$\begin{array}{c}\text { Spike } \\
\text { Recovery } \\
\%\end{array}$} & \multirow{2}{*}{$\begin{array}{c}\text { Spike } \\
\text { Duplicate } \\
\text { \% Recovery }\end{array}$} & \multirow{2}{*}{$\begin{array}{c}\text { Spike Dupl. } \\
\text { Precision } \\
\text { RPD }\end{array}$} & \multirow{2}{*}{$\begin{array}{l}\text { Blank } \\
\mu q / L\end{array}$} & \multirow{2}{*}{$\begin{array}{c}\text { Blank } \\
\text { Result } \\
\text { Qualifier }\end{array}$} \\
\hline & & & & & & $\begin{array}{c}\text { TOL } \\
\% \text { Recovery }\end{array}$ & $\begin{array}{c}\text { BFB } \\
\text { \% Recovery }\end{array}$ & $\begin{array}{c}\text { DCE } \\
\text { \% Recovery }\end{array}$ & & & & & \\
\hline S96V000039 & AP-105, 5AP-96-1A & $5.00 E+02$ & $\mathrm{U}$ & $5.00 \mathrm{E}+02$ & -0.4 & 104 & 99 & 106 & 104 & 104 & 0 & $1.00 \mathrm{E}+01$ & $\mathrm{U}$ \\
\hline $596 V 000040$ & AP-105, 5AP-96-2A & $5.00 E+02$ & $\mathrm{U}$ & $5.00 \mathrm{E}+02$ & -0.4 & 99 & 100 & 107 & $n / a$ & $\mathrm{na}$ & $\mathrm{n} / \mathrm{a}$ & $1.00 \mathrm{E}+01$ & $\mathrm{U}$ \\
\hline $596 \mathrm{V000041}$ & AP-105, 5AP-S6-3A & $5.00 E+02$ & $\bar{U}$ & $5.00 E+02$ & -0.4 & 100 & 100 & 106 & Na & na & $\mathrm{n} / \mathbf{a}$ & $1.00 \mathrm{E}+01$ & $\mathrm{U}$ \\
\hline S96V000042 & AP-105, 5AP-96-TB & $5.00 E+02$ & $\mathrm{U}$ & $5.00 E+02$ & -0.4 & 100 & 100 & 106 & na & na & n/a & $1.00 \mathrm{E}+01$ & $\bar{U}$ \\
\hline S96V000061 & AP-105, 5AP-96-OB & $5,00 E+02$ & U & $5.00 E+02$ & -0.4 & 100 & 100 & 105 & n/a & Na & $n / a$ & $1.00 \mathrm{E}+01$ & $\mathrm{U}$ \\
\hline
\end{tabular}

4-Methyl-2 pentanone

Procedure: LA-523-495

\begin{tabular}{|c|c|c|c|c|c|c|c|c|c|c|c|c|c|}
\hline \multirow{2}{*}{$\begin{array}{l}\text { Labcore } \\
\text { Number }\end{array}$} & \multirow{2}{*}{$\begin{array}{c}\text { Customer } \\
\text { Identification }\end{array}$} & \multirow{2}{*}{$\begin{array}{c}\text { Sample } \\
\text { Result } \\
\mu g^{\prime} L\end{array}$} & \multirow{2}{*}{$\begin{array}{l}\text { Sarmple } \\
\text { Result } \\
\text { Qualifier }\end{array}$} & \multirow{2}{*}{$\begin{array}{l}\text { Quantitation } \\
\text { Limik } \\
\mu g / L\end{array}$} & \multirow{2}{*}{$\begin{array}{l}\text { Continuing } \\
\text { Calibration } \\
\text { \% Difference }\end{array}$} & \multicolumn{3}{|c|}{ Surrogate } & \multirow{2}{*}{$\begin{array}{c}\text { Spike } \\
\text { Recovery } \\
\%\end{array}$} & \multirow{2}{*}{$\begin{array}{c}\text { Spike } \\
\text { Duplicate } \\
\text { \% Recovery }\end{array}$} & \multirow{2}{*}{$\begin{array}{c}\text { Spike Dupl. } \\
\text { Precision } \\
\text { RPD } \\
\end{array}$} & \multirow{2}{*}{$\begin{array}{l}\text { Blank } \\
\text { pal }\end{array}$} & \multirow{2}{*}{$\begin{array}{c}\text { Blank } \\
\text { Result } \\
\text { Qualifier }\end{array}$} \\
\hline & & & & & & $\begin{array}{c}\text { TOL } \\
\text { \% Recovery }\end{array}$ & $\begin{array}{c}\text { BFB } \\
\text { \% Recovery }\end{array}$ & $\begin{array}{c}\text { DCE } \\
\text { \% Recovery }\end{array}$ & & & & & \\
\hline S96V000039 & AP-105, 5AP-96-1A & $5.00 E+02$ & $\mathrm{U}$ & $5.00 \mathrm{E}+02$ & 0.8 & 104 & 99 & 106 & 104 & 108 & 4 & $1.00 E+01$ & $\bar{U}$ \\
\hline S96V000040 & AP-105, 5AP-96-2A & $5.00 \mathrm{E}+02$ & $\mathrm{U}$ & $5.00 \mathrm{E}+02$ & 0.8 & 99 & 100 & 107 & $n / a$ & $\pi / \mathbf{a}$ & $n / a$ & $1.00 \mathrm{E}+01$ & $\mathrm{U}$ \\
\hline S96V000041 & AP-105, 5AP-S6-3A & $5.00 E+02$ & $\mathrm{U}$ & $5.00 \mathrm{E}+02$ & 0.8 & 100 & 100 & 106 & n/a & Na & $n / a$ & $1.00 E+01$ & U \\
\hline S96V000042 & AP-105, 5AP-S6-TB & +02 & $\mathrm{U}$ & $5,00 \mathrm{E}+02$ & 0.8 & 100 & 100 & 106 & $n / a$ & nia & $n / a$ & $1.00 E+04$ & $\mathrm{U}$ \\
\hline S96V000061 & AP-105, 5AP-96-OB & $5.00 E+02$ & $\mathrm{U}$ & $5.00 E+02$ & 0.8 & 100 & 100 & 105 & na & $n / a$ & $\mathrm{~N} / \mathrm{a}$ & $1.00 E+01$ & $\mathrm{U}$ \\
\hline
\end{tabular}

Surrogates: $\quad$ QC Limits (CLP) Q (qualifiers):

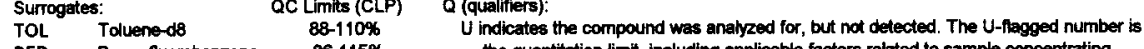

BFB Bromofluorobenzene $86-115 \%$ the quantitation limit, including applicable factors related to sample concentrating.

DCE 1,2-Dichloroethane-d4 76-114\% J indicates the estimated value. Spectra meet criteria, but response is below the

quantitation limit for the target compound. 


\section{WESD-WM-DP-202, REV. 1}

VOA

Acetone

Procedure: LA-623-405

\begin{tabular}{|c|c|c|c|c|c|c|c|c|c|c|c|c|c|}
\hline \multirow{2}{*}{$\begin{array}{l}\text { Labcore } \\
\text { Number }\end{array}$} & \multirow{2}{*}{$\begin{array}{c}\text { Customer } \\
\text { Identufication }\end{array}$} & \multirow{2}{*}{$\begin{array}{c}\text { Sample } \\
\text { Result } \\
\mu \mathrm{g} / \mathrm{L}\end{array}$} & \multirow{2}{*}{$\begin{array}{c}\text { Sample } \\
\text { Result } \\
\text { Qualifier }\end{array}$} & \multirow{2}{*}{$\begin{array}{c}\text { Quantitation } \\
\text { Limit } \\
\mu g / \mathrm{L}\end{array}$} & \multirow{2}{*}{$\begin{array}{c}\text { Continuing } \\
\text { Calibration } \\
\text { \% Difference }\end{array}$} & \multicolumn{3}{|c|}{ Surrogate } & \multirow{2}{*}{$\begin{array}{c}\text { Spike } \\
\text { Recovery } \\
\%\end{array}$} & \multirow{2}{*}{$\begin{array}{c}\text { Spike } \\
\text { Duplicate } \\
\text { \% Recovery }\end{array}$} & \multirow{2}{*}{$\begin{array}{l}\text { Spike Dupi. } \\
\text { Precision } \\
\text { RPD }\end{array}$} & \multirow{2}{*}{$\begin{array}{l}\text { Blank } \\
\text { eg/t }\end{array}$} & \multirow{2}{*}{$\begin{array}{l}\text { Blank } \\
\text { Result } \\
\text { Qualifier }\end{array}$} \\
\hline & & & & & & $\begin{array}{c}\text { TOL } \\
\text { \% Recovery }\end{array}$ & $\begin{array}{c}\text { BFB } \\
\text { \% Recovery }\end{array}$ & $\begin{array}{c}\text { DCE } \\
\text { \% Recovery }\end{array}$ & & & & & \\
\hline S96V000039 & AP-105, 5AP-96-1A & $5.50 \mathrm{E}+02$ & & $5.00 E+02$ & 0.9 & 104 & 99 & 106 & 82 & 82 & 0 & $1.00 \mathrm{E}+01$ & U \\
\hline S96V000040 & AP-105, 5AP-96-2A & $4.90 E+02$ & $\mathrm{~J}$ & $5.00 E+02$ & 0.9 & 99 & 100 & 107 & na & na & n/a & $1.00 \mathrm{E}+01$ & $\bar{U}$ \\
\hline s96V000041 & AP-105, 5AP-96-3A & $4.40 E+02$ & $j$ & $5.00 \mathrm{E}+02$ & 0.9 & 100 & 100 & 106 & $\mathrm{na}$ & $\mathrm{N} / \mathbf{a}$ & $n / a$ & $1.00 \mathrm{E}+01$ & U \\
\hline S96V000042 & AP-105, 5AP-96-TB & $5.00 E+02$ & $\bar{U}$ & $5.00 \mathrm{E}+02$ & 0.9 & 100 & 100 & 106 & na & $\mathrm{na}$ & Na & $1.00 \mathrm{E}+01$ & $\mathrm{U}$ \\
\hline$\$ 96 \mathrm{~V} 000061$ & AP-105, 5AP-96-OB & $5.00 E+02$ & $\mathrm{U}$ & $5.00 E+02$ & 0.9 & 100 & 100 & 105 & na & N/a & $\mathrm{Na}$ & $1.00 \mathrm{E}+01$ & U \\
\hline
\end{tabular}

2-Butanone

Procedure: LA-623-406

\begin{tabular}{|c|c|c|c|c|c|c|c|c|c|c|c|c|c|}
\hline \multirow{2}{*}{$\begin{array}{l}\text { Labcore } \\
\text { Number }\end{array}$} & \multirow{2}{*}{$\begin{array}{c}\text { Customer } \\
\text { Identification }\end{array}$} & \multirow{2}{*}{$\begin{array}{c}\text { Sample } \\
\text { Result } \\
\mu g / \mathrm{L}\end{array}$} & \multirow{2}{*}{$\begin{array}{l}\text { Sample } \\
\text { Result } \\
\text { Quaiffier }\end{array}$} & \multirow{2}{*}{$\begin{array}{l}\text { Quantitation } \\
\text { Limit } \\
\mu g / L\end{array}$} & \multirow{2}{*}{\begin{tabular}{|c|} 
Continuing \\
Calibration \\
$*$ Difference \\
\end{tabular}} & \multicolumn{3}{|c|}{ Surrogate } & \multirow{2}{*}{$\begin{array}{c}\text { Spike } \\
\text { Recovery } \\
\%\end{array}$} & \multirow{2}{*}{$\begin{array}{c}\text { Spike } \\
\text { Duplicate } \\
\text { \% Recovery }\end{array}$} & \multirow{2}{*}{$\begin{array}{l}\text { Spike Dupl. } \\
\text { Precision } \\
\text { RPD }\end{array}$} & \multirow{2}{*}{$\begin{array}{l}\text { Blank } \\
\mu g / L\end{array}$} & \multirow{2}{*}{$\begin{array}{c}\text { Blank } \\
\text { Result } \\
\text { Qualifier }\end{array}$} \\
\hline & & & & & & $\begin{array}{c}\text { TOL } \\
\text { \% Recovery }\end{array}$ & $\begin{array}{c}\text { BFB } \\
\text { \% Recovery }\end{array}$ & $\begin{array}{c}\text { DCE } \\
\text { \% Recovery }\end{array}$ & & & & & \\
\hline $596 \mathrm{~V} 000039$ & $A P-105,5 A P-96-1 A$ & $5.00 \mathrm{E}+02$ & $\mathrm{U}$ & $5.00 E+02$ & 8.0 & 104 & 99 & 106 & 88 & 88 & 0 & $1.00 \mathrm{E}+01$ & $U$ \\
\hline $596 \mathrm{~V} 000040$ & AP-105, 5AP-96-2A & $5.00 E+02$ & $\mathrm{U}$ & $5,00 E+02$ & 8.0 & 99 & 100 & 107 & $\mathrm{n} / \mathrm{a}$ & n/a & $n / a$ & $1.00 E+01$ & $\mathrm{U}$ \\
\hline $596 \mathrm{~V} 000041$ & AP-105, 5AP-96-3A & $5.00 \mathrm{E}+02$ & U & $5.00 E+02$ & 8.0 & 100 & 100 & 106 & $\mathrm{n} / \mathrm{a}$ & $\mathrm{Na}$ & n/a & $1.00 \mathrm{E}+01$ & U \\
\hline $596 \mathrm{~V} 000042$ & AP-105, 5AP-96-TB & $5.00 E+02$ & U & $5.00 E+02$ & 8.0 & 100 & 100 & 106 & $\mathrm{n} / \mathrm{a}$ & n/a & $\mathrm{n} / \mathrm{a}$ & $1.00 E+01$ & U \\
\hline $596 \mathrm{~V} 000061$ & AP-105, 5AP-96-OB & $5.00 \mathrm{E}+02$ & $\mathrm{U}$ & $5.00 \mathrm{E}+02$ & 8.0 & 100 & 100 & 105 & nia & $\mathrm{N} / \mathrm{a}$ & $\mathrm{N} / \mathrm{a}$ & $1.00 E+01$ & U \\
\hline
\end{tabular}

Tetrahydrofuran

Procedure: LA-523-40

\begin{tabular}{|c|c|c|c|c|c|c|c|c|c|c|c|c|c|}
\hline \multirow{2}{*}{$\begin{array}{l}\text { Labcore } \\
\text { Number }\end{array}$} & \multirow{2}{*}{$\begin{array}{c}\text { Customer } \\
\text { Identification }\end{array}$} & \multirow{2}{*}{$\begin{array}{c}\text { Sample } \\
\text { Result } \\
\mu g / L\end{array}$} & \multirow{2}{*}{$\begin{array}{l}\text { Sample } \\
\text { Result } \\
\text { Qualifier }\end{array}$} & \multirow{2}{*}{$\begin{array}{c}\text { Quantitation } \\
\text { Limit } \\
\mu g / L\end{array}$} & \multirow{2}{*}{$\begin{array}{l}\text { Continuing } \\
\text { Calibration } \\
\text { \% Difference }\end{array}$} & \multicolumn{3}{|c|}{ Surrogate } & \multirow{2}{*}{$\begin{array}{c}\text { Spike } \\
\text { Recovery } \\
\text { \% }\end{array}$} & \multirow{2}{*}{$\begin{array}{c}\text { Spike } \\
\text { Duplicate } \\
\text { \% Recovery }\end{array}$} & \multirow{2}{*}{$\begin{array}{c}\text { Spike Dupl. } \\
\text { Precision } \\
\text { RPD }\end{array}$} & \multirow{2}{*}{$\begin{array}{l}\text { Blank } \\
\text { hol }\end{array}$} & \multirow{2}{*}{$\begin{array}{l}\text { Blank } \\
\text { Resulk } \\
\text { Qualifier }\end{array}$} \\
\hline & & & & & & $\begin{array}{c}\text { TOL } \\
\text { \% Recovery }\end{array}$ & $\begin{array}{c}\text { BFB } \\
\text { \& Recovery }\end{array}$ & $\begin{array}{c}\text { DCE } \\
\text { \% Recovery }\end{array}$ & & & & & \\
\hline S96V000039 & AP-105, 5AP-96-1A & $5,00 E+02$ & $\bar{U}$ & $5.00 \mathrm{E}+02$ & 2.2 & 104 & 99 & 406 & 96 & 100 & 4 & $1.00 \mathrm{E}+01$ & $\bar{U}$ \\
\hline SS6V000040 & AP-105, 5AP-96-2A & $5.00 E+02$ & $\mathrm{U}$ & $5.00 \mathrm{E}+02$ & 2.2 & 99 & 100 & 107 & $\pi / a$ & na & $\mathrm{n} / \mathrm{a}$ & $1.00 E+01$ & U \\
\hline s96V000041 & AP-105, 5AP-96-3A & $5.00 E+02$ & $\bar{U}$ & $5.00 \mathrm{E}+02$ & 2.2 & 100 & 100 & 106 & $\mathrm{n} / \mathrm{a}$ & $n / a$ & $\mathrm{n} / \mathrm{a}$ & $1,00 E+01$ & $\mathbf{U}$ \\
\hline S96V000042 & AP-105, 5AP-96-TB & $5.00 E+02$ & $\mathrm{U}$ & $5.00 \mathrm{E}+02$ & 2.2 & 100 & 100 & 106 & na & Na & $\mathrm{n} / \mathrm{a}$ & $1.00 \mathrm{E}+0 \mathrm{i}$ & $\bar{U}$ \\
\hline S96V000061 & AP-105, 5AP-96-OB & $5.00 E+02$ & $\mathrm{U}$ & $5.00 \mathrm{E}+02$ & 2.2 & 100 & 100 & 105 & $n \sqrt{a}$ & $\mathrm{na}$ & $\mathrm{n} / \mathrm{a}$ & $1.00 E+01$ & U \\
\hline
\end{tabular}

Surrogates: $\quad$ QC Limits (CLP) Q (qualifiers):
TOL Toluene-d8
88-110\%
BFB Bromofluorobenzene $86-115 \%$
$U$ indicates the compound was analyzed for, but not detected. The U-flagged number is
DCE 1,2-Dichloroethane-d4 76-114\%
the quantitation limit, including applicable factors related to sample concentrating.
$J$ indicates the estimated value. Spectra meet criteria, but response is below the quantitation limit for the target compound.




\section{WE-SD-WM-DP-202, REV. 1}

\section{Semi-VOA}

2-Butoxyethanol

Procedure: LA-523-406

\begin{tabular}{|c|c|c|c|c|c|c|c|c|c|c|}
\hline $\begin{array}{l}\text { Labcore } \\
\text { Number }\end{array}$ & $\begin{array}{c}\text { Customer } \\
\text { Identification }\end{array}$ & $\begin{array}{c}\text { Sample } \\
\text { Result } \\
\mu g / L\end{array}$ & $\begin{array}{l}\text { Sample } \\
\text { Result } \\
\text { Qualifier }\end{array}$ & $\begin{array}{c}\text { Quantitation } \\
\text { Limit } \\
\mu g / L\end{array}$ & $\begin{array}{c}\text { Continuing } \\
\text { Calibration } \\
\text { \% Difference }\end{array}$ & $\begin{array}{c}\text { Spike } \\
\text { Recovery } \\
\%\end{array}$ & $\begin{array}{c}\text { Spike } \\
\text { Duplicate } \\
\text { \% Recovery }\end{array}$ & $\begin{array}{c}\text { Spike Dupl. } \\
\text { Precision } \\
\text { RPD }\end{array}$ & $\begin{array}{c}\text { Blank } \\
\mu g / L\end{array}$ & $\begin{array}{c}\text { Blank } \\
\text { Result } \\
\text { Qualifier }\end{array}$ \\
\hline S96V000043 & AP-105, 5AP-96-1B & $2.00 \mathrm{E}+03$ & $\mathrm{U}$ & $2.00 E+03$ & -4.7 & 88.0 & 100 & 13 & $2.00 \mathrm{E}+02$ & $\bar{U}$ \\
\hline S96V000044 & AP-105, 5AP-96-2B & $2.00 \bar{E}+03$ & $\mathrm{U}$ & $2.00 \mathrm{E}+03$ & -4.7 & $\mathrm{n} / \mathrm{a}$ & $\mathrm{n} / \mathrm{a}$ & $\mathrm{n} / \mathrm{a}$ & $2.00 \mathrm{E}+02$ & $\mathrm{U}$ \\
\hline S96V000045 & AP-105, 5AP-96-3B & $2.00 \mathrm{E}+03$ & $\mathrm{U}$ & $2.00 \mathrm{E}+03$ & -4.7 & $\mathrm{n} / \mathrm{a}$ & n/a & $n / a$ & $2.00 E+02$ & $\mathrm{U}$ \\
\hline S96V000046 & AP-105, 5AP-96-TB & $2.00 \mathrm{E}+02$ & U & $2.00 \mathrm{E}+02$ & -4.7 & $n / a$ & $\mathrm{n} / \mathrm{a}$ & $n / a$ & $2.00 \mathrm{E}+02$ & $\mathrm{U}$ \\
\hline S96V000062 & AP-105, 5AP-96-OB & $2.00 \mathrm{E}+02$ & $\mathrm{U}$ & $2.00 \mathrm{E}+02$ & -4.7 & $\mathrm{n} / \mathrm{a}$ & $n / a$ & $n / a$ & $2.00 E+02$ & $\mathrm{U}$ \\
\hline
\end{tabular}

Tri-n-butylphosphate

Procedure: LA-523-406

\begin{tabular}{|c|c|c|c|c|c|c|c|c|c|c|}
\hline $\begin{array}{l}\text { Labcore } \\
\text { Number }\end{array}$ & $\begin{array}{c}\text { Customer } \\
\text { Identification }\end{array}$ & $\begin{array}{c}\text { Sample } \\
\text { Result } \\
\mu g / L\end{array}$ & $\begin{array}{c}\text { Sample } \\
\text { Result } \\
\text { Qualifier }\end{array}$ & $\begin{array}{c}\text { Quantitation } \\
\text { Limit } \\
\mu \mathrm{g} / \mathrm{L}\end{array}$ & $\begin{array}{c}\text { Continuing } \\
\text { Calibration } \\
\% \text { Difference }\end{array}$ & $\begin{array}{c}\text { Spike } \\
\text { Recovery } \\
\%\end{array}$ & $\begin{array}{c}\text { Spike } \\
\text { Duplicate } \\
\text { \% Recovery }\end{array}$ & $\begin{array}{c}\text { Spike Dupl. } \\
\text { Precision } \\
\text { RPD }\end{array}$ & $\begin{array}{l}\text { Blank } \\
\mu g / L\end{array}$ & $\begin{array}{c}\text { Blank } \\
\text { Result } \\
\text { Qualifier }\end{array}$ \\
\hline S96V000043 & AP- 105,5 AP-96-1B & $2.10 \mathrm{E}+03$ & $\bar{B}$ & $2.00 \mathrm{E}+03$ & -4.2 & 91.6 & 88.0 & 4 & $2.70 \mathrm{E}+01$ & $J$ \\
\hline S96V000044 & $\mathrm{AP}-105,5 \mathrm{AP}-96-2 \mathrm{~B}$ & $2.40 \mathrm{E}+03$ & $\mathrm{~B}$ & $2.00 \mathrm{E}+03$ & -42 & $n / a$ & n/a & $\mathrm{n} / \mathrm{a}$ & $2.70 \tilde{E}+01$ & $\mathrm{~J}$ \\
\hline S96V000045 & AP-105, 5AP-96-3B & $1.50 \mathrm{E}+03$ & J日 & $2.00 \mathrm{E}+03$ & -4.2 & $\mathrm{n} / \mathrm{a}$ & $n / a$ & $\mathrm{n} / \mathrm{a}$ & $2.70 \mathrm{E}+01$ & $\mathrm{~J}$ \\
\hline S96V000046 & $A P-105,5 A P-96-7 B$ & $2.60 \mathrm{E}+01$ & $\mathrm{JB}$ & $2.00 E+02$ & -4.2 & $n / a$ & $\mathrm{n} / \mathrm{a}$ & $\mathrm{n} / \mathrm{a}$ & $2.70 \mathrm{E}+01$ & J \\
\hline S96V000062 & AP-105, 5AP-96-QB & $2.00 E+02$ & $\bar{U}$ & $2.00 \mathrm{E}+02$ & -4.2 & $n / a$ & $n / a$ & n/a & $2.70 \mathrm{E}+01$ & $\mathrm{~J}$ \\
\hline
\end{tabular}

\section{Surrogate Recovery}

\begin{tabular}{|c|c|c|c|c|c|c|c|c|c|}
\hline $\begin{array}{l}\text { Labcore } \\
\text { Number }\end{array}$ & $\begin{array}{c}\text { Customer } \\
\text { Identification }\end{array}$ & $\begin{array}{l}\text { S1 } \\
\%\end{array}$ & $\begin{array}{l}52 \\
\%\end{array}$ & $\begin{array}{l}\text { S3 } \\
\%\end{array}$ & $\begin{array}{l}\text { S4 } \\
\%\end{array}$ & $\begin{array}{l}\text { S5 } \\
\%\end{array}$ & $\begin{array}{l}56 \\
\%\end{array}$ & $\begin{array}{l}57 \\
\%\end{array}$ & $\begin{array}{l}\text { S8 } \\
\%\end{array}$ \\
\hline S96V000043 & AP-105, 5AP-96-1B & 0 & 0 & 0 & 42 & 91 & 65 & 0 & 94 \\
\hline S96V000044 & AP-105, 5AP-96-2B & 0 & 0 & 0 & 40 & 84 & 88 & 0 & 91 \\
\hline S96V000045 & $\mathrm{AP}-105,5 \mathrm{AP}-96-3 \mathrm{~B}$ & 0 & 0 & 0 & 38 & 84 & 67 & 0 & 84 \\
\hline S96V000046 & AP-105, 5AP-96-TB & 82 & 93 & 94 & 40 & 91 & 76 & 89 & 107 \\
\hline S96V000062 & AP-105, 5AP-96-OB & 74 & 88 & 89 & 34 & 90 & 70 & 80 & 108 \\
\hline
\end{tabular}

\section{Surrogates:}

\begin{tabular}{llc}
\multicolumn{2}{c}{ Surrogates: } & QC Limits (CLP) \\
S1 & 2-Fluorophenol-d5 & $21-110 \%$ \\
S2 & Phenol-d5 & $10-110 \%$ \\
S3 & 2-Chlorophenol-d4 & $33-110 \%$ \\
S4 & $1,2-D i c h l o r o b e n z e n e-d 4$ & $16-110 \%$ \\
S5 & Nitrobenzene-d5 & $35-114 \%$ \\
S6 & 2-Fluorobiphenyl & $43-116 \%$ \\
S7 & 2,4,6-Tribromophenol & $10-123 \%$ \\
S8 & Terphenyl-d14 & $33-141 \%$
\end{tabular}

$\mathrm{Q}$ (qualifiers):

$U$ indicates the compound was analyzed for, but not detected. The $U$-flagged number is the quantitation iimit, including applicable factors related to sample concentrating.

$J$ indicates the estimated value. Spectra meet criteria, but response is below the quantitation limit for the target compound. 
HNF
WHE-SD-WM-DP-202, REV. 1

Chain of Custody Forms 
HNE-SD-WM-DP-202, REV. 1

THIS PAGE WAS INTENTIONALLY LEFT BLANK 


\section{CHAIN-OF-CUSTODY RECORD FOR AUGER/GRAB SAMPLING}

: shipment Number $200-E-08$-TF (2) sample Number 541-96-1A (S) "31 supervioor RiJ. Pexzivic

1 Tonk AP.105 (5) Riser $10330^{\circ}$

(6) Cask/PiG Shipping Containar Serial Number $6003-0$

sdiation Survey Data For Caek/PlG

Over Top Dose Rate

Side Dowes Rate

Bottom Dous Rate

Smearable Contamination
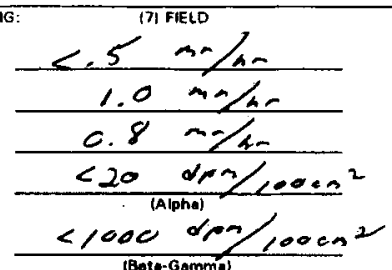

ACT *
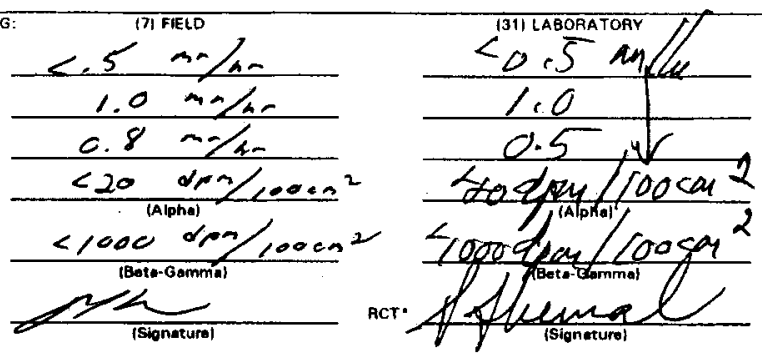

(8) Shipmont Doscription

A. Work Packevo Number

B. Caok/Pig Seal Number

C. Date and Tme Samplo

Fomovod trom Tank

D. Expected Llquid Content

E. Expected Solid Content

F. Dowe Rate Through Drll String

G. Expocted Sampto Longth IAwger/

volume IGRABI

\section{$E S-96-00219 / 0$ 10524}

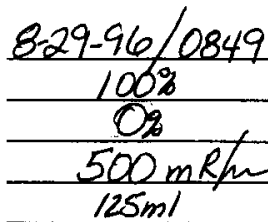

IINFORMA TION Include statement of labaretory teate to be performed.|

实

o) Field Commento

(32) Laboratory Commanta

1) Poimt of Oriain

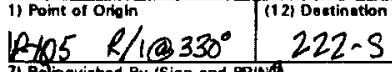

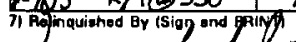

Drmes Luchs Itames lickik

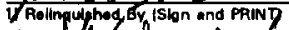

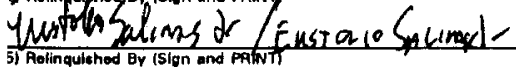

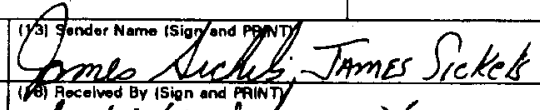
Eustoio Salimit

1221 Aacoivod ar (Sian and prinn

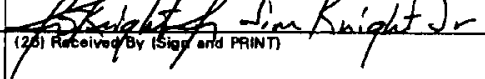
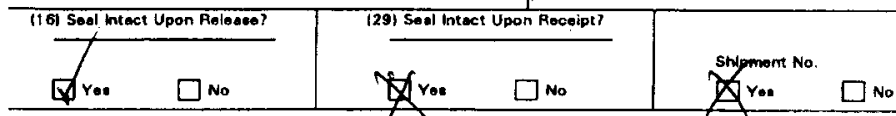

(30) Seal Date Consiotent with this Record?

STRIBUTION: White . Oftice of Sample Managendont Yollow - Recipient of Semple

Pink - Waste Tank Sempling

Goldenrod - Tonk Form Coerations.

Soel No. $\square$ No

Semplo No. $\square$ No 


\section{CHAIN-OF-CUSTODY RECORD FOR AUGER/GRAB SAMPLING}

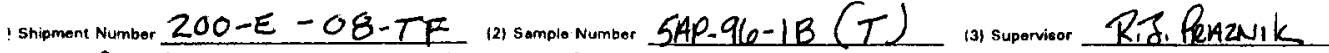
$6-5$ ") Tank AP-105 (5) Piser $10330^{\circ}$ (6) Cosk/PIG Shipping Container Serial Number
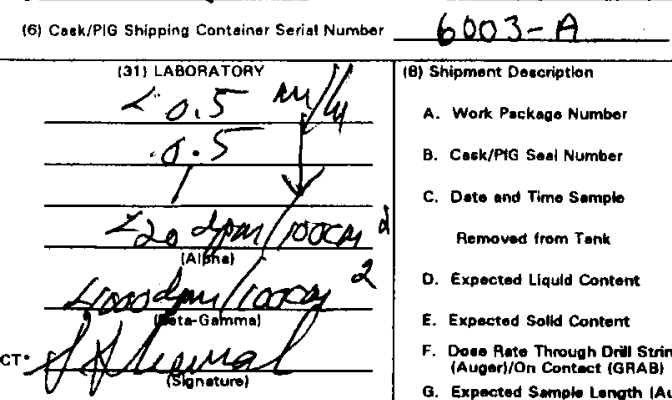

(6) Shipment Description

A. Work Packaga Number

B. Cosk/PTG Seal Number

$E S-96 \cdot 00219 / 0$

C. Deto and Time Sample Aemoved from Tank

D. Expected Liquid Content

E. Expocted Solld Content

F. Dous Aate Through Drill String

(Auger)iOn Contect (GAAB)

G. Expected Sumplo Lonoth (Augen) Volume (GPAB) 10525

"INFORMATION (Include staterment of laboratory teots to be periormed.

$\infty$

0) Fiold Comment:

(32) Laboratory Commente

11 Point of Origin

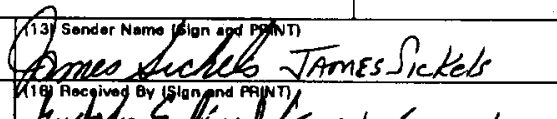

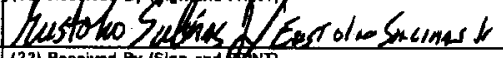

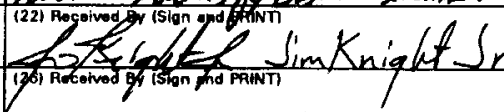

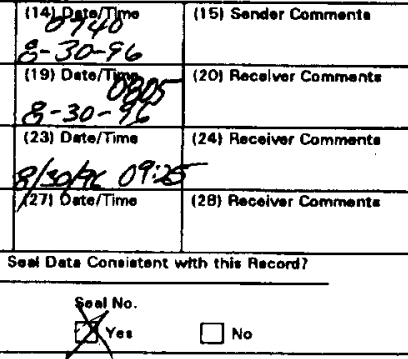

Yeras
Sample No. $\square$ No BC-6001-326 (01/96) 


\section{CHAIN-OF-CUSTODY RECORD FOR AUGER/GRAB SAMPLING}

: shipment Number 20020 A Tank -105 (5) Rioer $10330^{\circ}$ (2) Sampto Number
$5 A P-96-1 C(T)$
(3) suporisor Rid, Peaznil,

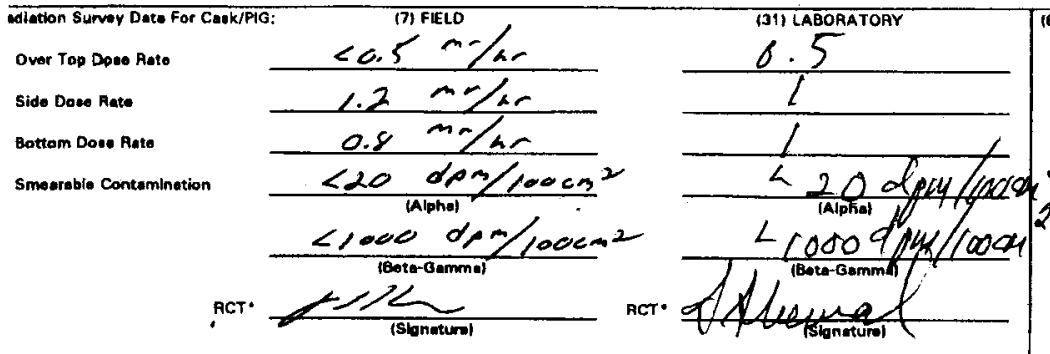

(6) Ceek/Plo shippino Containor Serial Number 6003E $/ \mathrm{TP}-2$

(日) Shipment Decorlption

A. Work Peckage Numbert

B. Ceak/PIG Soal Number

$E S-96 \cdot 00219 / 0$

C. Drte and Time samplo

Ramoved trom Tank

D. Expocted Llquld Content

E. Expeeted Solid Content

f. Does Rate Through Drill String

G. Expected Semplo Lenoth (Aupen) Volume 1ORABH 10526

I INFOAMATION (include etentement of ieborntory tonte to be pertormed.)

$\infty$

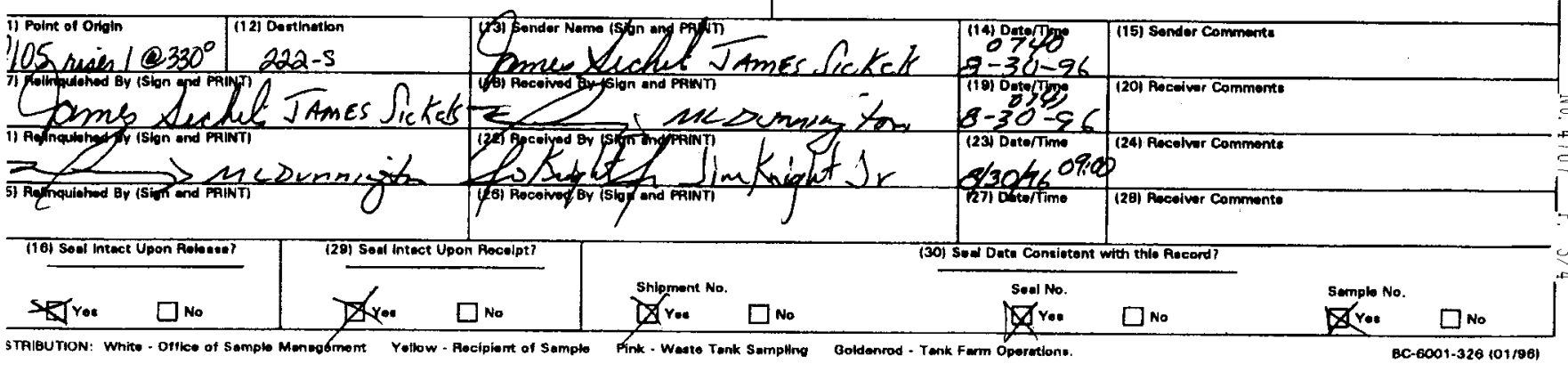




\section{CHAIN-OF-CUSTODY RECORD FOR AUGER/GRAB SAMPLING}

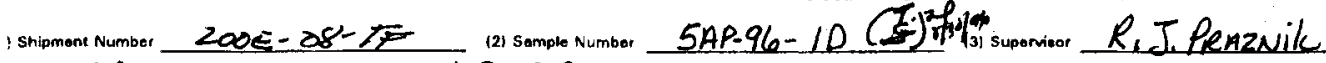

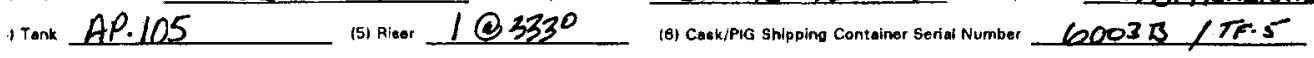

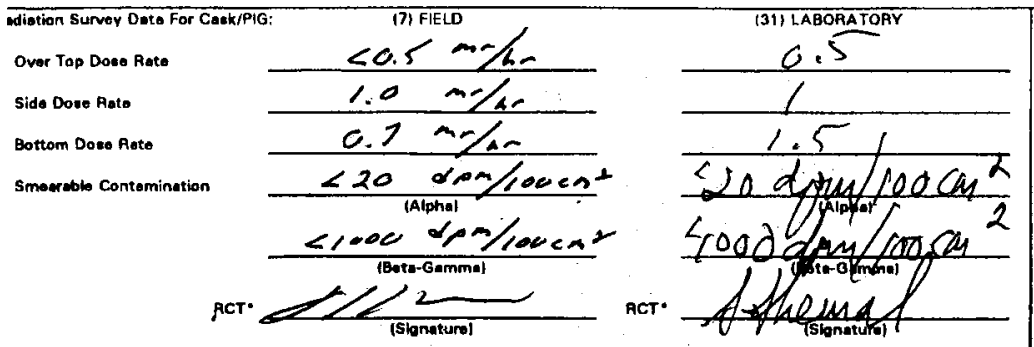

(B) Shipment Doscription

A. Work Packingo Number

8. Conk/PIG Soel Number

ES-96-00219/0

C. Date and Time Samplo

Rernoved from Tank

D. Expocted Liquid Content

E. Expoctod Solld Content

F. Doen Rate Trowgh Drill Strino

(Auger)/On Comet (GAAB)

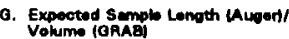
10527

I WFOAMATION (include ecetement of laboretory teet to be performed.)

$\infty$

of Find Commente

(32) Leboratory Commente
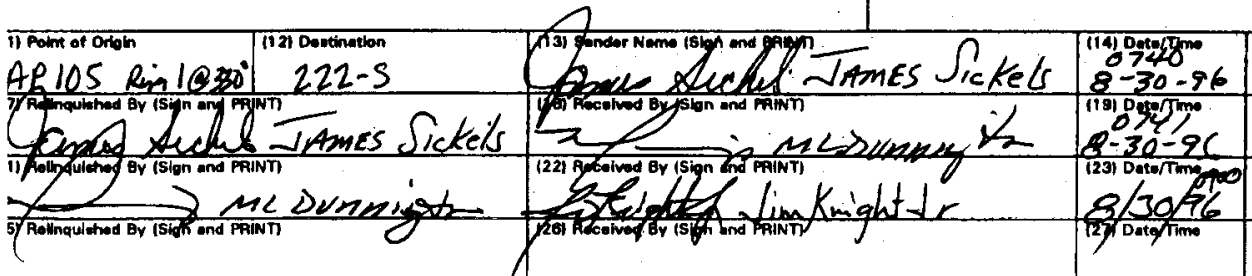

(16) Sondor Commonte

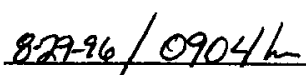
$106 \%$

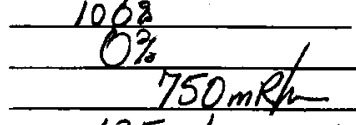
$125 \mathrm{~m}$ /

\begin{tabular}{|c|c|c|c|c|c|}
\hline (16) Soed Intect Upon Rolosase? & (29) Seel intect Upon Rocolpt? & & (30) Sosi Data Consirtent with this Plocord? & & \\
\hline Ero: & Exyo. $\quad \square \mathrm{Na}$ & $\begin{array}{l}\text { Shipgnent No. } \\
\text { tras } \square \text { No }\end{array}$ & Senjo. & Somple No. & $\square$ No \\
\hline
\end{tabular}




\section{CHAIN-OF-CUSTODY RECORD FOR AUGER/GRAB SAMPLING $6-2$

: shipment Numbar 2ODEOX-TF 1 Tank AP- 105 (5) Riser 1@90

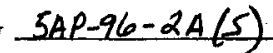

(3) supervioor R.J. ReAzNik

\section{4}

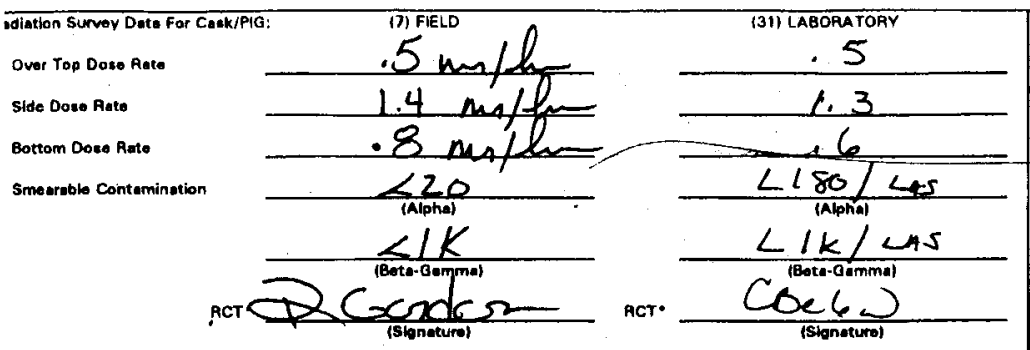

INFORMATION finclude etutemant of leboratery tente to be pertormod.I
(8) Shipmont Deccription

A. Work Peckeoe Number

B. Cack/PIO Sed Number

c. Dote and Timo Semplo

Flomoved from Tunk

D. Expoctod Uquid Cantent

E. Expected Solld Content

F. Dowe Rate Through Dritl Strine

(Avoen/On Comect topati)

- Expected Sample Length (Augent

Volume (GRAB)
$E 5-96-00219 / 0$ 10528 9-3-96/1105 his $100 \%$

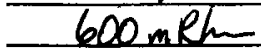

$125 \mathrm{ml}$.

\section{$\infty$}

of Fined commente

(32) Lebormeny Commente
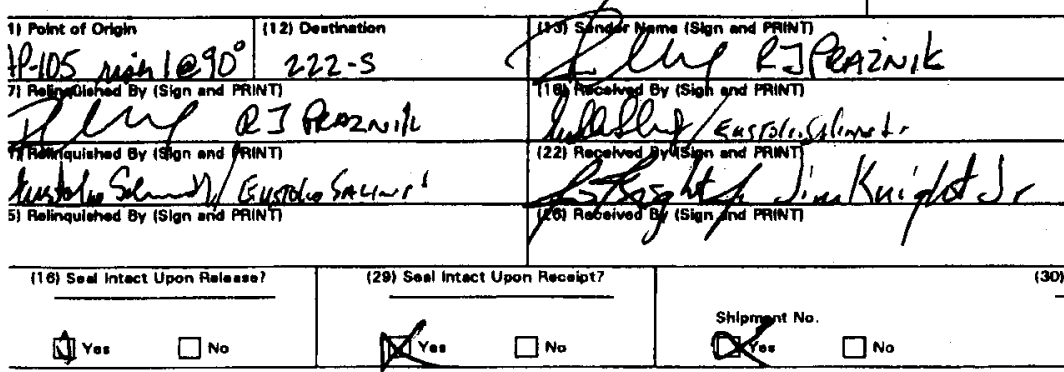

sThibution: Whito - Office of Sample Manegement Yollow - Rocipiont of Sample

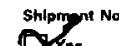

$\square$ No

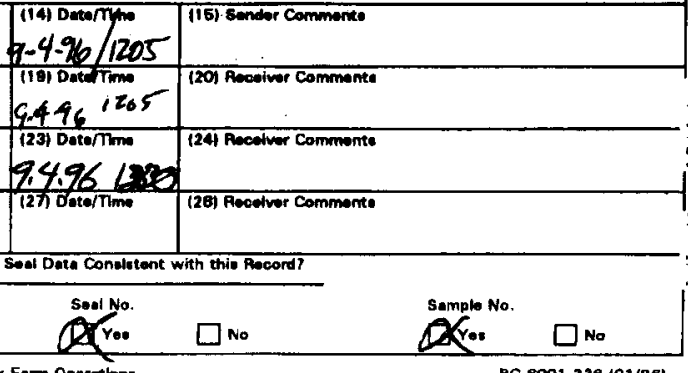


! shipmont Number 200E-08.TF "Tank AP-105 (5) Rleor $10.90^{\circ}$

idintion Surmy Onte for Cask/P/G:

Over Top Dose Rate

Side Doese Rate

Bottom Dose Ante

Smosroble Contembretion
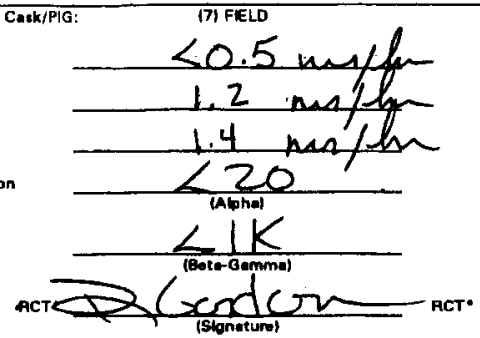

$5 A P-96-2 B(D)$

(3) supenvioor R.J.PRAZNIK

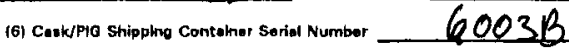

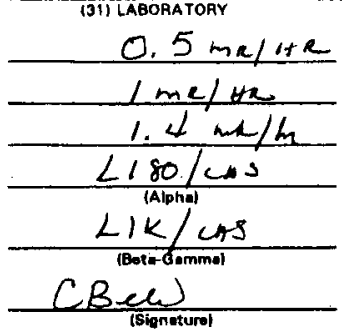

(ㅂ) Shipment Description

A. Work Peckege Number

B. Cenk/PIG Sent Number

C. Deto and Time Sampto

Romoved from Tank

D. Expectod Llquid Conterit

E. Expected Solid Content

F. Dose Rate Through Drit Sering

G. Expectud Samplo Lenoth (Auger)/

Volum torasi
$E S-96-00219 / 0$ 10529

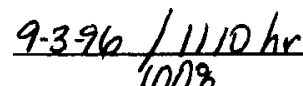

1008

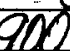

$125 \mathrm{~m} /$.

$\infty$

OT Fold Commente
Seal broke of for entering lab

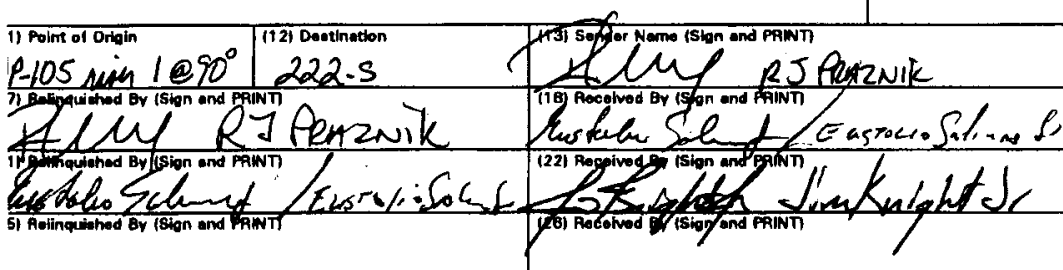

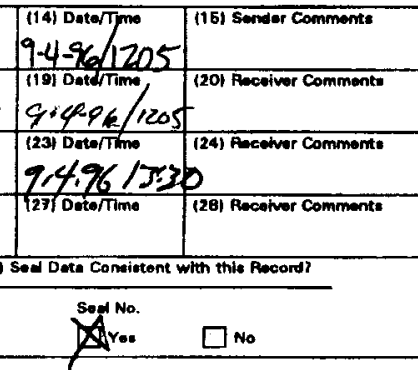

Somplo No.

Mryoe $\square$ No 
CHAIN-OF-CUSTODY RECORD FOR AUGER/GRAB SAMPLING

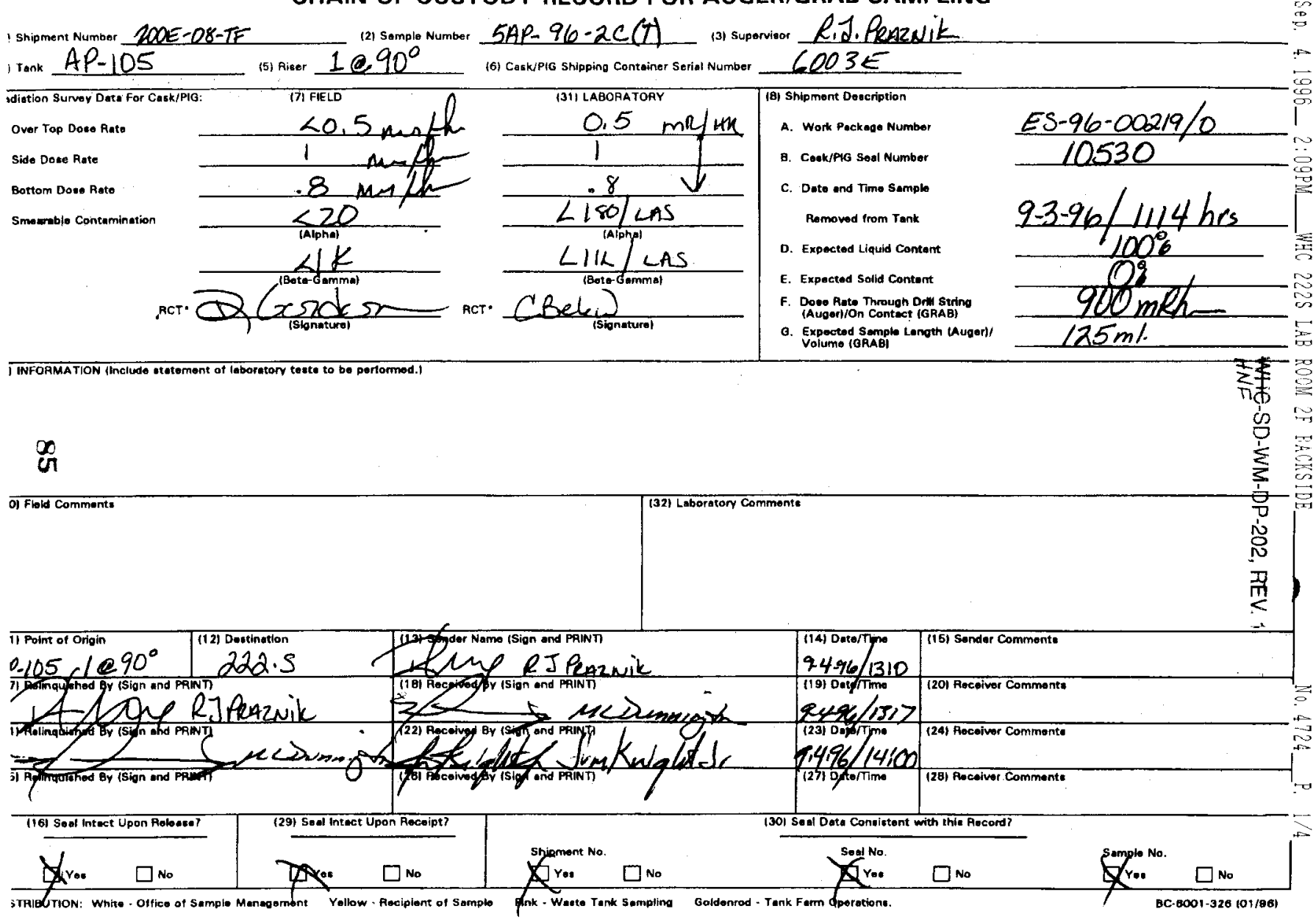


CHAIN-OF-CUSTODY RECORD FOR AUGER/GRAB SAMPLING

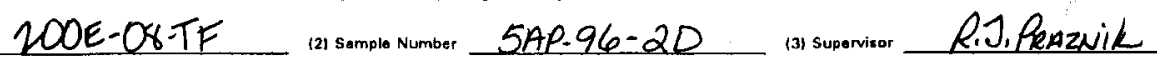

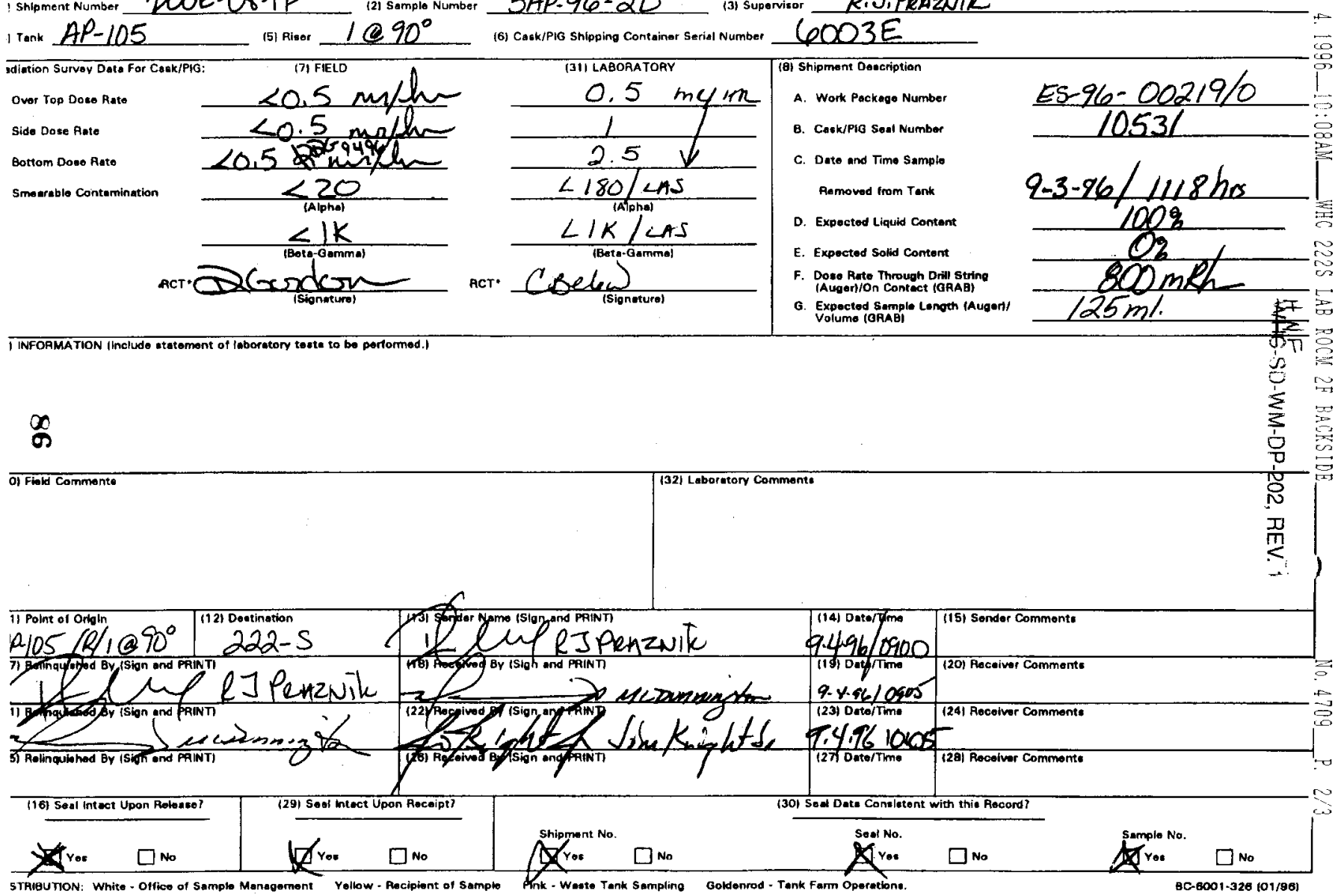




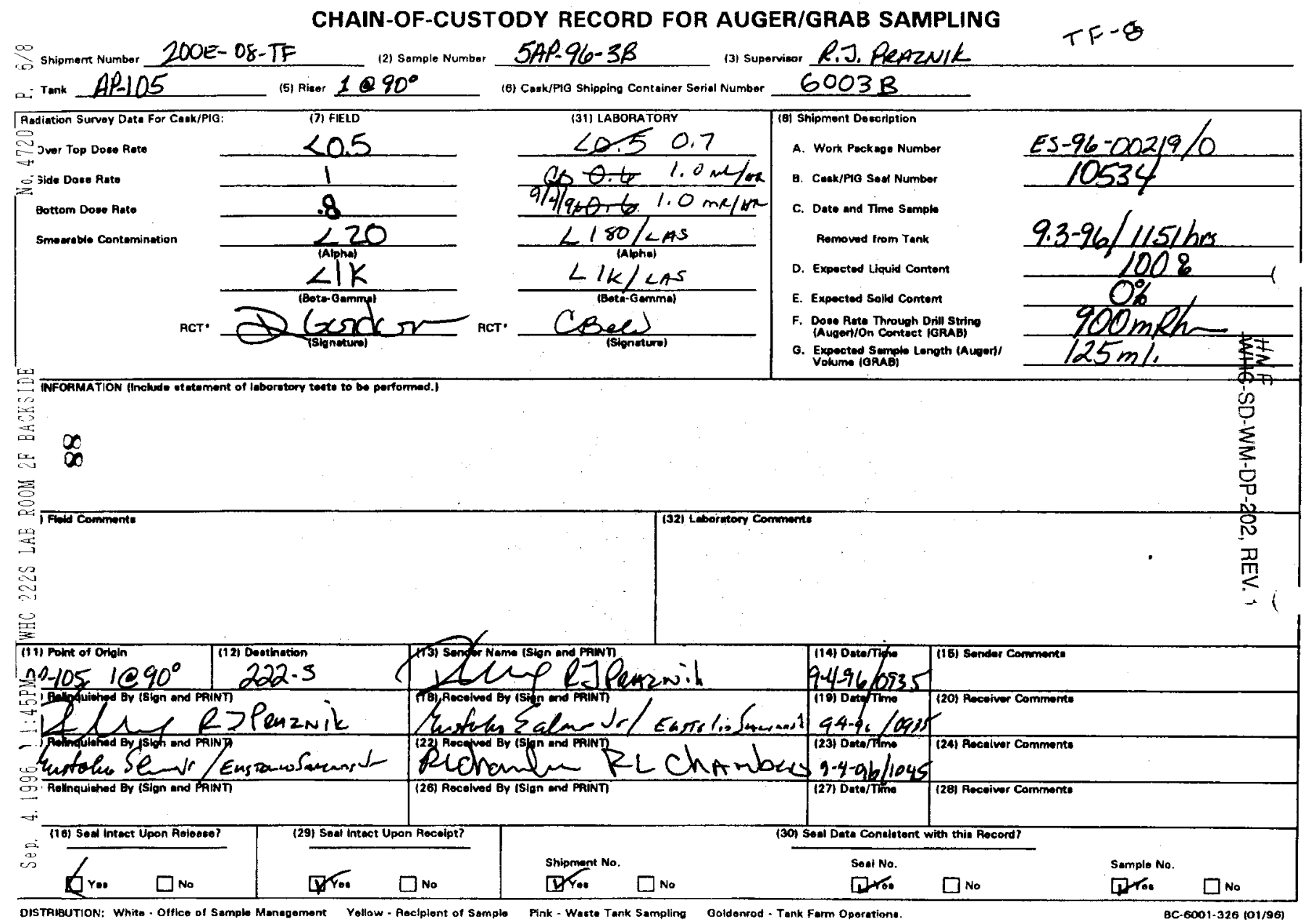




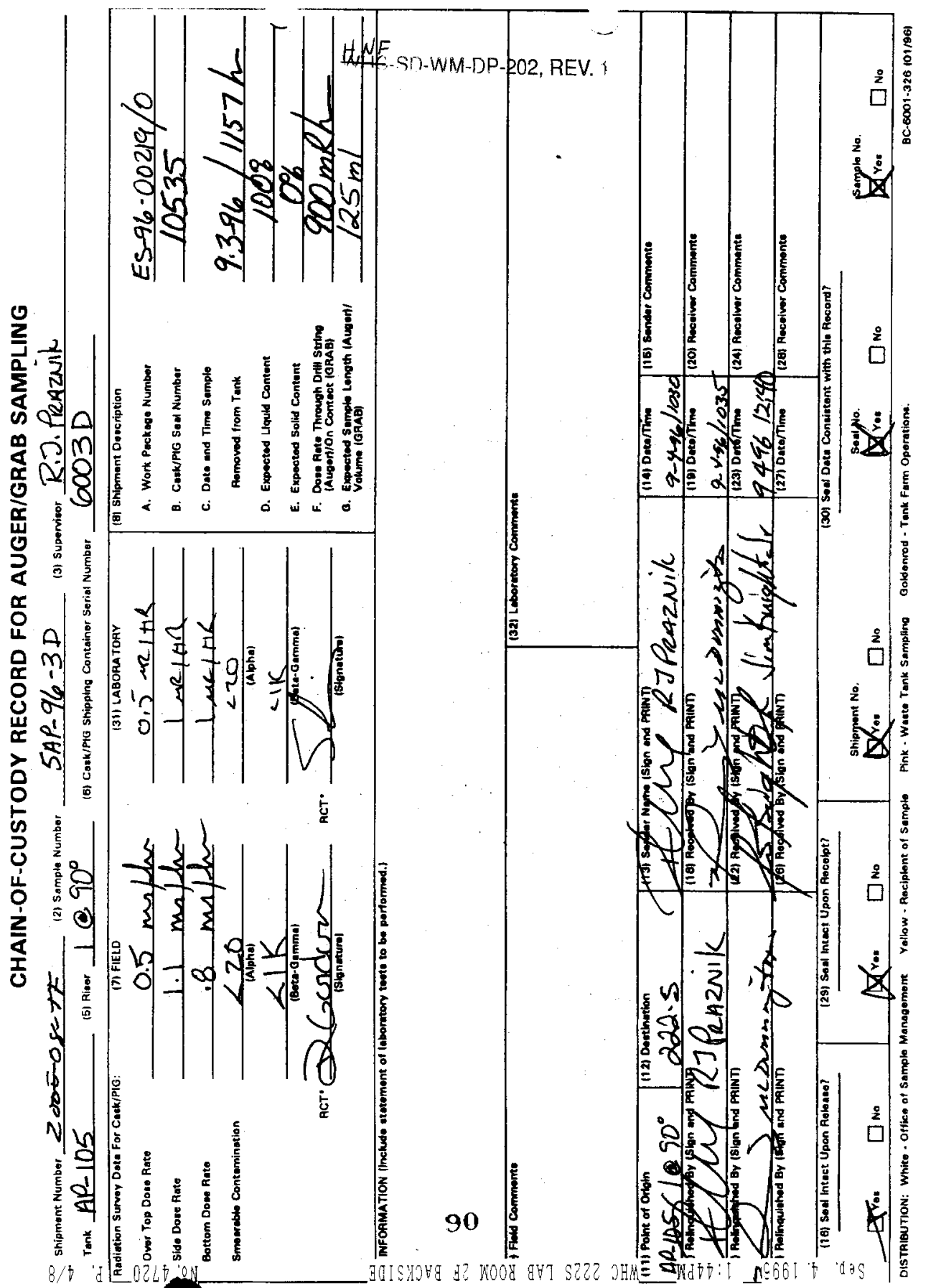


Shipment Number 200E-08TF Tenk AP-105 Rodiation Survey Datu For Cask/PIG:

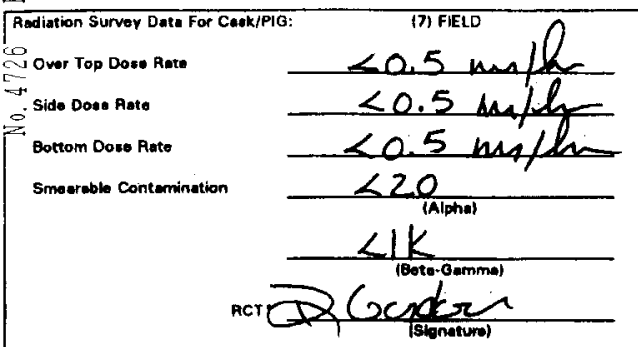
(2) Sample Number

5AP-96-IB $1(T)$ (3) sumonteor RiJ. PRAZNiK

西

(6) Cask/PIG Shipping Containar Serial Number $6003 \mathrm{~A}$

\begin{tabular}{|c|}
\hline $\begin{array}{l}\text { (31) LABORATOAY } \\
20.5\end{array}$ \\
\hline 20.5 \\
\hline$\angle 0,5$ \\
\hline$\angle 180 / \angle A J$ \\
\hline$\angle I K / \angle A J$ \\
\hline CABC(a) \\
\hline
\end{tabular}

\begin{tabular}{|c|c|}
\hline $181 \mathrm{si}$ & hipment Deceriotion \\
\hline & Work Packsoo Number \\
\hline B. & Cook/P1O Sed Number \\
\hline c. & Dete and Time Semple \\
\hline & Romoved Irom Tank \\
\hline D. & Expectod Lquid Content \\
\hline E. & Expoeted Solld Content \\
\hline & $\begin{array}{l}\text { Dowe Rate Though Drll Stritho } \\
\text { (Augert/On comter (GRAB) }\end{array}$ \\
\hline 0. & $\begin{array}{l}\text { Expocted Semplo Longth (Augert/ } \\
\text { Volume IGAABi }\end{array}$ \\
\hline
\end{tabular}
$E S-96-00219 / 0$ 10537

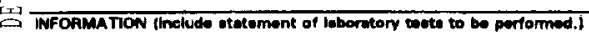

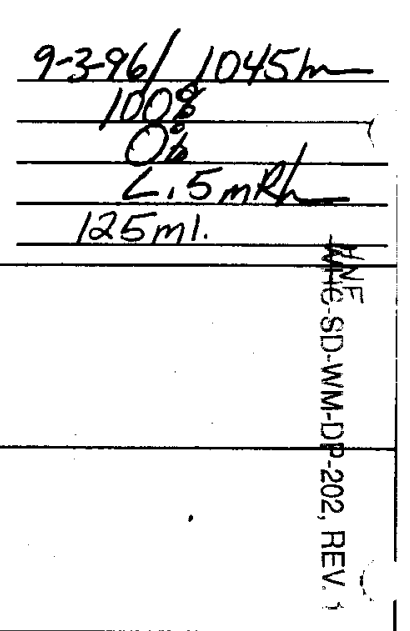

$\therefore$

$引$

吾 i Fold Commente

罚FED BLAKK

$\stackrel{\infty}{\infty}$

窟

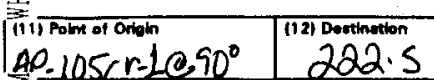

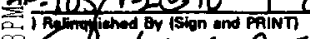

$\therefore$ Ifdel R.J.PenzNile

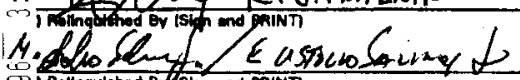

B.

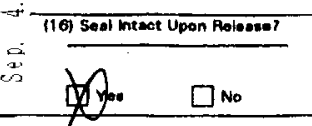

(29) Seel Intect Upon Receipt?

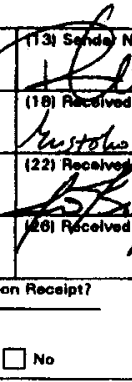

Nomo isty end Pativn

1321 Loboratory Comments

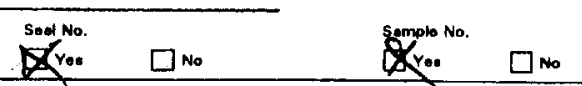

BC-6001-328 (01/96) 


\section{CHAIN-OF-CUSTODY RECORD FOR AUGER/GRAB SAMPLING}

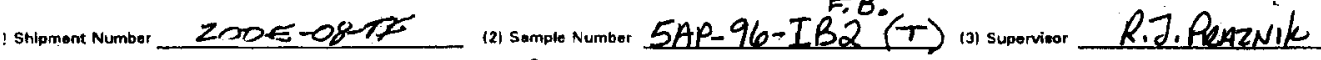
1 Tank AP-105 (5) Riser $1090^{\circ}$

(6) Cakk/PIO Shipping Conteiner Serial Number 6003E

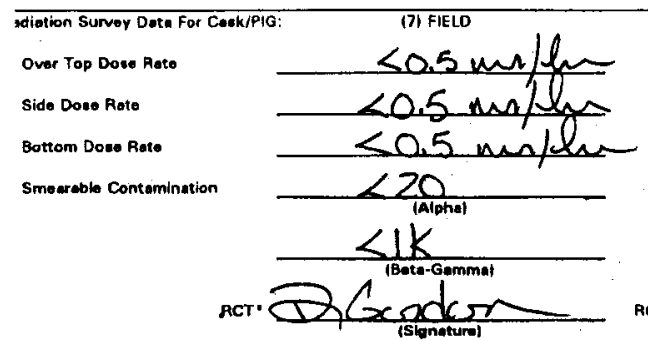

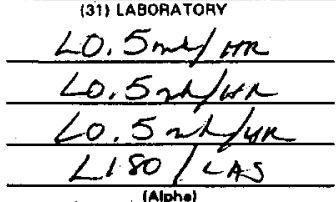

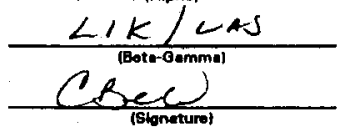

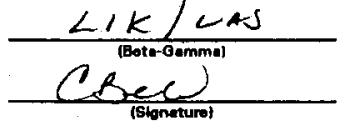

$$
\begin{aligned}
& \text { RCT }
\end{aligned}
$$

(8) Shipmomt Doecription

A. Work Packeos Numbor

B. Coek/PIG Sool Number

$E s-96-00219 / 0$

C. Date and Timo Samplo

Removed from Tenk

D. Expocted Liquid Contant

E. Expected Solld Content

F. Dowe Rete Through Drill string

(Augert/On Contect (GAAB)

O. Expocted Sumph Length (Augert)
Volume (ORAB) 10538

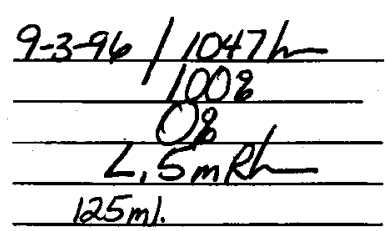

$125 \mathrm{ml}$

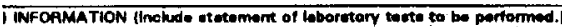

$\omega$

a Fied Commionts

(32) Libboratory Comments

FELW BLAWK

Troint of Driain

40105r-1090 $222-5$

ames Acchl Jameshickets

7

(14) 0atgon

青古

(1)

(2)


CHAIN-OF-CUSTODY RECQRP FOR AUGER/GRAB SAMPLING 200E-08-TF (2) sampio Number SAP-96-OB1(S) (31 supervior RiJ. PRAzNik

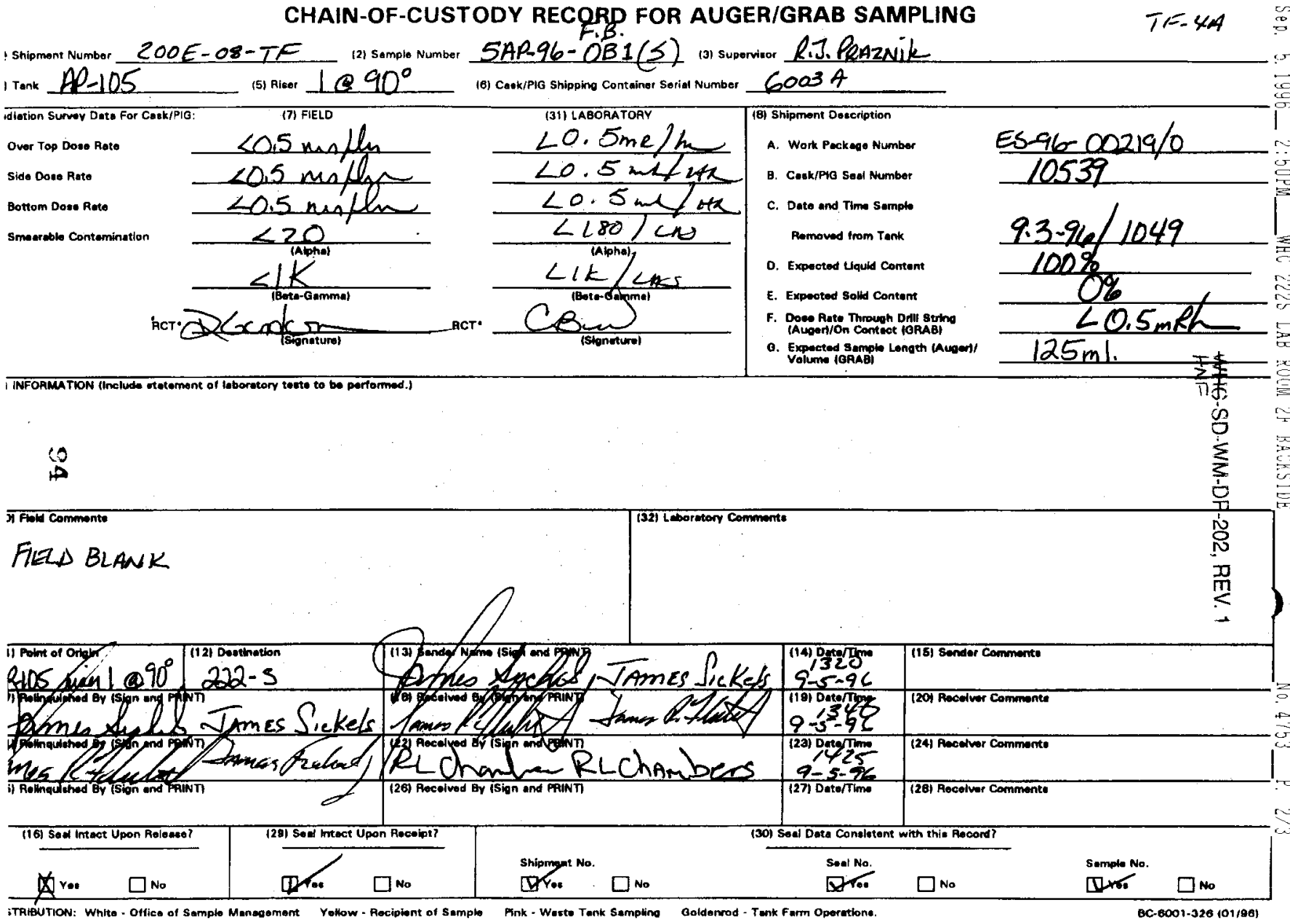


CHAIN-OF-CUSTODY RECORD FOR AUGER/GRAB SAMPLING

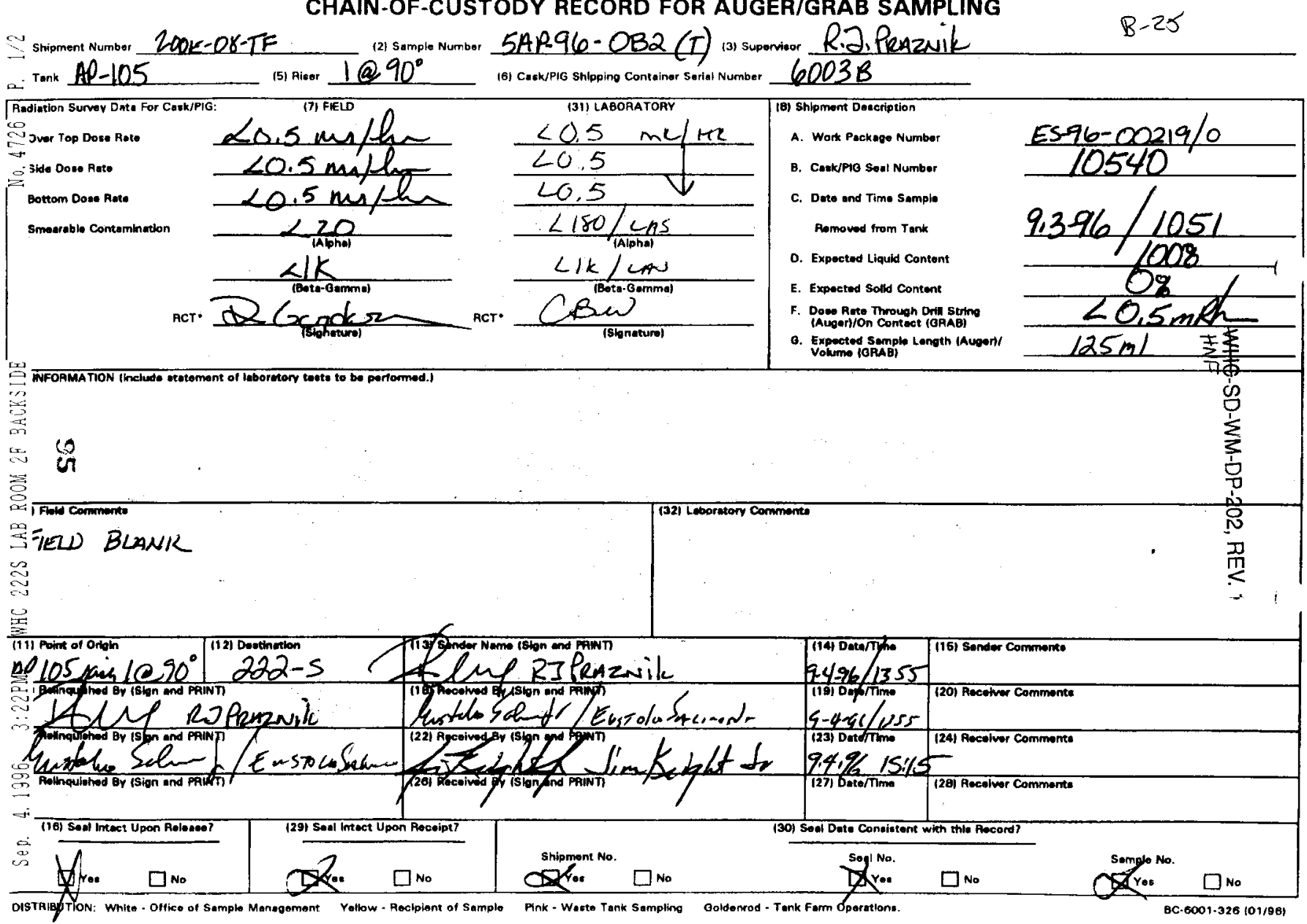


CHAIN-OF-CUSTODY RECORD FOR AUGER/GRAB SAMPLING

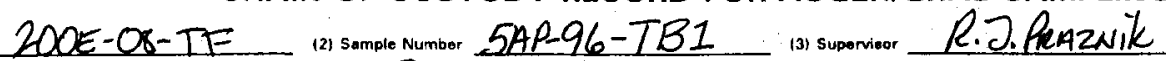

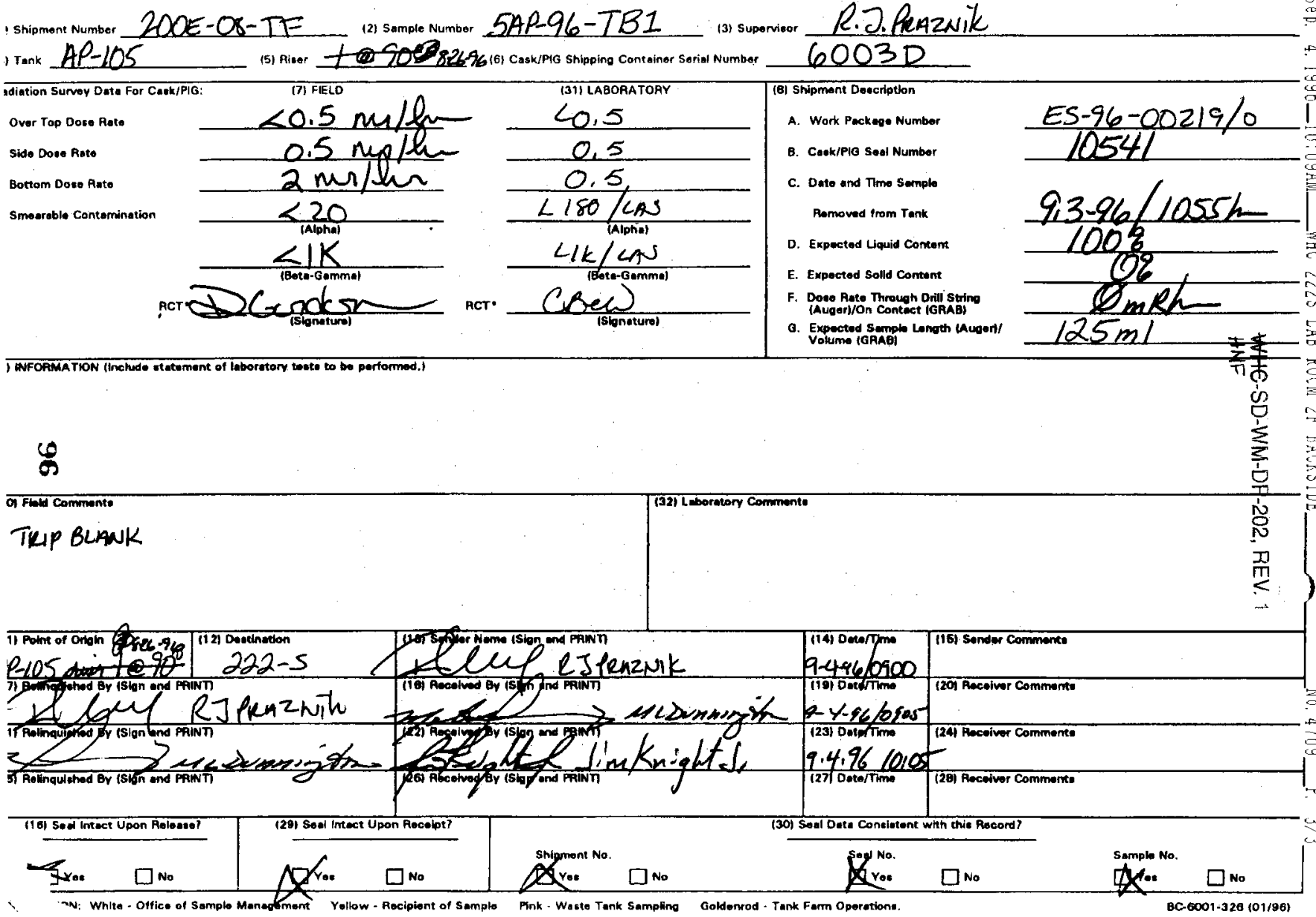


CHAIN-OF-CUSTODY RECGQRD FOR AUGER/GRAB SAMPLING

B-18

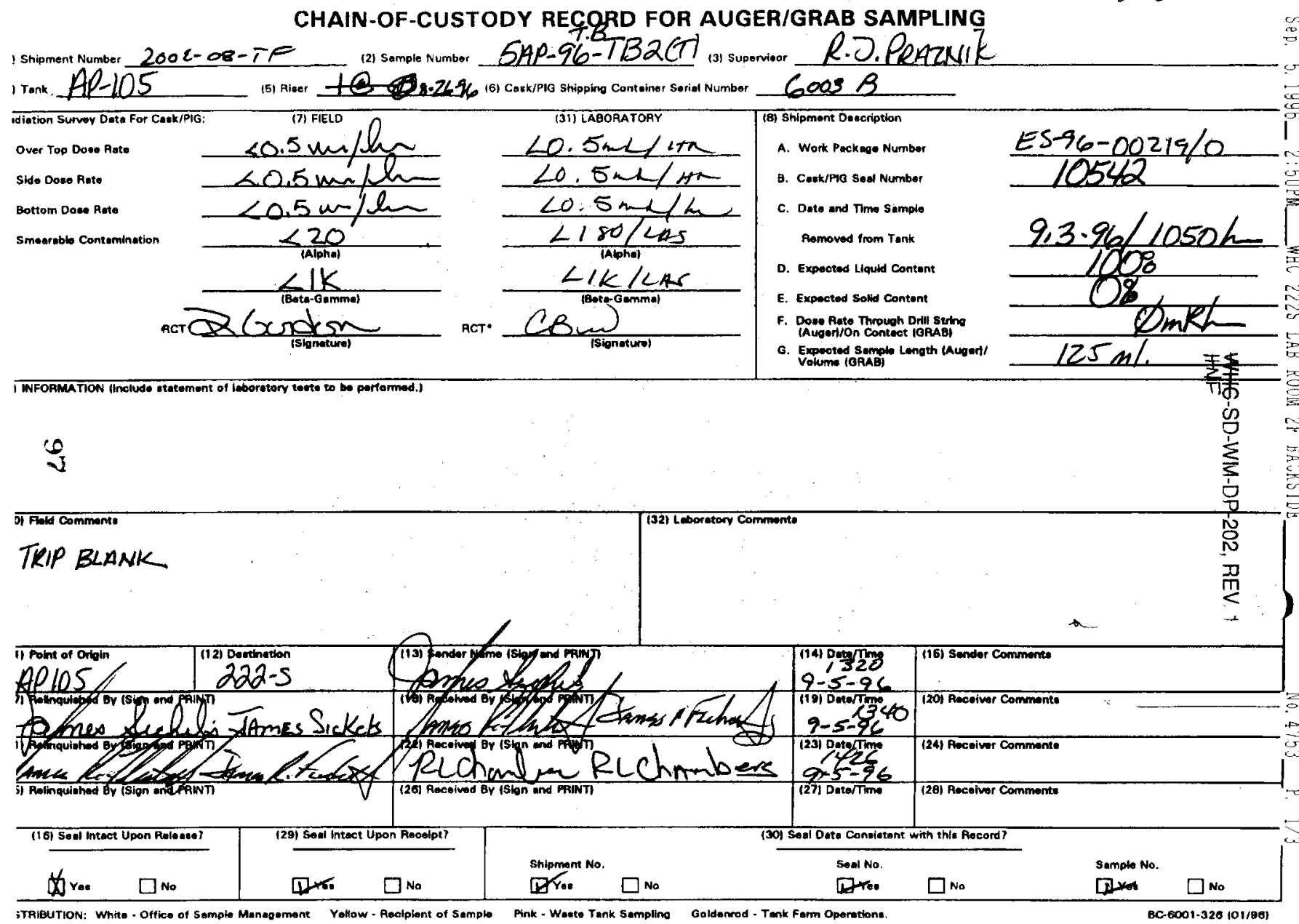


WHE-SD-WM-DP-202, REV. 1

Samp 7e Breakdown 


\section{LABCORE Data Entry Template for Worklist\# 12595}

Analyst: $\quad$ Instrument: NONE

Book \#

Method: LA-519-151 Rev/Mod _-2

Worklist Comment: @APPEAR2 FOR AP-105(CONTACT GEORGE MILLER)_ RTS!

\begin{tabular}{|c|c|c|c|c|c|c|c|c|c|c|}
\hline GROUP & PROJECT & S TYPE & SAMPLE\# & $R A$ & $\ldots \ldots-$ YEST $\cdots$ & MATR IX & ACTUAL & FOUND & DL & UNIT \\
\hline 96000853 & AP- 105 & 1 SAMPLE & s96v000055 & 0 & AAPPEARZ ORGVOLOZ & LIQUID & $\therefore$ N/A & & & $\mathrm{mL}$ \\
\hline 96000853 & $A P-105$ & 1 SAMPLE & S96V000055 & 0 & DAPPEAR2 AF'PEAR02 & LIQUID & N/A & & ted & \\
\hline 96000853 & $A P-105$ & 1 SAMPLE & S96V000055 & 0 & DAPPEAR2 DCISE-02 & LIQUID & $\mathrm{N} / \mathrm{A}$ & & & $\mathrm{mrad} /$ hour \\
\hline
\end{tabular}

\section{Final page for worklist \# 12595}

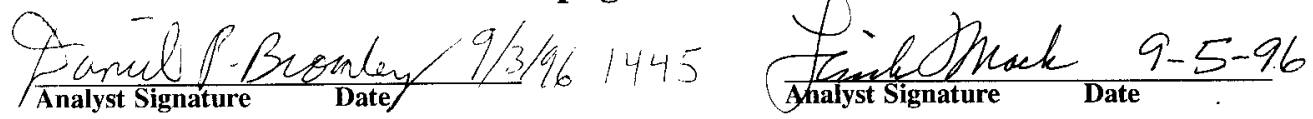

APPEARANLE

culor colorlej

ciarity clear

$10 \%$ solids

$25 \mathrm{~mL}$ tital volume

Units shown for $Q C$ (SPK \& STD) may not reflect the actual units. DL = Detection Limit, $S=$ Worklist Slot Number, $R=$ Replicate Number, $A=$ Aliquot Code. 
worklistrpt Version 2.1 05/15/95

$H N F$

-SO-WM-DP-202, REV.

Page: I 09/03/96 11:27

LABCORE Data Entry Template for Worklist\# 12596

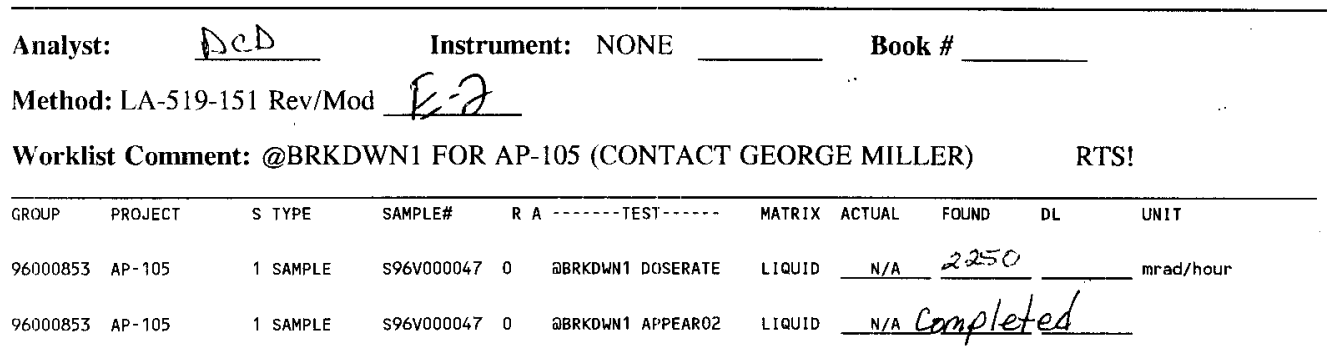

Final page for worklist \# 12596

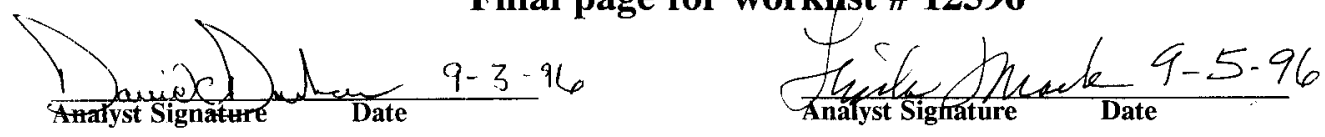

color: greenish yellow

clarity clear

$<1.0 \%$ solids

$\sim 125 \mathrm{ml}$ total volume $\rightarrow$ placed into $2-50 \mathrm{~mL}$ bottles

Organics: None disposed $225 \mathrm{~mL}$ sample per $P C$ instructions died

Data Entry Comments:

Units shown for QC (SPK \& STD) may not reflect the actual units. DL $=$ Detection Limit, $S=$ Worklist Slot Number, $R=$ Replicate Number, $A=$ Aliquot Code.

101 


\section{LABCORE Data Entry Template for Worklist\# 12712}

Analyst:

Instrument: NONE

Book \#

Method: LA-519-151 Rev/Mod EZ

Worklist Comment: AP-105. Phases/Solids,Color,Clarity,Mrad/hr. new

\begin{tabular}{|c|c|c|c|c|c|c|c|c|c|c|}
\hline GROUP & PROJECT & $\mathbf{s}$ & TYPE & SAMPLE\# & R A & A $\ldots \ldots$. TEST $\ldots \ldots$ & MATRIX & ACTUAL & FOUND & UNI T \\
\hline 96000855 & AP -105 & 1 & SAMPLE & $\$ 96 \mathrm{~V} 000056$ & 0 & AAPPEAR2 ORGVOLO2 & LIQUID & $\mathrm{N} / \mathrm{A}$ & c) & $\mathrm{mL}$ \\
\hline 96000855 & AP -105 & 1 & SAMPLE & s96V000056 & 0 & DAPPEAR2 APPEAR02 & LIQUID & $N / A$ & Carplote & \\
\hline 96000855 & $A P-105$ & 1 & SAMPLE & $\$ 96 \mathrm{~V} 000056$ & 0 & DAPPEAR2 DUSE-02 & LIQUID & $\mathrm{N} / \mathrm{A}$ & 3560 & $\mathrm{mrad} /$ hour \\
\hline 96000855 & $A P-105$ & 2 & SAMPLE & s96v000057 & 0 & DAPPEAR2 ORGVOLO2 & LIQUID & $N / A$ & 0 & $\mathrm{~mL}$ \\
\hline 9,000855 & AP -105 & 2 & SAMPLE & S96v000057 & 0 & DAPPEAR2 APPEAR02 & LIQUID & $N / A$ & Complete & \\
\hline 96000855 & AP -105 & 2 & SAMPLE & S96v000057 & 0 & DAPPEAR2 DUSE - 02 & LIQUID & $N / A$ & 3000 & $\mathrm{mrad} / \mathrm{hour}$ \\
\hline 96000855 & AP -105 & 3 & SAMPLE & s96v000053 & 0 & AAPPEAR2 ORGVOL02 & LIQUID & $\mathrm{N} / \mathrm{A}$ & 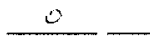 & $\mathrm{mL}$ \\
\hline 96000855 & AP- 105 & 3 & SAMPLE & s96V000053 & 0 & DAPPEAR2 APPE.AR02 & LIQUID & $N / A$ & Complete & \\
\hline 96000855 & $A P-105$ & 3 & SAMPLE & s96v000053 & 0 & DAPPEAR2 SAMFAMT2 & LIQUID & $N / A$ & 120 & $\mathrm{~mL}$ \\
\hline 96000855 & AP- 105 & 3 & SAMPLE & s96v000053 & 0 & DAPPEAR2 DOSE: 02 & LIQUID & N/A & 3000 & forad/hour \\
\hline
\end{tabular}

\section{Final page for worklist \# 12712}

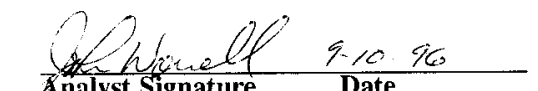

Analyst Signature

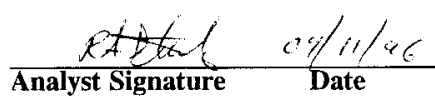
Appedarduce
$596 V 000056:$
$596 V 000057:$
$596 \vee 00005-3$
Cuen, No Salids,
Cie or. No solids
cuar, No Salids

Dutu Entry Comments:

Units shown for QC (SPK \& STD) may not reflect the actual units. DL $=$ Detection Limit, $S=$ Worklist Slot Number, $R=$ Replicate Number, $A=$ Aliquot Code. 


\section{LABCORE Data Entry Template for Worklist\# 12713}

Analyst:

Method: LA-519-151 Rev/Mod E-7

\section{Book \#}

Worklist Comment: AP-105. Doserate Worklist.(George Miller). new .

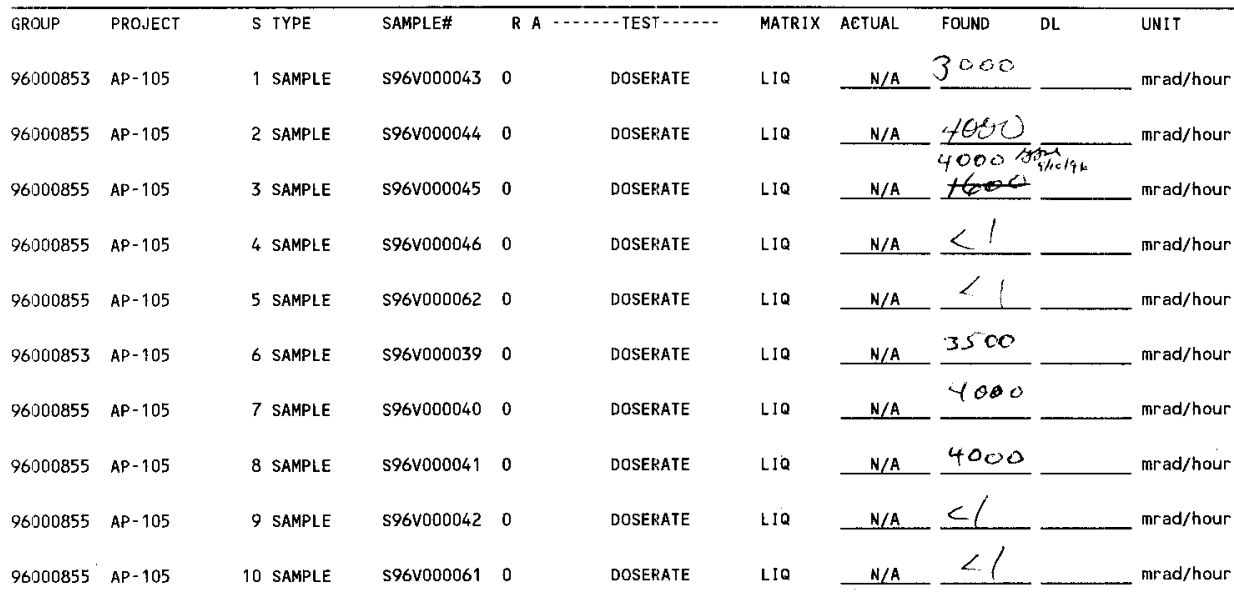

\section{Final page for worklist \# 12713}
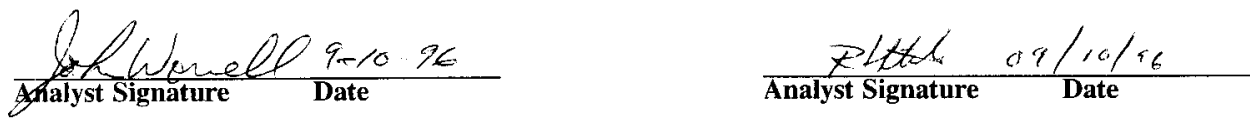

Data Entry Comments:

Units shown for $Q C$ (SPK \& STD) may not reflect the actual units. $D L=$ Detection Limit, $S=$ Worklist Slot Number, $R=$ Replicate Number, $A=$ Aliquot Code. 


\section{LABCORE Data Entry 'Template for Worklist\# 12714}

Analyst:

Instrument: NONE

Book \#

Method: LA-519-151 Rev/Mod $\_-\gamma$

Worklist Comment: AP-105. DOSERATE WORKLIST. (George Miller). new

\begin{tabular}{|c|c|c|c|c|c|c|c|c|c|c|}
\hline GRIDUP & PROJECT & S TYPE & SAMPLE\# & R A & $-\ldots$ TEST $\cdots . . .$. & MATRIX & ACTUAL & FOUND & $\mathrm{DL}$ & UNIT \\
\hline 961000855 & AP -105 & 1 SAMPLE & s96v000058 & 0 & DOSERATE & LIQUID & $N / A$ & $<$ & & $\mathrm{m} r \mathrm{ad} / \mathrm{hour}$ \\
\hline 961000855 & $A P-105$ & 2 SAMPLE & s96V000059 & 0 & DOSEFATE & LIQUID & $N / A$ & $<1$ & & $\mathrm{mrad} / \mathrm{hour}$ \\
\hline
\end{tabular}

\section{Final page for worklist \# 12714}
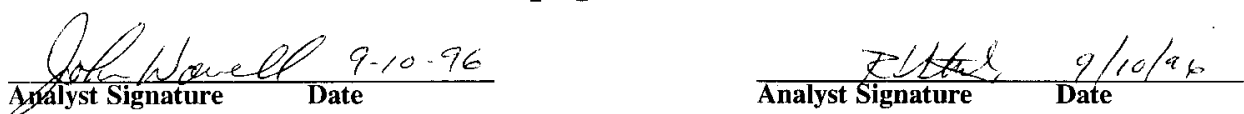

Data Entry Comments:

Units shown for $Q C$ (SPK \& STD) may not reflect the actual units. $O L=$ Detection Limit, $S=$ Worklist Slot Number, $R=$ Replicate Number, $A=$ Aliquot Code. 


\section{LABCORE Data Entry Template for Worklist\# 12715}

Analyst:

Instrument: NONE

Book \#

Method: LA-519-151 Rev/Mod E-

Worklist Comment: AP-105. BREAKDOWN WORKLIST. (George Miller). new

\begin{tabular}{|c|c|c|c|c|c|c|c|c|c|c|}
\hline GRDUP & PROJECT & S TYPE & SAMPLE\# & R A & $\cdots+$ TEST $\cdots \ldots$ & MATRIX & ACTUAL & FOUND & DL & UNIT \\
\hline 96000855 & AP. 105 & 1 SAMPLE & $596 \mathrm{~V} 0000 / 8$ & 0 & ABRKDWN1 DOSERATE & LIQUID & $\mathrm{N} / \mathrm{A}$ & 4000 & & $\mathrm{mrad} /$ hour \\
\hline 96000855 & AP -105 & 1 SAMPLE & s96v000048 & 0 & aBRKDWN1 AFPEAR02 & LIQUID & $N / A$ & C.mple & $t=$ & \\
\hline 961300855 & AP- 105 & 2 SAMPLE & $596 v 000049$ & 0 & DBRKDWN1 DOSERATE & LIQUID & $N / A$ & 4000 & & $\mathrm{mrad} / \mathrm{hour}$ \\
\hline 961000855 & $A P-105$ & 2 SAMPLE & s96v000049 & 0 & aBRKDWN1 APPEARO2 & LIQUID & $\mathrm{N} / \mathrm{A}$ & Contp & $\varepsilon$ & \\
\hline
\end{tabular}

\section{Final page for worklist \# 12715}

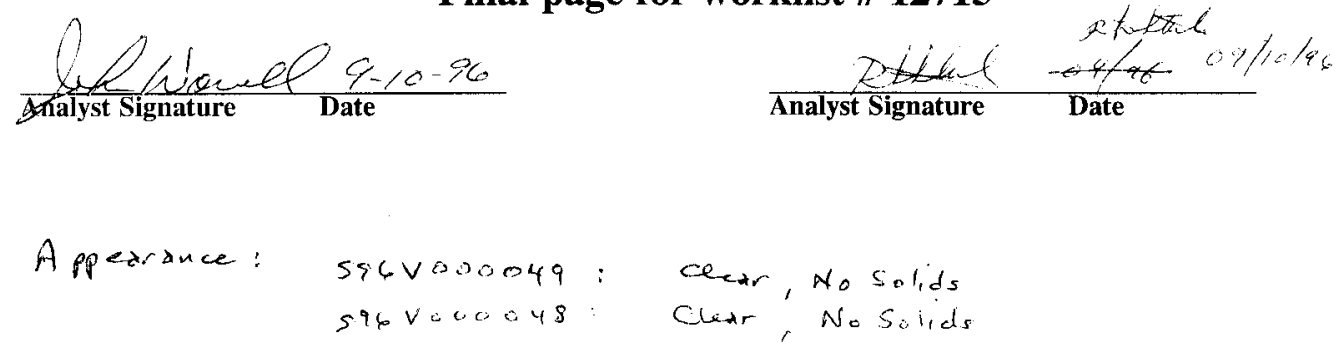

Data Entry Comments:

Uinits shown for $Q C$ (SPK \& STD) may not reflect the actual units. $D L=$ Detection Limit, $S=$ Worklist Slot Number, $R==$ Replicute Number, $A=$ Aliquot Code. 
HNF SD-WM-DF-202, REV. 1

Sample Preparations 
WF-SD-WM-DP-202, REV. 1

THIS PAGE WAS INTENTIONALLY LEFT BLANK 
worklistrpt Version $2.105 / 15 / 95$

$09 / 10 / 9674: 47$

\section{LABCORE Data Entry Template for Worklist\# 12716}

Page: $\quad 1$

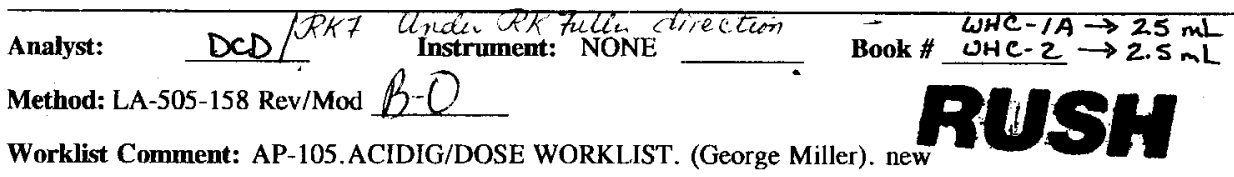

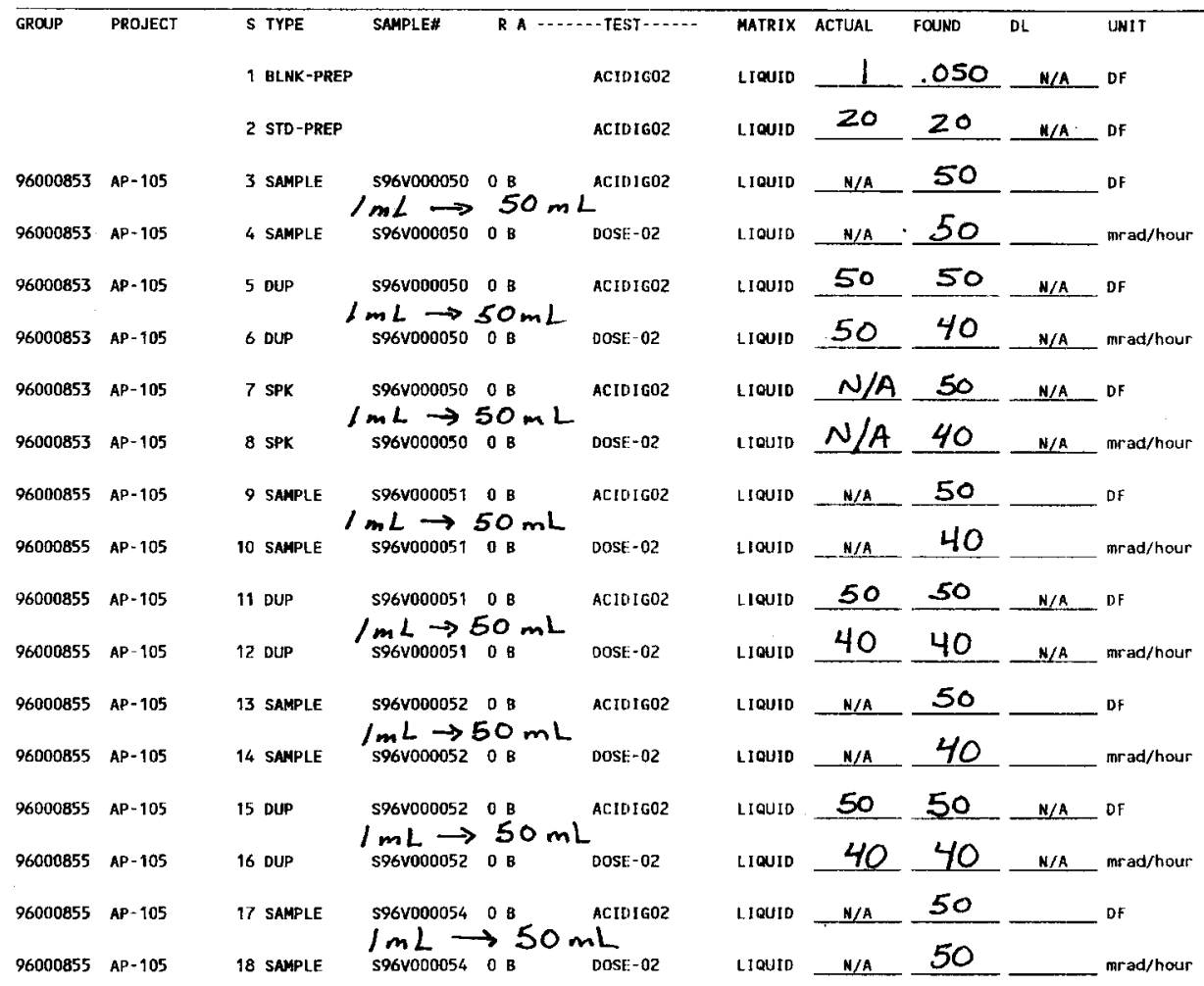

\section{Data Entry Comments:}

Units shown for QC (SPK \& STD) may not reflect the actual wnits. D $\check{L}=$ Detection Limit, $S=$ Worklist Slot Number, $R=$ Replicate Number, $A=$ Aliquot Code. 
wonklistrpt Version 2.1 05/15/95

\section{LABCORE Data Entry Template for Worklist\# 12716}

\begin{tabular}{|c|c|c|c|c|c|c|c|c|c|c|}
\hline GROUP & PROJECY & S TYPE & SAMPLE & $\overline{R A}$ & $\cdots-$ TEST & MATRIX & ACTUAL & FOUND & DL & UN!T \\
\hline 96000855 & $A P-105$ & 19 DUP & S96V000054 & $0 \mathrm{~B}$ & ACHDIGO2. & LtQuTo & 50 & 50 & N/A & $D F$ \\
\hline 96000855 & $A P-105$ & $20 \mathrm{Dup}$ & s96V000054 & $0 \mathrm{~B}$ & DOSE $=02$ & LIOUID & 50 & & N/A & $\mathrm{mrad} / \mathrm{hour}$ \\
\hline 96000855 & AP- 105 & 21 SAMPLE & s96v000060 & $\underset{\longrightarrow}{0 \mathrm{~B}}$ & $50^{\text {ACHDIGO2 }}$ & kIOUID & & 50 & & $D F$ \\
\hline 96000855 & AP- 105 & 22 SAMPLE & s96v000060 & $0 B$ & DOSE-02 & LIOUID & $N / A$ & & & $\mathrm{mrad} /$ hour \\
\hline 96000855 & AP- 105 & 23 DUP & $596 \mathrm{~V} 000060$ & $0 \mathrm{~B}$ & $A C I D I G O 2$ & LIQU]D & 50 & 50 & $N / A$ & $D F$ \\
\hline 96000855 & AP- 105 & 24 Dup & 596v000060 & 08 & DOSE -02 & LIOUID & & & N/A & mrad/hour \\
\hline
\end{tabular}

\section{Final page for worklist \# 12716}

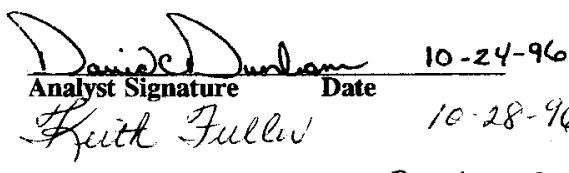

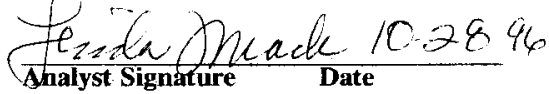
5962000047
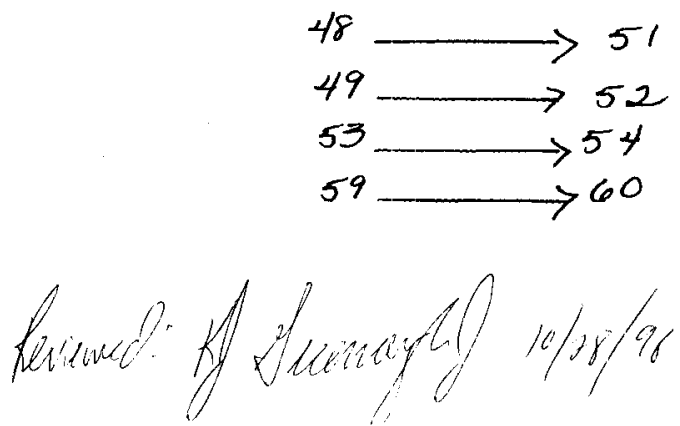

Data Entry Comments:

HPT: Gerry Gunuskey

Units shown for QC (SPK \& STD) may not reflect the actual units, $\overrightarrow{D L}=$ Detection Limit, $S=$ Worklist Slot Number, $R=$ Replicate Number, $A=$ Aliquot Code.

199 
HNF-SD-WM-DP-202, REV. 1

Inorganic Analyses 


\section{LABCORE Completed Worklist Report for Worklist\# 12917}

Analyst: pjm

Instrument: BA001

Book\# 133N16A

Method: $\angle A-510-112 \mathrm{Rev} / \mathrm{Mod}$

$\mathrm{C}-3$

Worklist Comment: AP-105 SPG. RCJ

\begin{tabular}{|c|c|c|c|c|c|c|c|}
\hline Seq Type & Sample & $\mathbf{R A}$ & Test & Matrix & Actual & Found & DL or Yield Unit \\
\hline $1 \mathrm{sto}$ & & 0 & sRe-0 1 & LTOUID & 1.39755 & 1.3784 & 98.630 Recovery \\
\hline 2 SAMLLE & S96V000048 & 0 & SPG-01 & LIQUID & $\mathbf{N} / \mathbf{A}$ & 1.2349 & $1.000-003$ sp.c. \\
\hline 3 DUP & $\$ 960000048$ & 0 & SPG-OL & LIoUID & 1.2349 & 1.2280 & $0.560 \mathrm{RPD}$ \\
\hline 4 SAMELE & $S 96 V 000049$ & 0 & SPG-01 & IIQUID & $\mathbf{N} / \mathbf{A}$ & 1.2347 & $1.000-003$ Sp.G. \\
\hline 5 DUt? & $896 \mathrm{~V} 000049$ & 0 & SPG 01 & Ixouts & 1.2347 & 1.2272 & 0.609 RPD \\
\hline 6 SAMPLE & $596 \vee 000047$ & 0 & SPG-01 & IIQUID & $\mathbf{N} / \mathbf{A}$ & 1.2294 & 1.000-003 Sp.G. \\
\hline 7 DUP & 8960000047 & 0 & SPG-01 & Itourd & 1.2294 & 1.2228 & $\because \quad 0.538 \mathrm{RPD}$ \\
\hline 8 SAMPLE & S96V000053 & 0 & SPG-01 & LIQUID & N/A & 1.2473 & $1.000-003$ sp.G. \\
\hline 9 Dष्ट: & E96V0000053 & 0 & SPG-01 & LIQUTtD & 1.2473 & 1.23 .56 & 0.942 RPD \\
\hline
\end{tabular}

\section{Final page for worklist\# 12917}

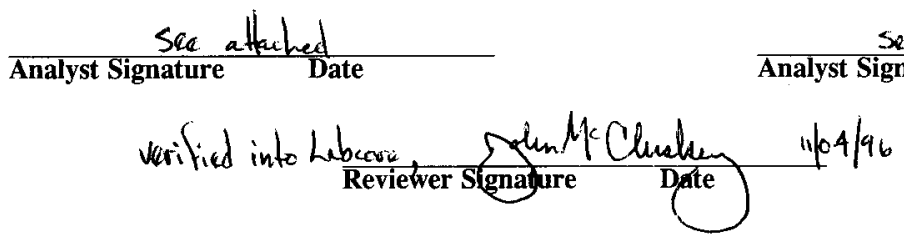


SPECIFIC GRAVITY: LA-510-112 (C-3)

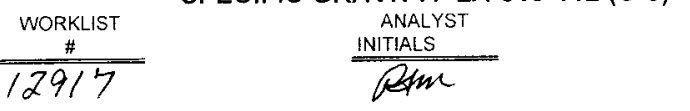

ANALYSIS
$\frac{\text { DATE }}{11 / 3 / 96}$

$\frac{\substack{\text { ANALYSIS } \\ \text { TIME }}}{0930}$

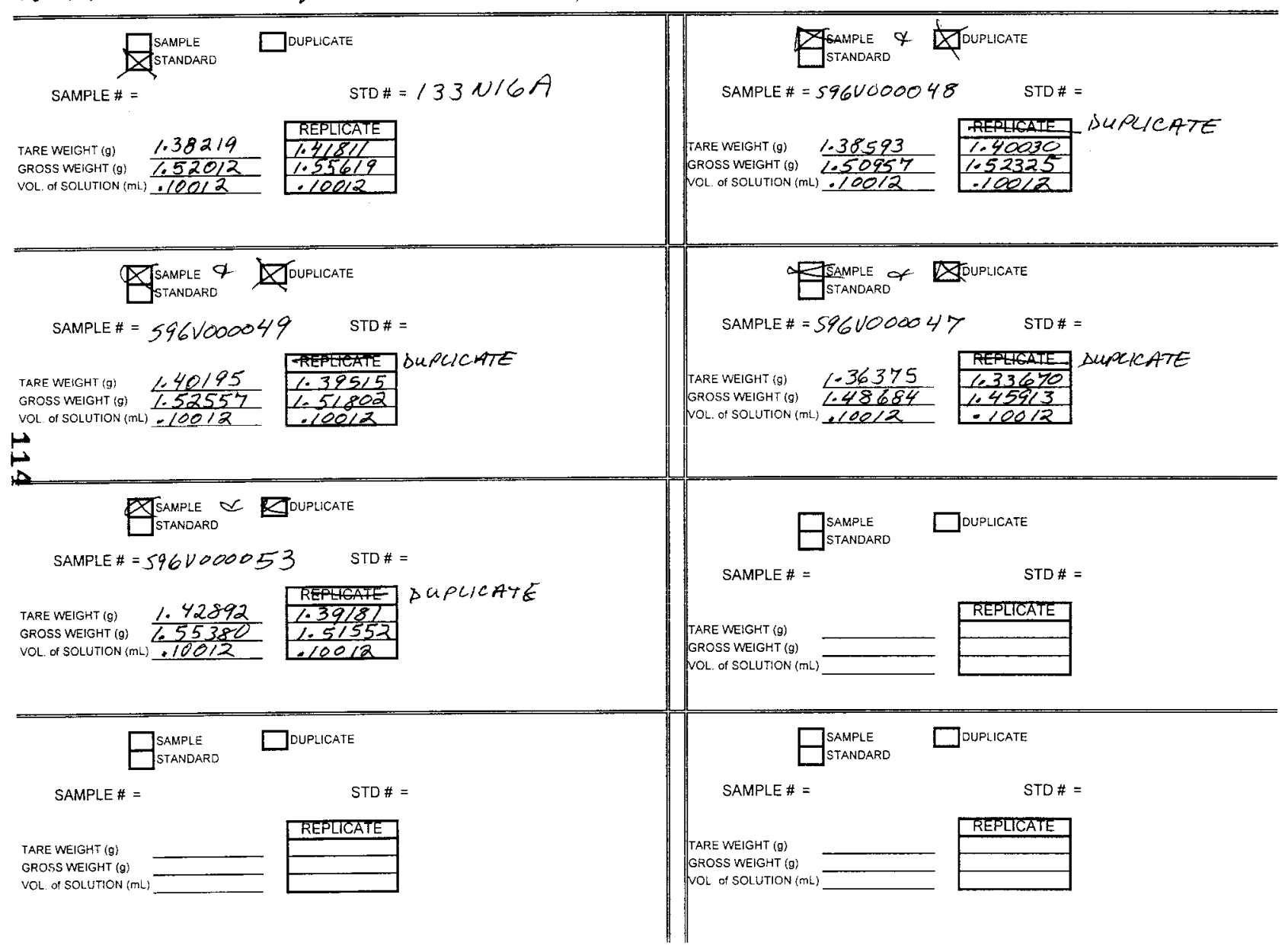




\section{SPECIFIC GRAVITY : LA-510-112 (C-3)}

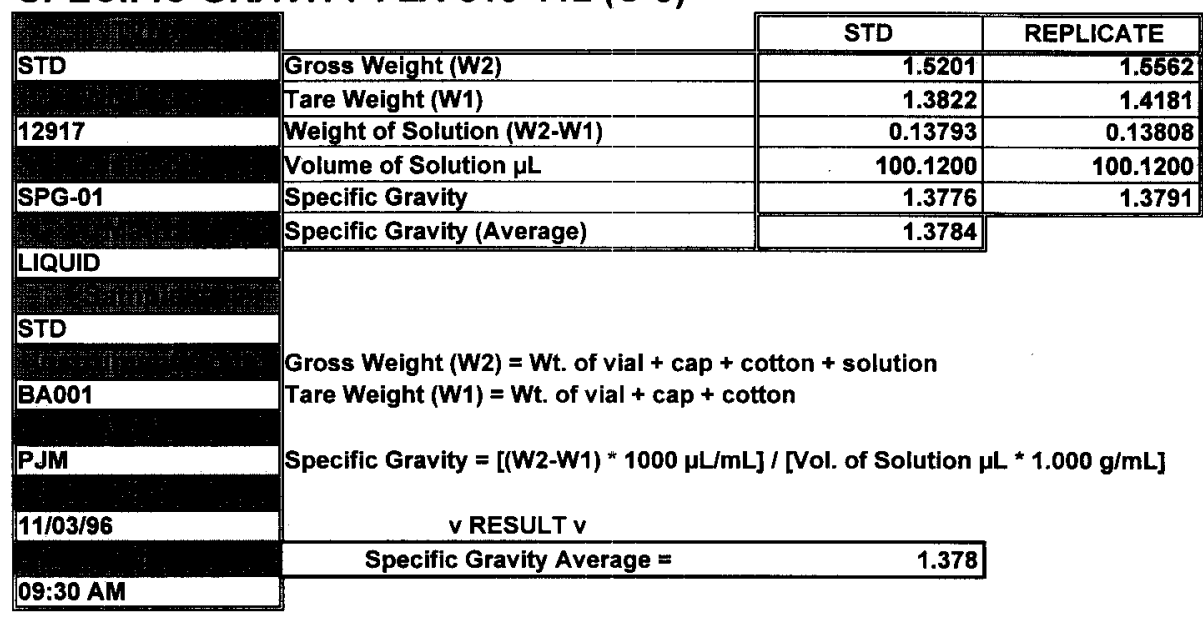

Data Entry by:

Approved by:

Form 510112L1 Rev. 1.1
Date:

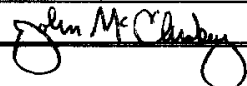

Date: $11 / 04196$

$11 / 03 / 96$

\section{5}




\section{HNFE-SD-WM-DP-202, REV. 1}

PLACE ANALYTICAL CARD IN BOX BELOW OR ATTACH TRAVELER

\section{SPECIFIC GRAVITY : LA-510-112 (C-3)}

\begin{tabular}{|c|c|c|c|}
\hline . & & SAMPLE & REPLICATE \\
\hline SAMPLE & Gross Weight (W2) & 1.5256 & 1.5180 \\
\hline $\mathrm{m}$ & Tare Weight (W1) & 1.4020 & 1.3952 \\
\hline 12917 & Weight of Solution (W2-W1) & 0.12362 & 0.12287 \\
\hline & Volume of Solution $\mu \mathrm{L}$ & 100.1200 & 100.1200 \\
\hline SPG-01 & Specific Gravity & 1.2347 & 1.2272 \\
\hline $4 \times 3 \times$, & Specific Gravity (Average) & 1.2310 & \\
\hline
\end{tabular}

\section{LIQUID}

\section{S96V000049}

Gross Weight $\left(W_{2}\right)=W t$. of vial + cap + cotton + solution

BA001

Tare Weight $(W 1)=W t$. of vial + cap + cotton

PJM

Specific Gravity $=[(W 2-W 1) * 1000 \mu \mathrm{L} / \mathrm{mL}] /[$ Vol. of Solution $\mu \mathrm{L} * 1.000 \mathrm{~g} / \mathrm{mL}]$

$11 / 03 / 96$

$\checkmark$ RESULT $v$

Specific Gravity Average =

09:30 AM

\begin{tabular}{|c|c|c|}
\hline Data Entry by: & Date: & $11 / 03 / 96$ \\
\hline Approved by: & Date: & $4 l_{0}+1 a_{6}$ \\
\hline Form 510112L1 Rev. 1.1 & & \\
\hline
\end{tabular}


SPECIFIC GRAVITY : LA-510-112 (C-3)

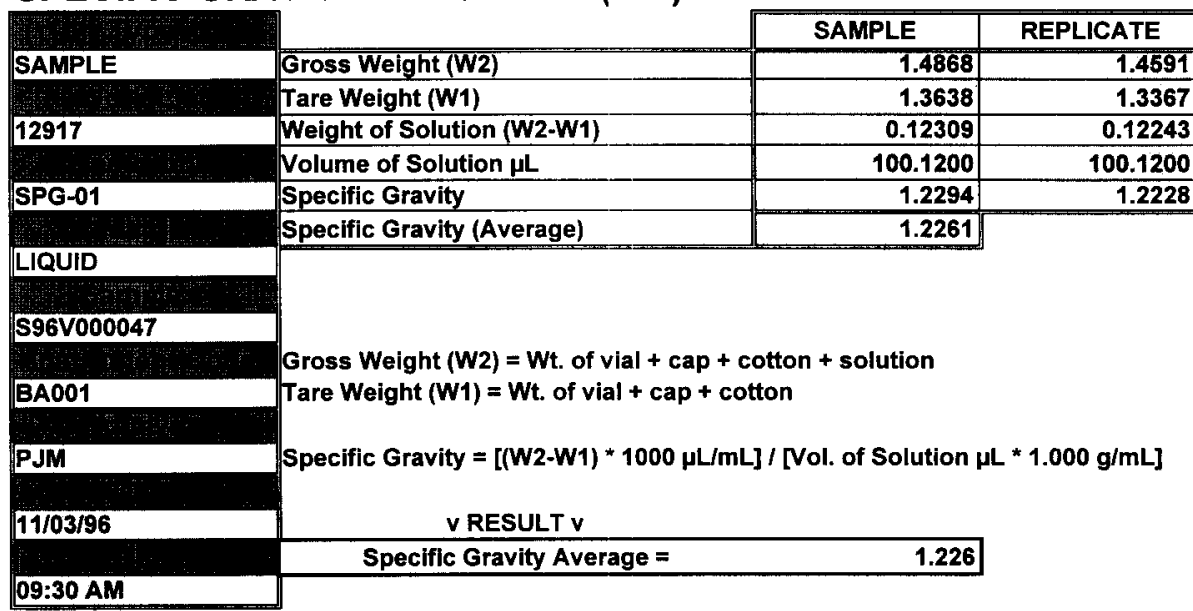

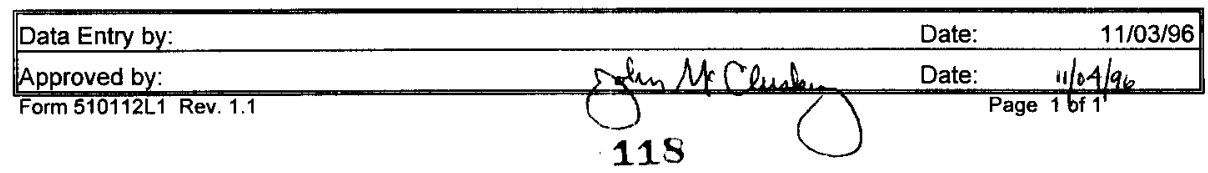




\section{LABCORE Data Entry Template for Worklist\# 12905}

Analyst: RAW Instrument: PH01 Book \# ISN17-D

Method: LA-212-106 Rev/Mod A-O

Worklist Comment: AP-105 PH. RCJ

\begin{tabular}{|c|c|c|c|c|c|c|c|c|c|c|}
\hline \multirow[t]{2}{*}{ GRDUP } & \multirow[t]{2}{*}{ PROJECT } & $S$ TYPE & SAMPLE\# & \multicolumn{3}{|c|}{ R A $\cdots$-TEST $\cdots$} & MATRIX & ACTUAL & FOUND & $\overline{D L}$ \\
\hline & & $1 \mathrm{STDPH}$ & & & & $\mathrm{PH}-01$ & LIQUID & 8.00 & 8.01 & $N / A$ \\
\hline 961300855 & AP- 105 & 2 SAMPLE & 5960000048 & 0 & $L_{0}$ & $\mathrm{PH}-01$ & LIQUID & $N / A$ & 13.49 & 0.01 \\
\hline 961000855 & $A P-105$ & 3 DUP & $596 \vee 000048$ & 0 & & PH-01 & LIQUID & 13.49 & 13.48 & $\mathrm{~N} / \mathrm{A}$ \\
\hline 961100855 & $A P-105$ & 4 SAMPLE & $596 \vee 000049$ & 0 & & PH-01 & LIQUID & $N / A$ & 13,45 & 0.01 \\
\hline 9611000855 & $A P-105$ & 5 DUP & $\$ 96 \mathrm{~V} 000049$ & 0 & & PH-01 & LIQUID & 13.45 & 13.46 & $\mathrm{~N} / \mathrm{A}$ \\
\hline 961100853 & $A P-105$ & 6 SAMPLE & $596 \mathrm{~V} 000047$ & 0 & 6 & $\mathrm{PH}-01$ & LIQUID & $N / A$ & 13.31 & 0.01 \\
\hline 96000853 & AP -105 & 7 DUP & $\$ 96 v 000047$ & 0 & & $\mathrm{PH}-01$ & LIQUID & 13.31 & $13: 35$ & $N / A$ \\
\hline
\end{tabular}

\section{Final page for worklist \# 12905}
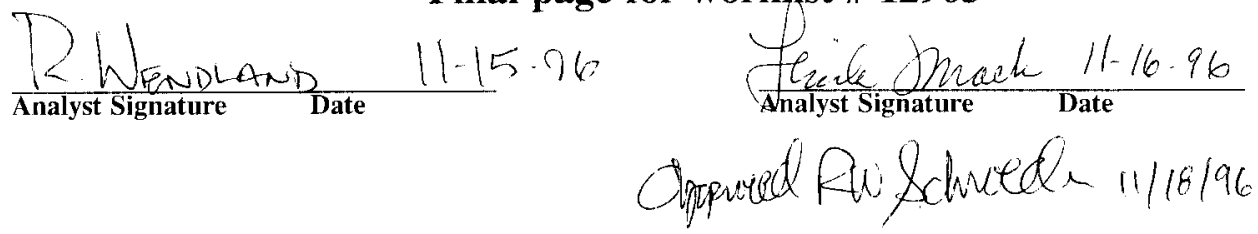

Data Entry Comments:

Units shown for $Q C$ (SPK \& STD) may not reflect the actual units. $D L=$ Detection Limit, $S=$ Worklist Slot Number, $R=$ Replicate Number, $A=$ Aliquot Code. 


\section{LABCORE Data Entry Template for Worklist\# 12904}

\section{Analyst: SeH Instrument: $\mathrm{PH} 01$ Book \# 79 N8}

Method: LA-211-102 Rev/Mod $\mathrm{CO}$

Worklist Comment: AP-105 OH. RCJ

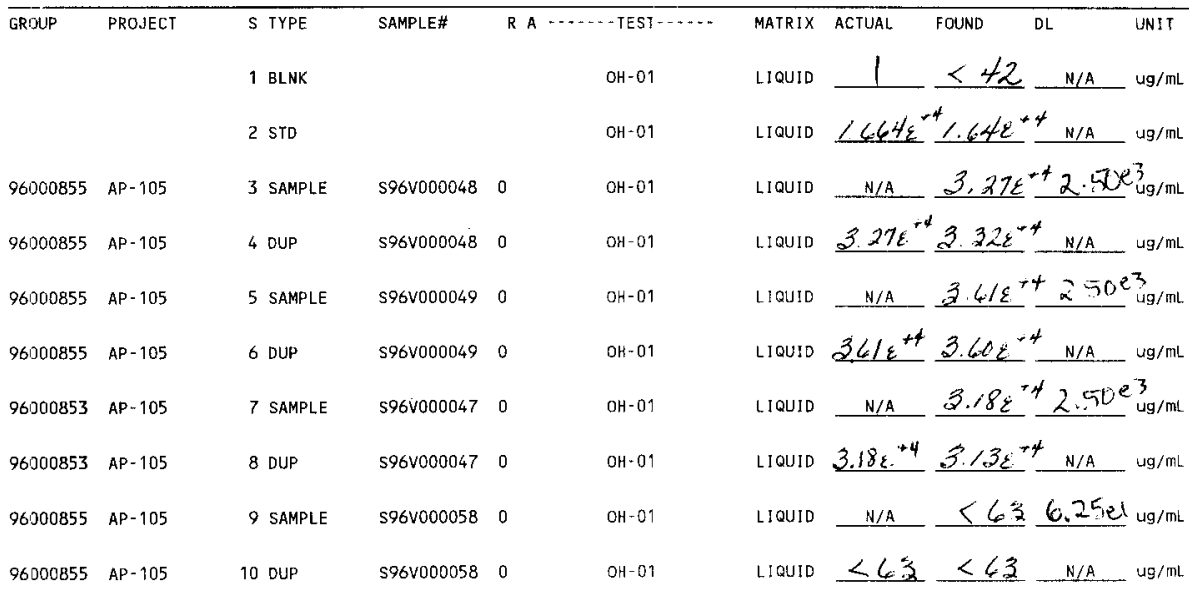

\section{Final page for worklist \# 12904}
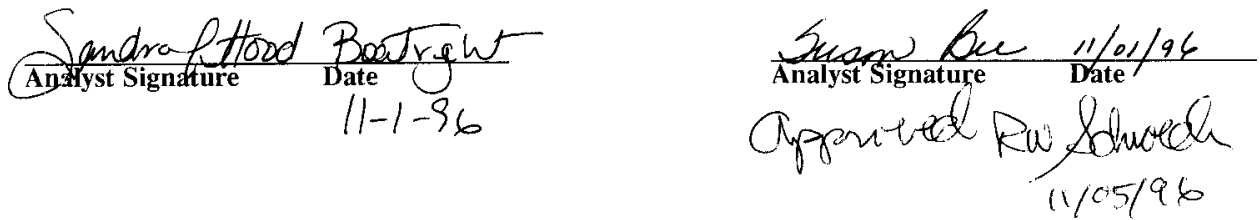

Data Entry Comments:

Units shown for $Q C$ (SPK \& STD) may not reflect the actual anits. $D L=$ Detection Limit, $S=$ Worklist Slot Number, $R=$ Replicate Number, $A=$ Aliquot Code. 


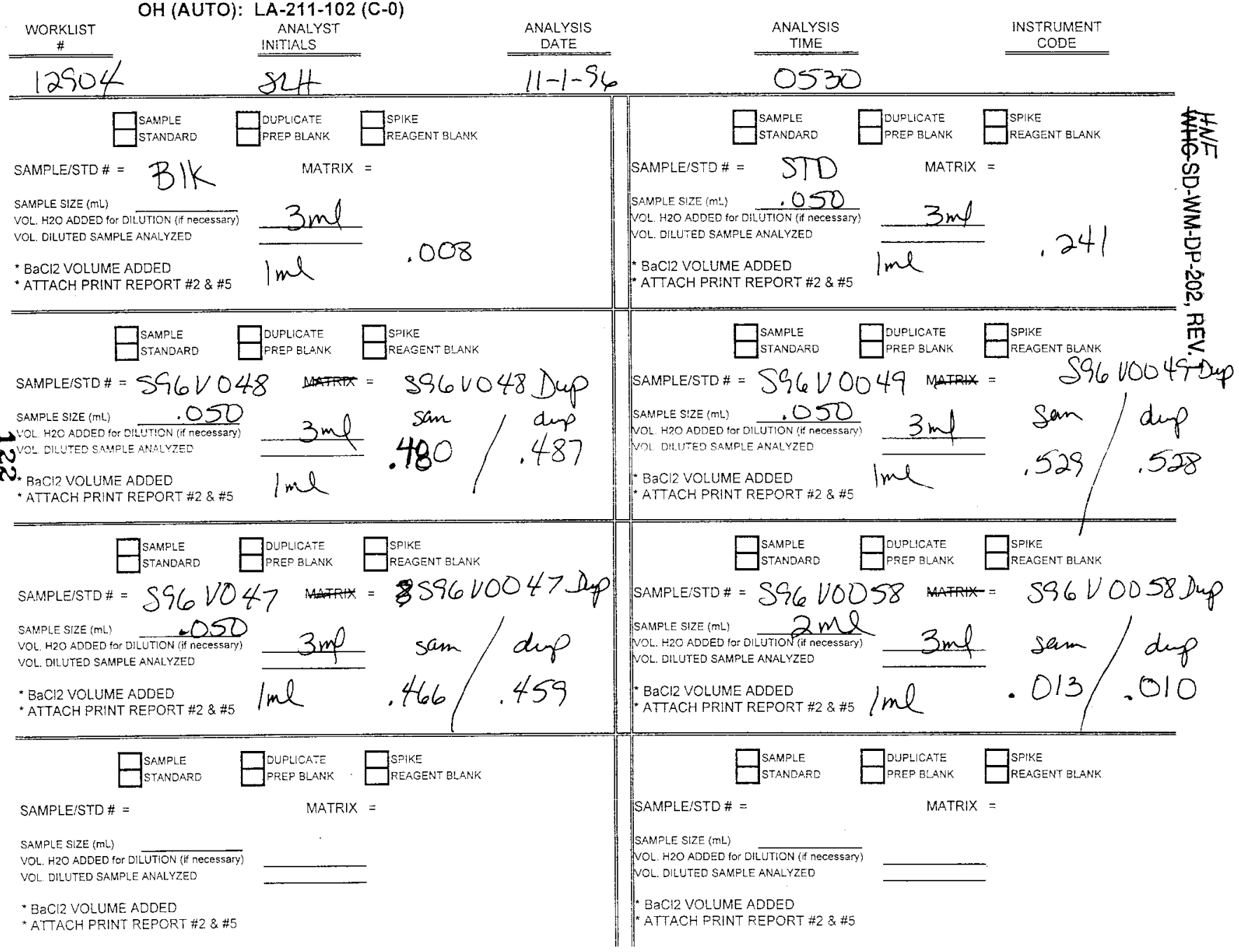




\begin{tabular}{|c|c|c|}
\hline \multicolumn{2}{|c|}{ OH (AUTO) : LA-211-102 (C-0) } & \multirow[t]{2}{*}{ BLANK } \\
\hline & Sample Size (mL) SS & \\
\hline BLANK & Concentration of $\mathrm{HNO} 3$ (Molarity) & 0.2005 \\
\hline & $\mathrm{HNO} 3$ Titrant at $\mathrm{OH}$ end-point in $\mathrm{mL}$ & 0.008 \\
\hline 12904 & Dilution Factor DF & 1 \\
\hline & Concentration of $\mathrm{OH}$ in Sample (Molarity) & 5.35E-04 \\
\hline $\mathrm{OH}-01$ & $\mathrm{OH}$ in Sample in $\mu \mathrm{g} / \mathrm{mL}$ (PPM) & $9.09 E+00$ \\
\hline LIQUID & & \\
\hline & Detection Limit $=125 \mu \mathrm{g} / \mathrm{SS}$ * DF & \\
\hline$-1 .$. & Detection Limit $(\mu \mathrm{g} / \mathrm{mL})$ & 4.17E+01 \\
\hline
\end{tabular}

\begin{tabular}{|c|c|c|}
\hline PHO1 & \multicolumn{2}{|c|}{$\mathrm{OH}$ Molarity $=\left((\mathrm{mL} \text { HNO3 })^{*}(\mathrm{M} \mathrm{HNO})\right) /$ Sample Size in $\mathrm{mL}^{*}$ Dilution Factor } \\
\hline & $\mu \mathrm{g} / \mathrm{mL}=(\mathrm{OH} \text { MOLARITY })^{*}(17.0 \mathrm{~g} / \mathrm{mole}$ & $/(1000 \mathrm{~mL} / \mathrm{L}))$ \\
\hline Thition & & BLANK \\
\hline 01:03 AM & Concentration of $\mathrm{OH}$ in Sample (Molarity) & 5.35E-04 \\
\hline & $\mathrm{OH}$ in Sample in $\mu \mathrm{g} / \mathrm{mL}$ (PPM) & $<42$ \\
\hline
\end{tabular}

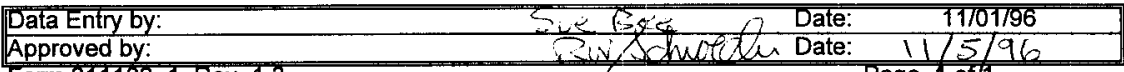
Form 211102_T Rev. 1.3 


\begin{tabular}{|c|c|c|}
\hline \multicolumn{2}{|c|}{ OH (AUTO) : LA-211-102 (C-0) } & STD \\
\hline & Sample Size (mL) SS & 0.050 \\
\hline \multirow{2}{*}{ STD } & Concentration of HNO3 (Molarity) & 0.2005 \\
\hline & HNO3 Titrant at $\mathrm{OH}$ end-point in $\mathrm{mL}$ & 0.241 \\
\hline \multirow{2}{*}{12904} & Dilution Factor DF & 1 \\
\hline & Concentration of $\mathrm{OH}$ in Sample (Molarity) & $9.66 \mathrm{E}-01$ \\
\hline $\mathrm{OH}-01$ & $\mathrm{OH}$ in Sample in $\mu \mathrm{g} / \mathrm{mL}$ (PPM) & $1.64 E+04$ \\
\hline \multicolumn{3}{|l|}{ LIQUID } \\
\hline \multirow{3}{*}{ STD } & Detection Limit $=125 \mu \mathrm{g} / \mathrm{SS}$ * DF & \\
\hline & & \\
\hline & Detection Limit $(\mu \mathrm{g} / \mathrm{mL})$ & $2.50 E+03$ \\
\hline \multirow[t]{2}{*}{ PH01 } & & \\
\hline & $\mathrm{OH}$ Molarity $=\left((\mathrm{mL} \mathrm{HNO} 3)^{*}(\mathrm{M} \mathrm{HNO})\right) / \mathrm{Sampl}$ & \\
\hline SLH & $\mathrm{OH}$ in $\mu \mathrm{g} / \mathrm{mL}=(\mathrm{OH} \text { MOLARITY })^{\star}(17.0 \mathrm{~g} / \mathrm{mole}$ & $1000 \mathrm{~mL} / L))$ \\
\hline \multirow[t]{2}{*}{$11 / 01 / 96$} & & \\
\hline & & STD \\
\hline \multirow[t]{2}{*}{ 01:03 AM } & Concentration of OH in Sample (Molarity) & $9.66 \mathrm{E}-01$ \\
\hline & $\mathrm{OH}$ in Sample in $\mu \mathrm{g} / \mathrm{mL}$ (PPM) & $1.64 E+04$ \\
\hline
\end{tabular}

Sue $\mathrm{BP}^{2}$ rensenatext 124
Date: $11701 / 96$

Date: $11 / 5 / 6$

Page tof' 


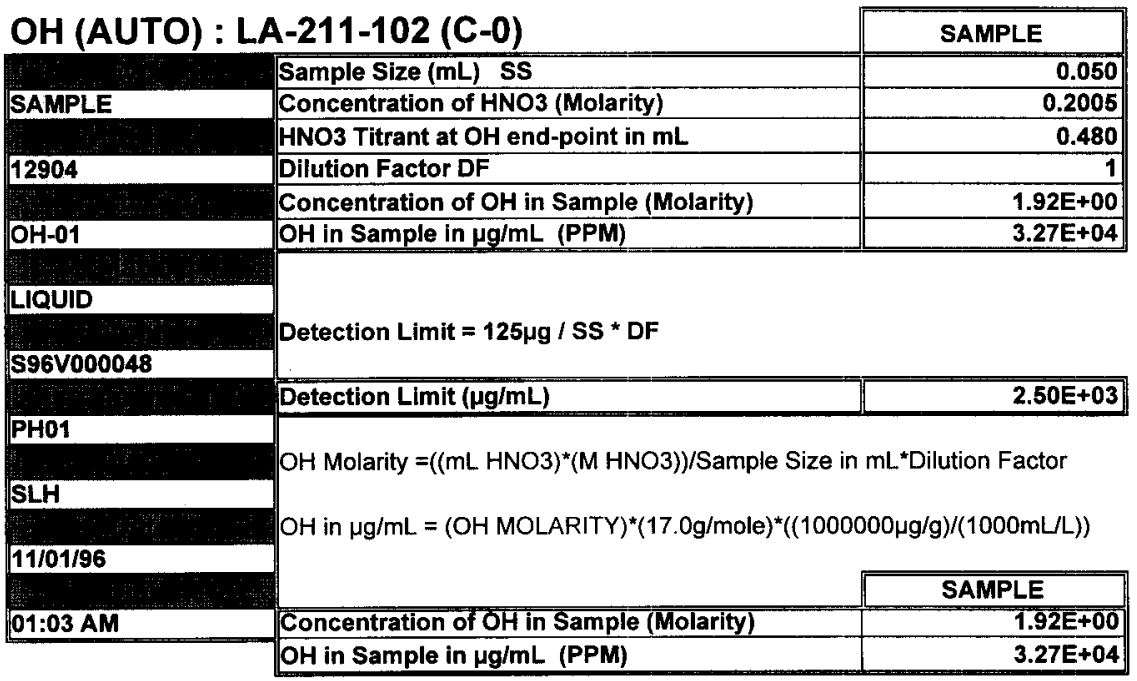

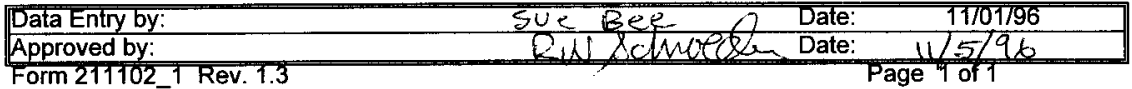




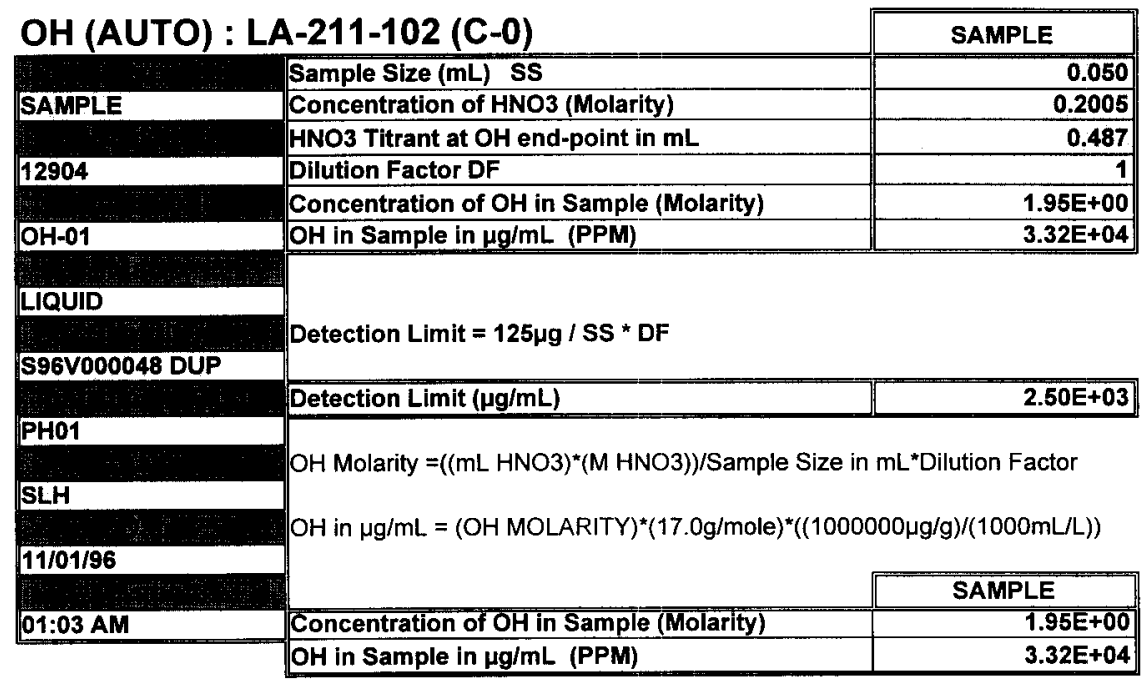

\begin{tabular}{|c|c|c|}
\hline Data Entry by: & Sue $6 e^{2}$ & $11 / 07 / 96$ \\
\hline Approved by: & Bu $x$ dovele & Date: $\quad 1105 / 96$ \\
\hline
\end{tabular}




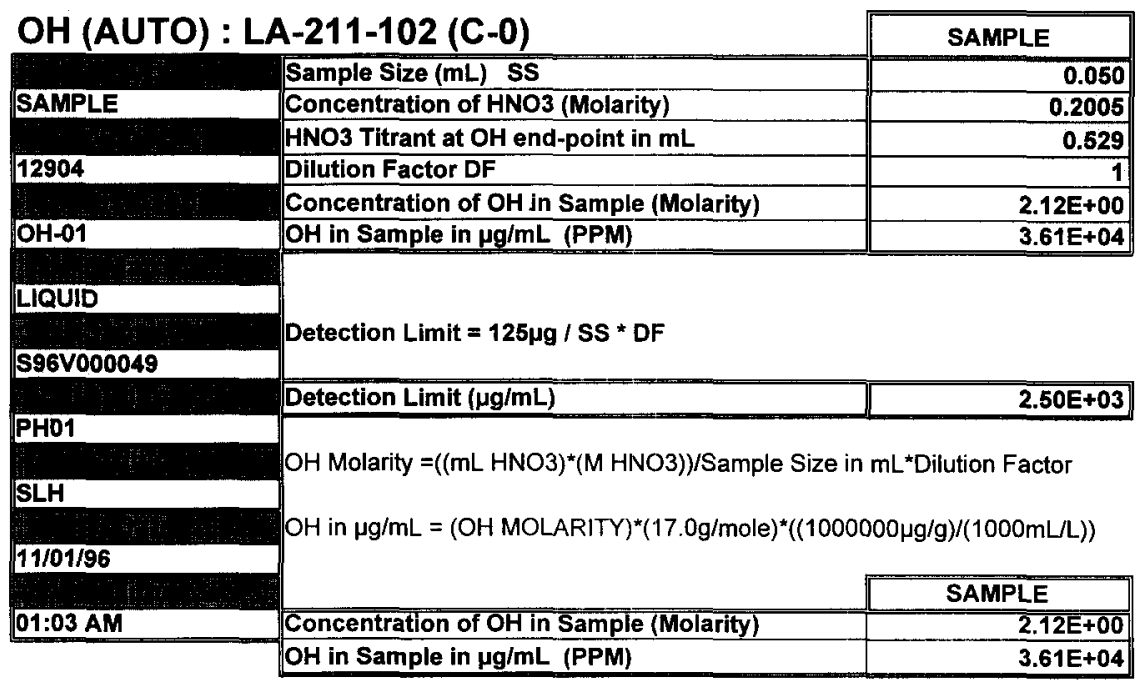




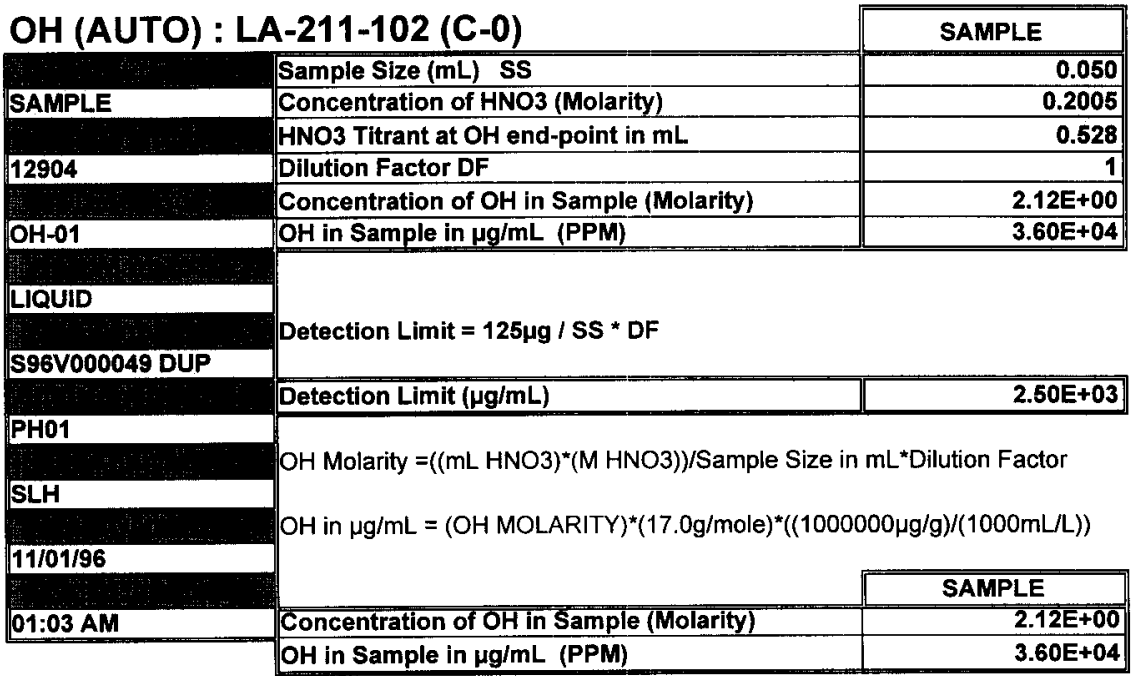

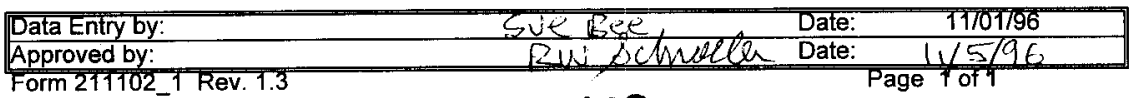




\begin{tabular}{|c|c|c|}
\hline \multicolumn{2}{|c|}{ OH (AUTO) : LA-211-102 (C-0) } & SAMPLE \\
\hline & Sample Size (mL) SS & 0.050 \\
\hline \multirow{2}{*}{ SAMPLE } & Concentration of HNO3 (Molarity) & 0.2005 \\
\hline & HNO3 Titrant at $\mathrm{OH}$ end-point in $\mathrm{mL}$ & 0.466 \\
\hline \multirow[t]{2}{*}{12904} & Dilution Factor DF & 1 \\
\hline & Concentration of OH in Sample (Molarity) & $1.87 \mathrm{E}+00$ \\
\hline $\mathrm{OH}-01$ & $\mathrm{OH}$ in Sample in $\mu \mathrm{g} / \mathrm{mL}$ (PPM) & $3.18 E+04$ \\
\hline \multirow{2}{*}{\multicolumn{3}{|c|}{ Detection Limit $=125 \mu \mathrm{g} / \mathrm{SS}$ * DF }} \\
\hline & Detection Limit $=125 \mu \mathrm{g} / \mathrm{SS}$ * DF & \\
\hline \multicolumn{3}{|c|}{ S96V000047 } \\
\hline & Detection Limit ( $\mu \mathrm{g} / \mathrm{mL}$ ) & $2.50 E+03$ \\
\hline \multirow[t]{2}{*}{ PH01 } & & \\
\hline & $\mathrm{OH}$ Molarity $=\left((\mathrm{mL} \mathrm{HNO})^{*}(\mathrm{M} H \mathrm{HNO})\right) / \mathrm{Sampl}$ & ilution Factor \\
\hline SLH & $\mathrm{OH}$ in $\mu \mathrm{g} / \mathrm{mL}=(\mathrm{OH} \text { MOLARITY })^{*}(17.0 \mathrm{~g} / \mathrm{mole}$ & 1)/(1000mL/L)) \\
\hline $01 / 96$ & & SAMPLE \\
\hline \multirow[t]{2}{*}{ 01:03 AM } & Concentration of $\mathrm{OH}$ in Sample (Molarity) & $1.87 \mathrm{E}+00$ \\
\hline & $\mathrm{OH}$ in Sample in $\mu \mathrm{g} / \mathrm{mL}$ (PPM) & $3.18 E+04$ \\
\hline
\end{tabular}

\begin{tabular}{|c|c|c|}
\hline Data Entry by: & Gye Bct & $17 / 01 / 96$ \\
\hline Approved by: & $\mathrm{RU}$ Xriwolloate: & $11 / 5 / 96$ \\
\hline
\end{tabular}




\begin{tabular}{|c|c|c|}
\hline OH (A & $-211-102(C-0)$ & SAMPLE \\
\hline & Sample Size (mL) SS & 0.050 \\
\hline SAMPLE & Concentration of HNO3 (Molarity) & 0.2005 \\
\hline & HNO3 Titrant at $\mathrm{OH}$ end-point in $\mathrm{mL}$ & 0.459 \\
\hline 12904 & Dilution Factor DF & \\
\hline & Concentration of $\mathrm{OH}$ in Sample (Molarity) & $1.84 \mathrm{E}+00$ \\
\hline $\mathrm{OH}-01$ & $\mathrm{OH}$ in Sample in $\mu \mathrm{g} / \mathrm{mL}$ (PPM) & $3.13 \mathrm{E}+04$ \\
\hline QUID & & \\
\hline & Detection Limit $=125 \mu \mathrm{g} / \mathrm{SS}$ * DF & \\
\hline 596 & & $250 \mathrm{~F}+03$ \\
\hline PH01 & & \\
\hline & $\mathrm{OH}$ Molarity $=\left((\mathrm{mL} \mathrm{HNO})^{*}(\mathrm{M} \mathrm{HNO})\right) / \mathrm{Samp}$ & ilution Factor \\
\hline & & \\
\hline & $\mathrm{OH}$ in $\mu \mathrm{g} / \mathrm{mL}=(\mathrm{OH} \text { MOLARITY })^{*}(17.0 \mathrm{~g} / \mathrm{mole}$ & (g)/(1000mL/L)) \\
\hline & & SAMPLE \\
\hline $01: 03 \mathrm{AM}$ & Concentration of OH in Sample (Molarity) & $1.84 E+00$ \\
\hline & $\mathrm{OH}$ in Sample in $\mu \mathrm{g} / \mathrm{mL}$ (PPM) & $3.13 E+04$ \\
\hline
\end{tabular}

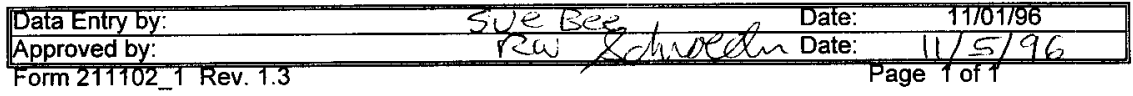




\begin{tabular}{|c|c|c|}
\hline \multicolumn{2}{|c|}{ OH (AUTO) : LA-211-102 (C-0) } & SAMPLE \\
\hline & Sample Size $(\mathrm{mL})$ ss & 2.000 \\
\hline \multirow{2}{*}{ SAMPLE } & Concentration of $\mathrm{HNO} 3$ (Molarity) & 0.2005 \\
\hline & HNO3 Titrant at $\mathrm{OH}$ end-point in $\mathrm{mL}$ & 0.013 \\
\hline \multirow[t]{2}{*}{12904} & Dilution Factor DF & 1 \\
\hline & Concentration of OH in Sample (Molarity) & $1.30 \mathrm{E}-03$ \\
\hline $\mathrm{OH}-01$ & $\mathrm{OH}$ in Sample in $\mu \mathrm{g} / \mathrm{mL}$ (PPM) & $2.22 E+01$ \\
\hline \multirow{2}{*}{ LIQUID } & & \\
\hline & Detection Limit $=125 \mu \mathrm{g} / \mathrm{SS}$ * DF & \\
\hline S96V000 & Detection Limit $(\mu \mathrm{g} / \mathrm{mL})$ & $6.25 \mathrm{E}+01$ \\
\hline
\end{tabular}

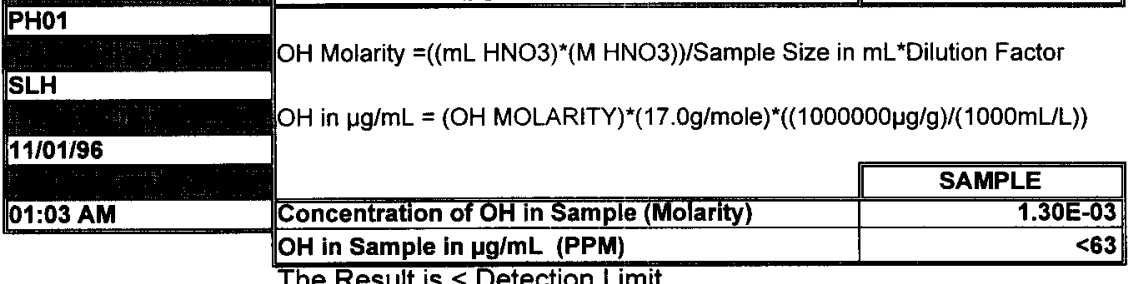

The Result is < Detection Limit Data Entry by:
Approved by: Form 211102_1 Rev. 1.3

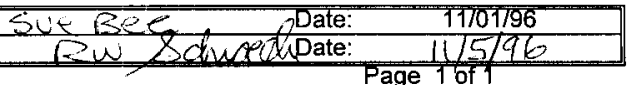

131 


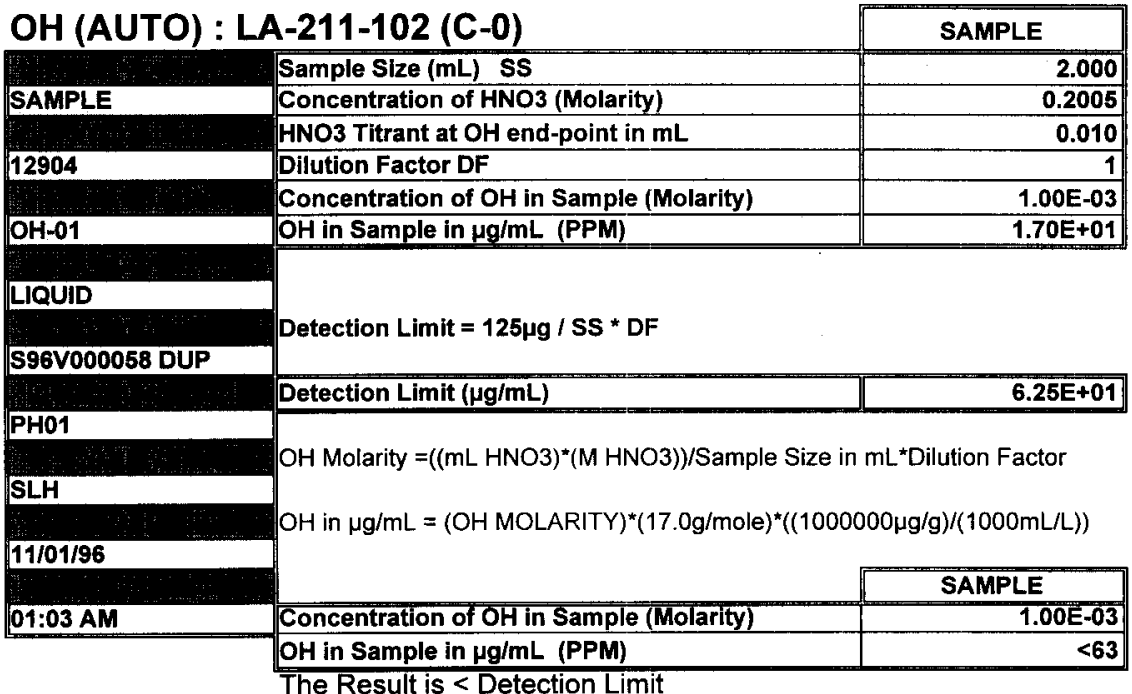

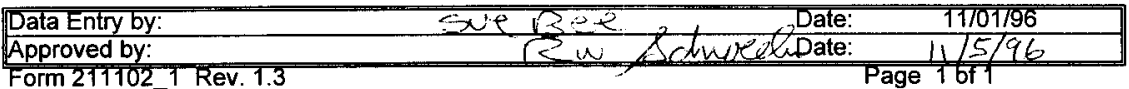




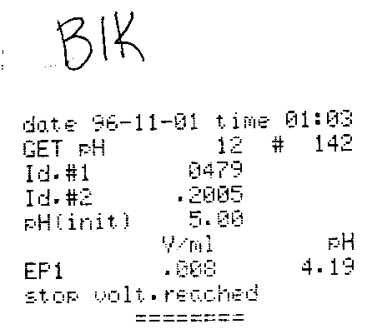

dote 5r-11-51 the $01: 09$

GET $\mathrm{SH}$ 12 \# $14 \mathrm{E}$

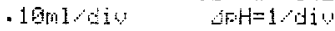

start i abot mil

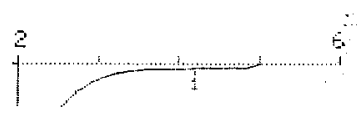

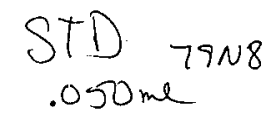

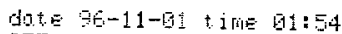

GET FH 12 \# 145

I‥ \# 1

Id. $\# 2$

949

FHlinit in

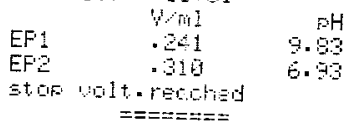

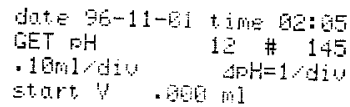

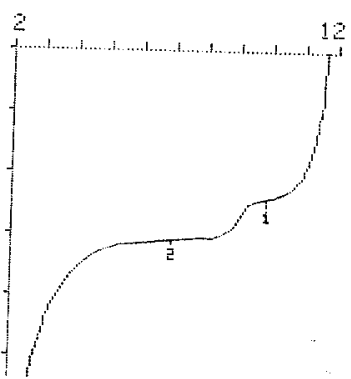




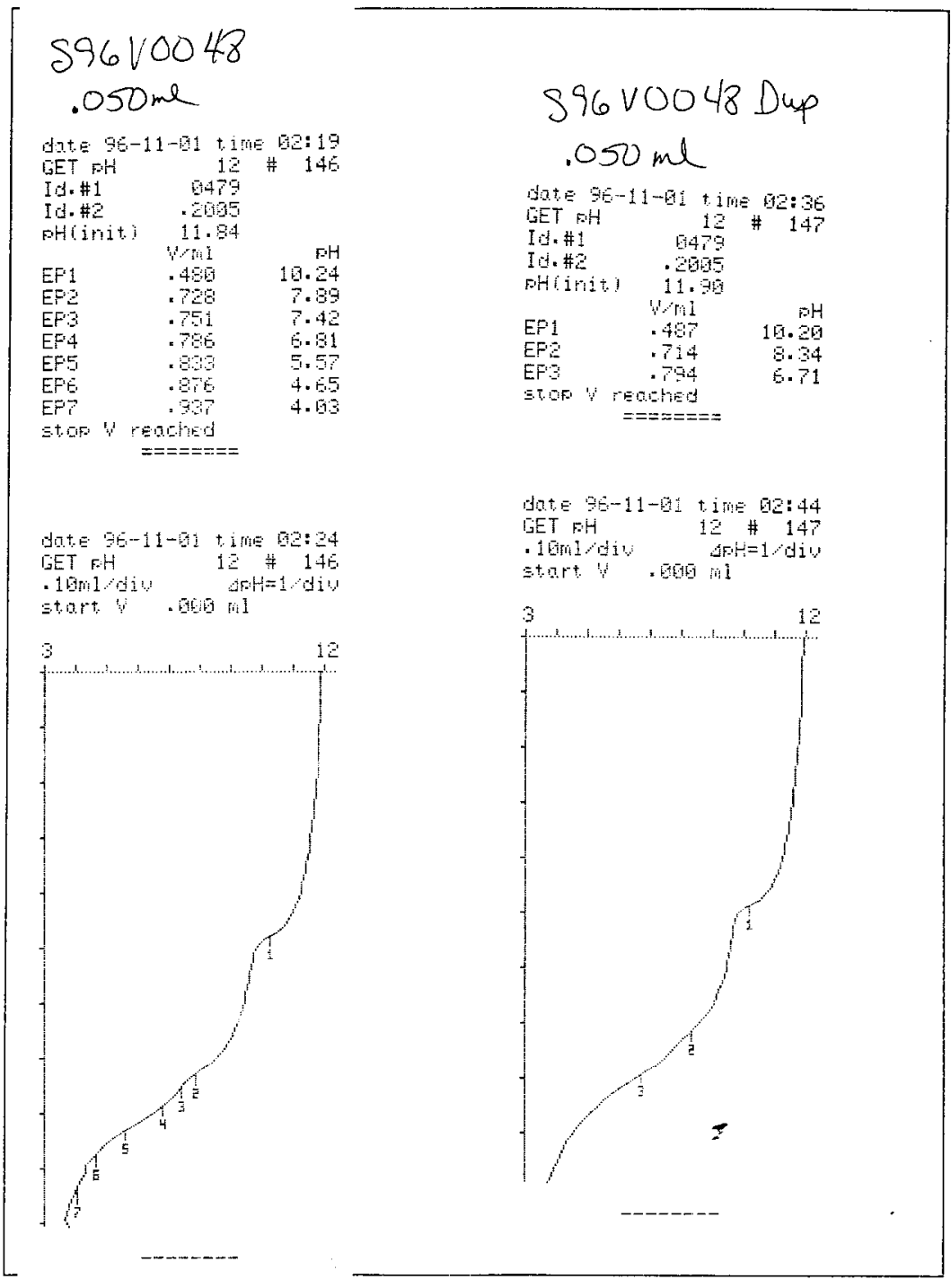


896.0049

Gote $96-11-61$ time $92: 50$

LET $\mathrm{FH}$ $1 \mathrm{Z}$ \# $14 \mathrm{~S}$

I 10 .\#1 1479

Id. $\# 2$. 2005

Hiniti 11.90

Whi $\mathrm{FH}$

EF1 $\quad .529 \quad 10.22$

$\mathrm{EF} \quad 775 \quad 8.47$

$\mathrm{EF}$

. 3

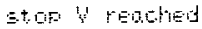

5. 47 $.050 \mathrm{ml}$

596 V0049 Dup

$.050 \mathrm{mh}$

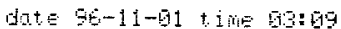

GET FH

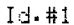

I d . \#

11.6

EF'

EF'

$E F Y$

Stop y renched

41
.525

- $7 \mathrm{~g}$

- Bet

$======$

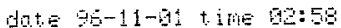

GET 12 \# 148

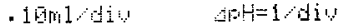

Etart $y$ aga m

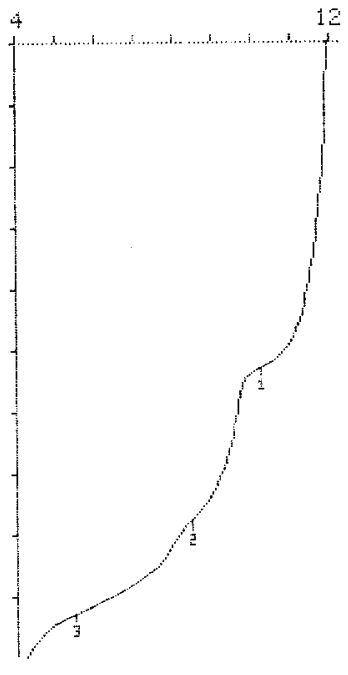

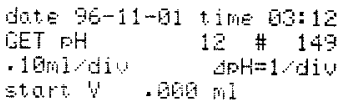

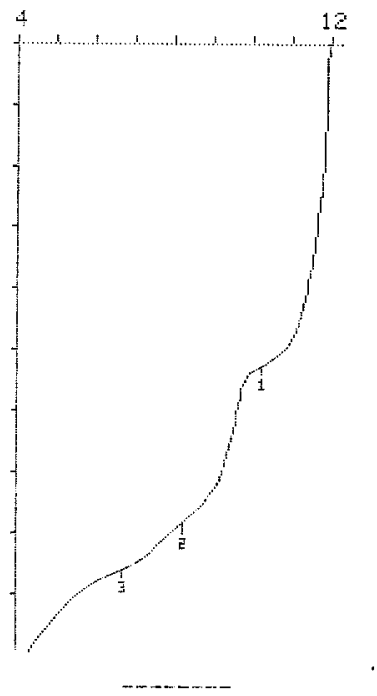




\section{HNFE-SD-WM-DP-202, REV. 1}
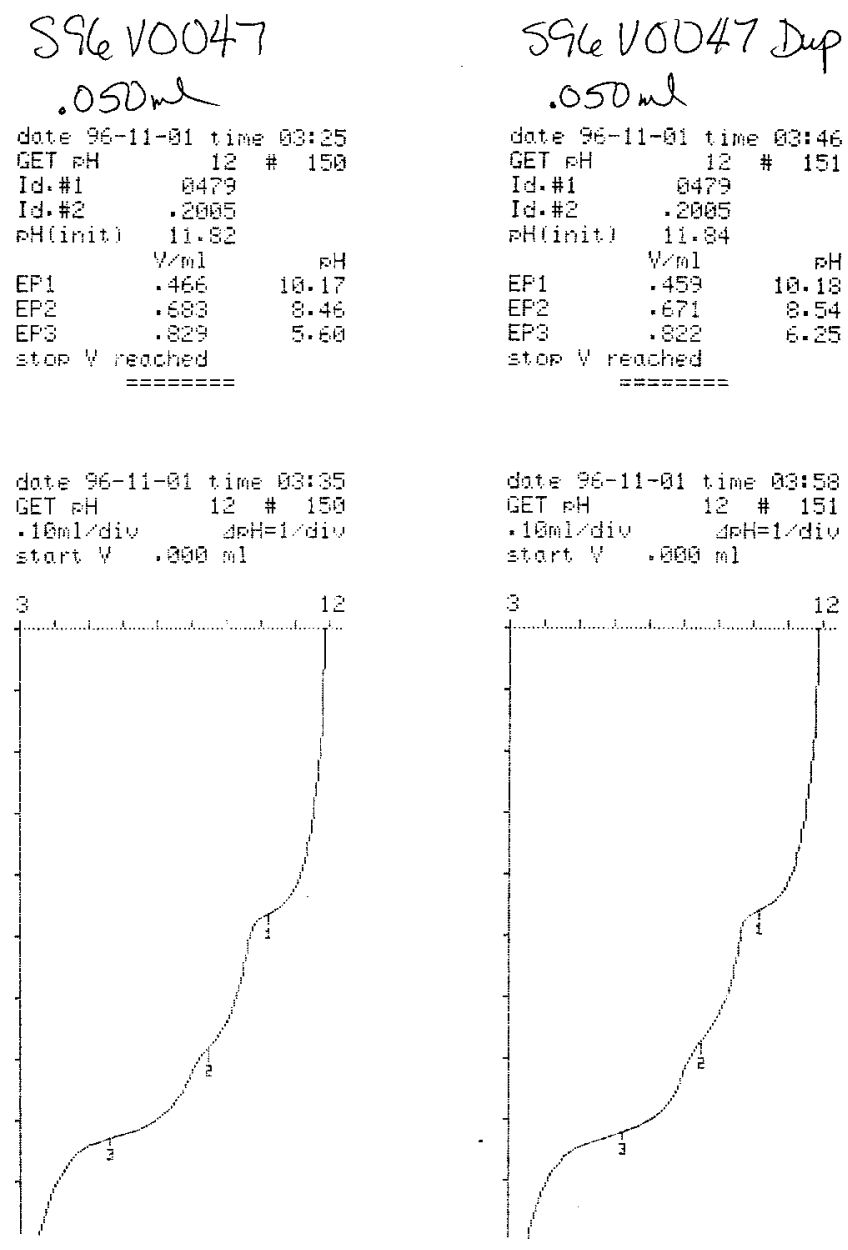


\section{8} $2 m e$

date 9t-11-61 time $9+29$

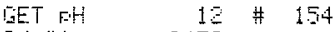

I]. \#1

I-日. He

FHtinit

2 两阳

5.95

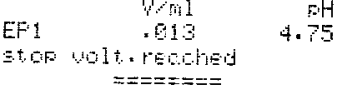

dnte 95-11-61 time 54t90

GET EH

12 * 154

- Ibmisiv

stort at an

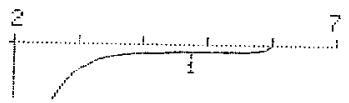

59610058

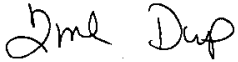

digte 9e-11-61 time 04:36

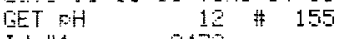

Id. \#1 1479

$\mathrm{IH}=\# 2 \quad .20 \mathrm{E}$

FHint 5.97

$\begin{array}{lll}\text { EP1 } & .919 & 5 \mathrm{H}\end{array}$

stop wit a tooched

$== \pm \#==$

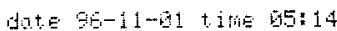

GET FH 12 मी 155

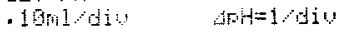

start $y$. mis

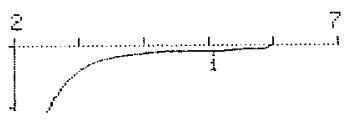




\section{LABCORE Data Entry Template for Worklist\# 12907}

\section{Analyst: RAW Instrument: NH301}

Method: LA-631-001 Rev/Mod B-2

Worklist Comment: AP-105 AMMONIA. RCJ

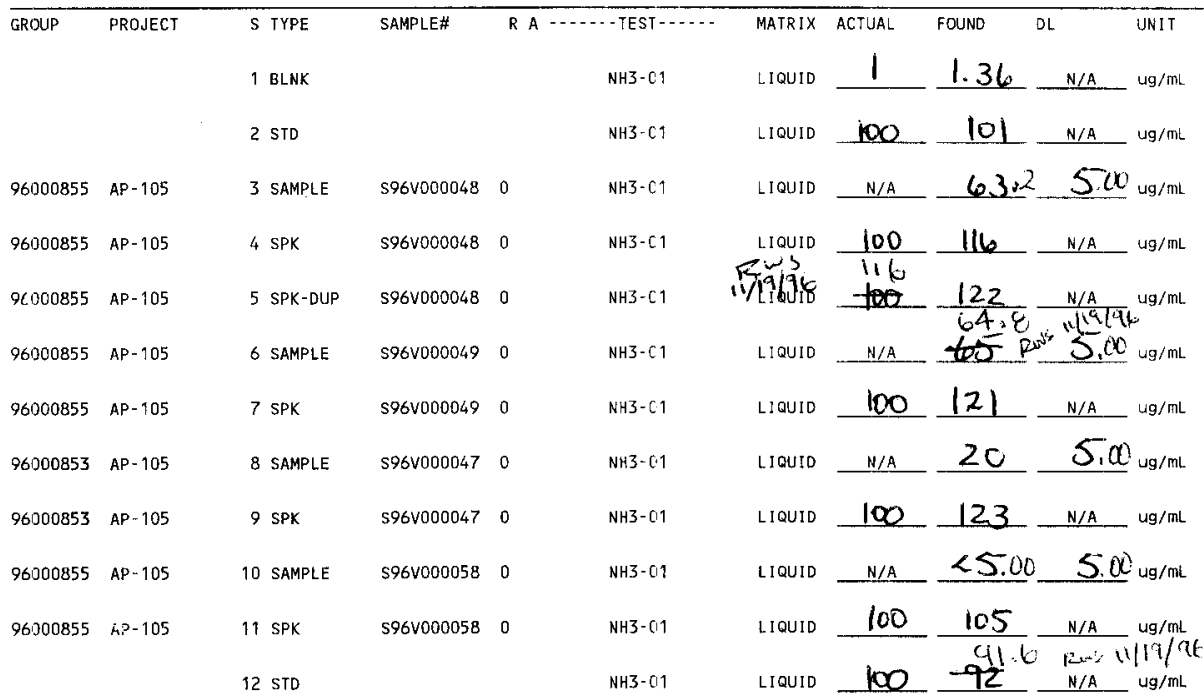

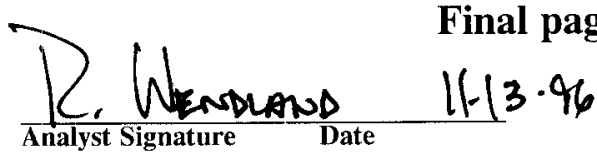

Final page for worklist \# 12907

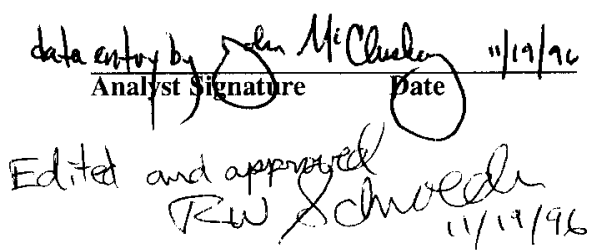

Duta Entry Comments:

Units shown for $Q C$ (SPK \& STD) may not reflect the actual units. $D L=$ Detection Limit, $S=$ Worklist Slot Number, $R=$ Replicate Number, $A=$ Aliquot Code. 
$H N F$

HHESD-WMi-DP-202, REV. 1

34 
落
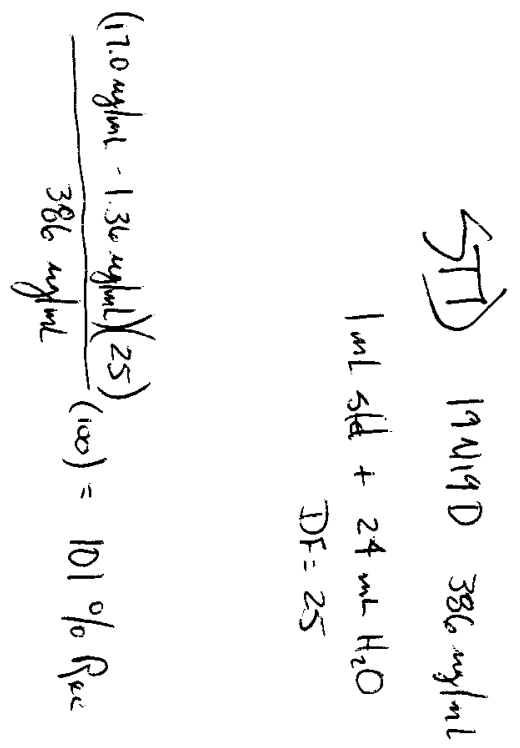

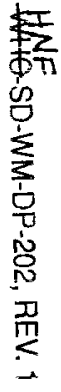


WE-SD-WM-DP-202, REV. 1

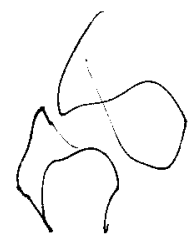

$$
(1.34-1.36)(25)<5 . \mathrm{mg} / \mathrm{mL}
$$

141 
HF

-SD-WM-CP-202, REV. 1

if 4 क

19.N19D $386 \mathrm{mg} / \mathrm{mL}$

$$
\begin{aligned}
& \text { ImL sample }+0.100 \mathrm{~mL} 19 / 19 \mathrm{D}+23.9 \mathrm{~mL} \mathrm{H}_{2} \mathrm{O} \\
& D F=25 \\
& \sqrt{5 u m p l i}^{\text {sus } 5} \\
& \frac{(25)(17.6 \mathrm{mg} / \mathrm{mL}-1.36 \mathrm{mg} / \mathrm{mL})-(0)(25)}{386 \mathrm{mg} / \mathrm{mL}}(100)=105 \% R_{\mathrm{kC}}
\end{aligned}
$$

142 
WHE-SD-WM-DP-¿OD, REV. 1

HNF

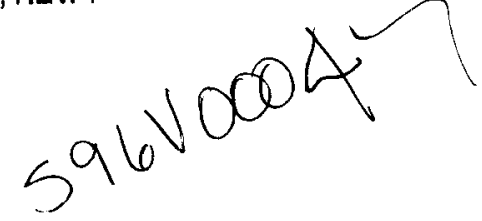

$$
\begin{gathered}
\mathrm{ImL}+24 m L \mathrm{H}_{2} \mathrm{O} \\
D F=25
\end{gathered}
$$

$(2.16 \mathrm{mg} / \mathrm{mL}-1.36 \mathrm{mg} / \mathrm{mL})(25)=20.0 \mathrm{mg} / \mathrm{mL}$ 
$\angle 96.907$ spike

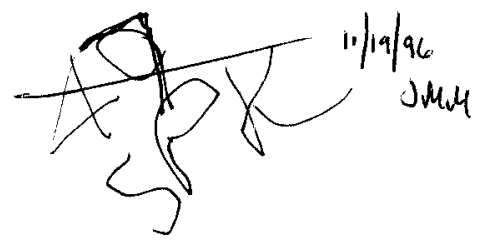

$19.19 D=386 \mathrm{mg} / \mathrm{mL}$

$1 \mathrm{~mL} \mathrm{sample}+0.100 \mathrm{~mL} 19 \mathrm{N19D}+23.9 \mathrm{~mL} \mathrm{H} \mathrm{O}$ $D F=25$

$$
\frac{(25)(21.1 \mathrm{mg} / \mathrm{mL}-1.36 \mathrm{ug} / \mathrm{mL})-(2.16 \mathrm{ug} / \mathrm{mL}-1.36 \mathrm{ug} / \mathrm{mL})(25)}{386 \mathrm{mg} / \mathrm{mL}}(100)=123 \% \mathrm{f}
$$


HNF

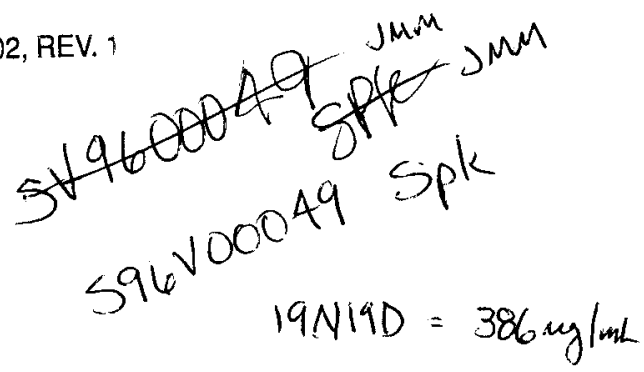

$$
\begin{gathered}
\text { ImL sample }+0.100 \mathrm{~mL} 19 / 19 D+23.9 \mathrm{~mL} \mathrm{H}_{2} \mathrm{O} \\
D F=25
\end{gathered}
$$

$$
\frac{(25)(22.6 \mathrm{mg} / \mathrm{mic}-1.36 \mathrm{mg} / \mathrm{mL})-(3.95 \mathrm{mg} / \mathrm{mL}-1.36 \mathrm{gg} / \mathrm{mL})(25)}{386 \mathrm{mg} / \mathrm{mL}}(100)=
$$$$
=121 \% \text { spike recovery }
$$ 
WNF-SD-WM-DP-202, REV. 1

salo

$$
\begin{gathered}
\text { lush smple }+\underbrace{24 m L H_{2} \mathrm{O}}_{\text {JMM }} \\
D F=25
\end{gathered}
$$

$(3.89 \mathrm{mg} / \mathrm{mL}-1.36 \mathrm{mg} / \mathrm{mL})(25)=63.2 \mathrm{mg} / \mathrm{mL}$

147 
WFE-SD-WM-DP-202, REV. 1

$$
\begin{aligned}
& \text { DP-202, REV. } 1 \\
& 596 \mathrm{NOO} \\
& 19 \mathrm{N19D}=386 \mathrm{ug} / \mathrm{mL} \\
& 1 \mathrm{~mL} \text { sample }+0.100 \mathrm{~mL} 19 \mathrm{~N} / 9 \mathrm{D}+23.9 \mathrm{~mL} \mathrm{H} \mathrm{C} \\
& D F=25
\end{aligned}
$$

$$
\begin{gathered}
\frac{(25)(21.8 \mathrm{mg} / \mathrm{mL}-1.36 \mathrm{~m} / \mathrm{mL})-(3.87 \mathrm{mg} / \mathrm{mL}-1.36 \mathrm{mg} / \mathrm{ml})(25)}{386 \mathrm{mg} / \mathrm{mL}} \\
=116 \% \text { spike vacevery }
\end{gathered}
$$

148 
WNE-SD-WM-DP-202, REV.

48

DaP SRR 19 N19D $=386 \mathrm{ug} / \mathrm{h}$

$$
\begin{aligned}
1 \mathrm{~mL} \text { sample } & +0.100 \mathrm{~mL} 19 N 190+23.9 \mathrm{mh} \\
D F & =25
\end{aligned}
$$

$$
R P D=\left|\frac{122-116}{\frac{122+116}{2}}\right|(100)=5 \% \text { RPD }
$$

$$
\frac{(25)(22.8 \mathrm{mg} / \mathrm{mL}-1.36 \mathrm{mg} / \mathrm{mL})-(3.89 \mathrm{mg} / \mathrm{mL}-1.36 \mathrm{mg} / \mathrm{mL})(25)}{386 \mathrm{mg} / \mathrm{mL}}(100)=122 \% \text { spike vacovecy }
$$

149 


\section{LABCORE Completed Worklist Report for Worklist\# 12912}

Analyst: vIm

Instrument: IC01

Book\# 32N20A

Method: $\quad \angle A-533-105 \operatorname{Rev} / \operatorname{Mod} D-1$

Worklist Comment: AP-105 IC. RCJ

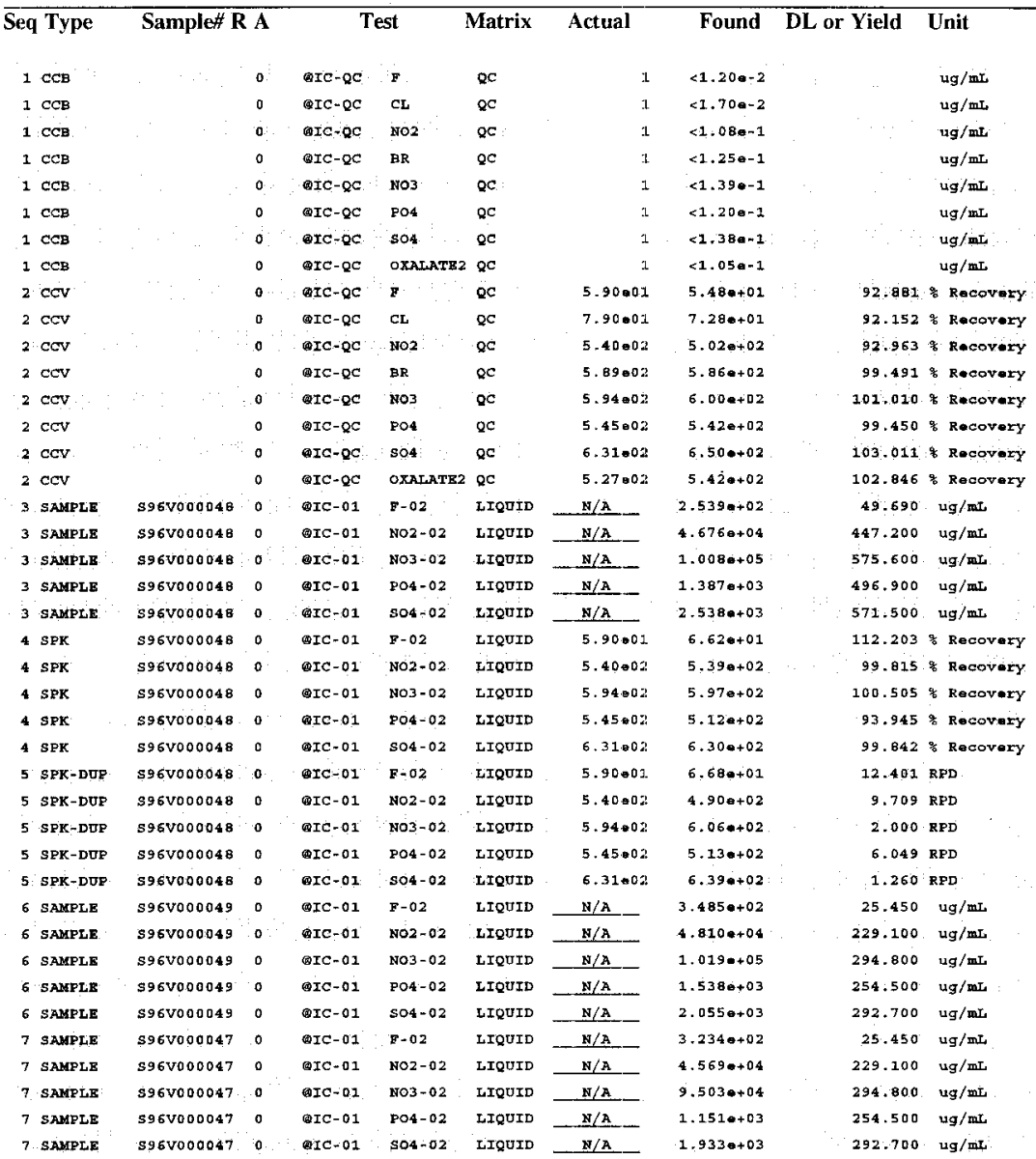




\section{LABCORE Completed Worklist Report for Worklist\# 12912}

\begin{tabular}{|c|c|c|c|c|c|c|c|c|c|}
\hline Seq Type & Sample\# R & & & st & Matrix & Actual & Found & DL or Yield & Unit \\
\hline B SAMPLE & S96V00005B & 0 & DIC-01 & F-02 & LIQUID & & 7.200 .02 & $7.20 \bullet-002$ & $\mathrm{ug} / \mathrm{mL}$ \\
\hline B SAMPLE & $596200005 B$ & 0 & aIC-01 & พ02-02 & LIQUID & $N / \mathbb{A}=$ & $6.4800-01$ & 0.648 & $\mathrm{ug} / \mathrm{mL}$ \\
\hline B SAMPLE & s96V00005B & 0 & $\operatorname{arc-01}$ & No3-02 & LIQUID & $\underline{w} / \mathrm{A}$ & $8.6 .30 \cdot-01$ & 0.834 & $\mathrm{ug} / \mathrm{mI}$ \\
\hline B SAMPLE & $s 96 \mathrm{~V} 000058$ & 0 & CoIC-01 & PO4-02 & LIQUID & $N / A$ & $7.2000-01$ & 0.720 & $\mathrm{ug} / \mathrm{mL}$ \\
\hline B SAMPLE & $\$ 960000058$ & 0 & OIC-01 & $504-02$ & IIQUID & $\mathrm{w} / \mathrm{A}$. & $1.4 .69 \mathrm{e}, 01$ & 0.828 & $\mathrm{ug} / \mathrm{m \tau}$ \\
\hline
\end{tabular}

Final page for worklist\# 12912

Analyst Signature Date

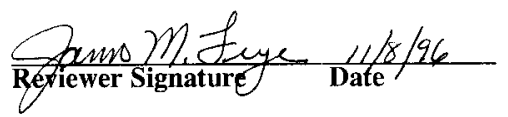


NEE-SD-WM-DP-202, REV. 1

$11 / 01 / 9609: 14$

Page: 1

A-0004 1

LABCORE Data Entry Template for Worklist\# 12912

Analyst: $\quad V(M$ Instrument: ICOO $\mp$ COI Book\# $32 N 20-A$

Method: LA-533-105 Rev/Mod D-1

Worklist Comment: AP-105 IC. RCJ

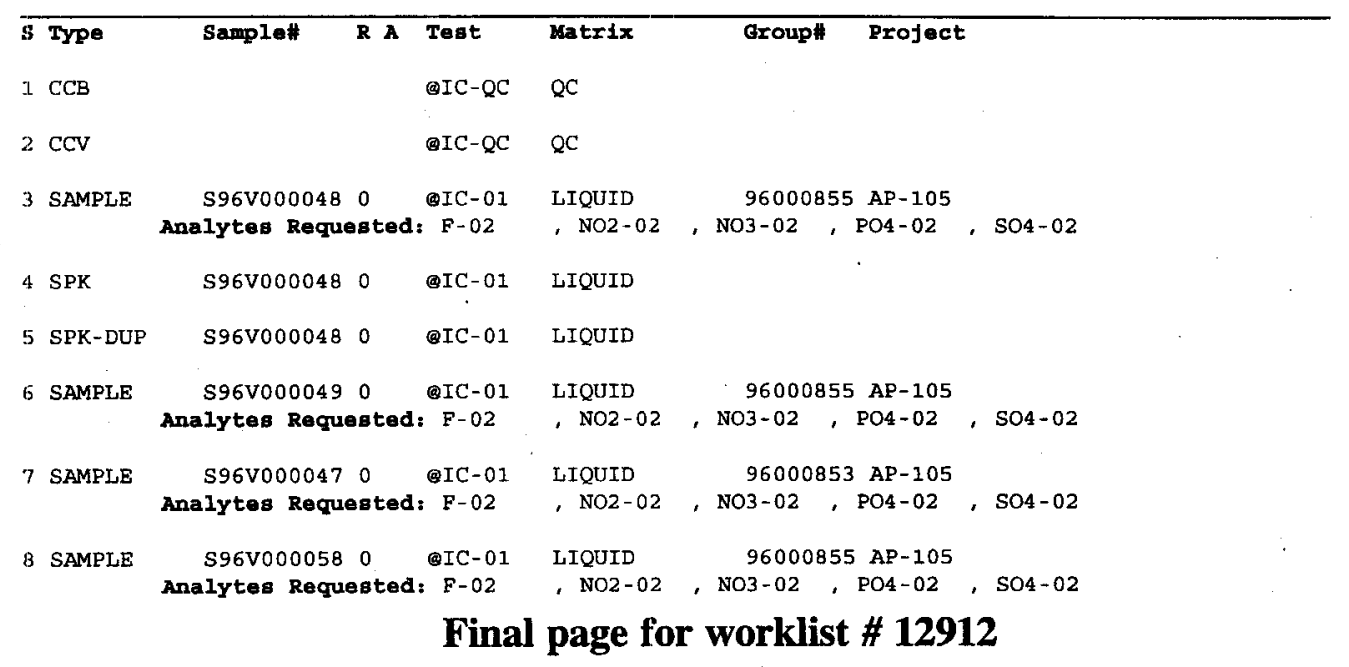

$\underset{\text { Analyst Signature }}{\|_{\text {Date }}}$

Analyst Signature Date

12912 NV. CST

Install

Uploaded IIt/lacemitige

Validated 1/8/96 smsuge

Data Entry Comments:

Soke dup calculation in to tone dreoust work, fix it in validation mene.

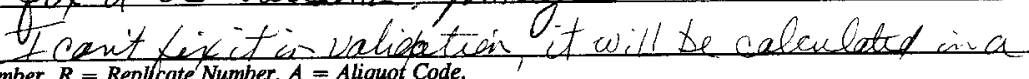
$S=$ Worklist Slot Number, $R=$ Replicate Number, $A=$ Aliquot Code.

separate query. 9 ins 
HNE-SD-WMM-DF-202, REV. 1

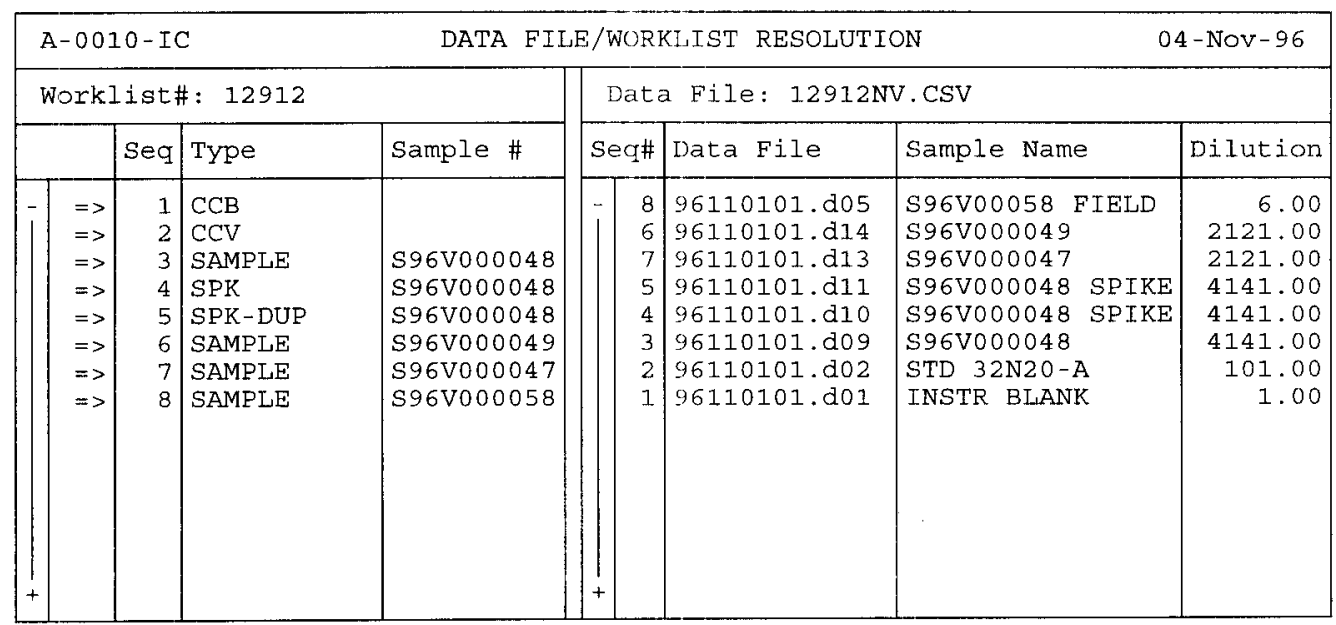

Save(F4) Abort(Shift-F3) ListFiles(Shift-F1) UploadFile(F8) 
Data Reprocessed on 11/04/1996 09:59:28

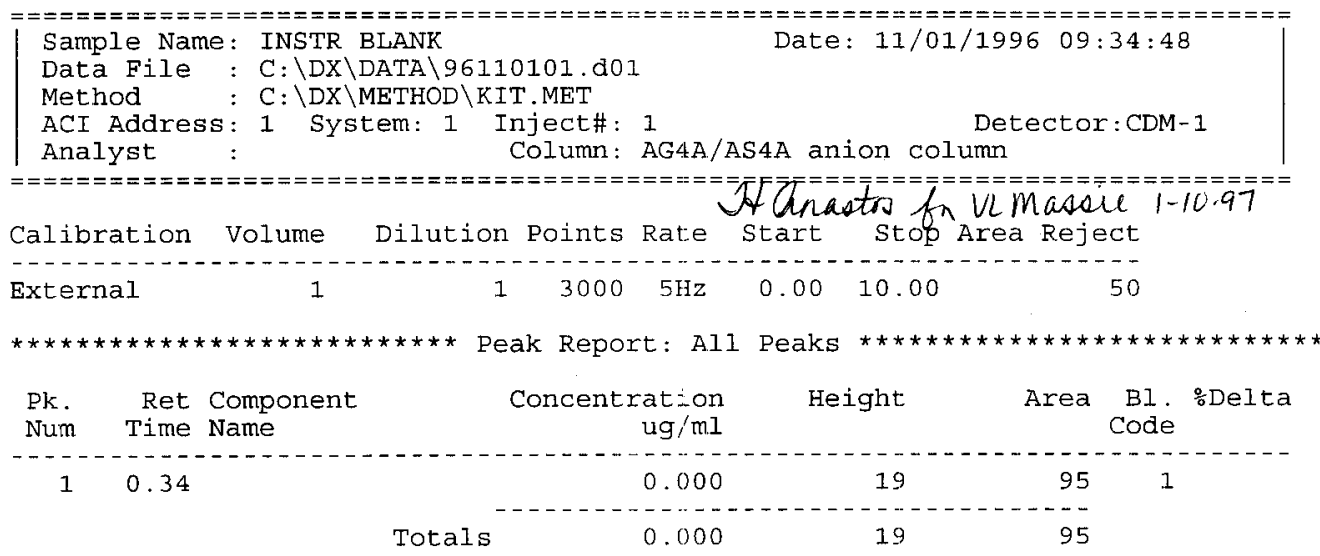

File: 96110101.d01 Sample: INSTR BLANK

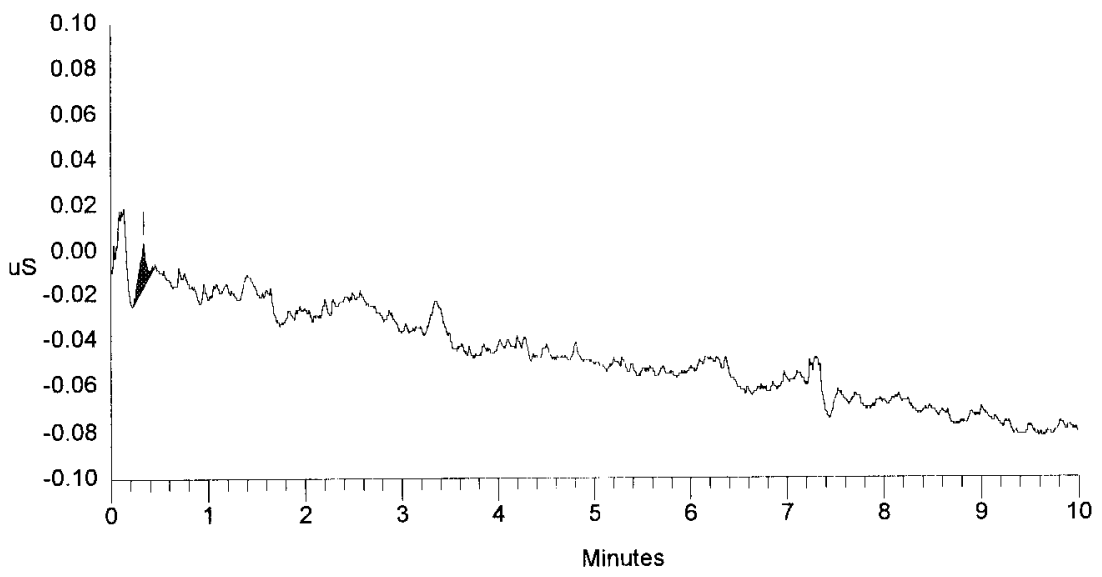

SIGNATURE ABOVE REPRESENTS CHEMICAL TECHNOLOGHGT/CHEMIST THAT COMPLETED/VERIFIED THE CALIBRATION/ANALYSIS ON PAGES 153 TO/62. 
Data Reprocessed on 11/04/1996 09:59:26

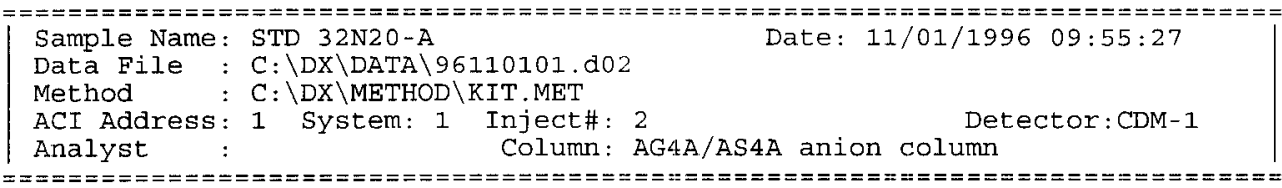

Calibration Volume Dilution Points Rate start stop Area Reject

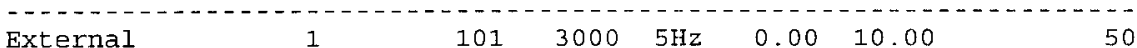

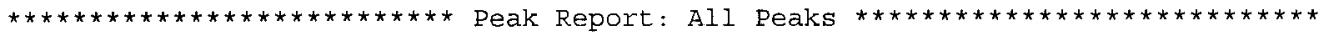

Pk. Ret Component Concentration Height Area Bl. Delta

Num Time Name

$\mathrm{ug} / \mathrm{ml}$

Area Bl. $\begin{aligned} & \text { BDelta } \\ & \text { Code }\end{aligned}$

$\begin{array}{lll}1 & 0.90 & \\ 2 & 1.06 & \text { fluoride } \\ 3 & 1.61 & \text { chloride } \\ 4 & 1.91 & \text { nitrite } \\ 5 & 2.89 & \text { bromide } \\ 6 & 3.28 & \text { nitrate } \\ 7 & 4.59 & \text { phosphate } \\ 8 & 6.03 & \text { sulfate } \\ 9 & 7.95 & \text { oxalate }\end{array}$

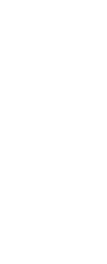

0.000

43

54.793

72.772

1756

501.926

1783

586.398

7285

$600 .: 86$

5148

$542 \cdot-46$

5602

650.421

1560

542.370

4714

2283

Totals

3551.012

30173

$\begin{array}{rrr}157 & 2 & \\ 0790 & 2 & 3.92 \\ 9637 & 1 & -0.41 \\ 6282 & 1 & -3.37 \\ 6024 & 1 & -3.13 \\ 6029 & 1 & -3.81 \\ 1697 & 1 & -6.96 \\ 6531 & 1 & -8.13 \\ 2946 & 1 & -3.21\end{array}$

File: 96110101.d02 Sample: STD 32N20-A

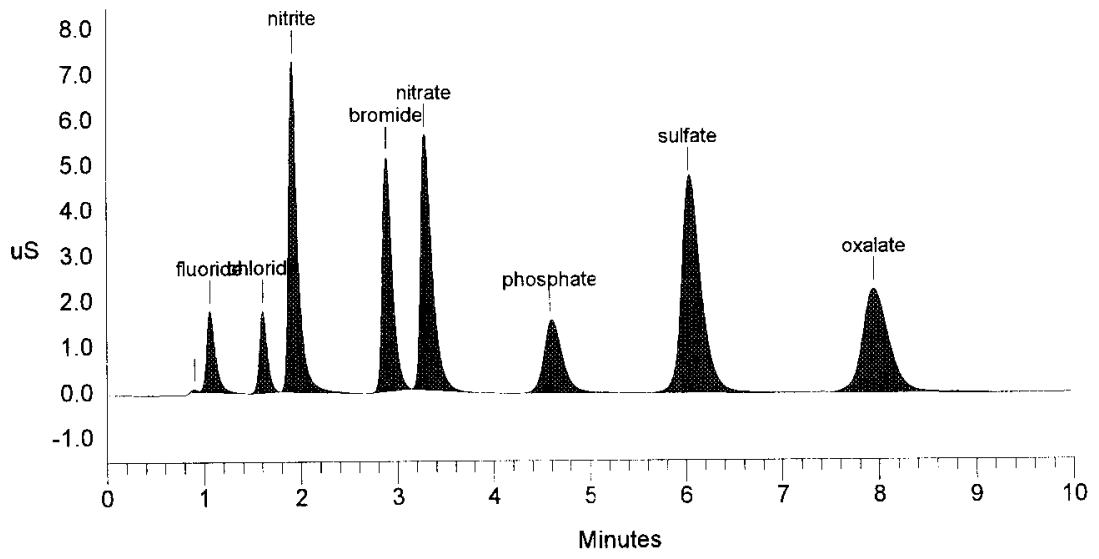


Data Reprocessed On 11/04/1996 09:59:24

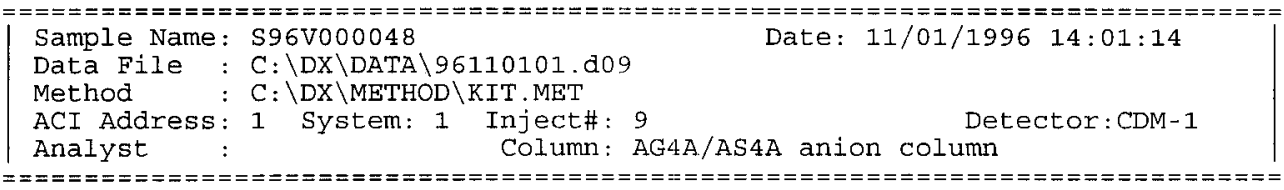

Calibration Volume Dilution Points Rate start stop Area Reject

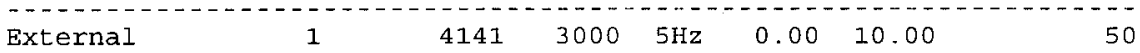

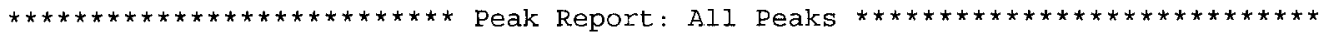

Pk. Ret Component Concentration Height Area Bl. \%Delta

Num Time Name ug/m.l Code

$\begin{array}{lll}1 & 0.89 & \\ 2 & 1.07 & \text { fluoride } \\ 3 & 1.59 & \text { chloride } \\ 4 & 1.90 & \text { nitrite } \\ 5 & 3.20 & \text { nitrate } \\ 6 & 4.61 & \text { phosphate } \\ 7 & 6.08 & \text { sulfate } \\ 8 & 8.00 & \text { oxalate }\end{array}$

$\begin{array}{rrrr}0.000 & 180 & 921 \\ 253.948 & 251 & 1024 \\ 2777.059 & 1533 & 8964 \\ 46760.209 & 16500 & 107657 \\ 100788.942 & 21879 & 202551 \\ & 1386.514 & 92 & 1342 \\ 2537.591 & 374 & 5807 \\ & 477.048 & 41 & 702 \\ \text { Totals } & 154981.311 & 40851 & 328968\end{array}$

File: 96110101.d09 Sample: S96V000048

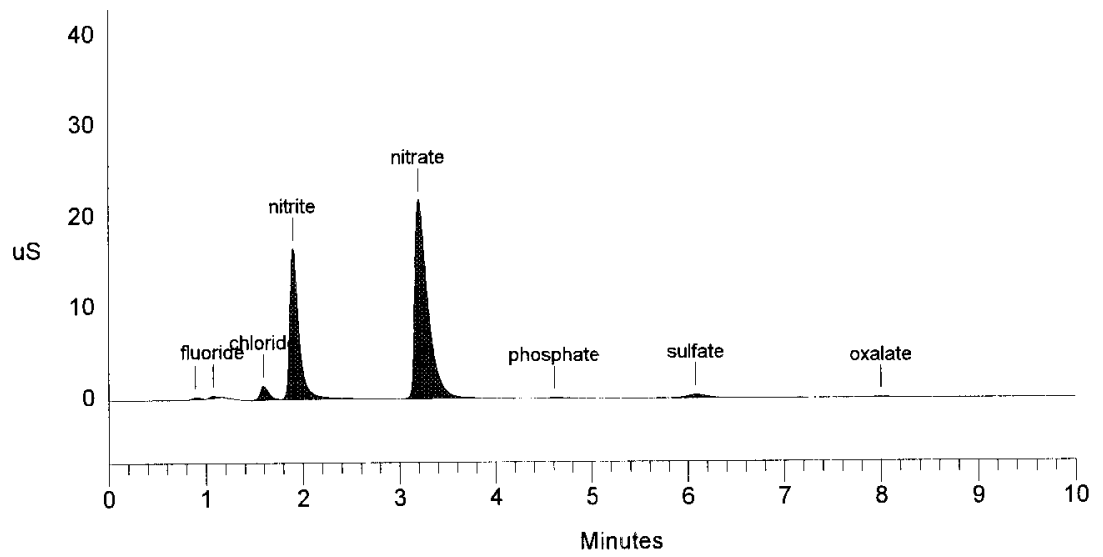


Data Reprocessed On 11/04/1996 09:59:22

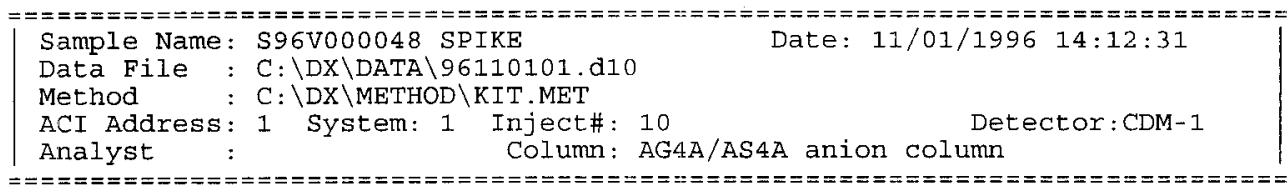

Calibration Volume Dilution Points Rate Start Stop Area Reject

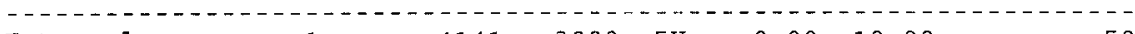

External

1

$\begin{array}{lllll}4141 & 3000 \quad 5 \mathrm{~Hz} \quad 0.00 \quad 10.00\end{array}$

50

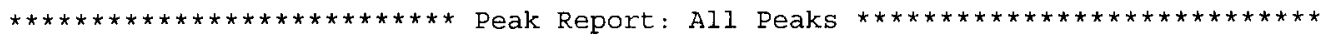

Pk. Ret Component Concentration Height Area Bl. $\frac{\text { Delta }}{}$

Num Time Name

$\mathrm{ug} / \mathrm{ml}$ Code

$\begin{array}{lll}1 & 0.90 & \\ 2 & 1.06 & \text { Eluoride } \\ 3 & 1.59 & \text { chloride } \\ 4 & 1.91 & \text { nitrite } \\ 5 & 2.87 & \text { bromide } \\ 6 & 3.19 & \text { nitrate } \\ 7 & 4.59 & \text { phosphate } \\ 8 & 6.03 & \text { sulfate } \\ 9 & 7.89 \text { oxalate }\end{array}$

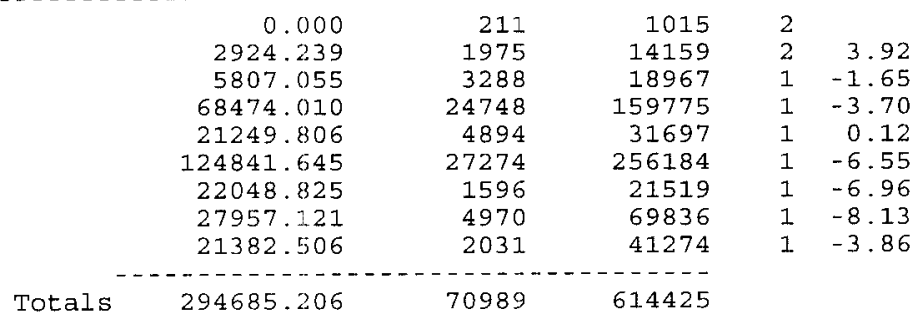

File: 96110101.d10 Sample: S96V000048 SPIKE

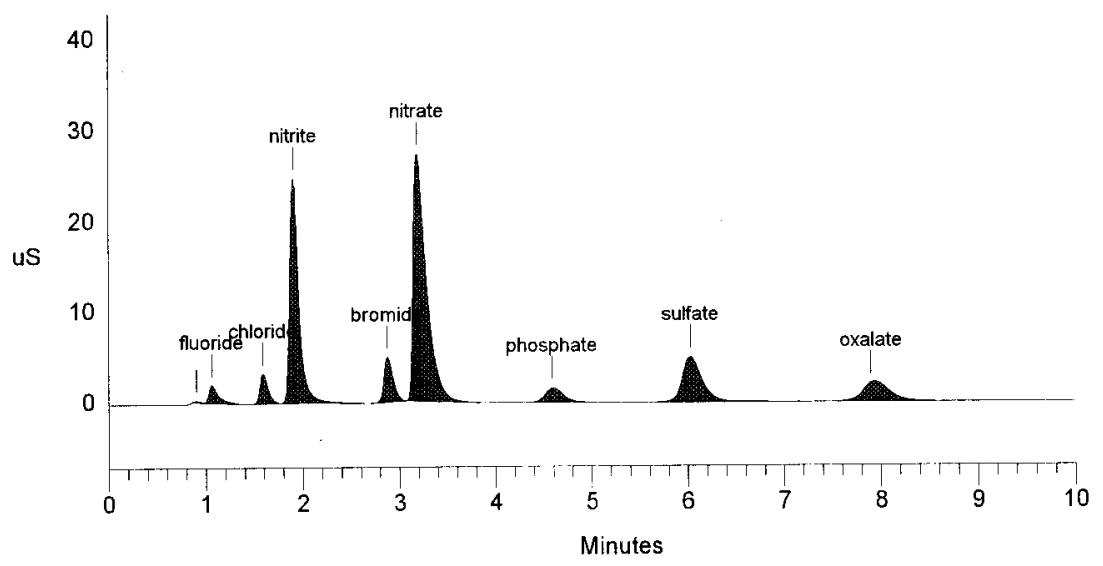


Data Reprocessed on 11/04/1996 09:59:20

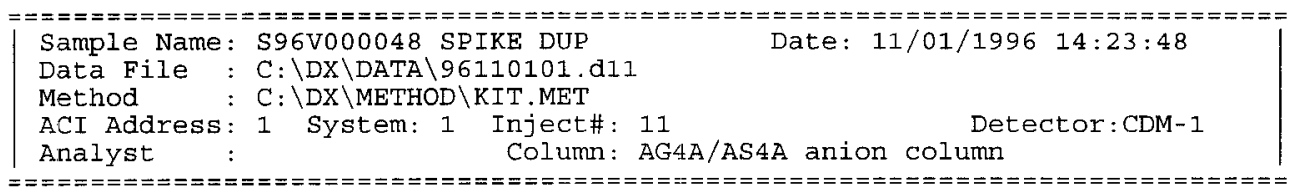

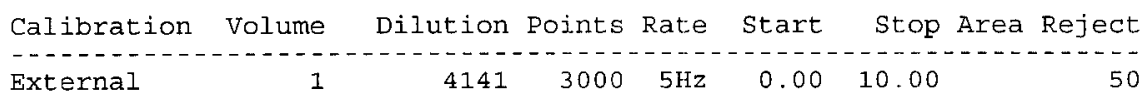

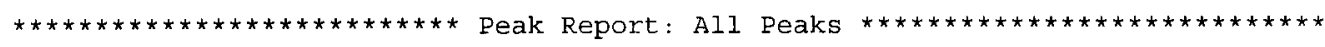

Pk. Ret Component $\quad \begin{gathered}\text { Concentration } \\ \text { ug/ml }\end{gathered}$ Height
Nume Name

$\begin{array}{rrrrr}0.000 & 222 & 1184 & 2 & \\ 2947.604 & 1998 & 14276 & 2 & 3.92 \\ 5686.671 & 3309 & 18564 & 1 & -1.65 \\ 66536.394 & 24692 & 155077 & 1 & -3.70 \\ 21309.040 & 4931 & 31789 & 1 & 0.12 \\ 125224.843 & 27187 & 257058 & 1 & -6.55 \\ 22062.747 & 1605 & 21533 & 1 & -6.96 \\ 28312.386 & 4973 & 70747 & 1 & -8.13 \\ 21170.129 & 2052 & 40858 & 1 & -3.86\end{array}$

Totals $293249.814 \quad 70971 \quad 611085$

File: 96110101.d11 Sample: S96V000048 SPIKE DUP

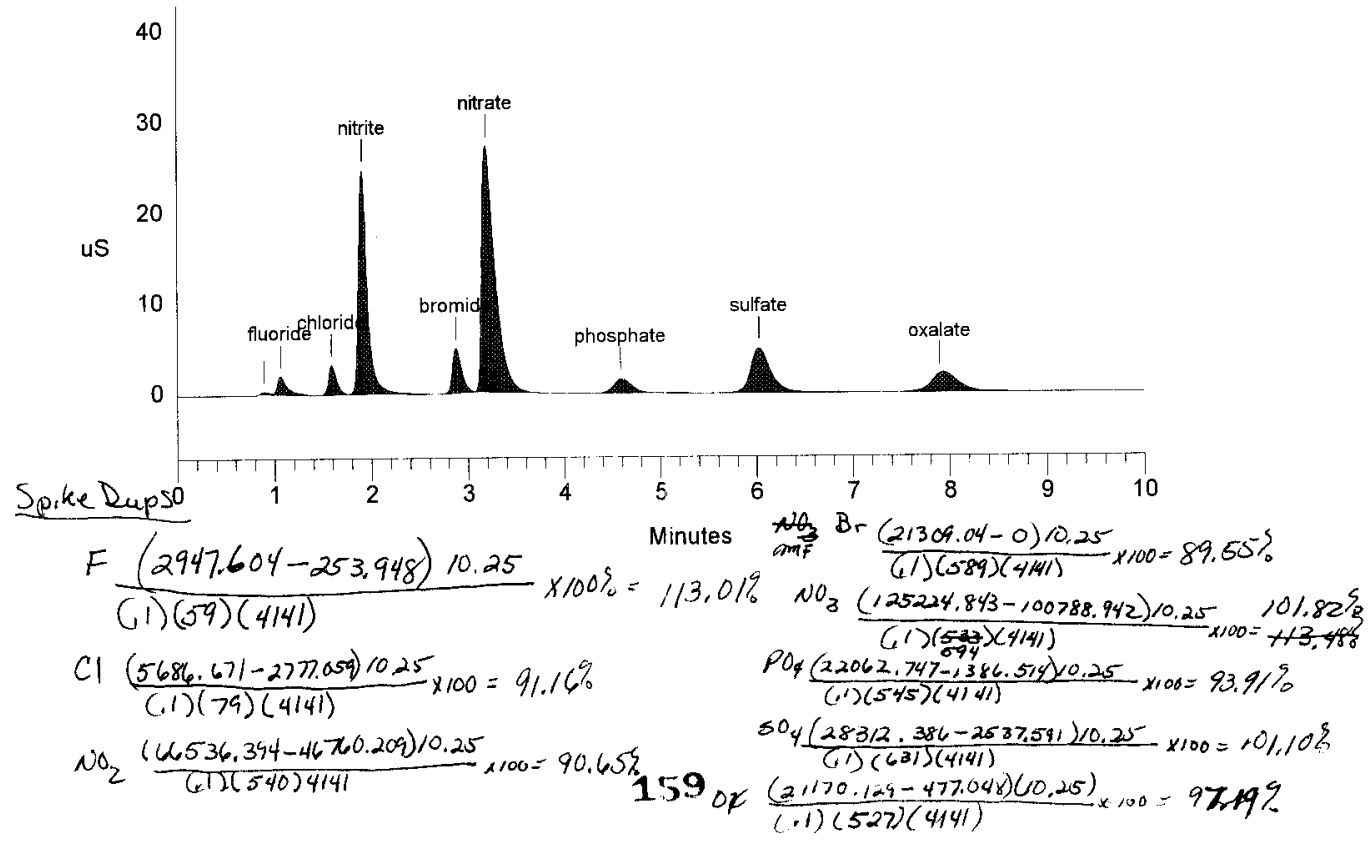


Data Reprocessed On 11/04/1996 09:59:18
Sample Name: S96V000047
Data File : C: $\backslash \mathrm{DX} \backslash \mathrm{DATA} \backslash 96110101 . \mathrm{d} 13$
Method
: $C: \backslash D X \backslash M E T H O D \backslash K I T . M E T$
ACI Address: 1 System: 1 Inject\#: 13
Analyst
Column: AG4A/AS4A anion column
Date: $11 / 01 / 199614: 49: 31$

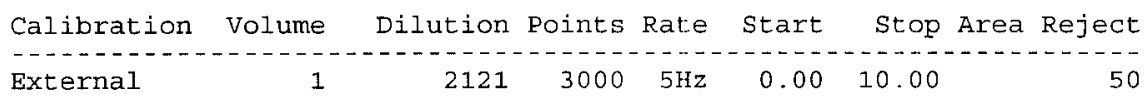

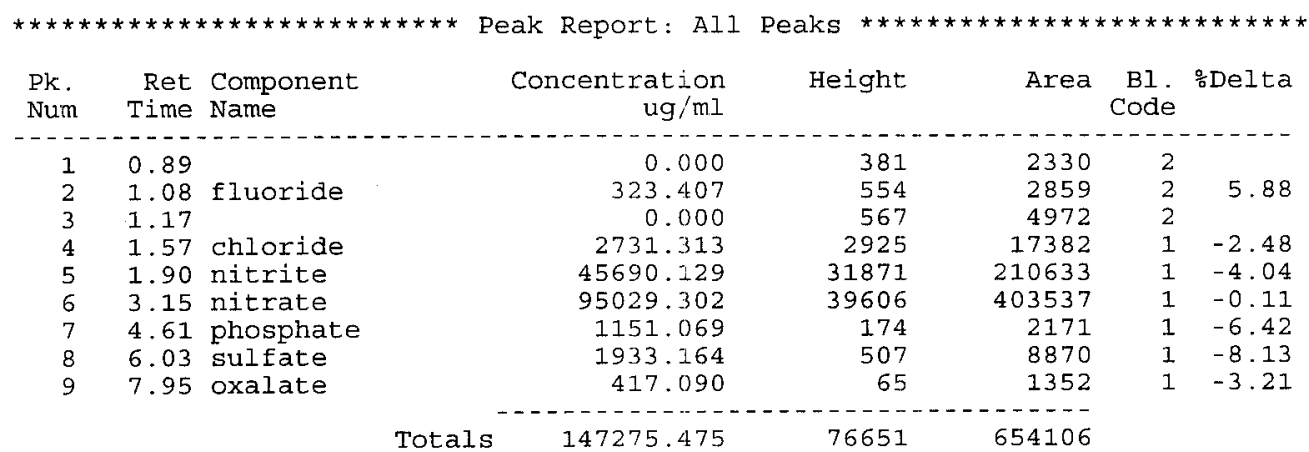

File: 96110101.d13 Sample: S96V000047

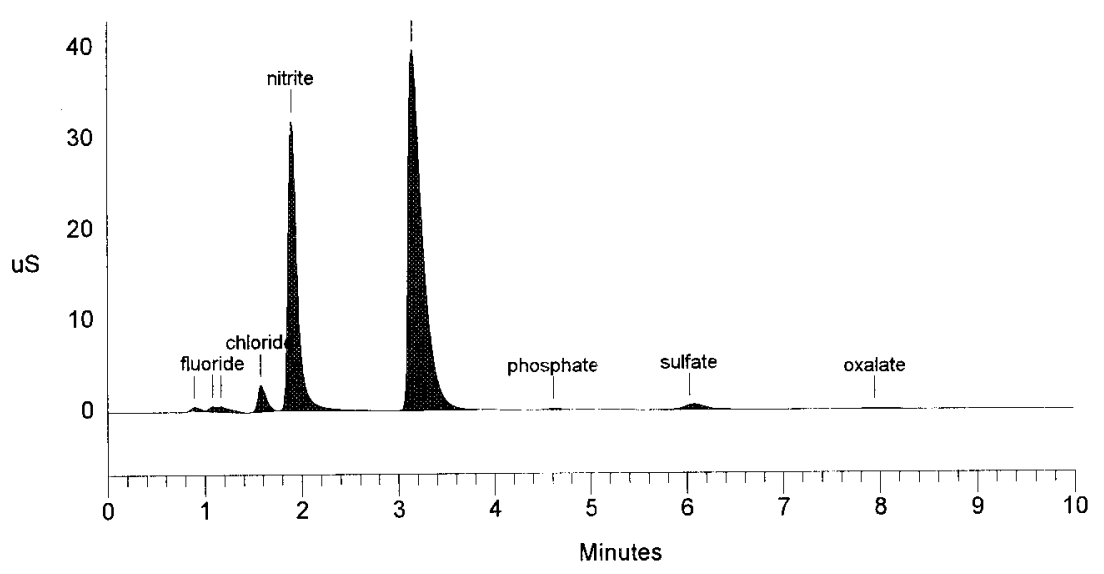


Data Reprocessed on 11/04/1996 09:59:16

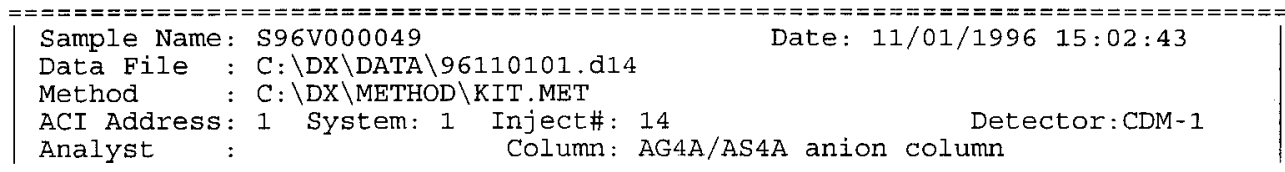

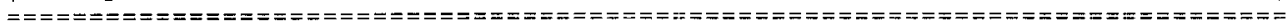

Calibration Volume Dilution Points Rate start stop Area Reject

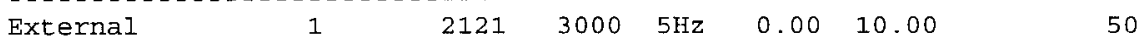

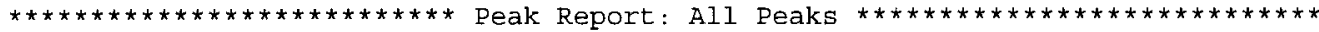
$\begin{array}{lrrr}\text { Pk. Ret Component } & \text { Concentration } & \text { Height: } & \text { Area Bl. } \\ \text { Num Delta } & \text { Time Name } / \mathrm{ml} & & \text { Code }\end{array}$

$\begin{array}{rrlrrrr}1 & 0.90 & 0.000 & 424 & 2572 & 2 \\ 2 & 1.08 \text { fluoride } & 348.537 & 592 & 3098 & 2 & 5.88 \\ 3 & 1.17 & 0.000 & 616 & 5365 & 2 & \\ 4 & 1.57 \text { chloride } & 2885.323 & 3172 & 18386 & 1 & -2.48 \\ 5 & 1.90 \text { nitrite } & 48097.247 & 34169 & 222320 & 1 & -4.04 \\ 6 & 3.14 \text { nitrate } & 101870.123 & 42509 & 438811 & 1 & 0.00 \\ 7 & 4.61 \text { phosphate } & 1538.337 & 196 & 2900 & 1 & -6.42 \\ 8 & 6.03 \text { sulfate } & 2054.880 & 545 & 9459 & 1 & -8.13 \\ 9 & 8.00 \text { oxalate } & 428.472 & 78 & 1395 & 1 & -2.56\end{array}$

Totals $157222.919 \quad 82300 \quad 704305$

File: 96110101.d14 Sample: S96V000049

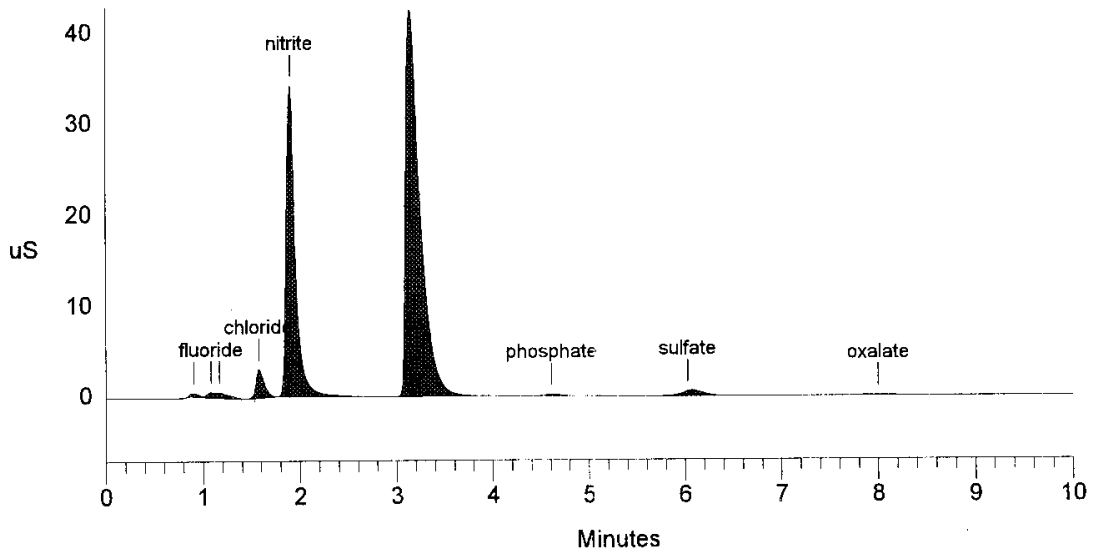


Data Reprocessed On 11/04/1996 09:59:13

\begin{tabular}{|c|c|c|}
\hline $\begin{array}{l}\text { Sample Name } \\
\text { Data File } \\
\text { Method }\end{array}$ & $\begin{array}{l}\text { S96V00058 FIEID BLANK } \\
\text { C: \DX \DATA } \backslash 96110101 . \text { d05 } \\
\text { C: } \backslash \text { DX } \backslash \text { METHOD } \backslash K I T . M E T\end{array}$ & Date: $11 / 01 / 199611: 43: 02$ \\
\hline $\begin{array}{l}\text { ACI Address } \\
\text { Analyst }\end{array}$ & $\begin{aligned} \text { Inject\#: } 5 \\
\text { Column: }\end{aligned}$ & $\begin{array}{l}\text { Detector: CDM-1 } \\
\text { on column }\end{array}$ \\
\hline
\end{tabular}

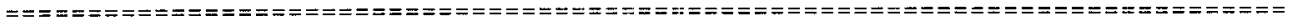

Calibration Volume Dilution Points Rate start stop Area Reject

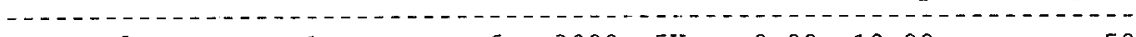

External

1

$\begin{array}{lllll}6 & 3000 \quad 5 \mathrm{~Hz} & 0.00 \quad 10.00\end{array}$

50

\begin{tabular}{|c|c|c|c|c|c|c|c|}
\hline $\begin{array}{l}\text { Pk. } \\
\text { Nuin }\end{array}$ & $\begin{array}{l}\text { Ret } \\
\text { Time }\end{array}$ & $\begin{array}{l}\text { Component } \\
\text { Name }\end{array}$ & $\begin{array}{r}\text { Concentration } \\
\mathrm{ug} / \mathrm{ml}\end{array}$ & Height & Area & $\begin{array}{l}\text { B1. } \\
\text { Code }\end{array}$ & $\therefore$ Delta \\
\hline$\ldots$ & & & & & & & -- \\
\hline 2 & 1.60 & chloride & 6.443 & 2738 & 14442 & 1 & -0.83 \\
\hline 3 & 3.35 & nitrate & 0.863 & 26 & 166 & 1 & -1.86 \\
\hline & 6.03 & sulfate & 14.689 & 1462 & 24714 & 1 & -8.13 \\
\hline & & & ----- & 4227 & ------ & & \\
\hline
\end{tabular}

File: 96110101.d05 Sample: S96V00058 FIELD BLANK

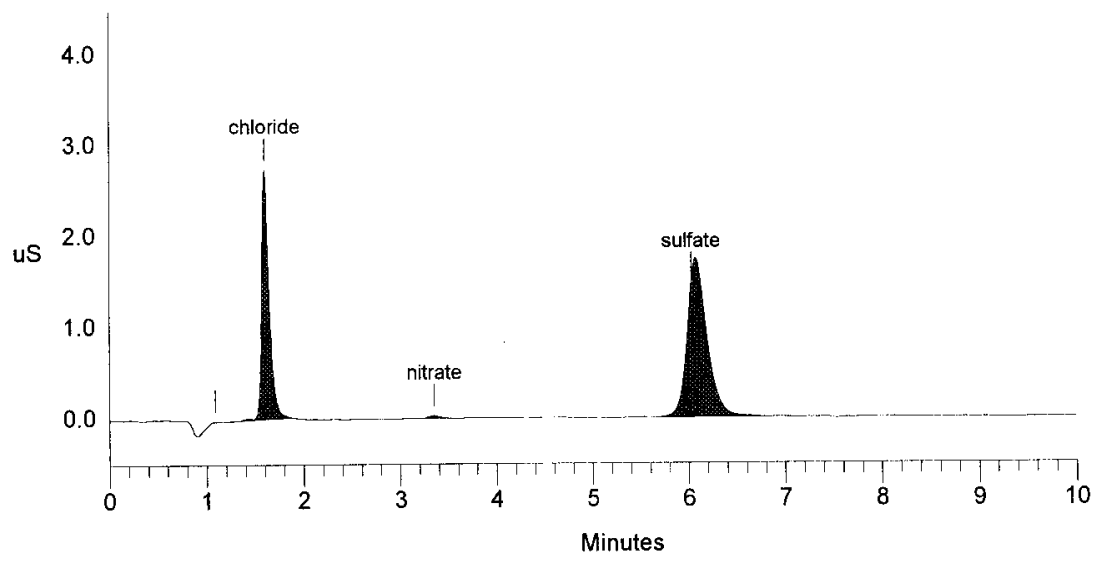




\section{LABCORE Data Entry Template for Worklist\# 14566}

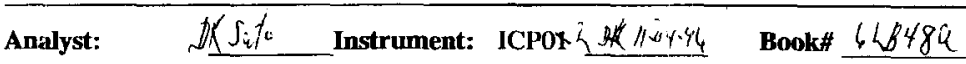

Method: LA-505-151/161 Rev/Mod B-1

2)

Worklist Comment: ICP AP-105 (LIQUID ACID DIGEST)

\begin{tabular}{|c|c|c|c|c|c|c|c|}
\hline $\bar{s}$ & TYPe & Sample: & R A & TAst & Matrix & Group\# & Project \\
\hline 1 & ICV & & & (ICP-QC & $Q C$ & & \\
\hline 2 & ICB & & & - ICP-QC & $Q C$ & & \\
\hline 3 & LLS & & & QICP-QC & $\mathrm{QC}$ & & \\
\hline 4 & ICSA & & & QICP-QC & $\mathrm{QC}$ & & \\
\hline 5 & ICSAB & & & QICP-QC & $\mathrm{QC}$ & & \\
\hline 6 & PREPSTDTJA & & & (ICP-B01 & LIQUID & & \\
\hline 7 & PREPBLKTJA & & & ICP-B01 & LIQUID & & \\
\hline
\end{tabular}

$$
\begin{aligned}
& 7 \text { PREPBLKTJA } \\
& \text { SURDil } \\
& 8 \text { SAMPLE }
\end{aligned}
$$$$
\text { 141104-96 }
$$$$
\text { S96V000050 O B OICP-B01 LIQUID }
$$$$
\text { Analytes Requested: }
$$

S96V000050 O B

S96V000050 O B S96V000050 O B

\section{1 sex-}

$12 \mathrm{CCV}$

$13 \mathrm{CCB}$

14 SAMPLE

15 DUP

16 SAMPLE

S96V000052 O B QICP-B01 LIQUID

Analytes Requested: $A L-B-01, C R-B-01$,

NI-B-01，SI-B-01 ,

96000853 AP- 105

FE-B-01， MN-B-01， NA-B-01 ,

U-B-01

(BICP-BOI LIQUID

@ICP-BOI LIQUID

बICP-QC QC

$@ I C P-Q C \quad Q C$

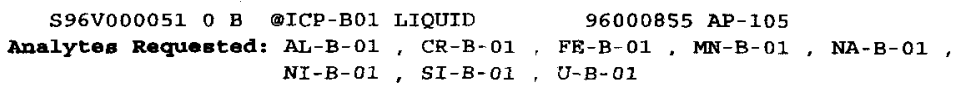

$N I-B-01, S I-B-O I, U-B-0 I$

96000855 AP-105

Data Entry Comments:

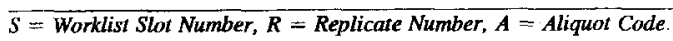




\section{LABCORE Data Entry Template for Worklist\# 14566}

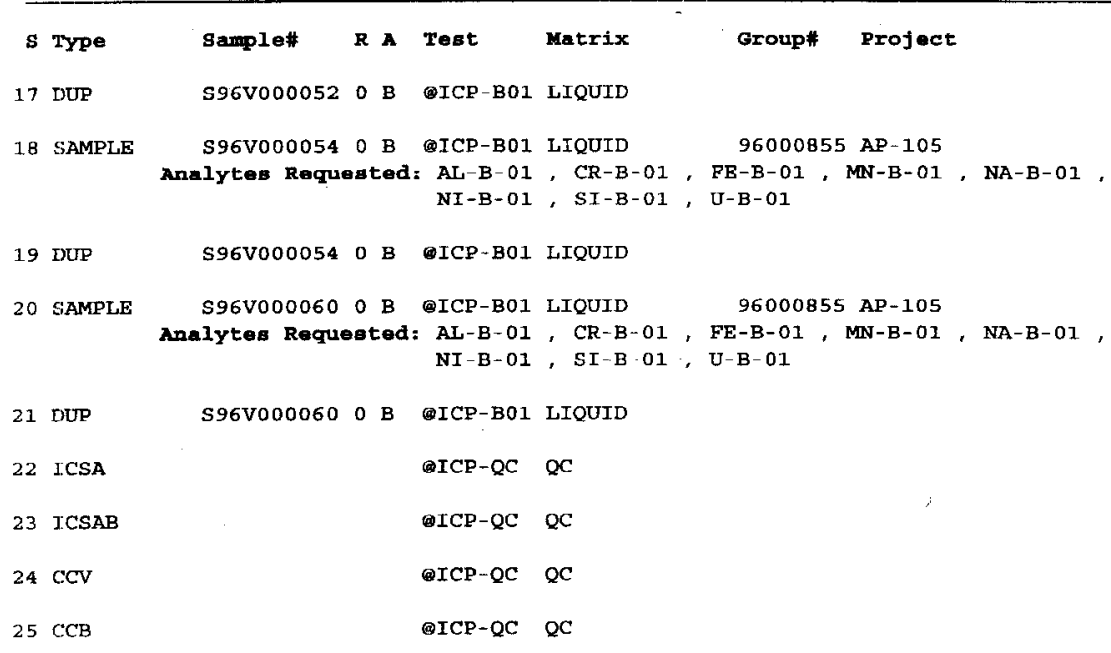

\section{Final page for worklist \# 14566}

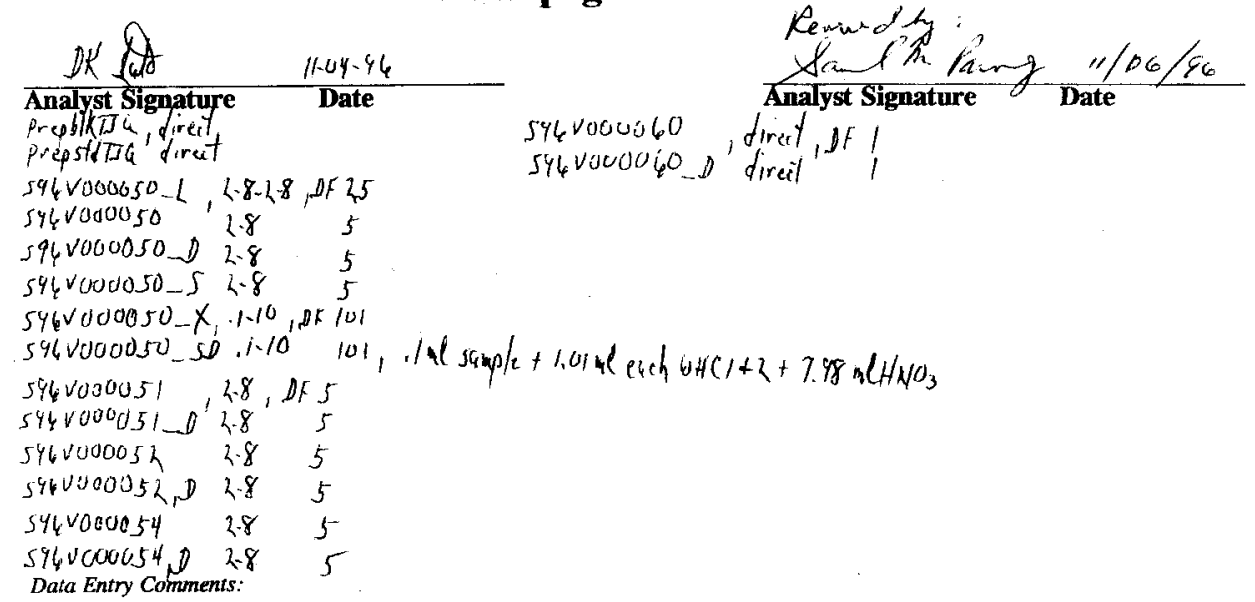

$\bar{S}=$ Worklist Slot Number, $R=$ Replicate Number, $A=$ Aliquot Code 
Kav. 18.1996 :03AM WHC 222S AAB FOM 2F HAYSIDE REV
Anatysis Report
Summary
HNFMon 11-04-96 12:03:45 PM
page 1

\begin{tabular}{|c|c|c|c|c|c|c|c|c|}
\hline A & Sample Name & File & Method & Date & Time & $O p I D$ & Type & Mode \\
\hline $\begin{array}{l}1 \\
2 \\
3 \\
4 \\
5 \\
6 \\
7 \\
8 \\
9 \\
10 \\
11 \\
12 \\
13 \\
14 \\
15 \\
16 \\
17 \\
18 \\
19 \\
20 \\
21 \\
22 \\
23 \\
24 \\
25 \\
26 \\
27 \\
28 \\
29 \\
30 \\
31 \\
32\end{array}$ & 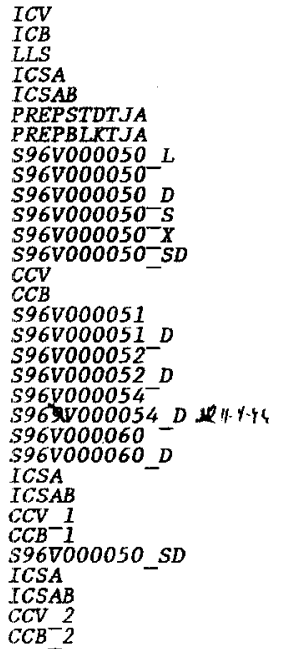 & $\begin{array}{l}961104 A \\
961104 A \\
961104 A \\
961104 A \\
961104 A \\
961104 A \\
961104 A \\
961104 A \\
961104 A \\
961104 A \\
961104 A \\
961104 A \\
961104 A \\
961104 A \\
961104 A \\
961104 A \\
961104 A \\
961104 A \\
961104 A \\
961104 A \\
961104 A \\
961104 A \\
961104 A \\
961104 A \\
961104 A \\
961104 A \\
961104 A \\
961104 A \\
961104 A \\
961104 A \\
961104 A \\
961104 A\end{array}$ & 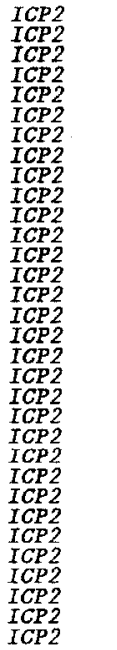 & $\begin{array}{l}11 / 04 / 96 \\
11 / 04 / 96 \\
11 / 04 / 96 \\
11 / 04 / 96 \\
11 / 04 / 96 \\
11 / 04 / 96 \\
11 / 04 / 96 \\
11 / 04 / 96 \\
11 / 104 / 96 \\
11 / 04 / 96 \\
11 / 04 / 96 \\
11 / 04 / 96 \\
11 / 04 / 96 \\
11 / 04 / 96 \\
11 / 04 / 96 \\
11 / 04 / 96 \\
11 / 04 / 96 \\
11 / 04 / 96 \\
11 / 04 / 96 \\
111 / 04 / 96 \\
11 / 04 / 96 \\
11 / 04 / 96 \\
11 / 04 / 96 \\
11 / 04 / 96 \\
11 / 04 / 96 \\
111 / 04 / 96 \\
11 / 04 / 96 \\
11 / 04 / 96 \\
11 / 04 / 96 \\
111 / 04 / 96 \\
11 / 04 / 96 \\
11 / 04 / 96\end{array}$ & $\begin{array}{l}09: 50 \\
09: 53 \\
09: 56 \\
09: 59 \\
10: 02 \\
10: 07 \\
10: 12 \\
10: 16 \\
10: 21 \\
10: 24 \\
10: 28 \\
10: 32 \\
10: 36 \\
10: 41 \\
10: 44 \\
10: 48 \\
10: 51 \\
10: 54 \\
11: 00 \\
11: 03 \\
11: 06 \\
11: 12 \\
11: 15 \\
11: 18 \\
11: 21 \\
11: 25 \\
11: 29 \\
11: 46 \\
11: 50 \\
11: 53 \\
11: 57 \\
12: 00\end{array}$ & $\begin{array}{l}D K S \\
D K S \\
D K S \\
D K S \\
D K S \\
D K S \\
D K S \\
D K S \\
D K S \\
D K S \\
D K S \\
D K S \\
D K S \\
D K S \\
D K S \\
D K S \\
D K S \\
D K S \\
D K S \\
D K S \\
D K S \\
D K S \\
D K S \\
D K S \\
D K S \\
D K S \\
D K S \\
D K S \\
D K S \\
D K S \\
D K S \\
D K S\end{array}$ & $\begin{array}{l}Q \\
0 \\
Q \\
Q \\
\\
S \\
S \\
S \\
S \\
S \\
S \\
S \\
S \\
Q \\
Q \\
S \\
S \\
S \\
S \\
S \\
S \\
S \\
S \\
Q \\
Q \\
Q \\
Q \\
S \\
Q \\
Q \\
Q \\
Q\end{array}$ & $\begin{array}{l}\text { CONC } \\
\text { CONC } \\
\text { CONC } \\
\text { CONC } \\
\text { CONC } \\
\text { CONC } \\
\text { CONC } \\
\text { CONC } \\
\text { CONC } \\
\text { CONC } \\
\text { CONC } \\
\text { CONC } \\
\text { CONC } \\
\text { CONC } \\
\text { CONC } \\
\text { CONC } \\
\text { CONC } \\
\text { CONC } \\
\text { CONC } \\
\text { CONC } \\
\text { CONC } \\
\text { CONC } \\
\text { CONC } \\
\text { CONC } \\
\text { CONC } \\
\text { CONC } \\
\text { CONC } \\
\text { CONC } \\
\text { CONC } \\
\text { CONC } \\
\text { CONC } \\
\text { CONC }\end{array}$ \\
\hline
\end{tabular}

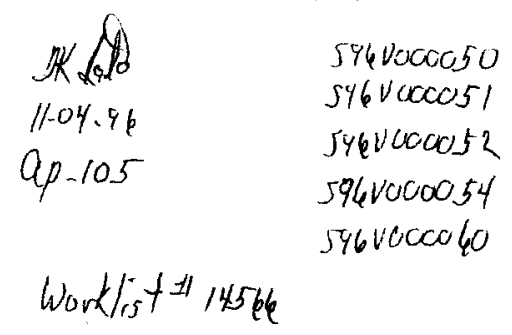

SIGNATURE ABOVE REPRESENTS CHEMICAL TECHNOLOGIST/CHEMIST THAT COMPLETED/VERIFIED THE CALIBRATIONANALYSIS ON PAGES 1105 TO 170 


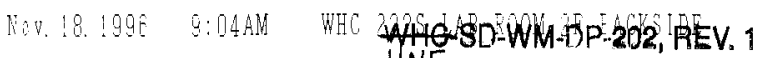

No 505

Mon 11-04-96 12:03:45 PM

Page 2

Anaiysis Report

Averages

HNF

\begin{tabular}{|c|c|c|c|c|c|c|c|}
\hline$\#$ & Sample Name & $A g$ & A1 & As & $B$ & $B a$ & $B e$ \\
\hline $\begin{array}{l}1 \\
2 \\
3 \\
4 \\
5 \\
6 \\
7 \\
8 \\
9 \\
10 \\
11 \\
12 \\
13 \\
14 \\
15 \\
16 \\
17 \\
18 \\
19 \\
20 \\
21 \\
22 \\
23 \\
24 \\
25 \\
26 \\
27 \\
28 \\
29 \\
30 \\
31 \\
32\end{array}$ & 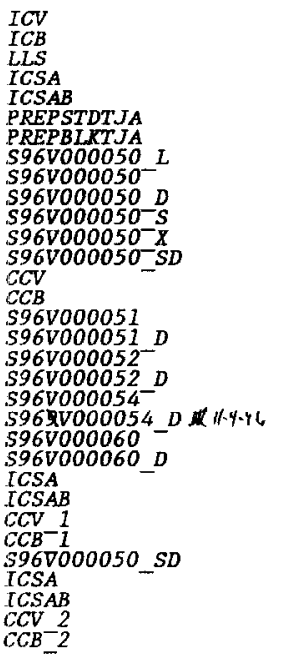 & $\begin{array}{l}4.995 \\
.0004 \\
.0202 \\
.0019 \\
.9579 \\
.5293 \\
.0007 \\
.1710 \\
.1605 \\
.1589 \\
.4531 \\
.1474 \\
9531 \\
5.031 \\
.0052 \\
.1692 \\
.1549 \\
.1556 \\
.1588 \\
.1637 \\
.1578 \\
-.0008 \\
-.0008 \\
.0016 \\
.9657 \\
4.993 \\
-.0005 \\
967.3 \\
.0109 \\
.9645 \\
4.977 \\
-.0004\end{array}$ & $\begin{array}{l}4.907 \\
.0030 \\
.0988 \\
245.0 \\
242.5 \\
4.492 \\
1310 \\
356.7 \\
348.4 \\
349.9 \\
351.7 \\
356.3 \\
1323 . \\
4.937 \\
.0034 \\
353.3 \\
334.0 \\
343.3 \\
351.0 \\
354.1 \\
346.1 \\
.1484 \\
1395 \\
246.5 \\
245.2 \\
4.903 \\
-10065 \\
1318 \\
243.1 \\
242.8 \\
4.862 \\
-.0089\end{array}$ & $\begin{array}{l}5.197 \\
-.0191 \\
.1921 \\
-.0029 \\
-.0361 \\
4.649 \\
-.0220 \\
-.6403 \\
-.4740 \\
-.3516 \\
4.498 \\
-1.898 \\
1038 \\
5.227 \\
-.0140 \\
-.4236 \\
-.3138 \\
-.3471 \\
-.3197 \\
-.4011 \\
.3301 \\
-.0088 \\
-.0217 \\
-.0539 \\
-.0030 \\
5.248 \\
1.0166 \\
1047 \\
-.0256 \\
-.0030 \\
5.205 \\
-.0167\end{array}$ & $\begin{array}{l}5.028 \\
.0034 \\
.1061 \\
.0060 \\
. .0007 \\
4.921 \\
.7079 \\
.7749 \\
.8063 \\
.6659 \\
5.137 \\
.8312 \\
990.2 \\
5.089 \\
.0010 \\
.7119 \\
.8087 \\
.6538 \\
.7700 \\
.7457 \\
.7167 \\
.6890 \\
.7670 \\
.0028 \\
.0009 \\
5.127 \\
.0019 \\
1004 \\
. .0035 \\
.0040 \\
5.038 \\
.0039\end{array}$ & $\begin{array}{l}4.947 \\
.0000 \\
.0983 \\
.0001 \\
4649 \\
4.401 \\
.0007 \\
.0040 \\
.0029 \\
.0025 \\
4.488 \\
10018 \\
1004 \\
5.012 \\
-0000 \\
.0024 \\
.0020 \\
.0025 \\
.0027 \\
.0020 \\
.0020 \\
.0011 \\
.0011 \\
.0001 \\
4705 \\
4.972 \\
.0001 \\
1010 \\
.0002 \\
4684 \\
4.932 \\
.0001\end{array}$ & $\begin{array}{l}5.139 \\
.0002 \\
.0104 \\
.0003 \\
.4756 \\
4.527 \\
.0002 \\
.0099 \\
.0051 \\
.0044 \\
4.658 \\
.0222 \\
1036 \\
5.168 \\
.0001 \\
.0061 \\
.0055 \\
.0055 \\
.0044 \\
.0051 \\
.0048 \\
.0002 \\
.0001 \\
.0000 \\
4801 \\
5.190 \\
.0000 \\
1042 \\
.0002 \\
4733 \\
5.119 \\
.0000\end{array}$ \\
\hline \# & Sample $\mathrm{Na}$ & $B i$ & $C a$ & $C d$ & $\mathrm{Ce}$ & Co & $C_{r}$ \\
\hline $\begin{array}{l}1 \\
2 \\
3 \\
4 \\
5 \\
6 \\
7 \\
8 \\
9 \\
10 \\
11 \\
12 \\
13 \\
14 \\
15 \\
16 \\
17\end{array}$ & $\begin{array}{l}I C V \\
\text { ICB } \\
\text { ILS } \\
\text { ICSA } \\
\text { ICSAB } \\
\text { PREPSTDTJA } \\
\text { PREPBLKTJA } \\
\text { S96V000050 L } \\
396 \mathrm{~V} 000050^{-} \\
\text {S96V000050 D } \\
\text { S96V000050-S } \\
\text { S96V000050 X } \\
\text { S96V000050 SD } \\
\text { CCV } \\
\text { CCB } \\
\text { S96V000051 } \\
\text { S96V000051 D }\end{array}$ & $\begin{array}{l}5.037 \\
-.0415 \\
.1755 \\
-.0329 \\
-.0450 \\
4.462 \\
-.0223 \\
-.1136 \\
.0513 \\
-.0024 \\
4.625 \\
-1.114 \\
1011 \\
5.193 \\
.0095 \\
-.1607 \\
-.0803\end{array}$ & $\begin{array}{l}5.025 \\
.0008 \\
.2108 \\
247.8 \\
249.3 \\
4.460 \\
.2104 \\
.5210 \\
.4058 \\
.3480 \\
4.841 \\
.5683 \\
987.5 \\
4.999 \\
.0023 \\
-3253 \\
.3857\end{array}$ & $\begin{array}{l}5.073 \\
-.0005 \\
.0092 \\
-.0000 \\
.9354 \\
4.410 \\
-.0010 \\
-.0201 \\
-.0007 \\
-.0073 \\
4.561 \\
.0406 \\
994.1 \\
5.059 \\
-.0008 \\
-.0015 \\
-.0013\end{array}$ & $\begin{array}{l}4.973 \\
.0044 \\
.1958 \\
.0076 \\
.0081 \\
4.547 \\
.0047 \\
-.0097 \\
-.0185 \\
-.0111 \\
4.702 \\
-.5651 \\
992.4 \\
4.999 \\
-.0019 \\
.0034 \\
-.0073\end{array}$ & $\begin{array}{l}5.098 \\
-.0024 \\
.0398 \\
-.0010 \\
.4653 \\
4.542 \\
.0026 \\
-.0774 \\
.0161 \\
=.0013 \\
4.643 \\
=.2262 \\
1.191 \\
5.097 \\
-.0029 \\
.0011 \\
.0117\end{array}$ & $\begin{array}{l}5.088 \\
-.0003 \\
.0199 \\
.0068 \\
4765 \\
4.429 \\
.0016 \\
4.363 \\
4.266 \\
4.267 \\
8.742 \\
4.261 \\
1000 \\
5.077 \\
-.0005 \\
4.311 \\
4.095\end{array}$ \\
\hline
\end{tabular}


W. 18. 1906 9:04AM WHC 2225 LAE DOOL $2 F$ EACBIDE

WHQSD-WM-1DP-202, REV. 1

HNF Mon 11-04-96 12:03:45 PM page 3

Analysis Report

Averages

$\mathrm{Ca}$

Bi

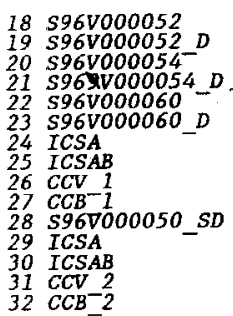

\# Sample Name

$\begin{array}{ll}1 & I C V \\ 2 & I C B \\ 3 & L L S\end{array}$

4 ICSA

5 ICSAB

6 PREPSTDTJA

7 PREPBLKTJA

$8596 \mathrm{~V} 000050 \mathrm{~L}$

9 S96V000050-

10 S96V000050 D

$11 \mathrm{~s} 96 \mathrm{V000050^{- } \mathrm { s }}$

$12 \mathrm{~s} 96 \mathrm{~V} 000050^{-\mathrm{X}}$

13 S96V000050-SD

$14 \mathrm{CCV}$

$15 C C B$

16 S96V000051

I) S96V000051 D

18 s96V000052

$19 S 96 V 000052 D$

$20596 \mathrm{V000054}$

21 S96 TV000054 D N 1.4 .94

$22596 \mathrm{~V} 000060$

$23596 V 000060 \quad D$

24 ICSA

25 ICSAB

$26 \mathrm{CCV} 1$

$27 \mathrm{CCB}^{-1}$

28 S96V000050 SD

29 ICSA

30 ICSAB

$31 \mathrm{CCV} 2$

$32 \mathrm{CCB}^{-2}$
$-.0936$

$-.1213 \quad .3738$

$.1540 \quad .3764$

$-.0198$

.0051

.0037

$-.0203$

5.088

$-.0013$

1020 .

$-.0408$

.0216

.0409

.5002

.4866

252.3

249.6

4.992

$-.0003$

$997 \cdot 1$

252.0

.0030

$\mathrm{Cu}$

Eu

5.182
-.0004
.0209
-.0105
.4743
4.449

.0029

.0313

.0302

.0105

4.583

$-.0000$

- 3386

5.240

-.0002

.0181

.0205

.0258

.0115

.0160

.0193

.0069

.0081

$-.0109$

.4820

5.237

$-.0007$

-.3203

.0201

.4726

5.149

$-.0004$

\section{$-.0011$}

.0003

$-.0344$

-.0362

$-.0014$

$-.0012$

$-.0007$

.0007

.0003

$-.0036$

$-.0580$

.3231

$-.0015$

-.0005
-.0029

0002

.0019

.0008

$-.0001$

$-.0006$

$-.0014$

$-.0007$

.0402

$-.0343$

-.0010

.0003

.3383

$-.0407$

$-.0397$

$-.0003$

.0002
Cd

$\mathrm{Ce}$

Co

$\begin{array}{llll}-.0014 & -.0054 & -.0055 & 4.222 \\ .0015 & -.0165 & .0037 & 4.304 \\ -.0054 & -.0001 & .0099 & 4.312 \\ .0001 & -.0149 & .0037 & 4.214 \\ -.0014 & -.0035 & -.0026 & .0006 \\ -.0013 & -.0012 & -.0044 & .0022 \\ -.0004 & .0145 & -.0022 & .0073 \\ .9399 & .0110 & .4651 & .4781 \\ 5.073 & 4.977 & 5.096 & 5.078 \\ 10019 & -.0011 & -.0035 & -.0007 \\ 1003 & 99911 & 1.503 & 1008 \\ .0008 & .0116 & -.0002 & .0092 \\ 5394 & .0119 & -4685 & .4783 \\ 5.052 & 4.939 & 5.094 & 5.067 \\ -.0010 & -.0078 & -.0025 & -.0014\end{array}$

$\mathrm{Fe}$

K

La

$-.0001$

5.042
.0006
.1032
93.52
92.96
4.551
.0443
.1103
.1329
.1273
4.594
.1103
986.5
5.034
.0001
.1358
.1218
.1320
.1129
.1485
.1872
.0429
.0359
94.22
93.45
5.011
90004
991.5
93.49
93.31
4.979
.0003

5. 000

$-.0794$

6171

.0509

.3327

4.469

.0616

64.42

66.68

68.73

71.87

47.82

1059

5.044

1613

80.72

77.33

67.08

68.14

67.95

67.19

.0371

.1692

.0573

.1268

5.059

.1528

1053

.1606

.2659

4.940

.0525
5. 028

$-.0001$

.1003

- .0050

$-.0039$

4.556

.0005

$-.0110$

$-.0006$

$-.0019$

4.726

$-.0766$

1004.

5.067

$-.0007$

$-.0028$

$-0032$

$-.0001$

$-.0021$

$-.0007$

$-.0045$

$-.0014$

$-.0003$

$-.0049$

$-.0045$

5.044

.0001

1009.

$-.0045$

- 0049

4.986

.0000
$L i$

$\mathrm{Cr}$

$-.0014$

Li

5. 059

0000

.0217

1. 040

4.595

.0011

.0012

.0018

4.870

$-.0712$

1027

5. 169

0000

$-0029$

-.0006

.0006

.0006

$-.0014$

-.0004

.0031

5.156

.0004

1028.

1.039

5.042

.0001 
Ho :8. :99e g:05AM WHC 2225 AREOSD-WM-DF-202, REV.

No. 965 P. 6.8

AnaIysis Report

Averages

HNF $11-04-96$ 12:03:45 PM

page 4

\begin{tabular}{|c|c|c|c|c|c|c|c|}
\hline \# & Sample Name & $M g$ & $\mathrm{Mn}$ & Mo & $\mathrm{Na}$ & & $N i$ \\
\hline $\begin{array}{l}10 \\
11 \\
12 \\
13 \\
14 \\
15 \\
16 \\
17 \\
18 \\
19 \\
20 \\
21 \\
22 \\
23 \\
24 \\
25 \\
26\end{array}$ & 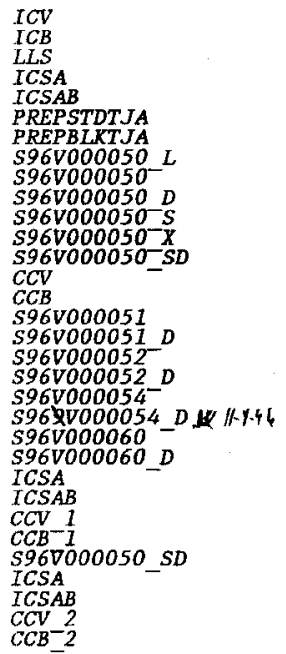 & $\begin{array}{l}4.845 \\
-.0065 \\
.1850 \\
254.3 \\
251.4 \\
4.741 \\
.0245 \\
.0958 \\
.0284 \\
.0471 \\
4.235 \\
.2260 \\
1004 \\
4.837 \\
.0049 \\
.0275 \\
.0282 \\
.0350 \\
.0126 \\
.0370 \\
.0134 \\
.0789 \\
.0848 \\
255.9 \\
2540 \\
4.798 \\
-01027 \\
1008 \\
251.8 \\
251.2 \\
4.748 \\
-.0073\end{array}$ & $\begin{array}{l}4.922 \\
-.0001 \\
.0192 \\
-.0069 \\
.4407 \\
4.320 \\
-.0001 \\
-.0060 \\
-.0008 \\
-.0016 \\
4.349 \\
.0465 \\
986.2 \\
4.949 \\
-.0002 \\
-.0018 \\
-.0012 \\
.0005 \\
-.0008 \\
-.0010 \\
-.0012 \\
-.0002 \\
-.0001 \\
-.0068 \\
.4423 \\
4.926 \\
-.0001 \\
993.6 \\
-.0059 \\
4457 \\
4.921 \\
-.0003\end{array}$ & $\begin{array}{l}5.074 \\
.0009 \\
.0963 \\
-.0075 \\
-.0139 \\
4.459 \\
-.0004 \\
.6041 \\
.6078 \\
.6103 \\
5.164 \\
.5915 \\
967.9 \\
5.084 \\
.0007 \\
.6250 \\
.5853 \\
.6061 \\
.6190 \\
.6181 \\
.6034 \\
.0008 \\
-.0014 \\
-.0121 \\
-.0135 \\
5.073 \\
.0002 \\
9839 \\
-.0083 \\
.0120 \\
5.074 \\
.0002\end{array}$ & $\begin{array}{l}4.885 \\
1.0033 \\
.2042 \\
197.7 \\
192.8 \\
5.235 \\
.9850 \\
2314 . \\
2252 \\
2258 \\
2251 \\
2306 \\
3281 \\
4.876 \\
-.0098 \\
2318 \\
2183 . \\
2202 . \\
2257 . \\
2288 . \\
2233 \\
19792 \\
1.099 \\
195.7 \\
194.1 \\
4.848 \\
-.0012 \\
3235 \\
192.1 \\
191.2 \\
4.778 \\
-.0049\end{array}$ & 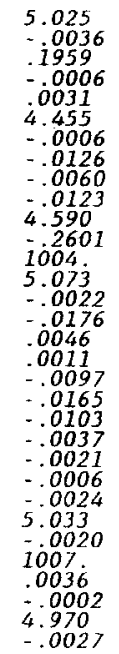 & $\begin{array}{l}5.028 \\
-.0037 \\
.0397 \\
-.0030 \\
.9221 \\
4.560 \\
.0000 \\
-.0407 \\
-.0058 \\
0174 \\
4.622 \\
9.9198 \\
982.1 \\
5.043 \\
.0017 \\
.0427 \\
.0415 \\
.0087 \\
.0046 \\
-.0163 \\
-.0110 \\
-.0018 \\
-.0075 \\
-.0036 \\
.9009 \\
4.996 \\
-00049 \\
9924 \\
.0115 \\
.9283 \\
5.004 \\
-.0037\end{array}$ \\
\hline & Sample & $P$ & h & $S$ & $s b$ & $\mathrm{Se}$ & $i$ \\
\hline $\begin{array}{l}12 \\
13 \\
14 \\
15\end{array}$ & $\begin{array}{l}I C V \\
I C B \\
I L L S \\
I C S A \\
I C S A B \\
P R E P S T D T J A \\
P R E P B L K T J A \\
S 96 V 000050-L \\
S 96 \mathrm{~V} 000050^{-} \\
S 96 V 000050 \quad D \\
S 96 V 000050^{-} S \\
S 96 V 000050^{-X} \\
S 96 V 000050^{-} S D \\
C C V \\
C C B \\
S 96 \mathrm{~V} 000051 \\
S 96 \mathrm{~V} 000051 \quad D\end{array}$ & $\begin{array}{l}5.163 \\
-.0037 \\
.4211 \\
.0202 \\
.0428 \\
4.682 \\
.0061 \\
11.81 \\
11.45 \\
11.61 \\
13.94 \\
12.55 \\
1039 \\
5.141 \\
.0109 \\
11.38\end{array}$ & $\begin{array}{l}5.055 \\
-.0046 \\
.2149 \\
.0322 \\
.9999 \\
4.314 \\
.0017 \\
.6105 \\
.7407 \\
.7024 \\
5.180 \\
.8121 \\
.013 \\
5.038 \\
-.0007 \\
.7221 \\
.6910\end{array}$ & $\begin{array}{l}5.063 \\
.0032 \\
.2024 \\
-.0491 \\
-.0460 \\
4.581 \\
.1295 \\
14.30 \\
14.06 \\
14.17 \\
18.65 \\
13.56 \\
1020 \\
5.056 \\
10.0053 \\
16.68 \\
15.85\end{array}$ & $\begin{array}{l}4.842 \\
.0017 \\
.1023 \\
.0025 \\
.0034 \\
4.441 \\
.0064 \\
.0664 \\
.0548 \\
.0533 \\
4.642 \\
-.1105 \\
985.7 \\
4.862 \\
.0046 \\
-.0103 \\
.0082\end{array}$ & $\begin{array}{l}4.794 \\
.0153 \\
.2046 \\
-.0341 \\
-.0142 \\
4.164 \\
.0103 \\
.0745 \\
.0894 \\
0343 \\
4.492 \\
.3433 \\
972.8 \\
4.027 \\
.0066 \\
.0493 \\
.0214\end{array}$ & $\begin{array}{l}4.974 \\
.0030 \\
Q .2096 \\
-.0072 \\
-.0012 \\
4.879 \\
1.173 \\
1.940 \\
1.889 \\
1.474 \\
5.162 \\
1.515 \\
1011 \\
4.975 \\
-.0035 \\
1.688 \\
1.672\end{array}$ \\
\hline
\end{tabular}


No: 121096 9:0EAM WEC 2225 LAE ROCSO-NM-DP-202, REV. 1

\begin{tabular}{|c|c|c|c|c|c|c|c|}
\hline \multicolumn{2}{|c|}{ Analysis Report } & Averages & \multicolumn{4}{|c|}{ Hon $11-04-96 \quad 12: 03: 45$ PM } & \multirow{2}{*}{$\begin{array}{l}\text { page } 5 \\
\text { Si }\end{array}$} \\
\hline$\#$ & Sample Name & $P$ & $\boldsymbol{P b}$ & $S$ & $S b$ & Se & \\
\hline $\begin{array}{l}18 \\
19 \\
20 \\
21 \\
22 \\
23 \\
24 \\
25 \\
26 \\
27 \\
28 \\
29 \\
30 \\
31 \\
32\end{array}$ & 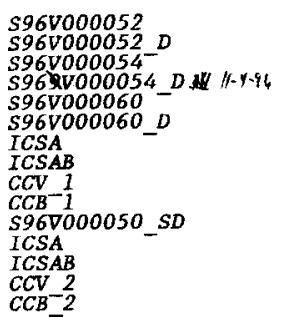 & $\begin{array}{l}11.40 \\
11.83 \\
11.99 \\
11.47 \\
.0002 \\
.0313 \\
.0090 \\
.0149 \\
5.303 \\
0102 \\
1052 \\
.0195 \\
.0219 \\
5.275 \\
.0020\end{array}$ & $\begin{array}{l}.7241 \\
.7624 \\
.7116 \\
.7486 \\
.0163 \\
.0091 \\
.0266 \\
.9927 \\
5.032 \\
.0058 \\
1025 \\
.0369 \\
1.007 \\
5.025 \\
-.0041\end{array}$ & $\begin{array}{l}14.09 \\
14.35 \\
14.41 \\
14.04 \\
1629 \\
.2074 \\
=.0487 \\
=.0522 \\
5.119 \\
=.0090 \\
1035 \\
=.0489 \\
=.0343 \\
5.087 \\
-.0060\end{array}$ & $\begin{array}{l}-.0107 \\
.0251 \\
.0104 \\
.0082 \\
.0043 \\
.0046 \\
.0118 \\
.0050 \\
4.845 \\
.0047 \\
1002 \\
.0116 \\
.0116 \\
4.835 \\
.0061\end{array}$ & $\begin{array}{l}.0991 \\
.1087 \\
.0730 \\
.0668 \\
.0194 \\
.0051 \\
-.0307 \\
-.0298 \\
4.937 \\
.0065 \\
975.2 \\
-.0022 \\
.0206 \\
4.867 \\
.0039\end{array}$ & $\begin{array}{l}1.673 \\
1.737 \\
1.818 \\
1.702 \\
1.363 \\
1.485 \\
-.0054 \\
-.0063 \\
4.960 \\
-.0011 \\
1027 \\
-.0042 \\
-.0022 \\
4.951 \\
-.0052\end{array}$ \\
\hline 借 & Sample $N$ & $S m$ & $S r$ & $T h$ & $T i$ & $T 1$ & $U$ \\
\hline $\begin{array}{l}1 \\
2 \\
3 \\
4 \\
5 \\
6 \\
7 \\
8 \\
9 \\
10 \\
11 \\
12 \\
13 \\
14 \\
15 \\
16 \\
17 \\
18 \\
19 \\
20 \\
21 \\
22 \\
23 \\
24 \\
25 \\
26 \\
27 \\
28 \\
29 \\
30 \\
31 \\
32\end{array}$ & 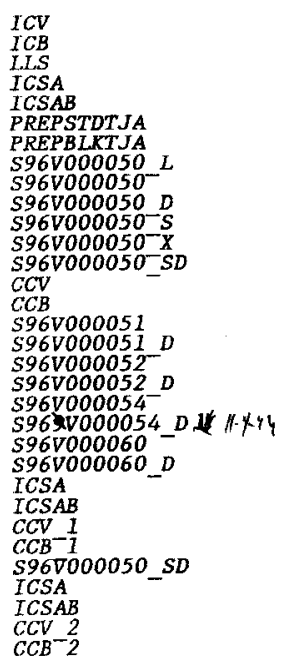 & $\begin{array}{l}4.856 \\
-.0013 \\
.1997 \\
-.0101 \\
.0007 \\
4.383 \\
-.0203 \\
=.1000 \\
-.0390 \\
=.0369 \\
4.484 \\
-1.191 \\
974.6 \\
4.892 \\
-.0105 \\
-.0793 \\
-.0368 \\
-.0054 \\
-.0243 \\
-.0497 \\
-.0430 \\
-.0270 \\
-.0178 \\
.0015 \\
-.0041 \\
4.887 \\
.0017 \\
979.8 \\
.0098 \\
-0053 \\
4.837 \\
-.0020\end{array}$ & $\begin{array}{l}4.888 \\
-.0001 \\
.0199 \\
.0019 \\
.0019 \\
4.386 \\
.0001 \\
.0000 \\
.0010 \\
.0012 \\
4.514 \\
.0150 \\
977.6 \\
4.917 \\
.0001 \\
.0007 \\
.0010 \\
.0017 \\
.0010 \\
.0010 \\
.0012 \\
.0018 \\
.0017 \\
.0019 \\
.0019 \\
4.903 \\
.0000 \\
981.7 \\
.0018 \\
.0018 \\
4.836 \\
.0001\end{array}$ & $\begin{array}{l}.0534 \\
.0000 \\
.0004 \\
.0007 \\
.0032 \\
.0466 \\
.0052 \\
.0477 \\
.0045 \\
.0141 \\
.0464 \\
.0031 \\
5.593 \\
.0597 \\
.0024 \\
.0046 \\
.0242 \\
.0037 \\
.0205 \\
. .0140 \\
.0092 \\
.0054 \\
.0008 \\
.0062 \\
.0041 \\
.0558 \\
.0042 \\
5.112 \\
.0010 \\
.0034 \\
.0596 \\
-.0001\end{array}$ & $\begin{array}{l}4.872 \\
.0002 \\
.0196 \\
.0015 \\
.0012 \\
4.092 \\
.0015 \\
.0001 \\
-.0001 \\
.0011 \\
4.138 \\
.0243 \\
887.1 \\
4.880 \\
-.0002 \\
.0011 \\
.0034 \\
.0011 \\
.0022 \\
-.0026 \\
-.0001 \\
.0008 \\
.0010 \\
.0007 \\
.0012 \\
4.882 \\
-.0000 \\
903.8 \\
.0010 \\
.0012 \\
4.833 \\
-.0002\end{array}$ & $\begin{array}{l}4.878 \\
.0028 \\
.3875 \\
-.0004 \\
0209 \\
4.796 \\
.0044 \\
-.0697 \\
.1370 \\
.0678 \\
4.339 \\
2.398 \\
950.4 \\
4.841 \\
-.0020 \\
-.1267 \\
-.1128 \\
-.0104 \\
-.0385 \\
-.0647 \\
-.1168 \\
-.0281 \\
.0008 \\
.0242 \\
.0076 \\
4.886 \\
-.0032 \\
958.8 \\
.0245 \\
-.0156 \\
4.859 \\
-.0063\end{array}$ & $\begin{array}{l}9.499 \\
.0055 \\
.4880 \\
-.0622 \\
-.0410 \\
8.556 \\
-.0762 \\
-.2046 \\
.1037 \\
.0891 \\
8.823 \\
-3.496 \\
69.49 \\
9.610 \\
-.0369 \\
.0083 \\
.0748 \\
.1990 \\
.1475 \\
.0620 \\
.0308 \\
-.0989 \\
-.0592 \\
-.0443 \\
.0446 \\
9.559 \\
.0070 \\
71.17 \\
-.0419 \\
-.0350 \\
9.424 \\
-.0048\end{array}$ \\
\hline
\end{tabular}



HNF-SH-WM-DE-282,REV 45 PM page 6

Analys is Report

Averages Zn

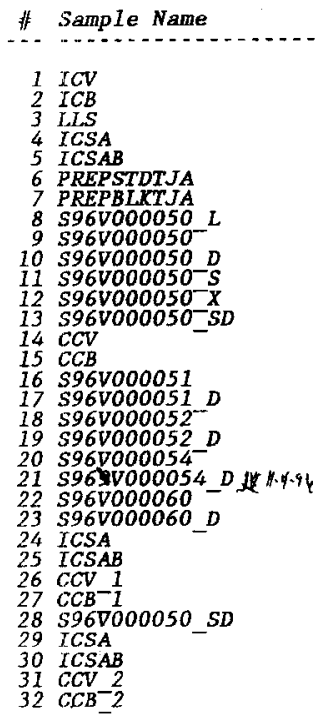

V $\quad Y$

5.040
.0004
.1025

$0067 \quad 5.106 \quad 4.922$

$\begin{array}{lll}5.0002 & -.0003\end{array}$

$\begin{array}{llll}-.0020 & .0064 & .0035 & -.0047\end{array}$

$\begin{array}{llll}.4647 & .0072 & .0572 & -.0026\end{array}$

$\begin{array}{llll}4.416 & .0048 & 4.374 & 4.530\end{array}$

$\begin{array}{llll}-.0034 & -.0010 & .0431 & .0004\end{array}$

$\begin{array}{llll}-.0098 & -.0078 & .0563 & -.0036\end{array}$

$\begin{array}{llll}-.0019 & -.0016 & .0624 & .0121\end{array}$

$\begin{array}{rrrr}-.0028 & -.0028 & .0438 & .0746\end{array}$

$\begin{array}{llll}4.526 & .0021 & 4.643 & .9840\end{array}$

$\begin{array}{llll}-.1806 & -.0618 & .0399 & -.1010\end{array}$

$\begin{array}{llll}.6275 & 1.419 & -3.037 & 989.4\end{array}$

$\begin{array}{llll}5.058 & .0064 & 5.107 & 4.948\end{array}$

$\begin{array}{llll}-.0018 & -.0005 & .0003 & -.0015\end{array}$

$\begin{array}{llll}-.0101 & -.0039 & 0476 & .0028\end{array}$

$\begin{array}{llll}-.0027 & -.0032 & .0553 & .0101\end{array}$

$\begin{array}{llll}.0016 & -.0013 & .0570 & .0166\end{array}$

$\begin{array}{llll}-.0011 & -.0020 & .0488 & .0132\end{array}$

$\begin{array}{llll}-.0046 & -.0032 & .0499 & .0129\end{array}$

$\begin{array}{llll}-.0055 & -.0035 & 0508 & .0073\end{array}$

$\begin{array}{llll}-.0045 & -.0014 & .0561 & -.0003\end{array}$

$\begin{array}{llll}-.0027 & -.0007 & .0527 & .0011\end{array}$

$\begin{array}{llll}.0000 & .0070 & .0036 & -.0029\end{array}$

$\begin{array}{llll}.4665 & .0069 & .9638 & -.0041\end{array}$

$\begin{array}{llll}5.049 & .0062 & 5.108 & 4.933\end{array}$

$\begin{array}{lllll}.0004 & .0000 & -.0013 & -.0000\end{array}$

$\begin{array}{llll}.7007 & 1.426 & -3.215 & 1004\end{array}$

$\begin{array}{llll}.0015 & .0074 & .0046 & -.0020\end{array}$

$\begin{array}{llll}.4677 & .0075 & .9655 & -.0023\end{array}$

$\begin{array}{llll}5.025 & .0067 & 5.110 & 4.895\end{array}$

$\begin{array}{lllll}-.0004 & -.0002 & -.0012 & -.0010\end{array}$

$M A_{A}$

11.04 .96 
Analyst: RAW Instrument: TOC01WB39937 Book 24NIZ-D stD

Method: LA-344-105 Rev/Mod D-1

$23 N 1 Z-8$ SPK

Worklist Comment: AP-105 TOC. RCJ

\begin{tabular}{|c|c|c|c|c|c|c|c|c|c|c|}
\hline \multirow[t]{2}{*}{ GROUP } & \multirow[t]{2}{*}{ PROJECT } & $\begin{array}{l}5 \text { TYPE } \\
1 \text { BLNK }\end{array}$ & \multirow[t]{2}{*}{ SAMPLE\# } & & R A $\cdots \cdots$ TEST $\cdots \cdots$ & $\begin{array}{l}\text { MATRIX } \\
\text { LIQUID }\end{array}$ & $\begin{array}{c}\text { ACTUAL } \\
1 \\
\end{array}$ & $\begin{array}{l}\text { FOUND } \\
0.70 \\
\end{array}$ & $\begin{array}{l}\text { DL } \\
\quad \mathrm{N} / \mathrm{A} \\
\end{array}$ & $\begin{array}{l}\text { UN1T } \\
\text { ug/mE }\end{array}$ \\
\hline & & 2 STD & & & Toc-01 & LIOUID & $3.00_{<<} 3$ & $28 x^{2} 3$ & $\mathrm{~N} / \mathrm{A}$ & ug/mL \\
\hline 96000855 & AP -105 & 3 SAMPLE & $596 \vee 000048$ & 0 & TOC- 01 & LIQUID & $N / A$ & $1.48 \mathrm{ez}$ & $5.50 \times$ & ug/mL \\
\hline 96000855 & AP -105 & $4 \mathrm{SPK}$ & s96v000048 & 0 & TOC- 01 & 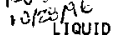 & $+4 t+2$ & 90.2 & $N / A$ & $\mathrm{ug} / \mathrm{mL}$ \\
\hline 96000855 & AP- 105 & 5 SPK-DUP & $596 v 000048$ & 0 & TOC-01 & LIQUID & 90.2 & 89.5 & N/A & $\mathrm{ug} / \mathrm{mL}$. \\
\hline 96000855 & AP- 105 & 6 SAMPLE & $596 \vee 000049$ & 0 & TOC-01 & LIQUID & N/A & $1.53 e^{3.3}$ & $5.5 x$ & ug/mL \\
\hline 96000853 & AP -105 & 7 SAMPLE & $596 v 000047$ & 0 & TOC -011 & LIQUID & N/A & $.50 \times 3$ & 5.501 & $\mathrm{ug} / \mathrm{m}$ \\
\hline 96000855 & AP- 105 & 8 SAMPLE & 596 V000053 & 0 & TOC-01 & LIQUID & N/A & 1.44 .3 & $5.50 k$ & $\mathrm{ug} / \mathrm{mL}$ \\
\hline 96000855 & AP- 105 & 9 SAMPLE & s96V000058 & 0 & TOC-01 & LIQUID & $N / A$ & 7.7000 & 5.500 & $\mathrm{ug} / \mathrm{mL}$ \\
\hline
\end{tabular}

\section{Final page for worklist \# 12910}

$1<N_{\text {end }}$ (andi $10-15.96$

Analyst Signature Date
RC $10 \cdot 2596$

Analy Signature Date

Page: $\quad l$ 
TOC- TOTAL ORGANIC CARBON ANALYSIS REPORT TICTOC REV 2.0

Sample: STD

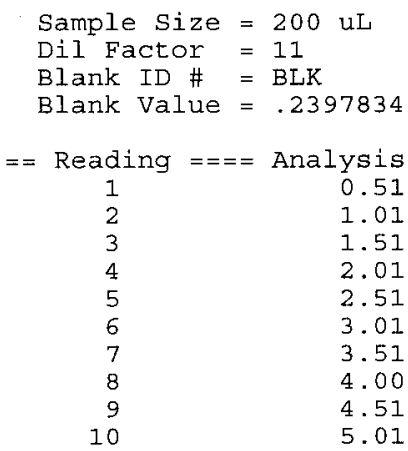

Date: $10 / 15 / 96$

Time: $09: 36: 47$

$\mathrm{ug} / \mathrm{minute} \mathrm{C}$

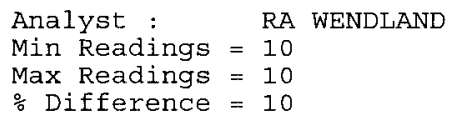

Time $====$ Coulometer $===z$ Difference $==$

1.60

34.80

47.30

51.10

52.30

52.70

52.90

53.00

53.10

53.20
0.00

95.40

26.43

7.44

2.29

0.76

0.38

0.19

0.19

0.19

BLANK VALUE $=1.2$ micrograms carbon BLANK FACTOR $=1.2 / 5.004517 \Rightarrow$ +2.4E-01 ug/min Carbon SAMPLE RESULTS : 


\section{WHESD-WM-DP-202, REV. 1 \\ HNF}

TOC- TOTAL ORGANIC CARBON ANALYSIS REPORT

TICTOC REV 2.0

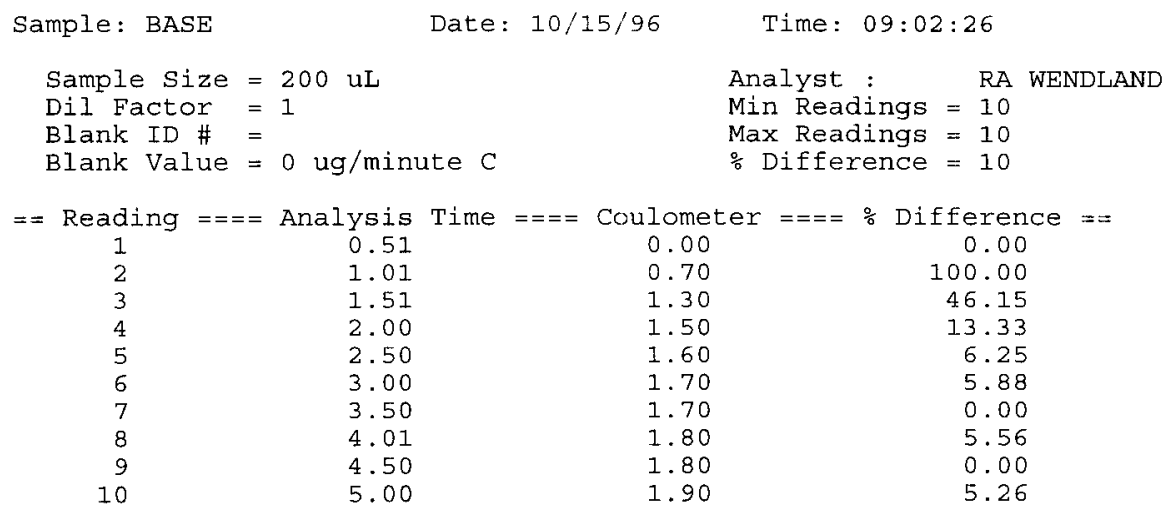

USER INPUT BLANK VALUE

BLANK VALUE $=0$ micrograms carbon

BLANK FACTOR $=0 / 0=$

$+0.0 \mathrm{E}+00 \mathrm{ug} / \mathrm{min}$ Carbon

SAMPLE RESULTS:

$\left(\begin{array}{l}1.9-0)(1) /(200) \\ 1.9-0)(1) /(200)(12)=\end{array}\right.$

+9.5E-03 g/L Carbon

$+7.9 \mathrm{E}-04$ Molar Carbon

Sample Run By:

RA WENDLAND 00000 


\section{HNF}

TOC- TOTAL ORGANIC CARBON ANALYSIS REPORT

TICTOC REV 2.0

$\ll$ BLANK ANALYSIS $\gg>$

Sample: BLK

Sample Size $=200 \mathrm{uL}$

Dil Factor $=1$

Blank ID \# = BIK

Blank Value $=\mathrm{N} / \mathrm{A}$
Date: $10 / 15 / 96$

Time: $09: 10: 36$

Analyst : RA WENDLAND

Min Readings $=10$

Max Readings $=10$

$\%$ Difference $=10$

$==$ Reading $====$ Analysis Time $==x$ Coulometer $===0 \%$ Difference $==$

$$
1
$$$$
2
$$

3

4

5

6

7

8

9

10
0.51

1.01

1.50

2.00

2.50

3.00

3.50

4.00

4.50

5.00
0.00

0.50

0.80

0.90

1.00

1.10

1.10

1. 20

1. 20

1. 20
0.00

100.00

37.50

11.11

10.00

9.09

0.00

8.33

0.00

0.00

BLANK VALUE $=1.2$ micrograms carbon

BLANK FACTOR $=1.2 / 5.004517=$

+2.4E-01 ug/min Carbon

Sample Run By:

RA WENDLAND 00000 
Sample: 48

Sample Size $=200 \mathrm{uL}$

Dil Factor $=11$

Blank ID \# = BLK

Blank Value $=.2397834 \mathrm{ug} / \mathrm{minute} \mathrm{C}$
Time: $10: 50: 19$

Analyst : RA WENDLAND

Min Readings $=10$

Max Readings $=10$

응 Difference $=10$

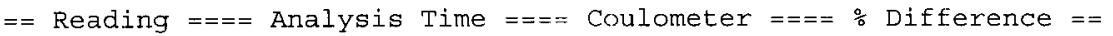

3

0.51

1.01

1.51

2.01

2.51

3.01

3.51

4.01

4.50

5.00
4.20

21.10

24.60

26.30

27.20

27.90

28.30

28.50

28.70

28.90
0.00

80.09

14.23

6.46

3.31

2. 51

1. 41

0.70

0.70

0.59

BLANK VALUE $=1.2$ micrograms carbon

BLANK FACTOR $=1.2 / 5.004517=$

$+2.4 \mathrm{E}-01 \mathrm{ug} / \mathrm{min}$ Carbon

SAMPLE RESULTS:

$(28.9-1.200044)(11) /(200)=$

$(28.9-1.200044)(11) /(200)(12)=$

$+1.52 \mathrm{E}+00$

9/L Carbon

$+1.27 E-01$ Molar Carbon

Sample Run By:

$\overline{R A}$ WENDLAND $\quad 00000$ 
TOC- TOTAL ORGANIC CARBON ANALYSIS REPORT

TICTOC REV 2.0

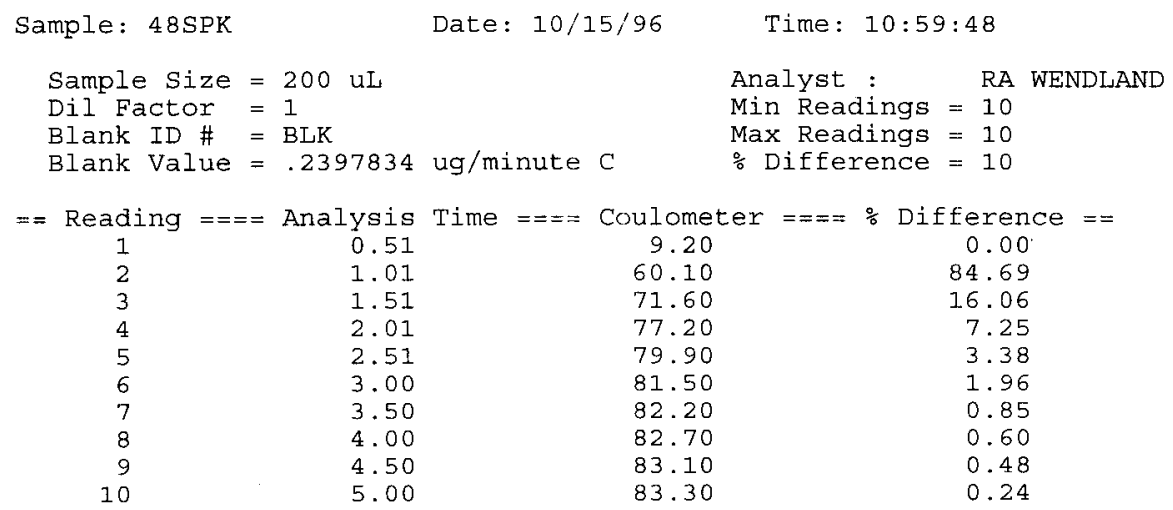

BLANK VALUE $=1.2$ micrograms carbon

BLANK FACTOR $=1.2 / 5.004517=\quad+2.4 \mathrm{E}-01 \quad \mathrm{ug} / \mathrm{min}$ Carbon

SAMPLE RESULTS:

$(83.3-1.19981)(1) /(200),(12)=$

$+4.11 \mathrm{E}-01 \mathrm{~g} / \mathrm{L}$ Carbon

$+3.42 E-02$ Molar Carbon

Sample Run By:

RA WENDLAND $\quad 00000$




\section{HNE-SD-WM-DH-202, REV. 1}

TOC- TOTAL ORGANIC CARBON ANALYSIS REPORT

TICTOC REV 2.0

Sample: 48DUPSPK

Dil Factor $=1$

Blank ID \# = BLK

Blank Value $=.2397834 \mathrm{ug} / \mathrm{minute} \mathrm{C}$
Sample Size $=200 \mathrm{uL}$

Date: $10 / 15 / 96$

Date: $10 / 15 / 96$

$==$ Reading $====$ Analysis Time $====$ Coul

$$
1
$$

2

3

4

5

6

7

8

9

10
0.51

1.01

1. 51

2.00

2. 50

3.00

3.50

4.00

4.50

5.00
9.80

61.90

70.80

75.40

78.10

79.90

81.00

81.80

82.30

82.80
Time: 11:06:58

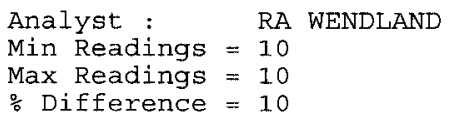




\section{HNF SO-WM-UP-202, REV. 1}

TOC- TOTAL ORGANIC CARBON ANALYSIS REPORT TICTOC REV 2.0

Sample: 49

Sample Size $=200 \mathrm{uL}$

Dil Factor $=11$

Blank ID \# = BLK

Blank Value $=.2397834 \mathrm{ug} /$ minute $\mathrm{C}$
Time: 13:01:59

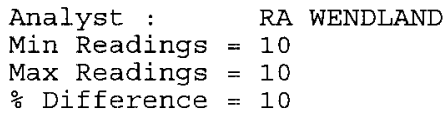

$==$ Reading $====$ Analysis Time $====$ Coulometer $====\div$ Difference $==$

$\begin{array}{rrrr}1 & 0.51 & 5.80 & 0.00 \\ 2 & 1.01 & 22.40 & 74.11 \\ 3 & 1.51 & 25.10 & 10.76 \\ 4 & 2.00 & 26.80 & 6.34 \\ 5 & 2.50 & 27.60 & 2.90 \\ 6 & 3.00 & 28.30 & 2.47 \\ 7 & 3.50 & 28.90 & 2.08 \\ 8 & 4.00 & 29.20 & 1.03 \\ 9 & 4.50 & 29.60 & 1.35 \\ 10 & 5.00 & 29.70 & 0.34\end{array}$

BLANK VALUE $=1.2$ micrograms carbon

BLANK FACTOR $=1.2 / 5.004517=$

+2.4E-01 ug/min Carbon

SAMPLE RESULTS:

$(29.7-1.199839)(11) /(200)=$

$(29.7-1.199839)(11) /(200)(12)=$

$+1.57 \mathrm{E}+00$

g/L Carbon

$+1.31 \mathrm{E}-01$

Molar Carbon

Sample Run By: 
TOC- TOTAL ORGANIC CARBON ANALYSIS REPORT

TICTOC REV 2.0

Sample: 47

Date: 10/15/96 Time: 10:11:59

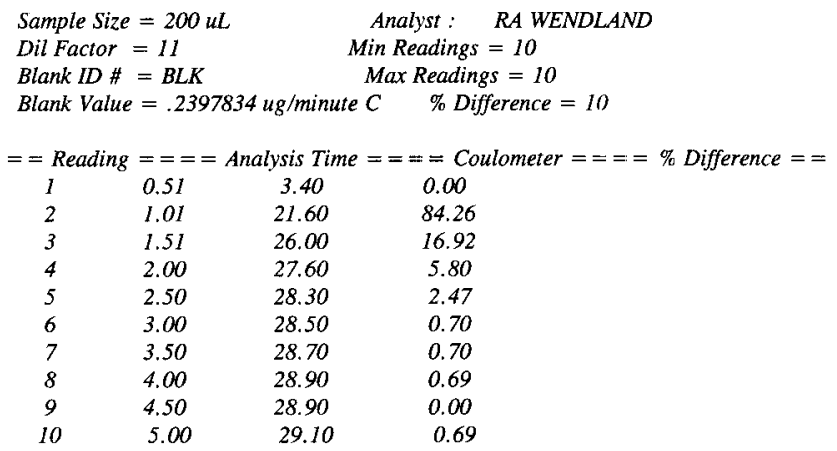

BLANK VALUE = 1.2 micrograms carbon

BLANK FACTOR $=1.2 / 5.004517=$

$+2.4 E-O I \quad$ ug/min Carbon

SAMPLE RESULTS:

$(29.1-1.200044)(11) /(200)=$

$+1.53 E+00 \quad \mathrm{~g} / \mathrm{L}$ Carbon

$(29.1-1.200044)(11) /(200)(12)=$

$+1.28 E \backsim 01$ Molar Carbon

Sample Run By:

RA WENDLAND

00000 
TOC- TOTAL ORGANIC CARBON ANALYSIS REPORT

TICTOC REV 2.0

Sample: 53

Date: 10/15/96 Time: 10:28:06

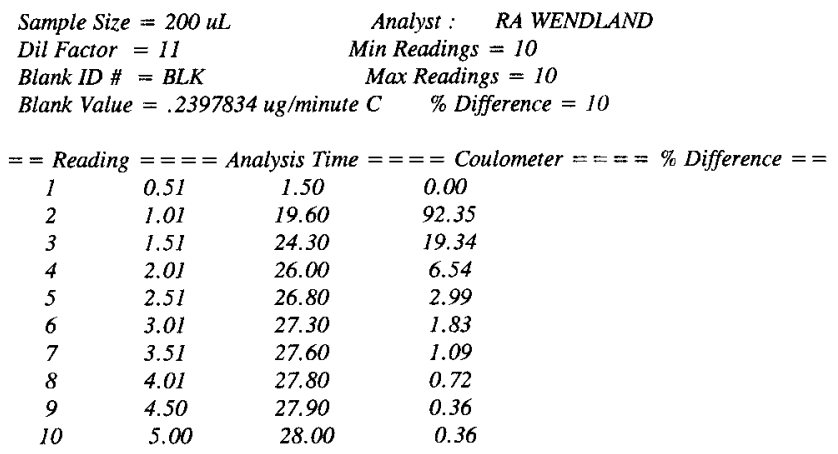

BLANK VALUE $=1.2$ micrograms carbon

$B L A N K$ FACTOR $=1.2 / 5.004517=$

$+2.4 E-01 \quad u g / \min$ Carbon

SAMPLE RESULTS:

$(28-1.200044)(11) /(200)=$

$(28-1.200044)(11) /(200)(12)=$

$+1.47 E+00 \quad g / L$ Carbon

$+1.23 E-01$ Molar Carbon

Sample Run By:

$R A$ WENDLAND

00000 


\section{HNFE-SD-WM-DP-202, REV. 1}

TOC- TOTAL ORGANIC CARBON ANALYSIS REPORT

TICTOC REV 2.0

Sample: 58

Sample Size $=200 \mathrm{uL}$

Dil Factor $=1.1$

Blank ID \# = BLK

Blank Value $=.2397834 \mathrm{ug} /$ minute $\mathrm{C}$
Date: $10 / 15 / 96$

Date: $10 / 15 / 96$

$==$ Reading $====$ Analysis Time $=== \pm$ Coul

$$
1
$$$$
2
$$

3

4

5

6

7

8

9

10
0.51

1. 01

1.51

2.01

2. 50

3.00

3.50

4.01

4.50

5.00
Time: 10:05:17

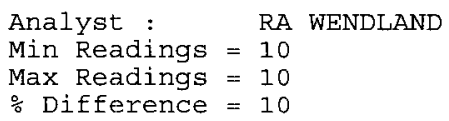

ulometer $====$ : Difference $==$

$$
0.40
$$

1.40

2.50

2.90

2.90

3.10

3. 10

3.20

3.30

3.30
0.00

71.43

44.00

13.79

0.00

6.45

0.00

3.13

3.03

0.00

BLANK VALUE $=1.2$ micrograms carbon

BLANK FACTOR $=1.2 / 5.004517=$

$+2.4 \mathrm{E}-01 \mathrm{ug} / \mathrm{min}$ Carbon

SAMPLE RESULTS:

$(3.3-1.200044)(1.1) /(200)=$

$+1.2 \mathrm{E}-02$

9/L Carbon

$(3.3-1.200044)(1.1) /(200)(12)=$

$+9.6 \mathrm{E}-04$

Molar Carbon

Sample Run By:

RA WENDLAND 00000

\section{1}




\section{HNF SD-WM-DP-202, REV. 1}

WORKBOOK PAGE: BLANK1

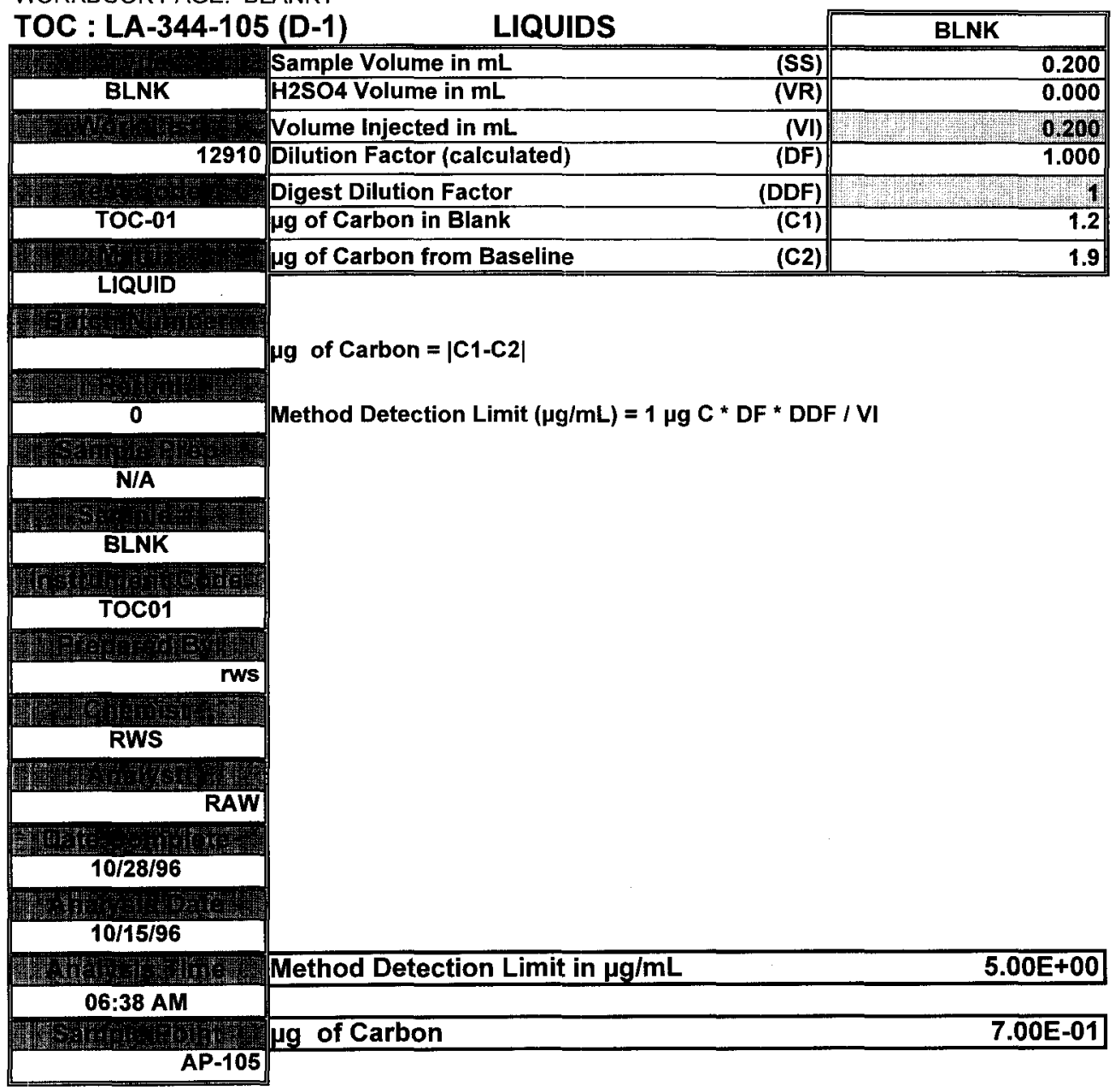

\begin{tabular}{|c|c|c|c|}
\hline Data Entered By: & rws & Date: & $10 / 28 / 96$ \\
\hline Signature of Chemist: & 8 & Date: & $10 / 28 / 96$ \\
\hline
\end{tabular}


WORKBOOK PAGE: STD2

TOC : LA-344-105 (D-1)

LIQUIDS

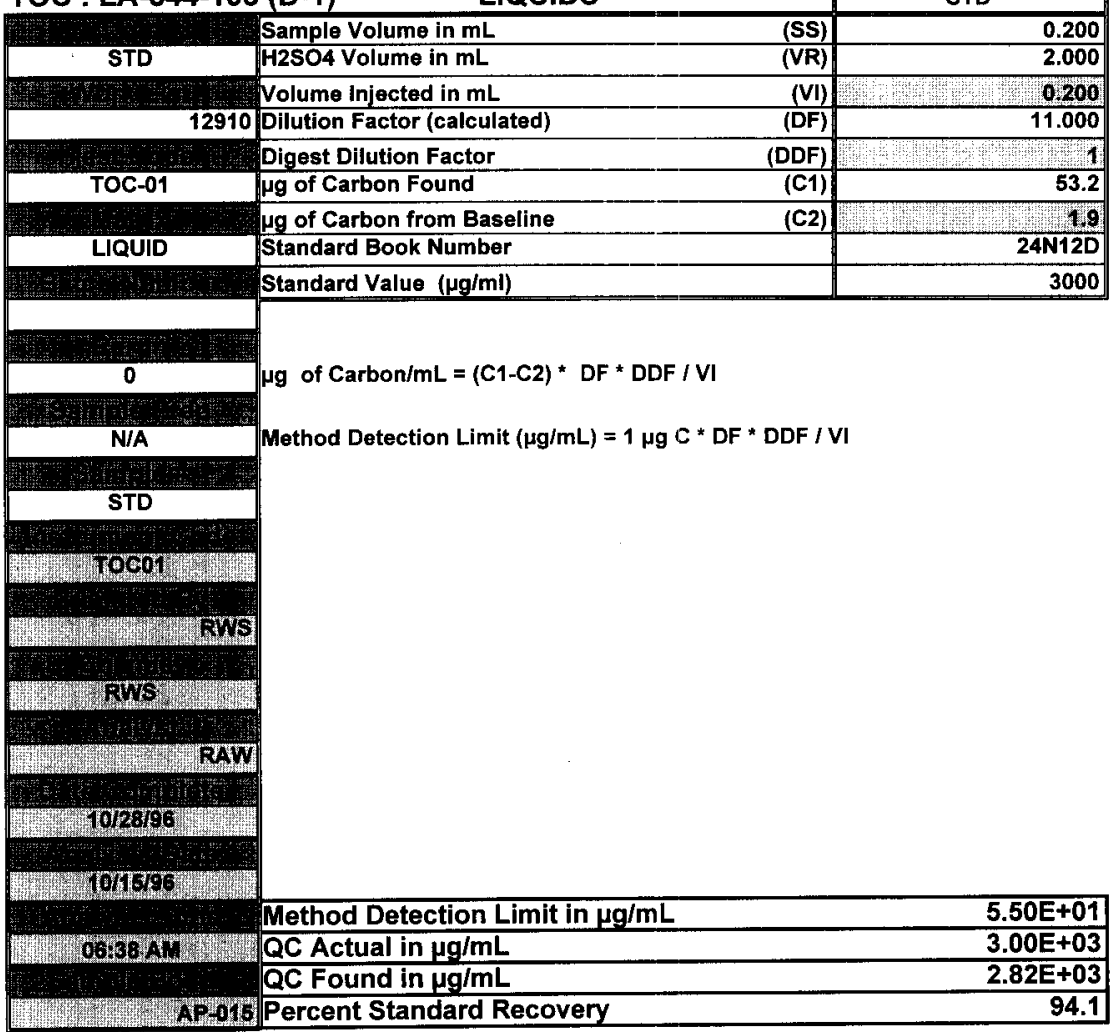

\begin{tabular}{|llll||}
\hline Data Entered By: & RWS & Date: & $10 / 28 / 96$ \\
\hline Signature of Chemist: & 344105ML & \\
\hline STANDARD WB1 REV 2.0 & &
\end{tabular}

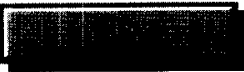

\section{3}




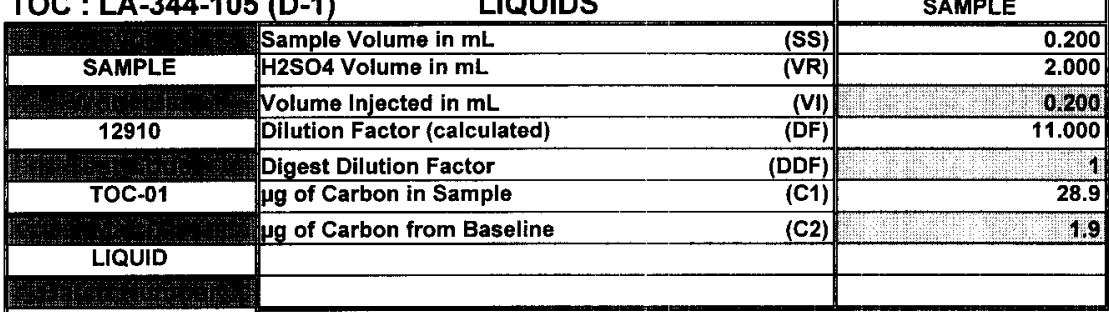

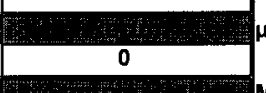

$\mu g$ of Carbon/g $=(C 1-C 2) * D F * 1000 /(V I * D g / L)$

Method Detection Limit $=1 \mu \mathrm{g} \mathrm{C} \mathrm{*} \mathrm{DF} \mathrm{*} \mathrm{DDF} \mathrm{/} \mathrm{VI}$

\section{N/A \\ $\frac{1}{596 \mathrm{~V} 000048}$}

Woor The

W RW RW

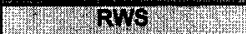

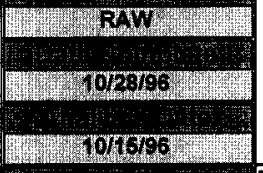

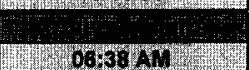

Method Detection Limit in $\mu \mathrm{g} / \mathrm{mL}$

\section{ug of Carbon $/ \mathrm{mL}$}

$1.48 \mathrm{E}+03$

\section{An 100}

\section{Data Entered By:}

Signature of Chemist:

\section{RWS}

xid

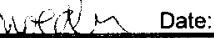

344105ML
Date: $\quad 10 / 28 / 96$ $16 / 28 / 46$ 
WORKBOOK PAGE: SPIKE5

TOC : LA-344-105 (D-1)

LIQUIDS

SPIKE

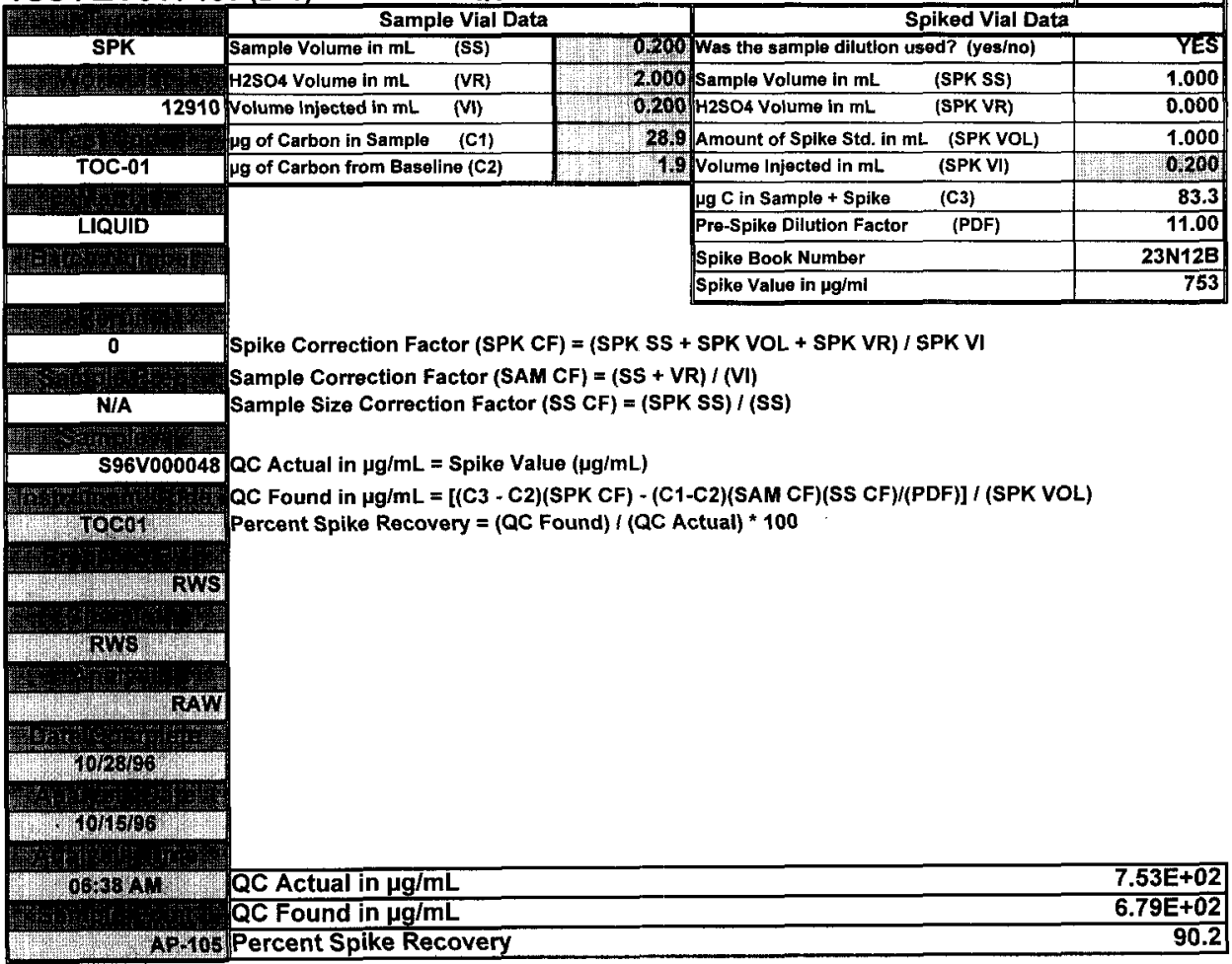

Data Entered By:

Signature of Chemist:

SPIKE.WB1 REV 2.0
RWS

Bu

$344105 \mathrm{ML}$
Date: $10 / 28 / 96$

Date: $16 / 28 / 96$

\section{5}


WORKBOOK PAGE: SPIKE5

$H N F$

WHE-SD-WM-LP-202, REV

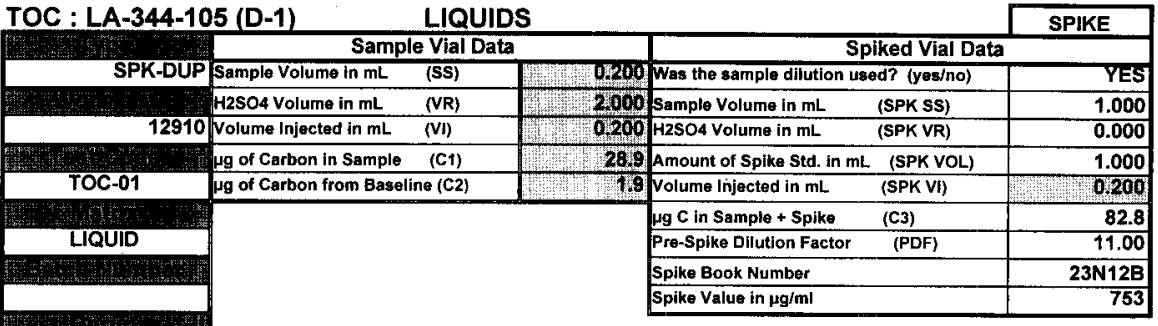

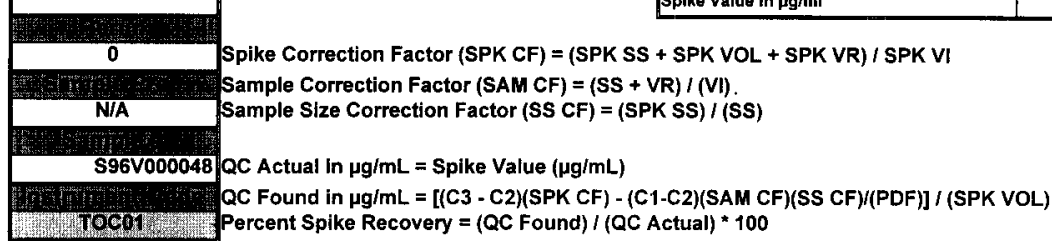

WWING

Wintring

WWrWWXW

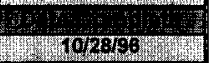

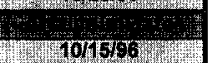

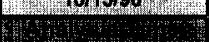

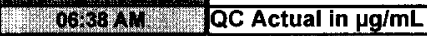

QC Found in $\mu \mathrm{g} / \mathrm{mL}$

$7.53 \mathrm{E}+02$

$6.74 E+02$

Drtrutwy

\begin{tabular}{|c|c|c|c|}
\hline Data Entered By: & RWS & Date: & $10 / 28 / 96$ \\
\hline Signature of Chemist: & Ge? & Date: & $10 / 28 / 9$ \\
\hline
\end{tabular}

\section{6}


HNF

ANG-SD-WM-LH-202, REV. ?

WORKBOOK PAGE: SAM3

TOC : LA-344-105 (D-1)

LIQUIDS

\begin{tabular}{|c|c|c|c|}
\hline & & & - \\
\hline SAMPLE & $\begin{array}{l}\text { Sample Volume in } \mathrm{mL} \\
\mathrm{H} 2 \mathrm{SO} \text {. Volume in } \mathrm{mL}\end{array}$ & (SS) & $\frac{0.200}{2.000}$ \\
\hline 12910 & \begin{tabular}{|l} 
Volume Injected in $\mathrm{mL}$ \\
Dilution Factor (calculated)
\end{tabular} & (VI) & Ure \\
\hline & Digest Dilution Factor & (DDF) & 1 \\
\hline TOC- & $\mathrm{g}$ of Carbon in Sample & (C1) & 29.7 \\
\hline & $\mu \mathrm{g}$ of Carbon from Baseline & (C2) & एका \\
\hline & & & \\
\hline & & & \\
\hline
\end{tabular}

0

$\mu g$ of Carbon/g $=(C 1-C 2) * D F * 1000 /(V I * D$ g/L $)$

Method Detection Limit $=1 \mu \mathrm{g} \mathrm{C} \mathrm{*} \mathrm{DF} \mathrm{*} \mathrm{DDF} \mathrm{/} \mathrm{VI}$

N/A

S96V000049

$4+1$

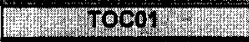

What

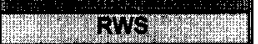

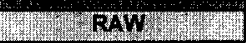

W.1.

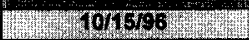

Method Detection Limit in $\mu \mathrm{g} / \mathrm{mL}$

$5.50 \mathrm{E}+01$

1. $0033 \mathrm{~g}$ WW

ug of Carbon/mL

$1.53 E+03$

XP103:

(1)

\begin{tabular}{|c|c|c|c|}
\hline Data Entered By: & RWS & Date: & $10 / 28 / 96$ \\
\hline Signature of Chemist: & 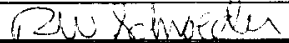 & Date: & $10 / 26 / 96$ \\
\hline
\end{tabular}

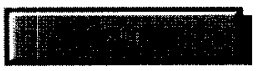


WORKBOOK PAGE: SAM3

TOC : LA-344-105 (D-1)

LIQUIDS

\begin{tabular}{|c|c|c|c|}
\hline IOC : LA-344-105 & LIQUI & & SAMPLE \\
\hline & Sample Volume in $\mathrm{mL}$ & (SS) & 0.200 \\
\hline SAMPLE & $\mathrm{H} 2 \mathrm{SO} 4$ Volume in $\mathrm{mL}$ & (VR) & 2.000 \\
\hline & Volume Injected in $\mathrm{mL}$ & (VI) & Hothy \\
\hline 129 & Dilution Factor (calculated) & (DF) & 11.000 \\
\hline & Digest Dilution Factor & (DDF) & 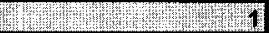 \\
\hline TOC & $\mu g$ of Carbon in Sample & (C1) & 29.1 \\
\hline & g of Carbon from Baseline & (C2) & W \\
\hline LI & & & \\
\hline & & & \\
\hline
\end{tabular}

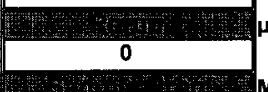

$\mathrm{ug}$ of Carbon/g $=(\mathrm{C} 1-\mathrm{C} 2) * \mathrm{DF} * 1000 /(\mathrm{VI}$ * $\mathrm{D} \mathrm{g} / \mathrm{L})$

Method Detection Limit $=1 \mu \mathrm{g} \mathrm{C}$ * DF * DDF / VI

\begin{tabular}{|l} 
N/A \\
S96V000047
\end{tabular}

16.

-

W.1. WW

(1)

H:-

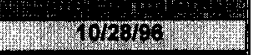

How

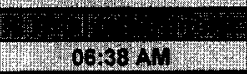

Method Detection Limit in $\mu \mathrm{g} / \mathrm{mL}$

$5.50 \mathrm{E}+01$

7WW AP 106

ug of Carbon $/ \mathrm{mL}$

$1.50 \mathrm{E}+03$

\begin{tabular}{|llll|}
\hline Data Entered By: & RWS & Date: & $10 / 28 / 96$ \\
\hline Signature of Chemist: & 344105ML & Date: $16 / 28 / 66$ \\
\hline SAMPLE. WB1 REV 2.0
\end{tabular}

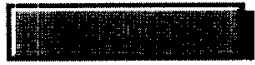




\section{HNF-SD-WM-DF-202, REV. 1}

WORKBOOK PAGE: SAM3

TOC : LA-344-105 (D-1)

\section{LIQUIDS}

SAMPLE

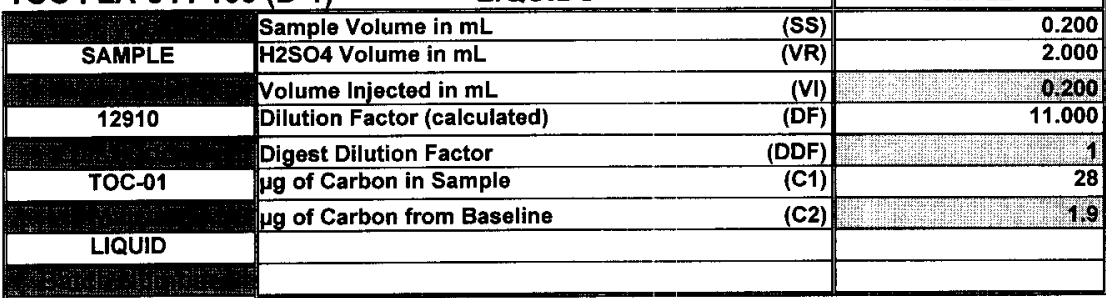

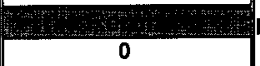

$\mu g$ of Carbon $/ g=(C 1-C 2) * D F * 1000 /(V) * D$ g/L $)$

$\frac{\text { N/A }}{\text { N/A }}$

Method Detection Limit $=1 \mu \mathrm{g} \mathrm{C}{ }^{*} \mathrm{DF}{ }^{*} \mathrm{DDF} / \mathrm{VI}$

S96V000053

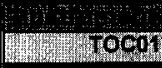

RWS

(1)

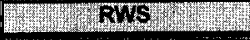

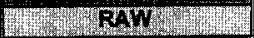

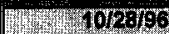

W 107 HF 6

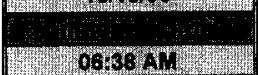

Method Detection Limit in $\mu \mathrm{g} / \mathrm{mL}$

$5.50 \mathrm{E}+01$

$\mu \mathrm{g}$ of Carbon/mL

$1.44 \mathrm{E}+03$

APTOS

\begin{tabular}{|c|c|c|c|}
\hline Data Entered By: & RWS & Date: & $10 / 28 / 96$ \\
\hline Signature of Chemist: & & Date: & \\
\hline
\end{tabular}


HNE-SD-WM-DP-202, REV. 1

WORKBOOK PAGE: SAM3

TOC : LA-344-105 (D-1)

LIQUIDS

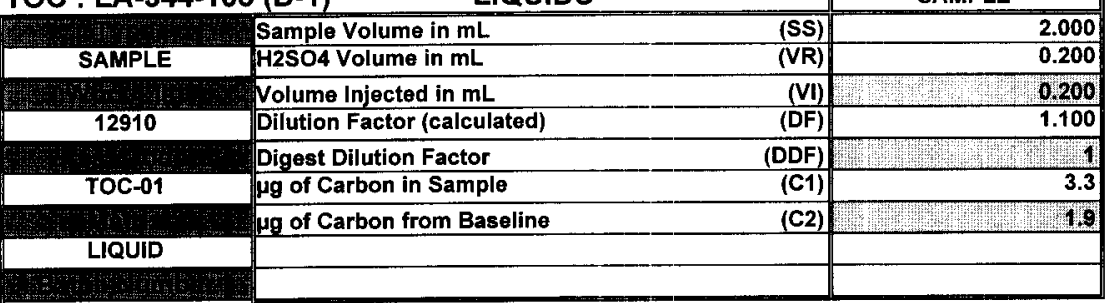

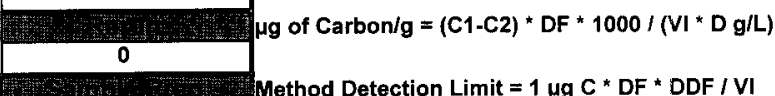

N/A

S96V000058

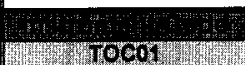

404030

W RWS

30

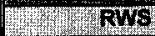

3.

WW RAW

Hown

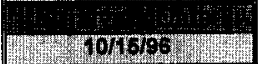

Method Detection Limit in $\mu \mathrm{g} / \mathrm{mL}$

5.50E+00

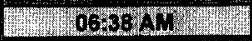

$4.4 \mathrm{pg}$ of Carbon $/ \mathbf{m L}$

$7.70 \mathrm{E}+00$

APt 105

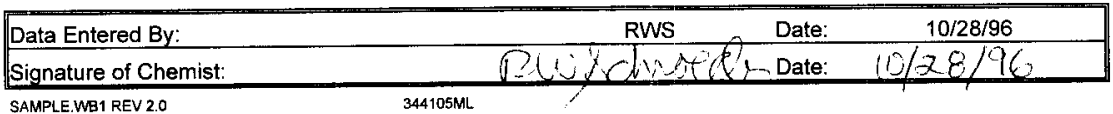

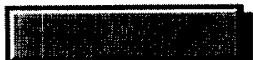




\section{LABCORE Data Entry Template for Worklist\# 12909}

\section{Analyst: $\quad$ Jis 2 \\ Instrument: TICOI $\mathbb{Z}_{2}$ \\ Book \#22N12F}

Method: LA-342-100 Rev/Mod C.C

Worklist Comment: AP-105 TIC. RCJ

\begin{tabular}{|c|c|c|c|c|c|c|c|c|c|c|c|}
\hline \multirow[t]{2}{*}{ GROUP } & PROJECT & & $\begin{array}{l}\text { TYPE } \\
\text { BLNK }\end{array}$ & SAMPLE\# & R A & $\begin{array}{c}\text { TIC }-02 \\
\text { T }\end{array}$ & $\begin{array}{l}\text { MATRIX } \\
\text { LIQUID }\end{array}$ & $\begin{array}{c}\text { ACTUAL } \\
\end{array}$ & $\begin{array}{l}\text { FOUND } \\
1.90 \\
\end{array}$ & $\begin{array}{r}\mathrm{DL} \\
\mathrm{N} / \mathrm{A} \\
\end{array}$ & $\begin{array}{l}\text { UNIT } \\
\mathrm{ug} / \mathrm{mL}\end{array}$ \\
\hline & & 2 & STD & & & $T[C-02$ & LIQU1D & $6.02^{27}$ & $5.94^{\circ}$ & $e^{2}{ }^{2} / \mathrm{A}$ & $\mathrm{ug} / \mathrm{mL}$ \\
\hline 96000855 & AP- 105 & 3 & SAMPLE & s $96 \mathrm{~V} 000048$ & 0 & TIC-02 & LIQUID & N/A & $275^{\mathrm{c3}}$ & 5 & $\mathrm{ug} / \mathrm{mL}$ \\
\hline 96000855 & AP- 105 & 4 & SPK & $596 \mathrm{~V} 000048$ & 0 & $\mathrm{~T}$ I C-02 & LIQUID & $\frac{2.78}{2+213}$ & $\frac{105.3}{174 c}$ & N/A & $\mathrm{ug} / \mathrm{mL}$ \\
\hline 96000855 & AP- 105 & 5 & SPK-DUP & $596 \mathrm{~V} 000048$ & 0 & TIC-02 & LIQUID & 1000 & 108 & $\mathrm{~N} / \mathrm{A}$ & $\mathrm{ug} / \mathrm{mL}$ \\
\hline 961000855 & AP -105 & 6 & SAMPLE & $596 v 000049$ & 0 & TIC-02 & LIQUID & N/A & $251^{23}$ & 5 & $\mathrm{ug} / \mathrm{mL}$ \\
\hline 96000855 & AP- 105 & 7 & SPK & $596 v 000049$ & 0 & $\mathrm{TIC}-\mathrm{O} 2$ & LIQUID & 100 & 102.6 & $N / A$ & $\mathrm{ug} / \mathrm{mL}$ \\
\hline 96000853 & $A P-105$ & 8 & SAMPLE & S96V000047 & 0 & $\mathrm{TIC}-02$ & LIQUID & N/A & $2.74^{\circ 3}$ & 5 & $\mathrm{ug} / \mathrm{mL}$ \\
\hline 96000853 & $A P-105$ & 9 & SPK & s96V000047 & 0 & TIC-CIZ & LIQUID & 100 & 100.6 & N/A & $\mathrm{ug} / \mathrm{mL}$ \\
\hline 96000855 & $A P-105$ & 10 & SAMPLE & $596 \mathrm{~V} 000058$ & 0 & TIC-02 & LIQUID & N/A & $7.00^{\circ}$ & $s$ & $\mathrm{ug} / \mathrm{mL}$ \\
\hline 96000855 & $A P-105$ & 19 & SPK & \$96V000058 & 0 & IIC-C12 & LIOUID & 100 & 101.7 & N/A & $\mathrm{ug} / \mathrm{mL}$ \\
\hline
\end{tabular}

\section{Final page for worklist \# 12909}
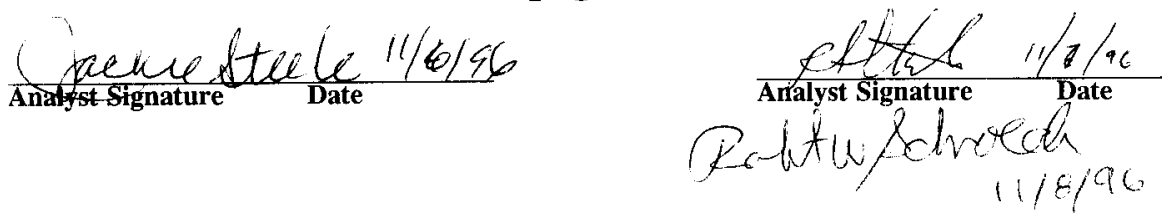

Data Entry Comments:

Units shown for $Q C$ (SPK \& STD) may not reflect the actual units. DL = Detection Limit, $S=$ Worklist Slot Number, $R=$ Replicate Number, $A=$ Aliquot Code . 
WHHE-SD-WNI-DP-202, REV. 1

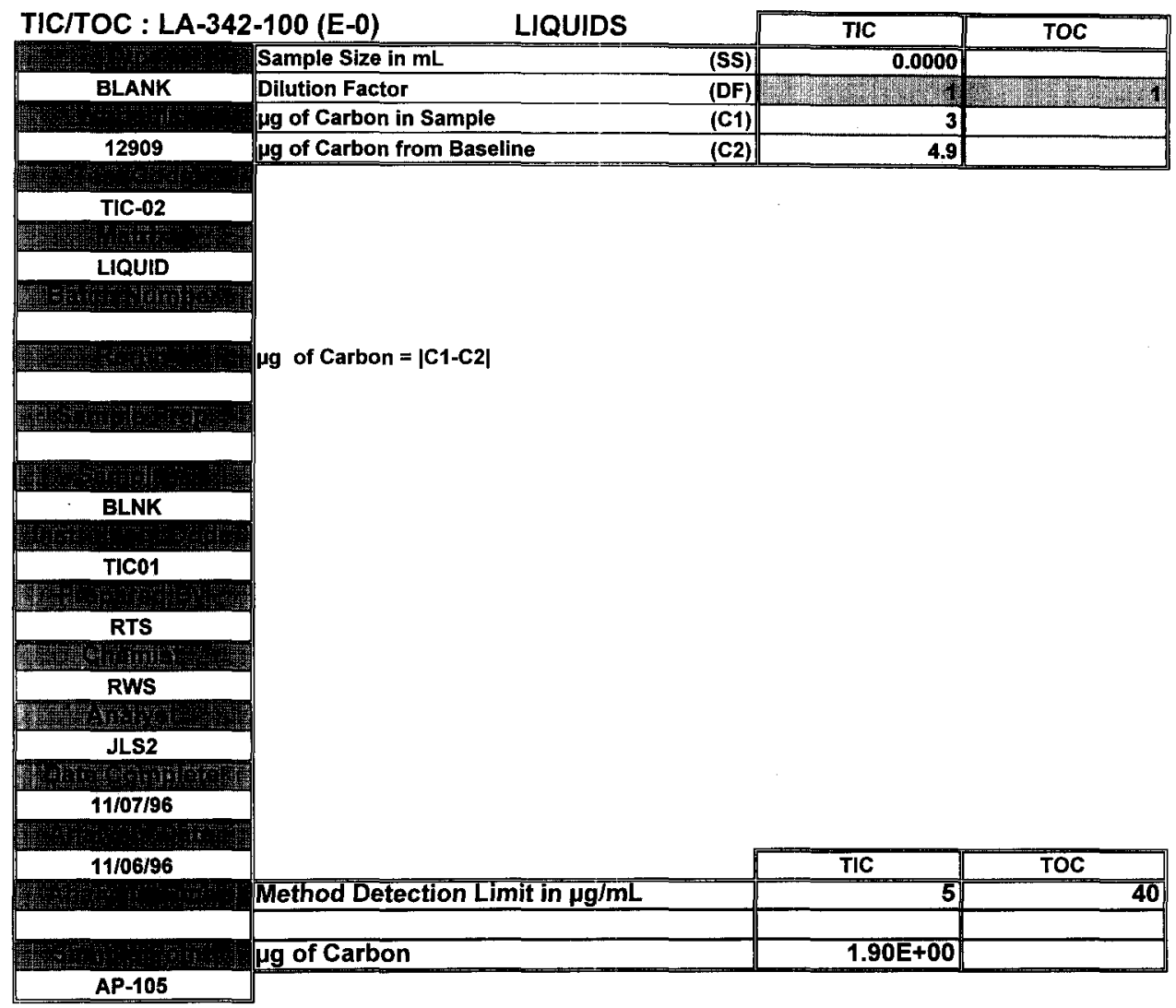

Data Entered By:

Signature of Chemist:

BLANK.WB1 REV 1.0
Dilution Factor

$\mu \mathrm{g}$ of Carbon in Sample

pg of Carbon from Baseline

pg of Carbon $=|\mathrm{C} 1-\mathrm{C} 2|$
(DF)

(C2) 


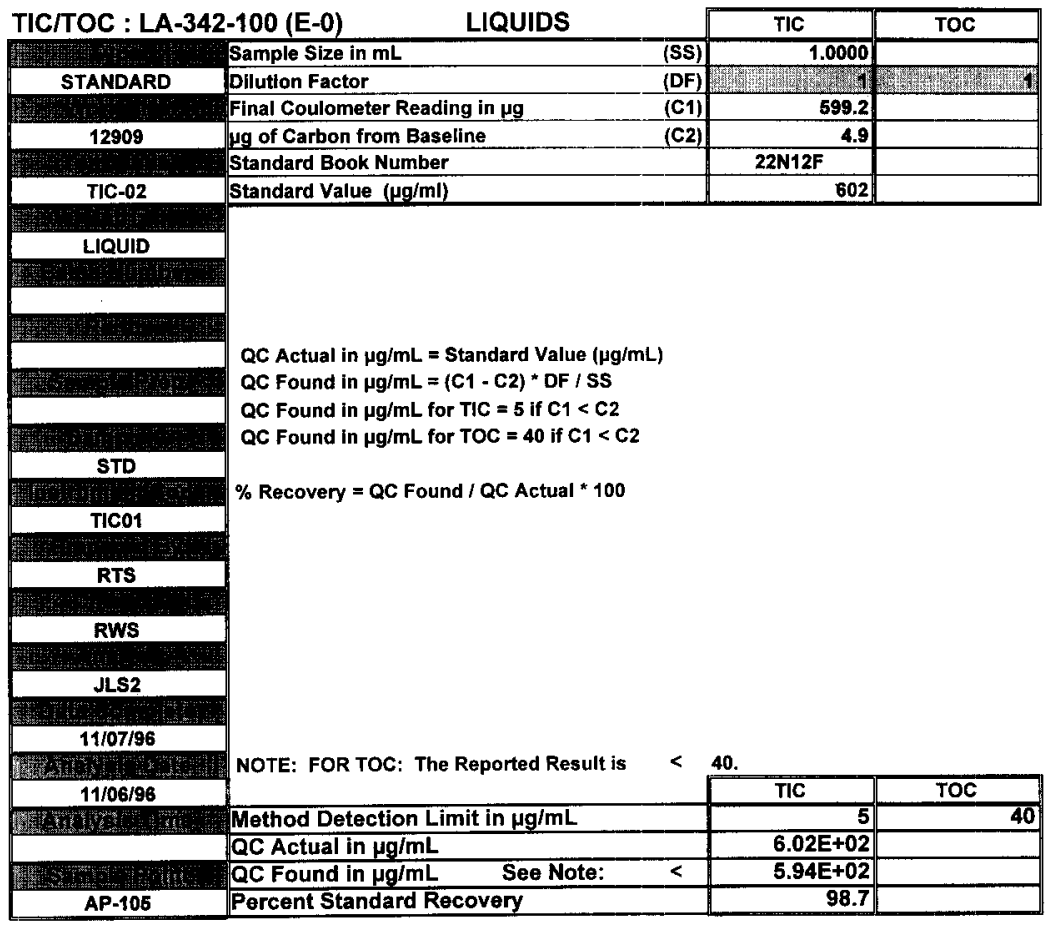

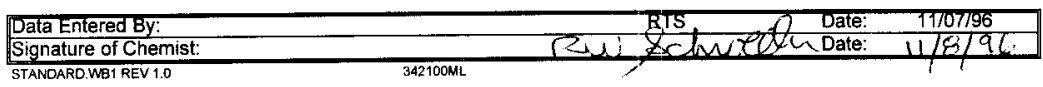




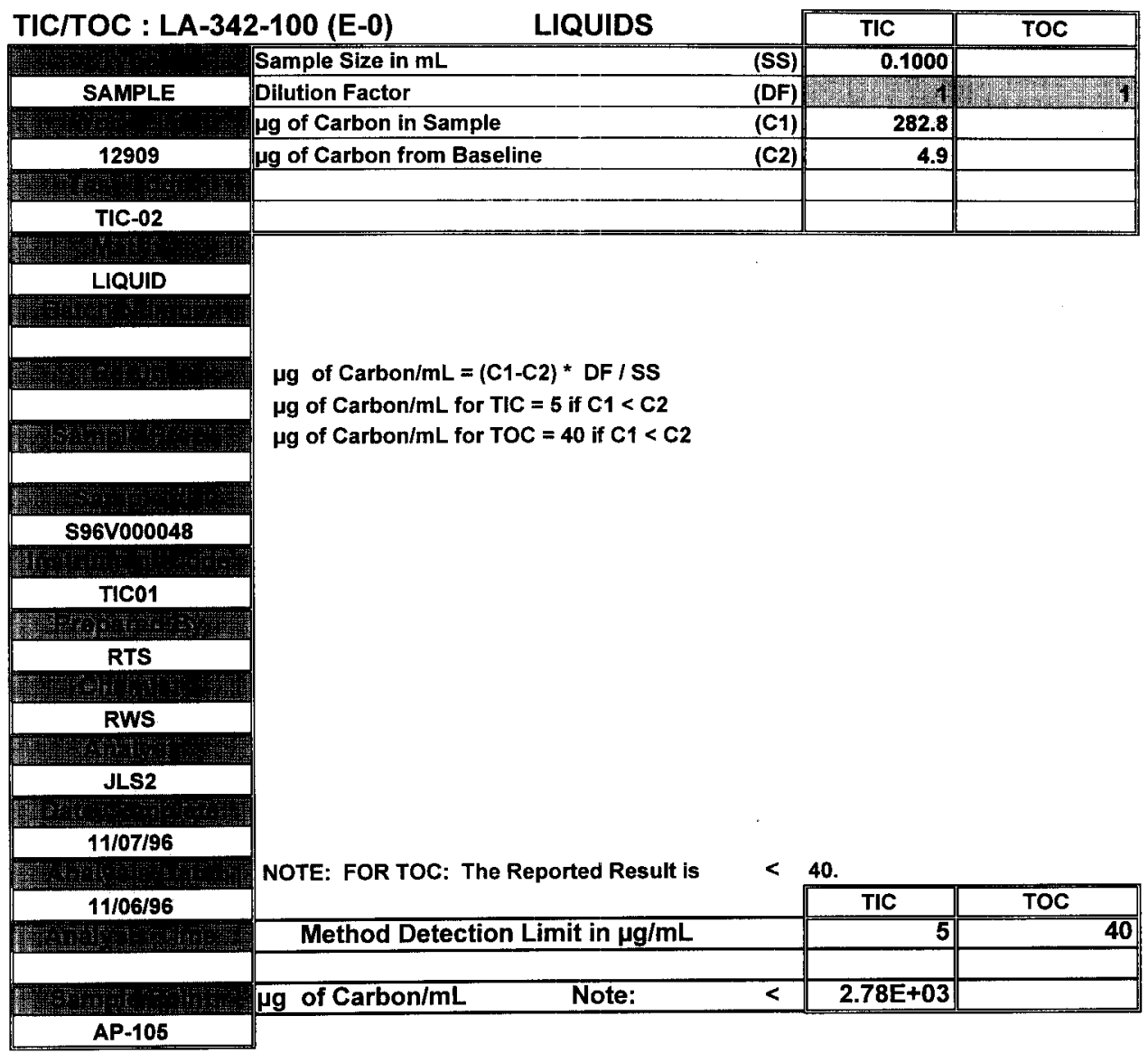

\begin{tabular}{|c|c|c|c|}
\hline Data Entered By: & RTS & Date: & $11 / 07 / 96$ \\
\hline Signature of Chemist: & Deu $8 \mathrm{ch}$ esth & Date: & $11 / 8 / 90$ \\
\hline
\end{tabular}


TIC/TOC : LA-342-100 (E-0)

LIQUIDS

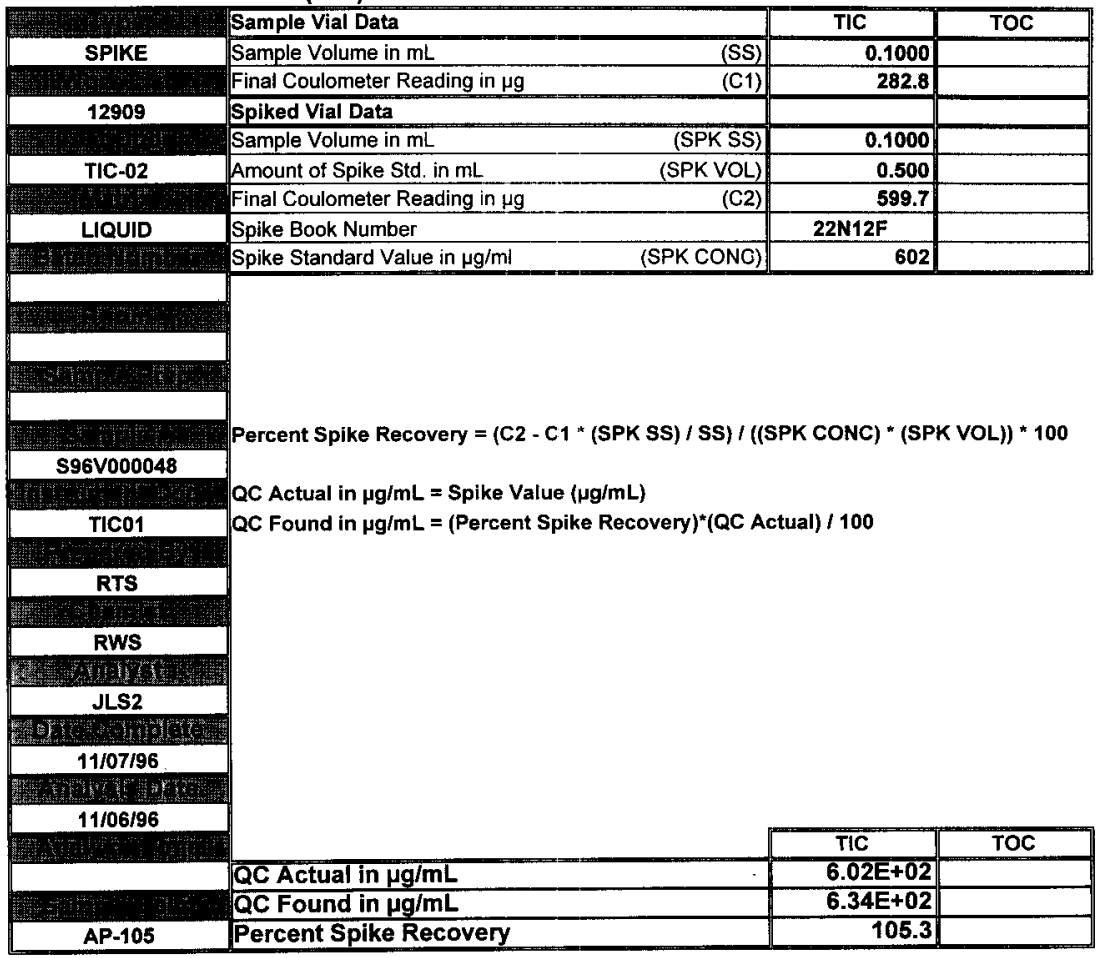

\begin{tabular}{|c|c|c|}
\hline Data Entered By: & RTS & Date: $\quad 11 / 07 / 96$ \\
\hline Signature of Chemist: & & Date: $1 / 8 / 96$ \\
\hline
\end{tabular}


TIC/TOC : LA-342-100 (E-0)

LIQUIDS

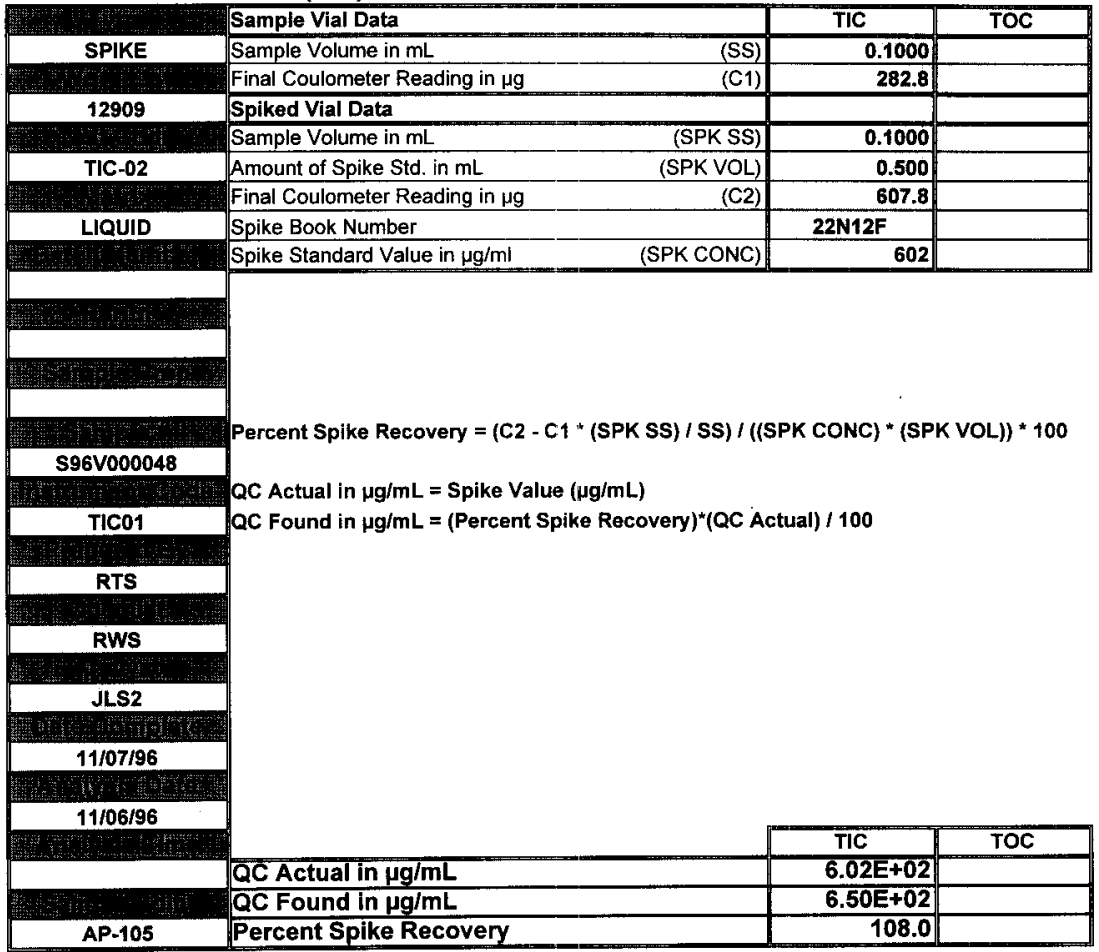

\begin{tabular}{|lll|}
\hline Data Entered By: & RTS & Date: $11 / 07 / 96$ \\
\hline Signature of Chemist: & 342100ML & Date: $11 / 8_{3} / 96$ \\
\hline SPIKE WB1 REV 1.1
\end{tabular}

\section{6}




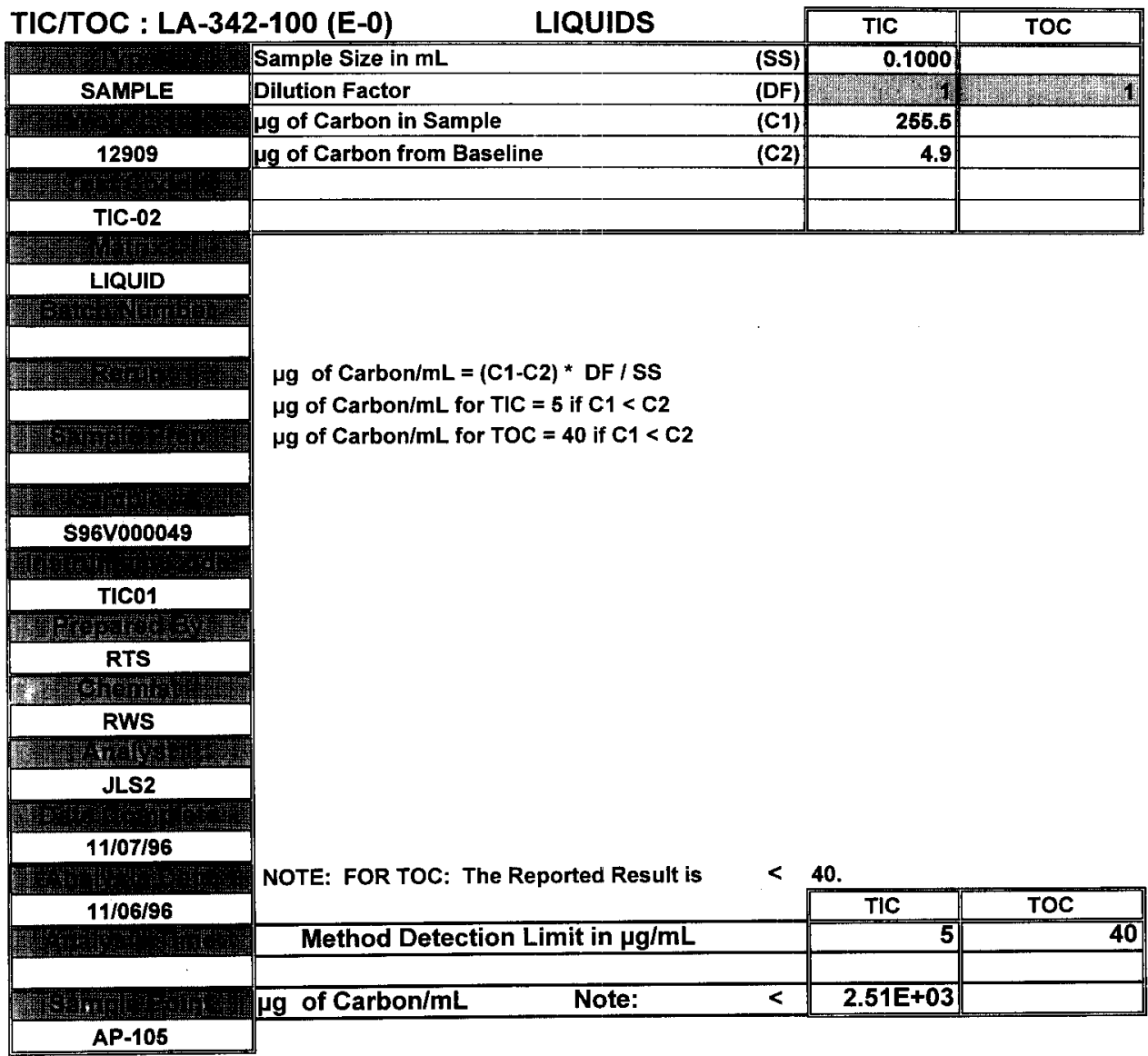

\begin{tabular}{|llll|}
\hline Data Entered By: & & RTS & Date: $11 / 07 / 96$ \\
\hline Signature of Chemist: & Rug & Date: $11 / 8 / 96$ \\
\hline SAMPLE WB1 REV 1.0
\end{tabular}

\section{7}


TIC/TOC : LA-342-100 (E-0)

LIQUIDS

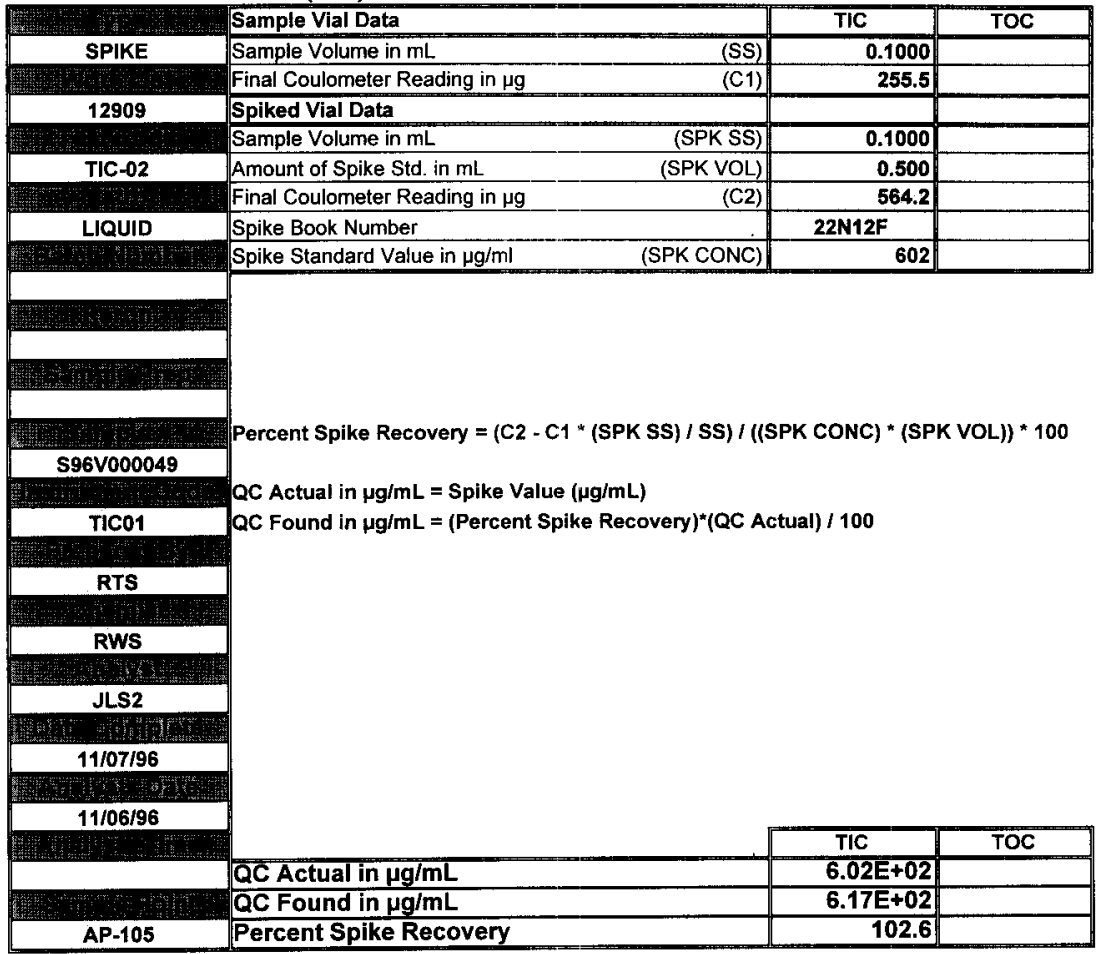

\begin{tabular}{|c|c|c|c|}
\hline Data Entered By: & RTS & Date: & $11 / 07 / 96$ \\
\hline Signature of Chemist: & Xdinote & Date: & $118 / 96$ \\
\hline
\end{tabular}

\section{8}




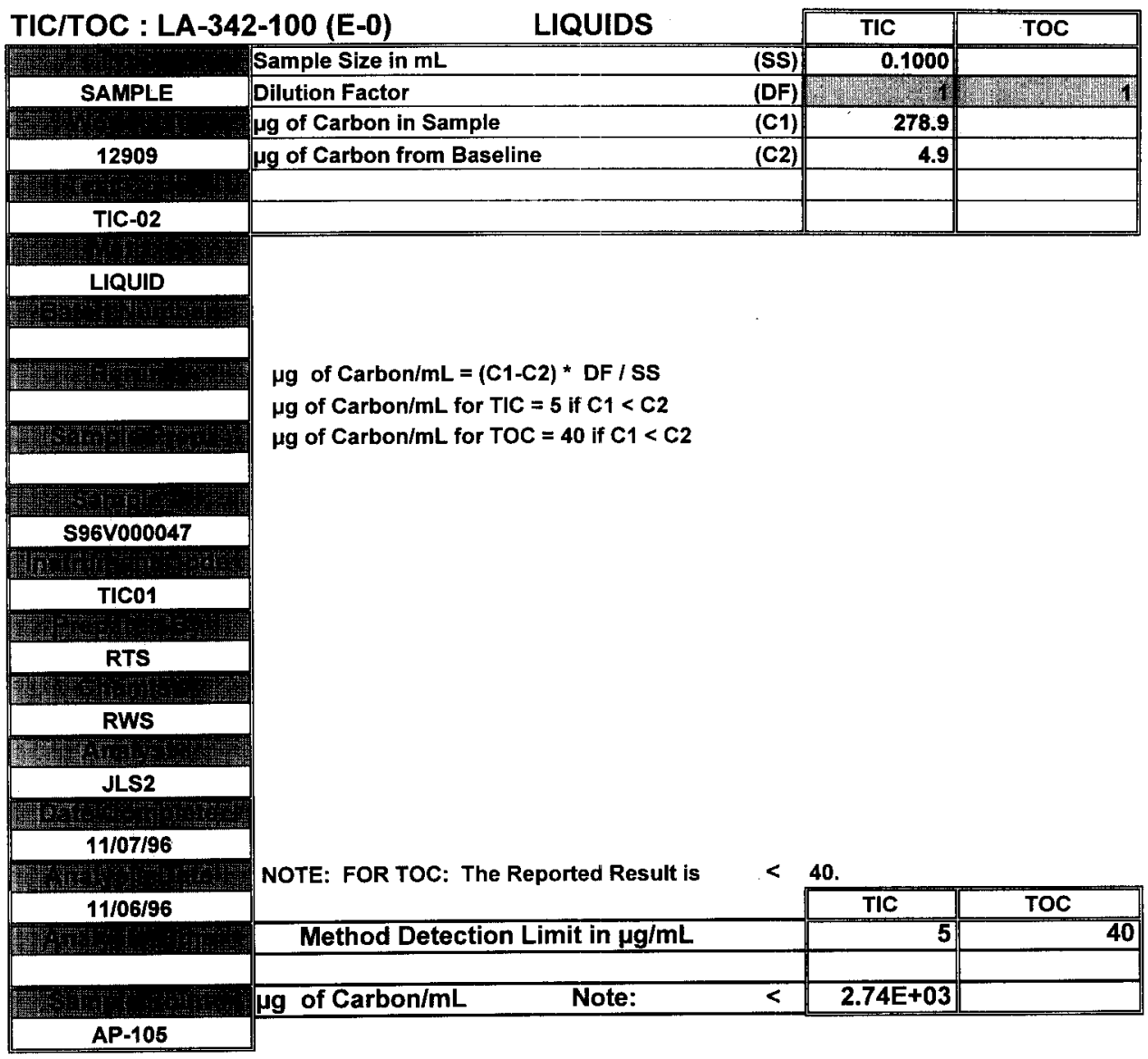

\begin{tabular}{|c|c|c|c|}
\hline Data Entered By: & RTS & Date: & $11 / 07 / 96$ \\
\hline Signature of Chemist: & sex & Date: & 118196 \\
\hline
\end{tabular}

\section{9}




\section{HNF-SD-WM-DP-202, REV. 1}

TIC/TOC : LA-342-100 (E-0)

LIQUIDS

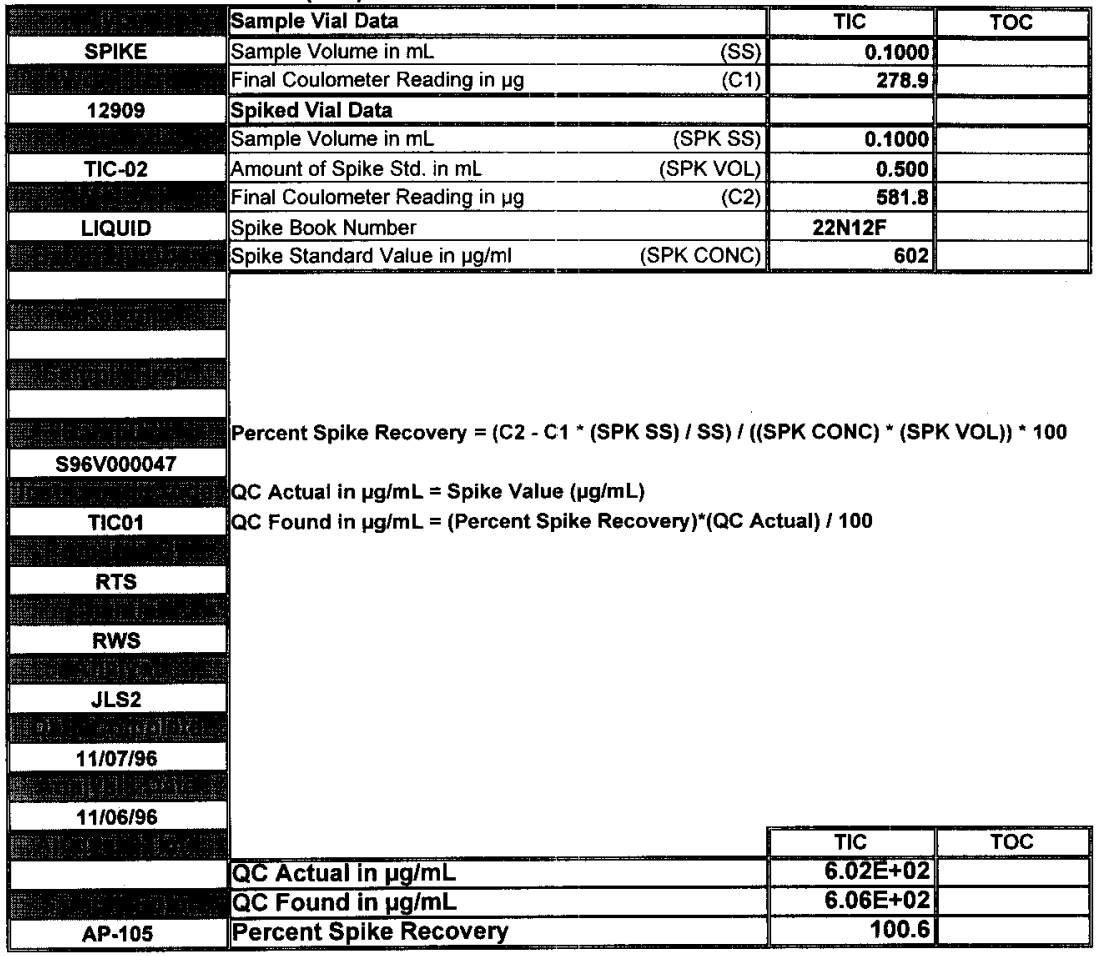

\begin{tabular}{l}
\hline Data Entered By: \\
\hline Signature of Chemist: \\
SPIKE.WB1 REV 1.1
\end{tabular}

\section{0}


HG-SD-WM-DP-202, REV. 1

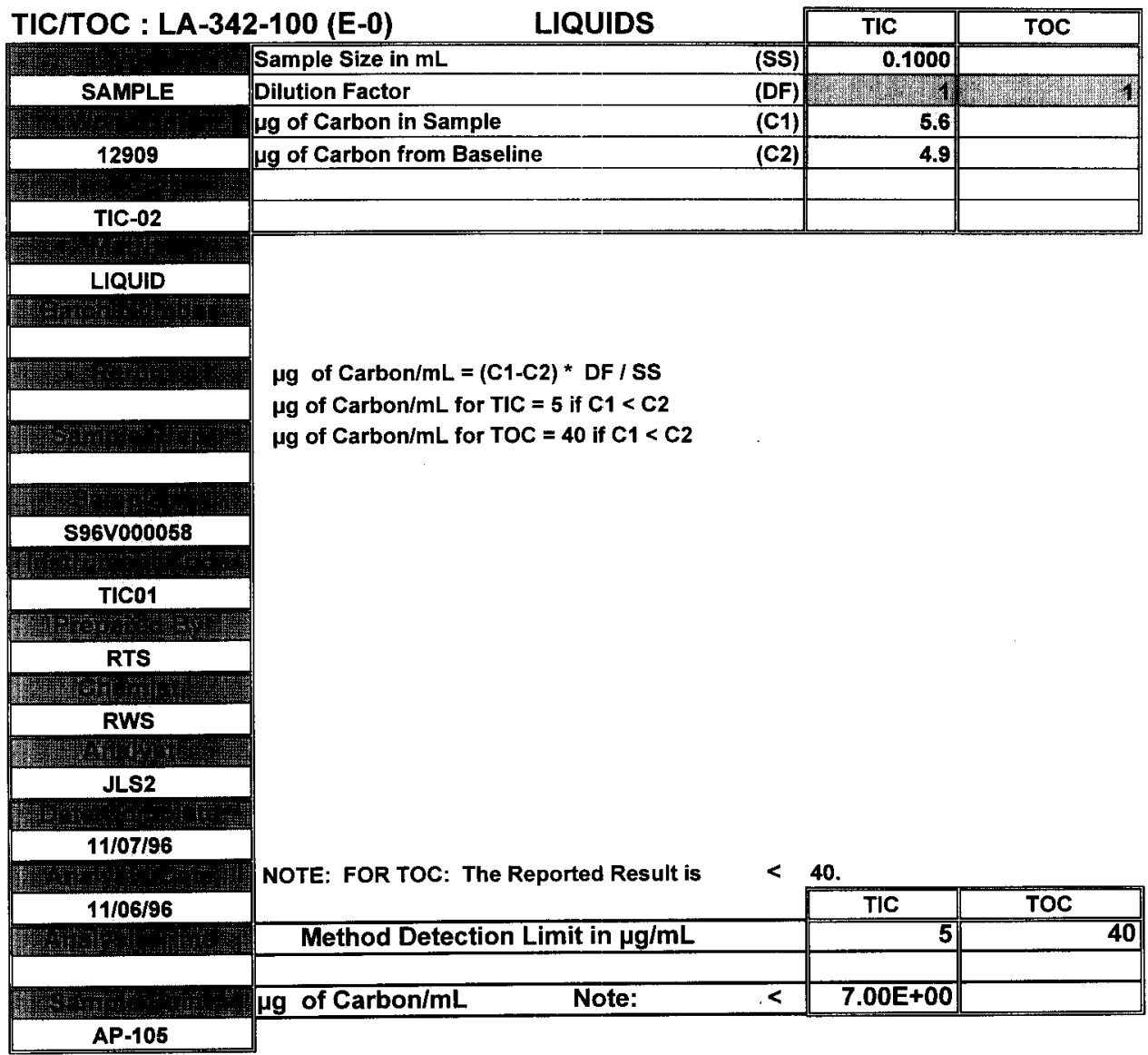

\begin{tabular}{|c|c|c|}
\hline Data Entered By: & RTS & $11 / 07 / 96$ \\
\hline Signature of Chemist: & ins anceln & Date: $\quad 11 / 9 / 91$ \\
\hline
\end{tabular}




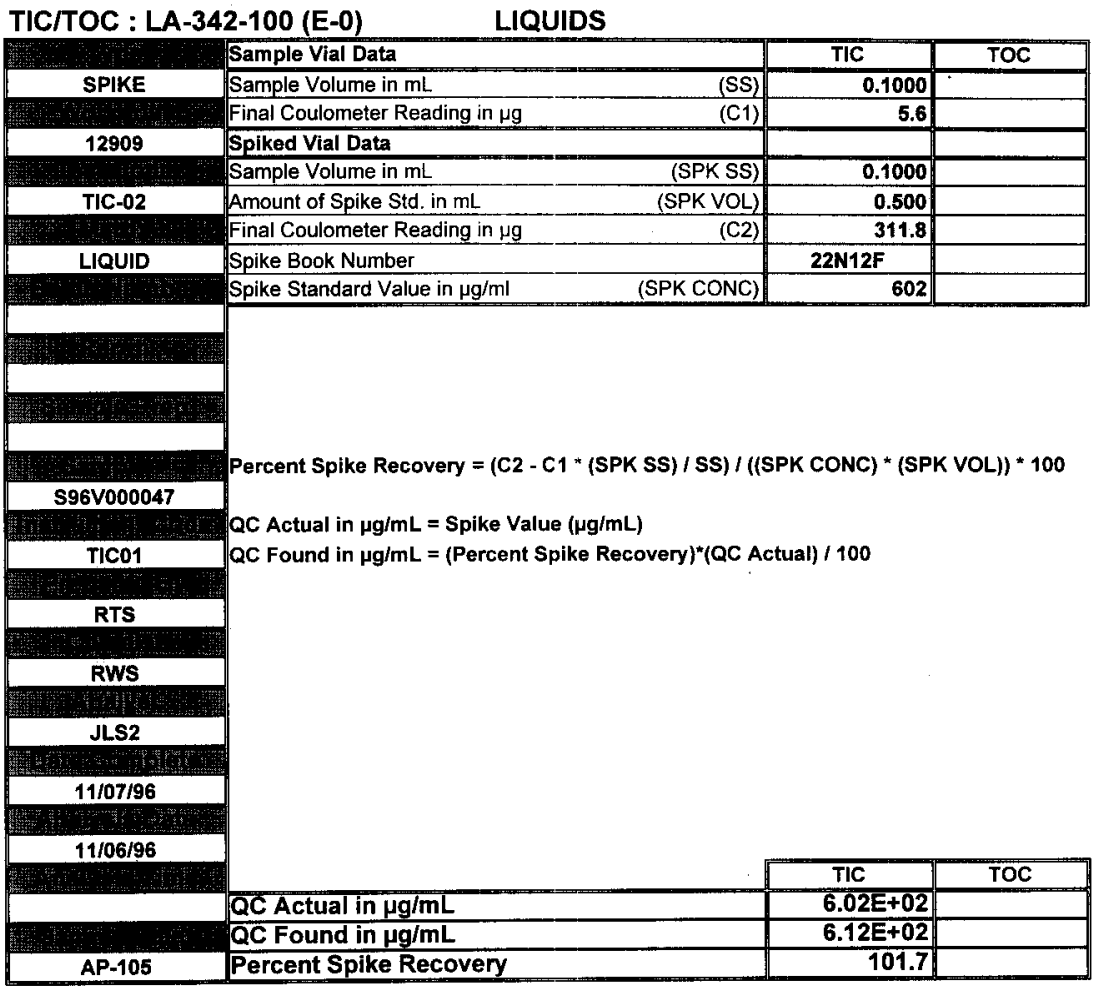

\begin{tabular}{|c|c|c|c|}
\hline Daita Entered By: & RTS & Date: & $11 / 07 / 96$ \\
\hline Signature of Chemist: & & Date: & $1 / 6 / 96$ \\
\hline
\end{tabular}




\section{HNF-SD-WM-OH-202, REV. 1}

TIC- TOTAL INORGANIC CARBON ANALYSIS REPORT

TICTOC REV 2.0

$\ll$ BLANK ANALYSIS $\gg>$

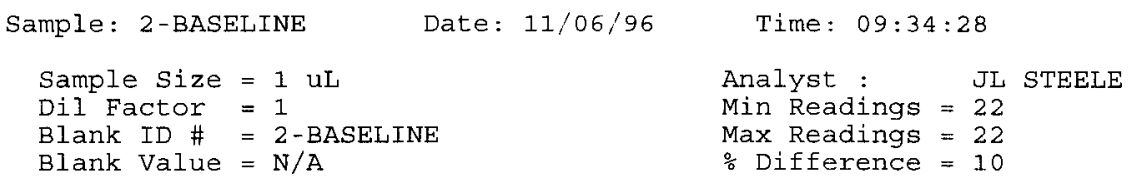

$==$ Reading $====$ Analysis Time $====$ Coulometer $====\frac{\circ}{0}$ Difference $==$

$\begin{array}{rrrr}1 & 0.51 & 0.70 & 0.00 \\ 2 & 1.01 & 1.30 & 46.15 \\ 3 & 1.51 & 1.70 & 23.53 \\ 4 & 2.01 & 1.90 & 10.53 \\ 5 & 2.51 & 2.30 & 17.39 \\ 6 & 3.00 & 2.40 & 4.17 \\ 7 & 3.50 & 2.70 & 11.11 \\ 8 & 4.00 & 2.90 & 6.90 \\ 9 & 4.50 & 3.10 & 6.45 \\ 10 & 5.00 & 3.30 & 6.06 \\ 11 & 5.50 & 3.40 & 2.94 \\ 12 & 6.00 & 3.60 & 5.56 \\ 13 & 6.50 & 3.70 & 2.70 \\ 14 & 7.00 & 3.90 & 5.13 \\ 15 & 7.50 & 4.00 & 2.50 \\ 16 & 8.00 & 4.20 & 4.76 \\ 17 & 8.50 & 4.30 & 2.33 \\ 18 & 9.00 & 4.80 & 10.42 \\ 19 & 9.50 & 4.80 & 0.00 \\ 20 & 10.00 & 4.90 & 2.04 \\ 21 & 10.50 & 4.90 & 0.00 \\ 22 & 11.00 & 4.90 & 0.00\end{array}$

BLANK VALUE $=4.9$ micrograms carbon

BLANK FACTOR $=4.9 / 10.99878=4.5 \mathrm{E}-01 \mathrm{ug} / \mathrm{min}$ Carbon

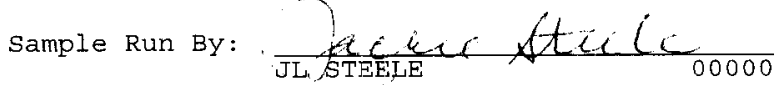




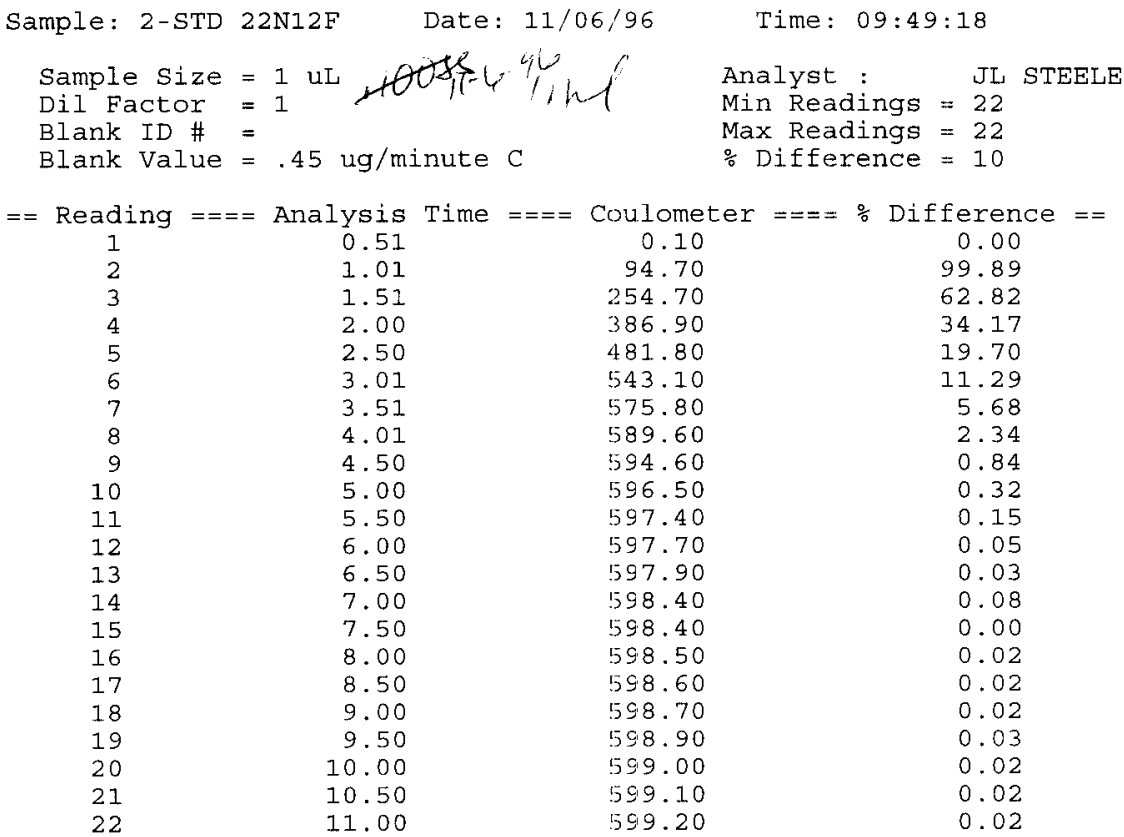

USER INPUT BLANK VALUE

BLANK VALUE $=4.94945$ micrograms carbon

BLANK FACTOR $=4.94945 / 10.99878:=$

+4.5E-01 ug/min Carbon

\section{SAMPLE RESULTS :}

$(599.2-4.949478)(1) /(1)(12)=$

$+5.943 \mathrm{E}+02 \mathrm{~g} / \mathrm{L}$ Carbon

$+4.952 \mathrm{E}+01$ Molar Carbon

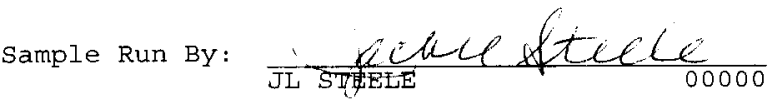




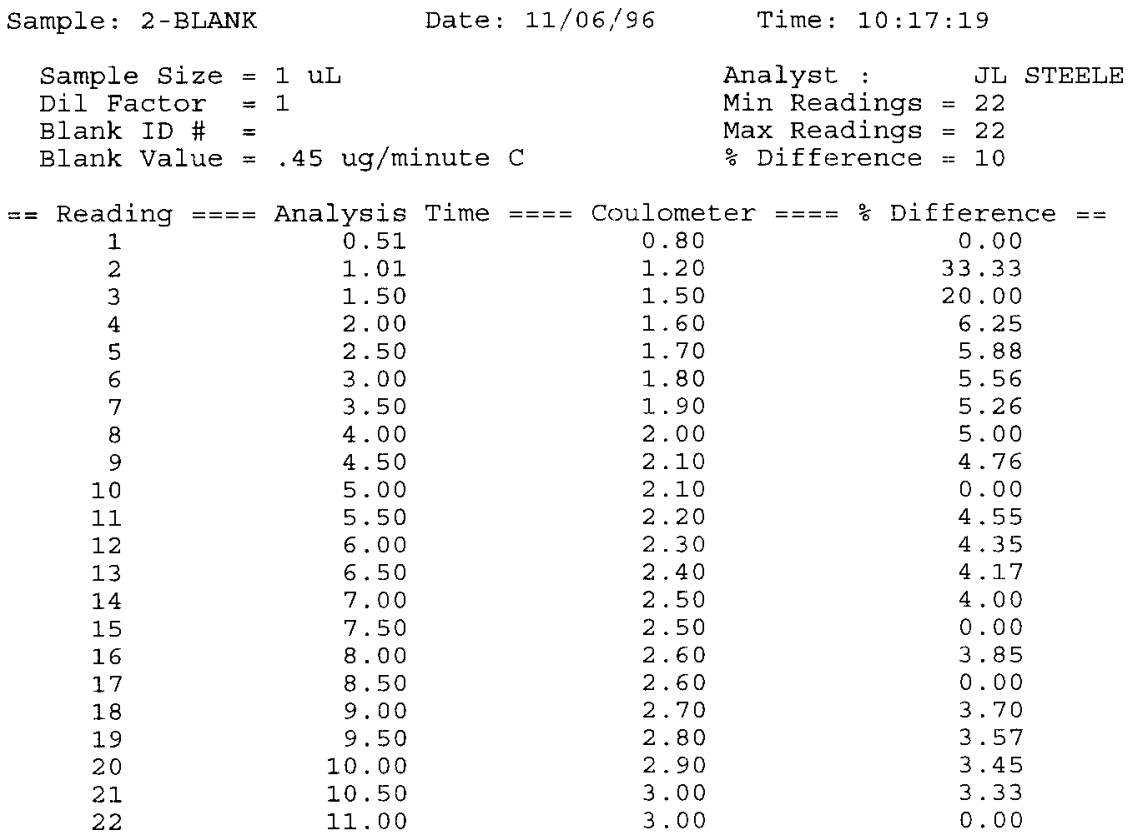

USER INPUT BLANK VALUE

BLANK VALUE $=4.94945$ micrograms carbon

BLANK FACTOR $=4.94945 / 10.99878:=\quad+4.5 \mathrm{E}-01 \quad \mathrm{ug} / \mathrm{min}$ Carbon

SAMPLE RESULTS:

$\begin{array}{lll}(3-4.949093)(1) /(1) & = & <5.00 \mathrm{E}-3 \mathrm{~g} / \mathrm{L} \text { Carbon } \\ 3-4.949093)(1) /(1)(12)= & <4.17 \text { E-4 Molar Carbon }\end{array}$

Sample Run By:

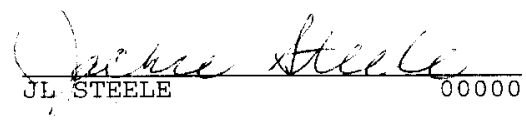




\section{HNF-SD-WM-DP-202, REV. 1}

TIC- TOTAL INORGANIC CARBON ANALYSIS REPORT

TICTOC REV 2.0

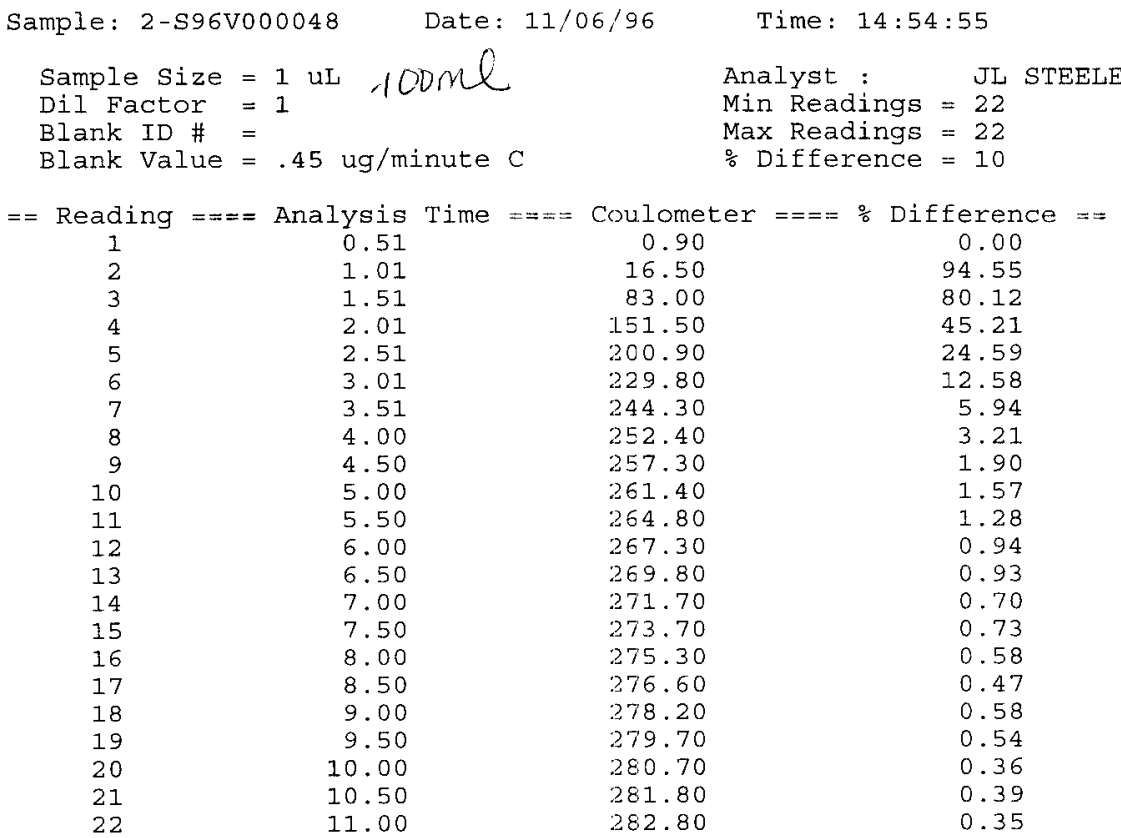

USER INPUT BLANK VALUE

BLANK VALUE $=4.94945$ micrograms carbon

BLANK FACTOR $=4.94945 / 10.99878=+4.5 \mathrm{E}-01 \quad \mathrm{ug} / \mathrm{min}$ Carbon

SAMPLE RESULTS:

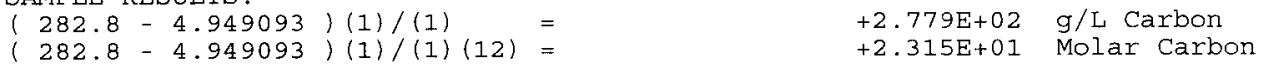

Sample Run BY:

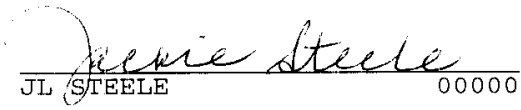




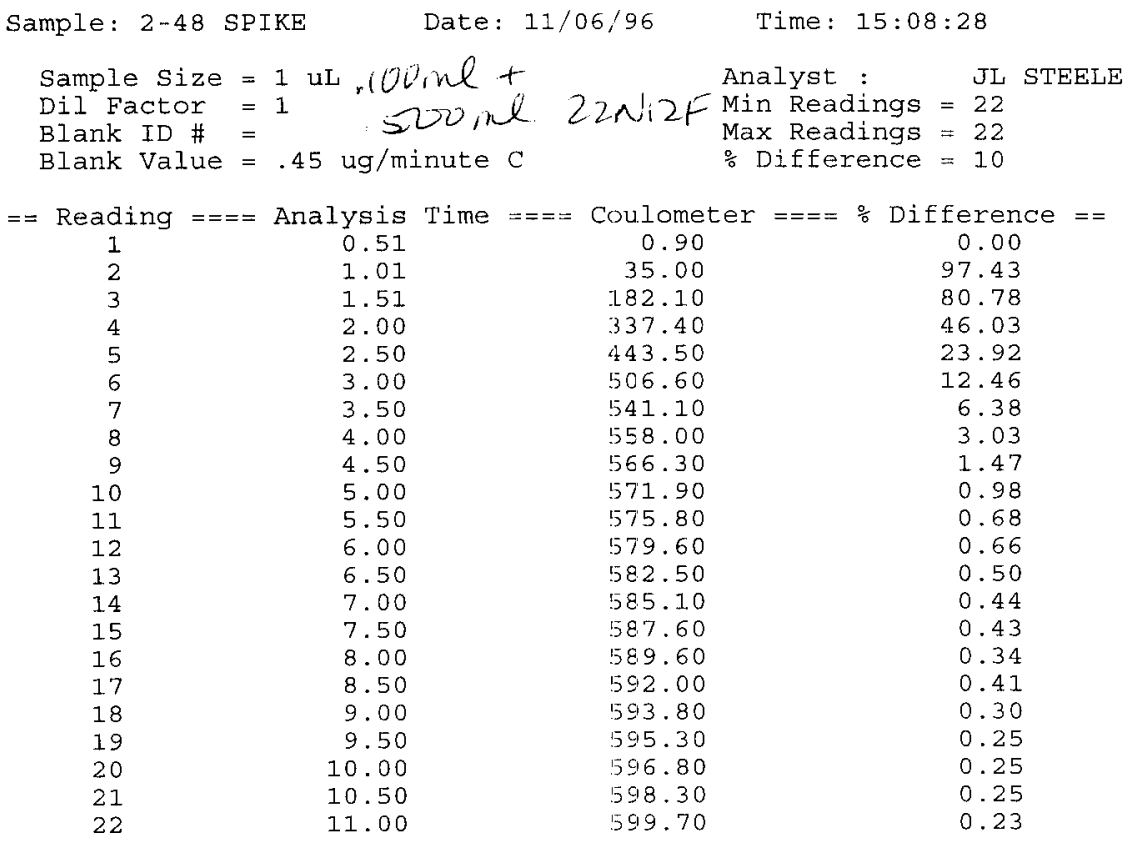

USER INPUT BLANK VALUE

BLANK VALUE $=4.94945$ micrograms carbon

BLANK FACTOR $=4.94945 / 10.99878=+4.5 \mathrm{E}-01 \quad \mathrm{ug} / \mathrm{min}$ Carbon

SAMPLE RESULTS :

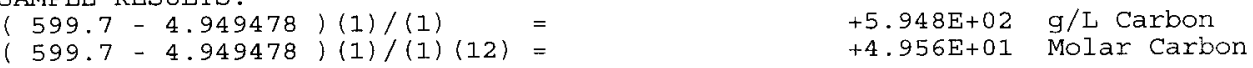

Sample Run By: $\frac{k e k e f \text { detele }}{\text { JETEELE }}$ 
Sample: 2-48 SPK DUP Date: $11 / 06 / 96$

Sample size $=1$ uL $: 00 \mathrm{ml}+$

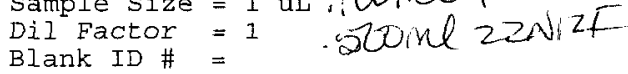

Blank Value $=.45 \mathrm{ug} /$ minute $\mathrm{C}$
Time: $15: 23: 34$

Analyst : JL STEELE

Min Readings $=22$

Max Readings $=22$

: Difference $=10$

$\begin{array}{cr}=\text { Reading }===\text { Analysis } \\ 1 & 0.51 \\ 2 & 1.01 \\ 3 & 1.51 \\ 4 & 2.00 \\ 5 & 2.50 \\ 6 & 3.00 \\ 7 & 3.50 \\ 8 & 4.00 \\ 9 & 4.50 \\ 10 & 5.00 \\ 11 & 5.50 \\ 12 & 6.00 \\ 13 & 6.50 \\ 14 & 7.00 \\ 15 & 7.50 \\ 16 & 8.00 \\ 17 & 8.50 \\ 18 & 9.00 \\ 19 & 9.50 \\ 20 & 10.00 \\ 21 & 10.50 \\ 22 & 11.00\end{array}$

Coulometer $====$ Difference $=:=$

1.30
35.70
187.40
349.80
455.50
515.40
547.10
562.10
570.50
575.70
580.50
584.20
587.70
590.60
593.50
596.00
598.40
600.50
602.60
504.30
606.10
507.80

0.00

96.36

80.95

46.43

23.21

11.62

5.79

2.67

1.47

0.90

0.83

0.63

0.60

0.49

0.49

0.42

0.40

0.35

0.35

0.28

0.30

0.28

USER INPUT BLANK VALUE

BLANK VALUE $=4.94945$ micrograms carbon

BLANK FACTOR $=4.94945 / 10.99878=+4.5 \mathrm{E}-01 \quad \mathrm{ug} / \mathrm{min}$ Carbon

SAMPLE RESULTS:

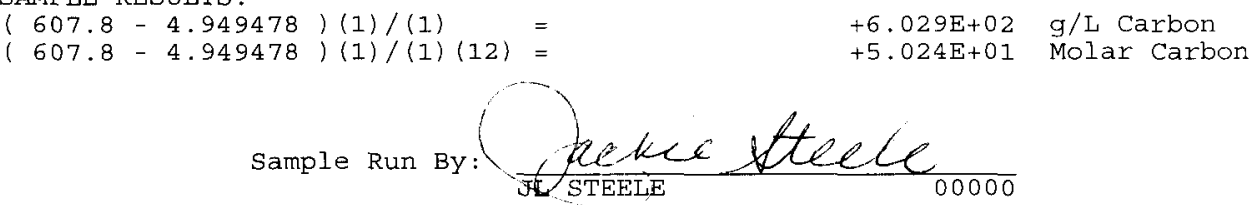


Sample: 2-S96V000049

Sample size $=1 \mathrm{uL}$

Dil Factor $=1$

Blank ID \# =

Blank Value $=.45 \mathrm{ug} / \mathrm{minute} \mathrm{C}$
Date: $11 / 06 / 96$

Time: $14: 15: 25$

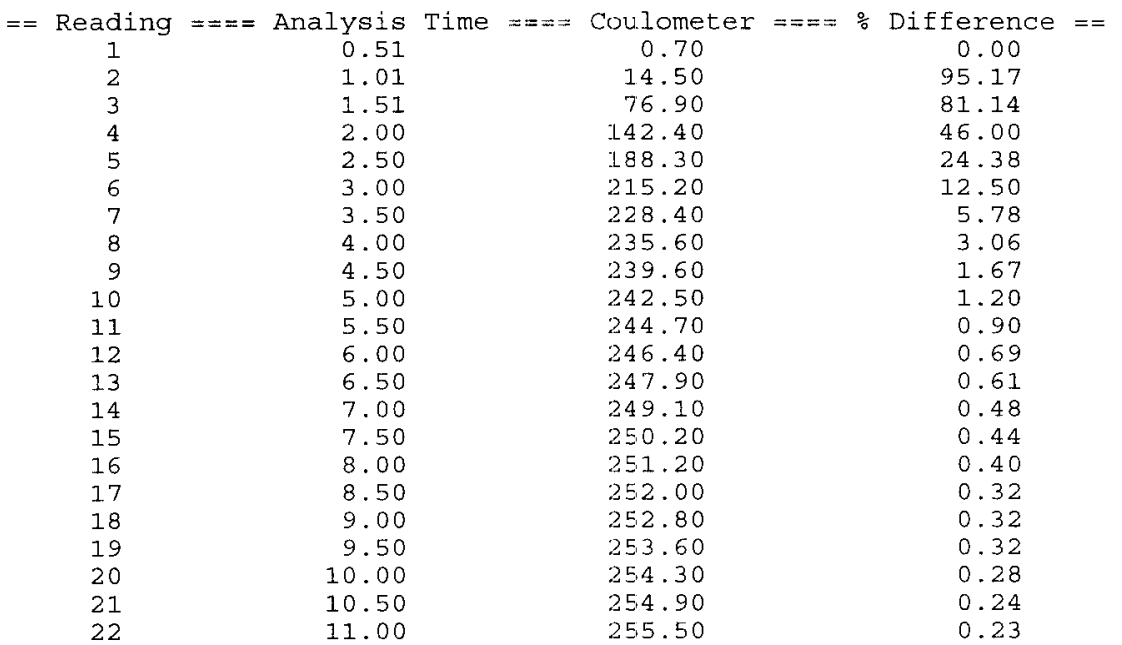

USER INPUT BLANK VALUE

BLANK VALUE $=4.94945$ micrograms carbon

BLANK FACTOR $=4.94945 / 10.99878=\quad+4.5 \mathrm{E}-01 \quad \mathrm{ug} / \mathrm{min}$ Carbon

SAMPLE RESULTS:

$\left(\begin{array}{l}255.5-4.949093)(1) /(1) \\ 255.5-4.949093)(1) /(1)(12)=\end{array}\right.$

$+2.506 \mathrm{E}+02 \mathrm{~g} / \mathrm{L}$ Carbon

$+2.088 \mathrm{E}+01$ Molar Carbon

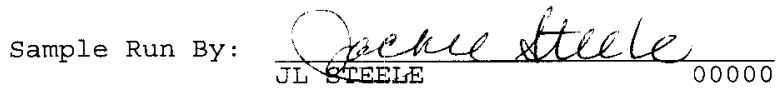




\section{HNFE-SD-WM-DP-202, REV}

TIC- TOTAL INORGANIC CARBON ANALYSIS REPORT

TICTOC REV 2.0

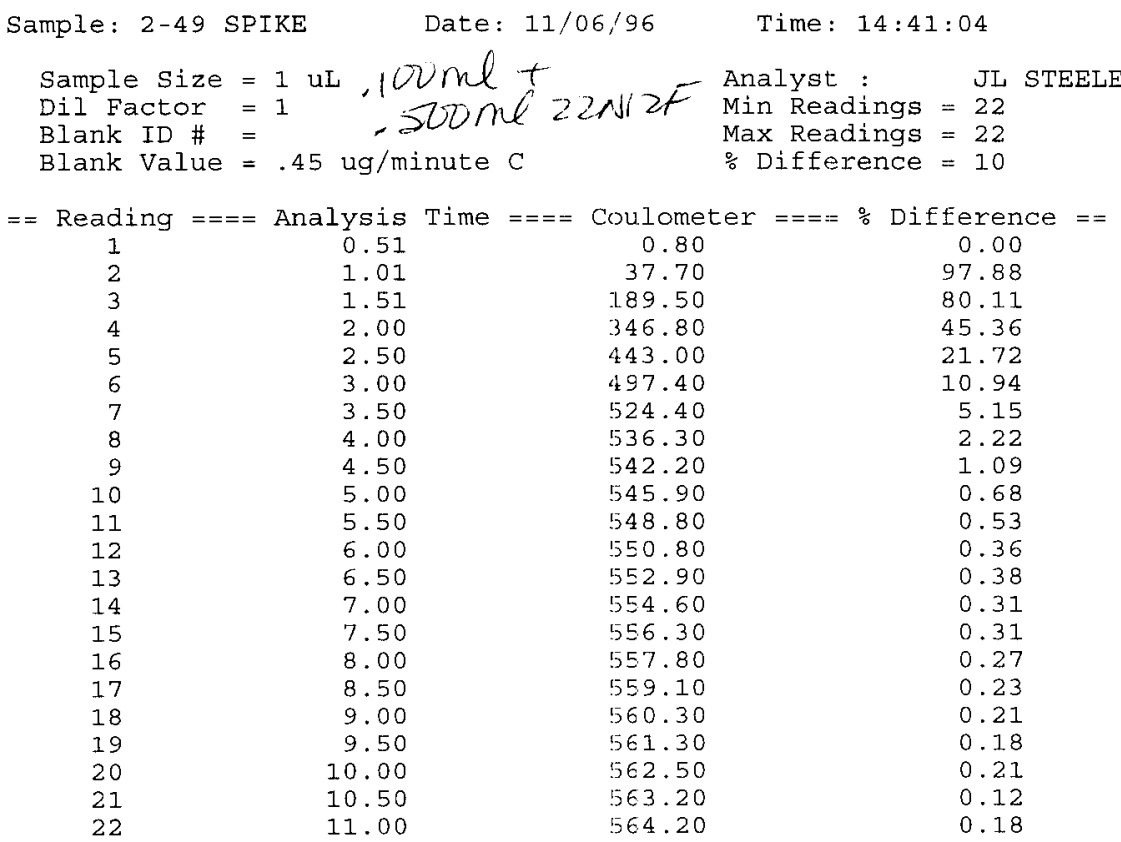

USER INPUT BLANK VALUE

BLANK VALUE $=4.94945$ micrograms carbon

BLANK FACTOR $=4.94945 / 10.99878=+4.5 \mathrm{E}-01 \mathrm{ug} / \mathrm{min}$ Carbon

SAMPLE RESULTS:

$\begin{array}{llll}(564.2-4.949039)(1) /(1) & = & +5.593 \mathrm{E}+02 \mathrm{~g} / \mathrm{L} \text { Carbon } \\ (564.2-4.949039)(1) /(1)(12)= & +4.660 \mathrm{E}+01 \text { Molar Carbon }\end{array}$

Sample Run By:

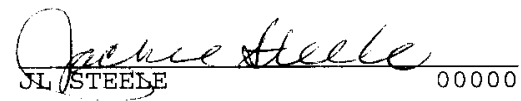




\section{HNF}

TIC- TOTAL INORGANIC CARBON ANALYSIS REPORT

TICTOC REV 2.0

Sample: 2-S96V000047 Date: $11 / 06 / 96$

Sample size $=1$ uL, 102 hin

Dil Factor $=1$

Blank ID \# =

Blank Value $=.45 \mathrm{ug} / \mathrm{minute} \mathrm{C}$
Time: $13: 48: 57$

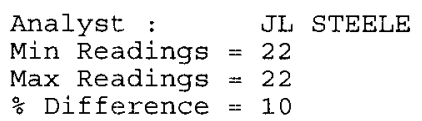

$\begin{array}{crcc}=\text { Reading }===\text { Analysis } & \text { Time }=== & \text { Coulometer }=== & \text { Difference }== \\ 1 & 0.51 & 0.20 & 0.00 \\ 2 & 1.01 & 10.50 & 98.10 \\ 3 & 1.50 & 72.70 & 85.56 \\ 4 & 2.00 & 146.10 & 50.24 \\ 5 & 2.50 & 201.30 & 27.42 \\ 6 & 3.00 & 235.60 & 14.56 \\ 7 & 3.50 & 253.80 & 7.17 \\ 8 & 4.00 & 262.60 & 3.35 \\ 9 & 4.50 & 267.20 & 1.72 \\ 10 & 5.00 & 269.60 & 0.89 \\ 11 & 5.50 & 271.30 & 0.63 \\ 12 & 6.00 & 272.60 & 0.48 \\ 13 & 6.50 & 273.70 & 0.40 \\ 14 & 7.00 & 274.50 & 0.29 \\ 15 & 7.50 & 275.20 & 0.25 \\ 16 & 8.00 & 275.80 & 0.22 \\ 17 & 8.50 & 276.50 & 0.25 \\ 18 & 9.00 & 277.00 & 0.18 \\ 19 & 9.50 & 277.50 & 0.18 \\ 20 & 10.00 & 278.00 & 0.18 \\ 21 & 10.50 & 278.40 & 0.14 \\ 22 & 11.00 & 278.90 & 0.18\end{array}$

USER INPUT BLANK VALUE

BLANK VALUE $=4.94945$ micrograms carbon

BLANK FACTOR $=4.94945 / 10.99878=+4.5 \mathrm{E}-01 \quad \mathrm{ug} / \mathrm{min}$ Carbon

SAMPLE RESULTS :

$(278.9-4.949039)(1) /(1)=$

$+2.740 \mathrm{E}+02 \mathrm{~g} / \mathrm{L}$ Carbon

$+2.283 \mathrm{E}+01$ Molar Carbon

Sample Run By: $\frac{\text { tetece tetele }}{00000}$ 


\section{HNFG-SD-WM-DP-202, REV. 1}

TIC- TOTAL INORGANIC CARBON ANALYSIS REPORT

TICTOC REV 2.0

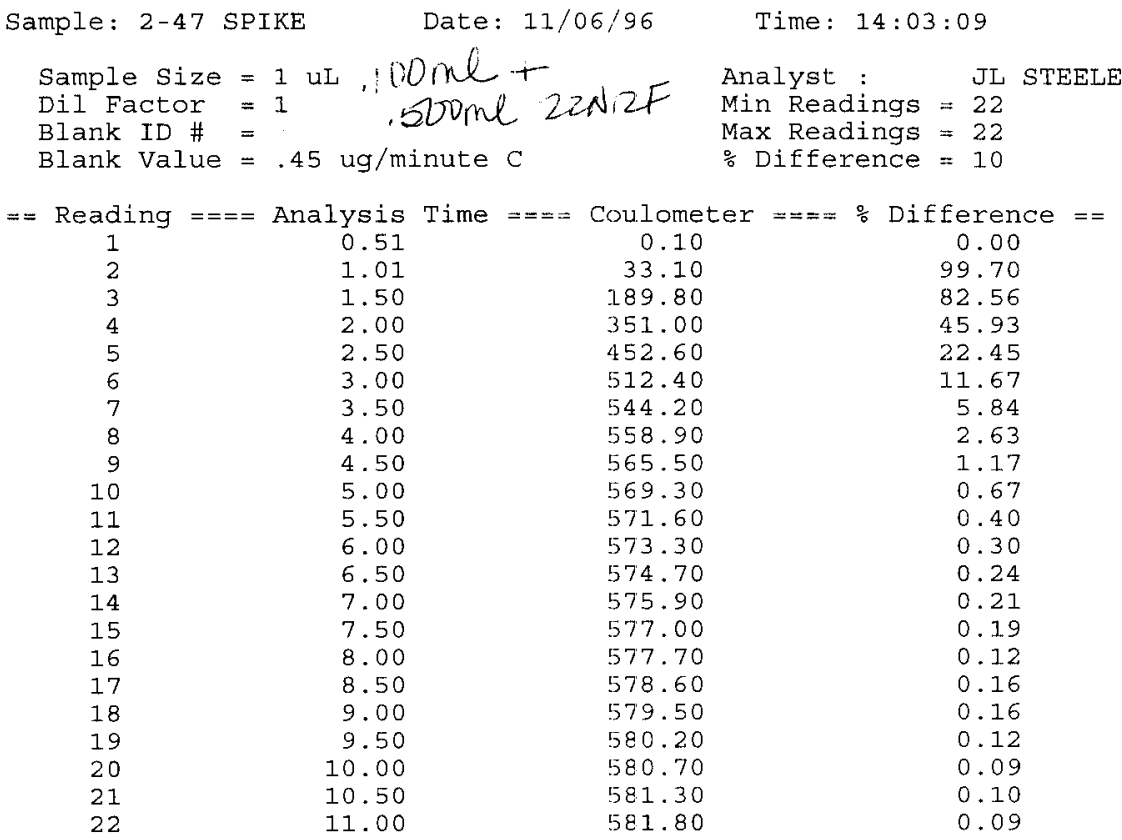

USER INPUT BLANK VALUE

BLANK VALUE $=4.94945$ micrograms carbon

BLANK FACTOR $=4.94945 / 10.99878 \Rightarrow+4.5 \mathrm{E}-01 \quad \mathrm{ug} / \mathrm{min}$ Carbon

SAMPLE RESULTS:

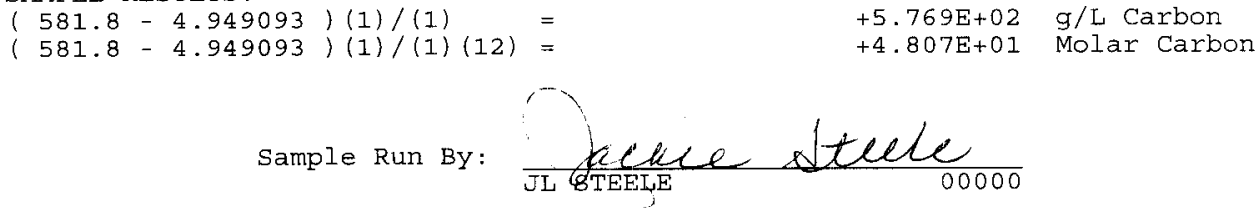




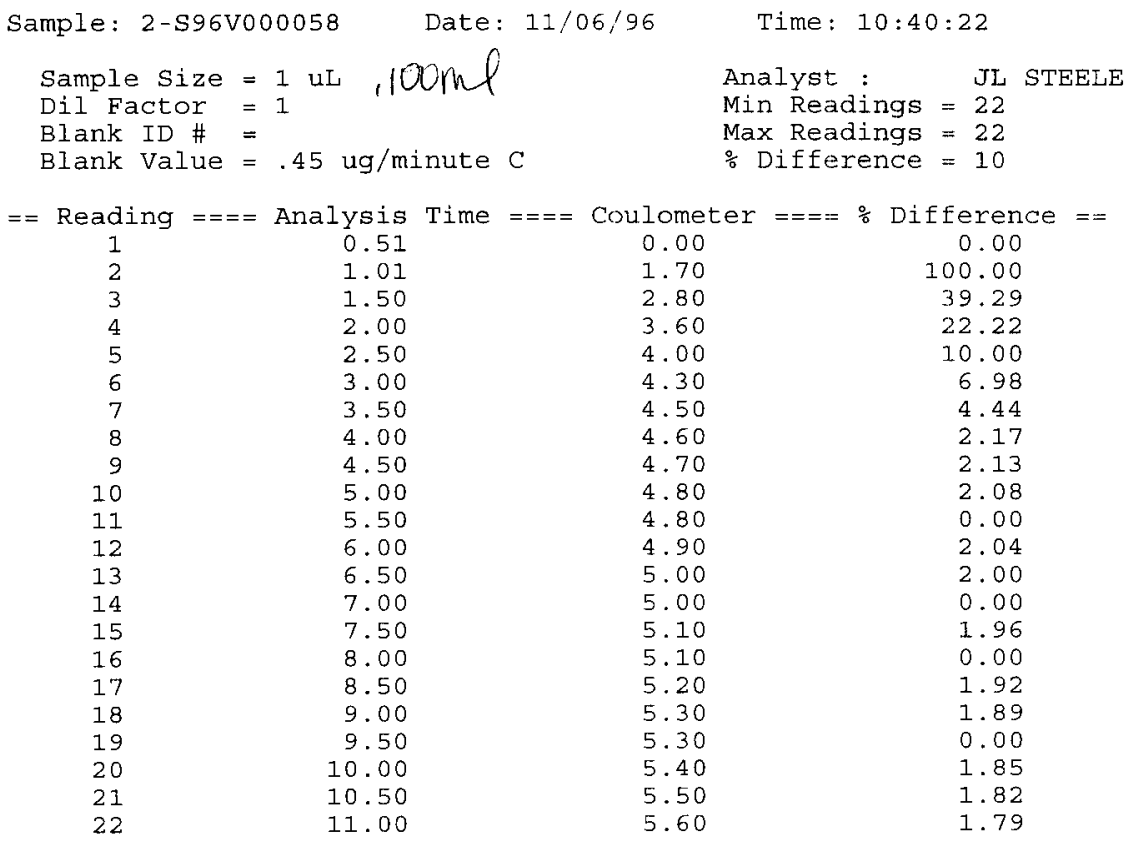

USER INPUT BLANK VALUE

BLANK VALUE $=4.94945$ micrograms carbon

BLANK FACTOR $=4.94945 / 10.99878:=\quad+4.5 \mathrm{E}-01 \quad \mathrm{ug} / \mathrm{min}$ Carbon

SAMPLE RESULTS:

$(5.6-4.948627)(1) /(1)=$

$+6.5 \mathrm{E}-01$

$\mathrm{g} / \mathrm{L}$ Carbon

$(5.6-4.948627)(1) /(1)(12)=$

+5.4E-02 Molar Carbon

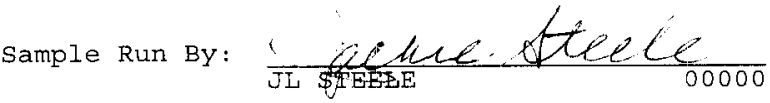



HNF
TIC- TOTAL INORGANIC CARBON ANALYSIS REPORT WHG-SD-WM-DP-202, REV
TICTOC REV 2.0

Sample: 2-458 SPIKE Date: 11/06/96 Time: 13:19:09

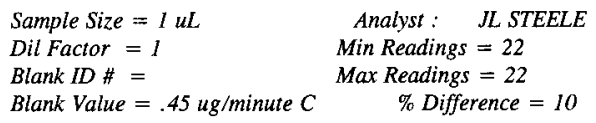




\section{LABCORE Data Entry Template for Worklist\# 12911}

Analyst:

Instrument: TOCO1

Book \# Z3NIZ-B SPR

Method: LA-344-105 Rev/Mod D - 1 $Z 4 N 1 Z-\$ S T D$

Worklist Comment: AP-105 TOTC. RCJ

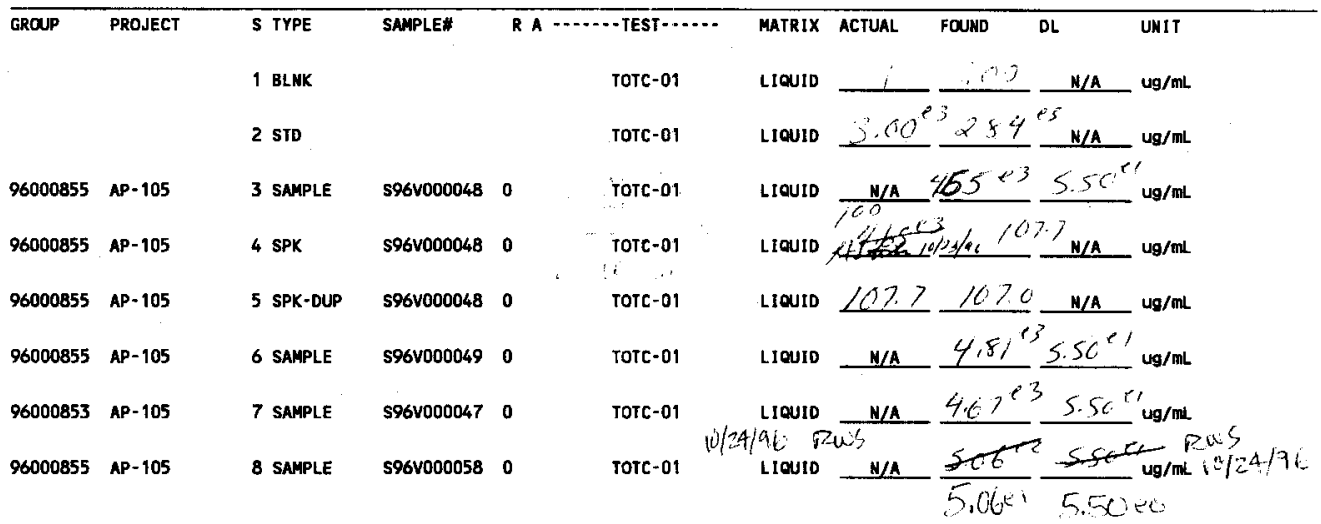

\section{Final page for worklist \# 12911}

Analyst Signature

Date

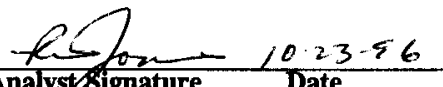

Analystosignature Date

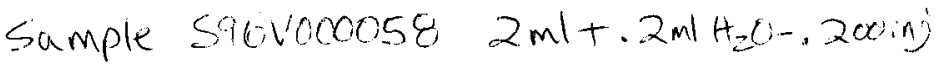

Data Entry Comments: $31 \mathrm{~K} \quad 0.20 \mathrm{O}, \mathrm{H} \mathrm{O}$ std $20 \mathrm{O}+2 \mathrm{ml}, \mathrm{O}-20 \mathrm{in}$ Samples s96vo00047,48\&41.200ml+2mLH,or-.20.nj.

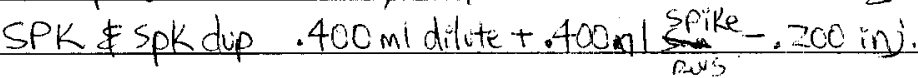

Units shown for $Q C$ (SPK \& STD) may not reflect the actual units. $D L=$ Detection Limit, $S=$ Worklist Slot Number,

$R=$ Replicate Number, $A=$ Aliquot Code. 


\section{TOC- TOTAL ORGANIC CARBON ANALYSIS REPORT TICTOC REV 2.0}

Sample: STD

Sample Size $=200 \mathrm{uL}$

Dil Factor $=11$

Blank ID \# = BLK

Blank Value $=.3796617 \mathrm{ug} / \mathrm{minute} \mathrm{C}$
Date: $10 / 16 / 96$

$\begin{array}{ll}\text { Analyst : } & \text { RA WENDLAND } \\ & \text { Min Readings }=10 \\ \text { Max Readings }=10 \\ \text { ug/minute } C \quad & \% \text { Difference }=10\end{array}$

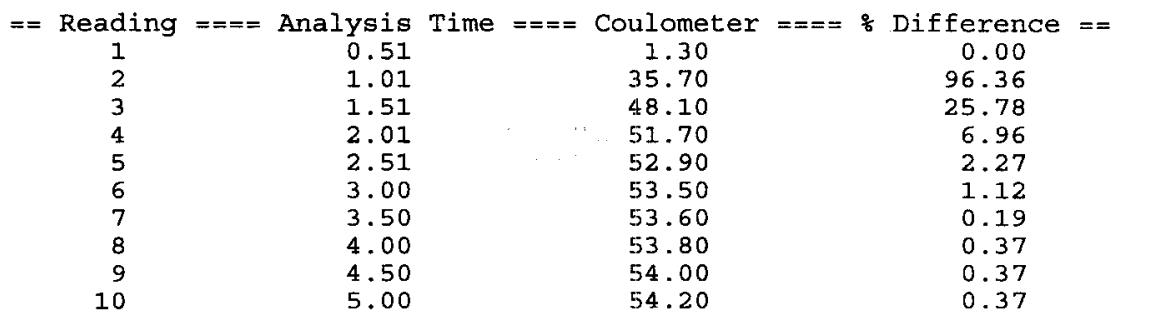

BLANK VALUE $=1.9$ micrograms carbon

BLANK FACTOR $=1.9 / 5.004456=$

SAMPLE RESULTS :

$(54.2-1.899745)(11) /(200)=$

$(54.2-1.899745)(11) /(200)(12)=$
$+3.8 \mathrm{E}-01$ ug/min Carbon

$+2.88 \mathrm{E}+00$

g/L Carbon

$+2.40 E-01$ Molar Carbon

Sample Run By:

RA WENDLAND 00000

SIGNATURE ABOVE REPAESENTS CHEMICAL TECHNOLOGIST/CHEMIST THAI

COMPLETEDNERIFIED THE CALIBAATIONANALYSIS ON PAGESQ $/ 16$ TO 232 
WIS-SC-WMi-DF-202, REV. 1

TOC- TOTAL ORGANIC CARBON ANALYSIS REPORT

TICTOC REV 2.0

Sample: BASE

Date: $10 / 16 / 96$

Time: 09:42:36

Sample Size $=200 \mathrm{uL}$

Analyst : RA WENDLAND

Dil Factor $=1$

Min Readings $=10$

Blank ID \# =

Blank Value $=0 \mathrm{ug} / \mathrm{minute} \mathrm{C}$

Max Readings $=10$

$\div$ Difference $=10$

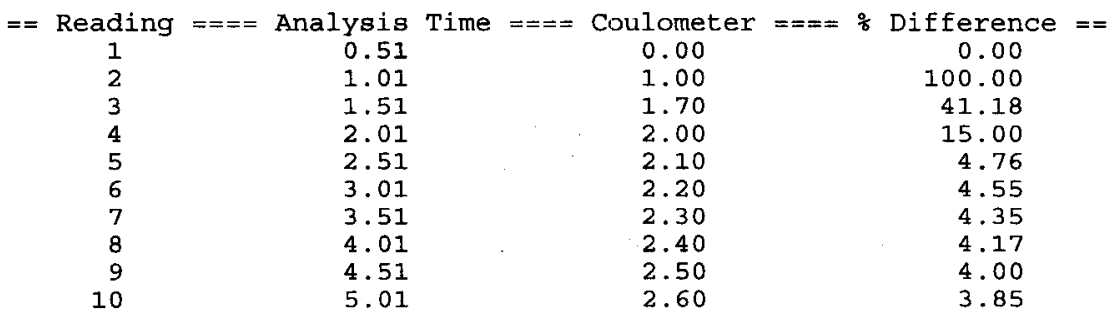

USER INPUT BLANK VALUE

BLANK VALUE = 0 micrograms carbon

BLANK FACTOR $=0 / 0=+0.0 E+00 \quad \mathrm{ug} / \mathrm{min}$ Carbon

SAMPLE RESULTS :

$\left(\begin{array}{l}2.6-0)(1) /(200) \\ (2.6-0)(1) /(200)(12)=\end{array}\right.$

$+1.3 E-02$

g/L Carbon

$+1.1 \mathrm{E}-03$

Molar Carbon

Sample Run By:

RA WENDLAND $\quad 00000$ 


\section{HNF-SD-WM-DP-202, REV. 1}

TOC- TOTAL ORGANIC CARBON ANALYSIS REPORT

TICTOC REV 2.0

$\ll$ BLANK ANALYSIS $\gg>$

Sample: BLK

Sample size $=200 \mathrm{uL}$

Dil Factor $=1$

Blank ID \# = BLK

Blank Value $=\mathrm{N} / \mathrm{A}$
Date: $10 / 16 / 96$
Time: 09:48:40

Analyst : RA WENDLAND

Min Readings $=10$

Max Readings $=10$

$\div$ Difference $=10$

$\begin{array}{crcc}=\text { Reading }===\text { Analysis Time }===\text { Coulometer }=== & \text { Difference }== \\ 1 & 0.51 & 0.10 & 0.00 \\ 2 & 1.01 & 0.60 & 83.33 \\ 3 & 1.51 & 1.00 & 40.00 \\ 4 & 2.01 & 1.10 & 9.09 \\ 5 & 2.51 & 1.30 & 15.38 \\ 6 & 3.01 & 1.40 & 7.14 \\ 7 & 3.51 & 1.50 & 6.67 \\ 8 & 4.01 & 1.60 & 6.25 \\ 9 & 4.50 & 1.80 & 11.11 \\ 10 & 5.00 & 1.90 & 5.26\end{array}$

BLANK VALUE $=1.9$ micrograms carbon

BLANK FACTOR $=1.9 / 5.004456=+3.8 \mathrm{E}-01 \mathrm{ug} / \mathrm{min}$ Carbon

Sample Run By:

RA WENDIAAND 00000 
Sample: 48

Sample Size $=200 \mathrm{uL}$

Dil Factor $=11$

Blank ID \# = BLK

Blank Value $=.3796617 \mathrm{ug} /$ minute $\mathrm{C}$
Date: $10 / 16 / 96$

Time: $10: 21: 06$

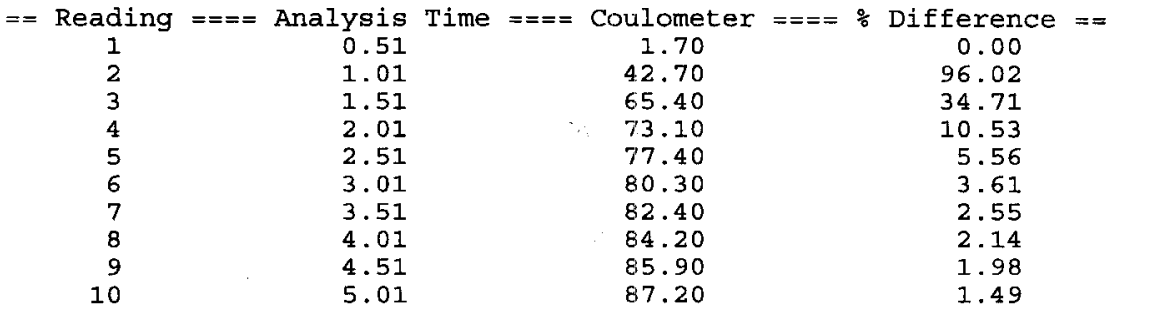

BLANK VALUE $=1.9$ micrograms carbon

BLANK FACTOR $=1.9 / 5.004456=+3.8 \mathrm{E}-01$ ug/min Carbon

SAMPLE RESULTS:

$\left(\begin{array}{l}87.2-1.900371)(11) /(200))(12)= \\ (87.2-1.900371)(11) /(200)(12)=\end{array}\right.$
Analyst : RA WENDLAND

Min Readings $=10$

Max Readings $=10$

$\because$ Difference $=10$ 
TOC- TOTAL ORGANIC CARBON ANALYSIS REPORT

TICTOC REV 2.0

Sample: 48SPK

$$
\begin{aligned}
& \text { Sample Size }=200 \mathrm{uL} \\
& \text { Dil Factor }=1 \\
& \text { Blank ID \# }=\text { BLK } \\
& \text { Blank Value }=.3796617 \mathrm{ug} / \text { minute } \mathrm{C}
\end{aligned}
$$

Time: 10:27:02

$$
\begin{array}{ll}
\text { Analyst : } & \text { RA WENDLAND } \\
\text { Min Readings }= & 10 \\
\text { Max Readings }= & 10 \\
\frac{\circ \text { Difference }}{}= & 10
\end{array}
$$

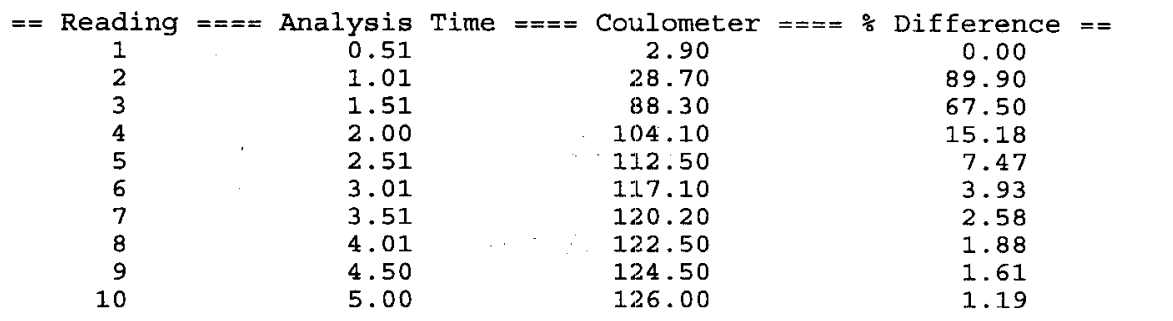

BLANK VALUE $=1.9$ micrograms carbon

BLANK FACTOR $=1.9 / 5.004456=$

+3.8E-01 ug/min Carbon

SAMPLE RESULTS:

$(126-1.900069)(1) /(200)=$

$+6.205 \mathrm{E}-01 \mathrm{~g} / \mathrm{L}$ Carbon

$(126-1.900069)(1) /(200)(12)=$

$+5.171 \mathrm{E}-02$ Molar Carbon

Sample Run By:

RA WENDIAND 00000 


\section{WFE-SD-WMI-DP-202, REV *}

TOC- TOTAL ORGANIC CARBON ANALYSIS REPORT

TICTOC REV 2.0

Sample: 48 DUPSPK

Sample Size $=200 \mathrm{uL}$

Dil Factor $=1$

Blank ID \# = BLK

Blank Value $=.3796617 \mathrm{ug} / \mathrm{minute} \mathrm{C}$
Date: $10 / 16 / 96$

Time: $10: 39: 52$

$\begin{array}{crcc}=\text { Reading }===\text { Analysis } & \text { Time }=== & \text { Coulometer }=== & \text { Difference }== \\ 1 & 0.51 & 3.00 & 0.00 \\ 2 & 1.01 & 72.40 & 95.86 \\ 3 & 1.51 & 97.60 & 25.82 \\ 4 & 2.01 & 107.70 & 9.38 \\ 5 & 2.51 & 11.3 .60 & 5.19 \\ 6 & 3.01 & 11.7 .30 & 3.1 .5 \\ 7 & 3.51 & 120.00 & 2.25 \\ 8 & 4.01 & 122.20 & 1.80 \\ 9 & 4.51 & 123.90 & 1.37 \\ 10 & 5.01 & 125.50 & 1.27\end{array}$

Analyst : RA WENDLAND

Min Readings $=10$

Max Readings $=10$

\% Difference $=10$

BLANK VALUE $=1.9$ micrograms carbon

BLANK FACTOR $=1.9 / 5.004456=+3.8 \mathrm{E}-01$ ug/min Carbon

SAMPLE RESULTS:

$(125.5-1.900417)(1) /(200)=$

$(125.5-1.900417)(1) /(200)(12)=$
$+6.180 \mathrm{E}-01 \mathrm{~g} / \mathrm{L}$ Carbon

$+5.150 \mathrm{E}-02$ Molar Carbon

Sample Run By:

RA WENDLAND 00000




\section{TOC- TOTAL ORGANIC CARBON ANALYSIS REPORT TICTOC REV 2.0}

Sample: 47

Sample Size $=200 \mathrm{uL}$

Dil Factor $=11$

Blank ID \# = BLK

Blank Value $=.3796617 \mathrm{ug} /$ minute $\mathrm{C}$
Date: $10 / 16 / 96$

Time: $10: 03: 03$

$==$ Reading $== \pm=$ Analysis Time $== \pm=$ Coulometer

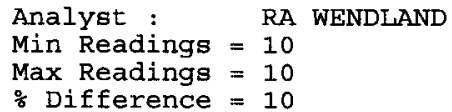

$\begin{array}{rr}1 & 0.51 \\ 2 & 1.01 \\ 3 & 1.51 \\ 4 & 2.01 \\ 5 & 2.51 \\ 6 & 3.01 \\ 7 & 3.51 \\ 8 & 4.01 \\ 9 & 4.51 \\ 10 & 5.01\end{array}$

1.00

26.70

61.50

73.00

78.00

80.80

82.80

84.50

86.10

87.50
Difference $==$

0.00

96.25

56.59

15.75

6.41

3.47

2.42

2.01

1.86

1.60
BLANK VALUE $=1.9$ micrograms carbon

BLANK FACTOR $=1.9 / 5.004456=$

\section{SAMPLE RESULTS:}

$(87.5-1.900417)(11) /(200)=$

$(87.5-1.900417)(11) /(200)(12)=$
$+3.8 E-01 \quad u g / m i n$ Carbon

$+4.71 E+00$

g/L Carbon

$+3.92 \mathrm{E}-01$ Molar Carbon

Sample Run By: 


\section{$H N F$ \\ WHE-SD-WM-DP-202, REV. 1 \\ TOC- TOTAL ORGANIC CARBON ANALYSIS REPORT \\ TICTOC REV 2.0}

Sample: 49

Sample Size $=200 \mathrm{uL}$

Dil Factor $=11$

Blank ID \# = BLK

Blank Value $=.3796617$ ug $/$ minute $\mathrm{C}$
Time: $10: 08: 29$

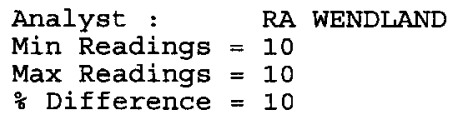

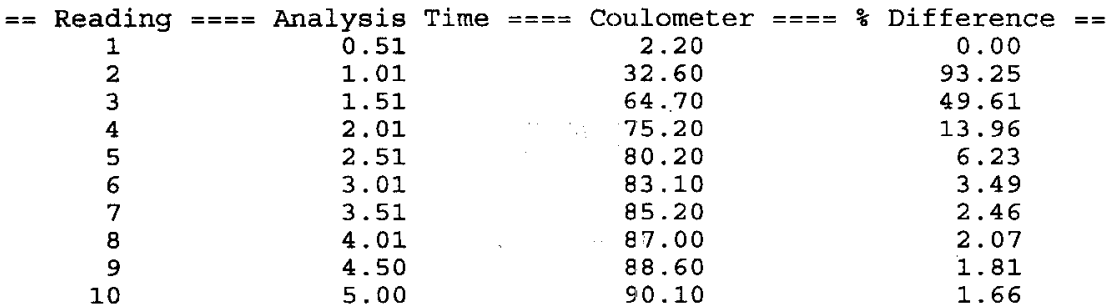

BLANK VALUE $=1.9$ micrograms carbon

BLANK FACTOR $=1.9 / 5.004456=+3.8 \mathrm{E}-01$ ug/min Carbon

SAMPLE RESULTS:

$(90.1-1.899676)(11) /(200)$
$(90.1-1.899676)(11) /(200)(12)=$

+4.85E+00 g/L Carbon

+4.04E-01 Molar Carbon

Sample Run By:

RA WENDLAND 00000 
TOC- TOTAL ORGANIC CARBON ANALYSIS REPORT TICTOC REV 2.0

Sample: 58

Sample Size $=200 \mathrm{uL}$

Dil Factor $=1.1$

Blank ID \# = BLK

Blank Value $=.3796617 \mathrm{ug} / \mathrm{minute} \mathrm{C}$

Date: $10 / 16 / 96$

$==$ Reading $====$ Analysis Time $====$ Could
0.51

1.01

1.50

2.01

2. 51

3.01

3.51

4.00

4.50

5.00
1.70

4.10

5.50

6.80

7.70

8.70

9.40

1.0 .40

11.00

11.80
- Time: $10: 14: 58$

$$
\begin{array}{ll}
\text { Analyst : } & \text { RA WENDLAND } \\
\text { Min Readings }=10 \\
\text { Max Readings }=10 \\
\% \text { Difference }=10
\end{array}
$$




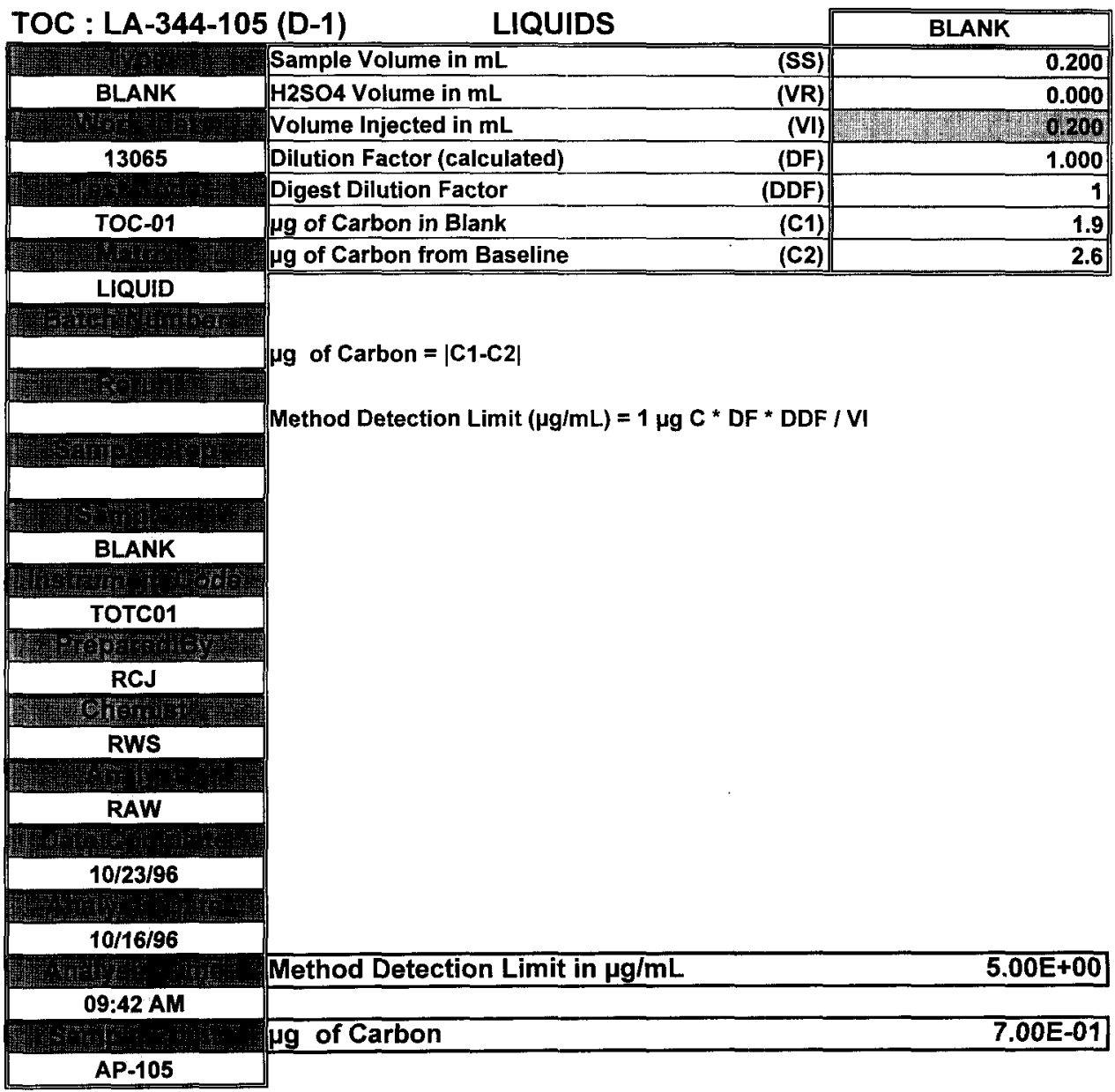

\begin{tabular}{|c|c|c|c|}
\hline Data Entered By: & RCJ & Date: & $10 / 23 / 96$ \\
\hline Signature of Chemist: & juiduraleh & Date: & $10 / 24 / 96$ \\
\hline
\end{tabular}


HNFE-SD-WM-DP-202, REV. 1

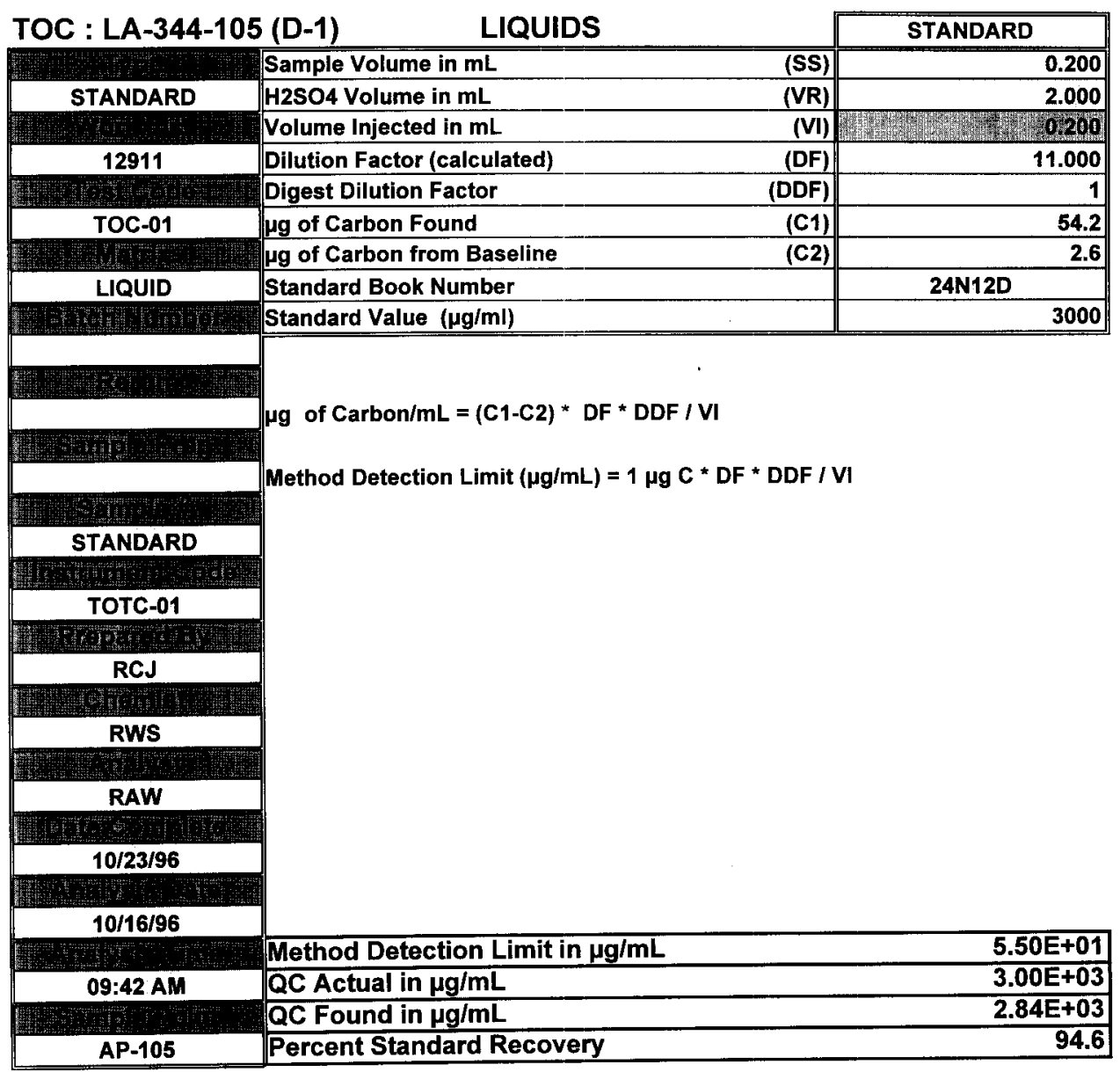

\begin{tabular}{|c|c|c|c|}
\hline Data Entered By: & $\mathrm{RCJ}$ & Date: & $10 / 23 / 96$ \\
\hline Signature of Chemist: & in schued & Date: & $10 / 24 / 96$ \\
\hline
\end{tabular}

\section{6}




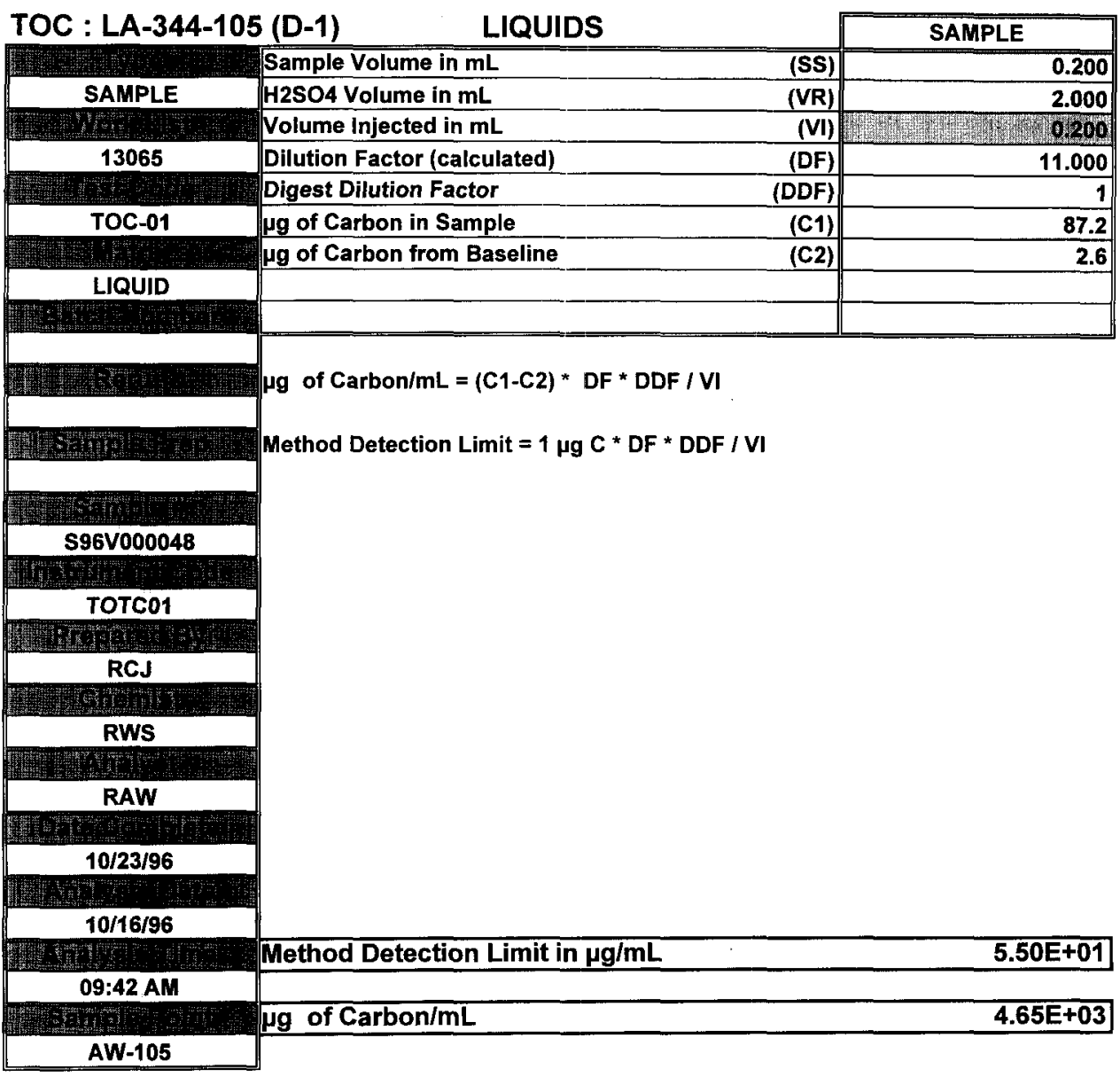

\begin{tabular}{|c|c|c|c|}
\hline Data Entered By: & RCJ & Date: & $10 / 23 / 96$ \\
\hline Signature of Chemist: & Penpliveden & Date: & $10 / 24 / 9 k$ \\
\hline
\end{tabular}

\section{7}


HNFGSD-WM-DIF-202, REV. 1

TOC : LA-344-105 (D-1)

LIQUIDS

\begin{tabular}{|c|c|c|c|c|}
\hline 1 & \multicolumn{2}{|c|}{ Sample Vial Data } & \multicolumn{2}{|c|}{ Spiked Vial Data } \\
\hline SPIKE & Sample Volume in $\mathrm{mL}$ & 0.200 & Was the sample dilution used? (yes/no) & YES \\
\hline & H2SO4 Volume in mL & 2.000 & Sample Volume in $\mathrm{mL}$ & 0.400 \\
\hline 12911 & Volume Injectod in $\mathrm{mL}$ (VI) & 0.200 & H2SO4 Volume in $\mathrm{mL}$ & 0.000 \\
\hline & $\mu g$ of Carbon in Sample (C1) & 87.2 & Amount of Spike Std. In mL (SPK VOL) & 0.400 \\
\hline TOC-01 & $\mu g$ of Carbon from Baseline (C2) & 2.6 & Volume Injected in $\mathrm{mL}$ & 280200 \\
\hline & & & pg C In Sample + Splke & 126 \\
\hline LIQUID & & & Pre-Spike Dilution Factor & 11.00 \\
\hline & & & Spike Book Number & 23N12B \\
\hline & & & Spike Value in $\mu \mathrm{g} / \mathrm{ml}$ & 753 \\
\hline
\end{tabular}

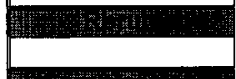

Spike Correction Factor (SPK CF) = (SPK SS + SPK VOL + SPK VR) / SPK VI

Sample Correction Factor (SAM CF) $=(\mathrm{SS}+\mathrm{VR}) /(\mathrm{VI})$

Sample Size Correction Factor (SS CF) $=$ (SPK SS) / (SS)

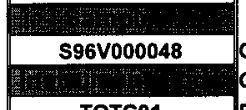

QC Actual in $\mu \mathrm{g} / \mathrm{mL}=$ Spike Value $(\mu \mathrm{g} / \mathrm{mL})$

QC Found in $\mu \mathrm{g} / \mathrm{mL}=[(\mathrm{C3}-\mathrm{C2})$ (SPK CF) - (C1-C2)(SAM CF)(SS CF)/(PDF) $/$ (SPK VOL)

\begin{tabular}{|c|}
\hline TOTC01 \\
\hline RCJ \\
\hline RWS \\
\hline RLY \\
\hline RAW \\
\hline $10 / 23 / 96$ \\
\hline $10 / 16 / 96$ \\
\hline 09:42 AM \\
\hline AP-106 \\
\hline
\end{tabular}

Percent Spike Recovery = (QC Found) $/$ (QC Actual) * 100

QC Actual in $\mu \mathrm{g} / \mathrm{mL}$

$7.53 E+02$

QC Found in $\mu \mathrm{g} / \mathrm{mL}$

8.11E+02

Percent Spike Recovery

107.7

\begin{tabular}{|c|c|c|}
\hline Data Entered By: & $\mathrm{RCJ}$ & Date: $\quad 10 / 23 / 96$ \\
\hline Signature of Chemist: & & Date: $10 / 24 / 7 i$ \\
\hline
\end{tabular}


HNFG-SD-WM-DP-202, REV. 1

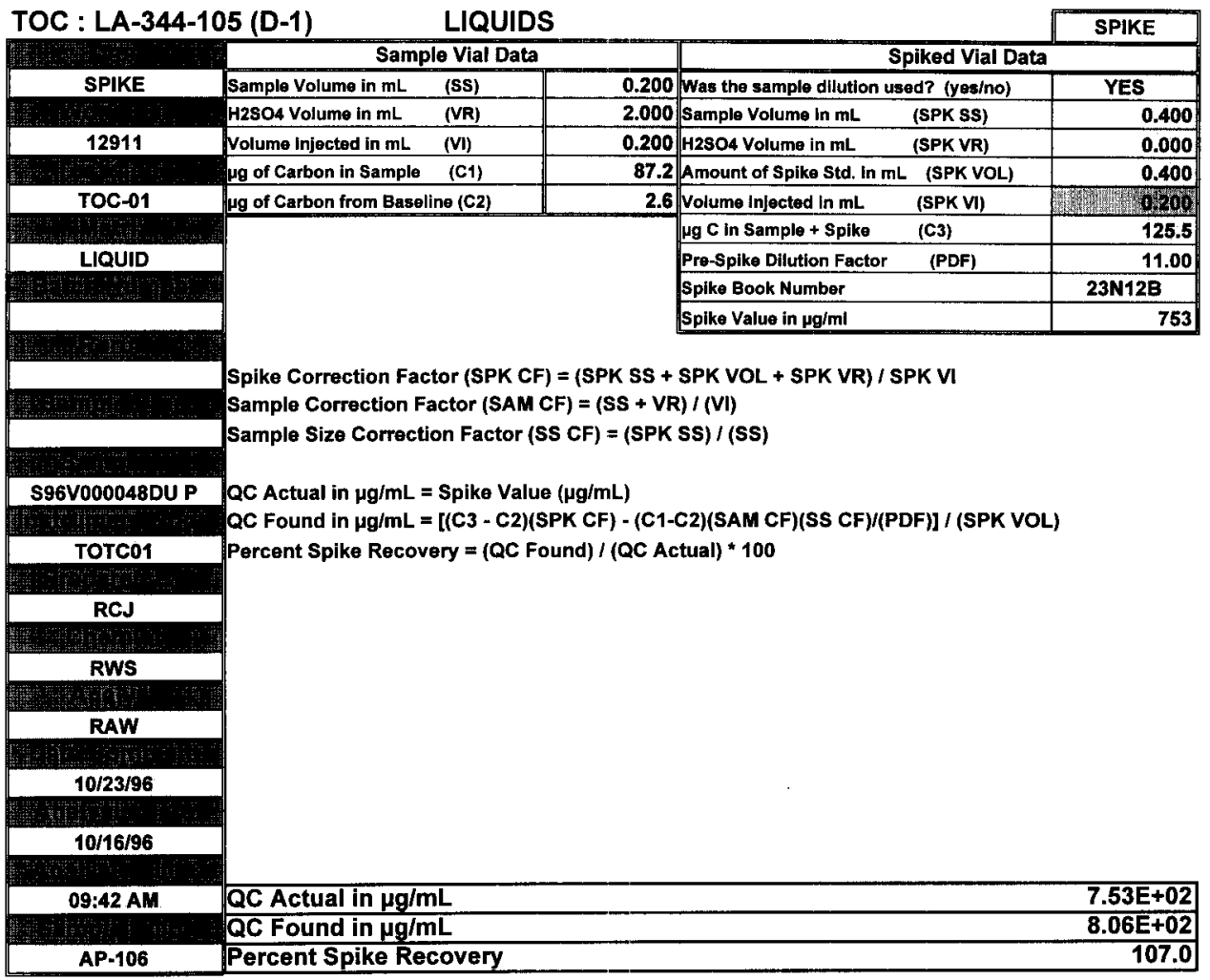

\begin{tabular}{|c|c|c|c|}
\hline Data Entered By: & RCJ & Date: & $10 / 23 / 96$ \\
\hline Signature of Chemist: & & Date: & $0 / 24 / 9$ \\
\hline
\end{tabular}


HF-SD-WM-DP-202, REV. 1

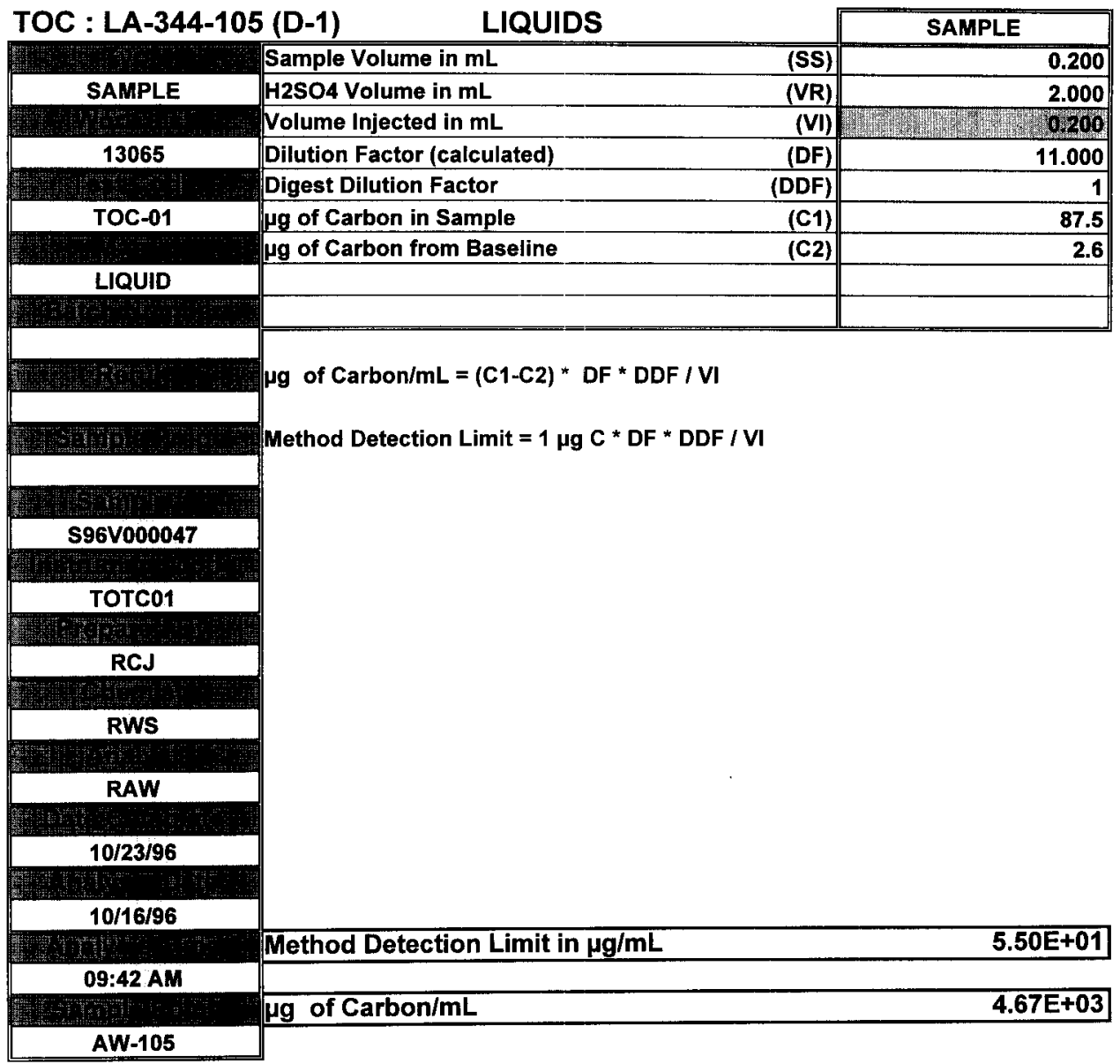

\begin{tabular}{|c|c|c|c|}
\hline Data Entered By: & $\mathrm{RCJ}$ & Date: & $10 / 23 / 96$ \\
\hline Signature of Chemist: & Sel $x d$ & Date: & $10 / 24 / 96$ \\
\hline
\end{tabular}

\section{0}




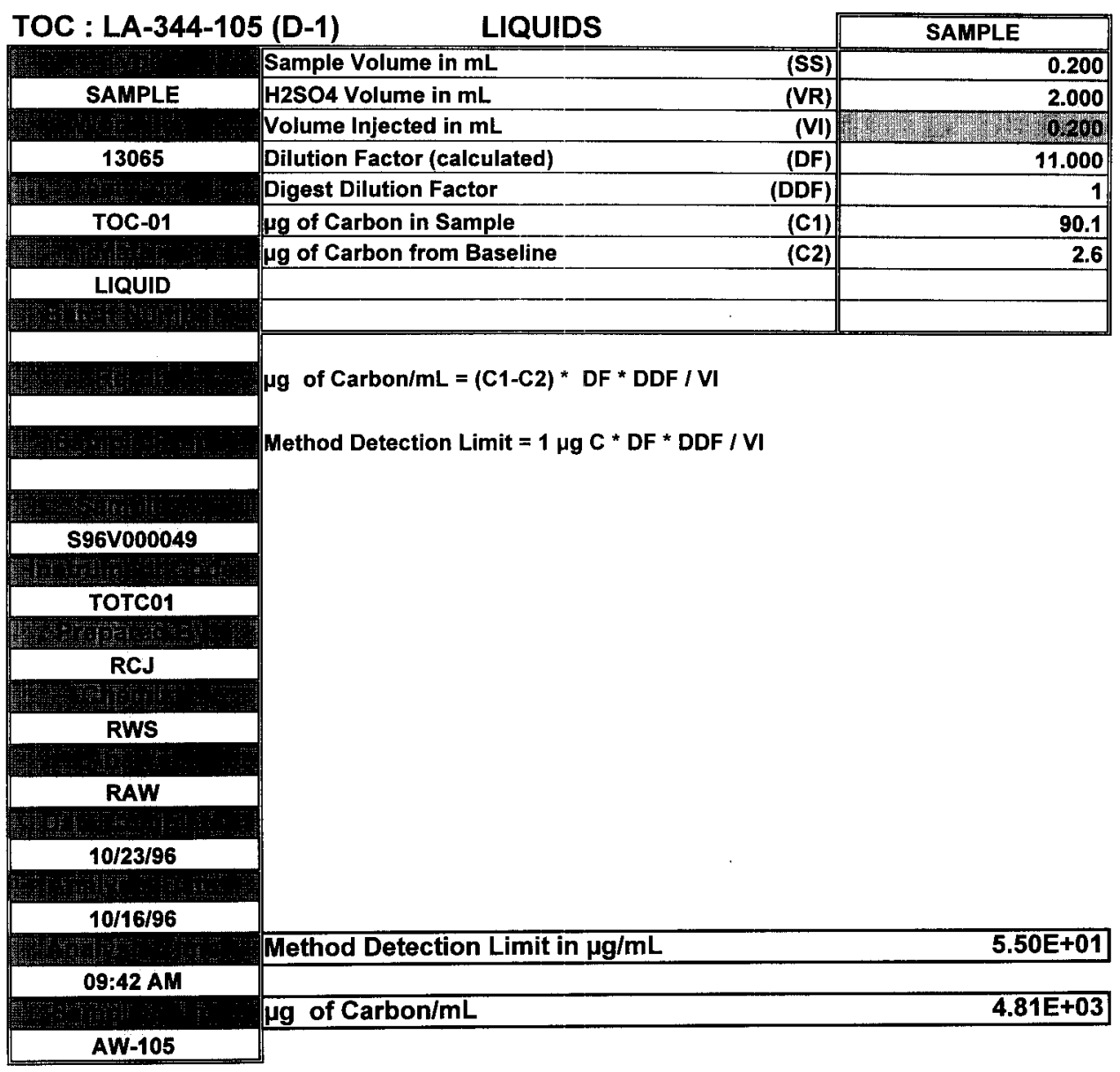

\begin{tabular}{|c|c|c|c|}
\hline Data Entered By: & RCJ & Date: & $10 / 23 / 96$ \\
\hline Signature of Chemist: & Ru' d-hores & Date: & $1012+196$ \\
\hline
\end{tabular}




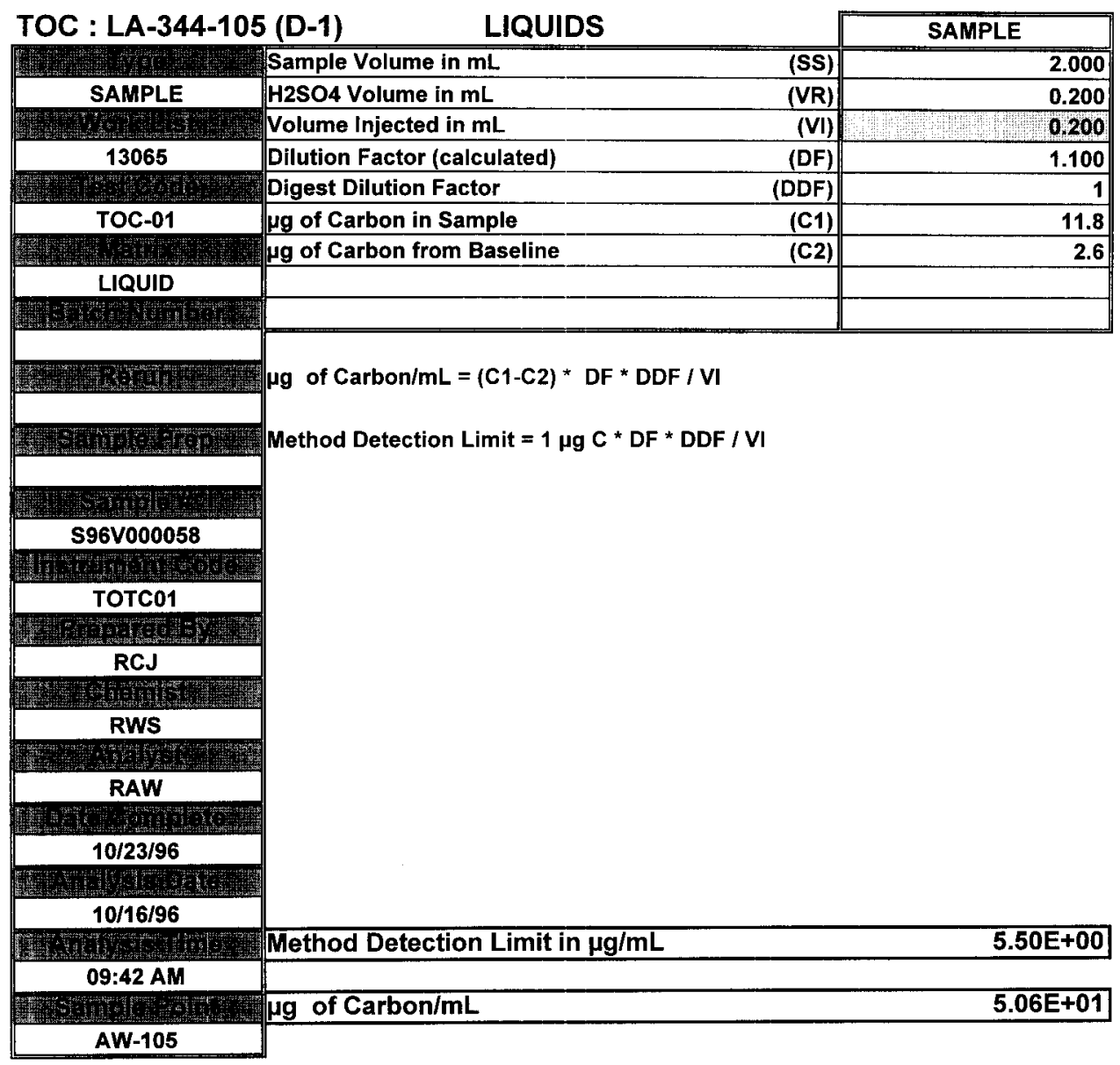

\begin{tabular}{|c|c|c|c|}
\hline Data Entered By: & RCJ & Date: & $10 / 23 / 96$ \\
\hline Signature of Chemist: & Rusdivecen & Date: & $10 / 24 / 91$ \\
\hline
\end{tabular}




\section{LABCORE Completed RadChem Report for Worklist\#: 12889}

Analyst: slh

Instrument: $\mathrm{U} 01$

Book\# 104056

Method: $19.925-\mathrm{NQ9}$ Rev/Mo

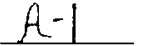

Worklist Comment: AP-105.0.1-10ml sample prep. For spike: $0.1 \mathrm{ml}$. new

\begin{tabular}{|c|c|c|c|c|c|c|c|c|c|}
\hline Seq Type & \multicolumn{3}{|c|}{ Sample\# R A } & Test & Matrix & Actual & Found & DL or Yield & Unit \\
\hline 1 STD: & & 0 & बu- 01 & $t-02$ & LIQTID & $6.398-02$ & $6.40 \mathrm{E}-02$ & $100 . \pm 56$ & \& kecovery \\
\hline $1 \leqslant \mathrm{TO}$ & & 0 & 01 & $\mathrm{U}-02 \mathrm{E}$ & LIQUID & 1 & $3.28 z+00$ & 3.280 & Ratio \\
\hline 2 BLMK & & 0 & QtV-01: & $0-02$ & LIQUID & 1 & $5.59 \mathrm{E}-2$ & $55.9000-003$ & $\mathrm{ug} / \mathrm{mit}$ \\
\hline 2 BLANK & & 0 & $\oplus 0-01$ & $\mathrm{U}-02 \mathrm{E}$ & LIQUID & 1 & $2.04 E+00$ & 2.040 & Ratio \\
\hline 3 SAMPLE & $\$ 96 v 000047$ & 0 & $\square 0-02$ & $0-02$ & LFQUID & N/A & $1.42 \mathrm{E}+01$ & $370.00-005$ & ug/mil \\
\hline 3 SAMPLE & 5960000047 & 0 & QU-01 & $\mathrm{U}-02 \mathrm{E}$ & LIQUID & N/A & $3.35 E+00$ & $0.00+000$ & \& Inat Exror \\
\hline 4 pUP. & 5960000047 & 0 & 001 & $0-02$ & LIOUID & $2.42 \mathrm{E}+1$ & $1.39 \mathrm{E}+1$ & 2.135 & RPD \\
\hline 4 DUP & s96v000047. & 0 & DU-01 & $\mathrm{U}-02 \mathrm{E}$ & LIQUID & 1 & $3.47 \mathrm{E}+00$ & 3.470 & Ratio \\
\hline 5 SPK & $\$ 96 v 000047$ & 0 & $00-01$ & $0-02$ & LIQUID & $5.498+01$ & $4.80 z+01$ & 87,432 & \& Recovery \\
\hline 6 SPR-DUP & s96v000047 & 0 & GU-01 & $\mathrm{U}-02$ & LIQDID & $9.80 E+01$ & 4. $97 \mathrm{E}+01$ & 3.480 & RPD \\
\hline
\end{tabular}

\section{Final page for worklist\# 12889}

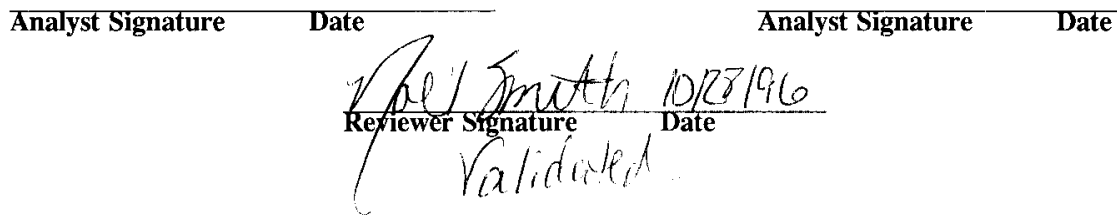




\section{LABCORE Data Entry Template for Worklist\# 12889}

Analyst: SLH C Instrument: U01

Method: LA-925-009 Rev/Mod it - l
Book\#

sut $10-26-96$

Worklist Comment: AP-105.0.1-10ml sample prep.For spike: $0.1 \mathrm{ml}$. new

S Type Sample\# R A Test Matrix Group\# Project
$1 \operatorname{STD}$
$@ \mathrm{U}-01$
IIQUID
2 BLNK
$\Leftrightarrow \mathrm{U}-01$
LIQUID
3 SAMPLE
S96V000047 0
$@ U-01$
LIQUID
$\mathrm{U}-02 \mathrm{E}$
$96000853 \mathrm{AP}-105$
Analytes Requested: U-02

LIQUID

5 SPK

S96V000047 0 @U-01

LIQUID

6 SPK -DUP

S96V000047 $0 \quad @ U-01$

LIQUID

\section{Final page for worklist \# 12889}
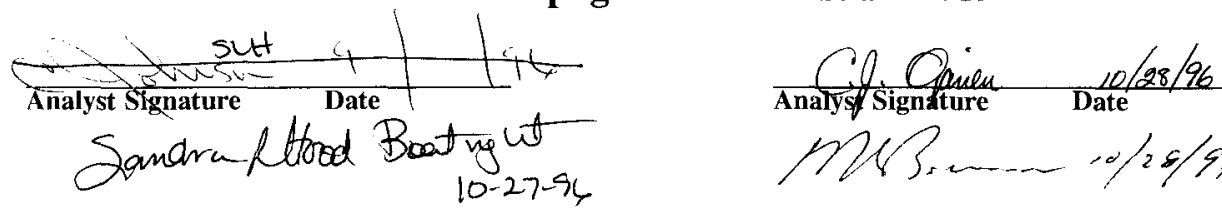

Date

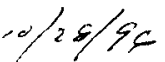

Data Entry Comments:

$S=$ Worklist Slot Number, $R=$ Replicate Number, $A=$ Aliquot Code. 
WORKBOOK PAGE: STD

HNF

Uranium by Phosphorescence: LA-925-009 (A-1) LIQUID/SOLID

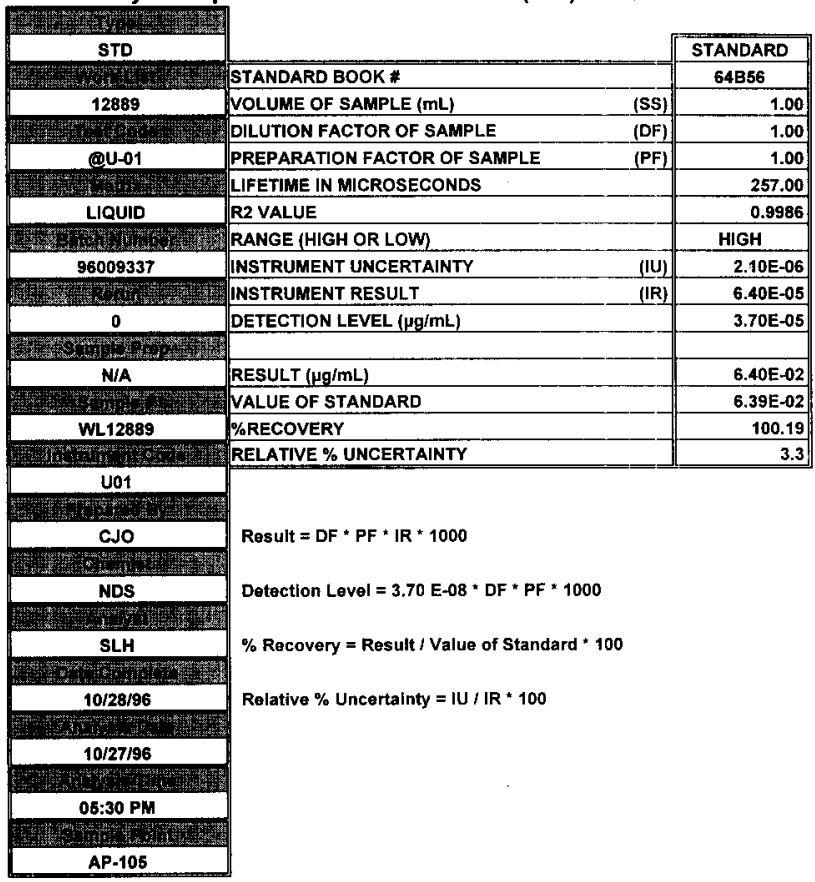

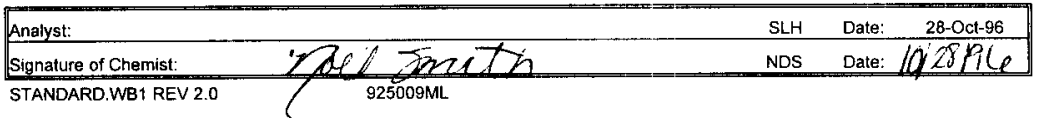




\section{HNFG-SD-WIM-DP-202, REV.}

WORKBOOK PAGE: BLANK2

Uranium by Phosphorescence: LA-925-009 (A-1) LIQUID/SOLID

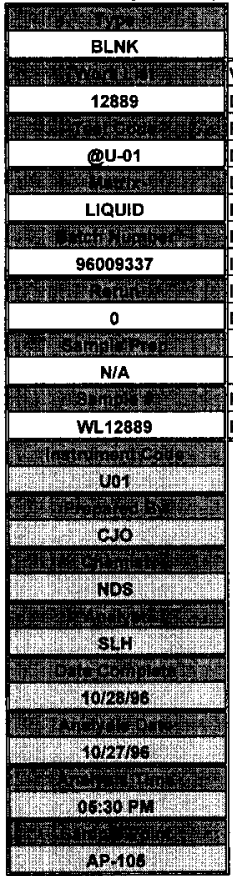

\begin{tabular}{|c|c|}
\hline & BLANK \\
\hline VOLUME OF SAMPLE $(\mathrm{mL})$ & 1.00 \\
\hline DILUTION FACTOR OF SAMPLE & 1.00 \\
\hline PREPARATION FACTOR OF SAMPLE & 100.00 \\
\hline DIGEST DILUTION FACTOR & 1.0000 \\
\hline LIFETIME IN MICROSECONDS & 225.00 \\
\hline R2 VALUE & 0.9963 \\
\hline RANGE (HIGH OR LOW) & Low \\
\hline INSTRUMENT UNCERTAINTY & 1.14E-08 \\
\hline INSTRUMENT RESULT & 5.59E-07 \\
\hline DETECTION LEVEL $(\mu \mathrm{g} / \mathrm{mL})$ & $3.70 E-03$ \\
\hline RESULT ( $\mu \mathrm{g} / \mathrm{mL})$ & $5.59 \mathrm{E}-02$ \\
\hline RELATIVE \% UNCERTAINTY & 2.0 \\
\hline
\end{tabular}

Result $=$ DF * PF * DDF *IR* 1000

Detection Level $=3.70$ E-08 * DF * PF * DDF * 1000

Relative \% Uncertainty $=$ IU / IR * 100

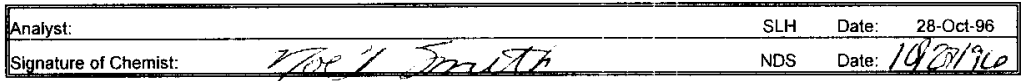

BLANK.WB1 REV 2.0

$925009 \mathrm{ML}$ 
HNF

WORKBOOK PAGE: SAM3

Uranium by Phosphorescence: LA-925-009 (A-1) LIQUID/SOLID

\begin{tabular}{|c|c|c|}
\hline SAMPLE & & SAMPLE \\
\hline & VOLUME OF SAMPLE (mL) & 1.00 \\
\hline 12889 & DILUTION FACTOR OF SAMPLE & 1.00 \\
\hline Mos & PREPARATION FACTOR OF SAMPLE & 100.00 \\
\hline Qu-01 & DIGEST DILUTION FACTOR & 1.0000 \\
\hline 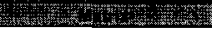 & LIFETIME IN MICROSECONDS & 153.00 \\
\hline LIQUID & R2 VALUE & 0.9991 \\
\hline 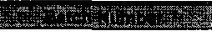 & RANGE (HIGH OR LOW) & HIGH \\
\hline 96009337 & INSTRUMENT UNCERTAINTY & 4.75E-06 \\
\hline Ds & INSTRUMENT RESULT & 1,42E-04 \\
\hline 0 & DETECTION LEVEL ( $\mathrm{g} / \mathrm{mL}$ ) & $3.70 \mathrm{E}-03$ \\
\hline & & \\
\hline N/A & CONCENTRATION IN SOLUTION (g/L) & $1.42 \mathrm{E}-02$ \\
\hline 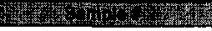 & RESULT ( $\mu g / \mathrm{mL})$ & $1.42 \mathrm{E}+01$ \\
\hline \$96V000047 & RELATIVE \% UNCERTAINTY & 3.3 \\
\hline
\end{tabular}

W.W

Yram $\mathrm{Cu}$

Result $=$ DF * PF * DDF * IR * 1000

Detection Level $=3.70 \mathrm{E}-08$ * DF * PF * DDF * 1000

Relative \% Uncertainty $=I U / I R * 100$

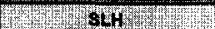

3.

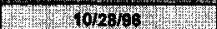

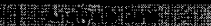

WII:

3)

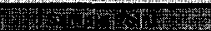

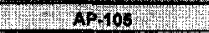

\begin{tabular}{|c|c|c|}
\hline Analyst: & SLH & 28-Oct-96 \\
\hline Signature of Chemist: & NDS & Date: $\angle 127 \not$ \\
\hline
\end{tabular}


WORKBOOK PAGE: DUP4 Uranium by Phosphorescence: LA-925-009 (A-1) LIQUID/SOLID

\begin{tabular}{|c|c|c|}
\hline DUP & & DUP \\
\hline hos & VOLUME OF SAMPLE (mL) & 1.00 \\
\hline 12889 & DILUTION FACTOR OF SAMPLE & 1.00 \\
\hline & PREPARATION FACTOR OF SAMPLE & 100.00 \\
\hline (Q)-01 & DIGEST DILUTION FACTOR & 1,0000 \\
\hline & LIFETIME IN MICROSECONDS & 155.00 \\
\hline LIQUID & R2 VALUE & 0.9986 \\
\hline M. & RANGE (HIGH OR LOW) & $\mathrm{HIGH}$ \\
\hline 96009337 & INSTRUMENT UNCERTAINTY & 4.82E-06 \\
\hline 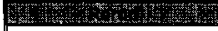 & INSTRUMENT RESULT & 1.39E-04 \\
\hline 0 & DETECTION LEVEL $(\mu \mathrm{g} / \mathrm{mL})$ & 3.70E-03 \\
\hline 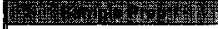 & & \\
\hline N/A & CONCENTRATION IN SOLUTION (g/L) & $1.39 \mathrm{E}-02$ \\
\hline Wy & RESULT $(\mu \mathrm{g} / \mathrm{mL})$ & $1.39 E+01$ \\
\hline S96V000047 & RELATIVE \% UNCERTAINTY & 3.5 \\
\hline
\end{tabular}

hy (F)

H. W.

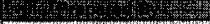

W.

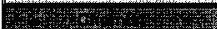

WW:

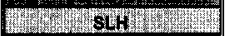

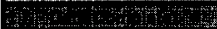

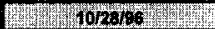

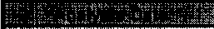

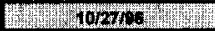

\%

Wrathex

1) $30 \%$

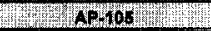

Detection Level $=3.70$ E-08 * DF * PF * DDF * 1000

Relative \% Uncertainty = IU /IR * 100

\begin{tabular}{|c|c|c|c|}
\hline Analyst: & SLH & Date: & 28-Oct-96 \\
\hline Signature of Chemist: & NDS & Date: & $\sqrt{3790}$ \\
\hline
\end{tabular}




\section{HNF-SD-WM-DP-202, REV.}

WORKBOOK PAGE: SPIKE5

Uranium by Phosphorescence: LA-925-009 (A-1) LIQUID/SOLID

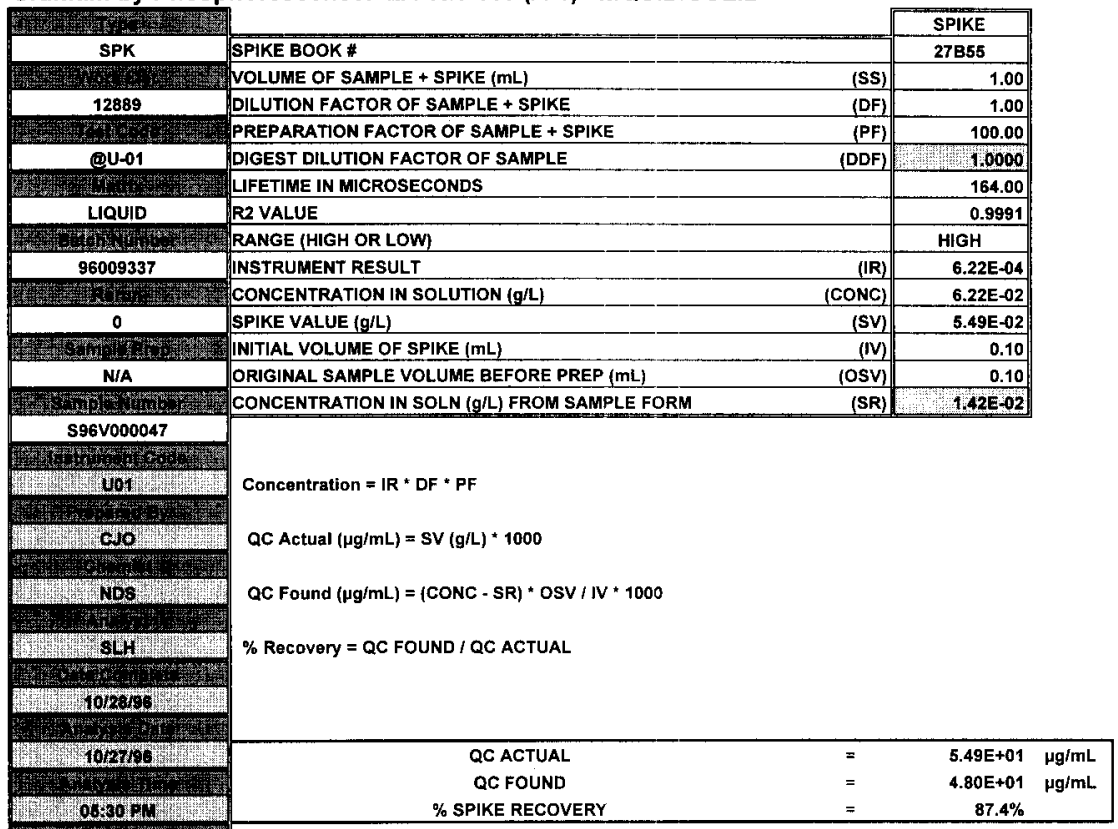

\begin{tabular}{|c|c|c|c|}
\hline Analyst: & SLH & Date: & 28-Oct-96 \\
\hline ature of Chemist: & & & \\
\hline
\end{tabular}


WORKBOOK PAGE: SP_DUP6

Uranium by Phosphorescence: LA-925-009 (A-1) LIQUID/SOLID

\begin{tabular}{|c|c|c|c|}
\hline 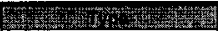 & & & SPK-DUP \\
\hline SPK-DUP & SPIKE BOOK \# & & 27B55 \\
\hline (n) & VOLUME OF SAMPLE + SPIKE (mL) & (SS) & 1.00 \\
\hline 12889 & DILUTION FACTOR OF SAMPLE + SPIKE & (DF) & 1.00 \\
\hline Hxum (x) & PREPARATION FACTOR OF SAMPLE + SPIKE & (PF) & 100.00 \\
\hline (9)-01 & DIGEST DILUTION FACTOR OF SAMPLE & (DDF) & 10000 \\
\hline 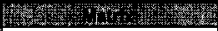 & LIFETIME IN MICROSECONDS & & 162.00 \\
\hline LIQUID & R2 VALUE & & 0.9991 \\
\hline Wx- & RANGE (HIGH OR LOW) & & HIGH \\
\hline 96009337 & INSTRUMENT RESULT & (IR) & $6.39 \mathrm{E}-04$ \\
\hline 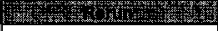 & CONCENTRATION IN SOLUTION (g/L) & (CONC) & $6.39 \mathrm{E}-02$ \\
\hline 0 & SPIKE VALUE (g/L) & (SV) & $5.49 \mathrm{E}-02$ \\
\hline W. & INITIAL, VOLUME OF SPIKE (mL) & (IV) & 0.10 \\
\hline N/A & ORIGINAL SAMPLE VOLUME BEFORE PREP (mL) & (OSV) & 0.10 \\
\hline (1) & CONCENTRATION IN SOLN (g/L) FROM SAMPLE FORM & (SR) & $1,42 \mathrm{E} 02$ \\
\hline
\end{tabular}

S96V000047

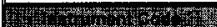

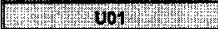

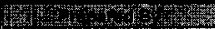

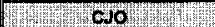

Y.4.

Why Wos

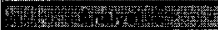

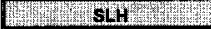

\%

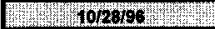

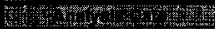

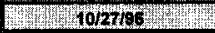

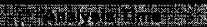

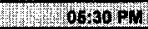

F.

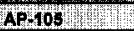

Concentration $=\mathrm{IR} * \mathrm{DF}$ * $\mathrm{PF}$

QC Actual $(\mu g / m L)=S V(g / L) * 1000$

QC Found $(\mu \mathrm{g} / \mathrm{mL})=\{$ CONC $-\mathrm{SR}) *$ OSV $/ \mathrm{IV} * 1000$

$\%$ Recovery = QC FOUND / QC ACTUAL

\begin{tabular}{|c|c|}
\hline Analyst: & 28-Oct-96 \\
\hline Signature of Chemist: & \\
\hline
\end{tabular}

$5.49 E+01 \mu \mathrm{g} / \mathrm{mL}$

4.97E+01 $\mu \mathrm{g} / \mathrm{mL}$

$90.5 \%$ 
WHE-SD-WM-DP-202, REV. 1

\section{URANIUM ANALYSIS}

$\stackrel{4}{*}$

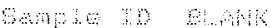

कथल

mer. matio two

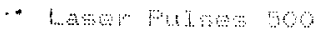

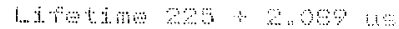

We " $9 \%$

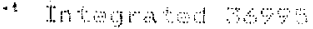

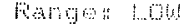

Daberme lop

क.

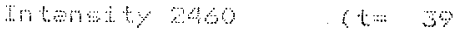

कno

bidution rator i marm.

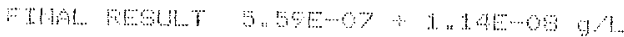

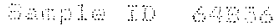

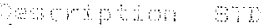

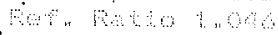

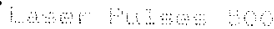

- aretan

-

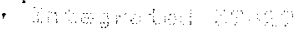

- $\quad \cdots, \quad \cdots$

$5, \cdots \quad \%$

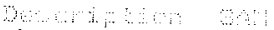

बत : $:$

,

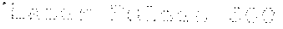

nमt me

at

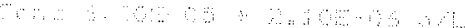

-

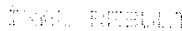

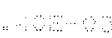

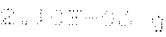

$\cdots$

....

1: - a

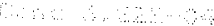

A

a $10, \cdots$ 
UNE SO-WM-DP-202, REV. 1

\author{
URANIUM ANALYSIS
}

Bampla I0 $9964004 \%$

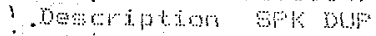

- Wer Ratio I. 06

- Laser rusas

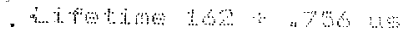
क

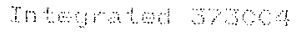

wase नाt

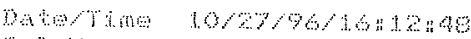

कI.

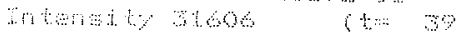

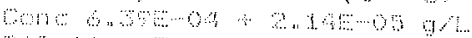

(1)

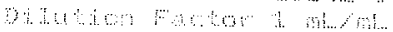




\section{LABCORE Completed RadChem Report for Worklist\#: 12891}

Analyst: dgg

Instrument: U01

Book\#641356

Method: CA-925-ARC Rev/Mod

$A-1$

Worklist Comment: AP-105. 0.1-10ml sample prep.Spike: $0.1 \mathrm{ml}$. new

\begin{tabular}{|c|c|c|c|c|c|c|c|c|c|}
\hline Seq Type & \multicolumn{3}{|c|}{ Sample\# R A } & Test & Matrix & Actual & Found & DL or Yield & Unit \\
\hline 1 sTD & & 0 & 001 & 0.02 & LIQUID & $6.39 \mathrm{E}-02$ & $6.42 \mathrm{E}-02$ & 100,469 & o Recovery \\
\hline $1 \mathrm{STD}$ & & 0 & $90-01$ & $0-025$ & LIQUID & 1 & $3.24 \mathrm{~s}+00$ & 3.240 & Ratio \\
\hline 2 BLIX & & 0 & $00-01$ & $0-02$ & LIOUID & 1 & $<4.07 \mathrm{E}-2$ & & ug/mL \\
\hline 2 BLANK & & 0 & Q0-01 & $0-02 \mathrm{E}$ & LIQDID & 1 & $N / A$ & & Ratio \\
\hline 3 SAMPLE & $596 \times 000040$ & 0 & $00+01$ & $0 \div 02$ & LIQUID & $\mathrm{N} / \mathrm{A}$ & $1.97 \mathrm{~B}+01$ & $407.00-004$ & $49 / \mathrm{min}$ \\
\hline 3 SAMPLI & S96V000048 & 0 & $00-01$ & $\mathrm{D}-02 \mathrm{E}$ & LIQUID & $N / A$ & $1.45 \mathrm{I}+00$ & $0.00+000$ & Ind nxror \\
\hline 4 DUP & s96v0000048 & 0 & $\infty 0-01$ & 0.02 & LTOUTD & $1.97 E+1$ & $1.738+1$ & 12.973 & RED \\
\hline 4 DDP & $596 \mathrm{V000048}$ & 0 & $00-01$ & $0-02 B$ & LIQVID & 1 & $1.45 \mathrm{~B}+00$ & 1.450 & Ratio \\
\hline $5 \mathrm{sFK}$ & 5960000040 & 0 & $00-01$ & $0-02$ & LTOUDD & $5.49 \mathrm{E}+01$ & $5.17 B+01$ & 94.171 & o Recovery \\
\hline 6 SPK-DUP & S96V000048 & 0 & $00-01$ & $0-02$ & LIQUID & $5,17 \mathbf{E}+01$ & $5.08 \mathrm{BE}+01$ & 1.756 & RED \\
\hline 7 SAMPLE & s96V000049 & 0 & $\square 0-01$ & $0-02$ & LrouID & $N / A$ & $1.678+01$ & $407.00-004$ & $\mathrm{ug} / \mathrm{met}$ \\
\hline 7 SAMPLB & 3960000049 & 0 & $00-01$ & $0-02 \mathrm{E}$ & LIQUID & $\mathbf{N} / \mathbf{A}$ & $1.53 \mathrm{E}+00$ & $0.00+000$ & : Inat Exrox \\
\hline
\end{tabular}

Final page for worklist\# 12891

Simature attached. Analyst Signature Date

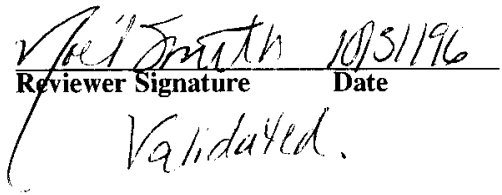




\section{LABCORE Data Entry Template for Worklist\# 12891}

Analyst: SUIf $\frac{10-27-26}{t}$ Instrument: U01

Book $\frac{-9+2+2}{2}-10.3$

Method: LA-925-009 Rev/Mod il

Worklist Comment: AP-105. 0.1-10ml sample prep.Spike: $0.1 \mathrm{ml}$. new

\begin{tabular}{|c|c|c|c|c|c|c|c|}
\hline$\overline{\mathrm{S}}$ & Type & Sample\# & R A & Test & Matrix & Group\# & Project \\
\hline 1 & STD & & & @U-01 & IIQUID & & \\
\hline 2 & BLNK & & & $@ U-01$ & LIQUID & & \\
\hline 3 & SAMPLE & S96V000048 & 0 & $@ U-01$ & LIQUID & 96000855 & AP- 105 \\
\hline & & Analytes Reque & ested: & : U-02 & , $\mathrm{U}-02 \mathrm{E}$ & & \\
\hline 4 & DUP & S96V000048 & 0 & $@ U-01$ & LIQUIID & & \\
\hline 5 & SPK & S96V000048 & 0 & $@ \mathrm{U}-01$ & LIQUTD & & \\
\hline 6 & SPK-DUP & $S 96 V 000048$ & 0 & $@ \mathrm{U}-01$ & LIQUID & & \\
\hline 7 & SAMPLE & S960000049 & $\begin{array}{c}0 \\
0 \sin \end{array}$ & $@ \mathrm{U}-01$ & LIQUID & 96000855 & AP- -105 \\
\hline
\end{tabular}

\section{Final page for worklist \# 12891}

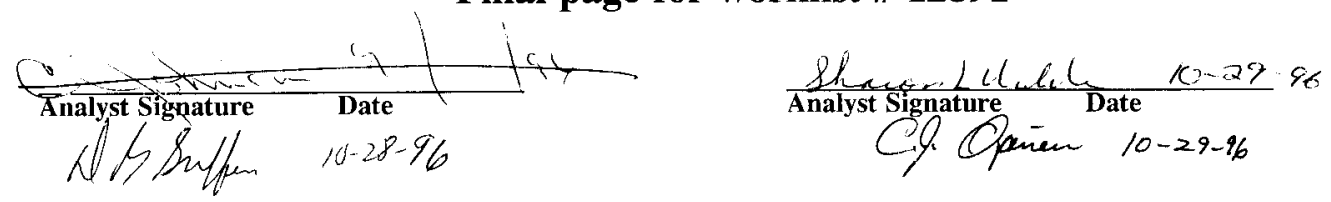

Data Entry Comments:

SLHB boled down

$\vec{S}=$ Worklist Slot Number, $R=$ Replicate Number, $A=$ Aliquot Code. 
WHAG-SD-WM-DP-202, REV. 1

Uranium wata wotvecinum ouses:

$10 / 28794$

kllst Number: 12891

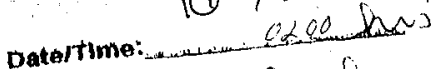

wo Bonk Numbor: 2 A BSS

spike vatume: 100 onf

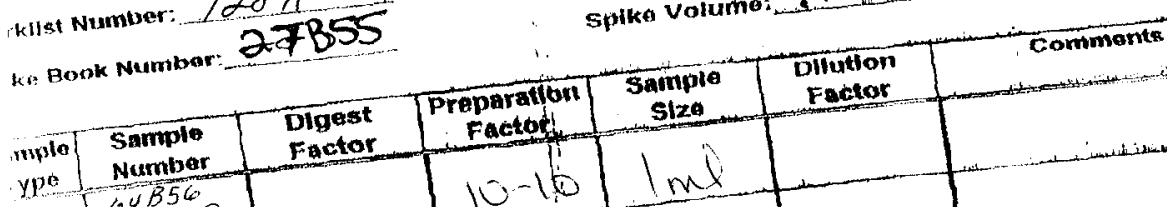

iiti) $\begin{gathered}64356 \\ 55830 \\ 542\end{gathered}$

(3i) NK

sal

Dif $\frac{-48}{48}$

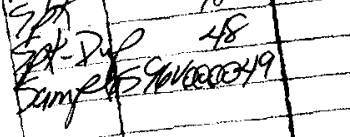

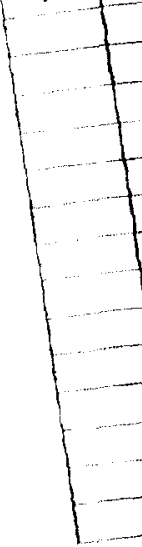

$-1$
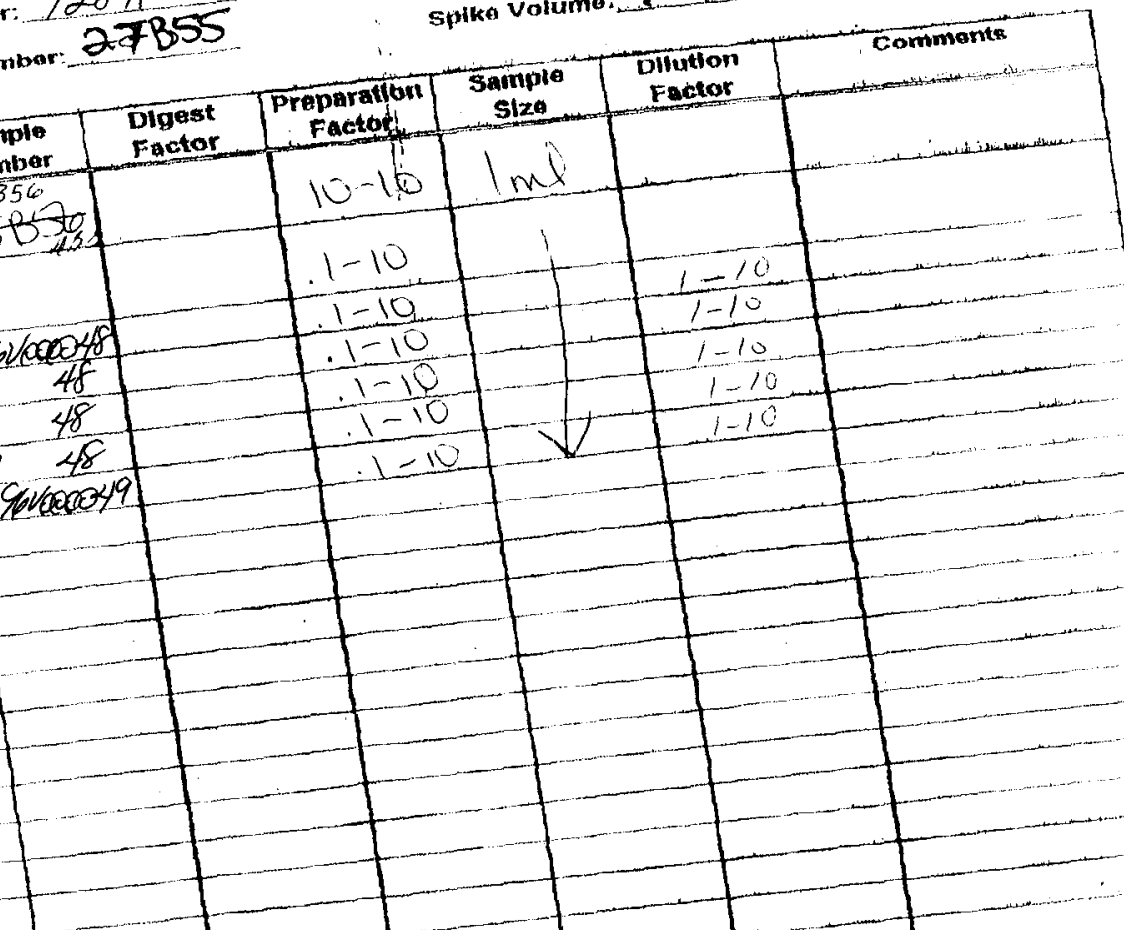
WF-SD-WM-DP-202, REV 1

$+$

\section{URANIUM ANALYSIS}

4

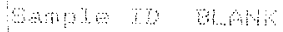

mon का का

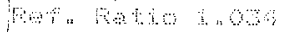

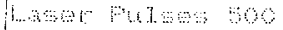

1. A to

2

और

(a)

anda

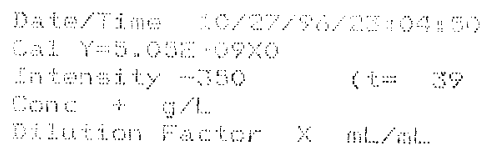

काष

औ-

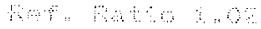

1.

1 \% 10

-

$\because \because \because)^{2}-\quad \square$

a

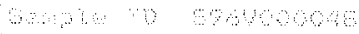

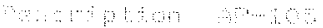

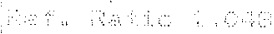

-

$\because+\cdots$

का का
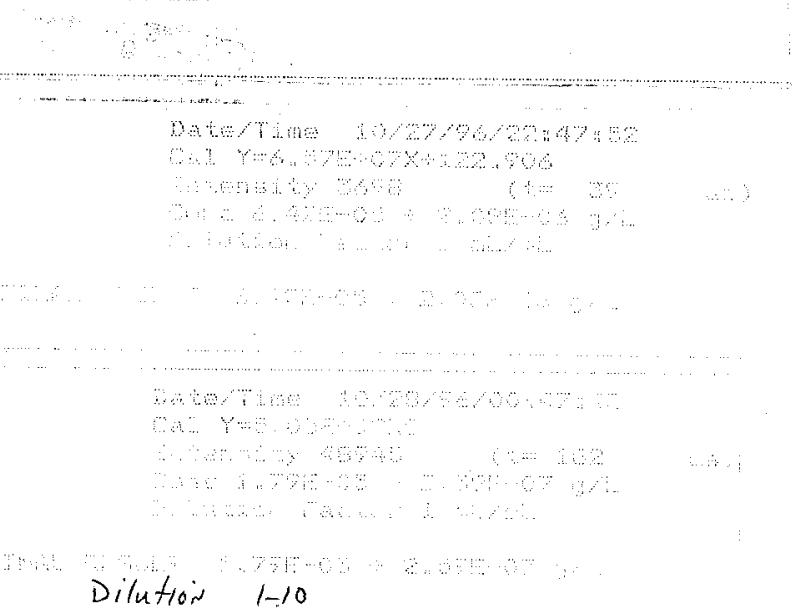

Dilution $1-10$

Dilution $1-10$ 


\section{HNE-SD-WM-DP-202, REV.}

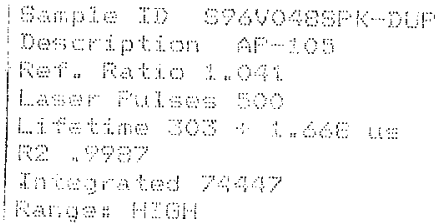

\section{URANIUM ANALYSIS}

URANTUM ANALYSIS

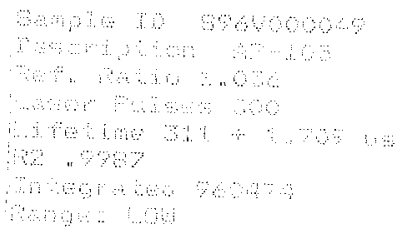

Am, का 9 क्येक?

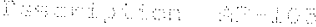

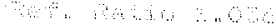

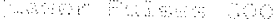

actore

+

and क्ष

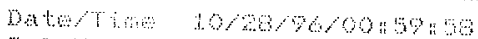

स म

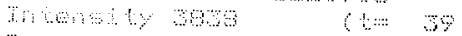

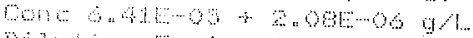

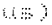

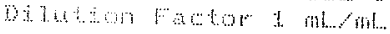

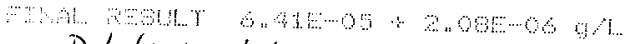

Dilution $1-10$
Dilution 1-10 n

a

In $10.8 \%$

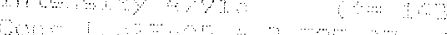

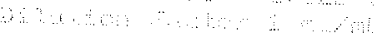

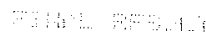


WORKBOOK PAGE: STD1

\section{Uranium by Phosphorescence: LA-925-009 (A-1) LIQUID/SOLID}

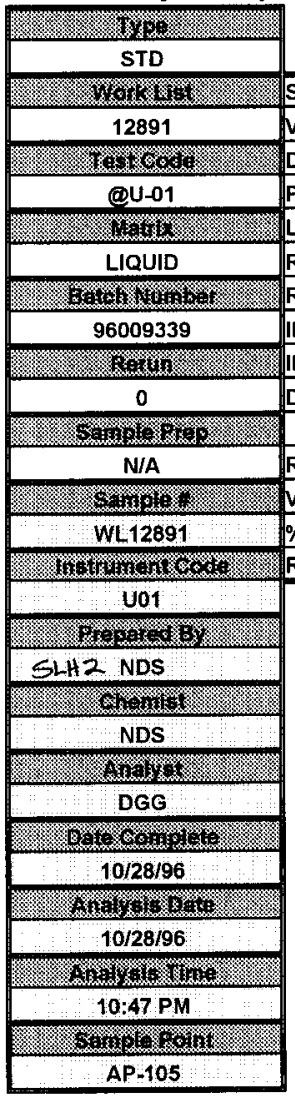

Result $=$ DF * PF * IR *1000

Detection Level $=3.70 \mathrm{E}-08$ * DF * PF * 1000

$\%$ Recovery $=$ Result $/$ Value of Standard * 100

Relative $\%$ Uncertainty $=$ IU / IR * 100

Analyst:
Signature of Chemist:
STANDARD.WB1 REV 2.0

DGG NDS
262.00 0.9989 HIGH

(IU)
2.08E-06

(IR) $6.42 \mathrm{E}-05$ $3.70 \mathrm{E}-05$ $6.42 \mathrm{E}-02$ $6.39 \mathrm{E}-02$ 100.50 RELATIVE \% UNCERTAINTY RESULT $(\mu \mathrm{g} / \mathrm{mL})$ 
WORKBOOK PAGE: BLANK2

\section{Uranium by Phosphorescence: LA-925-009 (A-1) LIQUID/SOLID}

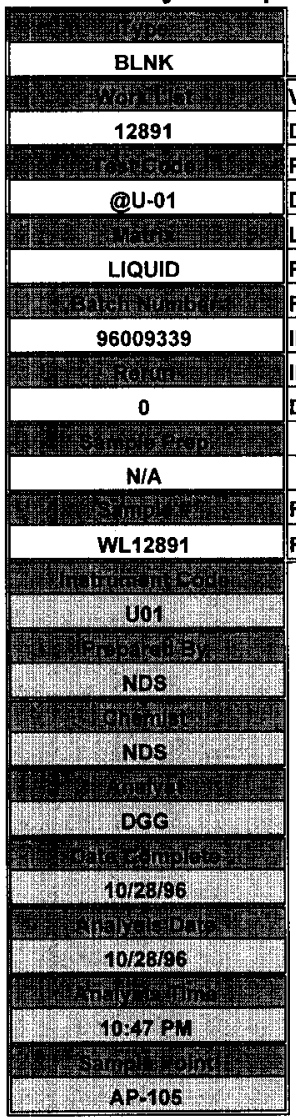

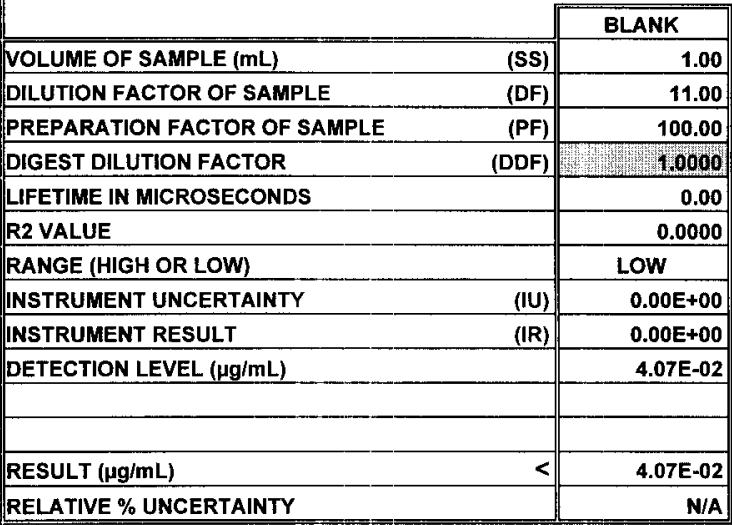

Result $=D F * P F * D D F * I R * 1000$

Detection Level $=3.70 \mathrm{E}-08$ * $\mathrm{DF}$ * PF * DDF * 1000

Relative \% Uncertainty $=$ IU $/ I R * 100$

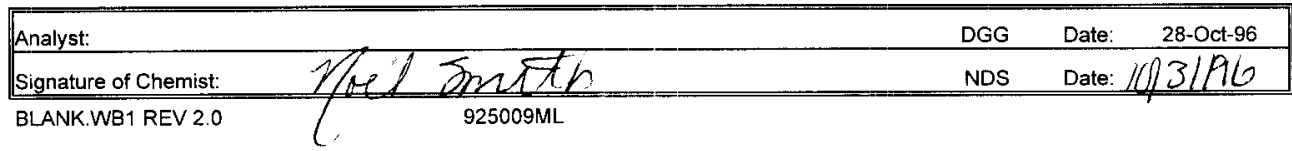


HNF-SD-WM-DP-202, REV. 1

WORKBOOK PAGE: SAM3

Uranium by Phosphorescence: LA-925-009 (A-1) LIQUID/SOLID

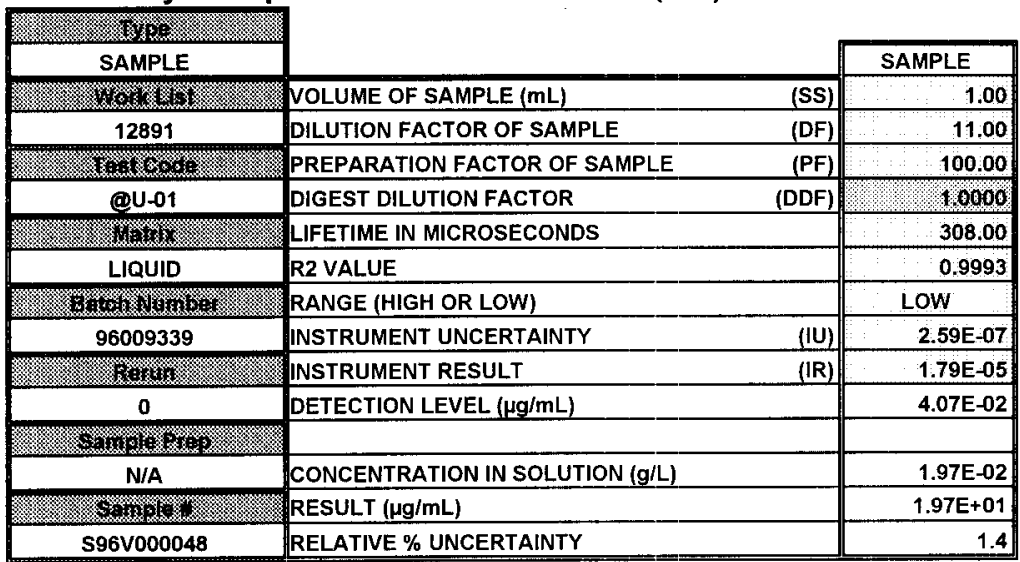

1.6. 10.28

10\%

3x w

Nos

3.t.

10185

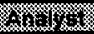

B6c

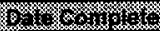

10226196

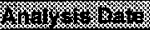

10128196

X

$10 \times 47 \mathrm{RM}$

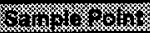

A. 105

\author{
Result $=D F * P F * D D F * I R * 1000$ \\ Detection Level $=3.70$ E -08 * DF * PF * DDF * 1000 \\ Relative \% Uncertainty $=$ IU $/$ IR * 100
}

\begin{tabular}{|c|c|c|c|}
\hline Analyst: & DGG & Date: & 28-Oct-96 \\
\hline Signature of Chemist: & NDS & Date: & $13 / 19$ \\
\hline
\end{tabular}

251 
HWFSD-WWM-DP-202, REV. 1

WORKBOOK PAGE: DUP4

Uranium by Phosphorescence: LA-925-009 (A-1) LIQUID/SOLID

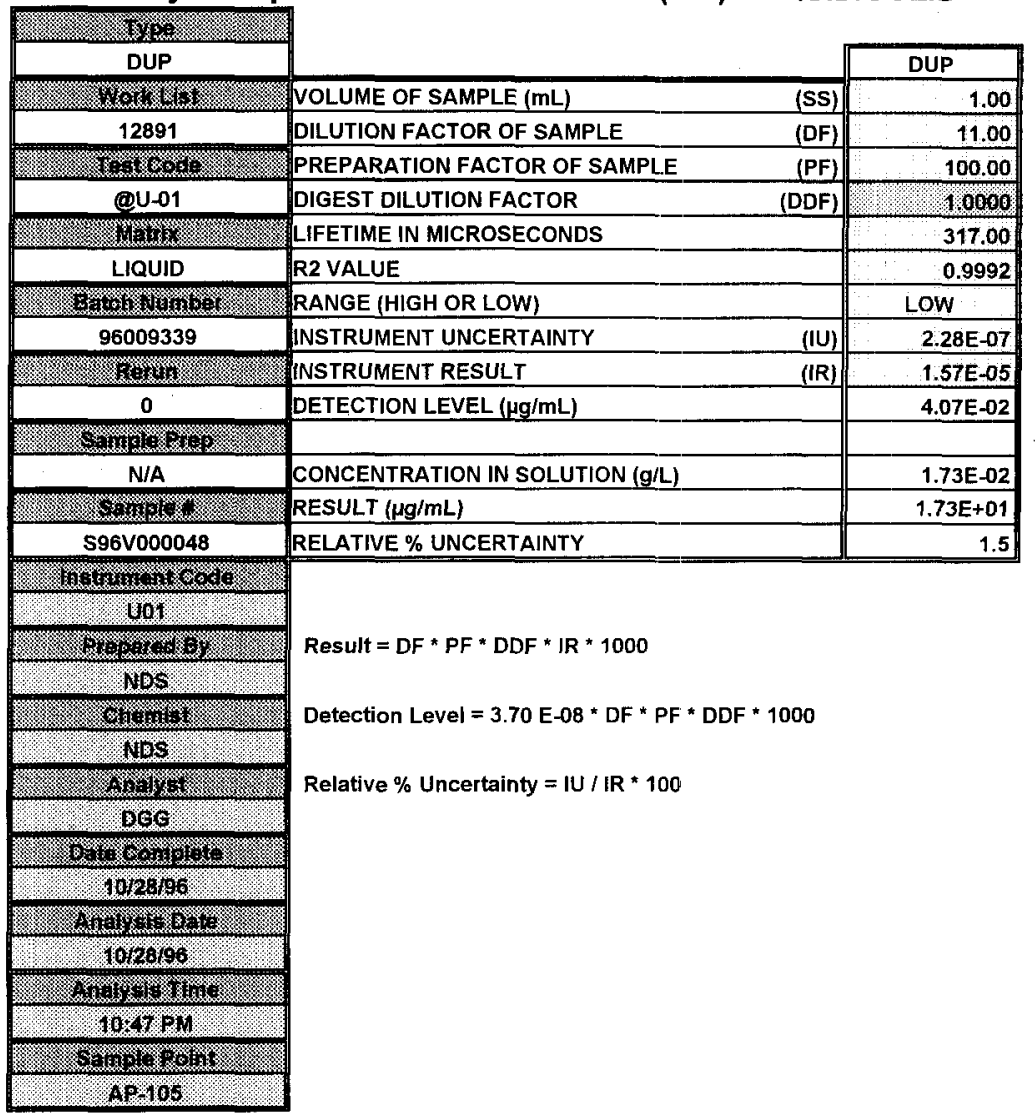

\begin{tabular}{|c|c|c|c|c|}
\hline Analyst: & & DGG & Date: & 28-Oct-96 \\
\hline Signature of Chemist: & $\operatorname{Sim} 1+t$ & NDS & Date: & 31196 \\
\hline
\end{tabular}


WORKBOOK PAGE: SPIKE5

HAE

Uranium by Phosphorescence: LA-925-009 (A-1) LIQUIDISOLID

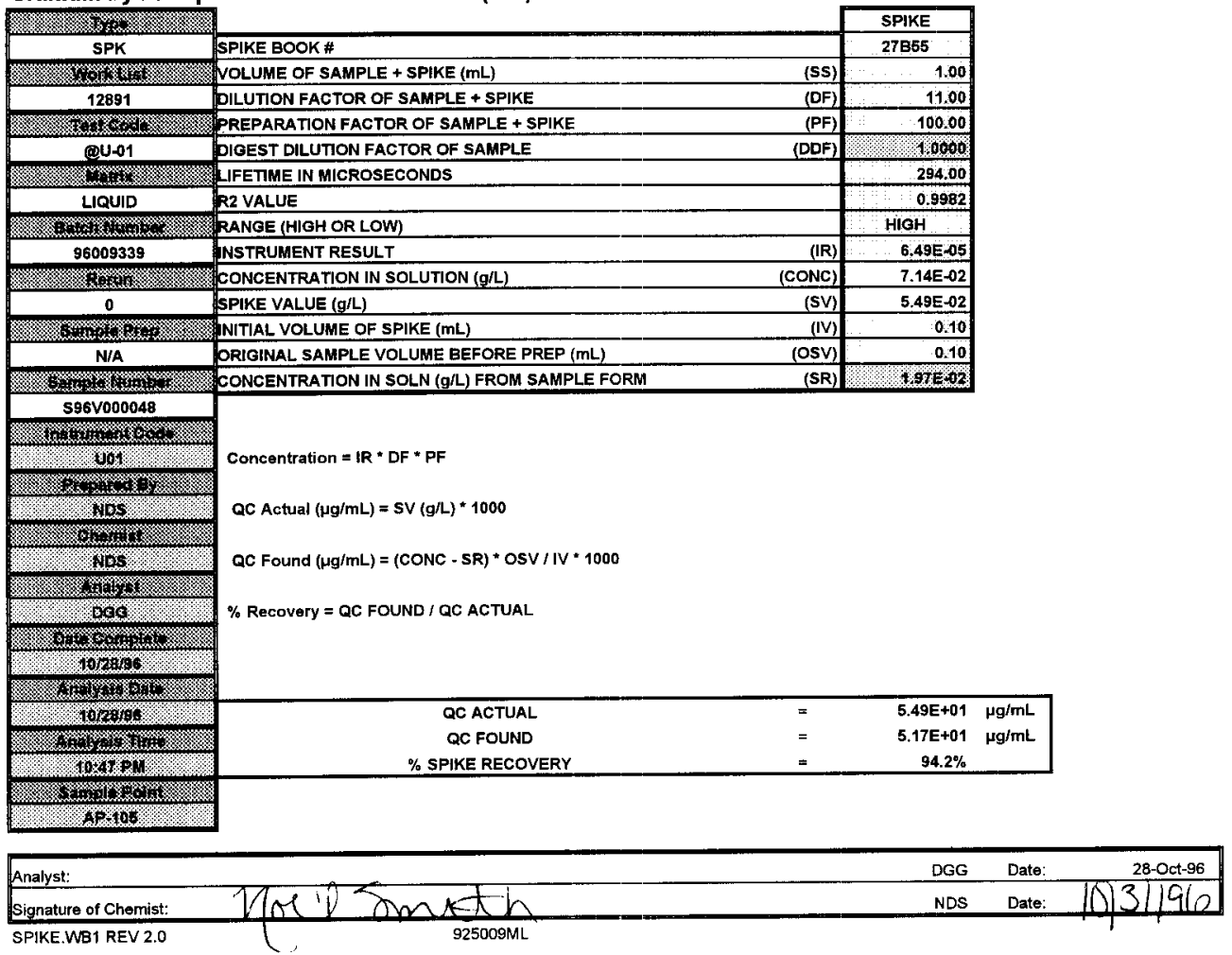


HNF-SD-WM-DP-202, REV. 1

WORKBOOK PAGE: SP_DUP6

Uranium by Phosphorescence: LA-925-009 (A-1) LIQUID/SOLID

\begin{tabular}{|c|c|c|c|c|}
\hline 8 & & & SPK-DUP & \\
\hline SPK-DUP & SPIKE BOOK \# & & 27855 & \\
\hline 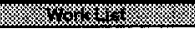 & VOLUME OF SAMPLE + SPIKE $(\mathrm{mL})$ & (SS) & 1,00 & \\
\hline 12891 & DILUTION FACTOR OF SAMPLE + SPIKE & (DF) & 11,00 & \\
\hline 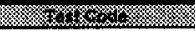 & PREPARATION FACTOR OF SAMPLE + SPIKE & (PF) & 100.00 & \\
\hline @บ-01 & DIGEST DILUTION FACTOR OF SAMPLE & (DDF) & 10000 & \\
\hline 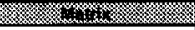 & LIFETIME IN MICROSECONDS & & 303.00 & \\
\hline LIQUAD & R2 VALUE & & 0.9987 & \\
\hline And & RANGE (HIGH OR LOW) & & $\mathrm{HIOH}$ & \\
\hline 96009339 & INSTRUMENT RESULT & (IR) & 6.41E.05 & \\
\hline 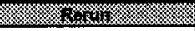 & CONCENTRATION IN SOLUTION $(g / L)$ & (CONC) & 7.05E-02 & \\
\hline 0 & SPIKE VALUE $(g / L)$ & (SV) & $5.49 E-02$ & \\
\hline $\operatorname{sos} 20$ & NITIAL VOLUME OF SPIKE (mL) & (IV) & 0.10 & \\
\hline N/A & ORIGINAL SAMPLE VOLUME BEFORE PREP $(\mathrm{mL})$ & (osv) & $0: 10$ & \\
\hline 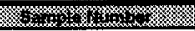 & CONCENTRATION IN SOLN (g/L) FROM SAMPLE FORM & (SR) & $192=02$ & \\
\hline S96V000048 & \multirow{12}{*}{ QC Found $(\mu \mathrm{g} / \mathrm{mL})=(\operatorname{CONC}-\mathrm{SR}) *$ OSV $/ \mathrm{IV} * 1000$} & & & \\
\hline$x+4 \times$ & & & & \\
\hline 1010 & & & & \\
\hline 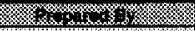 & & & & \\
\hline Wos & & & & \\
\hline 14 & & & & \\
\hline Nos & & & & \\
\hline-2 & & & & \\
\hline (306 & & & & \\
\hline $10 x+2$ & & & & \\
\hline $10 \mathrm{x}+10$ & & & & \\
\hline 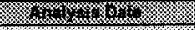 & & & & \\
\hline 6rasolo & QC ACTUAL & $=$ & $5.49 E+01$ & $\mu g / m L$ \\
\hline 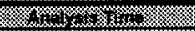 & QC FOUND & $=$ & $5.08 E+01$ & $\mu g / m L$ \\
\hline $10 \times 2 \%$ & $\%$ SPIKE RECOVERY & $=$ & $92.6 \%$ & \\
\hline
\end{tabular}

\%

4806

\begin{tabular}{|c|c|c|c|}
\hline Analyst: & DGG & Date: & 28-Oct-96 \\
\hline Signature of Chemist: & NDS & Date: & 1910 \\
\hline
\end{tabular}


WORKBOOK PAGE: SAM7

\section{Uranium by Phosphorescence: LA-925-009 (A-1) LIQUID/SOLID}

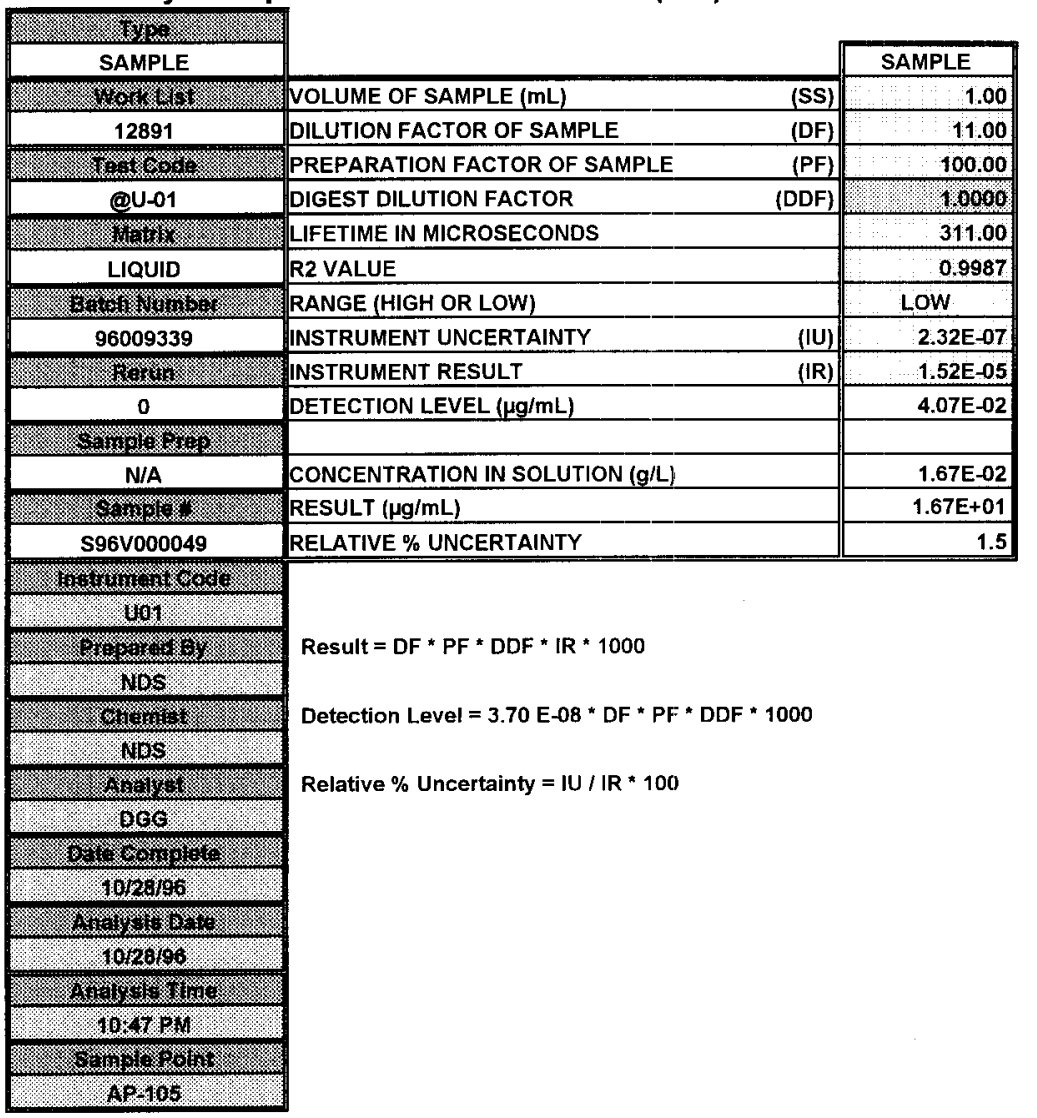

\begin{tabular}{|c|c|c|c|c|}
\hline Analyst: & & DGG & Date: & 28-Oct-96 \\
\hline Signature of Chemist: & Mon'd Jmath & NDS & Date: & 131196 \\
\hline
\end{tabular}




\section{LABCORE Completed RadChem Report for Worklist\#: 12892}

Analyst: $\mathrm{crj}$

Instrument: U01

Book\#

Method:

$\operatorname{Rev} / \operatorname{Mod}$

Worklist Comment: AP-105. 0.1-10ml sample prep.For spike: $0.1 \mathrm{ml}$. new

\begin{tabular}{|c|c|c|c|c|c|c|c|c|c|}
\hline$\overline{\text { Seq Type }}$ & \multicolumn{3}{|c|}{ Sample\# R A } & Test & Matrix & Actual & Found & DL or Yield & Unit \\
\hline STD & & 0 & au $=01$ & 1002 & LOUID & $5.10 \mathrm{E}-02$ & $5.33 \mathrm{E}-02$ & 104.510 & \% Recovery \\
\hline 1 STD & & 0 & au-01 & $\mathrm{U}-02 \mathrm{E}$ & LIQUID & 3 & $3.26 \mathrm{E}+00$ & 3.260 & Ratio \\
\hline 2 BLNK & & 0 & ol-01: & $4-02$ & 10010 & 1 & $6.10 \mathrm{E}-3$ & $61,000 e^{-004}$ & $\mathrm{uCt} / \mathrm{mL}$ \\
\hline 2 BLNK & & 0 & aU- 01 & U- 02E & LIQUID & 1 & $8.97 E+00$ & 8.970 & Ratio \\
\hline 3 SAMPLE & s960000058 & 0 & $a-01$ & 402 & LI QưID & $N / A$ & $3.70 \mathrm{E}-03$ & 370.0 e-005 & $u g / \mathrm{hL}$ \\
\hline 3 SAMPLE & $596 \vee 000058$ & 0 & QU- 01 & U-02E & LJQUID & $N / A$ & $N / A$ & $0.0 e+000$ & \% Inst Error \\
\hline 4 OUP & $\$ 96 \mathrm{~V} 000058$ & 0 & av-01 & $1-02$ & LIQUID & $\times 3.70 \mathrm{E}-3$ & $\times 3.70 \mathrm{e}-3$ & 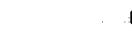 & PPO \\
\hline 4 DUP & $596 \mathrm{~V} 000058$ & 0 & $\mathrm{QU}-01$ & $U-02 E$ & LIQUID & 1 & $N / A$ & & Ratio \\
\hline 5 SPK: & $\$ 960000058$ & 0 & au 01 & $U-02$ & LIOUID & $5,69 E+01$ & $6,19 \mathrm{E}+01$ & 108.787 & \% Recovery \\
\hline 6 SPK-DUP & $596 \vee 000058$ & 0 & aU- 01 & $\mathrm{U}-02$ & LIQUID & $6.19 E+01$ & $5.86 \mathrm{E}+01$ & 5.477 & $\mathrm{PD}$ \\
\hline
\end{tabular}

Final page for worklist\# 12892

Ânalyst Signature Date

Analyst Signature Date

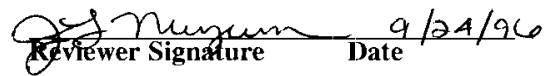




\section{LABCORE Data Entry Template for Worklist\# 12892}

\begin{tabular}{|c|c|c|}
\hline A nalyst: & $(\mathrm{R})$ Instrument: U01 & Book\# \\
\hline
\end{tabular}

Worklist Comment: AP-105. 0.1-10ml sample prep. For spike: $0.1 \mathrm{ml}$. new

\begin{tabular}{|c|c|c|c|c|c|c|c|}
\hline $\bar{s}$ & Type & Sample\# & $\mathrm{R} A$ & Test & Matrix & \multirow[t]{3}{*}{ Group\# } & \multirow[t]{3}{*}{ Project } \\
\hline 1 & STD & & & $@ \mathrm{U}-01^{\circ}$ & LIQIJ] D & & \\
\hline 2 & BLNK & & & $@ \mathrm{U}-01$. & LIQU]D & & \\
\hline \multirow[t]{2}{*}{3} & SAMPLE & S96V000058 & 0 & $@ \mathrm{U}-01$ & LIQUID & \multirow[t]{5}{*}{96000855} & \multirow[t]{5}{*}{ AP- 105} \\
\hline & & Analytes Reque & ested: & $=\mathrm{U}-02$ &,$\quad U-02 E$ & & \\
\hline 4 & DUP & S96V000058 & 0 & (ख) - 01 & LIQUID & & \\
\hline 5 & SEK & S96V000058 & 0 & $@ \mathrm{U}-01$ & LIQUID & & \\
\hline 6 & SPK-DUP & S96V000058 & 0 & $@ \mathrm{U}-01$ & LIQU]D & & \\
\hline
\end{tabular}

\section{Final page for worklist \# 12892}
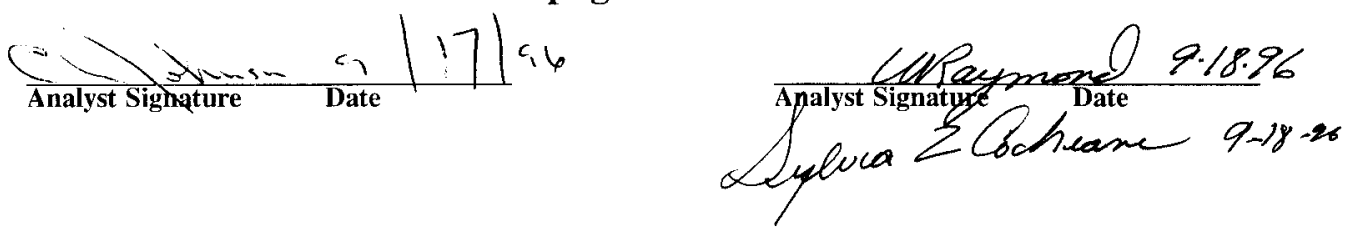

Data Entry Comments:

$\bar{S}=$ Worklist Stot Number, $R=$ Replicate Number, $A=$ Aliquot Code. 
ikllst Number: 12292 iko Book Number: $2 \partial$ BS 5

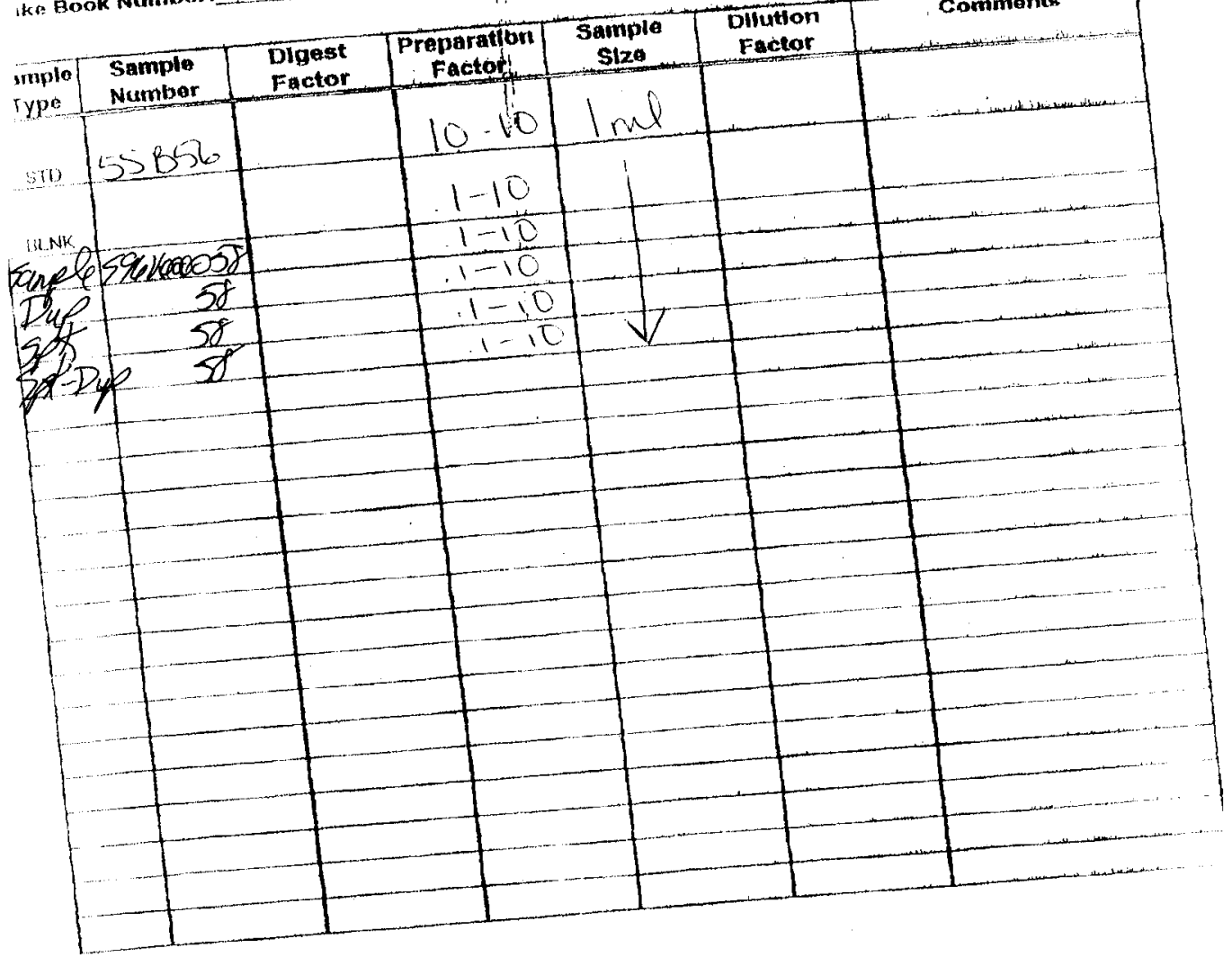




\section{URANIUM ANALYSIS}

Sample ID 55856

Description STD WL\#12892

Ref. Ratio 1.126

Laser Pulses 500

Lifetime $227+1.351$ us

$\mathrm{R} 2.9885$

Integrated 45387

Range: HIGH

Sample ID BLANK-FREF

Description BLK WL 12892

Ref. Ratio 1.109

Laser Pulses 500

Lifetime $180+11.193$ us $\mathrm{R} 2.9058$

Integrated 2883

Range: LOW
Date/Time 09/17/96/11:35:42

Cal $Y=7.3 e E+07 X-5.01 E+02$

Intensity $2920 \quad(t=39$

Cone $5.33 \mathrm{E}-05+1.74 \mathrm{E}-06 \mathrm{~g} / \mathrm{L}$

Dilution Factor $1 \mathrm{~mL} / \mathrm{mL}$

FINAL FESULT $5.33 \mathrm{E}-05+1.74 \mathrm{E}-0 \mathrm{~g} \mathrm{~g} / \mathrm{L}$

Date/Time $09 / 17 / 96 / 11: 38: 20$

Cal $Y=5.19 \mathrm{E}+09 \mathrm{X}-4.88 \mathrm{E}-04$

Intensity $184 \quad \quad(t=39$

Cone $6.10 \mathrm{E}-08+5.47 \mathrm{E}-09 \mathrm{~g} / \mathrm{L}$

Dilution Factor $1 \mathrm{~mL} / \mathrm{mL}$

FINAL RRSULT $6.10 \mathrm{E}-08+5.47 \mathrm{E}-09 \mathrm{~g} / \mathrm{L}$

Sample ID Seevoooos8

Deseription SAM WL\#12882

Ref. Ratio 1.114

Laser Pulses 500

Lifetime + us

$\mathrm{R} 2$

Integrated 295

Range: LOW

Sample $<$ Blank

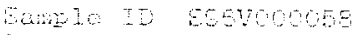

Detring

Nof. Fatio 1.10s

Reer Dusee son

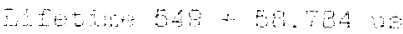

tent

rotegnated 2008

Sare: Dor
Date/Time 09/17/96/11:41:39

CaI $Y=5.19 \mathrm{E}+09 \mathrm{X}-4.88 \mathrm{E}-04$

Intensity 29

Conc $+\mathrm{g} / \mathrm{L}$

$(t=39$

Dilution Factor $1 \mathrm{mt} / \mathrm{mI}$

BINAL RESULT

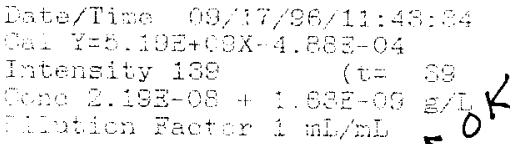

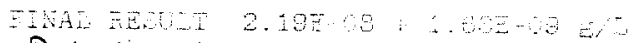

Zero will be used for ACE

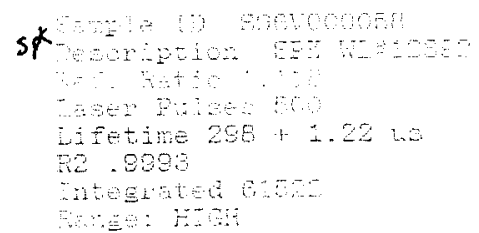

atartane

$6005.60 \mathrm{E}-05+1.70 \mathrm{E}-00 \mathrm{at}$

7.A.

$\ln )$

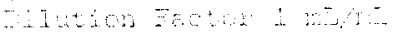

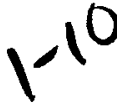


WORKBOOKPAGE: STD1

Uranium by Phosphorescence: LA-925-009 (A-1) LIQUID/SOLID

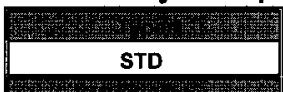

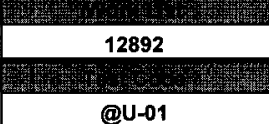

STANDARD BOOK \#

STANDARD

\begin{tabular}{|l|l|}
\hline 5NB56 & \\
\hline
\end{tabular}

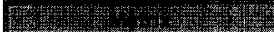

VOLUME OF SAMPLE (mL)

DILUTION FACTOR OF SAMPLE

(SS) PREPARATION FACTOR OF SAMPLE

LIQUID LIFETIME IN MICROSECONDS

(DF)
55B56

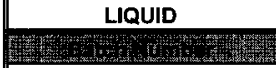

96009342

R2 VALUE

RANGE (HIGH OR LOW)

Hext.

INSTRUMENT UNCERTAINTY

INSTRUMENT RESULT

0

DETECTION LEVEL $(\mu \mathrm{g} / \mathrm{mL})$

The
N/A

RESULT $(\mu \mathrm{g} / \mathrm{mL})$

(PF)

1.00

1.00

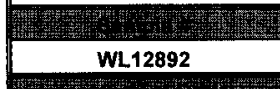

VALUE OF STANDARD

\%RECOVERY

RELATIVE \% UNCERTAINTY 1.00

19.

v01

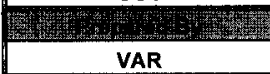

$\frac{5}{5 \text { JLN }}$

Result $=D F * P F * I R * 1000$

Detection Level $=3.70$ E-08 * DF * PF * 1000

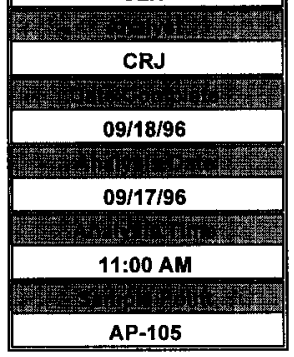

$\%$ Recovery $=$ Result $/$ Value of Standard $* 100$

Relative $\%$ Uncertainty $=$ IU $/$ IR * 100

\begin{tabular}{|lllll|}
\hline Analyst: & CRJ & Date: & $18-$ Sep-96 \\
\hline Signature of Chemist: & & JLN & Date: & $9 / 24 / \mathrm{ac}$ \\
\hline STANDARD.WB1 REV 2.0 & & &
\end{tabular}


HFE-SD-WM-DP-202, REV. 1

WORKBOOK PAGE: BLANK2

\section{Uranium by Phosphorescence: LA-925-009 (A-1) LIQUID/SOLID}

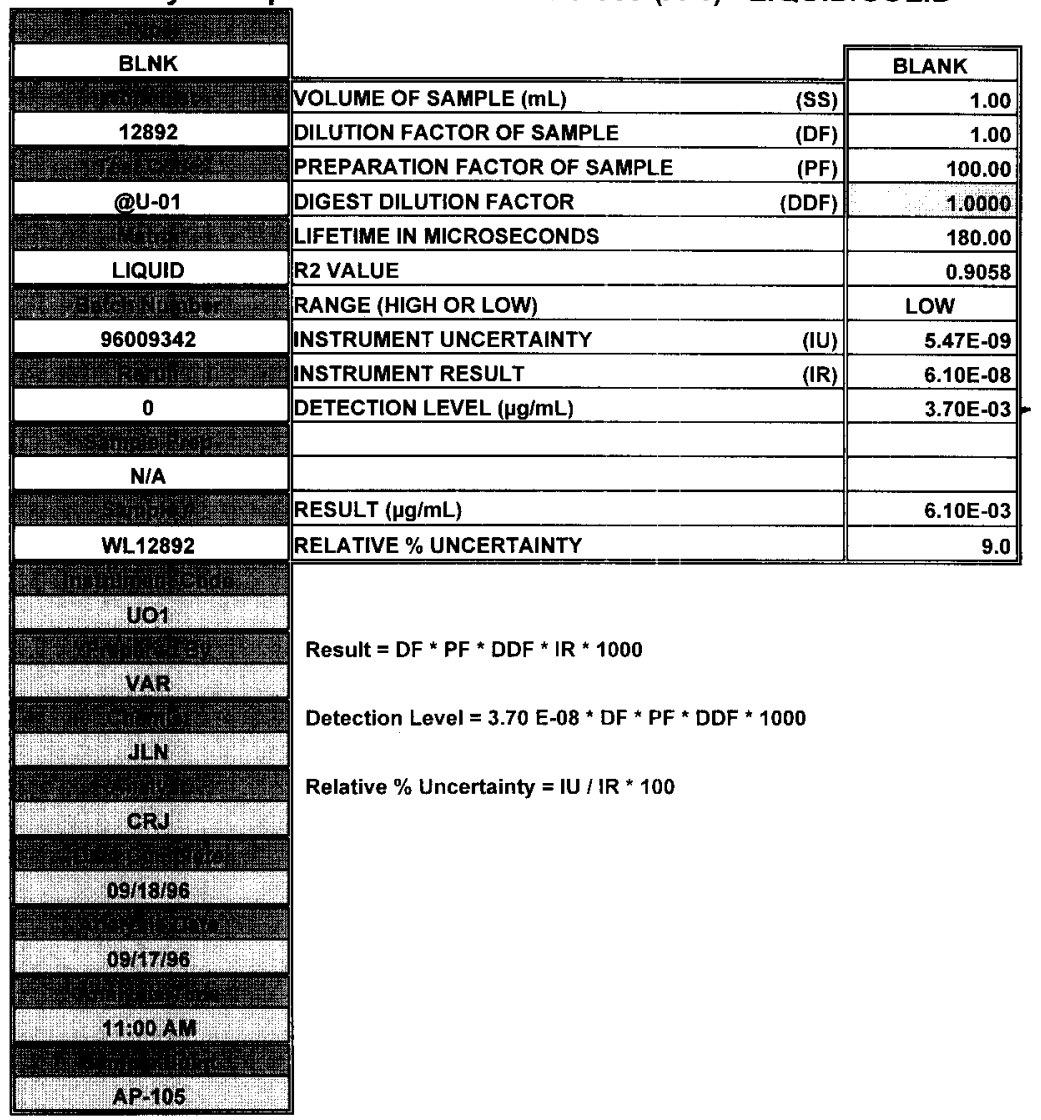

\begin{tabular}{|c|c|c|}
\hline Analyst: & $\mathrm{CRJ}$ & 18-Sep-96 \\
\hline Signature of Chemist: & $\mathrm{JLN}$ & Date: $9 / 2+/ 96$ \\
\hline
\end{tabular}


WORKBOOK PAGE: SAM3

\section{Uranium by Phosphorescence: LA-925-009 (A-1) LIQUID/SOLID}

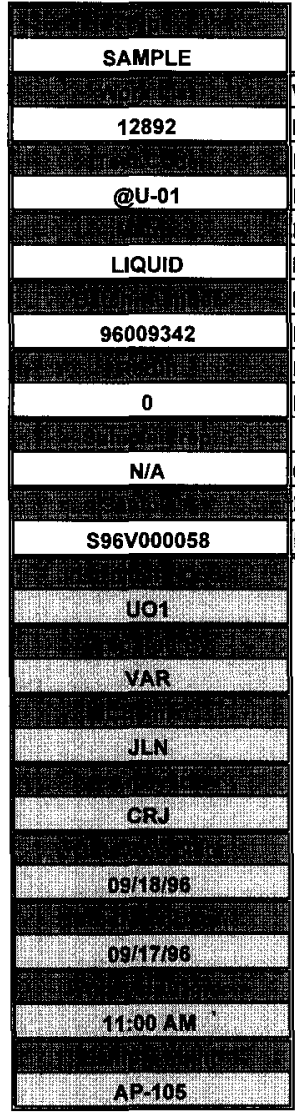

\begin{tabular}{|c|c|}
\hline & SAMPLE \\
\hline VOLUME OF SAMPLE $(\mathrm{mL})$ & 1.00 \\
\hline DILUTION FACTOR OF SAMPLE & 1.00 \\
\hline PREPARATION FACTOR OF SAMPLE & 100.00 \\
\hline DIGEST DILUTION FACTOR & 10000 \\
\hline LIFETIME IN MICROSECONDS & 0.00 \\
\hline R2 VALUE & 0.0000 \\
\hline RANGE (HIGH OR LOW) & LOW \\
\hline INSTRUMENT UNCERTAINTY & $0.00 \mathrm{E}+00$ \\
\hline INSTRUMENT RESULT & $0.00 E+00$ \\
\hline DETECTION LEVEL ( $\mu \mathrm{g} / \mathrm{mL})$ & $3.70 E-03$ \\
\hline CONCENTRATION IN SOLUTION $(\mathrm{g} / \mathrm{L})$ & $0.00 E+00$ \\
\hline RESULT ( $\mu \mathrm{g} / \mathrm{mL}$ ) & $3.70 \mathrm{E}-03$ \\
\hline RELATIVE \% UNCERTAINTY & N/A \\
\hline
\end{tabular}

Result $=$ DF * PF * DDF * IR * 1000

Detection Level $=3.70 \mathrm{E}-08$ * DF * PF * DDF * 1000

Relative $\%$ Uncertainty $=I U / I R * 100$

\begin{tabular}{|lll|}
\hline Snalyst: & CRJ & Date: \\
\hline Signature of Chemist: & JLN & Date: $9 / 24 / 94$ \\
\hline
\end{tabular}




\section{HNF-SD-WM-DP-202, REV. 1}

WORKBOOK PAGE: DUP4

\section{Uranium by Phosphorescence: LA-925-009 (A-1) LIQUID/SOLID}

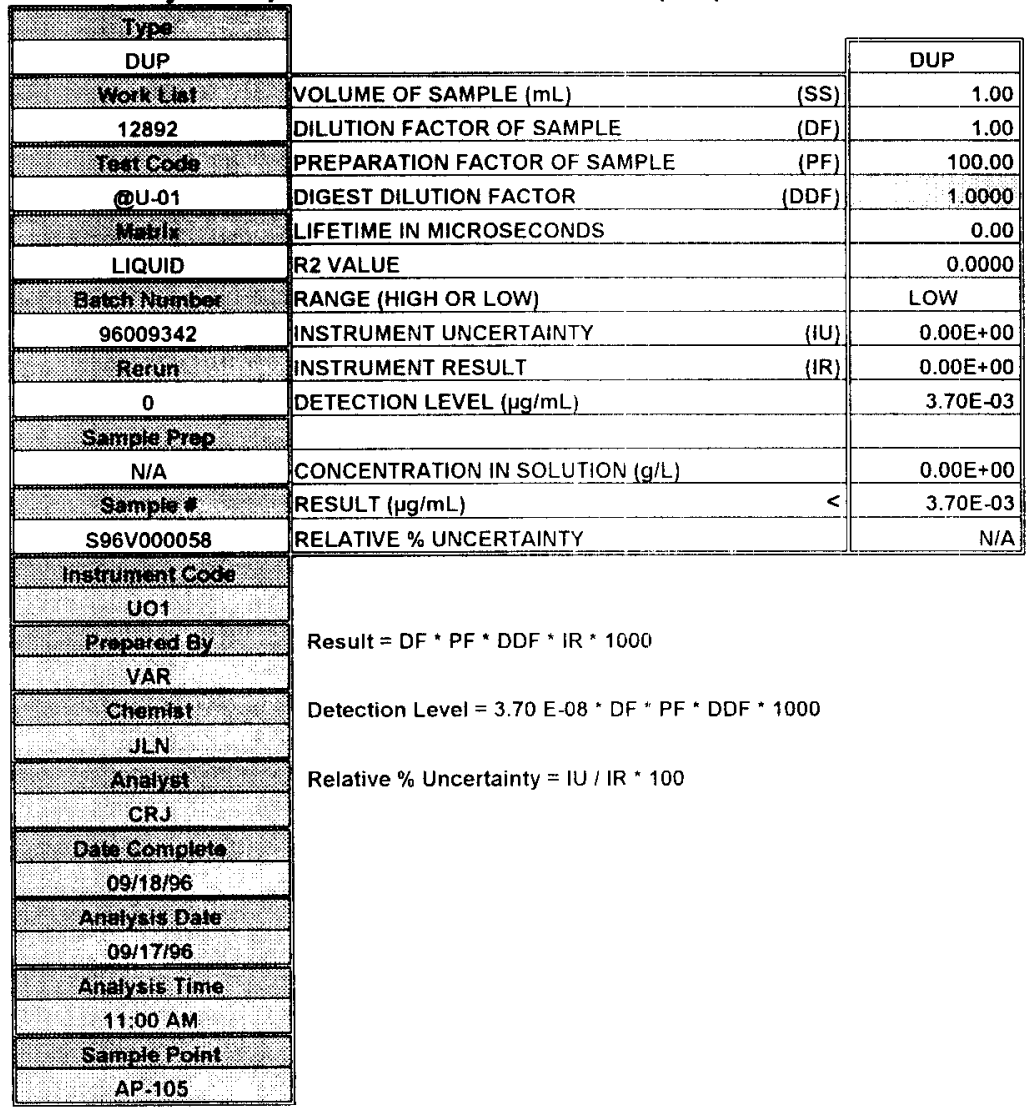

\begin{tabular}{|l|l|l|}
\hline Analyst: & CRJ Date & 18 -Sep-96 \\
\hline Signature of Chemist:
\end{tabular}


WNFGD-WM-DP-202, REV. 1

WORKBOOK PAGE: SPIKE5

Uranium by Phosphorescence: LA-925-009 (A-1) LIQUID/SOLID

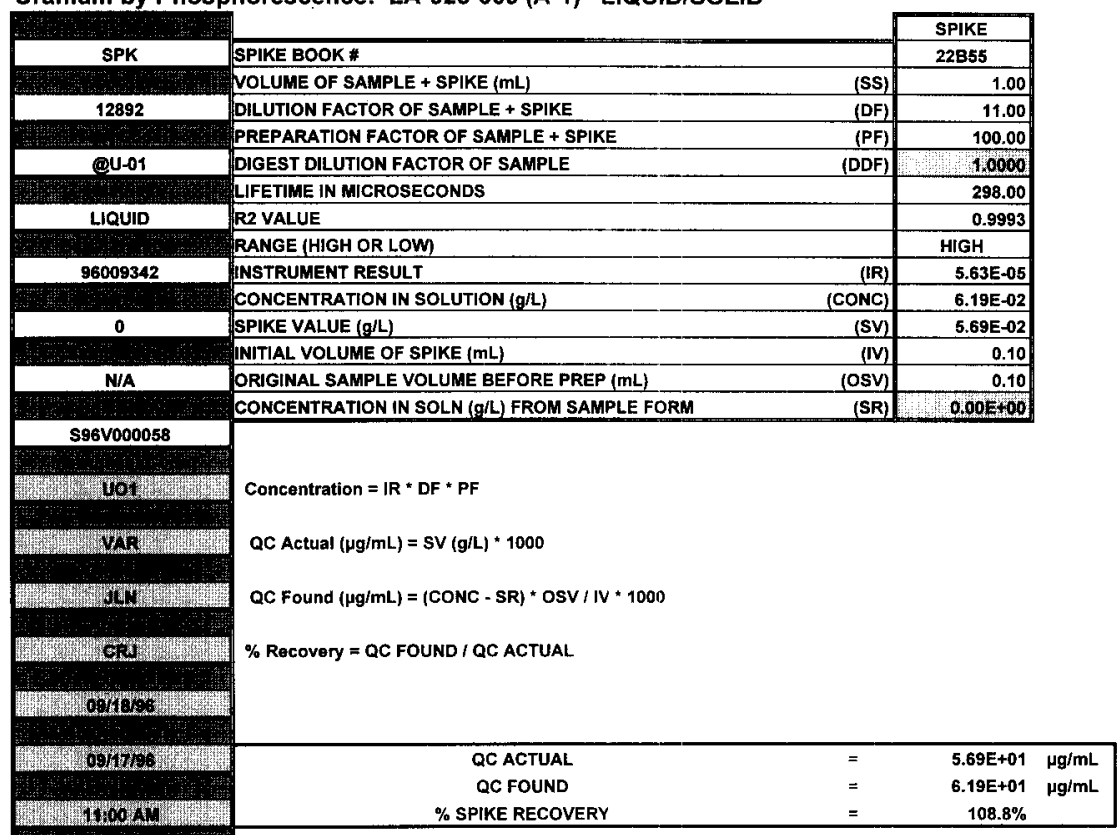

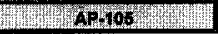

Analyst:
Signature of Chemist:
SPIKE.WB1 REV 2.0

\section{5}


WORKBOOK PAGE: SP.DUP6

Uranium by Phosphorescence: LA-925-009 (A-1) LIQUID/SOLID

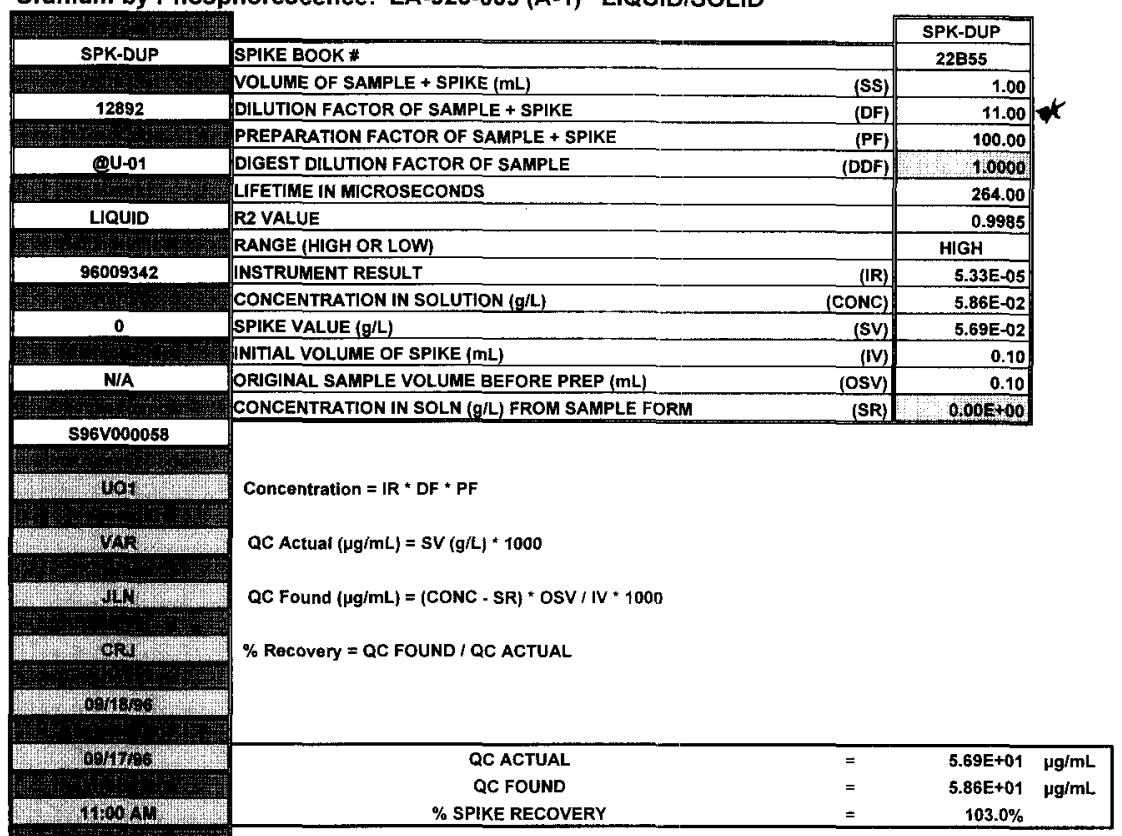

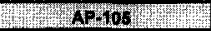

\begin{tabular}{|c|c|c|c|}
\hline Analyst: & CRJ & Date: & 18-Sep-96 \\
\hline Signature of Chemist: & JLN & Date: & \\
\hline
\end{tabular}

\section{6}


HNE-SD-WM-DP-202, REV. 1

Radiochemical Analyses

267 
WWE-WD-WM-DP-202, REV. 1

THIS PAGE WAS INTENTIONALLY LEFT BLANK

268 


\section{LABCORE Completed RadChem Report for Worklist\#: 15286}

Analyst: rag

Instrument: LSCO3

Book\#

Method:

$\operatorname{Rev} / \mathrm{Mod}$

Worklist Comment: See attached sheet for sample sizes. Rerun \#2. SLF

\begin{tabular}{|c|c|c|c|c|c|c|c|c|c|}
\hline Seq Type & \multicolumn{3}{|c|}{ Sample\# R A } & Text & Matrix & Actual & Found & DL or Yield & Unit \\
\hline I $\$ T 0$ & & 0 & aH3-01 & $H 3-01$ & LIOUID & $7.51 \mathrm{E}-04$ & $8.60 E-4$ & 114.514 & \% Recovery \\
\hline I STO & & 0 & aH3-01 & H3-01E & LIQUID & 1 & $9.40 \mathrm{E}-01$ & 0.940 & * Ct. Erro \\
\hline 2 BLNK & & 0 & ลН 3-01 & $\mathrm{H3}-01$ & LIQUID & 1 & $3.92 E-5$ & $39.200 e-006$ & $U C \mathrm{i} / \mathrm{mL}$ \\
\hline $2 \mathrm{BLNK}$ & & 0 & ан3-01 & $\mathrm{H3}-01 \mathrm{E}$ & LIQUID & $?$ & $5.66 \mathrm{E}+00$ & 5.660 & UCi $/ \mathrm{mL}$ \\
\hline 3 SAMPLE & s96v000047 & 0 & aH3-01 & H3-01 & LIQUID & $N / A$ & $4.65 E-03$ & $310.0 e-007$ & $u C i / m L$ \\
\hline 3 SAMPLE & s96v000047 & 0 & จH3-01 & H3-01E & LI OUIO & $N / A$ & $1.29 E+00$ & $100.0 e-006$ & $\%$ Ct. Error \\
\hline 4 SPK & s96v000047 & 0 & aH3.01 & H3-01 & LIQUID & $7.51 \mathrm{E}-04$ & $7.65 E-04$ & 101.864 & \% Recovery \\
\hline 5 SPK-DUP & s96V000047 & 0 & aH3-01 & H3-01 & LIQUID & $7.65 E-04$ & $7.86 \mathrm{E}-04$ & 2.708 & PD \\
\hline 6 SAMPLE & s96v000048 & 0 & ลH3-01 & $H 3-01$ & LIQUID & $N / A$ & $1.04 E-03$ & $282.0 e-008$ & $U C i / \mathrm{mL}$ \\
\hline 6 SAMPLE & $\$ 96 \vee 000048$ & 0 & aн3-01 & H3- D1E & LIQUID & $N / A$ & $8.30 \mathrm{E}-01$ & $100.0 \mathrm{e}-006$ & $\%$ ct. Error \\
\hline 7 SAMPLE & $596 v 000049$ & 0 & aH3-01 & H3-01 & LIQUID & $N / A$ & $2.76 \mathrm{E}-02$ & $312.0 e-007$ & UC $\mathrm{i} / \mathrm{mL}$ \\
\hline 7 SAMPLE & 5960000049 & 0 & อH3-01 & H3-01E & LIOUID & $N / A$ & $5.40 E-01$ & $100.0 e-006$ & \% ct. Error \\
\hline 8 SAMPLE & $596 v 000058$ & 0 & aH3-01 & H3-01 & LIOUID & $N / A$ & $8.98 \mathrm{E} \cdot 05$ & $309.0 \mathrm{e}-007$ & $u C \mathrm{i} / \mathrm{mL}$ \\
\hline 8 SAMPLE & $596 \vee 000058$ & 0 & อн3-01 & $H 3-01 E$ & LIOUID & $N / A$ & $5.34 E+00$ & $100.0 \mathrm{e}-006$ & \% ct. Error \\
\hline
\end{tabular}

Final page for worklist \# 15286

Analyst Signature Date Analyst Signature Date




\section{LABCORE Data Entry Template for Worklist\# 15286}

Analyst: RY Instrument: LSC00 6500 Book\# 44 B56

Method: LA-218-114 Rev/Mod B-C

Worklist Comment: See attached sheet for sample sizes. Rerun \#2. SLF

\begin{tabular}{|c|c|c|c|c|c|c|c|}
\hline $\mathbf{s}$ & Type & Sample\# & RA & Test & Matrix & Group\# & Project \\
\hline 1 & STD & & & $@ H 3-01$ & LIQUID & & \\
\hline 2 & BLNK & & & $@ H 3-01$ & LIQUID & & \\
\hline \multirow[t]{2}{*}{3} & SAMPLE & S96V0000047 & 0 & @H3-OI & LIQUID & 96000853 & $A P-105$ \\
\hline & & Analytes Reque & ested: & : $\mathrm{H3}-01$ &, $\mathrm{H} 3-01 \mathrm{E}$ & & \\
\hline 4 & SPK & S96V000047 & 0 & $@ \mathrm{H} 3-01$ & LIQUID & & \\
\hline 5 & SPK-DUP & $596 \mathrm{~V} 000047$ & 0 & $@ \mathrm{H} 3-01$ & LIQUID & & \\
\hline \multirow[t]{2}{*}{6} & SAMPLE & S96V000048 & 0 & $@ H 3-01$ & LIQUID & 96000855 & $A P-105$ \\
\hline & & Analytes Reque & ested: & : $\mathrm{H3}-01$ & , H3-OLE & & \\
\hline \multirow[t]{2}{*}{7} & SAMPLE & S96V000049 & 0 & @3 -01 & LIQUID & 96000855 & AP -105 \\
\hline & & Analytes Reque & ested: & : H3-01 &, $\mathrm{H} 3-01 \mathrm{E}$ & & \\
\hline 8 & SAMPLE & S96V000058 & 0 & $@ \mathrm{H} 3-01$ & LIQUID & 96000855 & AP -105 \\
\hline
\end{tabular}

\section{Final page for worklist \# 15286}

$\frac{\text { Ras hing ives } 12-1-16}{\text { Analyst Signature }}$

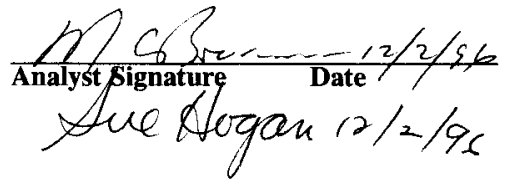

Data Entry Comments: $\quad 15 \mathrm{ml}$ sain $+1.5 \mathrm{ml}$ stel for spk samples 
WORKBOOK PAGE: STD1

HWFG-S[1-WM-DP-202, REV. 1

\begin{tabular}{|c|c|c|c|}
\hline H3 : LA-218-114 & TRITIUM LIQUIDS & & STANDARD \\
\hline & Disintegrations per minute from Counter & $\overline{(\text { (dpm) }}$ & 1909.67 \\
\hline STD & Sample Volume in $\mathrm{mL}$ & (SS) & 1 \\
\hline & Dilution Factor & (DF) & 1 \\
\hline 15286 & Digest Dilution Factor & (DDF) & 1 \\
\hline & Background Count Rate in Counts per Minute & (BKG cpm) & 21.44 \\
\hline @H3-01 & Background Count Time in Minutes & (BKG Time) & 50 \\
\hline 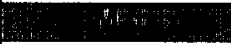 & Instrument Fractional Efficiency & (EFF) & $\overline{0.48}$ \\
\hline LIQUID & $\%$ Counting Error & & 0.94 \\
\hline Plate & Detection Limit & (Ld) & 6.47 \\
\hline 96011896 & Critical Level & (Lc) & 1.53 \\
\hline$(1+4)$ & Tritium Concentration in $\mu \mathrm{Ci} / \mathrm{L}$ & & 8.60E-01 \\
\hline 0 & Standard Book No. & & 64B56 \\
\hline Mit: & Standard Value in $\mu \mathrm{Ci} / \mathrm{mL}$ & & 7.5051E-04 \\
\hline
\end{tabular}

WL15286

Detection Limit (Ld) $=((2.72 /$ BKG Time $)+(2 *$ LC $)) /$ EFF

$\mathrm{H3} \mu \mathrm{Ci} / \mathrm{L}=\left(\mathrm{dpm}{ }^{*}(1000 \mathrm{~mL} / \mathrm{L}){ }^{*} \mathrm{DF}{ }^{*} \mathrm{DDF}\right) /(\mathrm{SS} *(2220000 \mathrm{dpm} / \mu \mathrm{Ci}))$

WC51586

$\mathrm{H} 3 \mu \mathrm{Ci} / \mathrm{mL} \quad=\mu \mathrm{Ci} / \mathrm{L} / 1000 \mathrm{~mL} / \mathrm{L}$

Detection Limit $(\mu \mathrm{Ci} / \mathrm{mL})=(\mathrm{Ld})(\mathrm{DF})(\mathrm{DDF}) /[(\mathrm{SS})(2220000 \mathrm{dpm} / \mu \mathrm{Ci})]$

MCB

SLF

\begin{tabular}{l|l} 
Tritium Concentration in $\mu \mathrm{Ci} / \mathrm{L}$ \\
\hline
\end{tabular}

8.60E-01

RAG

$12 / 02 / 96$

Tritium Concentration in $\mu \mathrm{Ci} / \mathrm{mL} \quad 8.60 \mathrm{E}-04$

Detection Limit in $\mu \mathrm{Ci} / \mathrm{mL} \quad 2.91 \mathrm{E}-06$

\% Counting Error 0.94

$12 / 01 / 96$

09:30 AM

AP-105

\begin{tabular}{|lllll|}
\hline Analyst: & RAG & Date: & 12/02/96 \\
\hline Signature of Chemist: & & SLF & Date: & 12/3/96 \\
\hline
\end{tabular}


WORKBOOK PAGE: BLANK2

\begin{tabular}{|c|c|c|c|}
\hline H3 : LA. & TRITIUM & & BLANK \\
\hline & Disintegrations per minute from Counter & (dpm) & 7.92 \\
\hline BLNK & Sample Volume in $\mathrm{mL}$ & (Ss) & \\
\hline & Dilution Factor & (DF) & 11 \\
\hline 15286 & Digest Dilution Factor & (DDF) & \\
\hline & Background Count Rate in Counts per Minute & (BKG cpm) & 2194 \\
\hline @H3-01 & Background Count Tíme in Minutes & (BKG Time) & Ho \\
\hline i: & Instrument Fractional Efficiency & (EFF) & 0.5434 \\
\hline LIQUID & $\%$ Counting Error & & 5.66 \\
\hline 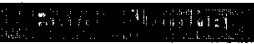 & Detection Limit & (Ld) & 5.72 \\
\hline 96011896 & Critical Level & (Lc) & 1.53 \\
\hline 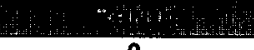 & Tritium Concentration in $\mu \mathrm{Ci} / \mathrm{L}$ & & 3.92E-02 \\
\hline
\end{tabular}

N/A

Critical Level $(L C)=2.33$ * the Square Root of (BKG cpm / BKG Time) Detection Limit $($ Ld $)=((2.72 /$ BKG Time $)+(2 *$ LC $)) /$ EFF $\mathrm{H} 3 \mu \mathrm{Ci} / \mathrm{L}=\left(\mathrm{dpm} *(1000 \mathrm{~mL} / \mathrm{L}){ }^{*} \mathrm{DF} * \mathrm{DDF}\right) /(\mathrm{SS} *(2220000 \mathrm{dpm} / \mu \mathrm{Ci}))$ WL15286 $\mathrm{H} 3 \mu \mathrm{Ci} / \mathrm{mL} \quad=\mu \mathrm{Ci} / \mathrm{L} / 1000 \mathrm{~mL} / \mathrm{L}$ Detection Limit $(\mu \mathrm{Ci} / \mathrm{mL})=(\mathrm{Ld})(\mathrm{DF})(\mathrm{DDF}) /[(\mathrm{SS})(2220000 \mathrm{dpm} / \mu \mathrm{Ci})]$ Mand

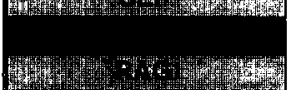

\begin{tabular}{lr}
\hline Tritium Concentration in $\mu \mathrm{Ci} / \mathrm{L}$ & $3.92 \mathrm{E}-02$ \\
\hline
\end{tabular}

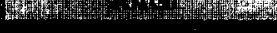

\begin{tabular}{|lr|}
\hline Tritium Concentration in $\mu \mathrm{Ci} / \mathrm{mL}$ & $3.92 \mathrm{E}-05$ \\
\hline Detection Limit in $\mu \mathrm{Ci} / \mathrm{mL}$ & $2.83 \mathrm{E}-05$ \\
\hline$\%$ Counting Error & 5.66 \\
\hline
\end{tabular}

MH:

\begin{tabular}{|lllll|}
\hline Analyst: & RAG & Date: & $12 / 02 / 96$ \\
\hline Signature of Chemist: & SLF & Date: & $12 / 3 / 96$ \\
\hline BLANK.WB1 REV 2.0 & & & \\
& $21811 \mathrm{NML}$ & & \\
& 272 & &
\end{tabular}


WORKBOOK PAGE: SAM3

WNEG-SE-WM-DP-202, REV. 1

\begin{tabular}{|c|c|c|c|}
\hline \multicolumn{3}{|c|}{ H3 : LA-218-114 (A-4), LA-218-115(A-0) } & SAMPLE \\
\hline & Disintegrations per minute from Counter & (dpm) & $\overline{937.56}$ \\
\hline SAMPLE & Sample Volume in $\mathrm{mL}$ & (SS) & 1 \\
\hline & Dilution Factor & (DF) & 11 \\
\hline 15286 & Digest Dilution Factor & (DDF) & WHWWW \\
\hline & Background Count Rate in Counts per Minute & (BKG cpm) & 2144 \\
\hline @H3-01 & Background Count Time in Minutes & (BKG Time) & 50 \\
\hline ith: & Instrument Fractional Efficiency & (EFF) & 0.4957 \\
\hline LIQUID & $\%$ Counting Error & & 1.29 \\
\hline 807 & Detection Limit & (Ld) & 6.27 \\
\hline 96011896 & Critical Level & (LC) & 1.53 \\
\hline & Tritium Concentration in $\mu \mathrm{Ci} / \mathrm{L}$ & & $4.65 \mathrm{E}+00$ \\
\hline
\end{tabular}

Critical Level $(L c)=2.33$ * the Square Root of (BKG cpm / BKG Time)

Detection Limit (Ld) $=\left((2.72\right.$ / BKG Time $)+\left(2{ }^{*}\right.$ Lc $\left.)\right) /$ EFF

$\mathrm{H} 3 \mu \mathrm{Ci} / \mathrm{L}=\left(\mathrm{dpm} *(1000 \mathrm{~mL} / \mathrm{L}) * \mathrm{DF}^{*} \mathrm{DDF}\right) /\left(\mathrm{SS}{ }^{*}(2220000 \mathrm{dpm} / \mu \mathrm{Ci})\right)$

$\mathrm{H} 3 \mu \mathrm{Ci} / \mathrm{mL} \quad=\mu \mathrm{Ci} / \mathrm{L} / 1000 \mathrm{~mL} / \mathrm{L}$

Detection Limit $(\mu \mathrm{Ci} / \mathrm{mL})=(\mathrm{Ld})(\mathrm{DF})(\mathrm{DDF}) /[(\mathrm{SS})(2220000 \mathrm{dpm} / \mu \mathrm{Ci})]$

PVy

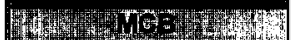

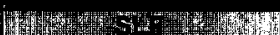

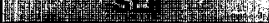

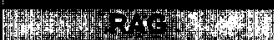

Tritium Concentration in $\mu \mathrm{Ci} / \mathrm{L} \quad 4.65 \mathrm{E}+00$

in

Tritium Concentration in $\mu \mathrm{Ci} / \mathrm{mL}$

$4.65 \mathrm{E}-03$

Detection Limit in $\mu \mathrm{Ci} / \mathrm{mL}$

$3.10 \mathrm{E}-05$

$\%$ Counting Error

1.29

mas

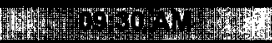

14axy

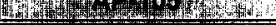

\begin{tabular}{|lllll|}
\hline Analyst: & RAG & Date: & $12 / 02 / 96$ \\
\hline Signature of Chemist: & 21811NML & SLF & Date: & $12 / 3 / 96$ \\
\hline SAMPLE.WB1 REV 2.0 & & &
\end{tabular}


WORKBOOK PAGE: SPIKE4

\section{HNE-SD-WM-DP-202, REV. 1}

\begin{tabular}{|c|c|c|c|}
\hline H3 : LA-218-11 & 4), LA-218 & & SPIKE \\
\hline & dpm of Sample from Counter & (dpm1) & 937.56 \\
\hline $\mathbf{S P}$ & dpm of Sample + Spike from Counter & (dpm2) & 1317.42 \\
\hline & Volume of Sample in Distillation Vial in $\mathrm{mL}$ & (Svol) & 1.5 \\
\hline 15286 & Volume of Spike in Distillation Vial in $\mathrm{mL}$ & (SPK vol) & 1.5 \\
\hline & Volume Transferred to Scintillation Vial in $\mathrm{mL}$ & (TR vol) & 1 \\
\hline @H3-01 & Spike Book Number & (Spk BN) & 64B56 \\
\hline & Spike Value in $\mu \mathrm{Cl} / \mathrm{mL}$ & (SPK val) & $7.5051 \mathrm{E}-04$ \\
\hline LIQUID & Background Count Rate in cpm & (BKG cpm) & 2444 \\
\hline & Background Count Time in Minutes & (BKG Time) & 50 \\
\hline 96011896 & Instrument Fractional Efficiency of Sample & (EFF) & 0.4935 \\
\hline & Detection Limit & (Ld) & 6.29 \\
\hline 0 & Critical Level & (Lc) & 1.53 \\
\hline 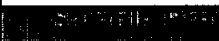 & $\mathrm{H} 3 \mu \mathrm{Ci} / \mathrm{mL}$ in Sample Vial & (Rsam) & 4.22E-04 \\
\hline N/A & $\mathrm{H} 3 \mu \mathrm{Ci} / \mathrm{mL}$ in Sample + Spike Vial & (Rs+s) & 5.93E-04 \\
\hline an & Vol. Ratio Sample Vol. to Total Vol. & (VRsam) & 5.00E-01 \\
\hline S96V000047 & Vol. Ratio Spike Vol. to Total Vol. & (VRspike) & 5.00E-01 \\
\hline
\end{tabular}

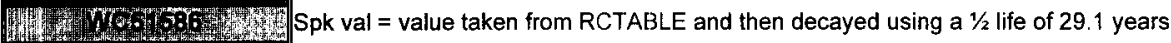

Detection Limit $(\mathrm{Ld})=\left((2.72\right.$ / BKG time $\left.)+\left(2{ }^{*} \mathrm{LC}\right)\right) / \mathrm{EFF}$

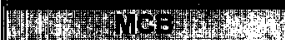

Critical Level $(L C)=2.33^{*}$ the Square Root of (BKG cpm / BKG time)

$\mathrm{H} 3 \mu \mathrm{Ci} / \mathrm{mL}$ in Sample Vial (Rsam) $=\mathrm{dpm} 1 /(\mathrm{TR}$ vol * $(2220000 \mathrm{dpm} / \mu \mathrm{Ci}))$

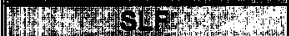

$\mathrm{H} 3 \mu \mathrm{Ci} / \mathrm{mL}$ in Sample + Spike Vial $(\mathrm{Rs}+\mathrm{s})=\mathrm{dpm} 2 /(\mathrm{TR}$ vol * $(2220000 \mathrm{dpm} / \mu \mathrm{Ci}))$

Volume Ratio of Sample Volume to Total Volume (VRsam) $=$ Svol / ( Svol + SPK vol )

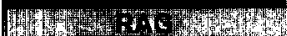

Volume Ratio of Spike Volume to Total Volume (VRspike) $=\mathrm{SPK}$ vol / ( Svol + SPK vol )

QC Actual in $\mu \mathrm{Ci} / \mathrm{mL}=\mathrm{SPK}$ val

Wraturam

Percent Spike Recovery $=$ QC Found / QC Actual *100

and

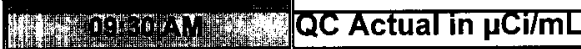

QC Found in $\mu \mathrm{CI} / \mathrm{mL} \quad$ = $7.65 \mathrm{E}-04$

WHF $101.9 \%$

स $12 / 3 / 96$


WORKBOOK PAGE: SP_DUP5

HNF

WHGSD-WM-LP-202, REV. 1

\begin{tabular}{|c|c|c|c|}
\hline \multicolumn{3}{|c|}{ H3 : LA-218-114 (A-4), LA-218-115(A-0) } & SPK-DUP \\
\hline & dpm of Sample from Counter & (dpm1) & 937.56 \\
\hline UP & dpm of Sample + Spike from Counter & (dpm2) & 1340.85 \\
\hline & Volume of Sample in Distillation Vial in $\mathrm{mL}$ & (Svol) & 1.5 \\
\hline 15286 & Volume of Spike in Distillation Vial in $\mathrm{mL}$ & (SPK vol) & 1.5 \\
\hline & Volume Transferred to Scintillation Vial in $\mathrm{mL}$ & (TR vol) & 1 \\
\hline @H3-01 & Spike Book Number & (Spk BN) & 64B56 \\
\hline & Spike Value in $\mu \mathrm{Ci} / \mathrm{mL}$ & (SPK val) & $7.5051 E-04$ \\
\hline LIQUID & Background Count Rate in cpm & (BKG cpm) & 2444 \\
\hline & Background Count Time in Minutes & (BKG Time) & 50 \\
\hline 96011896 & Instrument Fractional Efficiency of Sample & (EFF) & 0.4907 \\
\hline anting & Detection Limit & (Ld) & 6.33 \\
\hline 0 & Critical Level & (LC) & 1.53 \\
\hline & $\mathrm{H} 3 \mu \mathrm{Ci} / \mathrm{mL}$ in Sample Vial & (Rsam) & 4.22E-04 \\
\hline N/A & $\mathrm{H} 3 \mu \mathrm{Ci} / \mathrm{mL}$ in Sample + Spike Vial & (Rs+s) & 6.04E-04 \\
\hline & Vol. Ratio Sample Vol. to Total Vol. & (VRsam) & 5.00E-01 \\
\hline S96V000047 & Vol. Ratio Spike Vol. to Total Vol. & (VRspike) & 5.00E-01 \\
\hline
\end{tabular}

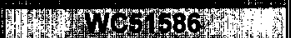

Spk val = value taken from RCTABLE and then decayed using a $1 / 2$ life of 29.1 years

Detection Limit $(\mathrm{Ld})=\left((2.72 / \mathrm{BKG}\right.$ time $\left.)+\left(2{ }^{*} \mathrm{LC}\right)\right) / \mathrm{EFF}$

ToB

Critical Level $(L c)=2.33^{*}$ the Square Root of (BKG cpm / BKG time)

$\mathrm{H} 3 \mu \mathrm{Ci} / \mathrm{mL}$ in Sample Vial (Rsam) $=\mathrm{dpm} 1 /(\mathrm{TR}$ vol * $(2220000 \mathrm{dpm} / \mu \mathrm{Ci}))$

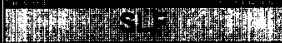

$\mathrm{H} 3 \mu \mathrm{Ci} / \mathrm{mL}$ in Sample + Spike Vial (Rs+s) $=\mathrm{dpm} 2 /(\mathrm{TR}$ vol * $(2220000 \mathrm{dpm} / \mu \mathrm{Ci}))$

Volume Ratio of Sample Volume to Total Volume (VRsam)= Svol / ( Svol + SPK vol )

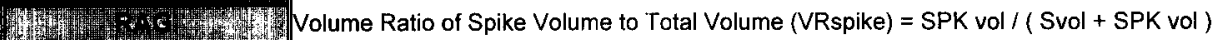

QC Actual in $\mu \mathrm{Ci} / \mathrm{mL}=\mathrm{SPK}$ val

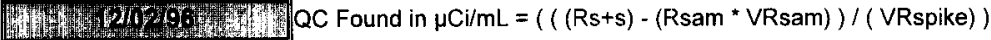

Percent Spike Recovery $=$ QC Found $/$ QC Actual *100

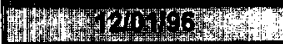

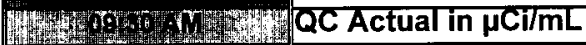

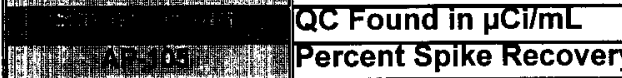

$=$

7.51E-04

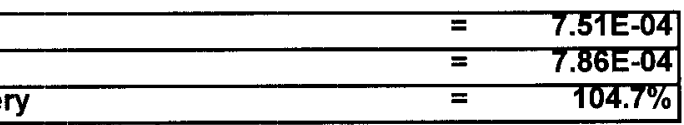

\&

$12 / 3 / 96$ 
WORKBOOK PAGE: SAM6

HNF

WHE-SD-WM-DP-202, REV. 1

\begin{tabular}{|c|c|c|c|}
\hline H3 : LA-218-114 (A-4), LA-218-115(A-0) & TRITIUM LIQUIDSI & TRITIUM LIQUIDS/ SOLIDS & SAMPLE \\
\hline & Disintegrations per minute from Counter & (dpm) & 204.84 \\
\hline SAMPLE & Sample Volume in $\mathrm{mL}$ & (SS) & 1 \\
\hline & Dilution Factor & (DF) & 1 \\
\hline 15286 & Digest Dilution Factor & (DDF) & 1 \\
\hline ... & Background Count Rate in Counts per Minute & (BKG cpm) & 2144 \\
\hline @H3-01 & Background Count Time in Minutes & (BKG Time) & 50 \\
\hline 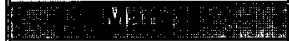 & Instrument Fractional Efficiency & (EFF) & 0.4954 \\
\hline LIQUID & $\%$ Counting Error & & 0.83 \\
\hline 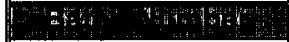 & Detection Limit & (Ld) & 6.27 \\
\hline 96011896 & Critical Level & (Lc) & 1.53 \\
\hline & Tritium Concentration in $\mu \mathrm{Ci} / \mathrm{L}$ & & $1.04 \mathrm{E}+00$ \\
\hline
\end{tabular}

Critical Level $(L c)=2.33^{*}$ the Square Root of (BKG cpm / BKG Time)

N/A

Detection Limit $($ Ld $)=((2.72 /$ BKG Time $)+(2 *$ Lc $)) /$ EFF

$\mathrm{H3} \mu \mathrm{Ci} / \mathrm{L}=\left(\mathrm{dpm}{ }^{*}(1000 \mathrm{~mL} / \mathrm{L}) *{ }^{*}{ }^{*} \mathrm{DDF}\right) /(\mathrm{SS} *(2220000 \mathrm{dpm} / \mu \mathrm{Ci}))$

$\mathrm{H} 3 \mu \mathrm{Ci} / \mathrm{mL} \quad=\mu \mathrm{Ci} / \mathrm{L} / 1000 \mathrm{~mL} / \mathrm{L}$

S96V000048

Detection Limit $(\mu \mathrm{Ci} / \mathrm{mL})=(\mathrm{Ld})(\mathrm{DF})(\mathrm{DDF}) /[(\mathrm{SS})(2220000 \mathrm{dpm} / \mu \mathrm{Ci})]$

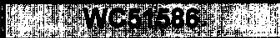

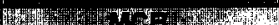

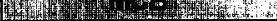

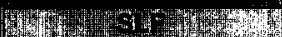

\begin{tabular}{|l|lr|} 
& Tritium Concentration in $\mu \mathrm{Ci} / \mathrm{L}$ & $1.04 \mathrm{E}+00$ \\
\hline & & \\
\hline & & \\
\hline & Tritium Concentration in $\mu \mathrm{Ci} / \mathrm{mL}$ & $1.04 \mathrm{E}-03$ \\
\hline & Detection Limit in $\mu \mathrm{Ci} / \mathrm{mL}$ & $2.82 \mathrm{E}-06$ \\
\hline \% Counting Error & 0.83 \\
\hline
\end{tabular}

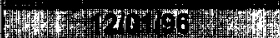

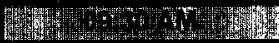

14.

Hes:

\begin{tabular}{|c|c|c|c|}
\hline Analyst: & RAG & Date: & $12 / 02 / 96$ \\
\hline Signature of Chemist: & SLF & Date: & $12 / 3 / 96$ \\
\hline SAMPLE.WB1 REV 2.0 & & & \\
\hline
\end{tabular}


WFG-SD-WM-DP-202, REV. 1

WORKBOOK PAGE: SAM7

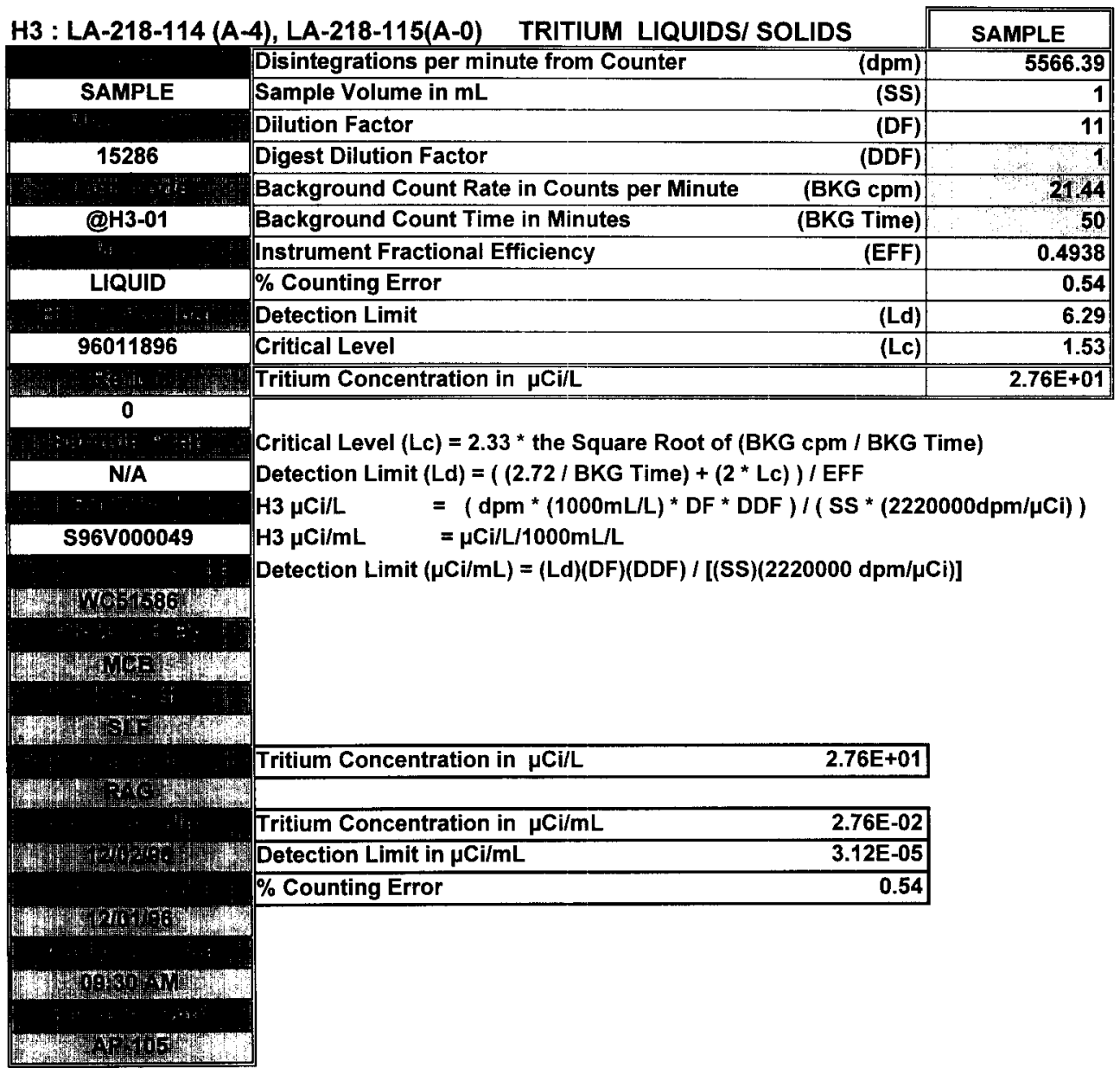

\begin{tabular}{|lllll|}
\hline Analyst: & & RAG & Date: & $12 / 02 / 96$ \\
\hline Signature of Chemist: & SLF & Date: & $12 / 3 / 96$ \\
\hline SAMPLE.WB1 REV 2.0 & & & \\
& & & \\
& $21811 \mathrm{NML}$ & &
\end{tabular}


WORKBOOK PAGE: SAMB

SD-WM-DP-202, REV. 1

\begin{tabular}{|c|c|c|c|}
\hline LA-218-114 & 4), LA-218-115(A-0) & LIDS & SAMPLE \\
\hline & Dis integrations per minute from Counter & $(\mathrm{dpm})$ & 18.13 \\
\hline LE & Sample Volume in $\mathrm{mL}$ & (SS) & \\
\hline & Dilution Factor & (DF) & 11 \\
\hline 15286 & Digest Dilution Factor & (DDF) & \\
\hline & Background Count Rate in Counts per Minute & (BKG cpm) & 244 \\
\hline @H3-01 & Background Count Time in Minutes & (BKG Time) & 50 \\
\hline n: & Instrument Fractional Efficiency & (EFF) & 0.4973 \\
\hline LIQUID & $\%$ Counting Error & & 5.34 \\
\hline 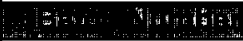 & Detection Limit & (Ld) & 6.25 \\
\hline 96011896 & Critical Level & (Lc) & 1.53 \\
\hline & Tritium Concentration in $\mu \mathrm{Ci} / \mathrm{L}$ & & $8.98 \mathrm{E}-02$ \\
\hline
\end{tabular}

Critical Level $(L c)=2.33^{*}$ the Square Root of (BKG cpm / BKG Time)

N/A

Detection Limit $($ Ld $)=((2.72 /$ BKG Time $)+(2 *$ Lc $)) /$ EFF

$\mathrm{H3} \mu \mathrm{Ci} / \mathrm{L}=(\mathrm{dpm} *(1000 \mathrm{~mL} / \mathrm{L}) * D F * \mathrm{DDF}) /\left(\mathrm{SS}^{*}(2220000 \mathrm{dpm} / \mu \mathrm{Ci})\right)$

$\mathrm{H} 3 \mu \mathrm{Ci} / \mathrm{mL} \quad=\mu \mathrm{Ci} / \mathrm{L} / 1000 \mathrm{~mL} / \mathrm{L}$

S96V000058

Detection Limit $(\mu \mathrm{Ci} / \mathrm{mL})=(\mathrm{Ld})(\mathrm{DF})(\mathrm{DDF}) /[(\mathrm{SS})(2220000 \mathrm{dpm} / \mu \mathrm{Ci})]$

Why

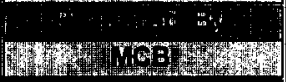

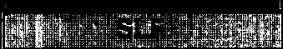

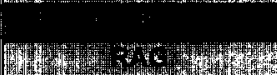

Tritium Concentration in $\mu \mathrm{Ci} / \mathrm{L} \quad 8.98 \mathrm{E}-02$

\begin{tabular}{|c|c|c|}
\hline \multirow{2}{*}{ WHA } & Tritium Concentration in $\mu \mathrm{Ci} / \mathrm{L}$ & 8.98E-02 \\
\hline & & \\
\hline & Tritium Concentration in $\mu \mathrm{Ci} / \mathrm{mL}$ & 8.98E-05 \\
\hline 0760 & Detection Limit in $\mu \mathrm{Ci} / \mathrm{mL}$ & 3.09E-05 \\
\hline & $\%$ Counting Error & 5.34 \\
\hline
\end{tabular}

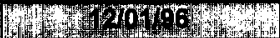

$\%$ Counting Error

5.34

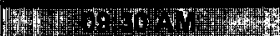

MTH

\begin{tabular}{|llll|}
\hline Analyst: & RAG & Date: $12 / 02 / 96$ \\
\hline Signature of Chemist: & 21811NML & SLF & Date: $12 / 3 / 96$ \\
\hline SAMPLE.WB1 REV 2.0 & & \\
\hline
\end{tabular}

278 


\section{LABCORE Completed RadChem Report for Worklist\#: 14423}

\section{Analyst: smf}

Instrument: $\mathrm{AB} 16$
Book\#

Method: $\lfloor A-508-101$ Rev/Mod $E-1$

Worklist Comment: AP-105 ABS

\begin{tabular}{|c|c|c|c|c|c|c|c|c|c|}
\hline Seq Туре & Sample\# & $\mathbf{R}$ & & Test & Matrix & Actual & Found & DL or Yield & Unit \\
\hline 1. STD & & 0 & QNB-01 & NupHAO1 & LIOOID & $2: 31 \mathrm{~g}-04$ & $2.36+-4$ & 103,030 & \& Rooovery \\
\hline 1 STD & & 0 & AAB-01 & ALPHAO $1 \mathrm{~B}$ & LIOUID & $\mathbf{2}$ & $3.48 \mathrm{H}+00$ & 3.480 & \& ct. Frro \\
\hline 1 sकb & & 0 & AAB-01 & BEM-01 & LTETID & $2.97 \times-03$ & $298 x-3$ & 100.337 & \& kooovery. \\
\hline $1 \mathrm{sTD}$ & & 0 & DAB-01 & BETA-01E & LIQUID & 1 & $6.928-01$ & 0.692 & of ct. Frro \\
\hline 2 BLMR-PREP & & 0 & $\triangle \mathrm{AB}-01$ & ALPHALO1 & LIQUIID & $:$ & $<1.023-2$ & & uct $/$ tht \\
\hline 2 RLNK-PREP & & 0 & ФAB-01 & ALPHAO1E & LIQUID & 1.00 & $5.008+02$ & 500.000 & $u C_{i} / \mathbf{m L}$ \\
\hline 2 BLNK-BREF & & 0 & AAB-O1 & BETA-01 & HTOID & 1 & $1.59 x-1$ & 0.159 & uct/mL \\
\hline 2 BLNK-PREP & & 0 & ABB- 01 & BETA-01E & LIQUID & 1.00 & $2.028+01$ & 20.200 & uCi/ml \\
\hline 3 BLMKK/BKG & & 0 & $\triangle A B-01$ & ATPHHOS & trootb & $1.00 \mathrm{E}+00$ & $1,11 \mathrm{E}+00$ & 1,110 & BLIXK/BKO \\
\hline 3 BLNK/BKO & & 0 & AAB- 01 & BESIA-01 & LIQUID & $1.00 \mathrm{~B}+00$ & $1.96 \mathrm{~B}+00$ & 1.960 & BLMK/BKo \\
\hline 4 sampro: & 8900000050 & 0 & $\triangle \mathrm{AB}-01$ & ALPHAOO 1 & HQOID & $s / A<$ & $1.02 \pi-02$ & $121,0-004$ & uci $/ \mathrm{mL}$ \\
\hline 4 SAMPLE & $596 \mathrm{V000050}$ & 0 & $\triangle A B-01$ & ALPHA $1 \mathrm{E}$ & IIQOID & $\mathrm{N} / \mathrm{A}$ & $5.00 \mathrm{~B}+02$ & $0.0 \bullet+000$ & \% ct. Exror \\
\hline 4 SAMPEI & s9.60000050 & 0 & $\triangle A B-01$ & BETR-01 & ITOUID & $N / a$ & $1.08 \mathrm{~F}+02$ & $371,00-004$ & uci $/ \mathrm{mL}$ \\
\hline 4 SAMPLE & S96V000050 & 0 & $\triangle A B-01$ & BETA-01E & LIQUID & $\mathbb{N} / \mathrm{A}$ & $1.27 B+00$ & $0.00+000$ & $\%$ Ct. Error \\
\hline 5 DOUP & 5960000050 & 0 & $\triangle A B-01$ & AEPHLOA & IIQUTP & $<1-02 \mathrm{E}-2$ & $<6,76 \mathrm{~B}-3$ & & PD: \\
\hline 5 DUP & 5960000050 & 0 & 3 CAB-01 & ALPHAO 1E & LIQOID & 1.00 & $5.00 \mathrm{~B}+02$ & 500.000 & \% ct. Erro \\
\hline 5 D Dop & s960000050 & 0 & $\triangle A B-01$ & BETR-O1 & WXorip & $1.0683+2$ & $300 \mathrm{OB}+2$ & $0.000=$ & PD: \\
\hline 5 DUP & 5960000050 & 0 & 3 AAB-01 & BETA-015 & LIQUID & 1.00 & $3.62 \mathrm{E}-01$ & 0.362 & \% ct. Erro \\
\hline 6 SAMBLE & S9600000s & 0 & 3. OAB- 01 & ALPHAO 1 & TIOOID & $\Delta / A<$ & $1.02 \mathrm{x}-02$ & $12100-004$ & $u c 1 / \mathrm{mb}$ \\
\hline 6 SAMPLY & 8960000051 & 0 & $3 A B-01$ & ALPHAO1B & LIQUID & $N / A$ & $5.00 \mathrm{~F}+02$ & $0.0 \bullet+000$ & o ct. Brror \\
\hline 6 SAMPLI & 596 V0000051 & 0 & $\quad 040-01$ & BETA O 1 & LIOEID & $N / 2$ & $1.12 \mathrm{E}+02$ & $371.0-004$ & uCt $/$ mot \\
\hline 6 SAMPLR & $596 \mathrm{~V} 000051$ & 0 & $3 A B-01$ & BETA-01B & LIQUID & $\underline{N} / \mathrm{A}$ & $3.55 \mathrm{~F}-01$ & $0.00+000$ & \& ct. Exror \\
\hline 7 DOP & $\$ 960000051$ & 0 & CAB- 01 & ACPHAO1 & LIOOID & $<1,02 \mathrm{~g}-2$ & $<5.05 \pi-3$ & & PDD \\
\hline 7 DUP & S96V000051 & 0 & $3 A B-01$ & ALPHAO1B & LIQUTD & 1.00 & $5.00 \mathrm{~F}+02$ & 500.000 & \% Ct. Rrro \\
\hline $7 \quad D Q P$ & $\$ 960000051$ & 0 & ONB-01 & BETA-01 & 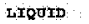 & $1.12 \pi+2$ & $1.06 \%+2$ & 5.505 & KPD \\
\hline 7 DUP & 5960000051 & 0 & IAB- 01 & BETA-018 & LIQUID & 1.00 & $3.63 \mathrm{~m}-01$ & 0.363 & \& ct. Erro \\
\hline 8 SAMPCS & 5960000052 & 0 & one-01 & ACPHAO 1 & LIQUTD & $\Delta / \mathbf{A}<$ & $5.05 \mathrm{k}-03$ & $1210-004$ & uct $/ \mathrm{mL}$ \\
\hline 8 SAMPLE & 5960000052 & 0 & 3 DAB-01 & ALPHAO $1 \mathrm{E}$ & LIQUID & $N / A$ & $5.00 \mathrm{E}+02$ & $0.0 \bullet+000$ & \& ct. Error \\
\hline S SAMELE & $\$ 960000052$ & 0 & $\triangle \mathrm{AB}-01$ & EETA O 1 & LIQJID: & $\mathbb{N} / \mathrm{A}$ & $1.07 \mathbf{E}+02$ & $371,00-004$ & UCt/mL \\
\hline 8 SAMPLR & 5960000052 & 0 & 3 DAB-01 & BETA-01E & LIODID & $\mathbb{N} / \mathrm{A}$ & $3.62 \mathrm{~B}-01$ & $0.0 \bullet+000$ & \& ct. Error \\
\hline 9. DOF & s9600000052 & 0 & $3 \bigcirc A B-01$ & ACPHAO 1 & LXQUID & $<5.05 \mathrm{E}-3$ & $45056-3$ & & RPD \\
\hline 9 DUP & 5960000052 & 0 & 3 AB-01 & ALPHAO $1 \mathrm{E}$ & EIQUID & 1.00 & $5.00 \mathrm{E}+02$ & 500.000 & \& ct. Erro \\
\hline 9 DDP & 5960000052 & 0 & OAR-O1 & BRTR 01 & LYOUTD: & $1.07 \mathrm{k}+2$ & 1,008 +2 & $0.930=$ & RPD \\
\hline 9 DUP & 5960000052 & 0 & 3 AB-01 & BETA-01E & LIQUID & 1.00 & $3.598-01$ & 0.359 & \% Ct, Erro \\
\hline 0 SAMELE: & $596 \times 000054$ & 0 & $3 \bigcirc A-01$ & ALPHAO1 & LTOUTD & $\mathbb{N} / \mathrm{A}$ & $5.05 x-0.3$ & $121,0=-004$ & nei/mit \\
\hline 0 SAMPLB & S96V000054 & 0 & $\Rightarrow A B-01$ & ALPHAO IE & LIQUID & $\mathrm{N} / \mathrm{A}$ & $5.00 \mathrm{E}+02$ & $0.00+000$ & \% ct. Error \\
\hline 0 SAMPES & 5960000054 & o & $\triangle \triangle A B-01$ & BETA-01 & EIOUTD & $\boldsymbol{k} / \mathrm{a}$ & $1.11 \mathrm{R}+02$ & $371,00-004$ & uej/int \\
\hline .0 SAMPLB & $596 \mathrm{~V} 000054$ & 0 & $3 \triangle A B-01$ & BETA-01E & LIQUID & $\mathbb{N} / \mathrm{A}$ & $3.55 \mathrm{~B}-07$ & $0.00+000$ & \& Ct, Error \\
\hline 1. DOP & S $96 \vee 000054$ & 0 & $\triangle A B=01$ & ALPHAOI & LTQJuxp & $<5.05 \mathrm{k}-3$ & $<5: 05:-3$ & : : : & $\mathrm{RPD}$ \\
\hline $1 \mathrm{CUP}$ & $S 96 V 000054$ & 0 & 3 AB-01 & ALPHA $01 \mathrm{E}$ & LIQUID & 1.00 & $5,00 \pi+02$ & 500.000 & ct. Erro \\
\hline 1 DUP. & 5960000054 & 0 & $\triangle A B+01$ & BETA-01 & EIQUID & 1. $11 \mathrm{~B}+2$ & $1.10 \mathrm{~B}+2$ & 0.90 & D \\
\hline
\end{tabular}




\section{LABCORE Completed RadChem Report for Worklist\#: 14423}

\begin{tabular}{|c|c|c|c|c|c|c|c|c|c|}
\hline Seq Type & Sample & $\mathbf{R}$ & & Test & Matrix & Actual & Found & DL or Yield & Unit \\
\hline 11 DOP & $S 96 V 000054$ & 0 & $B \quad \triangle A B-02$ & BETA-01E & LIQUID & 1.00 & $3,56 x-01$ & 0.356 & Ct. Exro \\
\hline 12 SAMPLI & 5960000060 & 0 & $B$ ans-01 & ALPBAO 1 & LIOUID & $N / A$ & $2.50 x-05$ & $597.00-007$ & $\mathbf{u c t} / \mathbf{m L}$ \\
\hline 12 SAMFLE & $\$ 960000060$ & 0 & B QAB-OL & ALPQAOIS & LTOUDD & $N / A$ & $5.005+02$ & $0.00+000$ & \& ct. Error \\
\hline 12 SAMPLI & $s 96 V 000060$ & 0 & $B A B-01$ & BETA- 01 & IIQTID & N/A & $3.02 \pi-04$ & $184,0=-006$ & $\mathrm{uct} / \mathrm{mL}$ \\
\hline 12 sampLa & $\$ 95 \vee 000060$ & 0 & $A B=01$ & $B B \times A-O D B$ & Hrooto & $\underline{N} / \boldsymbol{A}$ & $4.71 \mathrm{k}+01$ & $0.00+000$ & \& ct. Error \\
\hline $13 \mathrm{DOP}$ & S960000060 & 0 & B $A A B-01$ & ALPHAO 1 & IIQOID & $<2.50 \mathrm{R}-5$ & $<4.19 \mathrm{~K}-5$ & & RPD \\
\hline 13 DUP & 5950000060 & 0 & $B A B-O I$ & ACPHAOIS & IIOEID & 1,00 & $5,00 \mathrm{r}+02$ & 500,000 & $\because$ Ct Erro \\
\hline 13 DOP & S96V000060 & 0 & B $A B-01$ & BETA- 01 & IIQOID & $3.028-4$ & $2.19 \mathrm{E}-4$ & 31.862 & RPD \\
\hline 13 DUP & s96V000060 & 01 & exp 01 & BEFA OIB & LIODID & 2.00 & $5: 92 \mathrm{E}+01$ & 59.200 & \& Ct: Erro \\
\hline 14 SEK & 5960000060 & $\therefore$ & $B \quad \cos -01$ & ALPHA0 1 & IIQOID & $3.59 \mathrm{~B}-02$ & $3.15 \mathrm{E}-02$ & 87.744 & \& Recovery \\
\hline I4 $\triangle E K$ & s95V000060 & 0 & B $\quad A B-01$ & BENA - O1 & TrợD & $1.523 \div 01$ & $1.54 \mathrm{~B} 03$ & 101,316 & \& Recorury \\
\hline 15 SEK & $s 960000060$ & 0 & $B$ AAB-01 & ALPBAOI & LI & $3.15 \mathrm{~B}-02$ & $3.28 \mathrm{E}-02$ & 4.044 & RPD \\
\hline 5 S8X-DU? & S95V000060 & 0 & B $\quad \times B-01$ & Bgra 01 & LIQUTD & $1.54 \mathrm{~B} \div 01$ & $1.48 \mathrm{E}-01$ & 3,974 & RPD \\
\hline
\end{tabular}

Final page for worklist\# 14423

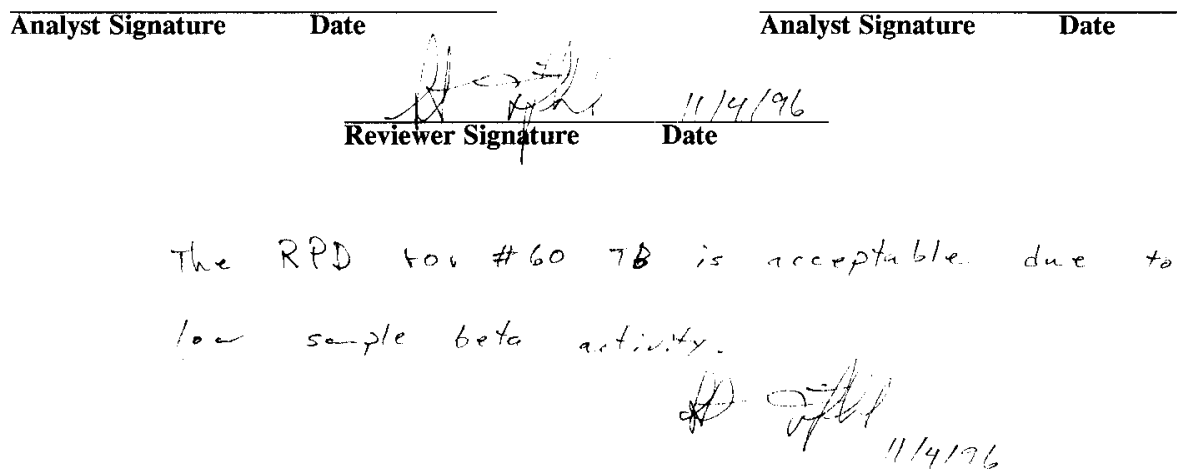




\section{LABCORE Data Entry Template for Worklist\# 14423}

\section{Analyst: \\ SMF Instrument: $\mathrm{AB} 00$

\#位 \\ Book\# 66856}

Method: LA-508-101 Rev/Mod E-l

Worklist Comment: AP-105 AB'S DETERMINE SS USING LUDLUM. RCJ

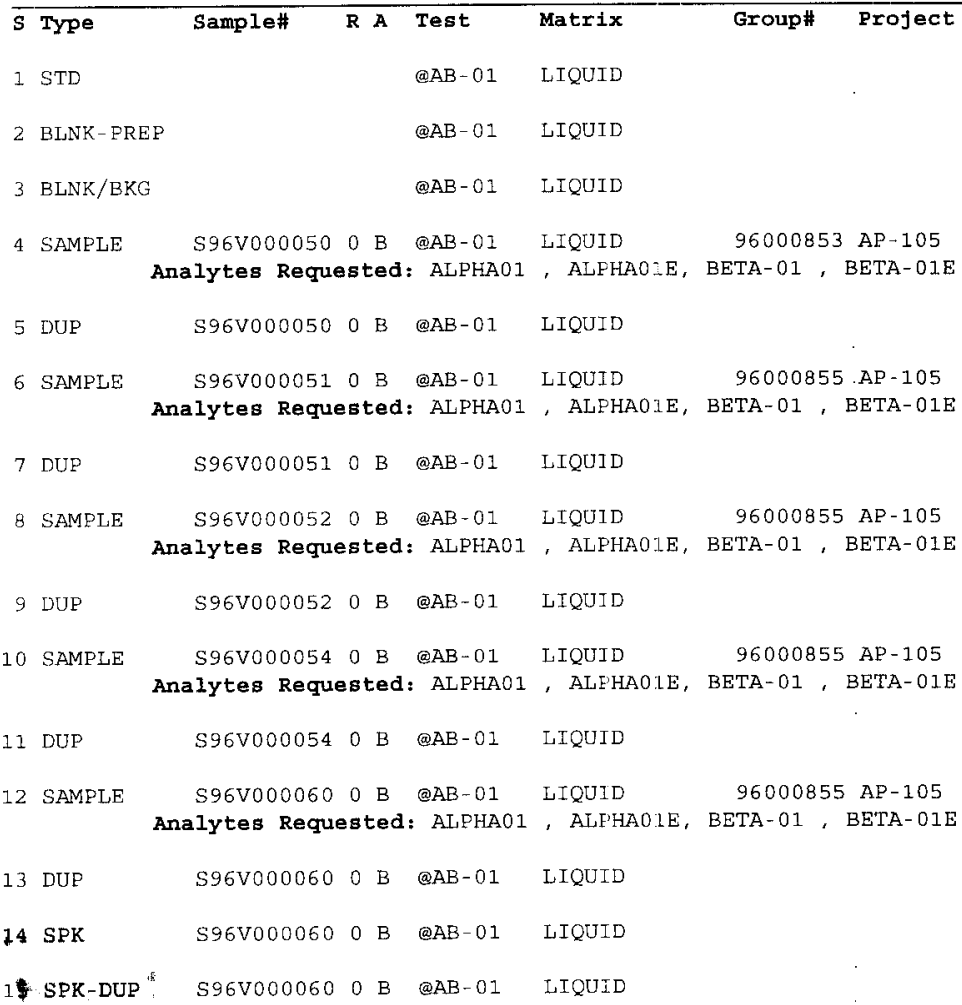

Data Entry Comments:

S96V000060 SPK \& SPK DUP - rum an SG6V00060 SAM

$S=$ Worklist Slot Number, $R=$ Replicate Number, $A=$ Aliquot $\bar{C}$ ode 


\section{LABCORE Data Entry Template for Worklist\# 14423}

S Type Sample\# R A Test Matrix Group\# Project

Final page for worklist \# 14423

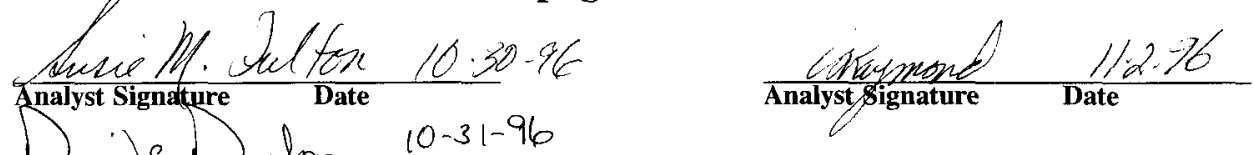


HNFG-SD-WM-DP-202, REV. 1

WORKBOOK PAGE: STD1

TB : LA-508-101 (E-1)

STD
ata
14423

LA-508-113 (A-2) STANDARD

DETECTOR NUMBER

DISH SIZE $(1,2$, or 5$)$

GROSS COUNTS

COUNT TIME in MINUTES

BACKGROUND in cpm

TB

SAMPLE SIZE in $\mathrm{mL}$

DILUTION FACTOR

STANDARD BOOK NUMBER

EFFICIENCY FACTOR

Lc, Rmax, or Rs,(SAMPLE RATE) as APPROPRIATE

LIQUID

Standard Vajue in $\mu \mathrm{Ci} / \mathrm{mL}$

Concentration in $\mu \mathrm{Ci} / \mathrm{L}$

Replicate Concentration in $\mu \mathrm{C} \mathrm{i} / \mathrm{L}$

AVERAGE CONCENTRATION in $\mu \mathrm{Ci} / \mathrm{L}$

\begin{tabular}{|r|r|r|}
\cline { 2 - 3 } \multicolumn{1}{c|}{} & STANDARD & REPLICATE \\
\hline (MS) & 16 & 16 \\
\hline (GC) & 2 & 2 \\
\hline (CT) & 85928 & 81493 \\
\hline BKG) & 30 & 30 \\
\hline (SS) & 15.3 & 15.3 \\
\hline (DF) & 1.000 & 1.000 \\
\hline d BN) & 1 & 1 \\
\hline (EFF) & 0.4195 & 0.4195 \\
\hline & 2848.967 & 2701.133 \\
\hline & $2.97 \mathrm{E}-03$ & \\
\hline \multicolumn{3}{|c|}{$3.06 \mathrm{E}+00$} \\
\hline
\end{tabular}

N/A

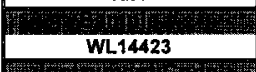

Rs (Sample Count Rate) $=(T C / C T)-B K G$

TOTAL BETA $\mu \mathrm{Ci} / \mathrm{L}=\mathrm{Rs} * 1000 \mathrm{mLL} * \mathrm{DF} /(\mathrm{EFF} * \mathrm{SS} * 2220000 \mathrm{dpm} / \mu \mathrm{Ci})$

TOTAL BETA $\mu \mathrm{Ci} / \mathrm{mL}=$ TOTAL BETA $\mu \mathrm{Ci} / \mathrm{L} / 1000 \mathrm{~mL} / \mathrm{L}$

WB27806
WBathen

Relative Counting Error $=[\mid($ The Square Root of TC $+B K G *$ CT) $/(T C-B K G * C T) \mid] * 1.96 * 100$

Detection Levels and Less Than Values are determined from Procedure LA-508-002

\section{VAR}

\begin{tabular}{|c|}
\hline $\mathbf{S L F}$ \\
$\mathbf{S M F}$ \\
\hline
\end{tabular}

$11 / 02 / 96$

TOTAL BETA CONCENTRATION in $\mu \mathrm{Ci} / \mathrm{mL}=$

$=2.98 \mathrm{E}-03$

(3)

\begin{tabular}{l} 
DETECTION \\
LEVEL \\
3.68E-06 \\
$\mu \mathrm{Ci} / \mathrm{mL}$ \\
\hline
\end{tabular}

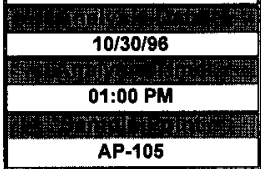

\begin{tabular}{|c|c|c|}
\hline Analyst: & VAR & Date: 02-Nov-96 \\
\hline Signature of Chemist: & & Date: $1 / 14 / 96$ \\
\hline
\end{tabular}




\section{WFE-SD-WM-DP-202, REV. 1}

WORKBOOK PAGE: STD2

AT : LA-508-101 (E-1)

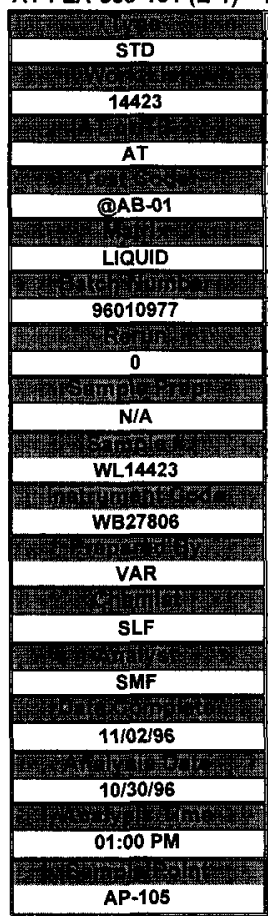

LA-508-113 (A-2) STANDARD

DETECTOR NUMBER

DISH SIZE 1,2 or 5

GROSS COUNTS

COUNT TIME in MINUTES

BACKGROUND in cpm

SAMPLE SIZE in $\mathrm{mL}$

DILUTION FACTOR

STANDARD BOOK NUMBER

EFFICIENCY FACTOR

Lc, Rmax, or Rs,(SAMPLE RATE) as APPROPRIATE

Standard Value in $\mu \mathrm{Ci} / \mathrm{mL}$

Concentration in $\mu \mathrm{CH} / \mathrm{L}$

Replicate Concentration in $\mu \mathrm{Ci} / \mathrm{L}$

AVERAGE CONCENTRATION in $\mu \mathrm{Ci} / L$

Rs (Sample Count Rate) $=(T C / C T)-B K G$

ALPHA TOTAL $\mu \mathrm{Ci} / \mathrm{L}=\mathrm{Rs} * 1000 \mathrm{mLL}$ * DF / (EFF* SS *2220000dpm/ $/ \mathrm{Ci}$ )

ALPHA TOTAL $\mu \mathrm{Ci} / \mathrm{mL}=$ ALPHA TOTAL $\mu \mathrm{Ci} / \mathrm{L} / 1000 \mathrm{~mL} / \mathrm{L}$

Relative Counting Error $=[$ [(The Square Root of TC + BKG *CT) / (TC - BKG * CT) $]$ ] $1.96 * 100$

Detection Levels and Less Than Values are delermined from Procedure LA-508-002.

ALPHA TOTAL CONCENTRATION in $\mu \mathrm{Ci} / \mathrm{mL}=2.38 \mathrm{E}-04$

RELATIVE COUNTING ERROR

2.3768E-01
STAMDARD

REPLICATE

(MS)

(CT)

(BKG)

(DF)

(Std BN)

(EFF)

$=$

2.31E-04

2.48E-01

2.27E-01

=

근

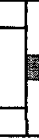

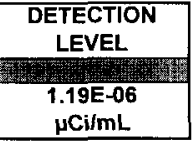

Analyst:
Signature of Chemist:
STANDARD.WB1 Rev. 1.0


WORKBOOK PAGE: BLANK3

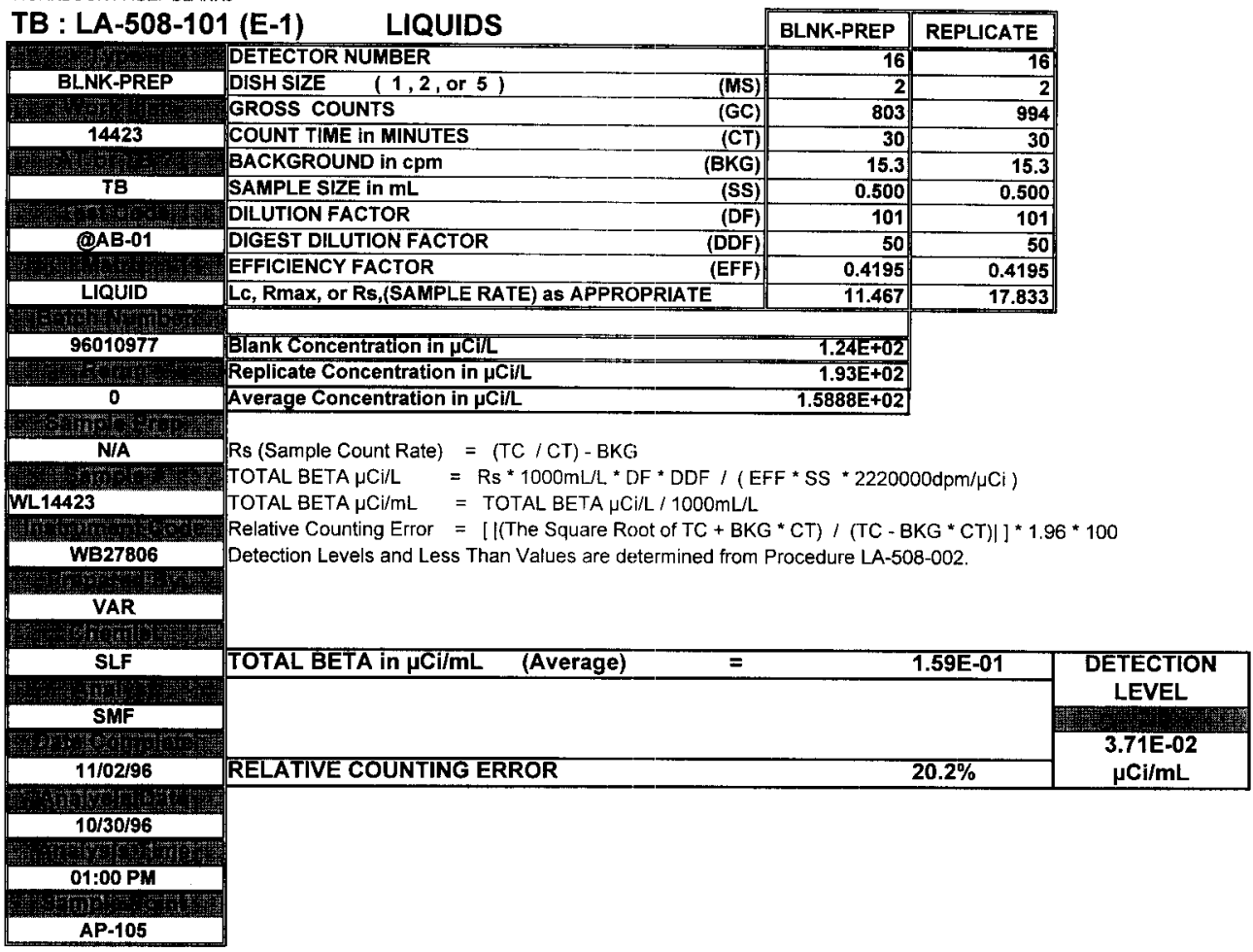

\begin{tabular}{|c|c|c|}
\hline Analyst: & SMF & Date: 02-Nov-96 \\
\hline Signature of Chemist: & SLF & Date: $1 / / 4 / 96$ \\
\hline
\end{tabular}

\section{5}


WORKBOOK PAGE: BLANK4

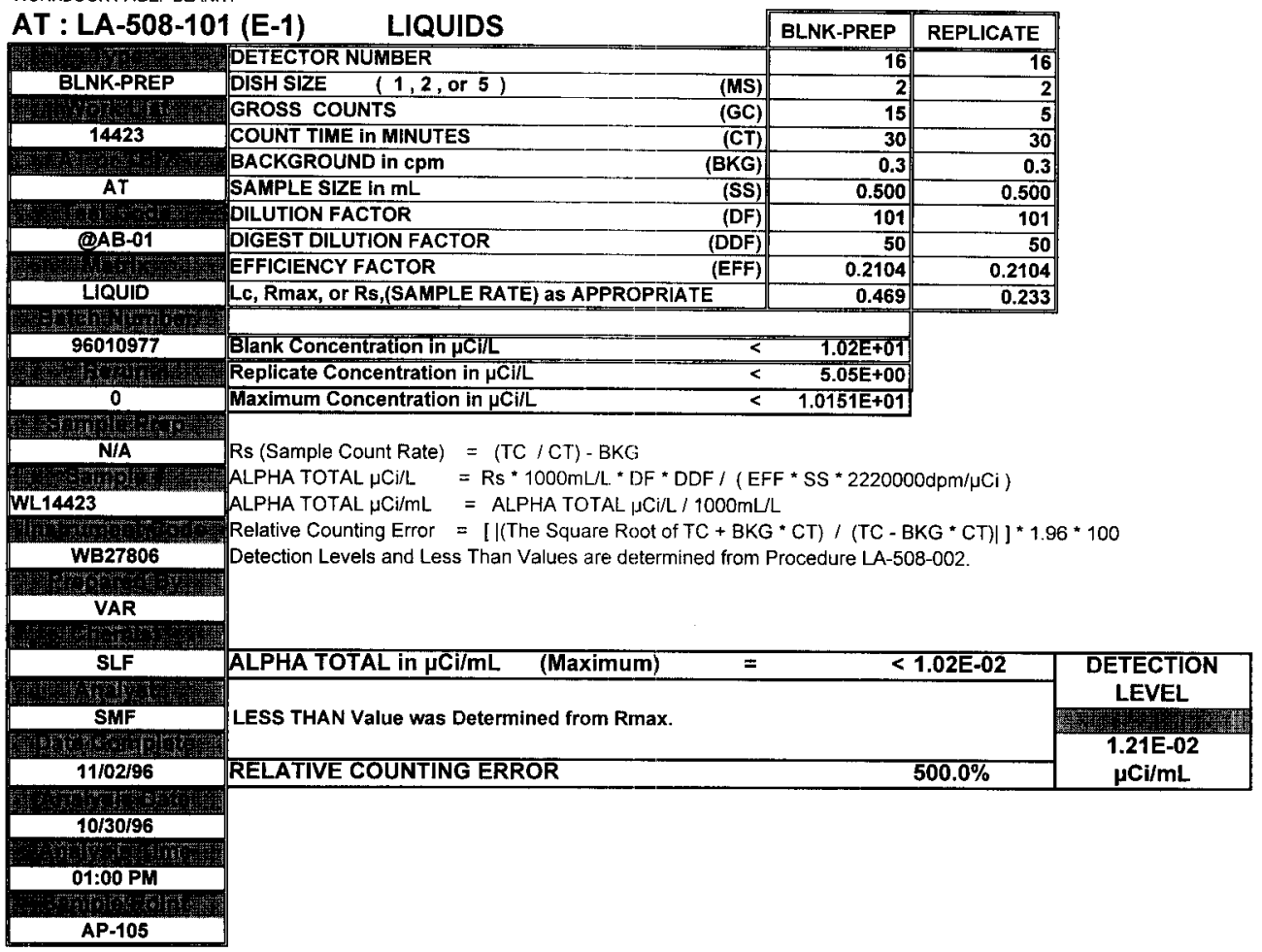

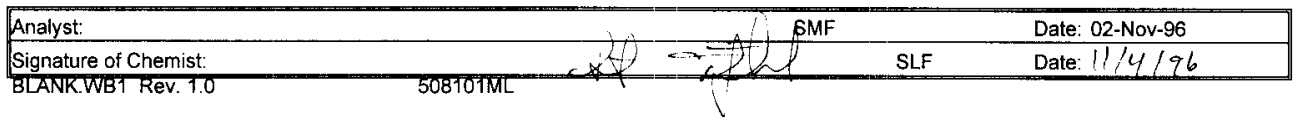


WORKBOOK PAGE: SAM7

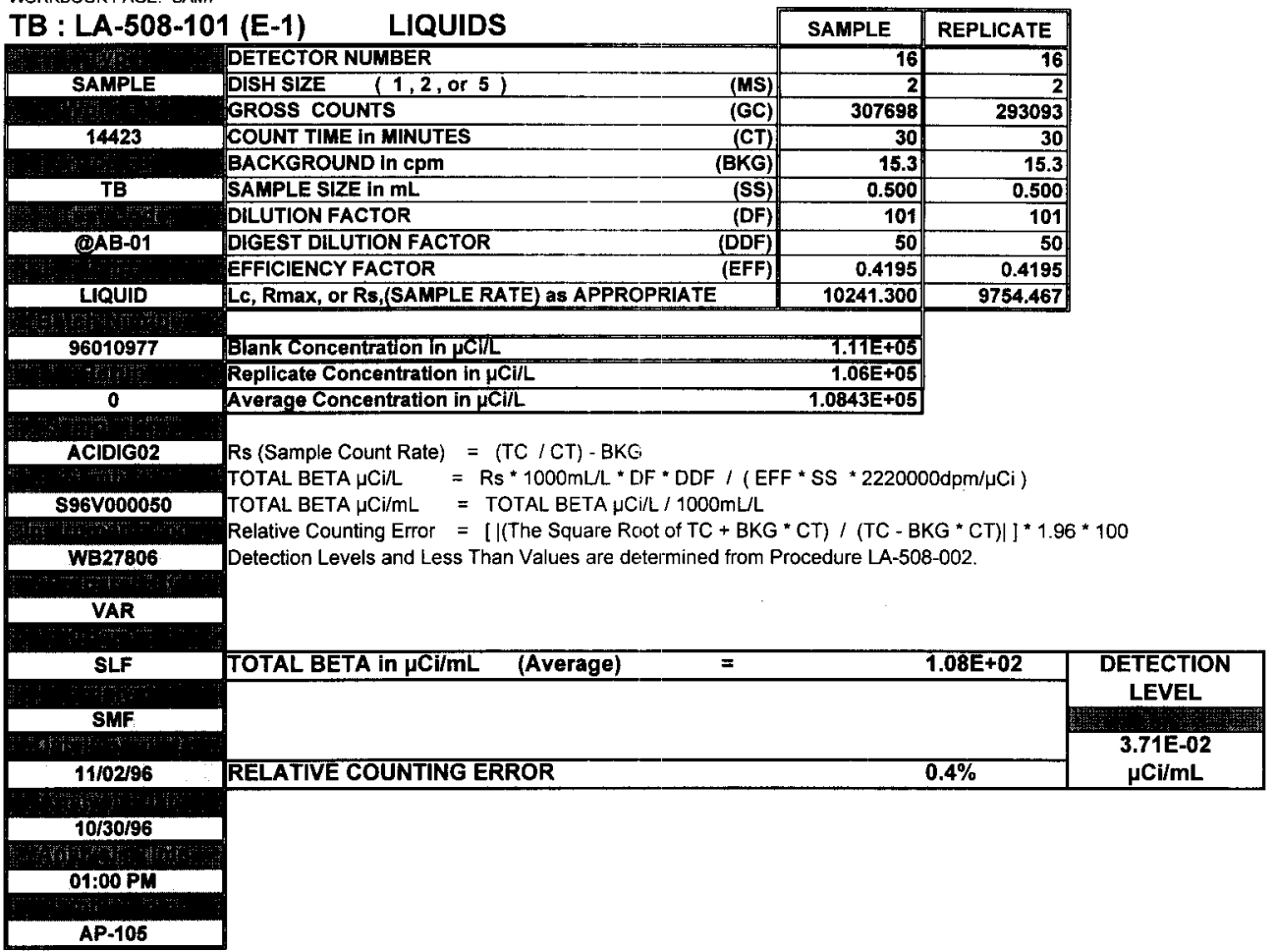

Analyst:
Signature of Chemist:
SAMPLE.WB1 Rev. 1.




\begin{tabular}{|c|c|c|c|c|}
\hline AT: LA-508-10 & LIQUIDS & SAMPLE & REPLICATE & \\
\hline ats: & DETECTOR NUMBER & 16 & 16 & \\
\hline SAMPLE & DISH SIZE $\quad(1,2$, or 5$)$ & 2 & 2 & \\
\hline & GROSS COUNTS & 15 & 4 & \\
\hline 14423 & COUNT TIME in MINUTES & 30 & 30 & \\
\hline 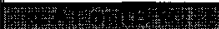 & BACKGROUND in cpm & 0.3 & 0.3 & \\
\hline AT & SAMPLE SIZE in mL & 0.500 & 0.500 & \\
\hline & DILUTION FACTOR & 101 & 101 & \\
\hline (QAB-01 & DIGEST DILUTION FACTOR & 50 & 50 & \\
\hline 37 & EFFICIENCY FACTOR & 0.2104 & 0.2104 & \\
\hline LIQUID & LC, Rmax, or Rs,(SAMPLE RATE) as APPROPRIATE & 0.469 & 0.233 & \\
\hline 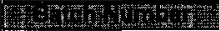 & & & & \\
\hline 96010977 & Blank Concentration in $\mu \mathrm{C} / \mathrm{L}$ & $1.02 \mathrm{E}+01$ & & \\
\hline Hol & Replicate Concentration in $\mu \mathrm{Ci} / L$ & $5.05 E+00$ & & \\
\hline 0 & Maximum Concentration in $\mu \mathrm{Ci} / \mathrm{L}$ & 1.0151E+01 & & \\
\hline & & & & \\
\hline ACIDIG02 & Rs (Sample Count Rate) $=(\mathrm{TC} / \mathrm{CT})-\mathrm{BKG}$ & & & \\
\hline 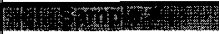 & ALPHA TOTAL $\mu \mathrm{Ci} / \mathrm{L}=\mathrm{Rs} * 1000 \mathrm{~mL} / \mathrm{L} * \mathrm{DF} * \mathrm{DDF} /(\mathrm{EF}$ & * SS * 222000 & $0 \mathrm{dpm} / \mu \mathrm{Ci})$ & \\
\hline S $96 \mathrm{~V} 000050$ & $=$ ALPHA TOTAL $\mu \mathrm{Ci} / \mathrm{L} / 1000 \mathrm{~mL}$ & & & \\
\hline mets & Relative Counting Error $=[\|($ The Square Root of $T C+B K G$ & $\mathrm{CT}) /(\mathrm{TC}-\mathrm{Bl}$ & $3 K G * C T) \mid] * 1.96$ & $\approx 100$ \\
\hline WB27806 & Detection Levels and Less Than Values are determined from & ocedure LA-50 & $8-002$ & \\
\hline VAR & & & & \\
\hline & & & & \\
\hline SLF & ALPHA TOTAL in $\mu \mathrm{Ci} / \mathrm{mL}$ & & $1.02 E-02$ & DETECTION \\
\hline 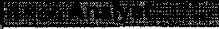 & & & & \\
\hline SMF & termined from Rmax. & & & Ply \\
\hline $11 / 02 / 96$ & RELATIVE COUNTING ERROR & & $500.0 \%$ & $\mu \mathrm{Ci} / \mathrm{mL}$ \\
\hline
\end{tabular}

$\frac{10 / 30 / 96}{15}$

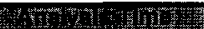

01:00 PM

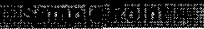

AP-105

\begin{tabular}{ll}
\hline Analyst: & Signature of Chemist: \\
\hline SAMPLE.WB1 Rev. 1. & $508101 \mathrm{ML}$
\end{tabular}


WORKBOOK PAGE: DUP9

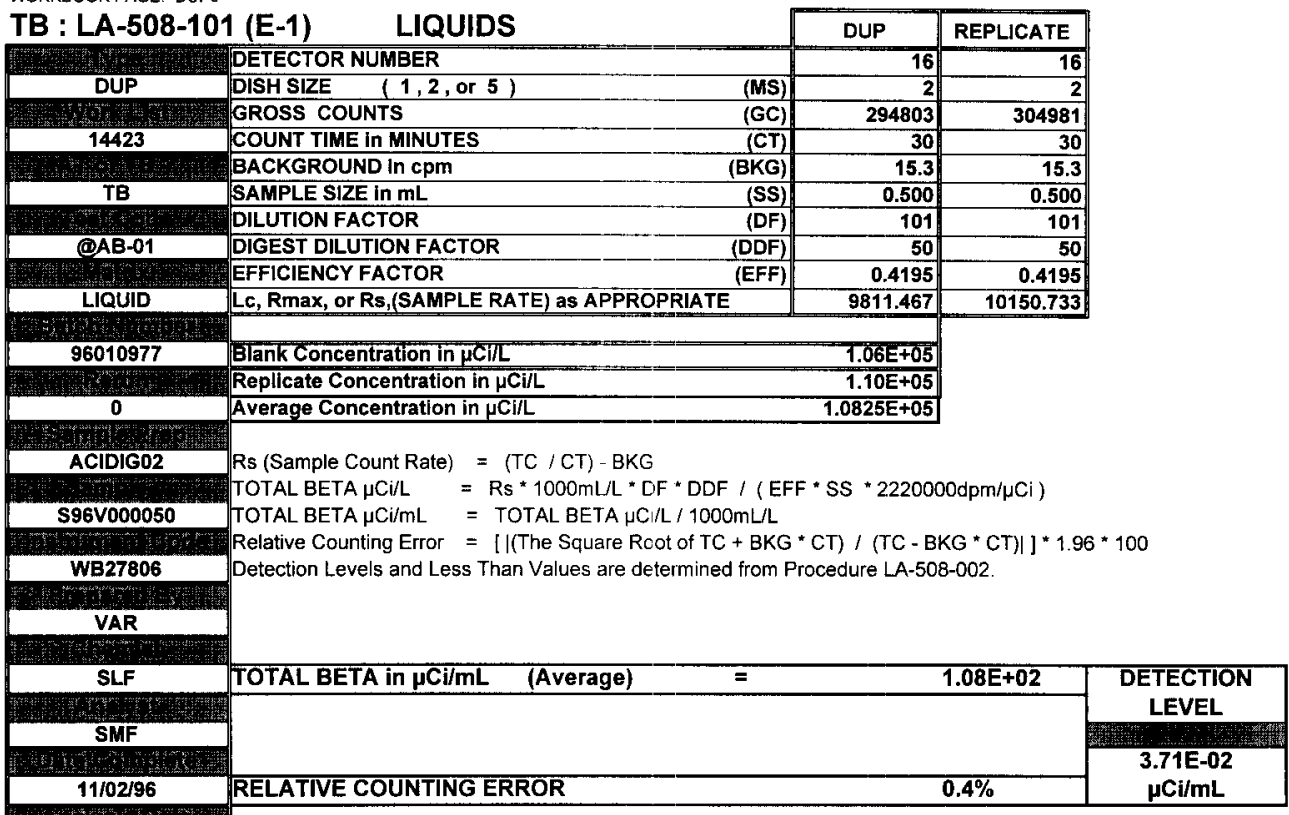

$10 / 30 / 96$

01:00 PM

AP-105

\begin{tabular}{l}
\hline Analyst: \\
\hline Signature of Chemist: \\
SAMPLE.WB1 Rev. 1.
\end{tabular}


WNE SD-WM-DF-202, REV. 1

WORKBOOK PAGE: DUP 10

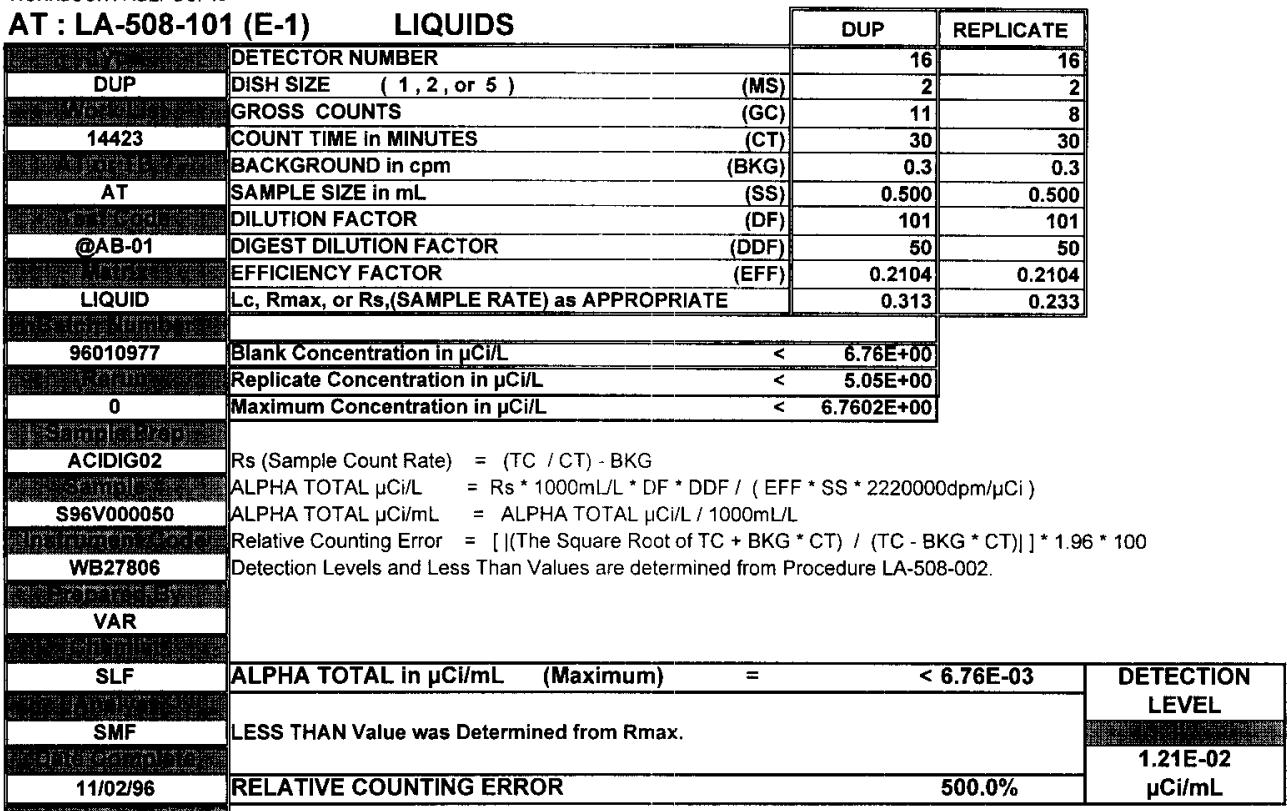

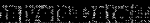

$10 / 30 / 96$

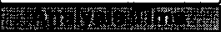

01:00 PM

$\frac{1}{\text { AP-105 }}$

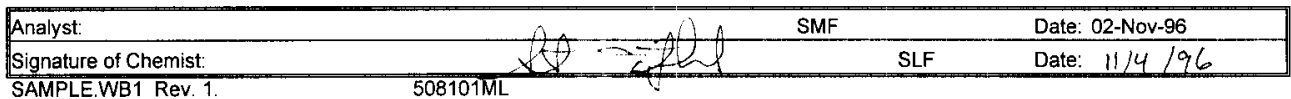


HNF

WORKBOOK PAGE: SAM11

\begin{tabular}{|c|c|c|c|c|}
\hline \multirow{2}{*}{\multicolumn{2}{|c|}{ LIC }} & & & \\
\hline & & SAMPLE & REPLICATE & \\
\hline & DETECTOR NUMBER & 16 & 16 & \\
\hline SAMPLE & $(1,2$, or 5$)$ & 2. & 2 & \\
\hline & GROSS COUNTS & 315357 & 305833 & \\
\hline 423 & COUNT TIME In MINUTES & 30 & 30 & \\
\hline & BACKGROUND in cpm & 15.3. & 15.3 & \\
\hline TB & SAMPLE SIZE in $\mathrm{mL}$ & 0.500 & 0.500 & \\
\hline & DILUTION FACTOR & 101 & 101 & \\
\hline @AB-01 & DIGEST DILUTIONFACTOR & 50 & 50 & \\
\hline & EFFICIENCY FACTOR & 0.4195 & 0.4195 & \\
\hline LIQUID & Le, Rmax, or Rs,(SAMPLE RATE) as APPROPRIATE & 10496.600 & 10179.133 & \\
\hline 96010977 & Blank Concentration in $\mu \mathrm{CilL}$ & $1.14 E+05$ & & \\
\hline 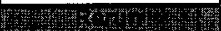 & Replicate Concentration in $\mu \mathrm{Ci} / \mathrm{L}$ & $1.10 E+05$ & & \\
\hline 0 & Average Concentration in $\mu \mathrm{Ci} / \mathrm{L}$ & $1.1212 \mathrm{E}+05$ & & \\
\hline & & & & \\
\hline ACIDIG02 & Rs (Sample Count Rate) $=(T C / C T)-B K G$ & & & \\
\hline & TOTAL BETA $\mu \mathrm{CI} / \mathrm{L}=\mathrm{RS} * 1000 \mathrm{~mL} / \mathrm{L}$ * DF * DDF / (EF) & $*$ SS * 222000 & Odpm/ $/ \mathrm{Ci}$ ) & \\
\hline S96V000051 & $=$ TOTAL BETA $\mu \mathrm{CI} / \mathrm{L} / 1000 \mathrm{~mL} \mathrm{~L}$ & & & \\
\hline 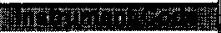 & Relative Counting Error $=[\mid(T$ The Square Root of TC + BKG & $\mathrm{CT}) /(\mathrm{TC}-\mathrm{Br}$ & $K G * C T) \mid] * 1.96$ & * 100 \\
\hline WB27806 & Detection Levels and Less Than Values are determined from $P$ & ocedure LA-508 & 8-002. & \\
\hline $\begin{array}{l}\text { VAR } \\
\text { VAR }\end{array}$ & & & & \\
\hline & & & & \\
\hline SLF & TOTAL BETA in $\mu \mathrm{Ci} / \mathrm{mL}$ & & $1.12 \mathrm{E}+02$ & DETECTION \\
\hline & & & & LEVEL \\
\hline SMF & & & & 7 \\
\hline 2/96 & RELATIVE COUNTING ERROR & & $0.4 \%$ & $\begin{array}{c}\text { 3.71E-02 } \\
\mu \mathrm{Ci} / \mathrm{mL}\end{array}$ \\
\hline
\end{tabular}

\begin{tabular}{|c|}
\hline $1 / 1,4.26$ \\
\hline $10 / 30 / 96$ \\
\hline $01: 00 \mathrm{PM}$ \\
\hline AP-105 \\
\hline
\end{tabular}

\begin{tabular}{|l} 
Analyst: \\
Signature of Chemist:
\end{tabular}


WORKBOOK PAGE: SAM12

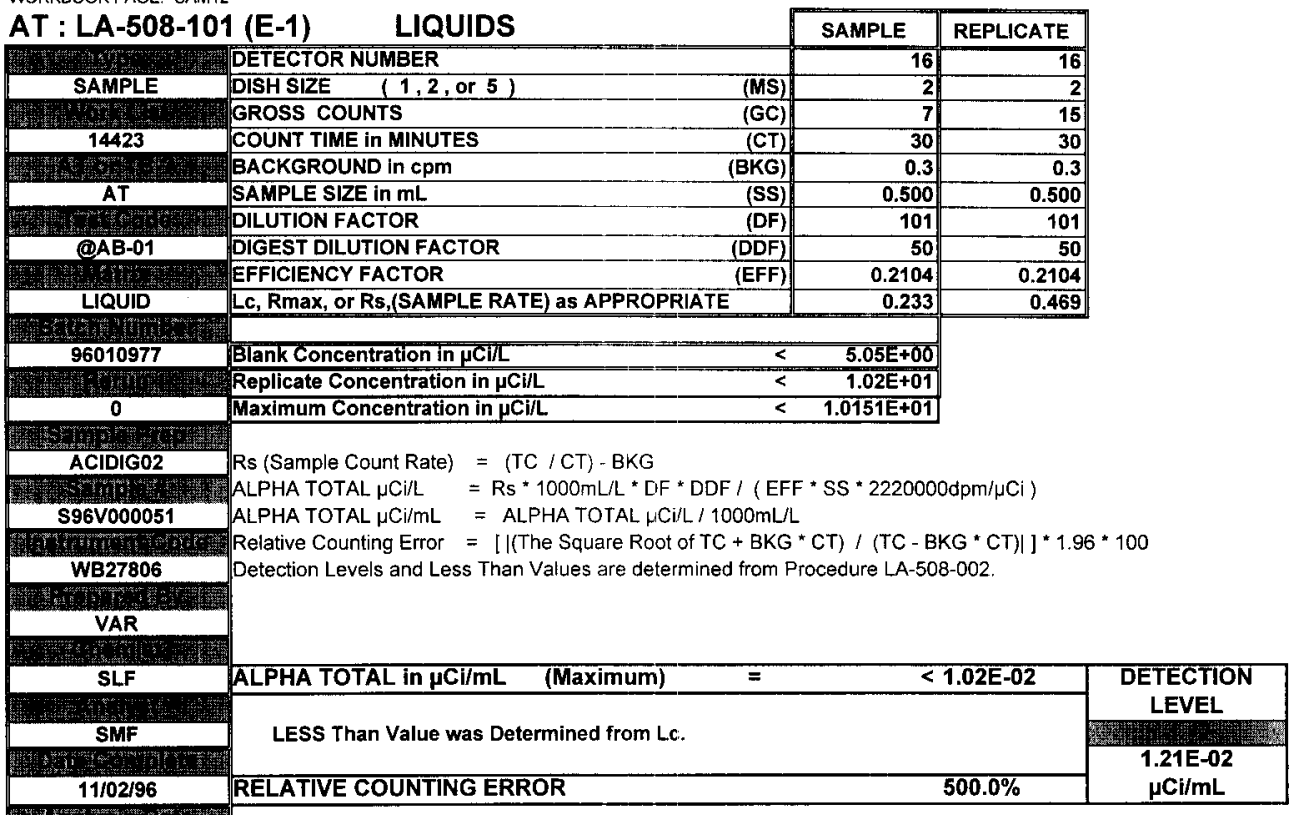

HNE SO-WM-DP-202, REV. 1

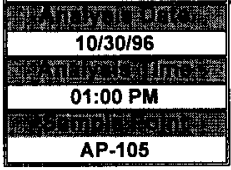

\begin{tabular}{l}
\hline Analyst: \\
\hline Signature of Chemist: \\
SAMPLE.WB1 Rev. 1.
\end{tabular}


WNFG-SD-WM-1)F-202, REV. 1

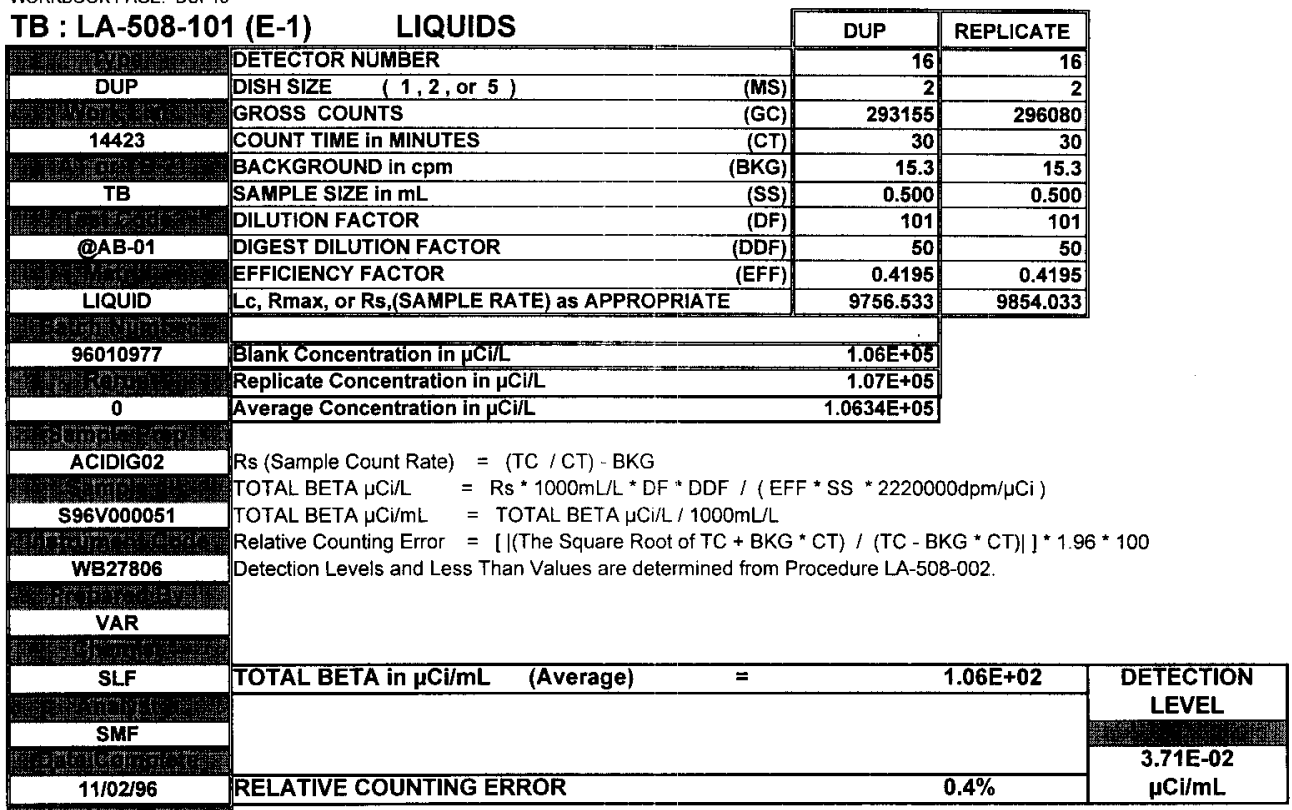

\begin{tabular}{|c|}
\hline 17 / \\
\hline $10 / 30 / 96$ \\
\hline 01:00 PM \\
\hline AP-105 \\
\hline
\end{tabular}

\begin{tabular}{|c|c|c|c|}
\hline Analyst: & & SMF & Date: 02-Nov-96 \\
\hline Signature of Chemist: & $6+2$ & SLF & Date: $11 / 4 / 96$ \\
\hline
\end{tabular}


WORKBOOK PAGE: DUP14

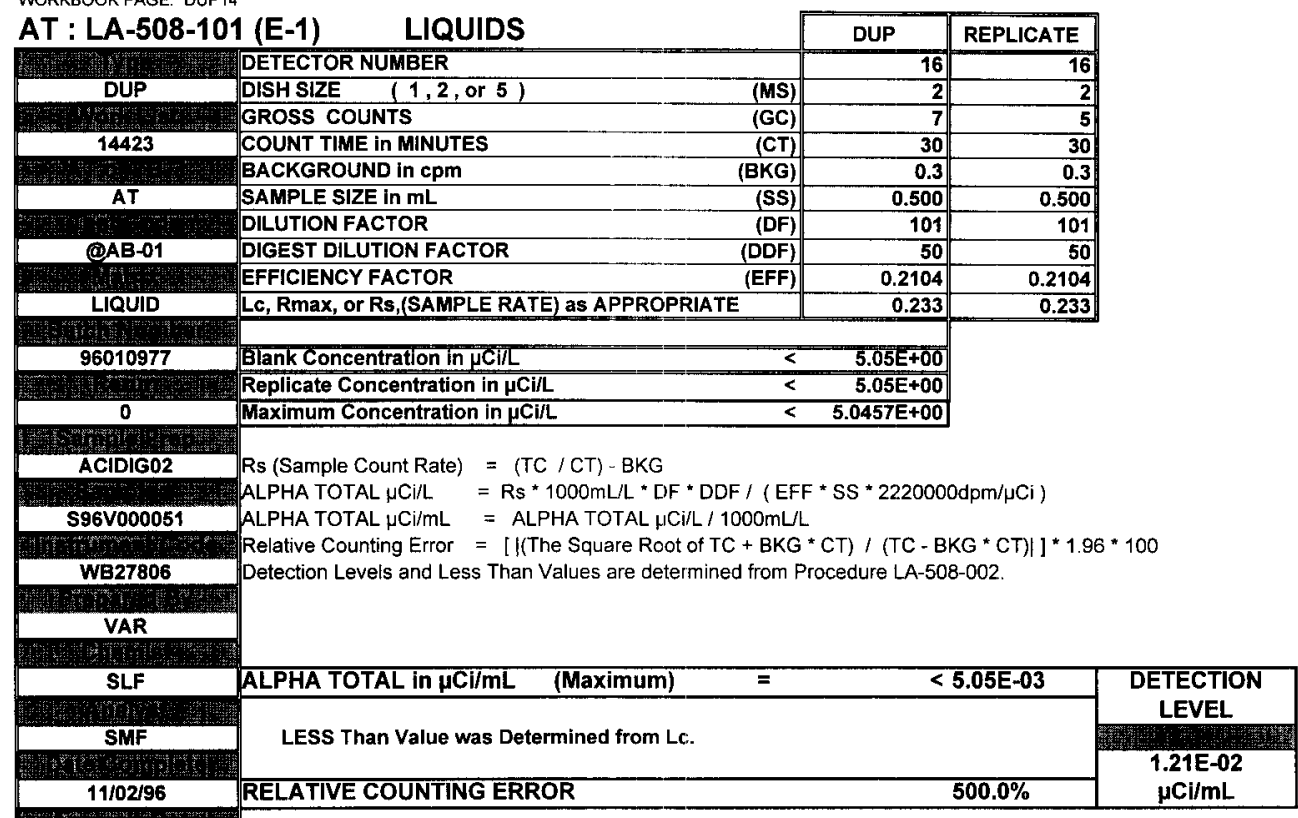

\begin{tabular}{|c|}
\hline $11 / 02 / 96$ \\
$10 / 30 / 96$ \\
\hline $01: 00$ PM \\
\hline AP-105 \\
\hline
\end{tabular}

Analyst:
Signature of Chemist:
SAMPLE.WB1 Rev. 1.


HNF STWM-SL-DP-202, REV.1

WORKBOOK PAGE: SAM15

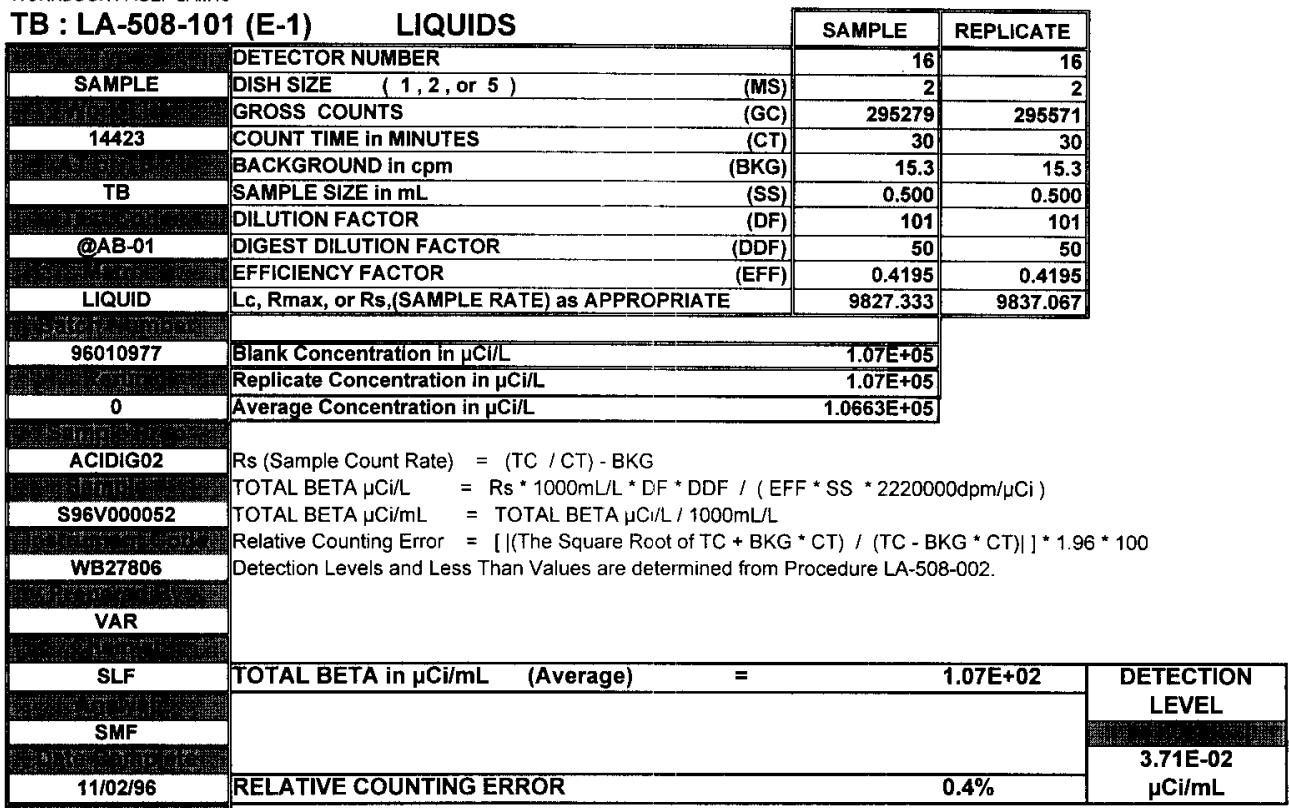

\begin{tabular}{|c|}
\hline 14 \\
\hline $10 / 30 / 96$ \\
$01: 00$ PM \\
\hline AP-105 \\
\hline
\end{tabular}

\begin{tabular}{|l}
\hline Analyst: \\
\hline Signature of Chemist: \\
\hline SAMPLE.WB1 Rev. 1.
\end{tabular}




\section{HFE-SD-WMA-IP-202, REV. 1}

WORKBOOK PAGE: SAM16

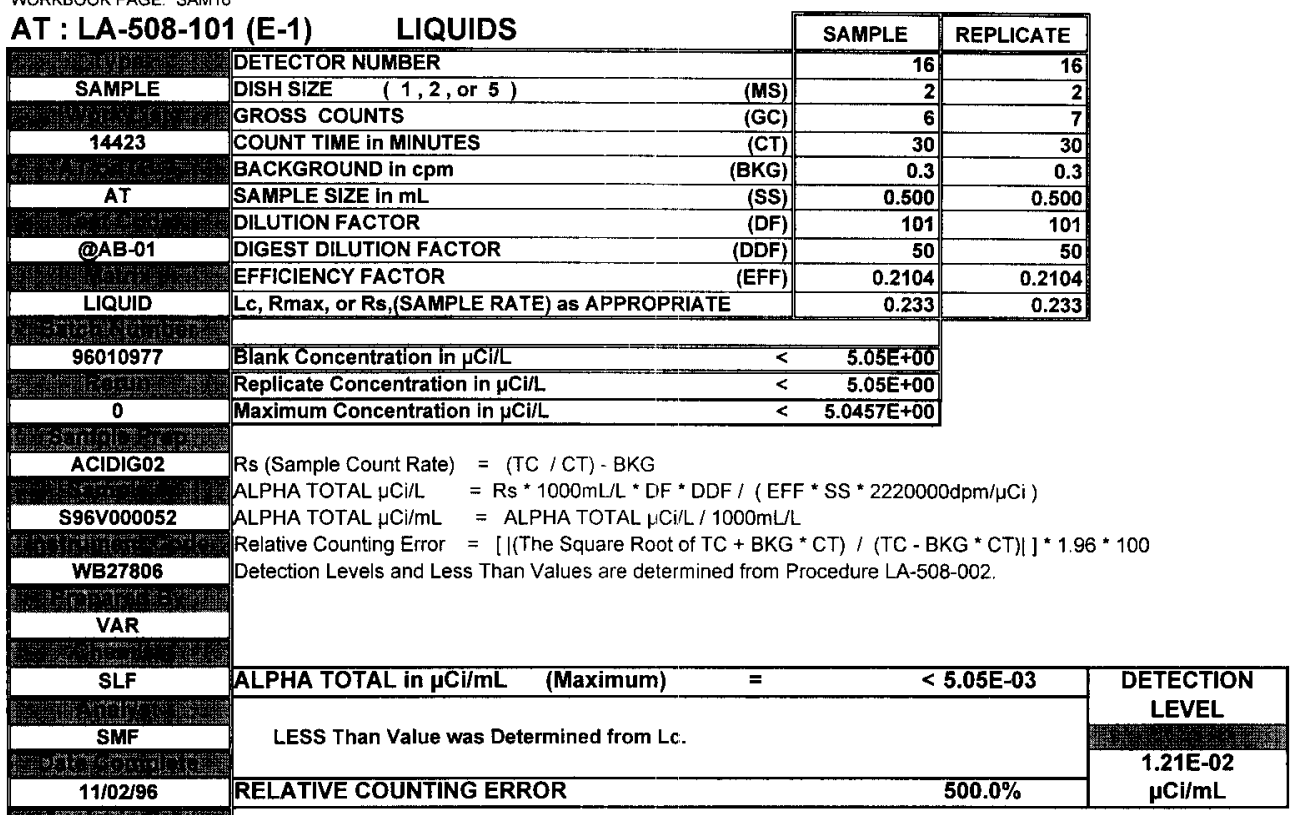

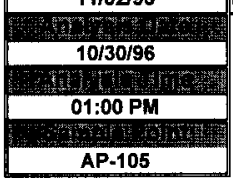

\begin{tabular}{|l|l|l|l|l|l|}
\hline Analyst: & SMF \\
\hline Signature of Chemist: & SLF \\
\hline SAMPLE.WB1 Rev. 1.
\end{tabular}


WORKBOOK PAGE: DUP17

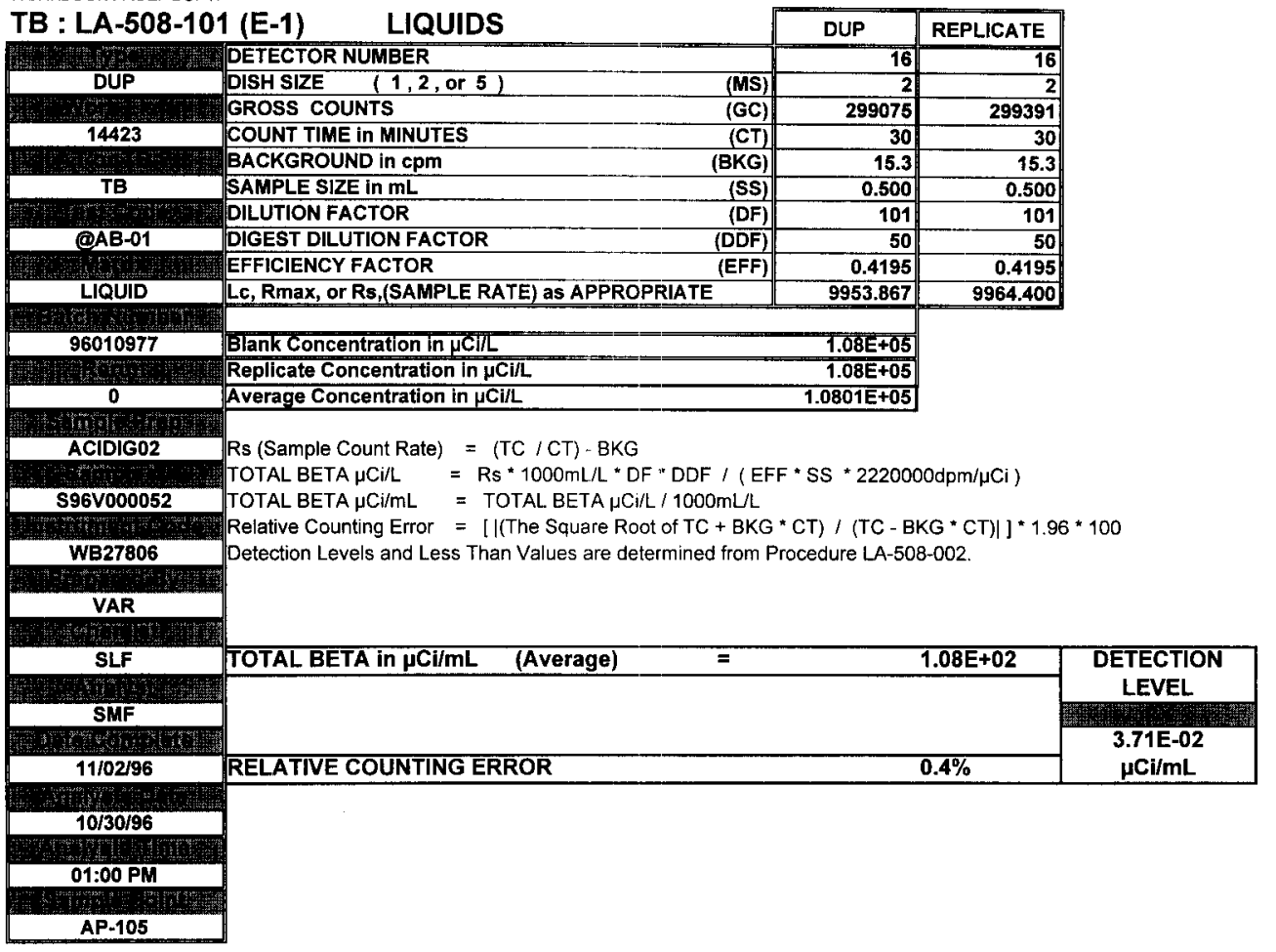

\begin{tabular}{|l|l|l|l|l|l|l|}
\hline Analyst: & Date: 02-Nov-96 \\
\hline Signature of Chemist: & $508101 \mathrm{ML}$ & SLF \\
\hline SAMPLE.WB1 Rev. 1.
\end{tabular}


WORKBOOK PAGE: DUP18

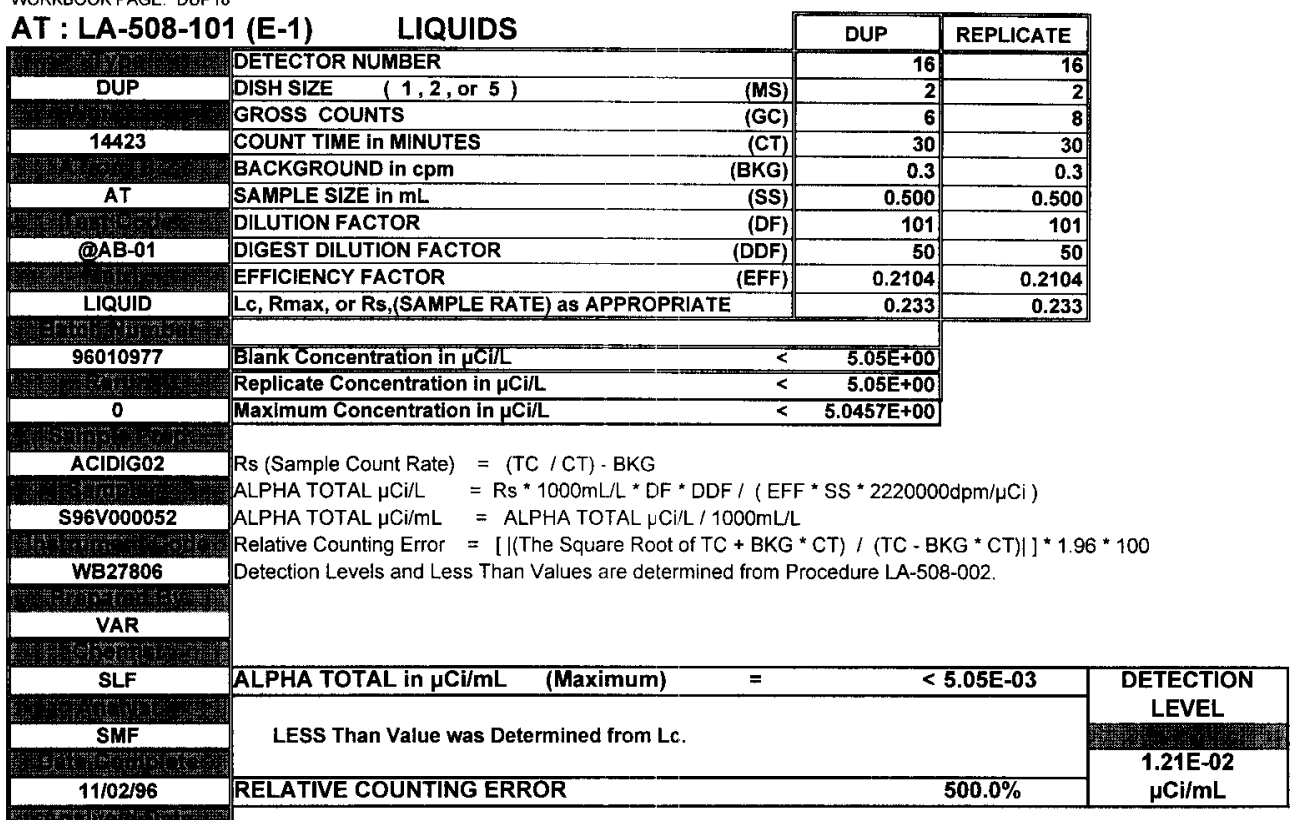

$10 / 30 / 96$

$3 \times 3 y^{2}$

01:00 PM

AP-105

\begin{tabular}{|c|c|c|}
\hline Analyst: & SMF & Date: 02-Nov-96 \\
\hline Signature of Chemist: & SLF & Date: $11 / 4 / 96$ \\
\hline
\end{tabular}




\section{$H N F$ \\ HHE-SD-WM-DF-202, REV. 1}

WORKBOOK PAGE: SAM19

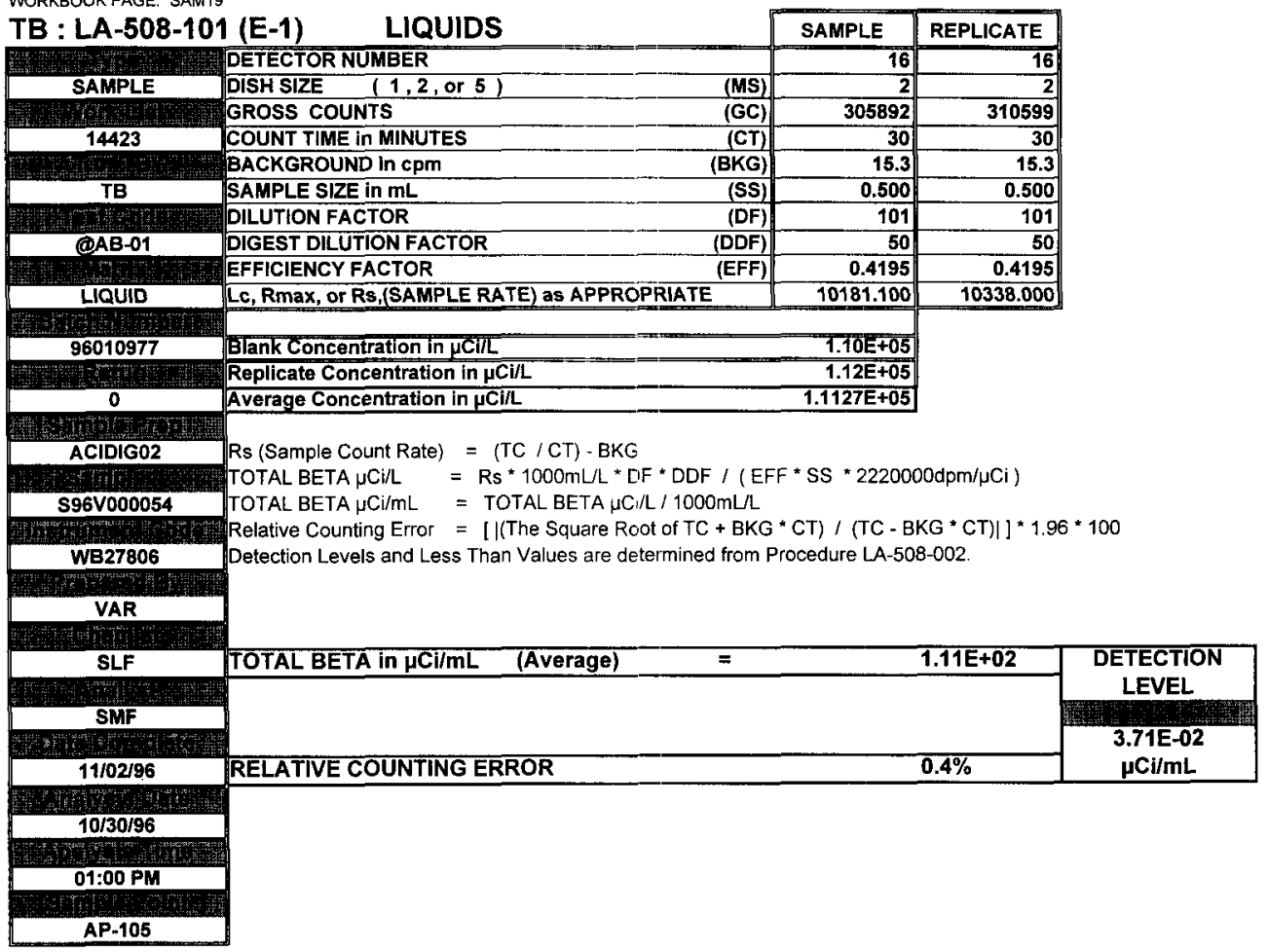

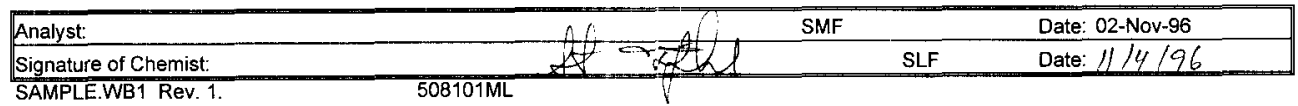


WF

WORKBOOK PAGE: SAM20

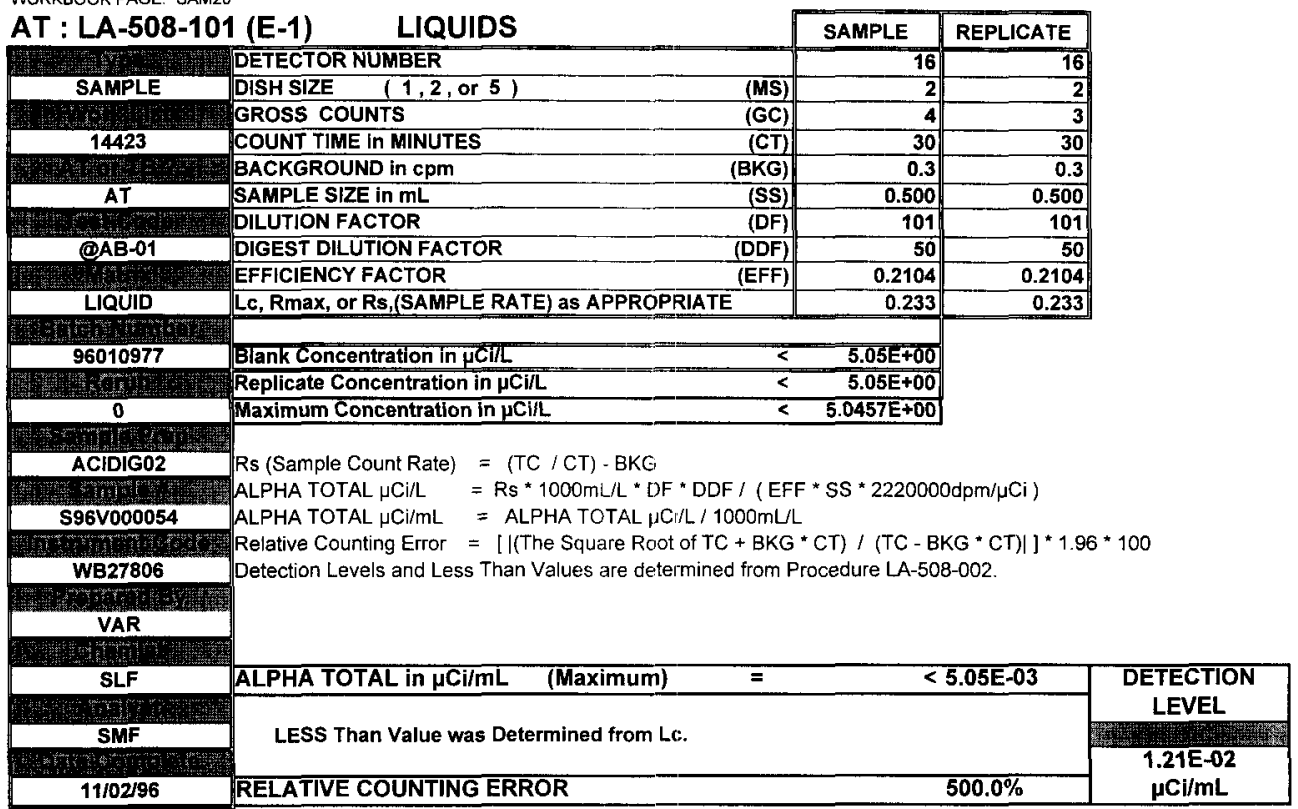

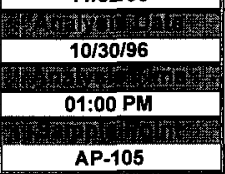

\begin{tabular}{|l|l|l|l|}
\hline Analyst: & Date: 02-Nov-96 \\
\hline Signature of Chemist: & $508101 \mathrm{ML}$ & SLF \\
\hline SAMPLE.WB1 Rev. 1. & Date: $1 / / 4 / 96$ \\
\hline
\end{tabular}


WORKBOOK PAGE: DUP2 1

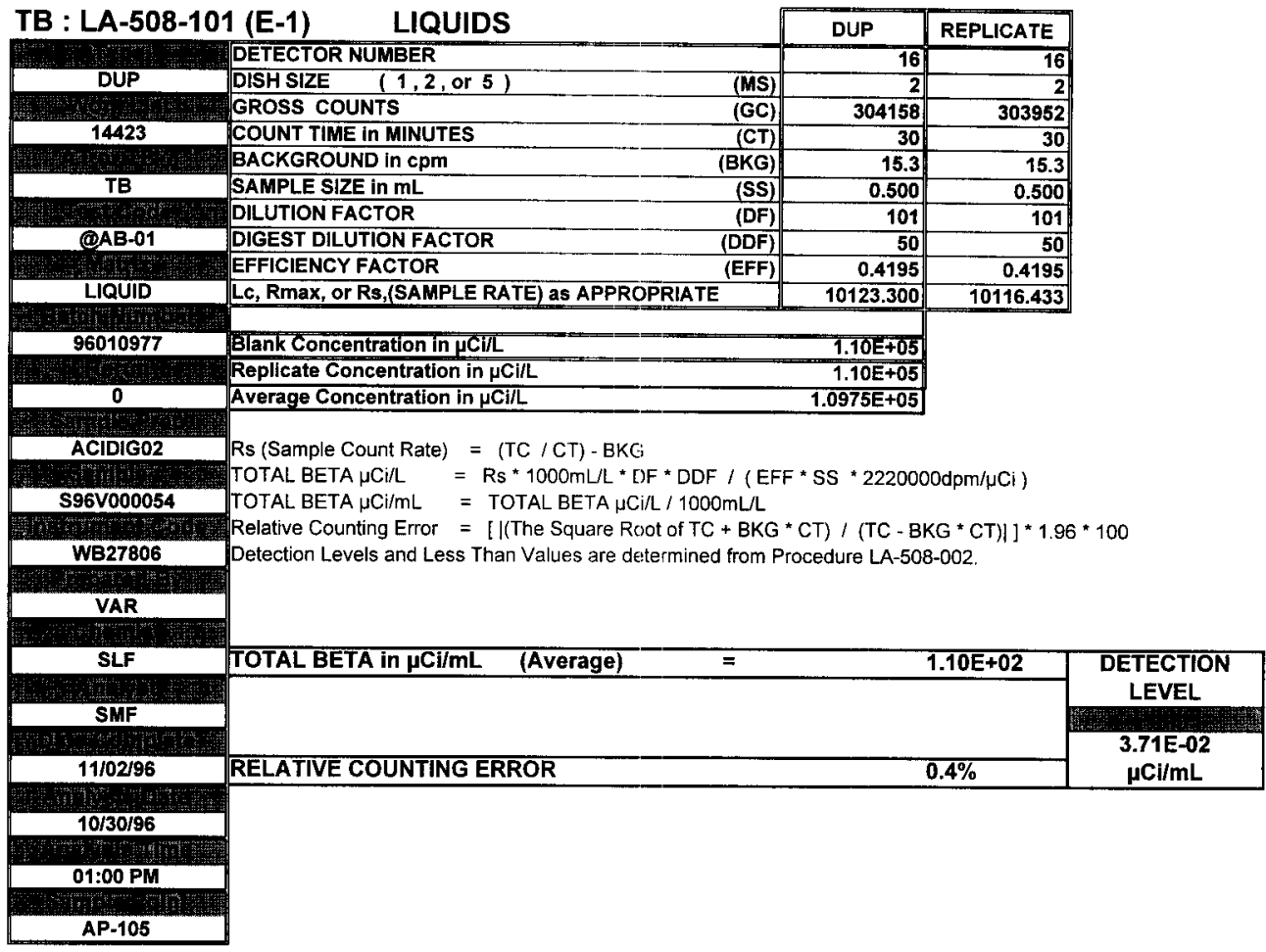

\begin{tabular}{|c|c|}
\hline Analyst: & Date: 02-Nov-96 \\
\hline Signature of Chemist: & Date: $/ 1 / 4 / 96$ \\
\hline
\end{tabular}


WORKBOOK PAGE: DUP22

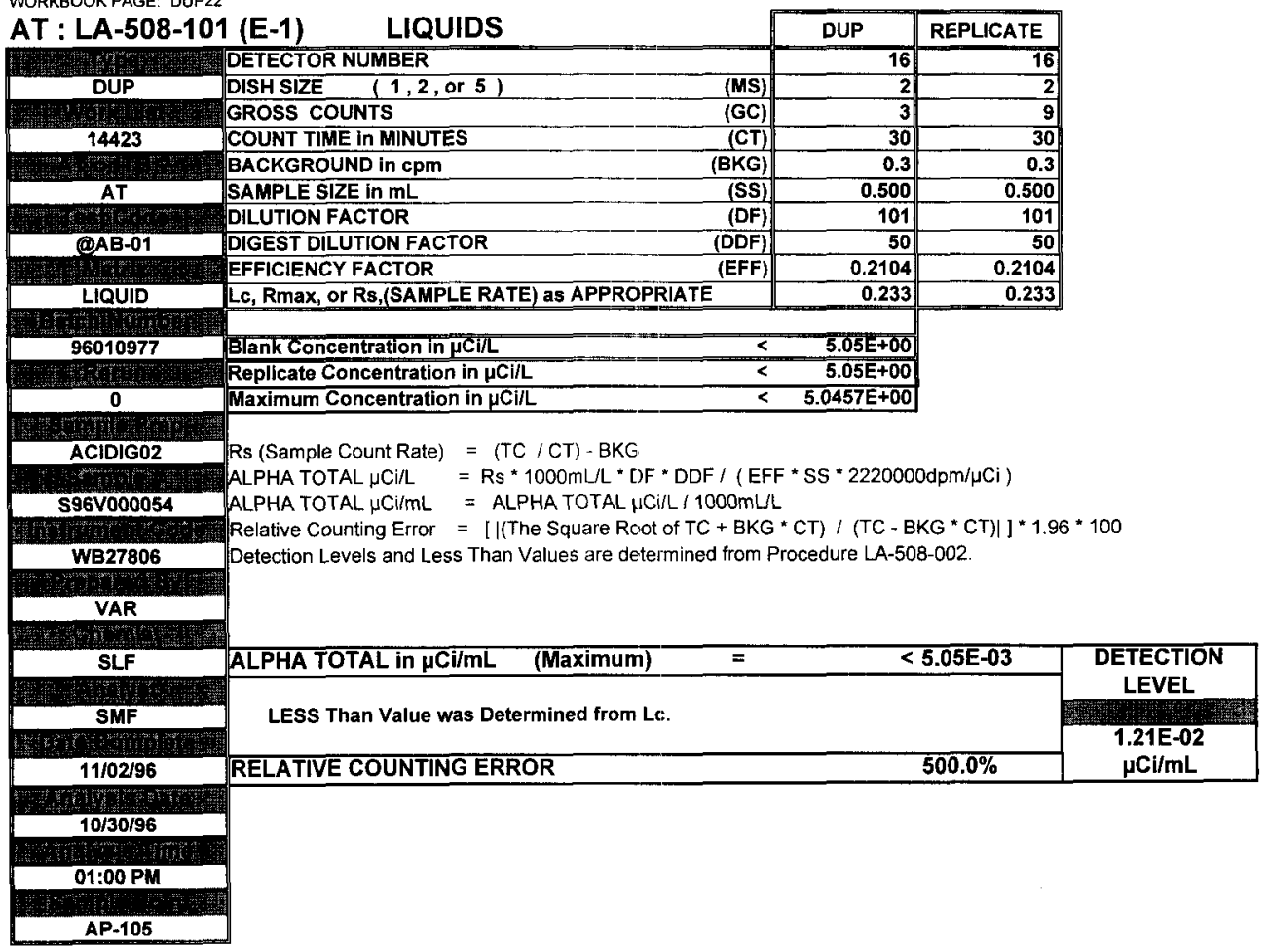

\begin{tabular}{l} 
Analyst: \\
\hline Signature of Chemist: \\
SAMPLEWWI Rev. 1.
\end{tabular}

\section{2}


WFIGSD-WM-DP-202, REV. 1

WORKBOOK PAGE: SAM23

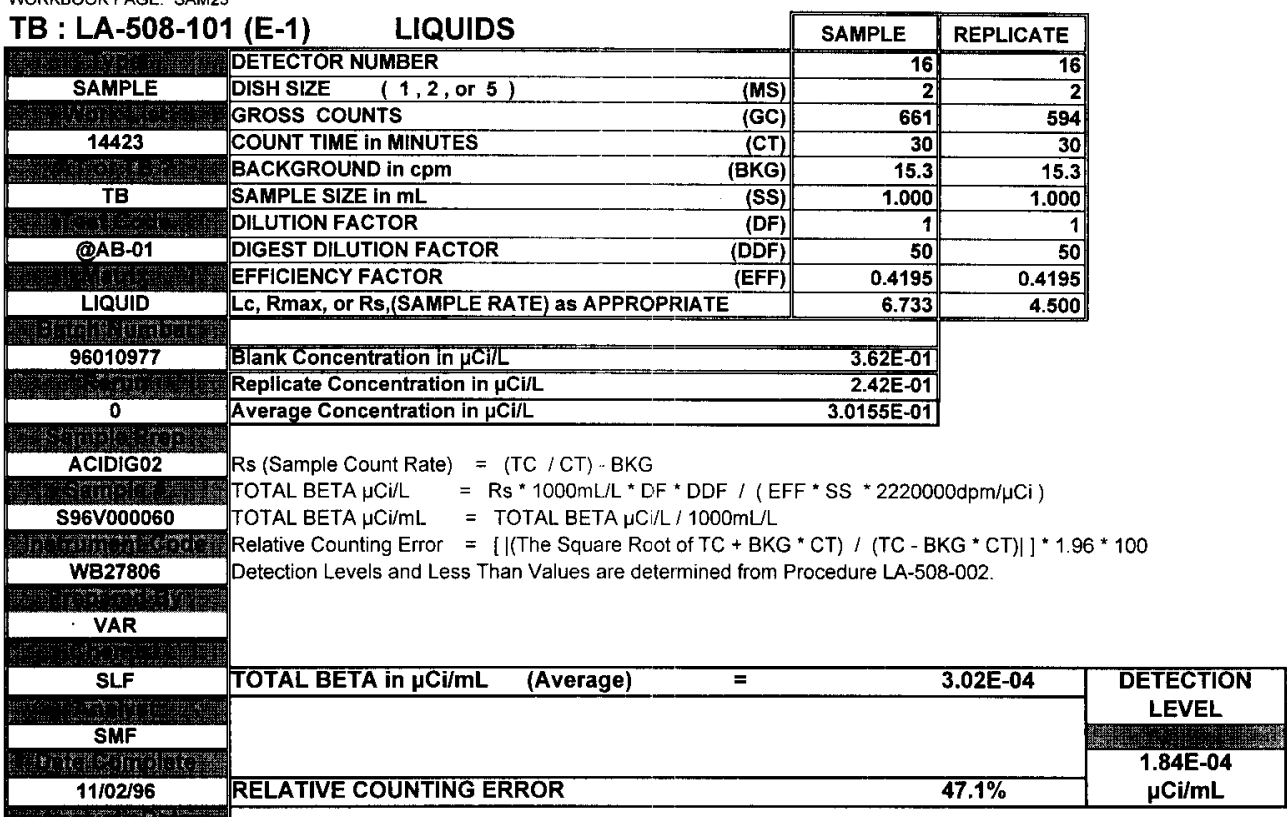

ग)

$10 / 30 / 96$

\%)

01:00 PM

H.

AP-105

\begin{tabular}{|llll|}
\hline Analyst: & SMF & Date: 02-Nov-96 & SLF \\
\hline Signature of Chemist: & $508101 \mathrm{ML}$ & Date: $/ 1 / 4 / 96$ \\
\hline SAMPLE.WB1 Rev. 1.
\end{tabular}


HNFE-SD-WM-DP-202, REV. 1

WORKBOOK PAGE: SAM24

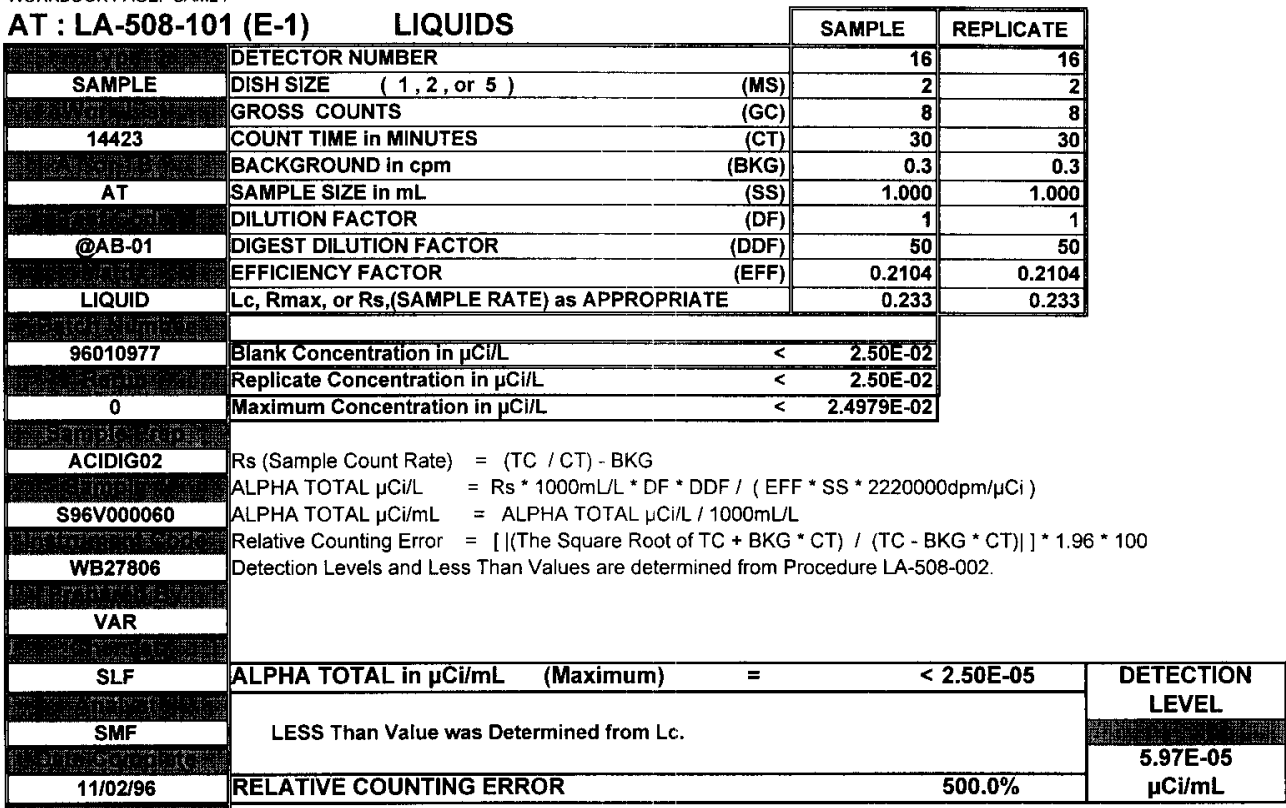

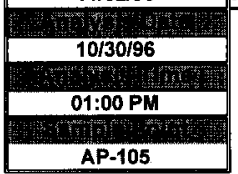

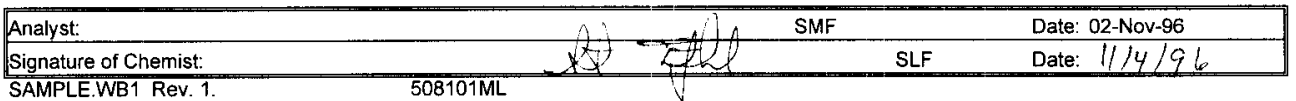


HNFG-SD-WM-DP-2C2, REV. \{

WORKBOOK PAGE: DUP25

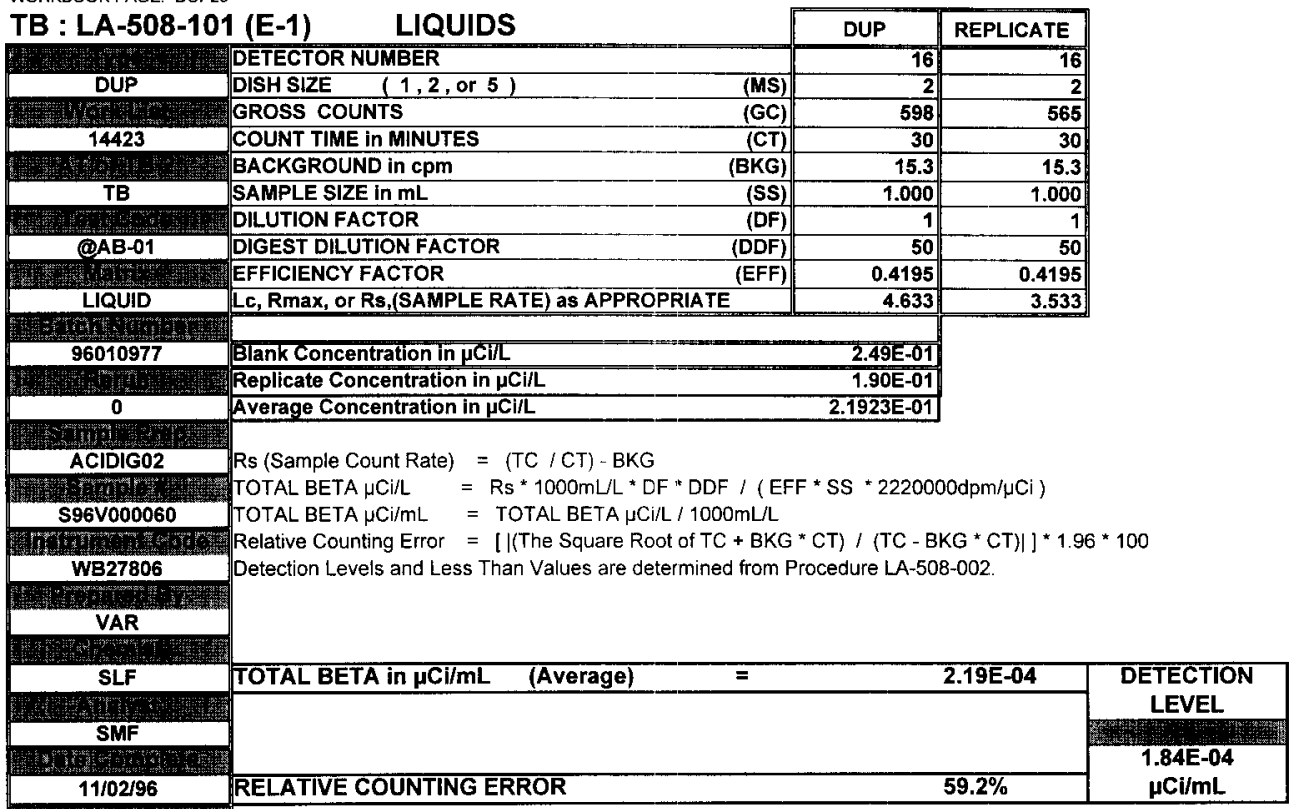

114

$10 / 30 / 96$

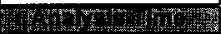

01:00 PM

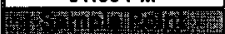

AP-105

\begin{tabular}{|c|c|c|}
\hline Analyst: & SMF & Date: 02-Nov-96 \\
\hline Signature of Chemist: & SLF & Date: ?1/4/96 \\
\hline
\end{tabular}

\section{5}




\section{HNFSO-WM-DP-202, REV.}

WORKBOOK PAGE: DUP26

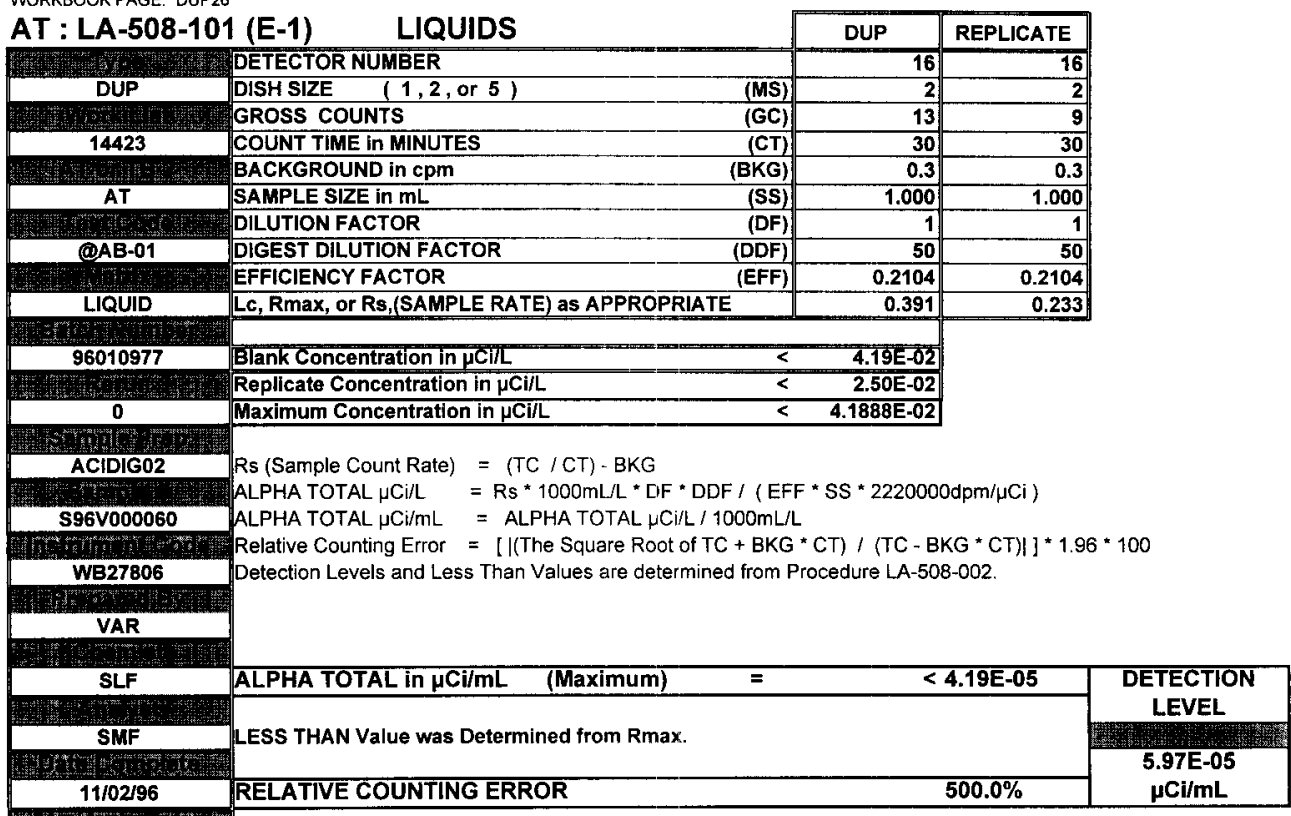

\begin{tabular}{|c|}
\hline $10 / 30 / 96$ \\
\hline $01: 00 ~ P M$ \\
\hline AP-105 \\
\hline
\end{tabular}

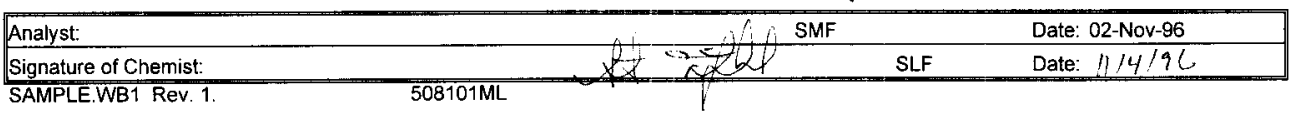




\section{HNF}

WORKBOOK PAGE: SPK27

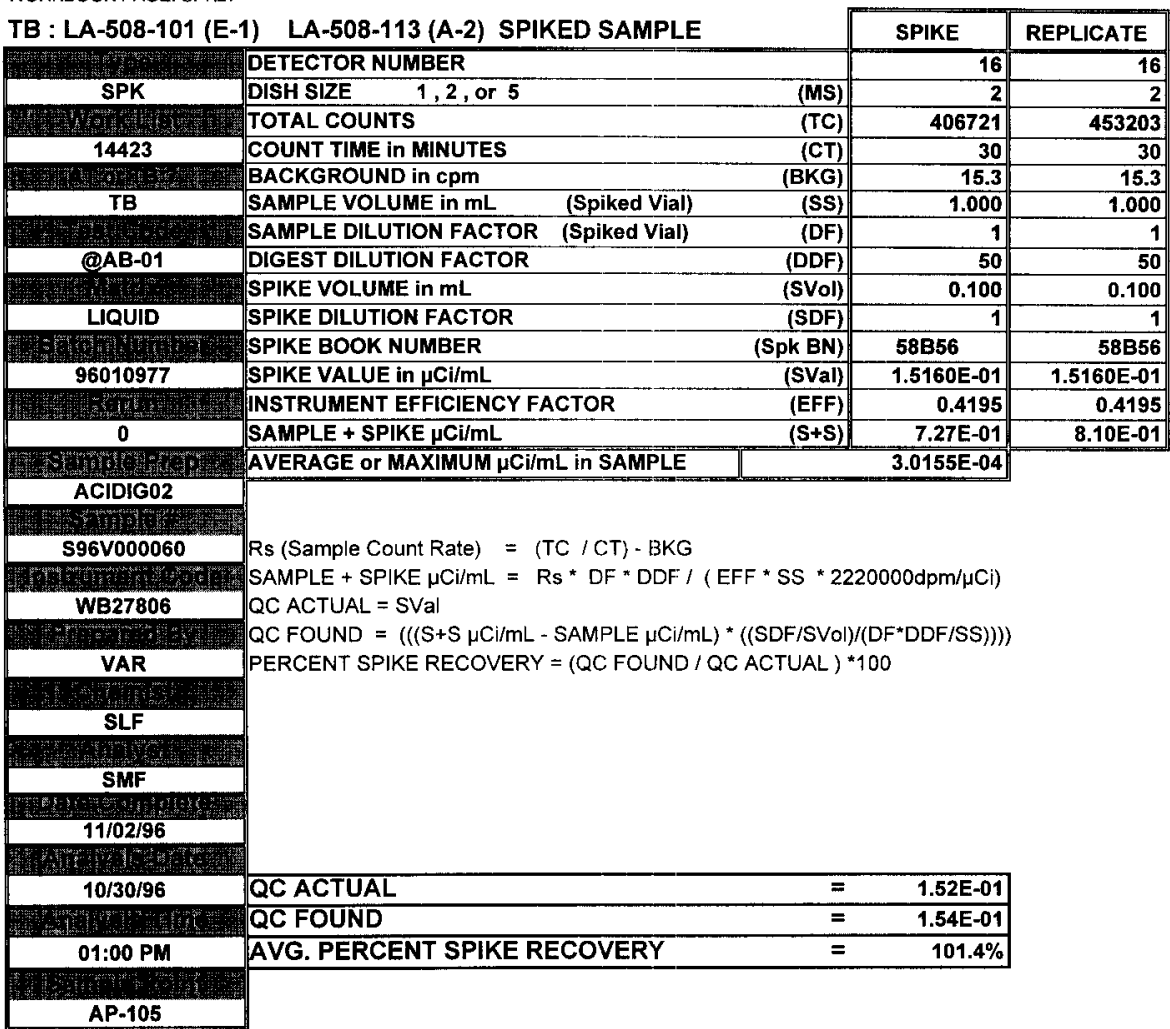

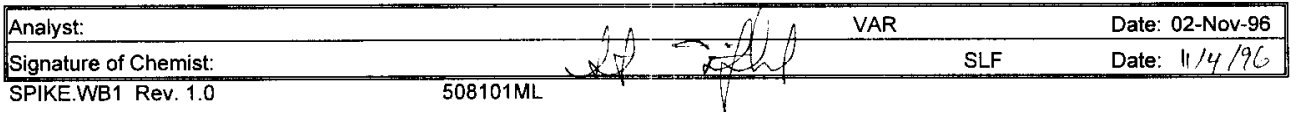


WORKBOOK PAGE: SPK28

$$
\text { WNFG-SD-WM-DP-202, REV. } 1
$$

\begin{tabular}{|c|c|c|c|}
\hline \multirow{2}{*}{\multicolumn{2}{|c|}{ AT : LA-508-101 (E-1) LA-508-113 (A-2) SPIKED SAMPLE }} & \\
\hline & & SPIKE & REPLICATE \\
\hline & DETECTOR NUMBER & 16 . & 16 \\
\hline SPK & DISH SIZE & 2 & 2 \\
\hline 18 & TOTAL COUNTS & 45394 & 42775 \\
\hline 14423 & COUNT TIME in MINUTES & 30 & 30 \\
\hline & BACKGROUND in cpm & 0.3 & 0.3 \\
\hline AT & SAMPLE VOLUME in $\mathrm{mL}$ & 1.000 & 1.000 \\
\hline & SAMPLE DILUTION FACTOR (Spiked Vial) & 1. & \\
\hline @ & DIGEST DILUTION FACTOR & 50 & 50 \\
\hline & SPIKE VOLUME in mL & 0.100 & 0.100 \\
\hline LIQUID & SPIKE DILUTION FACTOR & 1 & \\
\hline was & SPIKE BOOK NUMBER & $123 \mathrm{~B} 43$ & 123B43 \\
\hline 96010977 & SPIKE VALUE in $\mu \mathrm{Ci} / \mathrm{mL}$ & $3.5865 \mathrm{E}-02$ & $3.5865 \mathrm{E}-02$ \\
\hline & INSTRUMENT EFFICIENCY FACTOR & 0.2104 & 0.2104 \\
\hline o & SAMPLE + SPIKE $\mu \mathrm{Ci} / \mathrm{mL}$ & 1.62E-01 & $1.53 \mathrm{E}-01$ \\
\hline & AVERAGE or MAXIMUM $\mu \mathrm{Ci} / \mathrm{mL}$ in SAMPLE & 2.4979E-05 & \\
\hline ACIDIG02 & & & \\
\hline S96V000060 & Rs (Sample Count Rate) $=(\mathrm{TC} / \mathrm{CT})-\mathrm{BKG}$ & & \\
\hline Wn bus? & SAMPLE + SPIKE $\mu \mathrm{Ci} / \mathrm{mL}=\mathrm{Rs}^{*} \mathrm{DF}^{*} \mathrm{DDF} /(\mathrm{EFF} * \mathrm{SS} * 22$ & $000 \mathrm{dpm} / \mu \mathrm{Ci})$ & \\
\hline WB27806 & QC ACTUAL = SVal & & \\
\hline hemp: & QC FOUND $=\left(\left((S+S \mu \mathrm{Ci} / \mathrm{mL}-\mathrm{SAMPLE} \mu \mathrm{Ci} / \mathrm{mL}){ }^{*}((\mathrm{SD}\right.\right.$ & S))) $)$ & \\
\hline 8 & PERCENT SPIKE RECOVERY = (QC FOUND / QC ACTUAL $)$ & & \\
\hline
\end{tabular}

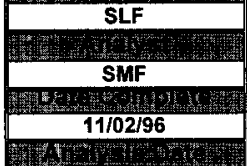

NOTE: Original Sample result was a LESS THAN value. Zero (0) was subtracted from the spiked value for QC found calculation.

\begin{tabular}{|c|c|c|c|}
\hline $10 / 30 / 96$ & QC ACTUAL. & $=$ & 3.59E-02 \\
\hline (7) 701091 & QC FOUND & $=$ & 3.15E-02 \\
\hline 01:00 PM & AVG. PERCENT SPIKE RECOVERY & $=$ & $87.7 \%$ \\
\hline
\end{tabular}

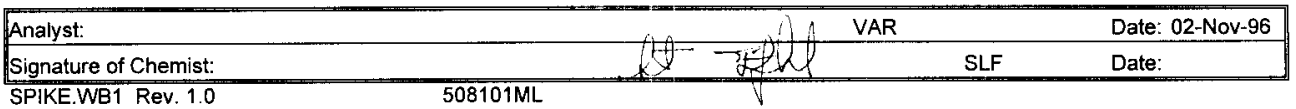




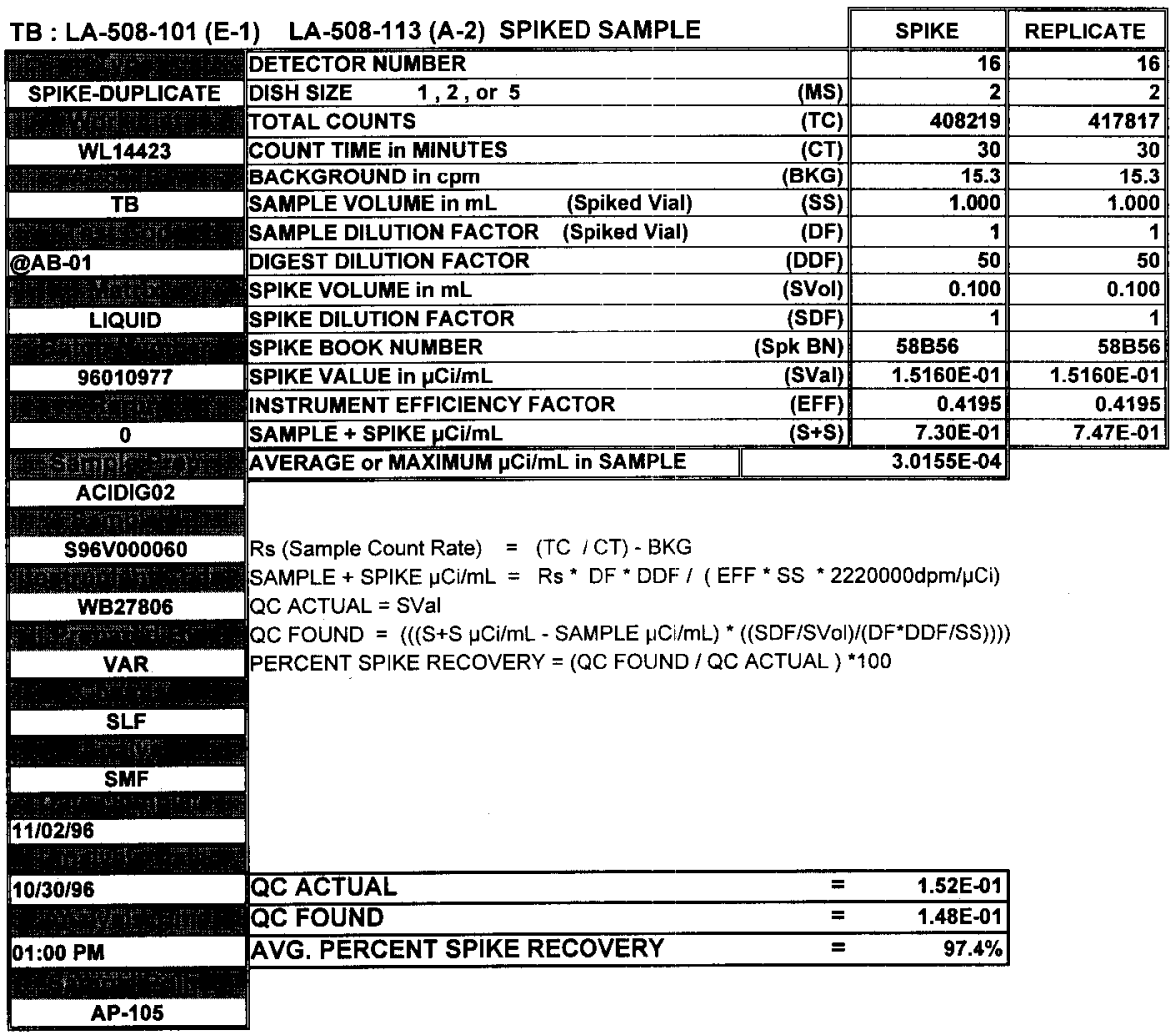

\begin{tabular}{|l|l|l|l|}
\hline Analyst: & Date: 02-Nov-96 \\
\hline Signature of Chemist: & $508101 \mathrm{ML}$ & SLF & Date: $11 / 4 / 91$ \\
\hline SPIKE.WB1 Rev. 1.0 & &
\end{tabular}




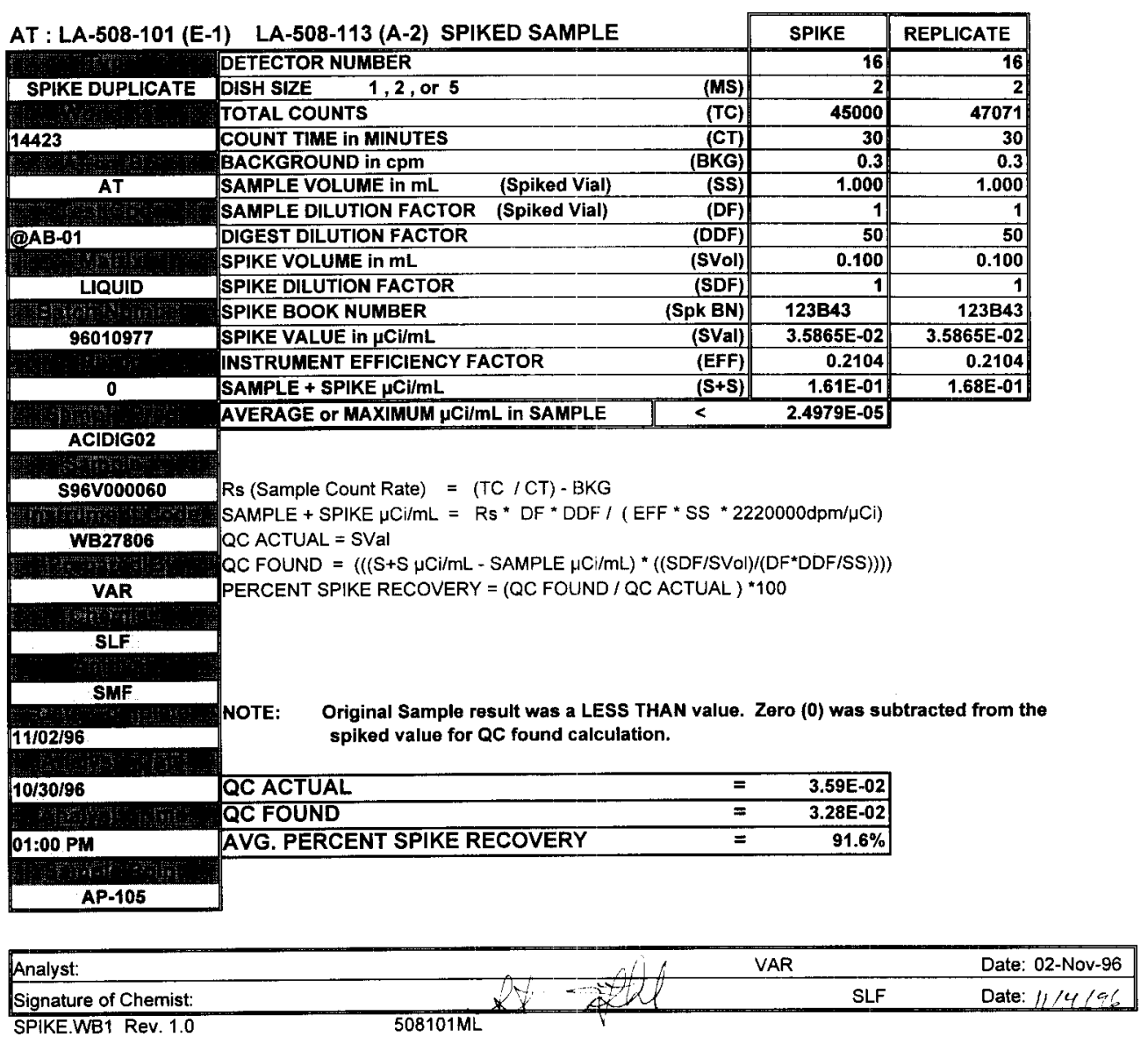




\section{LABCORE Completed RadChem Report for Worklist\#: 14453}

Analyst: scl

Method:

Worklist Comment: AP-105 GEA RCJ
Book\#

\begin{tabular}{|c|c|c|c|c|c|c|c|c|c|}
\hline$\overline{\text { Seq Type }}$ & Samplef & $\mathbf{R}$ & & Test & Matrix & Actual & Found & DL or Yield & Unit \\
\hline STD & & 0 & DGEA-03 & $\mathrm{CO60-02}$ & LIOUID & $7,882 \mathrm{e}-03$ & $7.820-03$ & 99,213 & HRecovery \\
\hline 1 SID & & 0 & QGEA- 03 & CO60-02E & LIQUID & 1 & 2.56 & 2.560 & * ct Error \\
\hline 1 STD & & 0 & 0 GEA 03 & $\operatorname{cs} 13702$ & 10010 & $7705 e-03$ & $7.420 \cdot 03$ & 06.301 & \% Recovery \\
\hline 1 STD & & 0 & QGEA-03 & CS13702E & LIQUID & 1 & 3.48 & 3.480 & * ct Error \\
\hline 2 BLMK -PREP & & 0 & OGEA 03 & $0060-02$ & Houlo & 1 & $1,14=4$ & & $4 \mathrm{Cl} / \mathrm{m}$ \\
\hline 2 BLNK-PREP & & 0 & DGEA-03 & $\mathrm{NB} 94-02$ & LIOUID & 1 & $<1.03 e-4$ & & $\mathrm{uCi} / \mathrm{mL}$ \\
\hline 2 BLNK -PREP & & 0 & QGEA- 03 & Ru10602 & tosio & 1 & $<2.17 \mathrm{e}-3$ & & uc $1 \mathrm{ml}$ \\
\hline 2 BLNK-PREP & & 0 & AGEA-03 & $\operatorname{cs} 13402$ & LIQUID & 1 & $<1.01 \mathrm{e}-4$ & & $\mathrm{uCi} / \mathrm{mL}$ \\
\hline 2 BL NK $P R E P$ & & 0 & AGEA 03 & $0 s 13702$ & $1 / 410$ & 1 & $2 ., 81 \mathrm{e} /$ & & uclom \\
\hline 2 BLNK-PREP & & 0 & DGEA-03 & CE 14402 & LIQUID & 1 & $<1.22 \mathrm{e}-3$ & & $\mathrm{LCi} / \mathrm{mL}$ \\
\hline 2 BLMK - PREP & & 0 & Q $\mathrm{GEA}-03$ & EU15402 & Hojo & 1 & $<3.45 \mathrm{e}-4$ & & $\mathrm{ucl} / \mathrm{ml}$ \\
\hline 2 BLNK - PREP & & 0 & DGEA-03 & EU15502 & LIOUID & 1. & $<3.02 e-4$ & & $\mathrm{uCi} / \mathrm{mL}$ \\
\hline 2 BLNK - PREP & & 0 & बGEA 03 & RA22602 & 10U10 & 1 & $2.08 \mathrm{e}-3$ & & $\mathrm{uct} / \mathrm{ml}$ \\
\hline 3 SAMPLE & s96V000050 & 0 B & DGEA-03 & C060-02 & LIOUID & $N / A$ & $6.071 e-03$ & $607.1 e-005$ & $\mathrm{uCi} / \mathrm{mL}$ \\
\hline 3 SAMPLE & $596 \mathrm{v000050}$ & $0 \%$ & OGEA- 03 & C060 02E & Louto & $N / A$ & no & $0,00+000$ & \% $\mathrm{ct}, \mathrm{error}$ \\
\hline 3 SAMPLE & s96v000050 & $\mathrm{OB}$ & DGEA-03 & $\mathrm{NB} 94-02$ & LIRUID & $\mathrm{N} / \mathrm{A}$ & $1.338 \mathrm{e}-02$ & $133.8 \mathrm{e}-004$ & UCi/mL \\
\hline 3 SAMPLE & s96 v000050 & $0 \mathrm{~B}$ & OGEA-03 & $6894-025$ & Lot 10 & $N / A$ & $n / a$ & $0,0 e+000$ & * ct, Error \\
\hline 3 SAMPLE & s96v000050 & O B & DGEA-03 & RU10602 & LIQUID & $N / A$ & $1.100 \mathrm{e}+00$ & $110,0 e-002$ & $\mathrm{uCi} / \mathrm{mL}$ \\
\hline 3 SAMPLE & s96v000050 & $0 \mathrm{~B}$ & DGEA-O3 & RU10602E & L 10010 & $N / A$ & $n / a$ & $0,0 e+000$ & \% ct, error \\
\hline 3 SAMPLE & $596 \vee 000050$ & 0 B & DGEA-03 & $\operatorname{cs} 13402$ & LIQUID & $N / A$ & $5.501 \mathrm{e}-02$ & $550.1 e-004$ & $\mathrm{uCi} / \mathrm{mL}$ \\
\hline 3 SAMPLE & $596 \vee 000050$ & 0 B & $\triangle G E A-03$ & CS13402E & LOUID & $\mathrm{K} / \mathrm{A}$ & $n / a$ & $0,0 e+000$ & $\% \mathrm{Ct}$. Error \\
\hline 3 SAMPLE & $596 v 000050$ & $O B$ & DGEA-03 & CS 13702 & LIQUID & N/A & $1.105 e+02$ & $0.0 \mathrm{e}+000$ & $\mathrm{UCi} / \mathrm{mL}$ \\
\hline 3 SAMPLE & 596 v000050 & 0 B & OGEA 03 & CS $13702 E$ & IOUID & $\mathrm{N} / \mathrm{A}$ & 0.330 & $0,0 \mathrm{e}+000$ & $\% \mathrm{ct}$ Error \\
\hline 3 SAMPLE & s96v000050 & $O B$ & DGEA-03 & CE14402 & LIQUID & N/A & $7.198 e-01$ & $719.8 \mathrm{e}-003$ & $\mathrm{uCi} / \mathrm{mL}$ \\
\hline 3 SAMPLE & 596 vo00050 & $0 \mathrm{~B}$ & DGEA 03 & CE $14402 \mathrm{E}$ & touro & $N / A$ & $\mathrm{n} / \mathrm{a}$ & $0.0 e+000$ & $\% \mathrm{Ct}$ Error \\
\hline 3 SAMPLE & $596 v 000050$ & O B & DGEA-03 & EU15402 & LIQUID & $\mathrm{N} / \mathrm{A}$ & $2.356 \mathrm{e}-02$ & $235.6 e-004$ & $\mathrm{uCi} / \mathrm{mL}$ \\
\hline 3 SAMPLE & $596 \times 000050$ & $0 B$ & OGEA 05 & EU15402E & 110110 & $N / A$ & $\mathrm{n} / \mathrm{s}$ & $0.0 e+000$ & $\%$ ct, Error \\
\hline 3 SAMPLE & s96V000050 & $0 \mathrm{~B}$ & aGEA-03 & EU15502 & LIQUID & $N / A$ & $2.024 e-01$ & $202.4 e-003$ & $\mathrm{uCi} / \mathrm{mL}$ \\
\hline 3 SAMPLE & $596 \times 000050$ & 08 & OGEA 03 & EU15502E & LIOUID & $N / A$ & $n / a$ & $0.0 e+000$ & X ct Error \\
\hline 3 SAMPLE & s96V000050 & $0 \mathrm{~B}$ & AGEA-03 & RA22602 & LIQUID & N/A $<$ & $1.427 \mathrm{e}+00$ & $142.7 \mathrm{e}-002$ & $\mathrm{UCi} / \mathrm{mL}$ \\
\hline 3 SAMPLE & s964000050 & 08 & QGEA 03 & $\mathrm{RA} 22602 \mathrm{E}$ & LIQULD & $\mathrm{N} / \mathrm{A}$ & ro & $0.0 \mathrm{e}+000$ & X CF Error \\
\hline 4 DUP & s96V000050 & $0 \mathrm{~B}$ & DGEA-03 & $0060-02$ & LIOUID & $<6.07 e-3$ & $<7.31 \mathrm{e}-3$ & & RPD \\
\hline 4 DUP & 8060000050 & $0 \mathrm{~B}$ & OGEA-03 & $606002 E$ & LOUTD & 1 & $\mathrm{n} / \mathrm{a}$ & & X ct Error \\
\hline 4 DUP & $\$ 96 \vee 000050$ & $0 \mathrm{~B}$ & DGEA-03 & NB94-02 & LIQUID & $<1.34 \mathrm{e}-2$ & $<1.34 \mathrm{e}-2$ & & RPD \\
\hline 4 DUP: & 8960000050 & 0 & QGEA-03 & $\mathrm{NB} 94-\mathrm{O} 2 \mathrm{E}$ & LOUID & 1 & no & & Y Ct Error \\
\hline 4 DUP & $S 96 \vee 000050$ & 08 & DGEA-03 & RU10602 & LIQUID & $<1.1000$ & $<1.11 e 0$ & & RPD \\
\hline 4 DUP & $\$ 960000050$ & 0 & OGEA 03 & $\mathrm{BU} 10602 \mathrm{E}$ & L10010 & 1 & $n / a$ & & \%ct Error \\
\hline 4 DUP & S96V000050 & $0 B$ & DGEA-03 & $C 513402$ & LIQUID & $<5.50 \mathrm{e}-2$ & $55.46 \mathrm{e}-2$ & & RPD \\
\hline $4 \mathrm{DUP}$ & 8960000050 & 0 & QGEA-03 & $0 \$ 13402 E$ & LIOU10 & $1:$ & $n / a$ & & \% Ct Error \\
\hline 4 DUP & s96v000050 & $O B$ & QGEA-03 & $\operatorname{cs} 13702$ & LIQUID & $1.10 e+02$ & $1.12 \mathrm{e}+02$ & 1.802 & RPO \\
\hline 4 DUP & 5960000050 & 0 & QGEA 03 & $C \$ 13702 E$ & LIOUID & 1 & 0,320 & 0.320 & \& ct error \\
\hline
\end{tabular}


LABCORE Completed RadChem Report for Worklist\#: 14453

\begin{tabular}{|c|c|c|c|c|c|c|c|c|c|}
\hline Seq Type & Sample & $\mathbf{R A}$ & & Test & Matrix & Actual & Found & DL or Yield & Unit \\
\hline 4 DUP & $596 \mathrm{~V} 000050$ & $0 \mathrm{~B}$ & DGEA-03 & CE14402 & LIQUID & $<7.20 e^{-1}$ & $<7.27 \mathrm{e}-1$ & & RPD \\
\hline 4 DUP & $596 \vee 000050$ & O B & DGEA-03 & CE14402E & LIQUID & 1 & $n / a$ & & $\%$ Ct Error \\
\hline 4 DUP & 596 vooooso & $0 \%$ & DEEA-03 & EU15402 & 1 10010 & $<2.36 \mathrm{e}-2$ & $2.82 \mathrm{e}, 2$ & & RPO \\
\hline 4 DUP & 6 V000050 & $O B$ & EEA-03 & EU15402E & LIQUID & 1 & $n / a$ & & $\% \mathrm{Ct} E$ \\
\hline bup & $6 v 000050$ & $0 \mathrm{~B}$ & EA-03 & 15502 & Lroulo & $<2.02 \mathrm{e}-1$ & $4 e-1$ & & RPD \\
\hline DUP & $66 v 000050$ & $0 \mathrm{~B}$ & EA-03 & J15502E & LIOUID & 1 & $n / a$ & & $\%$ ct E \\
\hline 4 oup & 6 vo & $0 \mathrm{~B}$ & $E A-03$ & RA22602 & LIOUID & $<1.43 \mathrm{eO}$ & $4.43=0$ & & RPD \\
\hline 4 DUP & 6 v0000050 & $O B$ & aGEA-03 & RA22602E & LIQUID & $!$ & $n / a$ & & \% Ct Err \\
\hline 5 SAMPLE & 6 vooodost & 08 & EEA- 03 & $\mathrm{CO60} 0 \mathrm{O2}$ & Llould & H/A & $7.572 \mathrm{e}-03$ & $757.2 \mathrm{e}-005$ & uct $/ \mathrm{m}$ : \\
\hline 5 SAMPLE & $6 v 000051$ & 08 & DGEA-03 & CO60-02E & LIQUID & N/A & $n / 8$ & $0.0 \mathrm{e}+000$ & \% ct. Err \\
\hline 5 SAMPLE. & 6 vooo0st & 08 & DGEA-03 & $\mathrm{AB} 94-\mathrm{OZ}$ & Loulo & N/A & $1.410 e-02$ & $141.0-004$ & $u C l / \mathrm{m}$ \\
\hline 5 SAMPLE & $6 \mathrm{~V} 000051$ & $0 \mathrm{~B}$ & DGEA-03 & NB94-02E & LIQUID & $N \angle A$ & $n / a$ & $0.0 \mathrm{e}+000$ & \% Ct. Er \\
\hline 5 SAMPLE & 60000051 & $0 B$ & बGEA - 03 & Ru10602 & HaulD & N/A & $e+0$ & $e-002$ & $\mathrm{uCs} / \mathrm{mL}$ \\
\hline 5 SAMPLE & 6 voo & $O B$ & QGEA-03 & RU10602E & LIOUID & $\mathrm{N} / \mathrm{A}$ & $\mathrm{n} /$ & +000 & $\% \mathrm{ct} \cdot \mathrm{Er}$ \\
\hline SAMPLE & $6 v 000051$ & $0 \mathrm{~A}$ & $\mathrm{GEA}-03$ & $\operatorname{cs} 13402$ & L routo & N/A & $5.593-02$ & 004 & $\mathrm{uCl} / \mathrm{mL}$ \\
\hline 5 SAMPLE & $96 \mathrm{v} 000051$ & O B & DGEA-03 & CS13402E & LIQUID & $N / A$ & $n / a$ & +000 & \% Ct. Err \\
\hline 5 SAMPLE & 5960000051 & $0 B$ & aGEA-03 & cs 13702 & LIOUID & N/A & $1.145 e+02$ & +000 & $\mathrm{HCl} / \mathrm{mL}$ \\
\hline 5 SAMPLE & $s 96 \mathrm{~V} 000051$ & O B & DGEA-03 & CS13702E & LIOUID & N/A & 0.320 & $0 \mathrm{e}+000$ & $\%$ ct. Erro \\
\hline 5 SAMPLE & $596 \mathrm{V000051}$ & 0 B & बGEA 03 & CE 14402 & LIOUID & N/A & 7.305001 & $730.5 e-003$ & scols \\
\hline 5 SAMPLE & s96v000051 & $O B$ & aGEA-03 & CE14402E & LIQUID & $\mathrm{N} / \mathrm{A}$ & $n / a$ & $e+000$ & $\% \mathrm{ct}$ \\
\hline 5 SAMPLE & s96vo00051 & O B & QGEA 03 & 15402 & LIOUID & $\mathrm{N} / \mathrm{A}$ & $e-02$ & .004 & uct \\
\hline 5 SAMPLE & s96v000051 & 0 B & QGEA-03 & EU15402E & LIOUID & $N / A$ & n & 00 & $x \mathrm{c}$ \\
\hline 5 SAMPEE & S96VOO & $0 B$ & GEA 03 & 15502 & 1.0uib & $\mathrm{N} / \mathrm{A}$ & $050 e-01$ & $205.00-003$ & cei/me \\
\hline SAMPLE & $66 \mathrm{VOO}$ & $\mathrm{OB}$ & GEA-03 & EU15502E & LIOUID & N/A & $n / a$ & $0.0 \mathrm{e}+000$ & \% ct. Error \\
\hline 5 SAMPLE & 596 V000051 & 08. & $\triangle G E A-03$ & RA22602 & lourd & N/A & $1.449 \mathrm{e}+00$ & $144.9 \mathrm{e}-002$ & $\mathrm{ucl} / \mathrm{mL}$ \\
\hline 5 SAMPLE & $596 v 000051$ & O B & QGEA-03 & RA22602E & LIOULD & N/A & $n / a$ & $.0 \mathrm{e}+000$ & \% Ct. Error \\
\hline 6 SAMPLE & $596 V 000052$ & $0 \mathrm{~B}$ & $\triangle C E A-03$ & $c 060-02$ & LOUID & N/A & $6.711 e-03$ & .005 & uci \\
\hline 6 SAMPLE & S96v000052 & O B & DGEA-03 & CO66-02E & LIQUID & N/A & $n / a$ & +000 & \% ct. Error \\
\hline 6 SAMPLE & $596 \times 00$ & $0 \mathrm{~B}$ & SEA-O3 & $94-02$ & LIOUID & $N / A$ & e-02 & 04 & ucI \\
\hline 6 SAMPLE & S96Vod & $O B$ & ac & 394-02E & LIQUID & N/A & a & $.0 \mathrm{e}+000$ & \% ct. Erro \\
\hline 6 SAMPLE: & 052 & 08 & GEA- 03 & 110602 & LIQUID & $\mathrm{N} / \mathrm{A}$ & $1.112 \mathrm{e}+00$ & $111.2 \mathrm{e}-002$ & $\mathrm{uCl} / \mathrm{mL}$ \\
\hline 6 SAMPLE & \$96v000052 & $O B$ & DGEA-03 & RU10602E & LIQUID & N/A & $n / a$ & $0.0 e+000$ & \% ct. Error \\
\hline 6 SAMPLE & $596 v 000052$ & 08 & DGEA- 03 & $\operatorname{cs} 13402$ & LIOUID & N/A & $5.546 \mathrm{e}-02$ & $554.6 \mathrm{e}-004$ & $\mathrm{uCj} / \mathrm{mL}$ \\
\hline 6 SAMPLE & S96V000052 & $O B$ & DGEA-03 & CS13402E & LIQUID & N/A & $n / a$ & $0.0 \mathrm{e}+000$ & \% ct. Error \\
\hline 6 SAMPLE: & 596 V000052 & 08 & DGEA- 03 & $\operatorname{cs} 13702$ & Clouid & $N / A$ & $120 e+02$ & $0.0 e+000$ & $\mathrm{uci} / \mathrm{mL}$ \\
\hline 6 SAMPLE & S96V000052 & $O B$ & DGEA-03 & CS13702E & LIQUID & N/A & 0.320 & 00 & Error \\
\hline 6 SAMPLE & $596 \vee 000052$ & $0 B$ & DCEA-03 & CE14402 & LIOUID & $N / A$ & $7.225 e-01$ & 722. & 0 \\
\hline 6 SAMPLE & 5960000052 & $O B$ & OGEA-03 & $C E 14402 E$ & LIQUID & N/ & & & $* 0$ \\
\hline 6 SAMPLE & 89660 & $0 B$ & GEA-O3 & EUT & LIOU10 & N/A & $631 \mathrm{e}-02$ & 04 & uci \\
\hline 6 SAMPLE & 052 & $O B$ & 33 & EU1 & LIQUID & $N / A$ & a & 000 & Error \\
\hline 6 SAMPLE & S96Vo & 0 B & QGE & EU15 & Luto & $\mathrm{N} / \mathrm{A}$. & $.044 \mathrm{e}-01$ & 204. & uct \\
\hline SAMPLE & 52 & O B & 23 & EU15502E & LIQUID & $N / A$ & $n / a$ & $0.0 \mathrm{e}+000$ & Error \\
\hline 6 SAMPLE & voo0052 & $0 B$ & aGEA-03 & RA22602 & Loulo & $N / A$ & $1.432 e+00$ & $143.2=002$ & $\mathrm{uCl} / \mathrm{nt}$ \\
\hline 6 SAMPLE & Sv000052 & O B & DGEA-03 & RA22602E & LIQUIO & $N / A$ & $n / a$ & $0.0 \mathrm{e}+000$ & * Ct. Error \\
\hline 7 SAMPLE & $6 \vee 000060$ & $\mathrm{O} B$ & $\triangle G E A-03$ & $\mathrm{cos} 0-02$ & LIQU1D & $N / A$ & $6.711 \mathrm{e}-03$ & $71,1 e-005$ & ueviont \\
\hline 7 SAMPLE & $596 \vee 000060$ & $O B$ & QGEA-03 & CO60-02E & LIQUID & N/A & $n / a$ & $.0 \mathrm{e}+000$ & $\%$ \\
\hline PLE & 6V0000060 & $0 \mathrm{~B}$ & DGEA D3 & $\mathrm{NB} 9402$ & Louto & $N / A$ & $e-03$ & $69.3 e-005$ & $u c j / m$ \\
\hline & v000060 & O B & A -03 & -02E & L10u1D & $\mathrm{N} / \mathrm{t}$ & $n / a$ & $0.0 \mathrm{e}+000$ & - Error \\
\hline SAMPLE & 5960000060 & $0 \mathrm{~B}$ & DGEA-03 & RU 0602 & LoOID & N/A & -01 & $105.8 \mathrm{e}-003$ & $\mathrm{uct} / \mathrm{m}$ \\
\hline
\end{tabular}




\section{LABCORE Completed RadChem Report for Worklist\#: 14453}

\begin{tabular}{|c|c|c|c|c|c|c|c|c|c|}
\hline Seq Туре & Sampl & $\mathbf{R}$ & & Test & Matrix & $\mathbf{a} \mid$ & & & Unit \\
\hline 7 SAMPLE & $596 \vee 000060$ & $O B$ & DGEA -03 & RU10602E & LIQUID & $\mathrm{N} / \mathrm{A}$ & $n / a$ & $0.0 e+000$ & * ct. Error \\
\hline 7 SAMPLE & S96v000060 & $O B$ & DGEA-03 & $\operatorname{cs} 13402$ & LIQUID & N/A & $4.177 e-03$ & $417.7 e-005$ & $u C \mathbf{i} / \mathbf{m L}$ \\
\hline 7 SAMPLE & s96V000060 & 08 & DGEA- 03 & $\$ 13402 E$ & Louro & N/A & $n$ & $0.0 e+000$ & y ct. Error \\
\hline 7 SAMPLE & $596 \mathrm{~V} 000060$ & $0 B$ & QGEA-03 & $\$ 13702$ & LIQUID & N/A & $e-02$ & $e-004$ & $u C i / m L$ \\
\hline 7 SAMPLE & $596 \vee 000060$ & 08 & $G E A-03$ & $02 \mathrm{E}$ & Ulouto & $\mathrm{N} / \mathrm{A}$ & $\mathrm{n} / \mathrm{a}$ & $e+000$ & $8 \mathrm{ct}$. Error \\
\hline 7 SAMPLE & $596 \vee 000060$ & $O B$ & QGEA-03 & CE 14402 & LIQUID & $N / A$ & $.239 \mathrm{e}-02$ & $523.9 \mathrm{e}-004$ & $u C \mathbf{i} / m L$ \\
\hline 7 SAMPLE & $596 \mathrm{~V} 000060$ & 08 & बCEA -03 & CE $14402 \mathrm{E}$ & Lloulo & N/A & $\mathrm{n} / \mathrm{a}$ & $0,0 e+000$ & \& ct, Error \\
\hline 7 SAMPLE & s96v000060 & 08 & DGEA-03 & EU15402 & LIQUID & $N / A$ & $1.528 \mathrm{e}-02$ & $152.8 \mathrm{e}-004$ & $U C \mathrm{C} / \mathrm{mL}$ \\
\hline 7 SAMPLE & S96V000060 & 08 & QGEA-O3 & EU15402E & Lroulo & $\mathrm{N} / \mathrm{A}$ & $n / 0$ & $0.0 \mathrm{e}+000$ & * ct. Error \\
\hline 7 SAMPLE & $\$ 96 \mathrm{~V} 000060$ & $0 \mathrm{~s}$ & DGEA-03 & EU15502 & LIQUID & $N / A$ & $1.802 e-02$ & $180.2 e-004$ & $\omega C \mathrm{i} / \mathrm{mL}$ \\
\hline 7 SAMPLE & $596 \mathrm{~V} 000060$ & 08 & DGEA-03 & $\mathrm{EU115502 \textrm {E }}$ & 40010 & $N / A$ & $\mathrm{n} / \mathrm{a}$ & $0.0 e+000$ & x ct, Error \\
\hline 7 SAMPLE & $s 96 \mathrm{~V} 000060$ & $O B$ & DGEA-03 & RA22602 & LIOUID & $N / A$ & $19 \mathrm{e}-01$ & $111.9 e-003$ & $\mathrm{uCi} / \mathrm{mL}$ \\
\hline HE & $896 \vee 000060$ & 68 & $A+03$ & 26022 & 10 & & $n$ & +000 & $\mathrm{Ct}, \mathrm{B}$ \\
\hline
\end{tabular}

\section{Comments Section:}

Comments for sample\# S96V000050 and test@GEA-03 .

$$
\mathrm{DL}=0=>\mathrm{n} / \mathrm{a} \text {. }
$$

Comments for sample\# S96V000051 and test@GEA-03 .

$\mathrm{DL}=0=>$ n/a.

Comments for sample\# S96V000052 and test @GEA-03 . $\mathrm{DL}=0=>\mathrm{n} / \mathrm{a}$.

Final page for worklist\# 14453

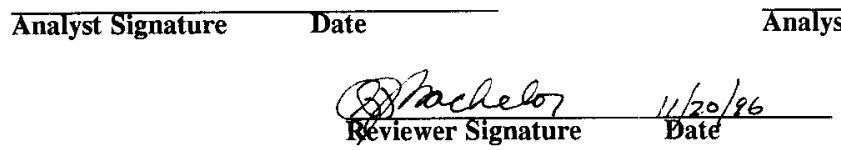




\section{LABCORE Data Entry Template for Worklist\# 14453}

Page: $\quad I$

Analyst: $\quad$ S.L $\quad$ Instrument: GEA00 Q
Method: LA-548-121 Rev/Mod $E-0$

Worklist Comment: AP-105 GEA RCJ

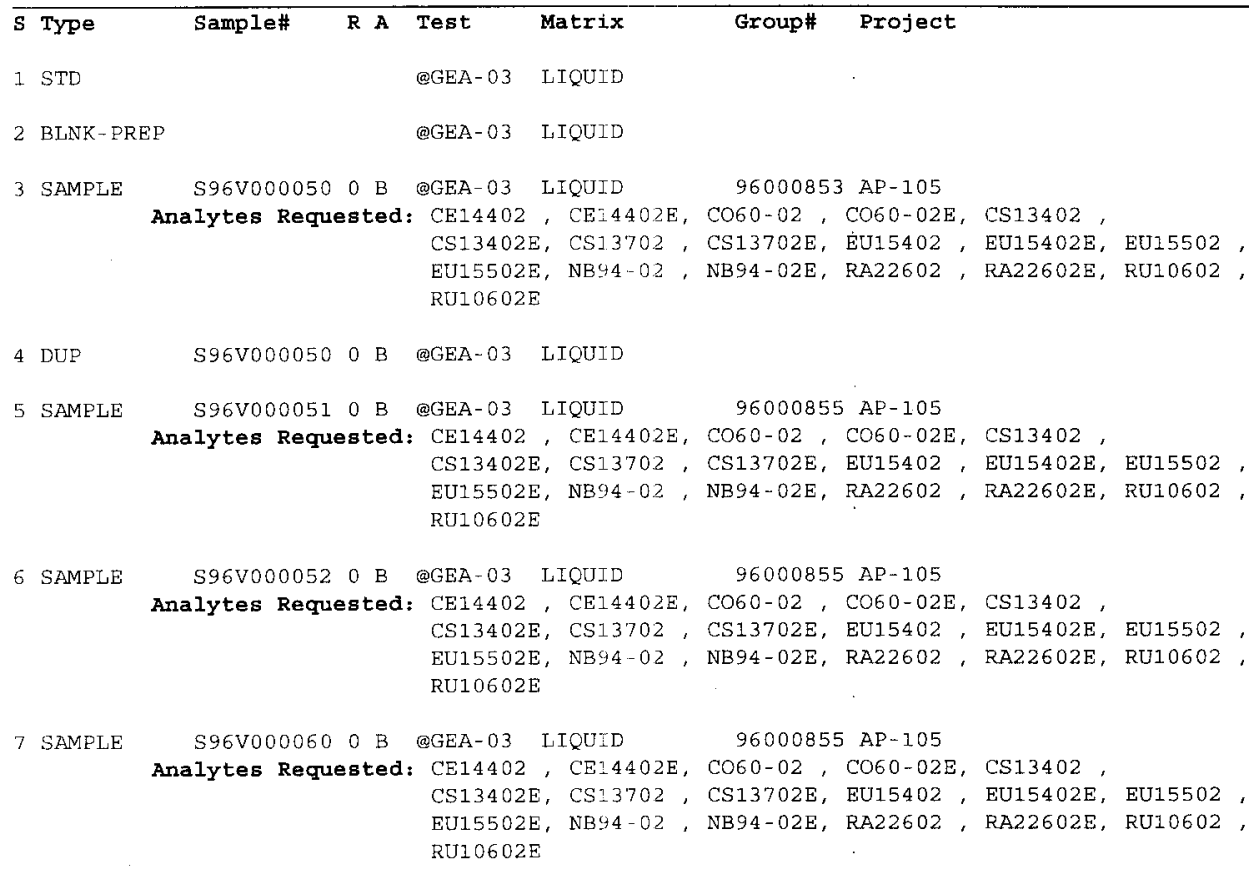

Final page for worklist \# 14453

$\frac{\text { Sue } \frac{\mathcal{L}}{\text { Analyst Signature }} \quad 11-19-96}{\text { Date }}$

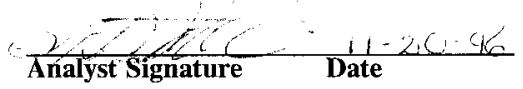

Datu Entry Comments:

$\bar{S}=$ Worklist Slot Number, $R=$ Replicate Number, $A=$ Aliquot Code. 


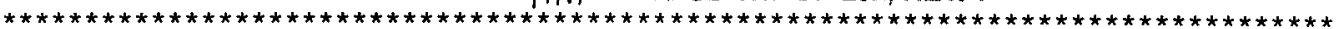

* 222-S Laboratory Counting Room 19-NOV-1996 14:31:58.14

worklist \#:

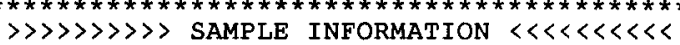

Sample ID:

Sample Size:

Dilution Factor:

$>>>>>>>>>$ COUNT INFORMATION $<<<<<<<<<~$

Detector ID:

File Number:

Geometry:

Count Time:

Real Time:

Dead Time:
14453

WL14453

$1.00000 \mathrm{E}-03 \mathrm{~L}$

$1.00000 \mathrm{E}+00$ dka300: [ spec.GEA2] 2g2942.cnf 42

$000: 50: 00.00 \mathrm{sec}$

0 00:50:05.32 sec

0.2 웅

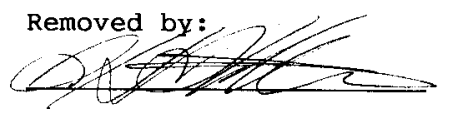

Verified by:

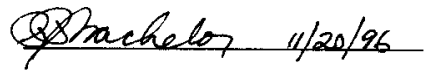

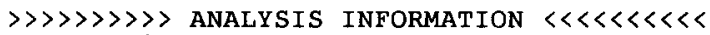

Sample Count Time:

Decayed to:

Standard Deviations:

Analysis Library:

Analyst:

Background Subtract:
19-NOV-1996 13:41:12.62

19-NOV-1996 13:41:12.62

2

ENVGEA

LMHS

DKA 300 : [ SPEC . GEA2 ] 2GBACK

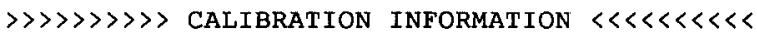

Date of last energy calibration: 21-MAR-1994 09:31:55.15

Date of last efficiency calibration: 21-MAR-1994 09:43:42.61

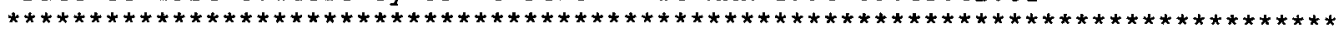

Post-NID Peak Search Report

It Energy Area FWHM Channel Left PW 8 Err Fit Nuclides Activity

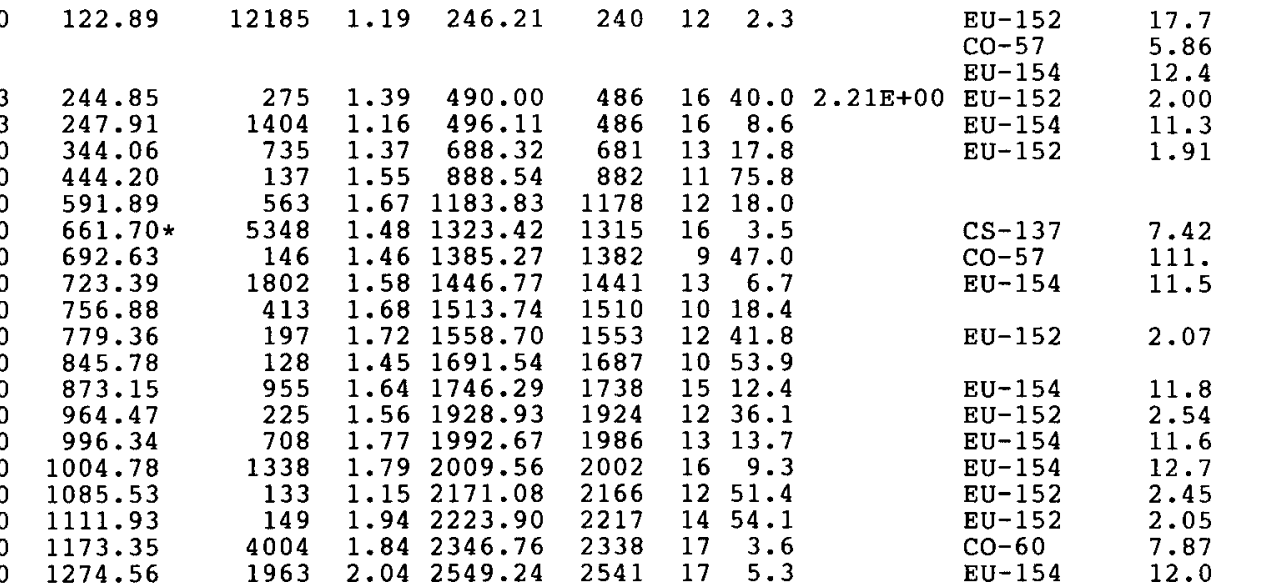


Post-NID Peak Search Report (continued)-SD-WM-DP-202, REV

Page : 2

Sample ID : WL14453

Acquisition date : 19-NOV-1996 13:41:12

\begin{tabular}{|c|c|c|c|c|c|c|c|c|c|c|}
\hline It & Energy & Area & FWHM & Channel & Left. & Pw & gErr & Fit & Nuclides & $\begin{array}{c}\text { Activity } \\
\text { uci/L }\end{array}$ \\
\hline $\begin{array}{l}0 \\
0 \\
0 \\
0\end{array}$ & $\begin{array}{l}1332.68 \\
1397.37 \\
1408.14 * \\
1596.61\end{array}$ & $\begin{array}{r}3569 \\
27 \\
184 \\
101\end{array}$ & $\begin{array}{l}1.95 \\
2.16 \\
2.39 \\
2.07\end{array}$ & $\begin{array}{l}2665.52 \\
2794.95 \\
2816.49 \\
3193.62\end{array}$ & $\begin{array}{l}2656 \\
2787 \\
2807 \\
3185\end{array}$ & $\begin{array}{l}21 \\
14 \\
21 \\
15\end{array}$ & $\begin{array}{r}3.6 \\
75.2 \\
17.4 \\
21.7\end{array}$ & & $\begin{array}{l}C O-60 \\
E U-152\end{array}$ & $\begin{array}{l}7.77 \\
2.01\end{array}$ \\
\hline
\end{tabular}


Summary of Nuclide Activity Sample ID : WL14453
INF-SD-WM-DH-202, REV.

Acquisition date : 19-NOV-1996 $13: 41: 12$

Total number of lines in spectrum

Number of unidentified lines

Number of lines tentatively identified by NID 20

$83.33 \%$

Nuclide Type :

\begin{tabular}{|c|c|c|c|c|c|c|}
\hline \multirow[t]{2}{*}{$\begin{array}{l}\text { Nuclide } \\
\text { EO-57 } \\
\text { CO-60 } \\
\text { CS-137 } \\
\text { EU-152 } \\
\text { EU-154 }\end{array}$} & $\begin{array}{r}\text { Hlife } \\
271.80 \mathrm{D} \\
5.27 \mathrm{Y} \\
30.00 \mathrm{Y} \\
13.54 \mathrm{Y} \\
8.59 \mathrm{Y}\end{array}$ & $\begin{array}{l}\text { Decay } \\
1.000 \\
1.000 \\
1.000 \\
1.000 \\
1.000\end{array}$ & $\begin{array}{c}\text { Wtd Mean } \\
\text { Uncorrected } \\
\text { uCi/L } \\
1.108 \mathrm{E}+02 \\
7.816 \mathrm{E}+00 \\
7.421 \mathrm{E}+00 \\
2.015 \mathrm{E}+00 \\
1.179 \mathrm{E}+01\end{array}$ & $\begin{array}{c}\text { Wtd Mean } \\
\text { Decay Corr } \\
\text { uCi/L } \\
1.108 \mathrm{E}+02 \\
7.816 \mathrm{E}+00 \\
7.421 \mathrm{E}+00 \\
2.015 \mathrm{E}+00 \\
1.179 \mathrm{E}+01\end{array}$ & \multirow[t]{2}{*}{$\begin{array}{c}\text { Decay Corr } \\
2-\text { Sigma Error } \\
0.520 \mathrm{E}+02 \\
0.200 \mathrm{E}+00 \\
0.258 \mathrm{E}+00 \\
0.211 \mathrm{E}+00 \\
0.038 \mathrm{E}+01\end{array}$} & \multirow[t]{2}{*}{$\begin{array}{l}\text { 2-Sigma } \\
\text { ofror Flags } \\
46.95130 \text { EuSB } \\
2.56 \\
3.48 \\
10.49 \\
3.25\end{array}$} \\
\hline & Total A & & $1.399 \mathrm{E}+02$ & $1.399 \mathrm{E}+02$ & & \\
\hline
\end{tabular}

Grand Total Activity : $1.399 \mathrm{E}+02 \quad 1.399 \mathrm{E}+02$

Flags: " $K "=$ Keyline not found

"M" = Manually accepted

"E" = Manually edited

"A" = Nuclide specific abn. limit 
Minimum Detectable Activity Report Sample ID : WL14453
WM-DP-202 REV.

Acquisition date : 19-NOV-1996 13:41:12
Nuclide

$\mathrm{BE}-7$

NA -22

NA -24

$\mathrm{K}-40$

CR-51

$\mathrm{MN}-54$

$\mathrm{CO}-56$

$\mathrm{CO}-58$

$\mathrm{FE}-59$

$\mathrm{SE}-75$

$\mathrm{SR}-85$

$\mathrm{Y}-88$

NB-94

ZRNB-95

RU-103

RURH-106

AG- $108 \mathrm{~m}$

CD-109

AG- $110 \mathrm{M}$

SN-113

$\mathrm{TE}-123 \mathrm{~m}$

SB- 124

SB- 125

TE-125m

I-131

CS- 134

BA- 140

LA- 140

CEPR-144

EU-155

HG- 203

TL-208

BI -212

PB-2 12

BI - 214

PB-2 14

RA-224

RA-226

AC -228

TH- 228

$\mathrm{TH}-229$

PA-233

UTH -233

PA-234M

TH-234

$\mathrm{U}-235$

NP -237

NP -239

PU-239

AM-241

AM- 243

\section{Bckgnd \\ Sum}

632 .

2060 .

21.

119.

664 .

286 .

422 .

319 .

264 .

707 .

514 .

6.

1035 .

2103 .

501 .

350 .

2113.

1284 .

449 .

564 .

797.

347 .

502 .

1176.

570 .

359 .

420 .

103.

825 .

1285 .

717 .

713 .

370 .

833.

411.

590 .

872 .

835.

397.

1182 .

1259 .

645 .

1248 .

433.

737.

822 .

1283 .

1251 .

800 .

863 .

892 .

\section{Energy \\ ( $\mathrm{keV}$ )}

477.59

1274.53

1368.55

1460.75

320.08

834.83

846.76

810.78

1099.25

264.66

514.01

1836.06

871.09

724.18

497.08

621.93

722.94

88.03

657.76

391.69

159.00

602.73

427.89

109.27

364.48

604.70

537.31

1596.21

133.51

105.31

279.20

277.36

727.18

238.63

609.31

351.92

240.99

186.10

911.21

84.37

88.47

312.17

245.34

1001.03

63.29

185.71

86.48

106.12

129.30

59.54

74.67

\section{MDA \\ ( $\mathrm{uCi} / \mathrm{L}$ )}

$1.0195 \mathrm{E}+00$

$4.4307 \mathrm{E}-01$

$4.7705 \mathrm{E}-02$

$1.1133 \mathrm{E}+00$

$7.7998 \mathrm{E}-01$

$1.1332 \mathrm{E}-01$

$1.3935 \mathrm{E}-01$

$1.1742 \mathrm{E}-01$

$2.4701 \mathrm{E}-01$

$1.2148 \mathrm{E}-01$

$1.0564 \mathrm{E}-01$

3. $2268 \mathrm{E}-02$

2. 2344 E-01

$6.2299 \mathrm{E}-01$

$1.0698 \mathrm{E}-01$

$1.9714 \mathrm{E}+00$

$3.0005 \mathrm{E}-01$

$2.7515 \mathrm{E}+00$

1. $2258 \mathrm{E}-01$

1. $3067 \mathrm{E}-01$

$6.3165 \mathrm{E}-02$

$9.7019 \mathrm{E}-02$

$2.8924 \mathrm{E}-01$

2. $5423 \mathrm{E}+01$

$9.8456 \mathrm{E}-02$

9. $9191 \mathrm{E}-02$

$3.9001 \mathrm{E}-01$

1. $2545 \mathrm{E}-01$

$9.5972 \mathrm{E}-01$

3. $5689 \mathrm{E}-01$

9. $1034 \mathrm{E}-02$

$1.1665 \mathrm{E}+00$

$1.7234 \mathrm{E}+00$

1. $6466 \mathrm{E}-01$

2. 3242E-0I

3. $9470 \mathrm{E}-01$

$1.8698 \mathrm{E}+00$

$1.6853 \mathrm{E}+00$

$5.4053 \mathrm{E}-01$

8. $1059 \mathrm{E}+00$

$3.9495 \mathrm{E}-01$

$1.9710 \mathrm{E}-01$

8. $3789 \mathrm{E}+01$

$2.7793 \mathrm{E}-01$

$3.7293 \mathrm{E}+00$

$1.0167 \mathrm{E}-01$

8. $2536 \mathrm{E}-01$

3. $3420 \mathrm{E}-01$

8. $2566 \mathrm{E}+02$

$6.4212 \mathrm{E}-01$

$1.9132 \mathrm{E}-01$ 


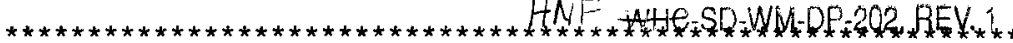

* 222-S Laboratory Counting Room 19-NOV-1996 15:46:41.33

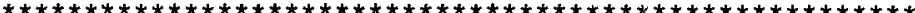

Worklist \#:

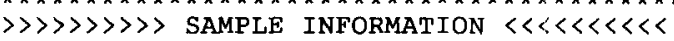

Sample ID:

Sample Size:

Dilution Factor:

$\gg>>>>>>>$ COUNT

\section{Detector ID:}

File Number:

Geometry:

Count Time:

Real Time:

Dead Time:
14453

WK 144 53-BLK

$2.50000 \mathrm{E}-04 \mathrm{~L}$

$1.00000 \mathrm{E}+00$ dka300: [ spec.GEA2 ]2g2943.cnf

42

0 00:50:00.00 sec

0 00:50:00.49 sec

$0.0 \%$

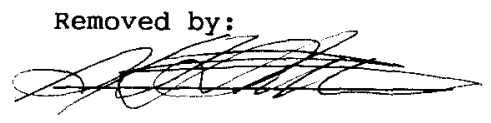

Verified by:

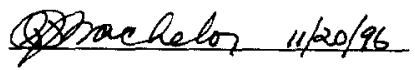

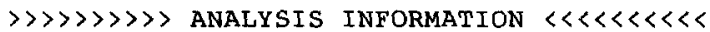

Sample Count Time: 19-NOV-1996 14:56:02.49

Decayed to:

Standard Deviations:

Analysis Library:

Analyst:

19-NOV-1996 14:56:02.49

Background Subtract:

2

ENVGEA

LMHS

DKA $300:$ [ SPEC. GEA2 ]2GBACK

$\gg \gg \gg \gg \gg \gg>$ CALIBRATION INFORMATION $<<<<<<<<<~$

Date of last energy calibration: 21-MAR-1994 09:31:55.15

Date of last efficiency calibration: 21-MAR-1994 09:43:42.61

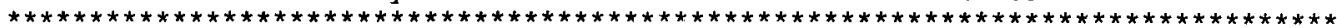

Post-NID Peak Search Report

It Energy Area FWHM Channel Left PW oferr Fit Nuclides Activity $\mathrm{uCi} / \mathrm{L}$

$\begin{array}{llllllll}0 & 526.53 & 21 & 1.37 & 1053.14 & 1049 & 9 & 69.5\end{array}$ 
Total number of lines in spectrum Number of unidentified lines

Number of lines tentatively identified by NID

1

$\star \star \star \star$ There are no nuclides meeting summary criteria

$$
0.00 \%
$$

Flags: "K" = Keyline not found

" $M$ " = Manually accepted

"E" = Manually edited

"A" = Nuclide specific abn. limit 
Minimum Detectable Activity Report Sample ID : WK14453-BLK

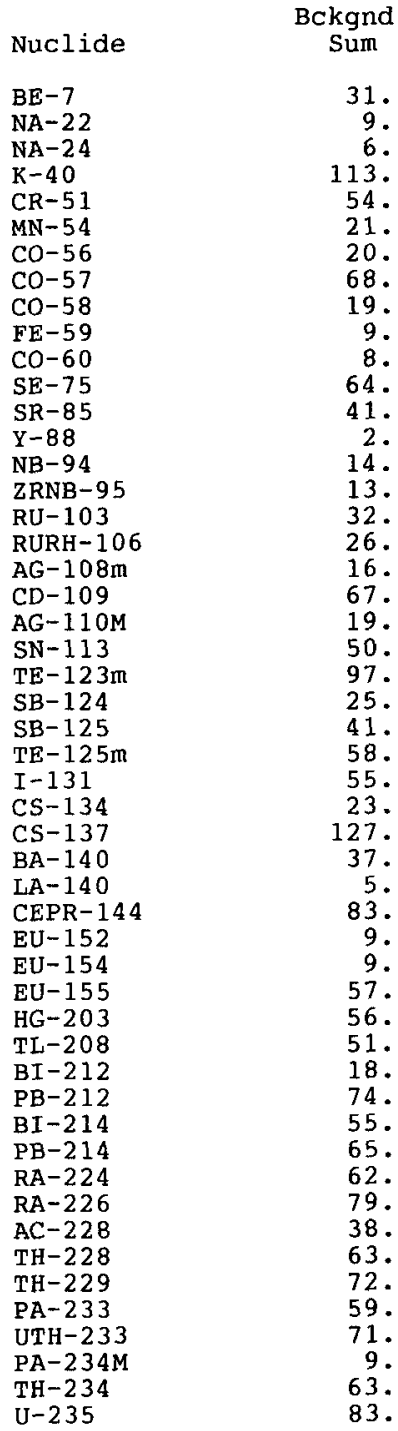

Energy

( kev)

477.59

1274.53

1368.55

1460.75

320.08

834.83

846.76

122.06

810.78

1099.25

1332.50

264.66

514.01

1836.06

871.09

724.18

497.08

621.93

722.94

88.03

657.76

391.69

159.00

602.73

427.89

109.27

364.48

604.70

661.66

537.31

1596.21

133.51

1408.01

1274.51

105.31

279.20

277.36

727.18

238.63

609.31

351.92

240.99

186.10

911.21

84.37

88.47

312.17

245.34

1001.03

63.29

185.71
Page : 3

M-DP-202, REV.

MDA

( $\mathrm{UCi} / \mathrm{L})$

9. $0110 \mathrm{E}-01$

$1.1912 \mathrm{E}-01$

1. $0694 \mathrm{E}-01$

4. $3397 \mathrm{E}+00$

8.8989E-01

1. $2212 \mathrm{E}-01$

$1.2146 \mathrm{E}-01$

7. $3806 \mathrm{E}-02$

$1.1396 \mathrm{E}-01$

1. $8079 \mathrm{E}-01$

$1.1447 \mathrm{E}-01$

1. $4651 \mathrm{E}-01$

$1.1861 \mathrm{E}-01$

7. $4519 \mathrm{E}-02$

1. $0318 \mathrm{E}-01$

1. $9728 \mathrm{E}-01$

$1.0765 \mathrm{E}-01$

2. $1661 \mathrm{E}+00$

$1.0364 \mathrm{E}-01$

2. $5119 \mathrm{E}+00$

$1.0028 \mathrm{E}-01$

1. $5526 \mathrm{E}-01$

8.8070E-02

1.0476E-01

3. $3197 \mathrm{E}-01$

2. $2513 \mathrm{E}+01$

1.2234 E-01

$1.0055 \mathrm{E}-01$

$2.9112 \mathrm{E}-01$

$4.6416 \mathrm{E}-01$

$1.1546 \mathrm{E}-01$

1. $2190 \mathrm{E}+00$

5. $9212 \mathrm{E}-01$

$3.4533 \mathrm{E}-01$

$3.0191 \mathrm{E}-01$

1. $0135 \mathrm{E}-01$

1. $2511 \mathrm{E}+00$

$1.5286 \mathrm{E}+00$

1. $9682 \mathrm{E}-01$

3. $4027 \mathrm{E}-01$

5. $2440 \mathrm{E}-01$

$1.9939 \mathrm{E}+00$

$2.0802 E+00$

$6.7085 \mathrm{E}-01$.

$7.4861 \mathrm{E}+00$

3. $7743 \mathrm{E}-01$

2. $3916 \mathrm{E}-01$

8. $0195 \mathrm{E}+01$

$1.6453 \mathrm{E}-01$

4. $3542 \mathrm{E}+00$

1. $2921 \mathrm{E}-01$ 
Minimum Detectable Activity Report (continued)

Nuclide

$$
\begin{aligned}
& \text { Bckgnd } \\
& \text { Sum }
\end{aligned}
$$

$\mathrm{NP}-237$

$\mathrm{NP}-239$

PU-239

AM-241

AM- 243
64.

60.

83.

61 .

79.
Energy

(keV)

86.48

106.12

129.30

59.54

74.67
MDA

( $\mathrm{UCi} / \mathrm{L}$ )

$7.3763 E-01$

$2.9374 \mathrm{E}-01$

$1.0611 \mathrm{E}+03$

$6.8556 \mathrm{E}-01$

2. 2711E-01 
* 222-S Laboratory Counting Room 19-NOV-1996 16:49:28.78 $>>>>>>>>>$ SAMPIE INFORMATION $<<<<<<<<<<~$

Worklist \#:

Sample ID:

Sample Size:

Dilution Factor:

$\gg \gg \gg \gg \gg \gg \gg$ COUNT

Detector ID:

File Number:

Geometry:

Count Time:

Real Time:

Dead Time:
14453

S96V50-SAM

2.50000E-04 L

1. $00000 \mathrm{E}+00$

INFORMATION $\ll \ll<\ll<\ll<~$

GEA2

dka 300: [ spec.GEA2 ]2g2944 . cnf

42

$000: 50: 00.00 \mathrm{sec}$

$0 \quad 00: 50: 53.75 \mathrm{sec}$

$1.8 \%$

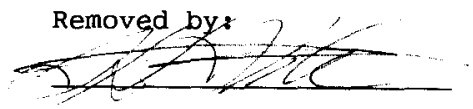

Verified by:

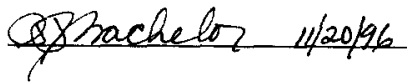

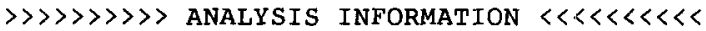

Sample Count Time: 19-NOV-1996 15:57:55.60

Decayed to:

Standard Deviations:

Analysis Library:

Analyst:

$19-N O V-1996 \quad 15: 57: 55.60$

2

ENVGEA

LMHS

Background Subtract: DKA300: [SPEC.GEA2]2GBACK

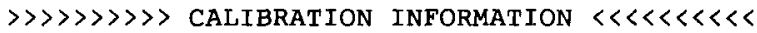

Date of last energy calibration: 21-MAR-1994 09:31:55.15

Date of last efficiency calibration: 21-MAR-1994 09:43:42.61

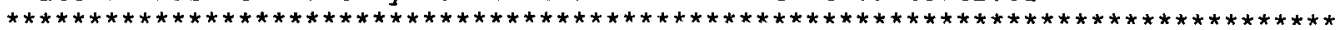

Post-NID Peak Search Report

It Energy Area FWHM Channel Left PW ster Fit Nuclides Activity uCi/L

0

$661.66 * 398314$

1.531323 .35

$\begin{array}{lll}1315 & 17 & 0.3\end{array}$

Cs- 137

2. $211 \mathrm{E}+03$ 
Total number of lines in spectrum Number of unidentified lines

Number of lines tentatively identified by NID $1 \quad 100.00 \%$

Nuclide Type :

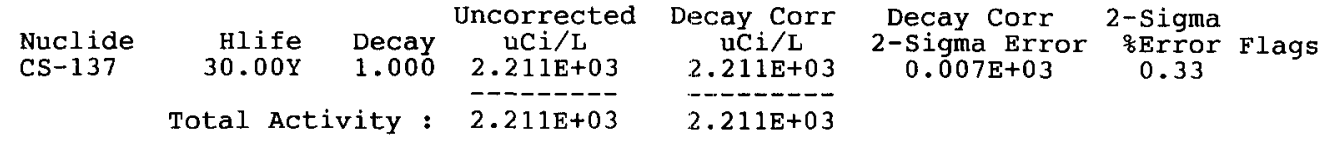

Grand Total Activity : $2.211 \mathrm{E}+03 \quad 2.211 \mathrm{E}+03$

Flags: "K" = Keyline not found

"M" = Manually accepted

"E" = Manually edited

"A" = Nuclide specific abn. limit 
Minimum Detectable Activity Report Sample ID : S96V50-SAM

Nuclide

BE-7
NA-22
NA-24
$\mathrm{K}-40$
CR-51
MN -54
CO-56
CO-57
CO-58
FE-59
CO-60
SE -75
SR-85
Y -88
NB- 94

ZRNB-95

RU-103

RURH-106

AG- $108 \mathrm{~m}$

CD-109

AG-110M

$\mathrm{SN}-113$

TE-123m

SB- 124

$\mathrm{SB}-125$

$\mathrm{TE}-125 \mathrm{~m}$

$\mathrm{I}-131$

CS -134

BA- 140

LA -140

CEPR-144

EU-152

EU-154

EU-155

HG- 203

TL-208

BI -212

PB- 212

BI -214

PB-214

$\mathrm{RA}-224$

RA- 226

AC -228

$\mathrm{TH}-228$

$\mathrm{TH}-229$

PA- 233

UTH-233

PA-234M

TH -234

$\mathrm{U}-235$

NP -237
Bckgnd
Sum

12731. 17.

9.

108.

10501.

107.

123.

11387.

120 .

48.

11574.

6373.

5.

93.

173.

7874 .

2725 .

171 .

9931.

9708.

11213.

11810 .

2844 .

12912 .

10593.

10506.

2763.

4723.

11597.

14.

17.

10333.

10971.

11133.

191.

13042 .

2696 .

10445.

12986 .

14953.

102.

9936.

9958.

10416.

12662 .

52.

9297.

14766.

9876 .

\section{Energy \\ (kev)}

477.59

1274.53

1368.55

1460.75

320.08

834.83

846.76

122.06

810.78

1099.25

1332.50

264.66

514.01

1836.06

871.09

724.18

497.08

621.93

722.94

88.03

657.76

391.69

159.00

602.73

427.89

109.27

364.48

604.70

537.31

1596.21

133.51

1408.01

1274.51

105.31

279.20

277.36

727.18

238.63

609.31

351.92

240.99

186.10

911.21

84.37

88.47

312.17

245.34

1001.03

63.29

185.71

86.48
Page : 3

Acquisition date : 19-NOV-1996 15:57:55

\section{MDA \\ ( $U \mathrm{C} i / L)$}

$1.8307 \mathrm{E}+01$

$1.6179 \mathrm{E}-01$

$1.2293 \mathrm{E}-01$

$4.2334 \mathrm{E}+00$

$1.2404 E+01$

$2.7728 \mathrm{E}-01$

$3.0078 \mathrm{E}-01$

$9.5441 \mathrm{E}-01$

$2.8745 \mathrm{E}-01$

$4.2316 \mathrm{E}-01$

$1.2142 \mathrm{E}-01$

$1.9658 \mathrm{E}+00$

$1.4877 \mathrm{E}+00$

$1.2172 \mathrm{E}-01$

$2.6753 \mathrm{E}-01$

$7.1546 \mathrm{E}-01$

$1.6958 \mathrm{E}+00$

$2.1996 \mathrm{E}+01$

$3.4149 \mathrm{E}-01$

$3.0604 \mathrm{E}+01$

2. $2796 \mathrm{E}+00$

$2.3309 \mathrm{E}+00$

$9.7260 E-01$

$1.1112 \mathrm{E}+00$

$5.8698 \mathrm{E}+00$

3. $0514 \mathrm{E}+02$

$1.6901 \mathrm{E}+00$

$1.1002 \mathrm{E}+00$

$5.2343 E+00$

$1.4899 \mathrm{E}-01$

$1.4395 \mathrm{E}+01$

$7.4556 \mathrm{E}-01$

$4.7114 \mathrm{E}-01$

$4.0477 \mathrm{E}+00$

1. $4240 \mathrm{E}+00$

$1.8432 \mathrm{E}+01$

$4.9541 \mathrm{E}+00$

$2.6066 \mathrm{E}+00$

2. $3819 \mathrm{E}+00$

$6.6993 \mathrm{E}+00$

$2.8868 \mathrm{E}+01$

$2.8533 \mathrm{E}+01$

$1.0963 \mathrm{E}+00$

9. $3997 \mathbf{E}+01$

$4.4425 \mathrm{E}+00$

$3.1679 \mathrm{E}+00$

$1.0677 \mathrm{E}+03$

$3.8491 \mathrm{E}-01$

$5.2966 \mathrm{E}+01$

$1.7234 \mathrm{E}+00$

$9.1583 \mathrm{E}+00$ 
Minimum Detectable Activity Report (continued) HW-SD-WM-DP-202, REV.

Sample ID : S96V50-SAM

Page : 4

Nuclide

NP -239

$\mathrm{PU}-239$

AM-241

AM- 243

\section{Bckgnd}

sum

10428.

11654 .

9525 .

9719.

Acquisition date : 19-NOV-1996 15:57:55

Energy

(kev)

106.12

129.30

59.54

74.67
MDA

( $\mathrm{UCi} / \mathrm{L}$ )

$3.8591 \mathrm{E}+00$

$1.2608 \mathrm{E}+04$

$8.5356 \mathrm{E}+00$

$2.5267 \mathrm{E}+00$ 


\section{* 222-S Laboratory Counting Room 19-NOV-1996 18:10:42.95} $\gg>\gg>>>>>$ SAMPLE INFORMATION $<<<<<<<<<~$

Worklist \#:

Sample ID:

Sample Size:

Dilution Factor:

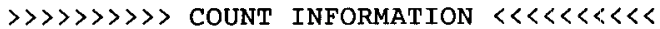

Detector ID:

File Number:

Geometry:

Count Time:

Real Time:

Dead Time:

\section{3}

S96V50-DUP

$2.50000 \mathrm{E}-04 \mathrm{~L}$

$1.00000 \mathrm{E}+00$

GEA2

dka 300: [spec.GEA2 ]2g2945.cnf 42

$000: 50: 00.00 \mathrm{sec}$

$0 \quad 00: 50: 54.35 \mathrm{sec}$

$1.8 \%$
Removed by:

Shunlal

Verified by:

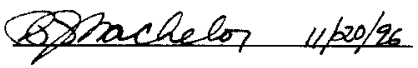

$\gg>>>>>>>$ ANALYSIS INFORMATION $<<<<<<<<<~$

Sample Count Time: 19-NOV-1996 17:19:11.29

Decayed to: 19-NOV-1996 17:19:11.29

Standard Deviations: 2

Analysis Library: ENVGEA

Analyst: $\quad$ LMHS

Background subtract: DKA300: [SPEC.GEA2]2GBACK

$>>>>>>>>>$ CALIBRATION INFORMATION $<<<<<<<<<~$

Date of last energy calibration: 21-MAR-1994 09:31:55.15

Date of last efficiency calibration: 21-MAR-1994 09:43:42.61

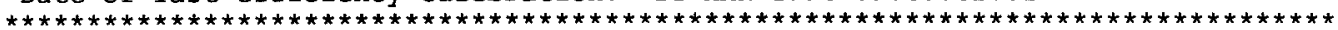

Post-NID Peak Search Report

It Energy Area FWHM Channel Left Pw $\%$ Err Fit Nuclides Activity $\mathrm{uCi} / \mathrm{L}$

CS -137

$2.239 \mathrm{E}+03$

1322.86

$\begin{array}{lll}23 & 1.50 & 2645.86\end{array}$

2643

$\begin{array}{ll}8 & 70.7\end{array}$ 
Summary of Nuclide Activity

Sample ID : S96V50-DUP
AHIE-SD-NM-DP-202, REV. 1

HNF Page : 2

Page : 22

Total number of lines in spectrum 2

Number of unidentified lines 1

Number of lines tentatively identified by NID 1 50.008

Nuclide Type :

\begin{tabular}{|c|c|c|c|c|c|c|}
\hline \multirow[t]{2}{*}{$\begin{array}{l}\text { Nuclide } \\
\text { CS-137 }\end{array}$} & $\begin{array}{r}\text { Hlife } \\
30.00 \mathrm{Y}\end{array}$ & $\begin{array}{l}\text { Decay } \\
1.000\end{array}$ & $\begin{array}{c}\text { Uncorrected } \\
\text { uCi } / \mathrm{L} \\
2.239 \mathrm{E}+03\end{array}$ & $\begin{array}{c}\text { Decay Corr } \\
\text { uCi } / \mathrm{L} \\
2.239 \mathrm{E}+03\end{array}$ & $\begin{array}{c}\text { Decay Corr } \\
\text { 2-Sigma Error } \\
0.007 \mathrm{E}+03\end{array}$ & $\begin{array}{l}\text { 2-Sigma } \\
\text { \%Error Flags } \\
0.32\end{array}$ \\
\hline & otal AC & ty : & $2.239 E+03$ & $2.239 \mathrm{E}+03$ & & \\
\hline
\end{tabular}

Grand Total Activity : $2.239 \mathrm{E}+03 \quad 2.239 \mathrm{E}+03$

Flags: " $K "=$ Keyline not found

"E" = Manually edited

" $M "$ = Manually accepted

"A" = Nuclide specific abn. limit 
Minimum Detectable Activity Report Sample ID : \$96V50-DUP

Nuclide

$\mathrm{BE}-7$

$\mathrm{NA}-22$

NA -24

$\mathrm{K}-40$

CR-51

MN -54

CO -56

CO- 57

CO- 58

FE-59

CO-60

$\mathrm{SE}-75$

SR-85

$\mathrm{Y}-88$

NB-94

ZRNB-95

RU -103

RURH-106

AG $-108 \mathrm{~m}$

CD-109

AG- $110 \mathrm{M}$

SN-113

$\mathrm{TE}-123 \mathrm{~m}$

SB-124

SB- 125

TE- $125 \mathrm{~m}$

I -131

CS -134

BA- 140

LA- 140

CEPR-144

EU-152

EU-154

EU- 155

HG- 203

TL-208

BI- 212

PB- 212

BI -214

$\mathrm{PB}-214$

$\mathrm{RA}-224$

RA- 226

AC -228

$\mathrm{TH}-228$

TH-229

PA- 233

UTH-233

$\mathrm{PA}-234 \mathrm{M}$

$\mathrm{TH}-234$

U-235

$\mathrm{NP}-237$

\section{Bckgnd \\ sum}

12675.

25 .

5 .

94 .

10475 .

122 .

112 .

11511.

128 .

51 .

13.

11913.

6614 .

3.

94.

185.

7994 .

2792 .

182 .

10041 .

9641 .

11247 .

11767 .

2803 .

12920 .

10681 .

10707 .

2725 .

4748 .

11831 .

7.

25 .

10450 .

11095 .

11244 .

211 .

13219 .

2846 .

10592 .

13141 .

15104 .

84.

10099 .

10029 .

10690 .

12624 .

59.

9556 .

14953 .

10115.

\section{Energy \\ (kev)}

477.59

1274.53

1368.55

1460.75

320.08

834.83

846.76

122.06

810.78

1099.25

1332.50

264.66

514.01

1836.06

871.09

724.18

497.08

621.93

722.94

88.03

657.76

391.69

159.00

602.73

427.89

109.27

364.48

604.70

537.31

1596.21

133.51

1408.01

1274.51

105.31

279.20

277.36

727.18

238.63

609.31

351.92

240.99

186.10

911.21

84.37

88.47

312.17

245.34

1001.03

63.29

185.71

86.48
Page : 3

Acquisition date : 19-NOV-1996 17:19:11

MDA

( $\mathrm{UCi} / \mathrm{L}$ )

$1.8267 \mathrm{E}+01$

$1.9473 \mathrm{E}-01$

$9.3957 \mathrm{E}-02$

$3.9611 \mathrm{E}+00$

1. $2389 \mathrm{E}+01$

$2.9556 \mathrm{E}-01$

$2.8714 \mathrm{E}-01$

9.5960E-01

$2.9771 \mathrm{E}-01$

4. $3336 \mathrm{E}-01$

$1.4619 \mathrm{E}-01$

$1.9944 \mathrm{E}+00$

$1.5156 \mathrm{E}+00$

$9.0457 \mathrm{E}-02$

2. $6873 \mathrm{E}-01$

7. $3982 \mathrm{E}-01$

$1.7086 \mathrm{E}+00$

$2.2266 \mathrm{E}+01$

3. $5269 \mathrm{E}-01$

$3.0774 \mathrm{E}+01$

$2.2717 \mathrm{E}+00$

$2.3345 \mathrm{E}+00$

$9.7080 \mathrm{E}-01$

$1.1032 \mathrm{E}+00$

$5.8717 \mathrm{E}+00$

$3.0641 \mathrm{E}+02$

$1.7062 \mathrm{E}+00$

$1.0926 \mathrm{E}+00$

$5.2483 \mathrm{E}+00$

$8.5479 \mathrm{E}-02$

1. $4540 \mathrm{E}+01$

$5.1817 \mathrm{E}-01$

$5.6485 \mathrm{E}-01$

$4.0705 \mathrm{E}+00$

1. $4320 \mathrm{E}+00$

$1.8524 \mathrm{E}+01$

$5.2087 \mathrm{E}+00$

$2.6243 \mathrm{E}+00$

$2.4473 \mathrm{E}+00$

$6.7471 \mathrm{E}+00$

$2.9041 \mathrm{E}+01$

$2.8677 \mathrm{E}+01$

$9.9654 \mathrm{E}-01$

$9.4765 \mathrm{E}+01$

$4.4584 \mathrm{E}+00$

3. $2093 \mathrm{E}+00$

$1.0661 \mathrm{E}+03$

4. $1073 \mathrm{E}-01$

$5.3701 \mathrm{E}+01$

1. $7343 \mathrm{E}+00$

$9.2684 \mathrm{E}+00$ 
Minimum Detectable Activity Report (continuete-SD-WM-DP-202, REV. 1 Page : 4 Sample ID : S96V50-DUP Acquisition date : 19-NOV-1996 17:19:11
Nuclide
NP -239
$\mathrm{PU}-239$
AM-24 1
AM-2 43

Bckgnd

Sum

10397.
$11960^{\circ}$
9439.
9634.

10397.

9439.

9634 .

$\begin{aligned} & \text { Energy } \\ & \text { (kev) }\end{aligned}$
106.12
129.30
59.54
74.67
MDA ( $u \mathrm{Ci} / \mathrm{L}$ )

$3.8535 \mathrm{E}+00$

$1.2772 \mathrm{E}+04$

$8.4968 \mathrm{E}+00$

2. $5157 \mathrm{E}+00$ 
$>>>>>>>>>$ SAMPLE INFORMATION $<<<<<<<<<$

Worklist \#:

Sample ID:

Sample Size:

Dilution Factor:

$$
>>>>>>>>\text { COUNT }
$$

Detector ID:

File Number:

Geometry:

Count Time:

Real Time:

Dead Time:
14453

S96V51-SAM

$2.50000 \mathrm{E}-04 \mathrm{~L}$

$1.00000 \mathrm{E}+00$
HNFE-SD-WRM-DE-202 REV. 1

sLuale

Verified by:

GEA2

dka $300:[$ spec.GEA2 ] 2g2946.cnf

42

$000: 50: 00.00 \mathrm{sec}$

$000: 50: 55.61 \mathrm{sec}$

$1.8 \%$

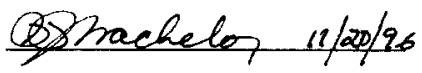

$>>>>>>>>$ ANALYSIS INFORMATION $<<<<<<<<<~$

Sample Count Time: 19-NOV-1996 18:51:48.98

Decayed to:

Standard Deviations: 2

Analysis Library: ENVGEA

Analyst: SLH2

Background Subtract: DKA300:[SPEC.GEA2]2GBACK

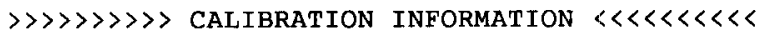

Date of last energy calibration: 21-MAR-1994 09:31:55.15

Date of last efficiency calibration: 21-MAR-1994 09:43:42.61

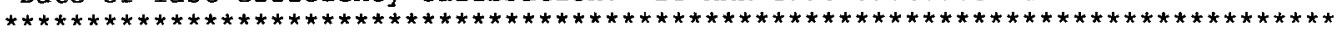

Post-NID Peak Search Report

It Energy Area FWHM Channel Left Pw oErr Fit Nuclides Activity $\mathrm{uCi} / \mathrm{L}$

$\begin{array}{rrrrrrrrrr}0 & 661.68 * & 412033 & 1.53 & 1323.37 & 1315 & 17 & 0.3 & C S-137 & 2.287 \mathrm{E}+03 \\ 0 & 1322.77 & 42 & 2.85 & 2645.69 & 2641 & 11 & 47.9 & & \end{array}$


Total number of lines in spectrum 2

Number of unidentified lines

Number of lines tentatively identified by NID $1 \quad 50.00 \%$

Nuclide Type :

\begin{tabular}{|c|c|c|c|c|c|c|c|}
\hline \multirow[t]{2}{*}{$\begin{array}{l}\text { Nuclide } \\
\text { CS-137 }\end{array}$} & $\begin{array}{r}\text { Hlife } \\
30.00 Y\end{array}$ & $\begin{array}{l}\text { Decay } \\
1.000\end{array}$ & $\begin{array}{c}\text { Uncorrected } \\
\text { uCi } / \mathrm{L} \\
2.287 \mathrm{E}+03\end{array}$ & $\begin{array}{c}\text { Decay Corr } \\
\text { uCi } / \mathrm{L} \\
2.287 \mathrm{E}+03\end{array}$ & $\begin{array}{c}\text { Decay Corr } \\
\text { 2-Sigma Error } \\
0.007 \mathrm{E}+03\end{array}$ & $\begin{array}{c}2 \text {-Sigma } \\
\text { s. Error } \\
0.32\end{array}$ & Flags \\
\hline & Total Act & ity : & $2.287 \mathrm{E}+03$ & $2.287 E+03$ & & & \\
\hline
\end{tabular}

Grand Total Activity : $2.287 \mathrm{E}+03 \quad 2.287 \mathrm{E}+03$

Flags: " $K$ " = Keyline not found

"M" = Manually accepted

$" E "$ = Manually edited

"A" = Nuclide specific abn. limit 
Minimum Detectable Activity Report Sample ID : S96V51-SAM

Nuclide

\begin{tabular}{|c|c|}
\hline 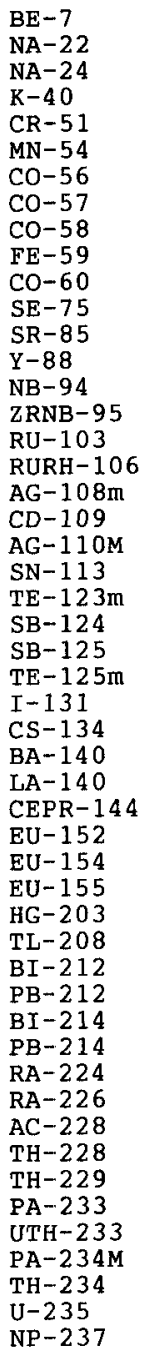 & $\begin{array}{r}12767 . \\
18 . \\
5 . \\
116 . \\
10762 . \\
113 . \\
129 . \\
11845 . \\
140 . \\
39 . \\
14 . \\
12078 . \\
6565 . \\
6 . \\
103 . \\
171 . \\
8220 . \\
2856 . \\
157 . \\
10360 . \\
9744 . \\
11613 . \\
11817 . \\
2879 . \\
13150 . \\
10957 . \\
10956 . \\
2856 . \\
4952 . \\
3 . \\
11946 . \\
9 . \\
10 . \\
10601 . \\
11340 . \\
11416 . \\
198 . \\
13459 . \\
2855 . \\
10721 . \\
13333 . \\
15420 . \\
112 . \\
10250 . \\
10350 . \\
10783 . \\
13019 . \\
71 . \\
9696 . \\
15255 . \\
10190 .\end{array}$ \\
\hline
\end{tabular}

\section{Energy ( $\mathrm{keV}$ )}

477.59

1274.53

1368.55

1460.75

320.08

834.83

846.76

122.06

810.78

1099.25

1332.50

264.66

514.01

1836.06

871.09

724.18

497.08

621.93

722.94

88.03

657.76

391.69

159.00

602.73

427.89

109.27

364.48

604.70

537.31

1596.21.

133.51

1408.01

1274.51

105.31

279.20

277.36

727.18

238.63

609.31

351.92

240.99

186.10

911.21

84.37

88.47

312.17

245.34

1001.03

63.29

185.71

86.48
MDA

( $\mathrm{UCi} / \mathrm{L})$

$1.8333 \mathrm{E}+01$

1. $6668 \mathrm{E}-01$

$9.3958 \mathrm{E}-02$

4. $3879 \mathrm{E}+00$

1. $2558 \mathrm{E}+01$

$2.8442 \mathrm{E}-01$

$3.0815 \mathrm{E}-01$

$9.7342 \mathrm{E}-01$

3. $1053 \mathrm{E}-01$

3. $7849 \mathrm{E}-01$

$1.5143 \mathrm{E}-01$

$2.0082 \mathrm{E}+00$

1. $5100 \mathrm{E}+00$

1. 2912E-01

$2.8199 \mathrm{E}-01$

$7.0962 \mathrm{E}-01$

1. $7327 \mathrm{E}+00$

2. $2519 \mathrm{E}+01$

3. 2729E-01

3. $1259 \mathrm{E}+01$

$2.2838 \mathrm{E}+00$

$2.3722 \mathrm{E}+00$

$9.7286 \mathrm{E}-01$

$1.1178 \mathrm{E}+00$

$5.9238 \mathrm{E}+00$

$3.1034 \mathrm{E}+02$

$1.7260 \mathrm{E}+00$

$1.1185 \mathrm{E}+00$

$5.3601 \mathrm{E}+00$

8. $5479 \mathrm{E}-02$

1. $4610 \mathrm{E}+01$

$5.9430 \mathrm{E}-01$

4. $8212 \mathrm{E}-01$

$4.0997 \mathrm{E}+00$

$1.4478 \mathrm{E}+00$

1. $8665 \mathrm{E}+01$

$5.0450 \mathrm{E}+00$

$2.6480 \mathrm{E}+00$

$2.4513 \mathrm{E}+00$

$6.7895 \mathrm{E}+00$

$2.9252 \mathrm{E}+01$

$2.8976 \mathrm{E}+01$

$1.1483 \mathrm{E}+00$

$9.5474 \mathrm{E}+01$

$4.5291 \mathrm{E}+00$

$3.2232 \mathrm{E}+00$

$1.0827 \mathrm{E}+03$

$4.4923 \mathrm{E}-01$

$5.4092 \mathrm{E}+01$

$1.7518 \mathrm{E}+00$

9. $3027 \mathrm{E}+00$ 
Minimum Detectable Activity Report (continueNG-SD-WM-DP-202, REV. 1 Page : 4 Sample ID : S96V51-SAM

Acquisition date : 19-NOV-1996 18:51:48

Nuclide

NP -239

PU-239

AM-241

$\mathrm{AM}-243$

\section{Bckgnd}

sum

$$
\begin{array}{r}
10677 . \\
12062 . \\
9607 . \\
9896 .
\end{array}
$$

\section{Energy}

(kev)

106.12

129.30

59.54

74.67

\section{MDA}

( $\mathrm{UCi} / \mathrm{L})$

3. $9050 \mathrm{E}+00$

1. $2826 \mathrm{E}+04$

8. $5719 \mathrm{E}+00$

2. $5496 \mathrm{E}+0 \mathrm{O}$ 
Worklist \#:

Sample ID:

Sample Size:

Dilution Factor:

$>>>>>>>>$ COUNT INFORMATION $<<<<<<<<<$

Detector ID:

File Number:

Geometry:

Count Time:

Real Time:

Dead Time:
14453

S96V52-SAM

2. 50000E-04 L

1. $00000 \mathrm{E}+00$

GEA2

dka300:[spec.GEA2]2g2947.cnf

42

0 00:50:00.00 sec

$0 \quad 00: 50: 54.37$ sec

1. $8 \%$
Removed by:

SLClete

Verified by:

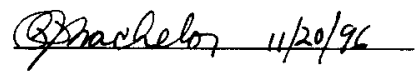

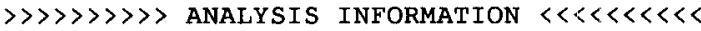
Sample Count Time:
$\begin{array}{ll}19-N O V-1996 & 19: 46: 02.35 \\ 19-N O V-1996 & 19: 46: 02.35\end{array}$
Decayed to:
Standard Deviations:
Analysis Library:
Analyst:
2
ENVGEA
SLH2
Background subtract: DKA300: [SPEC.GEA2]2GBACK

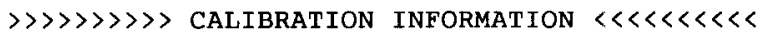 \\ Date of last energy calibration: $2 . L$-MAR-1994 09:31:55.15 \\ Date of last efficiency calibration: 2 l-MAR-1994 09:43:42.61}

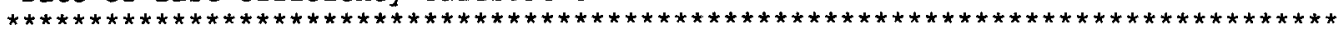

Post-NID Peak Search Report

\begin{tabular}{|c|c|c|c|c|c|c|c|c|c|c|}
\hline It & Energy & Area & FWHM & Channel & Left & Pw & 음 & Fit & Nuclides & $\begin{array}{c}\text { Activity } \\
\text { uCi/L }\end{array}$ \\
\hline 0 & $661.68 *$ & 403065 & 1.54 & 1323.38 & 1315 & 17 & 0.3 & & $\mathrm{CS}-137$ & $2.237 \mathrm{E}+03$ \\
\hline
\end{tabular}


Summary of Nuclide Activity

Sample ID : S96V52-SAM
$W N E$

AtHE-SD-WMi-DP-202, REV.

Page : 2

Acquisition date : 19-NOV-1996 19:46:02

Total number of lines in spectrum 1

Number of unidentified lines

Number of lines tentatively identified by NID $1 \quad 100.00 \%$

Nuclide Type :

\begin{tabular}{|c|c|c|c|c|c|c|c|}
\hline $\begin{array}{l}\text { Nuclide } \\
\text { CS-137 }\end{array}$ & $\begin{array}{r}\text { Hlife } \\
30.00 Y\end{array}$ & $\begin{array}{l}\text { Decay } \\
1.000\end{array}$ & $\begin{array}{c}\text { wtd Mean } \\
\text { Uncorrected } \\
\text { uCi/L } \\
2.237 \mathrm{E}+03\end{array}$ & $\begin{array}{c}\text { Wtd Mean } \\
\text { Decay Corr } \\
\text { uCi } / \mathrm{L} \\
2.237 \mathrm{E}+03\end{array}$ & $\begin{array}{c}\text { Decay Corr } \\
\text { 2-Sigma Error } \\
0.007 \mathrm{E}+03\end{array}$ & $\begin{array}{c}2 \text {-Sigma } \\
\text { s:Error } \\
0.32\end{array}$ & Flags \\
\hline & Total Act & ity : & $2.237 \mathrm{E}+03$ & $2.237 \mathrm{E}+03$ & & & \\
\hline
\end{tabular}

Grand Total Activity : $2.237 \mathrm{E}+03$

$2.237 \mathrm{E}+03$

Flags: "K" = Keyline not found

"M" = Manually accepted

"E" = Manually edited

"A" = Nuclide specific abn. limit 
Minimum Detectable Activity Report Sample ID : S96V52-SAM

Nuclide

$\mathrm{BE}-7$

NA -22

$\mathrm{NA}-24$

$\mathrm{K}-40$

CR-51

$\mathrm{MN}-54$

$\mathrm{CO}-56$

$\mathrm{CO}-57$

$\mathrm{CO}-58$

FE -59

CO-60

$\mathrm{SE}-75$

$\mathrm{SR}-85$

$Y-88$

NB-94

ZRNB-95

RU-103

RURH-106

AG $-108 \mathrm{~m}$

$\mathrm{CD}-109$

AG- $110 \mathrm{M}$

$\mathrm{SN}-113$

$\mathrm{TE}-123 \mathrm{~m}$

SB-124

SB-125

TE $-125 \mathrm{~m}$

$I-131$

CS-134

$\mathrm{BA}-140$

LA-140

CEPR-144

EU-152

EU-154

EU-155

HG -203

TL-208

BI -212

PB-2 12

BI-214

PB-214

RA-224

RA-226

AC- 228

$\mathrm{TH}-228$

$\mathrm{TH}-229$

PA-233

UTH -233

$\mathrm{PA}-234 \mathrm{M}$

$\mathrm{TH}-234$

U-235

NP -237

\section{Bckgnd \\ sum}

12628 .

21 .

4.

105.

10621 .

115.

122 .

11430 .

122 .

31 .

11 .

11728 .

6426 .

2 .

97.

194 .

8036.

2786 .

214 .

10157 .

9391 .

11285.

11665 .

2842 .

13075 .

10744 .

10793 .

2809 .

4683 .

6.

11685.

5 .

22 .

10540 .

11220 .

11281.

188 .

13204 .

2795 .

10615 .

12972 .

15069 .

72 .

10031 .

10147.

10734 .

12687 .

56.

9424 .

14986 .

10090 .

\section{Energy (kev)}

477.59

1274.53

1368.55

1460.75

320.08

834.83

846.76

122.06

810.78

1099.25

1332.50

264.66

514.01

1836.06

871.09

724.18

497.08

621.93

722.94

88.03

657.76

391.69

159.00

602.73

427.89

109.27

364.48

604.70

537.31

1596.21

133.51

1408.01

1274.51

105.31

279.20

277.36

727.18

238.63

609.31

351.92

240.99

186.10

911.21

84.37

88.47

312.17

245.34

1001.03

63.29

185.71

86.48
Page : 3

Actuisition date : 19-NOV-1996 19:46:02

\section{MDA \\ $(\mathrm{uCi} / \mathrm{L})$}

$1.8233 \mathrm{E}+01$

$1.8094 \mathrm{E}-01$

$7.9266 \mathrm{E}-02$

4. $1860 \mathrm{E}+00$

1. $2475 \mathrm{E}+01$

2.8711E-01

$2.9965 \mathrm{E}-01$

$9.5622 \mathrm{E}-01$

2. $9015 \mathrm{E}-01$

3. $3908 \mathrm{E}-01$

$1.3423 \mathrm{E}-01$

$1.9788 \mathrm{E}+00$

$1.4939 \mathrm{E}+00$

$7.4519 \mathrm{E}-02$

$2.7417 \mathrm{E}-01$

$7.5789 \mathrm{E}-01$

1. $7131 \mathrm{E}+00$

2. $2239 \mathrm{E}+01$

$3.8154 \mathrm{E}-01$

$3.0951 \mathrm{E}+01$

2. $2421 \mathrm{E}+00$

$2.3384 \mathrm{E}+00$

$9.6661 \mathrm{E}-01$

1. $1106 \mathrm{E}+00$

$5.9068 \mathrm{E}+00$

$3.0731 \mathrm{E}+02$

$1.7131 \mathrm{E}+00$

$1.1092 \mathrm{E}+00$

$5.2121 \mathrm{E}+00$

$1.2089 \mathrm{E}-01$

$1.4450 \mathrm{E}+01$

$4.5305 \mathrm{E}-01$

$5.2618 \mathrm{E}-01$

$4.0879 \mathrm{E}+00$

$1.4401 \mathrm{E}+00$

$1.8554 \mathrm{E}+01$

4. $9143 \mathrm{E}+00$

$2.6228 \mathrm{E}+00$

$2.4254 \mathrm{E}+00$

$6.7543 \mathrm{E}+00$

$2.8853 \mathrm{E}+01$

$2.8643 \mathrm{E}+01$

$9.2257 \mathrm{E}-01$

$9.4449 \mathrm{E}+01$

$4.4844 \mathrm{E}+00$

3. $2160 \mathrm{E}+00$

$1.0688 \mathrm{E}+03$

3. $9854 \mathrm{E}-01$

$5.3329 \mathrm{E}+01$

$1.7362 \mathrm{E}+00$

9. $2571 \mathrm{E}+00$ 
Minimum Detectable Activity Report (continued)

Nuclide

$\begin{array}{rr}N P-239 & 10493 . \\ P U-239 & 11760 . \\ A M-241 & 9351 . \\ A M-243 & 9684 .\end{array}$

Bckgnd sum

10493 .

1760 .

9684 .
Energy

(kev)

106.12

129.30

59.54

74.67
MDA ( $\mathrm{UCi} / \mathrm{L}$ )

$3.8712 \mathrm{E}+00$

$1.2665 \mathrm{E}+04$

$8.4569 \mathrm{E}+00$

$2.5221 \mathrm{E}+00$ 
* 222-S Laboratory Counting Room

$>>>>>>>>>>$ SAMPLE INFORMATION $<<<<<<<<<<$

Worklist \# :

Sample ID:

Sample Size:

Dilution Factor:

$\gg \gg \gg \gg \gg \gg \gg$ COUNT

Detector ID:

File Number:

Geometry:

Count Time:

Real Time:

Dead Time:
14453

S97V60-SAM

$2.50000 \mathrm{E}-04 \mathrm{~L}$

$1.00000 \mathrm{E}+00$
Removed by:

$8 k i_{t} \ell_{L} l$

Verified by:

GEA2

dka $300:[$ spec.GEA2 ]2g2948.cnf

42

0 00:50:00.00 sec

$000: 50: 00.48 \mathrm{sec}$

$0.0 \%$

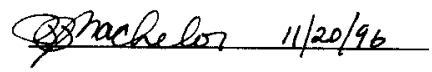

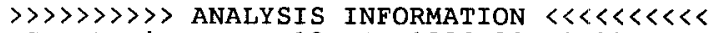

Sample Count Time: 19-NOV-1996 20:59:39.72

Decayed to: 19-NOV-1996 20:59:39.72

Standard Deviations: 2

Analysis Library: ENVGEA

Analyst: $\quad$ SLH2

Background Subtract: DKA300:[SPEC.GEA2 ]2GBACK

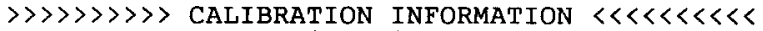

Date of last energy calibration: 21-MAR-1994 09:31:55.15

Date of last efficiency calibration: 21-MAR-1994 09:43:42.61

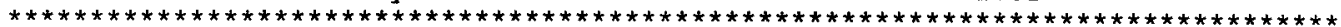

Post-NID Peak Search Report

It Energy Area FWHM Channel Left Pw oferr Fit Nuclides Activity $\mathrm{uCi} / \mathrm{L}$

032.74 *

$\begin{array}{lll}78 & 2.18 & 66.03\end{array}$

$61 \quad 1386.5$ 
Total number of lines in spectrum Number of unidentified lines

Number of lines tentatively identified by NID 0

**** There are no nuclides meeting summary criteria

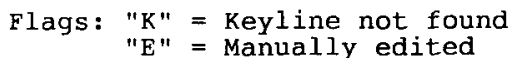

"N" = Manually accepted

" $A^{\prime} "$ = Nuclide specific abn. limit 
Minimum Detectable Activity Report Sample ID : S97V60-SAM

Nuclide $\quad \begin{gathered}\text { Bckgnd } \\ \text { Sum }\end{gathered}$

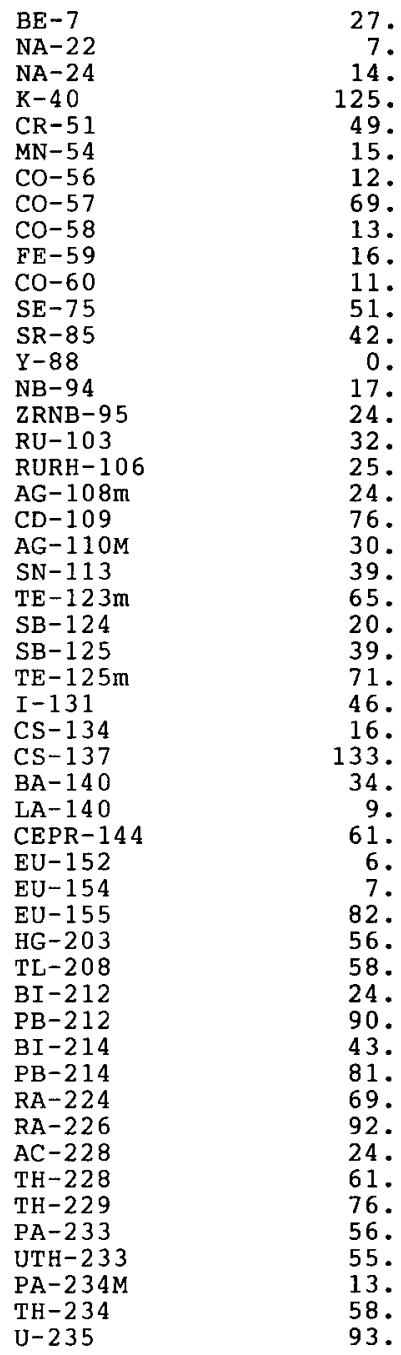

\section{Energy (kev)}

477.59

1274.53

1368.55

1460.75

320.08

834.83

846.76

122.06

810.78

1099.25

1332.50

264.66

514.01

1836.06

871.09

724.18

497.08

621.93

722.94

88.03

657.76

391.69

159.00

602.73

427.89

109.27

364.48

604.70

661.66

537.31

1596.21

133.51

1408.01

1274.51

105.31

279.20

277.36

727.18

238.63

609.31

351.92

240.99

186.10

911.21

84.37

88.47

312.17

245.34

1001.03

63.29

185.71
Page : $\quad 3$

\author{
MDA \\ ( $\mathrm{uCi} / \mathrm{L}$ )
}

8.4955E-01

$1.0487 \mathrm{E}-01$

1. $5504 \mathrm{E}-01$

4. $5555 \mathrm{E}+00$

8.5132E-01

$1.0353 \mathrm{E}-01$

$9.4030 \mathrm{E}-02$

$7.4301 \mathrm{E}-02$

$9.3766 \mathrm{E}-02$

$2.4437 \mathrm{E}-01$

1. $3423 \mathrm{E}-01$

1. $3107 \mathrm{E}-01$

1. $2120 \mathrm{E}-01$

$0.0000 \mathrm{E}+00$

$1.1385 \mathrm{E}-01$

$2.6494 \mathrm{E}-01$

$1.0773 \mathrm{E}-01$

$2.1158 \mathrm{E}+00$

$1.2726 \mathrm{E}-01$

$2.6811 \mathrm{E}+00$

$1.2646 \mathrm{E}-01$

$1.3781 \mathrm{E}-01$

7.2203E-02

$9.2170 \mathrm{E}-02$

$3.2107 \mathrm{E}-01$

$2.4954 \mathrm{E}+01$

$1.1221 \mathrm{E}-01$

8. 3545E-02

$2.9774 \mathrm{E}-01$

4. $4204 \mathrm{E}-01$

$1.4857 \mathrm{E}-01$

$1.0477 \mathrm{E}+00$

4. $9890 \mathrm{E}-01$

3. $0552 \mathrm{E}-01$

3. $6033 \mathrm{E}-01$

1. $0162 \mathrm{E}-01$

$1.3256 \mathrm{E}+00$

$1.7488 \mathrm{E}+00$

2. $1651 \mathrm{E}-01$

2. $9939 \mathrm{E}-01$

$5.8583 \mathrm{E}-01$

$2.0991 \mathrm{E}+00$

$2.2372 \mathrm{E}+00$

$5.3631 \mathrm{E}-01$

7. $3458 \mathrm{E}+00$

3. $8750 \mathrm{E}-01$

2. $3232 \mathrm{E}-01$

$7.0145 \mathrm{E}+01$

$1.8963 \mathrm{E}-01$

$4.1671 \mathrm{E}+00$

1. $3696 \mathrm{E}-01$ 
Minimum Detectable Activity Report (continue HftC-SD-WM-DP-202, REV. 1 Page : 4 Sample ID : S97V60-SAM

Acquisition date : 19-NOV-1996 20:59:39

Nuclide

$\mathrm{NP}-237$

$\mathrm{NP}-239$

PU-239

$A M-241$

AM-2 43

\section{Bckgnd}

Sum

72 .

73.

60 .

61 .

68 .
Energy

(kev)

86.48

106.12

129.30

59.54

74.67
MDA

( $\mathrm{uCi} / \mathrm{L}$ )

7. $8172 \mathrm{E}-01$

3. $2342 \mathrm{E}-01$

$9.0607 \mathrm{E}+02$

6.8495E-01

2. 1151E-01 


\title{
LABCORE Completed RadChem Report for Worklist\#: 12887
}

Analyst: slh

\author{
Instrument: GEA06
}

Book\#

Method: Rev/Mod

Worklist Comment: AP-105. Sample size $=1.0 \mathrm{ml}$. new

\begin{tabular}{|c|c|c|c|c|c|c|c|c|c|}
\hline Seq Typé & Sample & $\mathbf{R A}$ & & Test & Matrix & Actual & Found & DL or Yield & Unit \\
\hline 1 STP & & 0 & $01129-01$ & $\pi 129+01$ & ITOUTD & 7. $8618-04$ & 5.70z-4 & 72.519 & \& Recovary \\
\hline ITt & & 0 & - $1129-01$ & I129-01C & LIQUID & 100 & $6.075+01$ & 60.700 & * Recovery \\
\hline 1 STP & & 0 & $0 T 129-01$ & $1129-013$ & LIQUID & 1.00 & $128 z+00$ & 1280 & Ct Krro \\
\hline 2 BLNK & & 0 & I129-01 & $1129-01$ & LIOUID & 1 & $<1.46 \mathrm{E}-5$ & & $\mathrm{uCt} / \mathrm{mL}$ \\
\hline 2 Bunk & & 0 & $1129-01$ & I129-01E & LIQDID & 2.00 & $0.00 \mathrm{E}+00$ & 0.000 & $\mathrm{UCA} / \mathrm{mL}$ \\
\hline 3 SAMPLEF & 5960000048 & 0 & QI129-01 & $1129-01$ & LIQUUID & $\mathbf{N} / \mathbf{A}$ & $1.40 \mathrm{E}-04$ & $522.00-008$ & $\mathrm{uCl} / \mathrm{mL}$ \\
\hline 3 saypat & s96v000048 & 0 & $4+129-01$ & $+229-0 x \mathrm{C}$ & ITQUTD & N/A & $5.0 \mathrm{BB}+02$ & $0.00+000$ & \& Rocovery. \\
\hline 3 SAMPLE & S96v000048 & 0 & $01129-01$ & I129-01B & LIQUTD & $\mathbf{n} / \mathbf{A}$ & $3,25 \mathrm{~F}+00$ & $0.00+000$ & \& Ct. Error \\
\hline 4 DUT & 8960000048 & 0 & $01129-01$ & $I 129-0 \mathrm{t}$ & IIOUTD & $1.408-4$ & $1<4 E-4$ & 2.817 & RPD \\
\hline $4 \mathrm{DUP}$ & s96v000048 & 0 & $01129-01$ & I129-01C & LIQUID & 100 & $4.985+01$ & 49.800 & \& Recovery \\
\hline 4 DIP & 5960000048 & 0 & $0 \pi 129-01$ & $7129-013$ & LIQUID & 1.00 & $3.15 \mathrm{~F}+00$ & 3.150 & Q Ct Erro \\
\hline $5 \mathrm{SPR}$ & $s 960000048$ & 0 & TI129-01 & $1129-01$ & LIQUID & $7.86 \mathrm{~B}-0.4$ & $6.23 \mathrm{~F}-04$ & 79.262 & \& Recovery \\
\hline $6 S \mathrm{SP}-\mathrm{DtP}$ & 5960000048 & 0 & $\$ 1129-01$ & I129-01 & IIQUID & $6 \cdot 23 \mathrm{~B}-04$ & $6,16 \mathrm{x}-04$ & 1.130 & PQPD \\
\hline 7 SAMPLE & 5960000049 & 0 & $01129-01$ & $1129-01$ & LIQUID & $N / A$ & $1.19 \mathrm{R}-04$ & $433.00-008$ & $\mathrm{uCl} / \mathrm{ms}$ \\
\hline 7 saupts: & 5960000049 & 0 & $0+129-01$ & I129-01C & tretto & $w / A$ & $6.13 x+01$ & $0.00+000$ & R Rocovery \\
\hline 7 SAMPLE & s96v000049 & 0 & $01129-01$ & I129-01E & IIQUID & $N / A$ & $3.26 \mathrm{E}+00$ & $0.00+000$ & \& Ct. Error \\
\hline
\end{tabular}

\section{Final page for worklist\# 12887}

\section{Analyst Signature}

Date
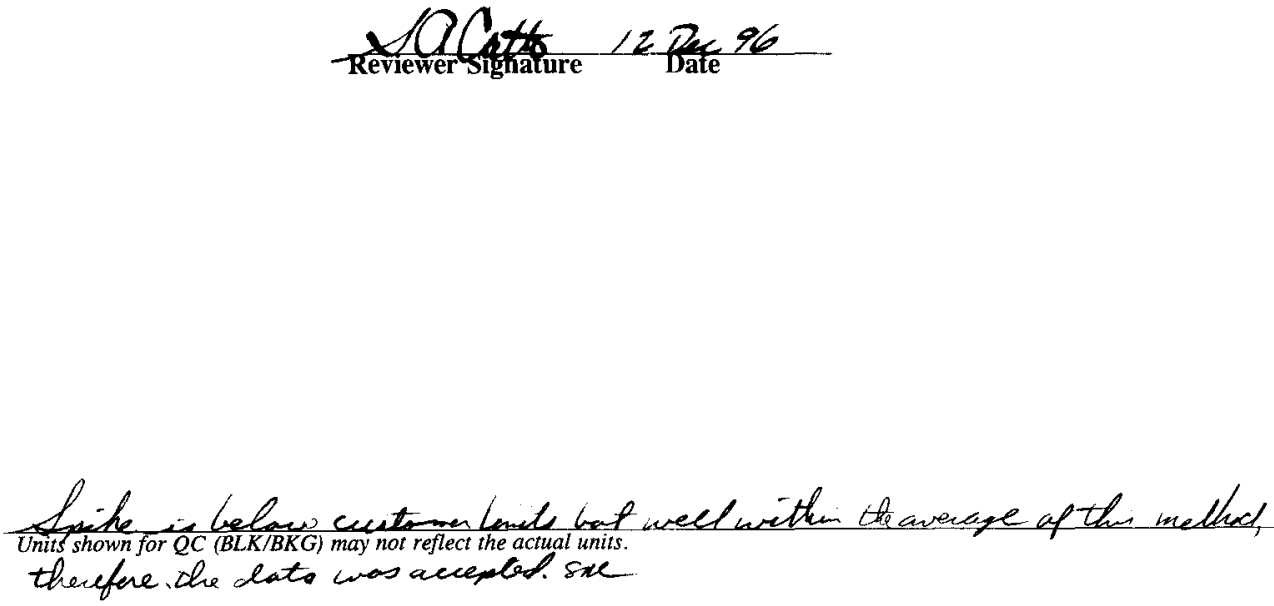


\section{LABCORE Data Entry Template for Worklist\# 12887}

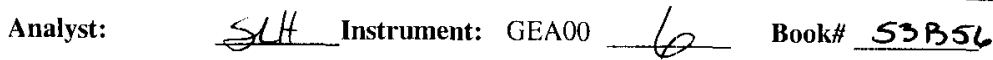

Method: LA-378-103 Rev/Mod C-O

Worklist Comment: AP-105. Sample size $=1.0 \mathrm{ml}$. new

\begin{tabular}{|c|c|c|c|c|c|c|c|}
\hline $\bar{s}$ & TYpe & Sample\# & $\mathrm{RA}$ & Test & Matrix & Group\# & Project \\
\hline 1. & STD & & & $@ 1129-01$ & IIQUID & & \\
\hline 2 & BLNK & & & $@ I 129-01$ & LIQUID & & \\
\hline \multirow[t]{2}{*}{3} & SAMPIE & S96V000048 & 0 & $@ I 129-01$ & LIQUID & 96000855 & $A P-105$ \\
\hline & & Analytes Reque & ested: & $=1129-0 I$ & I $129-01 \mathrm{C}$ & I129-01E & \\
\hline 4 & DUP & $596 \vee 000048$ & 0 & $@ 1129-01$ & LIQUTD & & \\
\hline 5 & SPK & S96V000048 & 0 & @I129-01 & LIQUID & & \\
\hline 6 & SPK-DUP & S96V000048 & 0 & $@ I 129-01$ & LIQUID & & \\
\hline 7 & SAMPLE & S96V000049 & 0 & @I $129-01$ & LIQUID & 96000855 & $A P-105$ \\
\hline & & Analytes Reque & sted: & $=$ I129-01 & , I129-01C. & I129-01E & \\
\hline
\end{tabular}

\section{Final page for worklist \# $\mathbf{1 2 8 8 7}$}
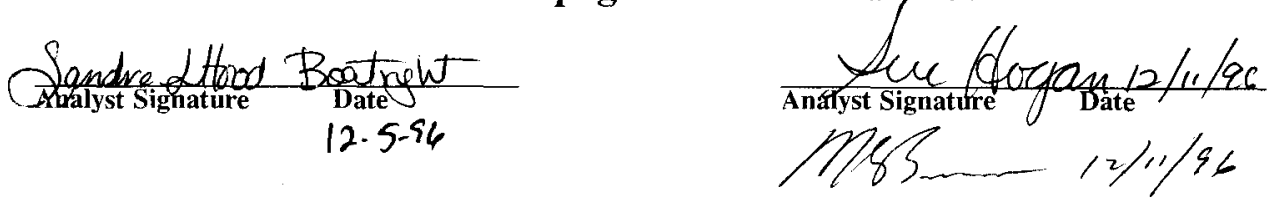

Data Entry Comments:

$S=$ Worklist Slot Number, $R=$ Replicate Number, $A=$ Aliquot Code. 
WORKBOOK PAGE: STQ1

\begin{tabular}{|c|c|c|}
\hline |-129: LA-378-10 & IODINE-129 & STANDARD \\
\hline 28 & GROSS WEIGHT & 2.889 \\
\hline STD & TARE WEIGHT & 2atog \\
\hline 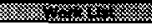 & MASS PRECIPITATE & 0.0186 \\
\hline 12887 & 1-129 $\mu \mathrm{Ci} / \mathrm{SAMPLE}$ from GEA & 30004 \\
\hline 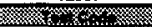 & SAMPLE VOLUME in $\mathrm{mL}$ & 1,0000 \\
\hline PI129-01 & DILLUTION FACTOR & 10000 \\
\hline 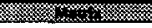 & DIGEST DILUTION FACTOR & 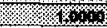 \\
\hline LIQUID & CARRIER FRACTIONAL RECOVERY & 0.6066 \\
\hline $\sin$ & COUNT TIME IN MINUTIES & 100 \\
\hline 96009327 & COUNTING UNCERTAINTY & 1,29 \\
\hline In:m & STANDARD BOOK NUMBER & Wasce \\
\hline o & STANDARD VALUE in $\mu \mathrm{Ci} / \mathrm{mL}$. & $7.865-04$ \\
\hline 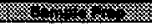 & & \\
\hline NOT LISTED & & \\
\hline 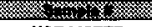 & & \\
\hline NOT LISTED & One $\mathrm{mL}$ of the solution is equivalent to 0.0305 grams. & \\
\hline Wotstost & Carrier Percent Recovery = (ut2-w11) 100100305 & \\
\hline 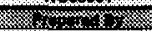 & $1-129 \mu \mathrm{Ci} / \mathrm{mL}=(\mathrm{l}-129 \mathrm{GEA})(\mathrm{DF})(\mathrm{DOF}) /[(\mathrm{Rec})(\mathrm{SS})]$ & \\
\hline SEH & & \\
\hline 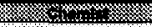 & & \\
\hline SAC & & \\
\hline Q & & \\
\hline stik & & \\
\hline 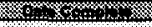 & & \\
\hline Timises & & \\
\hline (2) & & \\
\hline 122006 & & \\
\hline 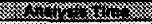 & lodine $129 \ln \mu \mathrm{Cl} / \mathrm{mL}$ & 6.70E-04 \\
\hline 14toon & Carrier Percent Rocovery & $60.7 \%$ \\
\hline 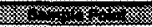 & Counting Uncertainty & $1.3 \%$ \\
\hline Ap os & Standard Recovery & 72.6\% \\
\hline
\end{tabular}

\begin{tabular}{|c|c|c|}
\hline Analyst: & SLH & $12 / 11 / 96$ \\
\hline Signature of Chemist: & $S A C$ & DateEC : 2 MSfi \\
\hline
\end{tabular}




\section{HNE \\ A4G SD-WM-DP-202, REV. i}

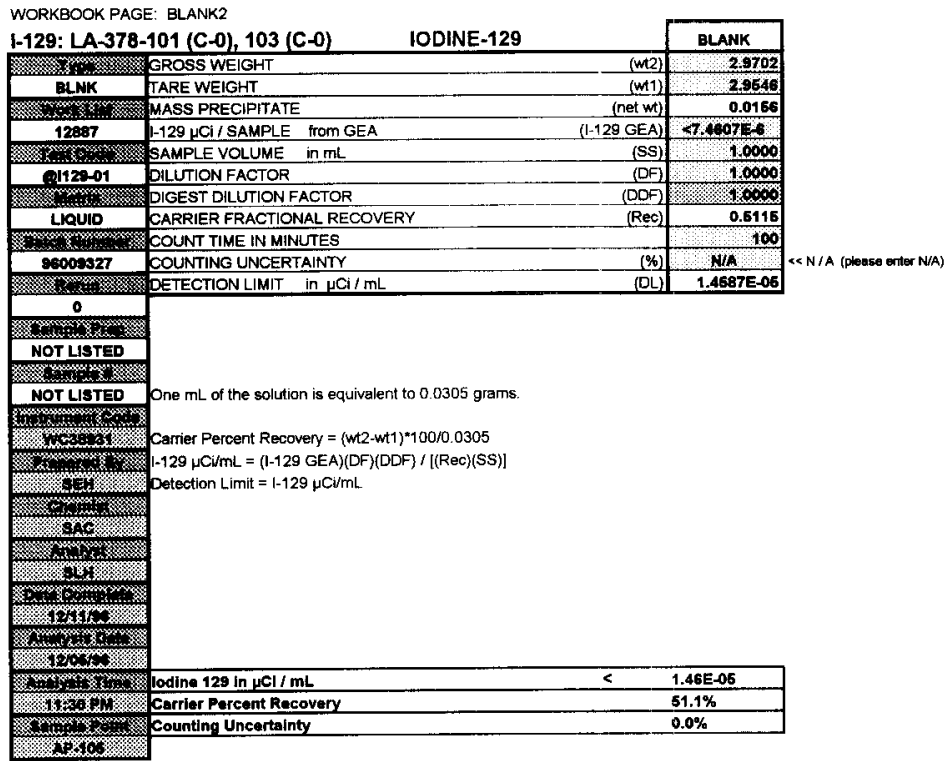

\begin{tabular}{|c|c|c|}
\hline Analyst: & SLH & Date: $12 / 11 / 96$ \\
\hline Signature of Chemist: & SAC & Dato \\
\hline
\end{tabular}

\section{6}




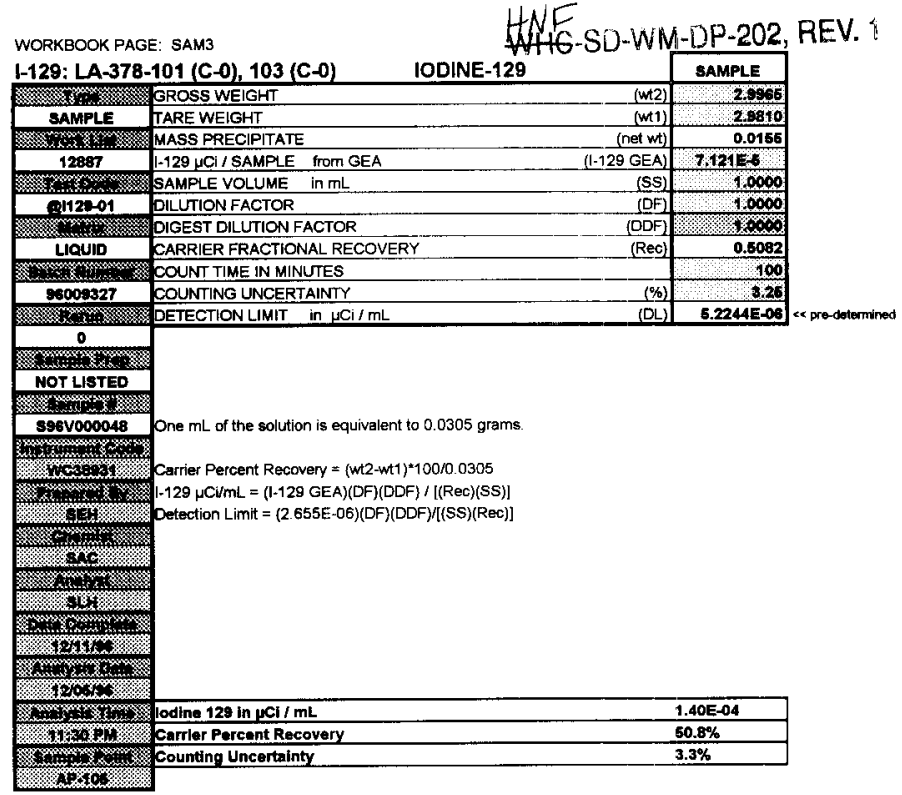

\begin{tabular}{|c|c|c|}
\hline Anatyst: & SLH & Date: $\quad 12 / 11 / 96$ \\
\hline Signature of Chermist: & SAC & Date:C: $12 \quad 193$ \\
\hline
\end{tabular}


WORKBOOK PAGE: DUP4

|-129: LA-378-101 (C-0), 103 (C-0)

IODINE-129

DUPLICATE

\begin{tabular}{|c|c|c|c|}
\hline 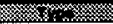 & GROSS WEIGHT & (M2) & 2.8801 \\
\hline DUP & TARE WEIGHT & $(w+1)$ & 2.8649 \\
\hline 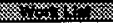 & MASS PRECIPITATE & (net wt) & 0.0152 \\
\hline 12897 & 1-129 $\mu \mathrm{Ci} / \mathrm{SAMPLE}$ from GEA & $(1-129 \mathrm{GEA})$ & $7,195=6$ \\
\hline 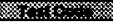 & SAMPLE VOLUME in $\mathrm{mL}$. & (SS) & 10000 \\
\hline :12:-01 & DILUTION FACTOR & (DE) & 1.0000 \\
\hline 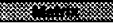 & DIGEST DILUTION FACTOR & (DDF) & $200 \%$ \\
\hline LIQUID & CARRIER FRACTIONAL RECOVERY & $(\operatorname{Rec})$ & 0.4984 \\
\hline 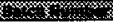 & COUNT TIME IN MINUTES & & 100 \\
\hline 26009327 & COUNTING UNCERTAINTY & {$[\%)$} & 3,16 \\
\hline 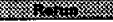 & DETECTION LIMIT in $\mu \mathrm{Ci} / \mathrm{mL}$. & (믄) & 6.3275E-06 \\
\hline
\end{tabular}
0

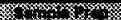 \\ NOT LISTED \\ nom \\ s96v000048}

One $\mathrm{mL}$ of the solution is equivalent to 0.0305 grams

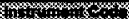

Carrier Percent Recovery $=(w t 2-w+1)^{* 100 / 0.0305}$

Fu $1-129 \mu \mathrm{Ci} / \mathrm{mL}=(\mathrm{l}-129 \mathrm{GEA})(\mathrm{DF})(\mathrm{DDF}) /[(\mathrm{Rec})(\mathrm{SS})]$

.

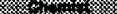

.

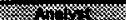

\%

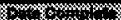

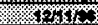

\%

singosis

Yodine $129 \mathrm{ln} \mathrm{HCl} / \mathrm{mL} \quad 1.44 \mathrm{E}-04$

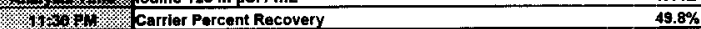

Counting Uncertainty $3.2 \%$

(n) $\mathrm{mos}$.

\begin{tabular}{|c|c|c|}
\hline Analyst: & SLH & Date: $\quad 12 / 11 / 96$ \\
\hline Signature of Chemist: & SAC & Date ${ }^{\mathrm{t}} \mathrm{r}, \mathrm{I}$ \\
\hline
\end{tabular}


Date : 25-SEP-1996 10*56

Client ID: VBLKOT

Sample Info:

Purge Volume: 5.0

Column phase: db-624
Instrument: troi.i

Operator: den

Column diameter: 0.25

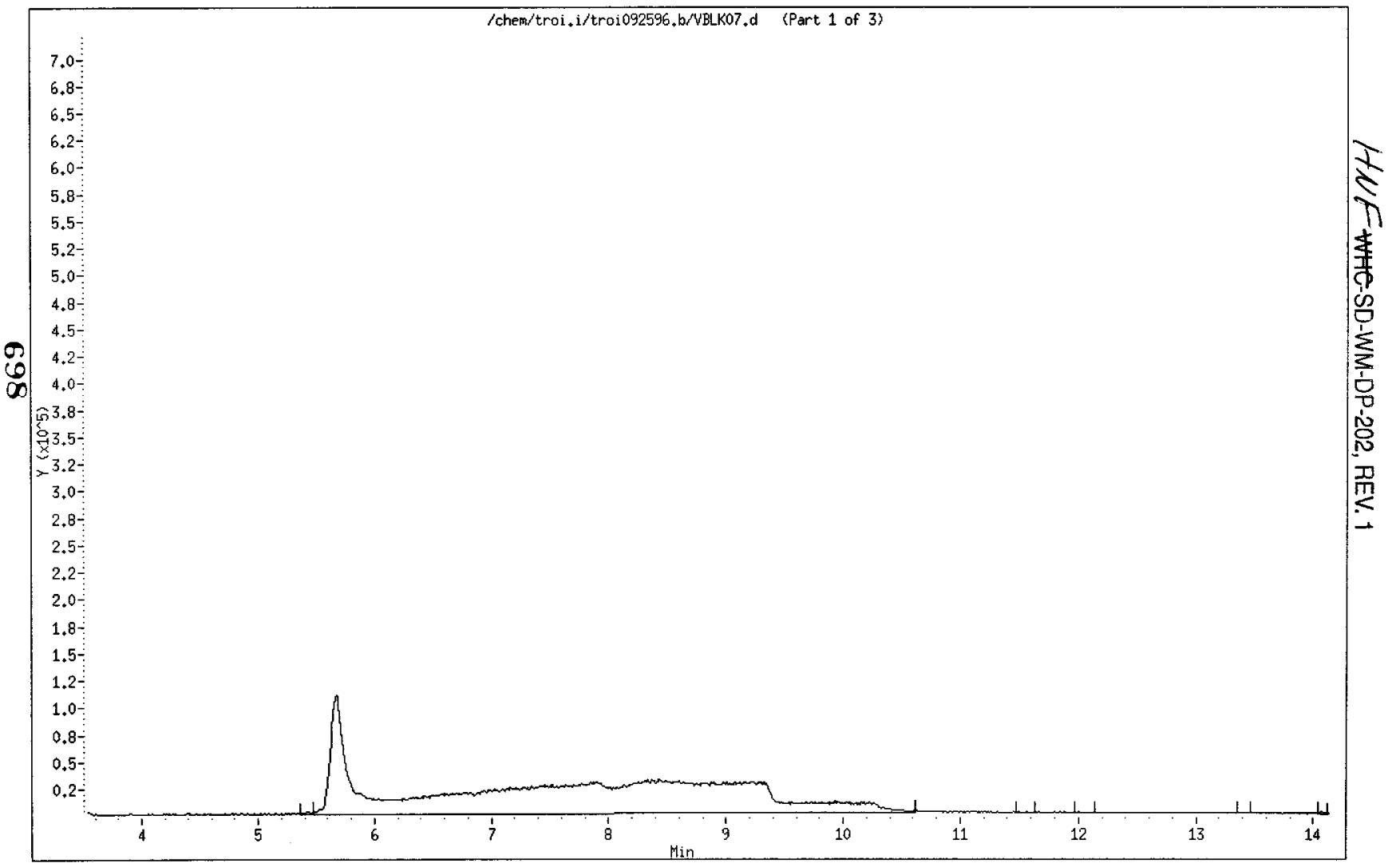


IH F F C-SD-WM-DP-202, REV. 1

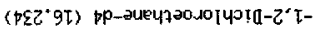
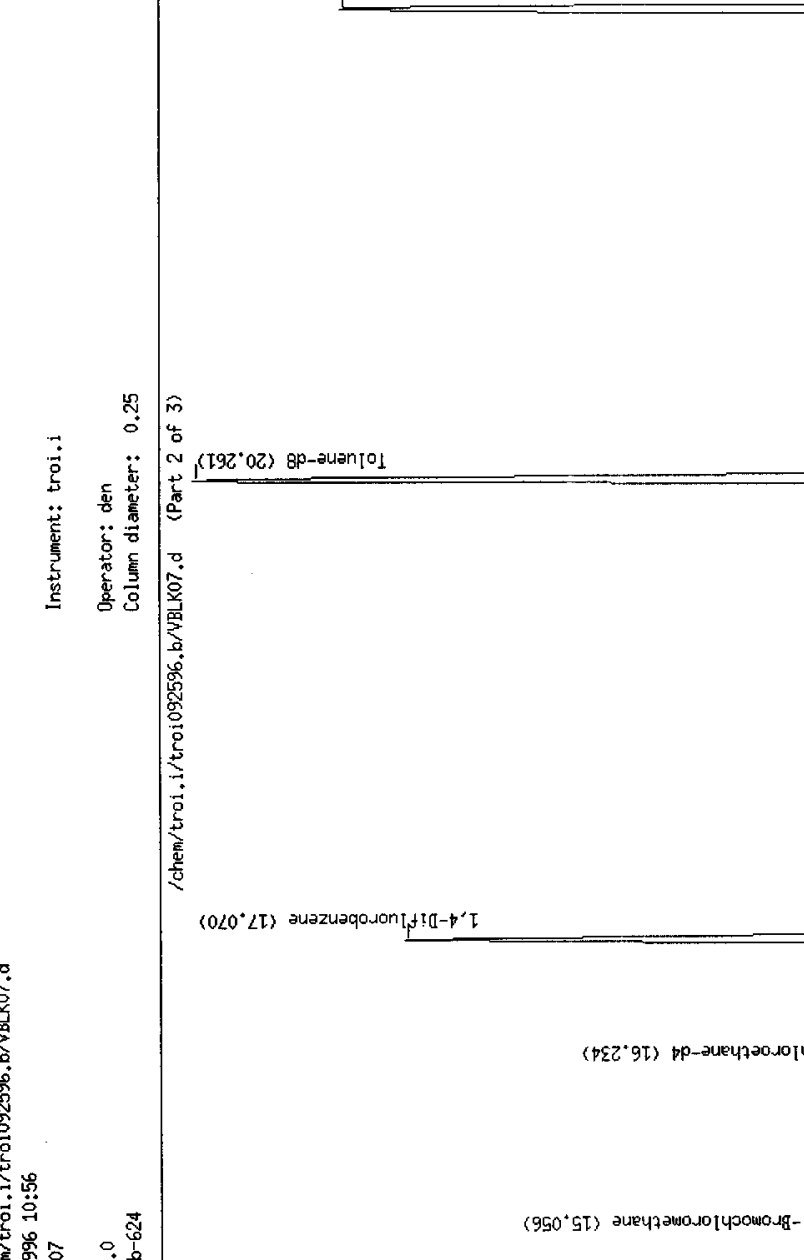
Date : 25-SEP-1996 10:56

Client ID: VBLKOT

Sample Info:

Purge Volume: 5.0

Column phase: $d b-624$

Instrument: troi i

Operator: den

Column dianeter: 0.25

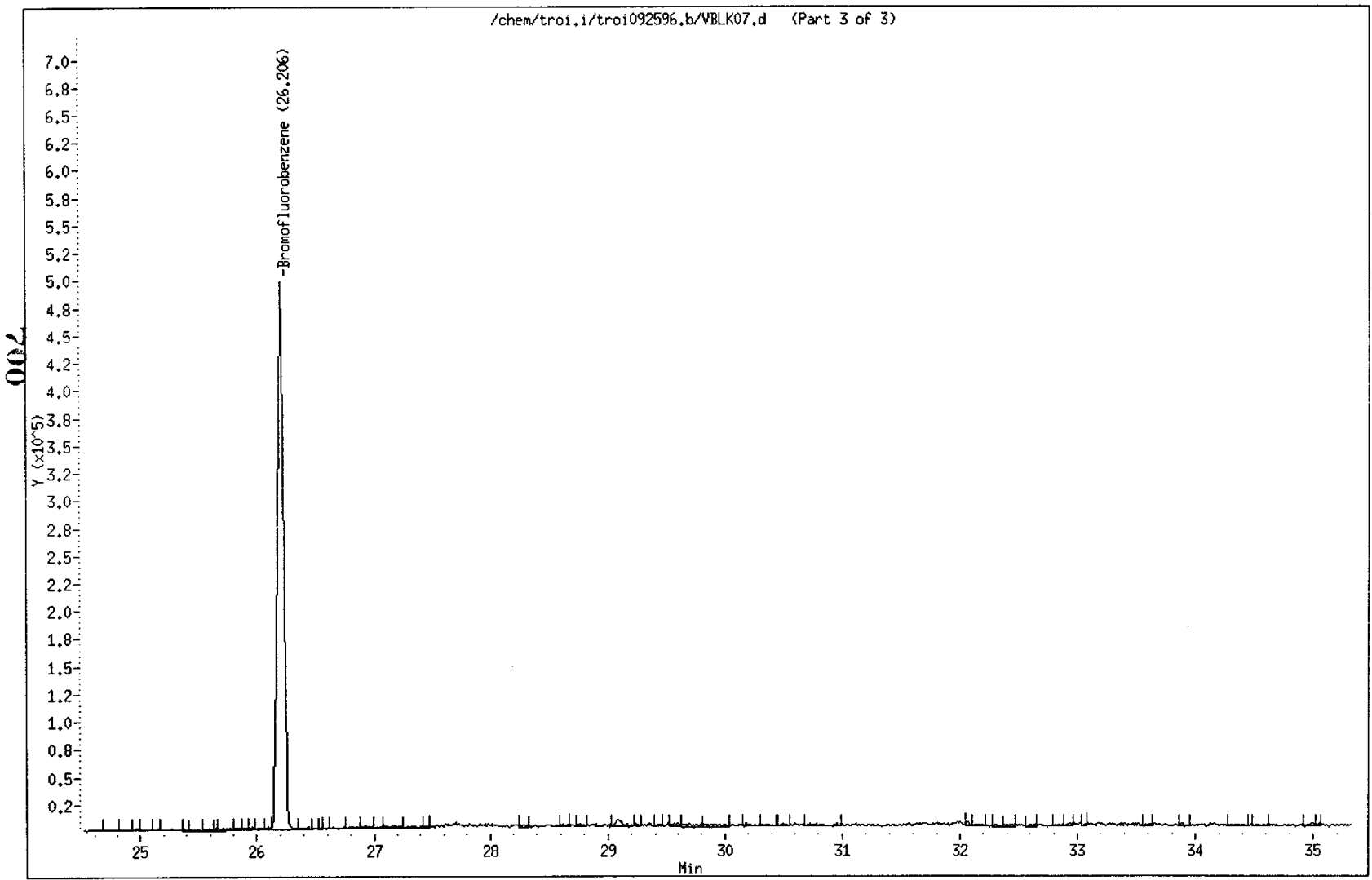


HNF SD-WM-DP. 202, REV.1

WORKBOOK PAGE: SPIKE5

\begin{tabular}{|c|c|c|c|}
\hline & \multirow{2}{*}{ SPIKE } & \\
\hline 1-129: LA-378 & $101(C-0), 103(C-0)$ & & \\
\hline 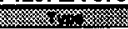 & GROSS WEIGHT & 30007 & \\
\hline SPK & TARE WEIGHT & 29865 & \\
\hline 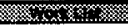 & MASS PRECIPITATE & 0.0142 & \\
\hline 12887 & SAMPLE + SPIKE I-129 $\mu \mathrm{Ci} /$ SAMPLE from GEA & 3.63E 4 & \\
\hline 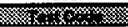 & SPIKE VOLUME in $\mathrm{mL}$ & 1000 & \\
\hline Ri120-01 & DILUTION FACTOR & 10000 & \\
\hline ming & DIGEST DILUTION FACTOR & $\$ 80 \%$ & \\
\hline LQUID & CARRIER FRACTIONAL RECOVERY & 0.4666 & \\
\hline 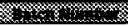 & SPIKE BOOK NUMBER & \$Bse & \\
\hline 98009327 & ACTIVITY OF SPIKE in $\mu \mathrm{Ci} / \mathrm{mL}$. & $7.86 E-04$ & \\
\hline 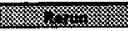 & COUNTING UNCERTAINITY & 129 & \\
\hline 0 & SAMPLE VOLUME USED IN SPIKE TEST in ML & 100000 & \\
\hline 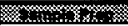 & SAMPLE l-129 $\mu \mathrm{CI} / \mathrm{SAMPLE}$ from GEA & $7.121 E-5$ & \& importid from sample page \\
\hline NOT LISTED & SAMPLE DILUTION FACTOR & 1.0000 & \&s imported from sample peage \\
\hline 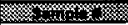 & SAMPLE VOLUME in $\mathrm{mL}$ & 1.0000 & \& importod from semplo pape \\
\hline $390 \mathrm{v} 000048$ & SAMPLE RECOVERY & 0.6082 & \& importod trom sample page \\
\hline 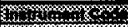 & COUNT TIME IN MINUTES & 100 & \\
\hline Hosting & & & \\
\hline $3 \times x+\sin x$ & & & \\
\hline 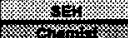 & One $\mathrm{mL}$ of the solution is equivalent to 0.0305 grams. & & \\
\hline xusto & Carrier Percent Recovery $=((w 2-w t 1)(100)) / 0.0305$ & & \\
\hline 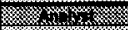 & 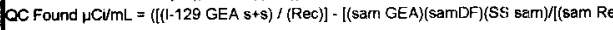 & c)(samss)]] * [(Spk DF/SS & spk)] \\
\hline 834 & QC Actual $\mu \mathrm{C} / \mathrm{mL}=$ Activity of Spike & & \\
\hline$x_{1}, x_{1}$ & \% Spike Recovery $=(O C$ Fourd $/ O C$ Actual $) * 100$ & & \\
\hline 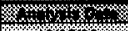 & & & \\
\hline 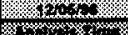 & & & \\
\hline mas & $\overline{C C}$ Actual in $\mu \mathrm{Ci} / \mathrm{mL}$ & $7.86 E-04$ & \\
\hline 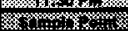 & ac Found in uci/mL & 6.23E-04 & \\
\hline 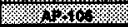 & Percent Spike Recovery & $79.3 \%$ & \\
\hline
\end{tabular}

Analyst: 24 Cat5


WORKBOOK PAGE: SP_DUP6

\begin{tabular}{|c|c|c|c|}
\hline \multicolumn{2}{|l|}{ VORK } & \multirow{2}{*}{ SPK-DUP } & \\
\hline 1-129: LA-378 & $-101(C-0), 103(C-0)$ & & \\
\hline 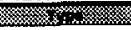 & GROSS WEIGHT & 20746 & \\
\hline SPK-DUP & TARE WEIGHT & 2.8609 & \\
\hline 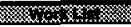 & MASS PRECIPITATE & 0.0178 & \\
\hline 12887 & SAMPLE + SPIKE I-129 $\mu$ Cl / SAMPLE from GEA & $4416-4$ & \\
\hline 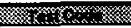 & SPIKE VOLUME in $\mathrm{mL}$ & 10000 & \\
\hline P1128-01 & DILLTION FACTOR & 10000 & \\
\hline xem & DIGEST DILUTION FACTOR & stis: & \\
\hline LIQU!D & CARRIER FRACTIONAL RECOVERY & 0.6836 & \\
\hline 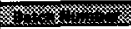 & SPIKE BOOK NUMBER & osesto & \\
\hline 0000327 & ACTIVITY OF SPIKE in $\mu \mathrm{Ci} / \mathrm{mL}$ & 7.86E-04 & \\
\hline 3. & COUNTING UNCERTAINITY & 1,18 & \\
\hline 0 & SAMPLE VOLUME USED IN SPIKE TEST in $\mathrm{mL}$ & 100000 & \\
\hline 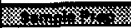 & SAMPLE I-129 $\mu \mathrm{Ci} / \mathrm{SAMPLE}$ from GEA & $7.121 E-6$ & \&< imporited from sumple papo \\
\hline NOT LISTED & SAMPLE DILUTION FACTOR. & 1.0000 & ex imporied from sample papos \\
\hline 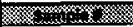 & SAMPLE VOLUME in $\mathrm{mL}$ & 1.0000 & « imported from sample pape \\
\hline $396 \mathrm{~V} 000048$ & SAMPLERECOVERY & 0.5082 & ex Imporied from sample papes \\
\hline (3) & COUNT TIME IN MINUTES & 100 & \\
\hline 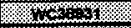 & & & \\
\hline 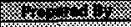 & & & \\
\hline 804 & One mL of the solution is equivalent to 0.0305 grams. & & \\
\hline $\operatorname{sen} 20$ & & & \\
\hline 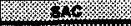 & Carrier Percent Recovery $=((w t 2-w 11)(100)) / 0.0305$ & & \\
\hline 40 & 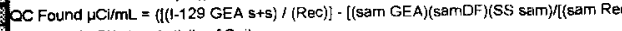 & c) $($ samSS $)]]) *[($ Spk DF/SS & spk)] \\
\hline (1) & QC Actual $\mu \mathrm{Cl} / \mathrm{mL}=$ Activity of Spike & & \\
\hline 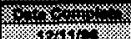 & * Spike Recovery $=(O C$ Found /OC Actual $) * 100$ & & \\
\hline Kathon- & & & \\
\hline 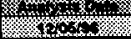 & & & \\
\hline 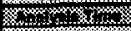 & & & \\
\hline 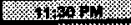 & ac Actual in $\mu \mathrm{C} / \mathrm{mL}$ & 7.88E-04 & \\
\hline 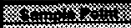 & OC Found in $\mu \mathrm{Ci} / \mathrm{mL}$ & $6.16 E-04$ & \\
\hline$\%$ \% $10 \%$ & Percent Spike Recovery & $78.4 \%$ & \\
\hline
\end{tabular}

\begin{tabular}{|lllll}
\hline Analyst: SLE & SLH & Date: & $12 / 11 / 96$ \\
\hline
\end{tabular}




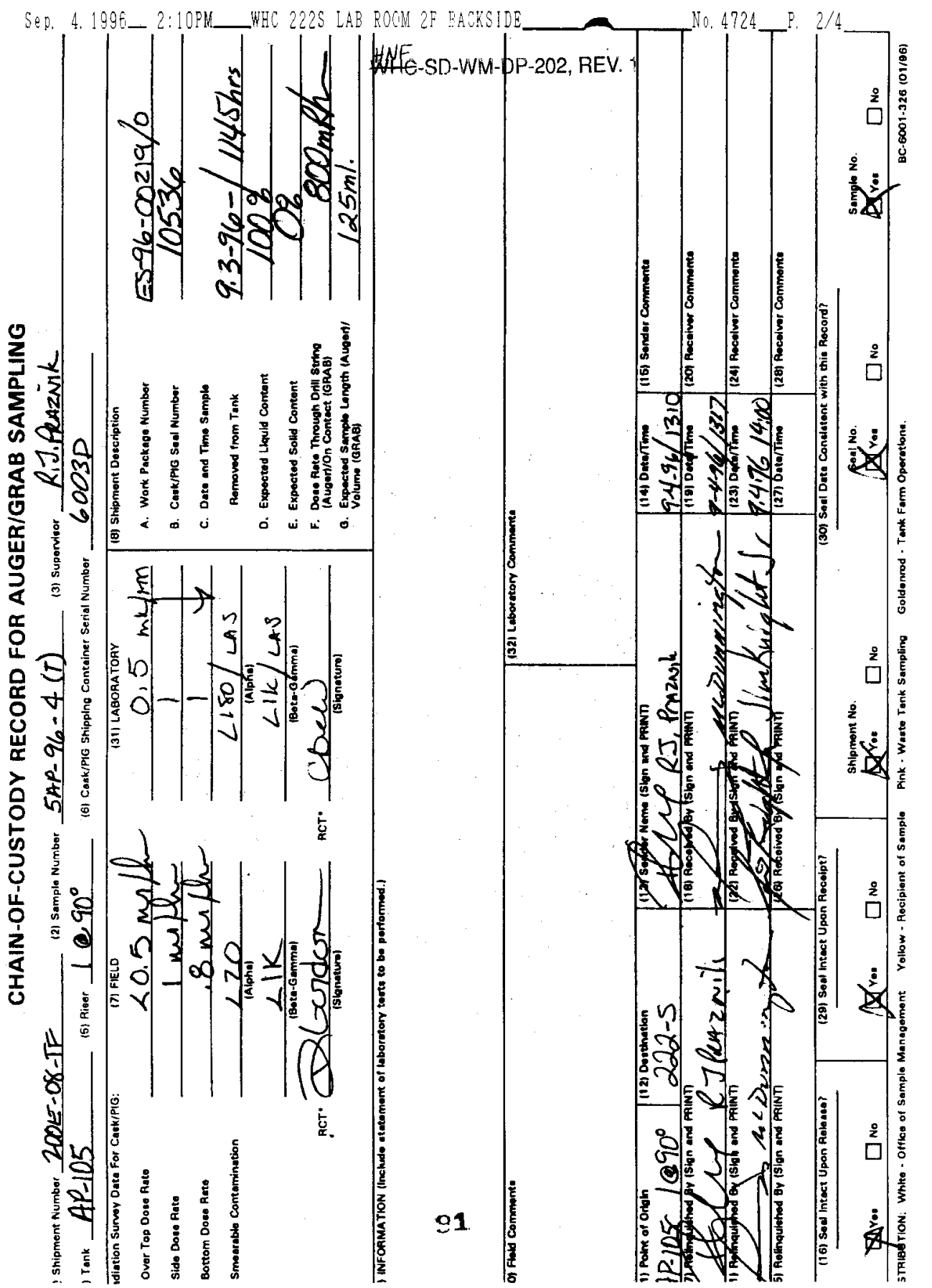


HNF

WORKBOOK PAGE: BLANK2

\begin{tabular}{|c|c|c|c|c|c|c|}
\hline$-220-101$ & Sr-89/90 : LA-220-101 (D- &, 102 & 3), 104( & $D-1)$ & & BLNK-PREP \\
\hline & DETECTOR NUMBER & & 12 & CARRIER ADDED in $\mathrm{mL}$ & (CVA) & 1.000 \\
\hline -PREF & TOTAL COUNTS & (TC) & 66 & GROSS WEIGHT & (W2) & 7.7455 \\
\hline & COUNT TIME in MINUTES & (CT) & 10 & TARE WEIGHT & (W1) & 7.6542 \\
\hline 3408 & BACKGROUND in cPm & (BKG) & 4.1 & NET WEIGHT & (W3) & 0.0913 \\
\hline & SAMPLE VOLUME in $\mathrm{mL}$ & (SS) & 2.500 & DELTA TIME (HOURS) & (DT) & 8.98 \\
\hline & DILUTION FACTOR & DF & 1 & & & \\
\hline & DIGEST DILUTION FACTOR & (DDF) & 50 & & & \\
\hline QUID & SAMPLE COUNT RATE & (Rs) & 2.50 & SR-90 EFFICIENCY FACTOR & (c1) & 0.4180 \\
\hline & CRITICAL LEVEL & (Lc) & 1.22 & Y-90 EFFICIENCY FACTOR & (C2) & 0.4660 \\
\hline & TIME OF SEPARATION & (ST) & $11: 45$ & $R \max$ & & N/A \\
\hline & DATE OF SEPARATION & (SD) & $12 / 08 / 96$ & DETECTION LIMIT & (Ld) & 2.53 \\
\hline 0 & TIME OF COUNT & (TOC) & $20: 44$ & Sr-89/90 CONC in $\mu \mathrm{Ci} / \mathrm{L}$ & & $5.3503 \mathrm{E}-02$ \\
\hline & DATE OF COUNT & (DOC) & $12 / 08 / 96$ & & & \\
\hline N/A & & & & & & \\
\hline & & & & & & \\
\hline & & & & & & \\
\hline & Sample Count Rate $(\mathrm{Rs})=(\mathrm{To}$ & tal Count & TC) / Coun & tt Time (CT)) - Background in cp & (BKG) & \\
\hline WB27811 & $\mathrm{Sr}-89 / 90$ CONC in $\mu \mathrm{Ci} / \mathrm{L}$ & Replace & with RMAX if & RS $<=L c$ and RS $>=0$ or Replace RS w & $L e$ if $R S<0$ & \\
\hline & $R^{\star} D^{\star} D D F /\left(\left(C 1+C 2^{*}(1-e\right.\right.$ to $t$ & e power & ((-natural I & $\left.\log 2)\left(64.2^{*} \mathrm{DT}()\right)\right)^{*} \mathrm{SS}^{*} \mathrm{REC}{ }^{*} 2220$ & & \\
\hline VAR & NOTE: 64.2 = Half Life for $Y-9$ & and $\mathrm{Re}$ & $=$ Fractiona & al Carrier Recovery ((W2-W1) / & (A* 0.1000$)$ & \\
\hline & Relative Counting Error $=($ The & Square & ot of $(T C+$ & BKG * CT) $\left./\left(T C-B K G{ }^{*} \mathrm{CT}\right)\right)^{\star} 1$ & & \\
\hline SAC & Percent Carrier Recovery $=(\mathrm{N}$ & et Weigh & Expected w & veight) * 100 & & \\
\hline & NOTE: Expected weight $=\mathrm{CV}$ & ${ }^{*} 0.1$ & & & & \\
\hline JDS & Detection Levels and Less The & In Values & re determin & hed from Procedure LA-508-002. & & \\
\hline $12 / 09 / 96$ & Delta Time (hours $)=\langle(\mathrm{DOC}-s$ & D) * 24) & (TOC - ST) & 100 & & \\
\hline & & & & & & DETECTION \\
\hline $12 / 08 / 96$ & Sr-89/90 CONCENTRATION & & & $5.35 E-05$ & $\mu \mathrm{Ci} / \mathrm{mL}$ & LEVEL \\
\hline & & & & & & \\
\hline 08:15 PM & RELATIVE COUNTING ERRO & & & $81.1 \%$ & & $5.42 \mathrm{E}-05$ \\
\hline & & & & & & $\mu \mathrm{Cl} / \mathrm{mL}$ \\
\hline P.105 & PERCENT CARRIER RECOV & ERY & & $91.3 \%$ & & \\
\hline
\end{tabular}

\begin{tabular}{|llll|}
\hline Analyst: & JDS & Date: \\
\hline Signature of Chemist: & SAC & Date: & DEC TI \\
\hline BLANK.WB1 REV 2.0 & 22010NML & Date & \\
\hline
\end{tabular}

